\title{
Causes and consequences of dilated cardiomyopathy
}

Citation for published version (APA):

Verdonschot, J. (2021). Causes and consequences of dilated cardiomyopathy: integrating genotype and phenotype to redefine disease diagnostics and therapeutics. [Doctoral Thesis, Maastricht University]. Gildeprint Drukkerijen. https://doi.org/10.26481/dis.20210108jv

Document status and date:

Published: 01/01/2021

DOI:

10.26481/dis.20210108jv

Document Version:

Publisher's PDF, also known as Version of record

\section{Please check the document version of this publication:}

- A submitted manuscript is the version of the article upon submission and before peer-review. There can be important differences between the submitted version and the official published version of record.

People interested in the research are advised to contact the author for the final version of the publication, or visit the DOI to the publisher's website.

- The final author version and the galley proof are versions of the publication after peer review.

- The final published version features the final layout of the paper including the volume, issue and page numbers.

Link to publication

\footnotetext{
General rights rights.

- You may freely distribute the URL identifying the publication in the public portal. please follow below link for the End User Agreement:

www.umlib.nl/taverne-license

Take down policy

If you believe that this document breaches copyright please contact us at:

repository@maastrichtuniversity.nl

providing details and we will investigate your claim.
}

Copyright and moral rights for the publications made accessible in the public portal are retained by the authors and/or other copyright owners and it is a condition of accessing publications that users recognise and abide by the legal requirements associated with these

- Users may download and print one copy of any publication from the public portal for the purpose of private study or research.

- You may not further distribute the material or use it for any profit-making activity or commercial gain

If the publication is distributed under the terms of Article $25 \mathrm{fa}$ of the Dutch Copyright Act, indicated by the "Taverne" license above, 


\section{CAUSES AND CONSEQUENCES OF DILATED CARDIOMYOPATHY}

Integrating genotype and phenotype to redefine disease diagnostics and therapeutics

PROEFSCHRIFT

ter verkrijging van de graad van doctor aan de Universiteit Maastricht, op gezag van de Rector Magnificus, Prof. Dr. Rianne M. Letschert

volgens het besluit van het College van Decanen,

in het openbaar te verdedigen

op vrijdag 8 januari 2021 om 12.00 uur

door

Job Antonius Jozef Verdonschot

Geboren op 3 november 1988 te Maasbracht-Beek 


\section{Promotores}

Prof. Dr. S.R.B. Heymans

Prof. Dr. H.G. Brunner

\section{Copromotores}

Dr. I.P.C. Krapels

Dr. M.R. Hazebroek

\section{Beoordelingscommissie}

Prof. Dr. P.G.A. Volders (voorzitter)

Prof. Dr. N. Hübner (Charité Medical School, Berlin, Germany)

Prof. Dr. J.P. van Tintelen (Universitair Medisch Centrum Utrecht)

Dr. D. Linz

Dr. M.W. Nabben

Financial support by the Dutch Heart Foundation for the publication of this thesis is gratefully acknowledged.

Financial support for the printing of this thesis as provided by the following sponsors is gratefully acknowledged:

Hart en vaat onderzoeksfonds Limburg | Boehringer-Ingelheim | TOMTEC Imaging Systems $\mathrm{GmbH}$ | Olink Proteomics AB.

\section{ISBN:}

978-94-6419-060-1

Graphic design:

Jelle Janssen

Layout by:

Job Verdonschot

\section{Printed by:}

Gildeprint te Enschede 
$-3-$ 


\section{CONTENTS}

Chapter 1

Chapter 2

Chapter 3

EDITORIAL

Chapter 4

Chapter 5

Chapter 6

EDITORIAL
General introduction

Page 7

\section{PART I - Clinical consequences of genetics}

Implications of genetic testing in dilated

Page 29

caradiomyopathy

Circ Genet Genom Precis Med; 2020

Titin cardiomyopathy leads to altered mitochondrial

Page 71 energetics, increased fibrosis and long-term life-threatening arrhytmias.

Eur Heart J; 2018

Titin cardiomyopathy: why we need to go big to understand the giant.

A mutation update for the FLNC gene in myopathies and cardiomyopathies

Page 111

Hum Mutat; 2020 (Editor's Choice)

Mutations in PDLIM5 are rare in dilated cadriomyopathy but are emerging as potential disease modifiers.

Mol Genet Genomic Med; 2020

Clinical phenotype and genotype associations with improvement in left ventricular function in dilated cardiomyopathy

Circ Heart Fail; 2018

Linking clinical parameters and genotype in dilated cardiomyopathy 


\section{PART II - Redefining disease diagnostics and therapeutics}

Chapter 7

Chapter 8

Chapter 9

Chapter 10

Chapter 11

Chapter 12

EDITORIAL

Chapter 13

Chapter 14
Distinct cardiac transcriptomic clustering in titin and lamin

A/C-associated dilated cardiomyopathy patients

Circulation; 2020

Phenotypic clustering of dilated cardiomyopathy patients high-

lights etiological differences

Eur Heart J; 2020

Cardiac inflammation impedes response to cardiac resynchronization therapy in patients with idiopathic dilated cardiomyopathy Circ Arrhytm Electrophysiol; 2020

Metabolic profiling associates with disease severity in nonischemic dilated cardiomyopathy

J Card Fail; 2020

Role of targeted therapy in dilated cardiomyopathy: the challenging road toward a personalized approach

J Am Heart Assoc; 2019

\section{PART III - Screening of asymptomatic family members}

Value of speckle tracking-based deformation analysis in the screening of asymptomatic dilated cardiomyopathy relatives

JACC Cardiovasc Imaging; 2020

The Value of Strain in Familial Dilated Cadriomyopathy Screening

General discussion

Summary, Nederlandse samenvatting, Valorization, Dankwoord, About the author, List of publications
Page 327

Page 195

Page 201

Page 227

Page 247

Page 271

Page 301

Page 341 


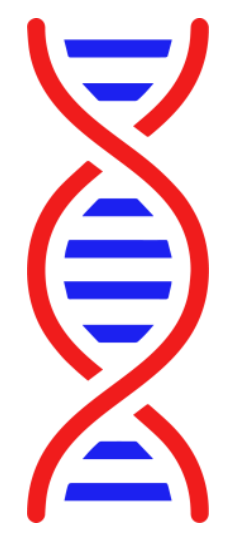

- 6 - 


\section{CHAPTER 1}

General introduction 


\subsection{The complexity and global burden of dilated cardiomyopathy}

Dilated cardiomyopathy (DCM) is defined as the presence of left ventricular or biventricular dilatation and systolic dysfunction in the absence of abnormal loading conditions (hypertension, valvular disease) or coronary artery disease sufficient to cause global systolic impairment ${ }^{1-4}$. The clinical definition of DCM is based solely on abnormal cardiac structure and function. DCM is treated as a monomorphic disease through standard heart failure therapy with the aim to restore cardiac function ${ }^{5,6}$. This approach does not capture the extensive etiological heterogeneity of DCM. Pathogenic gene variants, acquired diseases, and their combination can all lead to DCM ${ }^{7-9}$. A significant subgroup of DCM patients has an established genetic etiology ${ }^{10}$. A pathogenic gene variant is found in $\sim 20 \%$ of DCM patients, and genetic analysis is considered a first-tier test in dilated $D C M$ 7, 11, 12. Previous studies focused genetic testing largely on familial forms of DCM, in the absence of acquired diseases 13. Accumulating evidence suggests that an acquired or non-familial disease does not exclude a genetic predisposition ${ }^{14-16}$. There is currently no study performing genetic testing with standardized variant interpretation in an unselected, prospective cohort of DCM patients including both familial and non-familial forms- in which also acquired diseases are wellphenotyped. Also, it remains to be shown if therapy response and subsequent prognosis vary between etiological subgroups of DCM.

\section{1a Definitions, classifications and nomenclature of DCM}

Multiple attempts were made to include the etiological heterogeneity in definitions and classifications of DCM to better guide diagnostics and therapy. None reached global consensus due to financial, ethical and logistical considerations. The World Health Organization (WHO) traditionally defined cardiomyopathies purely by structural and hemodynamic phenotypes (Box 1). Subsequent cardiomyopathy classifications schemes from the American Heart Association (AHA) and the European Society of Cardiology (ESC) relied

\section{BOX 1: Historical aspects of dilated cardiomyopathy}

Origin of cardiomyopathy classification

The first classification system for cardiomyopathies originated from the World Health Organization (WHO) and was published in $1980^{18}{ }^{19}$. The system was completely based on the observed structural and hemodynamic phenotype: dilated, hypertrophic and restrictive cardiomyopathy. All cardiomyopathies were initially considered idiopathic, and therefore defined as 'heart muscle diseases of unknown cause'. The first pathogenic gene variants were described in the early 90s, thus the cardiomyopathy was no longer idiopathic in many patients. Cardiomyopathies were then redefined as "diseases of the myocardium associated with cardiac dysfunction" in the WHO 1996 classification system ${ }^{1}$. Arrhythmogenic right ventricular cardiomyopathy (ARVC) was introduced and the category 'unclassified cardiomyopathies' was added. Although DCM is purely defined as 'dilatation and impaired contraction of the left or both ventricles', the diverse etiologies are mentioned and introduced as separate entities 'specific cardiomyopathies'.

The evolving genetic knowledge eventually formed the basis of the most recent cardiomyopathy classifications of the American Heart Association (AHA) in 2006 and the European Society of Cardiology (ESC) in $2008^{3,17}$. The American statement made a distinction between primary and secondary cardiomyopathies, in which secondary implies that the cardiomyopathy is part of a multi-systemic disease such as sarcoidosis or amyloidosis. Primary cardiomyopathies were classified as 'Genetic', 'Mixed' and 'Acquired'. DCM was placed in the mixed category, although a genetic component was described as infrequent. DCM remained mainly idiopathic, as specific etiologies were classified as 'acquired' cardiomyopathies (e.g. toxic cardiomyopathy). The American classification was strongly based on etiology. The main goal of the European classification was to enhance clinical utility, and patients were grouped together based on their morphological and functional phenotypes (in line with the WHO recommendation). Further classification was mainly based on genetic and familial disease. The European classification acknowledged the potential of genetics to prevent disease progression and detection in family members. 


\section{Evolution of genetics in dilated cardiomyopathy (Figure 1)}

Dilated cardiomyopathy as familial disease

The first cohort study in the mid-80s detected a familial form in $6.5 \%$ of DCM patients ${ }^{20}$. Familial DCM was thought to be a rare clinical entity before this study. The first detailed clinical examination of DCM relatives was published in 1992, concluding that the true prevalence of familial DCM was more close to $20 \%{ }^{21}$. The high percentage of familial DCM showed the importance of clinical screening as many relatives of DCM patients can be asymptomatic despite having cardiac abnormalities. In 1998, familial DCM was clustered into subgroups based on their clinical presentation: [1] DCM with muscular dystrophy; [2] juvenile DCM with a rapid progressive course in male relatives without muscular dystrophy; [3] DCM with segmental hypokinesia of the left ventricle; [4] DCM with conduction defects; and [5] DCM with sensorineural hearing loss ${ }^{22}$. The patient clusters formed the basis for genotype-phenotype correlations: it was suggested that the clusters shared a genetic predisposition.

\section{Prevalence of familial and genetic dilated cardiomyopathy over time}

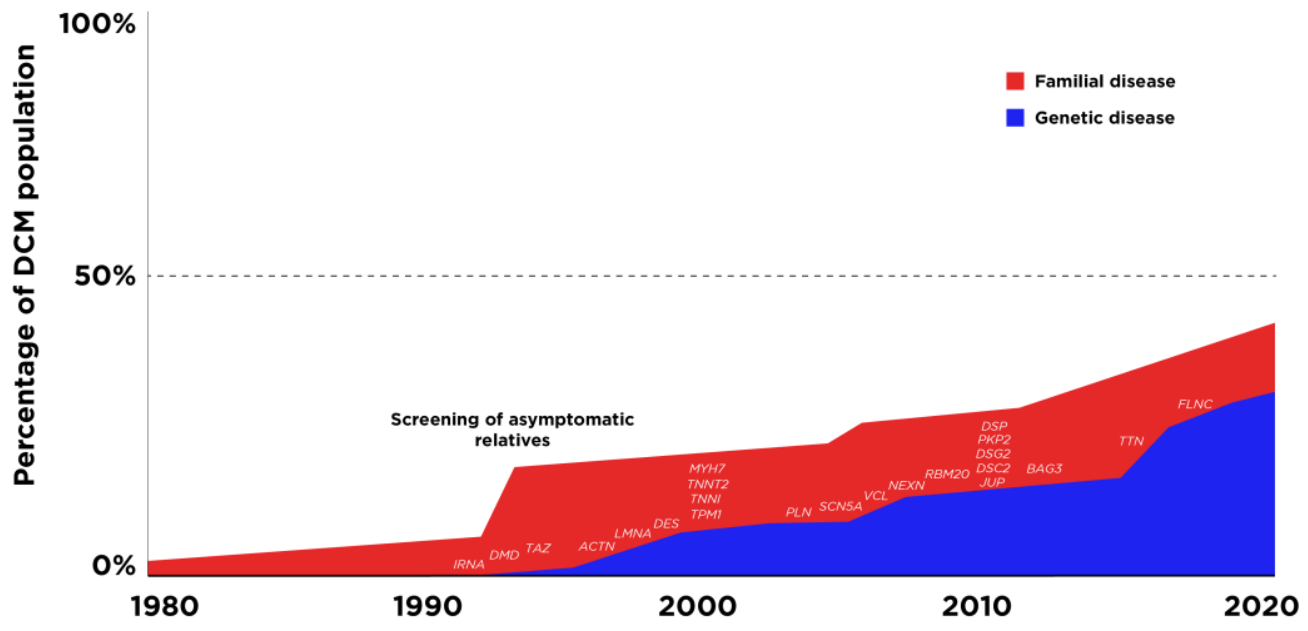

Figure 1. Prevalence of familial and genetic dilated cardiomyopathy over time. Timeline indicates the discovery of specific genes as monogenic cause of DCM

\section{Dilated cardiomyopathy as genetic disease}

-1990-2000: A more elaborate study showed that the genetic landscape of familial DCM is genetically phenotypically very heterogeneous ${ }^{23}$. In parallel, the first genes associated with DCM were discovered in the 90s: dystrophin $(D M D)^{24,25}$, desmin $(D E S)^{26}$, tafazzin $(T A Z)^{27}$, lamin $\mathrm{A} / \mathrm{C}(L M N A)^{28}$ and actin $(A C T C)^{29}$. The first discovered genes were related to the described patient clusters: an X-linked gene $(D M D)$ underlying a progressive disease in teenage boys with clinical signs of skeletal myopathy (cluster 2). The maternally inherited mitochondrial DNA (tRNA ${ }^{\text {Leu(UUR) }}$ ) related to a syndromic form of DCM (cluster 5) 30. Missense mutations in the Rod domain of LMNA were related to DCM with conduction defects and high disease penetrance (cluster 4$)^{28}$.

- 2000-2010: A genome-wide linkage study determined the sarcomere genes (MYH7, TNNT2, TNNI, TPM1) as important genetic contributors to DCM in $2000^{31}$. Many monogenic causes of DCM were discovered in the following decade (2000-2010). These genes form now an essential part of nowadays gene panels: sodium voltage- gated channel alpha subunit $5(S C N 5 A)^{32}$, RNA binding motif protein $20(R B M 20)^{33}$, the desmosomal genes (PKP2, DSP, DSG2, DSC2 and $J U P)^{34}$, phospholamban $(P L N)^{35}$, nexilin $(N E X N)^{36}$ and vinculin $(V C L)^{37}$.

- 2010-2020: In the last decade, novel methods of gene sequencing enabled the pursuit of monogenic causes of DCM: e.g. next generation sequencing (NGS) and genome-wide association studies (GWAS) led to the discovery of bcl2-associated athanogene $3(B A G 3)^{38}$. The discovery with most impact was the relation between truncating titin variants (TTNtV) and DCM ${ }^{39}$. Although the association was already suggested in 2002, NGS was necessary to properly sequence TTN in large populations, establishing TTNtV as the most important genetic contributor to DCM ${ }^{40}$. It has since been established that only a subset of TTNTV are pathogenic ${ }^{41}$. From the few 'new' DCM genes that were discovered in the following years, only the truncating variants in filamin $\mathrm{C}$ (FLNC) had a major impact on clinical genetic testing in $D_{C M}{ }^{42}$. It is possible that the finding of $F L N C$ concludes the era of finding major monogenic causes of DCM now that over 60 genes have been associated with the disease ${ }^{7}$. Instead of expanding the number of genes causative for DCM, we are now at the point that we have gathered enough knowledge to re- evaluate the true pathogenicity of variants in many genes discovered in the past decades, before advancing to complex polygenic and multifactorial models of DCM. 
more heavily on the distinction between genetic and non-genetic causes ${ }^{3,17}$. The main goal was to make the classification scheme practical for clinical utility by focusing on the morphological and functional phenotypes. Neither of the two classification schemes mention specific causes in the overall definition and classification of DCM.

The World Health Federation (WHF) endorsed a comprehensive classification system for cardiomyopathy called MOGE(S), which maintained the morpho-functional identification but a prominent role for the genetic basis and other etiologies of the disease ${ }^{2}$. It addressed 5 attributes of a cardiomyopathy, as shown in Box 2. The MOGE(S) classification attracted clinical attention, and was proven to be prognostic relevant as it could project geneenvironment interactions in DCM patients ${ }^{8,43}$. This classification system was not adopted in routine clinical practice, due to the complexity and expertise needed to properly use and apply the classification. The need for a concise but comprehensive classification system is still unmet, which will better represent the complex heterogeneity of DCM.

\section{BOX 2: MOGE(S) classification for cardiomyopathies ${ }^{2}$}

[M] stands for morpho-functional phenotype. In line with the previous classification systems, the classic division in dilated, hypertrophic, restrictive and arrhythmogenic phenotypes forms the basis of the nosology.

[0] denotes organ involvement. As the cardiomyopathy can be part of a multi-systemic disease

(a secondary cardiomyopathy according to the AHA classification), other organs can be involved in the disease.

[G] for genetic transmission, which can be autosomal dominant/recessive, X-linked, matrilineal,

but also unknown or sporadic.

[E] for etiology, in which $\mathrm{G}$ denotes genetic disease with a detected genetic variant.

Also non-genetic etiologies can be included under this nosologic descriptor.

[S] represents disease stage, including both the American College of Cardiology/American Heart Association stage (A to D) followed by New York Heart Association (NYHA) functional classes (I to IV).

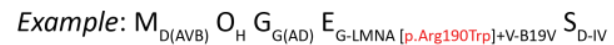

This describes a patient with dilated cardiomyopathy with an atrio-ventricular block (M) without any other organ involvement (O). It is described as a familial disease with an autosomal dominant inheritance pattern (G), genetic testing found a pathogenic LMNA variant and a parvovirus B19 (B19V) was found in the heart as etiologies (E).

The patient is currently in stage D with NYHA class IV.

\section{1b Epidemiology}

Although DCM was classified as a rare disease in the previous millennium, it is now regarded a globally common disease with a high burden. The change is due to an indisputable increase in disease prevalence and recognition, but also due to the changing definition and classification of DCM. A reliable population-based epidemiological study to estimate the prevalence and incidence of DCM is missing. An early small study in 1985 in Minnesota calculated a prevalence of $1: 2,500$ individuals ${ }^{44}$, but this seems to be severely underestimated ${ }^{45}$. Three approaches were undertaken to estimate the true prevalence of DCM $^{10}$ (Box 3). Current data support an approximate prevalence of 1:250-500. The Global Burden of Disease (GBD) Study estimated the global prevalence of cardiomyopathies around 2,5 million patients in $2015^{46}$, which is a significant increase compared to 2005 .

Overall, young DCM patients have a better prognosis compared to the older ischemic cardiomyopathy patients. The cardiomyopathy-associated mortality decreased over the period from 1990 to 2017, based on the latest values from the GBD Study ${ }^{51}$. An observed decrease of $6 \%$ deaths per 100,000 individuals was specifically noted in the Netherlands 
(Figure 2). The decrease in mortality was accompanied by decreased morbidity over the past decades. Survival of patients with DCM improved due to the increased use of heart failure therapy such as $\beta$-blockers and angiotensin-converting enzyme (ACE) inhibitors, implantable cardioverter defibrillators (ICD) and heart transplantation ${ }^{52,53}$. Despite the improvement of heart failure therapy, the effects are not well characterized for specific DCM subgroups, i.e. it is unknown how different etiologies and their interactions impact the response to generic heart failure therapy. DCM is now the leading cause of heart transplantation, the mortality and morbidity is still substantial and is no longer decreasing (Figure 2) ${ }^{54}$.

\section{BOX 3: Three approaches to estimate the true prevalence of DCM ${ }^{10}$}

1. Relating it to the revised prevalence of HCM with the Minnesota ratio

The ratio DCM:HCM in the Minnesota study was 2:1 ${ }^{44}$. The revised prevalence of HCM is $1: 500{ }^{47}$.

This would make the prevalence of DCM approximately 1:250.

2. Extrapolation from the USA heart failure population

About $30-50 \%$ of the systolic heart failure population is due to non-ischemic DCM, over 1 million individuals.

About $75 \%$ of the non-ischemic DCM is assigned as 'idiopathic' in the large clinical trials of heart failure,

leaving 750,000 individuals with DCM in the USA.

This would make the prevalence of DCM approximately 1:400.

3. Use the population-based prevalence of $L V$ dysfunction as a surrogate for DCM

2,042 asymptomatic individuals above the age of 45 years underwent cardiac screening between 1997 and $2000{ }^{48}$.

$6 \%$ had LV dysfunction ( $<50 \%$ ) and $2 \%$ had moderate-to-severe LV dysfunction ( $<40 \%) .0 .8 \%$ of the persons

between 45-54 years had moderate-to-severe LV dysfunction without an identifiable cause. If only half of these patients would have DCM, the prevalence would be approximately 1:250. These numbers were comparable to later studies in the MESA and Framingham study ${ }^{49,50}$.

\subsection{Impact of genetics for family members}

Finding a genetic etiology mostly does not change the therapeutic management of the index patient (Table 1) ${ }^{12,55,56}$. But it can have profound impact on family members ${ }^{12}$. Early detection of disease in asymptomatic family members is the principal outcome of genetic testing in DCM patients $4,5,11,57,58$. Severe cardiac dysfunction can be present in relatives without any obvious signs or symptoms for extended periods of time ${ }^{59}$. In fact, the first sign of DCM can be sudden cardiac death ${ }^{60}$. First-degree relatives of a DCM patient are always advised to undergo cardiac screening. The risk for developing DCM is determined by the genetic results of the proband and family member, and their age (Figure 3 ). Cardiac screening should be repeated over time in a frequency based on genetic status and age. Exact recommendations on the frequency and age of discontinuation for serial screening are mostly absent and conflicting, leaving the cardiologist to decide $4,5,11-13,57,58$.

Treatment can begin as soon as abnormal cardiac function manifests, preventing progression of the disease and the occurrence of sudden cardiac death. In order to make cardiac screening of relatives more effective, better knowledge of disease penetrance and more sensitive cardiac screening tools are both required. Better understanding of variant classification and disease penetrance of specific gene variants together with sensitive and specific cardiac screening tools, will help to improve cardiac screening of DCM relatives. 

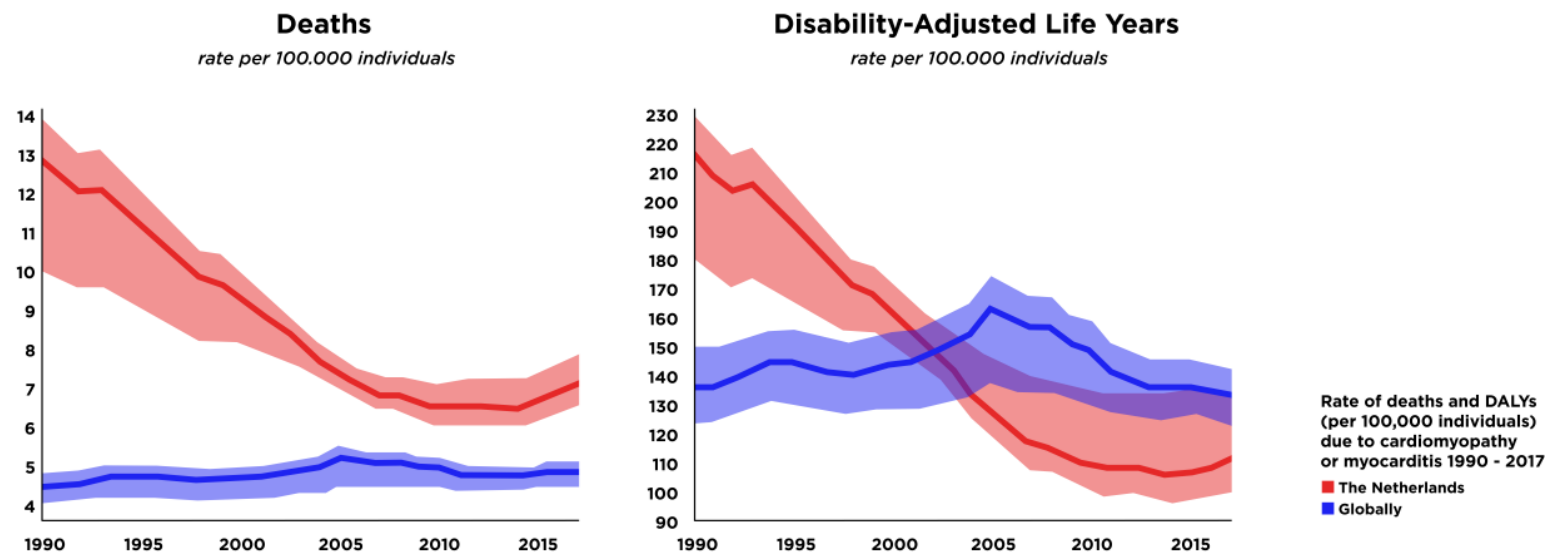

Figure 2. Mortality and morbidity of cardiomyopathy. The graphs show the rate of deaths and DALYs per 100,000 individuals. Data and figure from https://vizhub.healthdata.org/gbd-compare/. Accessed 27 May 2020.

\subsection{Diagnostic and therapeutic trajectories of a dilated cardiomyopathy patient}

The limited therapeutic consequences of genetics for the patient themselves are reflected in the guidelines: only the finding of a pathogenic LMNA variant will influence clinical-decision making for the cardiologist by decreasing the threshold for implantable cardioverterdefibrillator (ICD) therapy, because there is a high arrhythmic risk before left ventricular dysfunction in carriers (level B-non randomized; Table 1) ${ }^{5,11-13,55,56,58,61-66}$. Other genes (e.g. $D E S, S C N 5 A$ and $P L N$ ) are occasionally mentioned to have a prognostic role, but are not followed by clear recommendations due to insufficient genotype-phenotype knowledge; i.e. there is no convincing evidence of specific clinical consequences associated with pathogenic non-LMNA gene variants 5, 12,58,61. Observational evidence of a high arrhythmic risk is emerging for FLNC and TTN variants, irrespective of left ventricular dysfunction (level C) ${ }^{42,67}$.

Additional evidence of the clinical consequences of genes such as FLNC and TTN, will provide more insight into a specific phenotype on which therapy could be adjusted.

The recommended diagnostic and therapeutic trajectories of DCM patients do not deviate from the standard heart failure patient ${ }^{5,6}$. Although not implemented in the official guidelines, both the AHA and the ESC published position statements on specific trajectories for DCM patients $4,13,68$. The heterogeneity of DCM necessitates a deliberate analysis of every aspect of the individual and their family members as well as an integrated probabilistic interpretation of cardiac investigations.

\section{3a Diagnostics in dilated cardiomyopathy}

Elucidation of the etiology in DCM can directly influence clinical-decision making for the patient and their family members ${ }^{68}$. Accurate phenotyping in addition to cardiac characterization is therefore essential in DCM. The first step is to exclude coronary artery disease or valvular disease as cause for the observed cardiac dysfunction. The further diagnostic process is generally separated into a basic and second-level evaluation steps. The 
Table 1. Overview of European and American guidelines and statement regarding recommendations on genetics and clinical decision-making.

\begin{tabular}{|c|c|c|c|c|c|c|c|c|}
\hline Year & Association & Domain & $\begin{array}{l}\text { Type of } \\
\text { article }\end{array}$ & Subject & $\begin{array}{c}\text { Therapeutic consequences of } \\
\text { genetics }\end{array}$ & $\begin{array}{l}\text { Level of } \\
\text { evidence }\end{array}$ & Suggestions & Implicated genes \\
\hline 2010 & ESC & Genetics & $\begin{array}{l}\text { Position } \\
\text { Statement }\end{array}$ & $\begin{array}{l}\text { Genetic counseling } \\
\text { and testing in } \\
\text { cardiomyopathies }\end{array}$ & $\begin{array}{l}\text { Early prophylactic defibrillator } \\
\text { implantation owing to a high } \\
\text { risk of sudden death (for } \\
\text { example, in LMNA) }\end{array}$ & - & - & LMNA \\
\hline 2011 & $\mathrm{CCS} / \mathrm{CHRS}$ & Genetics & $\begin{array}{l}\text { Position } \\
\text { Statement }\end{array}$ & $\begin{array}{l}\text { Genetic testing in } \\
\text { cardiac arrhythmias } \\
\text { associated with } \\
\text { sudden cardiac death }\end{array}$ & - & - & - & - \\
\hline 2011 & HRS/EHRA & Genetics & $\begin{array}{c}\text { Expert } \\
\text { Consensus } \\
\text { Statement }\end{array}$ & $\begin{array}{l}\text { Genetic testing for } \\
\text { cardiomyopathies }\end{array}$ & $\begin{array}{l}\text { A therapeutic role for DCM } \\
\text { genetic testing exists for } \\
\text { patients with DCM and } \\
\text { prominent CCD that often } \\
\text { stems from mutations in } L M N A \text {. } \\
\text { Early or pre-emptive use of an } \\
\text { implantable cardiac defibrillator } \\
\text { has been advocated. }\end{array}$ & - & $\begin{array}{l}\text { There is no } \\
\text { prognostic role of } \\
\text { genetic testing for } \\
\text { DCM except for the } \\
\text { increased risk of } \\
\text { sudden cardiac death } \\
\text { in } L M N A \text { - and } D E S \text { - } \\
\text { mediated disease. }\end{array}$ & LMNA, DES \\
\hline 2012 & $\mathrm{AHA}$ & Genetics & $\begin{array}{c}\text { Policy } \\
\text { Statement }\end{array}$ & $\begin{array}{l}\text { Genetics and } \\
\text { cardiovascular } \\
\text { disease }\end{array}$ & - & - & - & - \\
\hline 2013 & $\mathrm{ACC} / \mathrm{AHA}$ & Heart Failure & Guideline & $\begin{array}{l}\text { Management of } \\
\text { heart failure }\end{array}$ & - & - & - & - \\
\hline 2015 & ESC & Arrhythmias & Guideline & $\begin{array}{l}\text { Management of } \\
\text { ventricular } \\
\text { arrhythmias and the } \\
\text { prevention of sudden } \\
\text { cardiac death }\end{array}$ & $\begin{array}{l}\text { An ICD should be considered in } \\
\text { patients with DCM and a } \\
\text { confirmed disease-causing } \\
\text { LMNA mutation and clinical risk } \\
\text { factors }\end{array}$ & Ila; B & - & $\angle M N A$ \\
\hline 2016 & ESC & Heart Failure & Guideline & $\begin{array}{l}\text { Diagnosis and } \\
\text { treatment of heart } \\
\text { failure }\end{array}$ & $\begin{array}{l}\text { Determination of the genotype } \\
\text { is important, since some forms } \\
\text { [e.g. mutations in } L M N A \text { and }\end{array}$ & - & - & LMNA, PLN \\
\hline
\end{tabular}




\begin{tabular}{|c|c|c|c|c|c|c|c|c|}
\hline & & & & & $\begin{array}{l}P L N] \text { are related to a poorer } \\
\text { prognosis. }\end{array}$ & & & \\
\hline 2016 & ESC & Heart Failure & $\begin{array}{l}\text { Position } \\
\text { Statement }\end{array}$ & $\begin{array}{l}\text { Revised definition of } \\
\text { dilated } \\
\text { cardiomyopathy and } \\
\text { hypokinetic non- } \\
\text { dilated } \\
\text { cardiomyopathy }\end{array}$ & $\begin{array}{l}\text { When a definite causative } \\
\text { LMNA mutation is identified, } \\
\text { early indication for primary } \\
\text { prevention by ICD implantation } \\
\text { should be considered (guided } \\
\text { by risk factors). }\end{array}$ & - & - & LMNA \\
\hline 2017 & $\begin{array}{l}\text { AHA/ACC/ } \\
\text { HRS }\end{array}$ & Arrhythmias & Guideline & $\begin{array}{l}\text { Management of } \\
\text { ventricular } \\
\text { arrhythmias and the } \\
\text { prevention of sudden } \\
\text { cardiac death }\end{array}$ & $\begin{array}{l}\text { In patients with DCM due to a } \\
\text { LMNA mutation who have } 2 \text { or } \\
\text { more risk factors (NSVT, LVEF } \\
<45 \% \text {, nonmissense mutation, } \\
\text { and male sex), an ICD can be } \\
\text { beneficial if meaningful survival } \\
\text { of greater than } 1 \text { year is } \\
\text { expected. }\end{array}$ & Ila; B-NR & - & LMNA \\
\hline 2018 & HFSA & Genetics & Guideline & $\begin{array}{l}\text { Genetic evaluation of } \\
\text { cardiomyopathy }\end{array}$ & $\begin{array}{l}\text { In patients with } \\
\text { cardiomyopathy and significant } \\
\text { arrhythmia or known risk of } \\
\text { arrhythmia, an ICD may be } \\
\text { considered before the LVEF falls } \\
\text { below } 35 \% \text {. }\end{array}$ & C & $\begin{array}{l}\text { Genetic DCM with } \\
\text { high arrhythmic risk } \\
\text { before LV } \\
\text { dysfunction should } \\
\text { be re-evaluated in } \\
\text { the therapeutic } \\
\text { guidelines }\end{array}$ & $\begin{array}{c}\text { LMNA DES; FLNC; } \\
\text { SCN5A }\end{array}$ \\
\hline 2019 & HFA & Heart Failure & $\begin{array}{l}\text { Position } \\
\text { Statement }\end{array}$ & $\begin{array}{l}\text { Heart failure in } \\
\text { cardiomyopathies }\end{array}$ & $\begin{array}{c}\text { Lower treshold for ICD therapy } \\
\text { in } L M N A \text { and } P L N \text {-associated } \\
\text { DCM. Eplerenone and } \\
\text { perindopril in DMD }\end{array}$ & - & $\begin{array}{l}\text { CRISPR/Cas9 gene } \\
\text { repair is promising } \\
\text { for the future } \\
\text { treatment of } \\
\text { monogenic primary } \\
\text { DCM }\end{array}$ & $\angle M N A ; P L N ; D M D$ \\
\hline
\end{tabular}

Abbreviations: ESC indicates European Society of Cardiology; CCS, Canadian Cardiovascular Society; CHRS, Canadian Heart Rhythm Society; HRS, Heart Rhythm Society; EHRA, European Heart Rhythm Society; AHA, American Heart Association; ACC, American College of Cardiology; HFSA, Heart Failure Society of America; HFA, Heart Failure Association; DCM, Dilated Cardiomyopathy; ICD, Implantable Cardioverter-Defibrillator; CCD, Cardiac Conduction Disease; NSVT, Non-Sustained Ventricular Tachycardia; $L V E F$, Left Ventricular Ejection Fraction. 


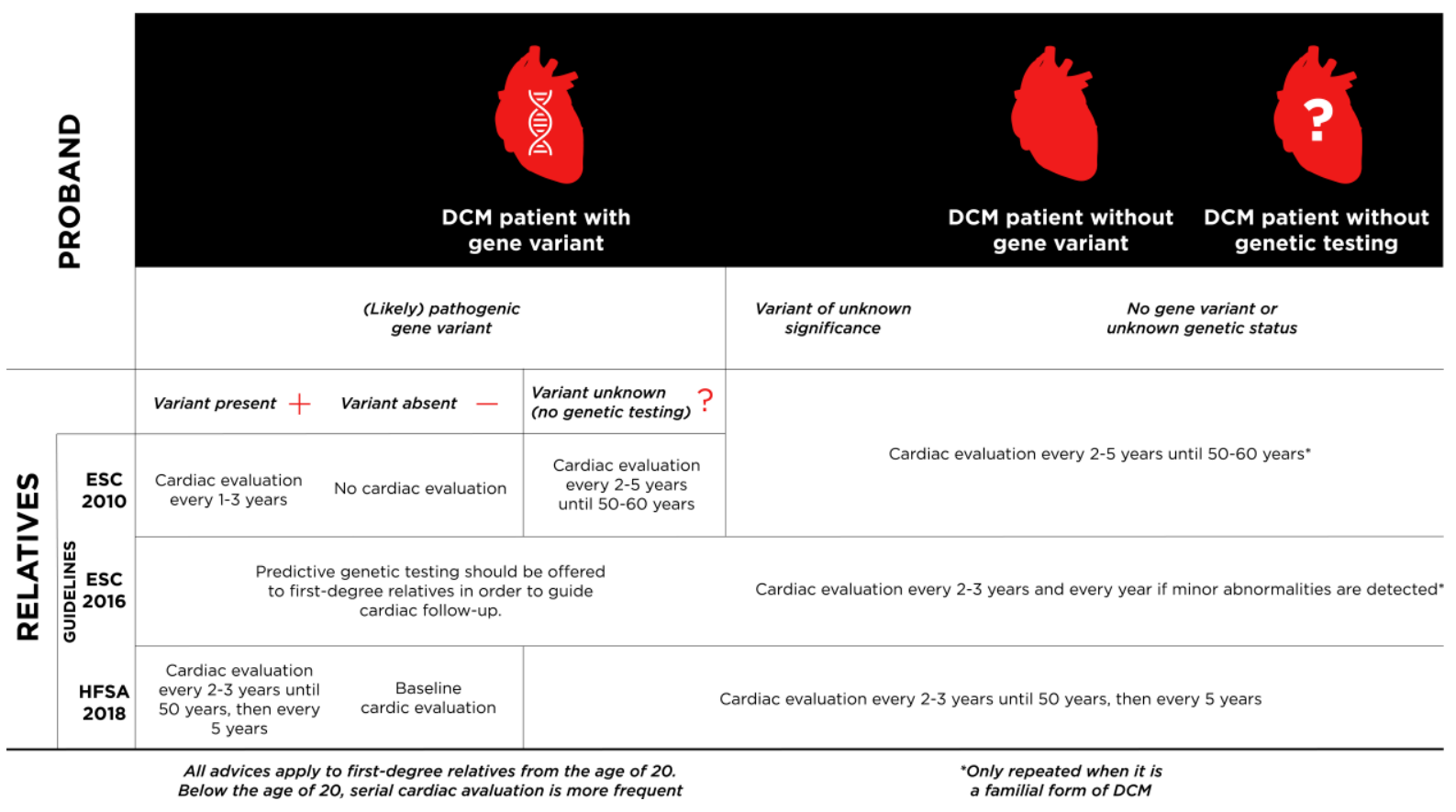

Figure 3. Cardiac screening advice for the 6 categories of first-degree DCM relatives, based on three guidelines. Genotype-phenotype correlations are not included in this overview (e.g. LMNA).

initial work-up is aimed to determine the baseline cardiac function and the identification of clinical clues which can be further characterized during second-level diagnostics (Figure 4). Second-level diagnostics require biochemical analysis (e.g. organ and non-organ specific serum autoantibodies, virus titers), more advanced imaging (e.g. cardiovascular magnetic resonance imaging) and endomyocardial biopsies (e.g. amyloid, and viral- or immunemediated inflammatory cardiomyopathy). Genetic testing is also considered a second-level test, as it is indicated after clinical clues for a familial or syndromic form of DCM ${ }^{68}$. A targeted two-level diagnostic approach has been proposed ${ }^{4,13}$, although a complete diagnostic workup would probably provide better insight in DCM etiology. Current indications for secondlevel diagnostics, including genetic testing, may be too strict. Genetic testing should be considered upon baseline evaluation in every DCM patient.

\section{$1.3 b$ Treatment of dilated cardiomyopathy}

Guidelines for the therapeutic trajectory of DCM follows that of general heart failure, based on optimal heart failure medication, including ICD and cardiac resynchronization therapy (CRT) ${ }^{5,69}$. The improved insight into the underlying etiologies of DCM has not yet let to implementation of etiology-directed treatment.

Despite advances in heart failure therapy, only half of the DCM patients respond adequately ${ }^{70}$. Possible explanations include: [1] the indications for therapy are not specific enough (i.e. the right patient is not getting the right medication) or [2] current therapies do not target pathways which drive disease progression (i.e. the right medication is not available). Most therapy is based on cardiac function (left ventricular ejection fraction) and symptoms of the patient (NYHA class). Stratification on these clinical parameters leads to the same treatment for a very heterogeneous patient population. Important knowledge-gaps that prevent a more targeted approach include: [1] insufficient knowledge of the molecular pathways of cardiomyocyte damage, inflammation or fibrosis, and [2] insufficient knowledge regarding the dynamics of these processes to allow optimal timing. 


\section{Dilated cardiomyopathy patient}

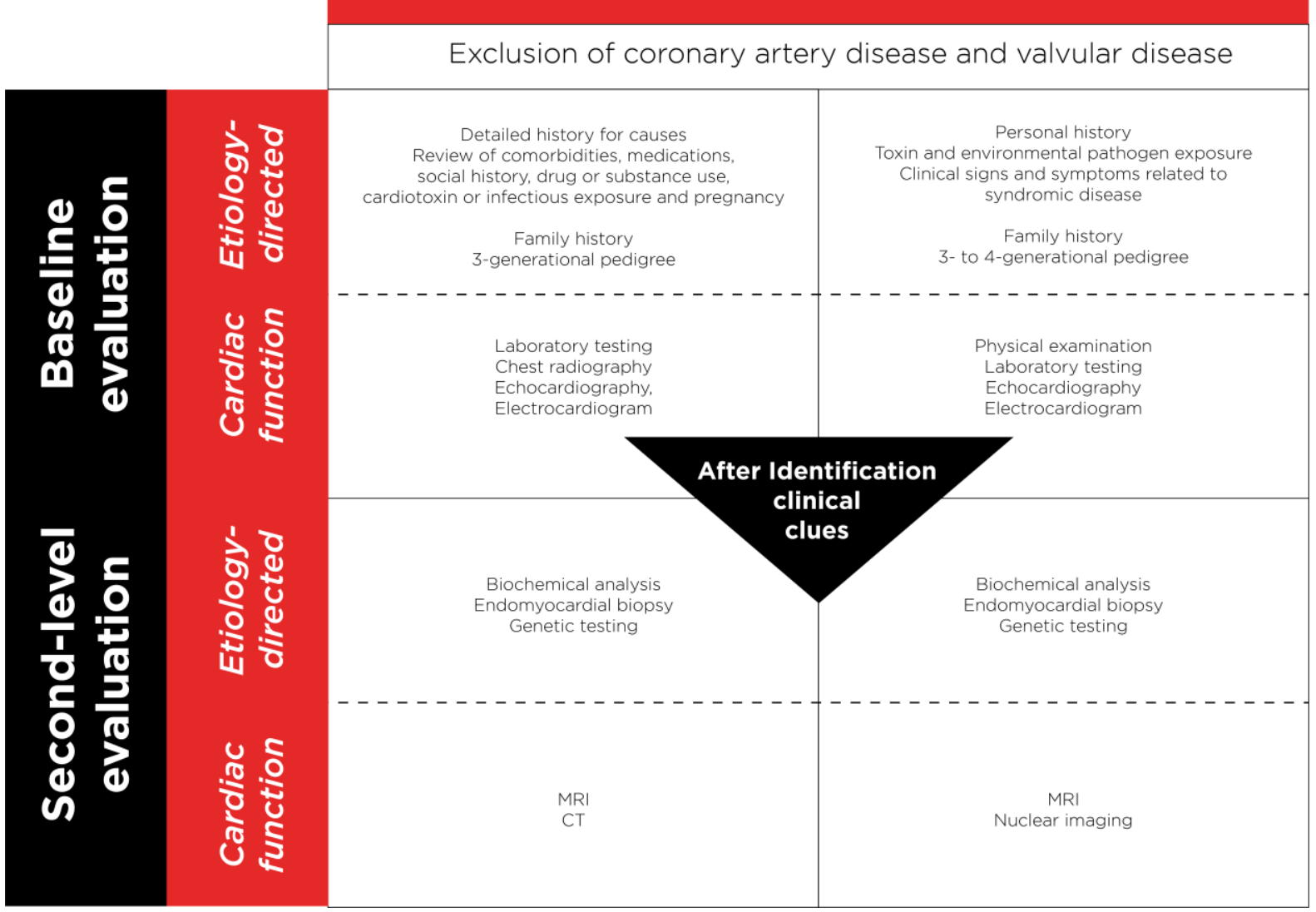

Figure 4. Diagnostic work-up for dilated cardiomyopathy patients as depicted in the DCM position statements of the American Heart Association (AHA) and European Society of Cardiology (ESC).

\section{3c Therapeutic consequences of genetics in dilated cardiomyopathy}

The main benefit of genetic testing is that it allows early detection of disease in asymptomatic relatives. With the exception of $L M N A$, finding a genetic factor does not change the therapeutic course for the DCM patient. Integration of genetic information into the therapeutic course of DCM patients is an important topic of ongoing research ${ }^{71}$. The potential influence of genetics on patient treatment can be broadly divided into three different approaches:

- Amelioration of the clinical consequences: based on genotype-phenotype knowledge

- Targeting of the molecular consequences at the cellular level: based on structural and intracellular knowledge

- Address the genetic perturbation: based on in-depth genetic and transcriptomic knowledge

No randomized-controlled trials evaluated clinical decision-making in genetic DCM. There is evidence from well-designed and executed non-randomized controlled studies (level Ila 
evidence) for early ICD implantation in DCM patients with a LMNA mutation who have two or more risk factors: non-sustained ventricular tachycardia, left ventricular ejection fraction below 45\%, non-missense mutation, and male sex (Table 1) ${ }^{55,56}$. Future studies on phenotypic and outcome characteristics in carriers of other specific gene variants may drive differentiated therapy. Gene therapy carries promise of curative potential in monogenic DCM, but remain far from clinical implementation ${ }^{72-74}$.

\subsection{Integration of genetics in guidelines concerning dilated cardiomyopathy}

Current guidelines on genetic investigation of DCM patients focus on familial disease in the absence of established environmental factors $4,13,55$. Screening recommendations for DCM relatives vary between guidelines, and can be non-specific. Treatment of DCM is stratified by clinical characteristics, and not by etiology. If genetic etiology impacts prognosis and treatment response, this could inspire more tailored therapeutic regimens. Thereby taking the variable penetrance and etiological fraction per gene into account, i.e. integrating genetic variants with comorbidities, acquired diseases and gender to evaluate the clinical course per patient. In the future, classification of DCM will likely integrate etiologies and phenotype to provide prognostically and therapeutically relevant subgroups.

\section{4a Indications for genetic testing in dilated cardiomyopathy}

Guidelines disagree on which patients are eligible for genetic testing (Figure 5). With the exception of the Heart Failure Society of America (HFSA), genetic testing is primarily recommended in familial forms of DCM. European guidelines also include patients with idiopathic DCM or the suspicion of a syndromic form, with a prerequisite of a family structure that permits segregation analysis ${ }^{11,13}$. American guidelines are in general more conservative, stating that genetic testing is only recommended in established or probable familial disease as determined by clinical testing of first-degree relatives $4,13,55,57$. Current indications reflect the evidence gathered from traditional studies performing genetic testing in familial DCM. The HFSA is the first to deviate from this dogma, but evidence from a study performing genetic testing in an unselected, prospective cohort of DCM could induce a change in recommendations and guidelines ${ }^{12}$.

\subsection{Maastricht registry of genetic dilated cardiomyopathy patients}

All research performed in this thesis was performed using the clinical and genetic data, and the biological material of the Maastricht Cardiomyopathy Registry and the Biobank Clinical Genetics Maastricht. The extensive data in both registries allowed us to investigate the outstanding questions in the field of genetics in DCM (Table 2).

The Maastricht Cardiomyopathy Registry is an ongoing prospective observational single center study, initiated in 2004 by the cardiology department of the Maastricht University Medical Centre (MUMC). The study protocol was approved by the local Institutional Review Board. All new patients over the age of 18 years old, diagnosed with DCM are approached for inclusion upon informed consent. To ensure inclusion of primary DCM patients, and no other cardiomyopathies according to the current classification schemes ${ }^{3,17}$, the following exclusion criteria are used: [1] significant coronary artery disease (stenosis $>50 \%$ ) using coronary angiography which is explanatory for the cardiomyopathy; [2] primary valvular disease (mitral regurgitation grade $\geq 3$, aortic regurgitation grade $\geq 2$, or aortic stenosis $<1 \mathrm{~cm}^{2}$ ); [3] hypertensive heart disease; [4] congenital heart disease; [5] (suspected) 
acute myocarditis; [6] (likely) diagnosis of arrhythmogenic right ventricular dysplasia; [7] (likely) diagnosis of hypertrophic cardiomyopathy; and [8] peripartum cardiomyopathy.

All patients undergo a complete diagnostic work-up, including baseline and secondlevel diagnostics (Figure 3). Baseline information and follow-up data are collected in an online database management system, including genetic testing results. Since 2012, every consecutive DCM patient is screened using a gene-panel consisting of 47 DCM-associated genes, named the core-panel. Patients who had genetic testing before 2012 were invited for retrospective genetic screening using the corepanel. DCM patients who had genetic testing using the corepanel, formed the patient cohort of the research performed in this thesis. Patient recruitment remains ongoing and currently $>1000$ DCM patients have been genetically screened using the corepanel in Maastricht. In addition, blood samples and cardiac biopsies are collected at baseline and stored in the central Maastricht Biobank.

Indication for genetic testing in dilated cardiomyopathy

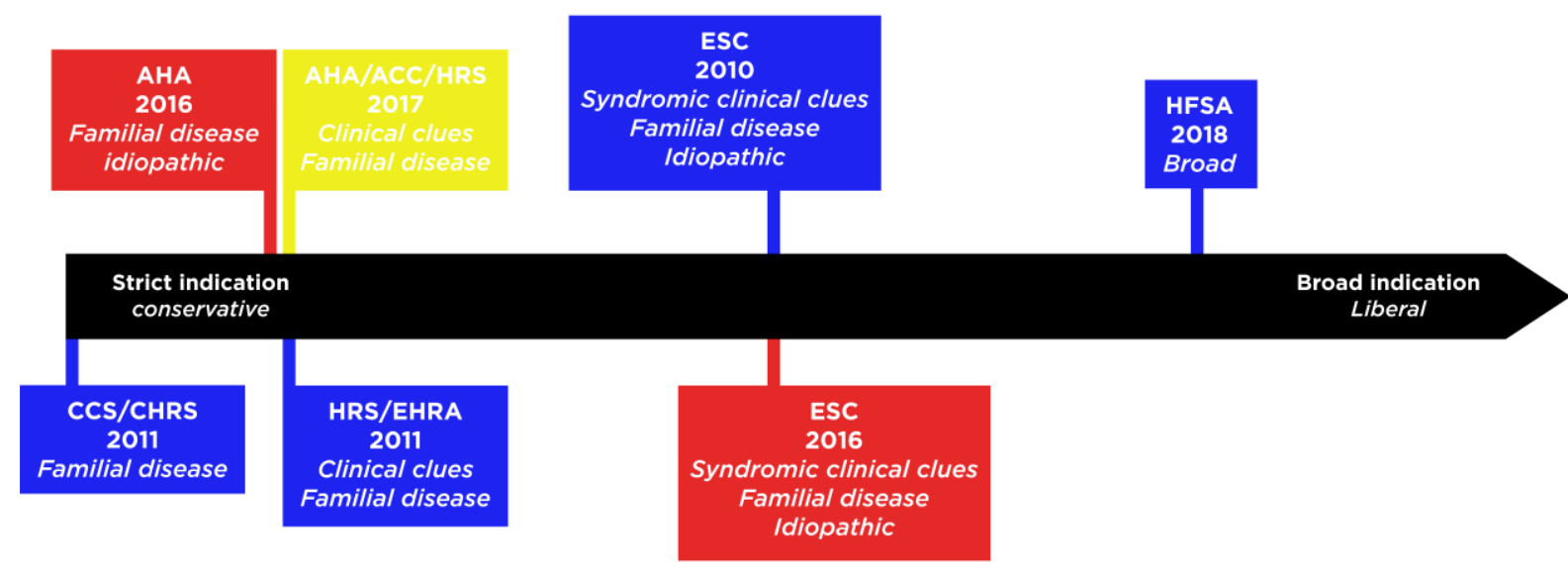

No recommendation on indication for genetic testing

Guideline/statement regarding

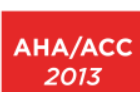

Genetics

Heart Failure

Arrhythmias

Figure 5. Indications for genetic testing among a variety of American and European guidelines and position statements regarding dilated cardiomyopathy.

\subsection{Scope of this dissertation}

The extensive possibilities to detect genetic variation in patients, require adequate interpretation and clinical translation to benefit the wellbeing of the patient and their relatives. Current therapeutic implications of genetics in DCM patients are limited. Increased knowledge on the clinical consequences of specific genes and the underlying pathobiology of genetic DCM, are two topics which could increase the therapeutic implications of genetic information.

This dissertation evaluates current indications for genetic testing by a systematical assessment of the genetic results in an unselected, prospective DCM cohort. In addition, it aims to contribute information on genotype-phenotype correlations of specific, highly prevalent DCM-associated genes. The genotype-phenotype knowledge is correlated to medical therapy and survival of the patient to investigate the prognostic role of genetic testing in DCM. We build on the current paradigm of DCM and address the limitations of current classification systems by utilizing novel approaches in patient classification and 
integrating the transcriptome with the genotype and phenotype. The resulting prognostic information and therapeutic recommendations per gene direct the treating cardiologist towards a personalized approach for every patient with (genetic) DCM.

Table 2. Overview of outstanding research questions in the field of genetics in dilated cardiomyopathy

\begin{tabular}{l} 
Outstanding research questions \\
The role of genetics in the clinical care of dilated cardiomyopathy \\
\hline Definition and classification of dilated cardiomyopathy \\
- Does it have therapeutic and prognostic value to integrate (genetic) etiology with cardiac function \\
and comorbidities to redefine DCM patient subgroups? \\
Clinical consequences of genetics in dilated cardiomyopathy \\
- Does therapy response and outcome vary between genetic subgroups of DCM? \\
- Which genes, besides LMNA, have unambiguous clinical consequences that are preventable or \\
Implementation of genetics in dilated cardiomyopathy guidelines \\
- $\quad$ What is the genetic yield of comprehensive genetic testing in an unselected DCM cohort? \\
- Does genetic testing at baseline evaluation provide additional value in patient and family care? \\
- Which pathogenic variant carriers will benefit the most from device implantation before left \\
ventricular dysfunction? \\
Screening of asymptomatic family members \\
- Are there differences in disease penetrance per gene, and which other (environmental) factors \\
determine disease development in gene carriers? \\
Does a family member with normal cardiac volumes has no cardiac dysfunction? Are there more \\
sensitive and specific screening tools to detect early disease?
\end{tabular}

\subsection{Outline of this thesis}

This thesis is subdivided into three parts, each of which addresses a different aspect of the implications of genetic testing in dilated cardiomyopathy (Table 3): Part I: clinical consequences of genetics, Part II: redefining disease diagnostics and therapeutics, and Part III: screening of asymptomatic family members.

Part I of this thesis will discuss genetic testing in patients with DCM, with a focus on genotypephenotype correlations. Chapter 2 re-evaluates the indications for genetic testing, the pathogenicity of variants in implicated genes, the prognostic value of genotype-phenotype correlations, and segregation analysis within families. Chapters 3 and $\mathbf{4}$ provide more in-depth discussion of the clinical and molecular consequences of truncating variants in titin (TTN) and filamin C (FLNC) respectively. Chapter 5 describes a novel bio-informatic method to find a genetic contributor to the DCM phenotype. Part I concludes with chapter 6 , which reports on the therapeutic response in relation to the genotype of DCM patients.

Part II integrates genotype and phenotype, together with transcriptomics and metabolomics, in order to unravel underlying molecular differences between DCM patients with different etiologies. Chapter 7 investigates the transcriptomic profile in the hearts of genetic DCM patients and associates it to their specific geno- and phenotype. These associations are put in a new perspective in chapter $\mathbf{8}$, in which a new method for classification of DCM patients is proposed, highlighting the importance of etiology integrated with cardiac function and comorbidities. Chapter 9 evaluates the predictive value of second-level diagnostics on the treatment response of DCM patients receiving cardiac resynchronization therapy. Chapter 10 shows how the clinical presentation of DCM is reflected in the plasma metabolites. Chapter 
11 concludes Part II by summarizing results from published clinical trials pursuing a more targeted approach for therapy in DCM.

Part III describes the implications of genetic testing for relatives of a DCM patient. Chapter 12 describes an alternative clinical method to detect the phenotype in asymptomatic relatives of DCM patients, who are advised to undergo frequent cardiac screening. Chapter 13 contains a general discussion of the results described in this thesis and provides an outlook for the future of genetics in DCM.

Table 3. Overview of the general introduction and related thesis chapters on the specific introduction sections.

\section{Introduction section}

1.1a Definitions, classifications and nomenclature of DCM

1.1b Epidemiology

1.2 Impact of genetics for family members

1.3a Diagnostics in dilated cardiomyopathy

1.3b Treatment of dilated cardiomyopathy

1.3c Therapeutic consequences of genetics in dilated cardiomyopathy

1.4a Indications for genetic testing in dilated cardiomyopathy

1.5 Maastricht registry of genetic dilated cardiomyopathy patients
Related thesis chapter

7-9 and 11

5

2 and 12

$2,9,10$ and 12

3, 6-10 and 11

2-4 and 6

2-10 


\section{REFERENCES}

1. Richardson $\mathrm{P}$, McKenna $\mathrm{W}$, Bristow $\mathrm{M}$, et al. Report of the 1995 World Health Organization/International Society and Federation of Cardiology Task Force on the Definition and Classification of cardiomyopathies. Circulation. 1996;93:841-842.

2. Arbustini E, Narula N, Tavazzi L, et al. The MOGE(S) classification of cardiomyopathy for clinicians. J Am Coll Cardiol. 2014;64:304-18.

3. Elliott $P$, Andersson $B$, Arbustini $E$, et al. Classification of the cardiomyopathies: a position statement from the European Society Of Cardiology Working Group on Myocardial and Pericardial Diseases. Eur Heart J. 2008;29:270-6.

4. Bozkurt B, Colvin M, Cook J, et al. Current Diagnostic and Treatment Strategies for Specific Dilated Cardiomyopathies: A Scientific Statement From the American Heart Association. Circulation. 2016;134:e579-e646.

5. Ponikowski P, Voors AA, Anker SD, et al. 2016 ESC Guidelines for the diagnosis and treatment of acute and chronic heart failure: The Task Force for the diagnosis and treatment of acute and chronic heart failure of the European Society of Cardiology (ESC)Developed with the special contribution of the Heart Failure Association (HFA) of the ESC. Eur Heart J. 2016;37:2129-200.

6. Yancy CW, Jessup M, Bozkurt B, et al. 2013 ACCF/AHA guideline for the management of heart failure: a report of the American College of Cardiology Foundation/American Heart Association Task Force on Practice Guidelines. $J$ Am Coll Cardiol. 2013;62:e147-239.

7. Japp AG, Gulati A, Cook SA, et al. The Diagnosis and Evaluation of Dilated Cardiomyopathy. J Am Coll Cardiol. 2016;67:2996-3010.

8. Hazebroek MR, Moors S, Dennert R, et al. Prognostic Relevance of Gene-Environment Interactions in Patients With Dilated Cardiomyopathy: Applying the MOGE(S) Classification. J Am Coll Cardiol. 2015;66:131323.

9. Piran S, Liu P, Morales A, et al. Where genome meets phenome: rationale for integrating genetic and protein biomarkers in the diagnosis and management of dilated cardiomyopathy and heart failure. J Am Coll Cardiol. 2012;60:283-9.

10. Hershberger RE, Hedges DJ and Morales A. Dilated cardiomyopathy: the complexity of a diverse genetic architecture. Nature reviews Cardiology. 2013;10:531-47.

11. Charron P, Arad M, Arbustini E, et al. Genetic counselling and testing in cardiomyopathies: a position statement of the European Society of Cardiology Working Group on Myocardial and
Pericardial Diseases. Eur Heart J. 2010;31:271526.

12. Hershberger RE, Givertz MM, Ho CY, et al. Genetic Evaluation of Cardiomyopathy-A Heart Failure Society of America Practice Guideline. J Card Fail. 2018;24:281-302.

13. Pinto YM, Elliott PM, Arbustini E, et al. Proposal for a revised definition of dilated cardiomyopathy, hypokinetic non-dilated cardiomyopathy, and its implications for clinical practice: a position statement of the ESC working group on myocardial and pericardial diseases. Eur Heart J. 2016;37:1850-8.

14. Ware JS, Amor-Salamanca A, Tayal U, et al. Genetic Etiology for Alcohol-Induced Cardiac Toxicity. J Am Coll Cardiol. 2018;71:2293-2302.

15. Ware JS, Li J, Mazaika E, et al. Shared Genetic Predisposition in Peripartum and Dilated Cardiomyopathies. N Engl J Med. 2016;374:23341.

16. Garcia-Pavia P, Kim Y, Restrepo-Cordoba MA, et al. Genetic Variants Associated With Cancer Therapy-Induced Cardiomyopathy. Circulation. 2019;140:31-41.

17. Maron BJ, Towbin JA, Thiene G, et al. Contemporary definitions and classification of the cardiomyopathies: an American Heart Association Scientific Statement from the Council on Clinical Cardiology, Heart Failure and Transplantation Committee; Quality of Care and Outcomes Research and Functional Genomics and Translational Biology Interdisciplinary Working Groups; and Council on Epidemiology and Prevention. Circulation. 2006;113:1807-16.

18. McKenna WJ, Maron BJ and Thiene G. Classification, Epidemiology, and Global Burden of Cardiomyopathies. Circulation research. 2017;121:722-730.

19. Report of the WHO/ISFC task force on the definition and classification of cardiomyopathies. British heart journal. 1980;44:672-3.

20. Michels VV, Driscoll DJ and Miller FA, Jr. Familial aggregation of idiopathic dilated cardiomyopathy. Am J Cardiol. 1985;55:1232-3.

21. Michels VV, Moll PP, Miller FA, et al. The frequency of familial dilated cardiomyopathy in a series of patients with idiopathic dilated cardiomyopathy. N Engl J Med. 1992;326:77-82.

22. Grunig $E$, Tasman JA, Kucherer $H$, et al. Frequency and phenotypes of familial dilated cardiomyopathy. J Am Coll Cardiol. 1998;31:18694.

23. Mestroni L, Rocco C, Gregori D, et al. Familial dilated cardiomyopathy: evidence for genetic and phenotypic heterogeneity. Heart Muscle Disease Study Group. J Am Coll Cardiol. 1999;34:181-90. 
24. Towbin JA, Hejtmancik JF, Brink P, et al. X-linked dilated cardiomyopathy. Molecular genetic evidence of linkage to the Duchenne muscular dystrophy (dystrophin) gene at the Xp21 locus. Circulation. 1993;87:1854-65.

25. Muntoni F, Cau M, Ganau A, et al. Brief report: deletion of the dystrophin muscle-promoter region associated with X-linked dilated cardiomyopathy. N Engl J Med. 1993;329:921-5.

26. Li D, Tapscoft T, Gonzalez O, et al. Desmin mutation responsible for idiopathic dilated cardiomyopathy. Circulation. 1999;100:461-4.

27. Bione S, D'Adamo P, Maestrini E, et al. A novel Xlinked gene, G4.5. is responsible for Barth syndrome. Nature genetics. 1996;12:385-9.

28. Fatkin D, MacRae C, Sasaki T, et al. Missense mutations in the rod domain of the lamin $A / C$ gene as causes of dilated cardiomyopathy and conduction-system disease. $N$ Engl J Med. 1999;341:1715-24.

29. Olson TM, Michels VV, Thibodeau SN, et al. Actin mutations in dilated cardiomyopathy, a heritable form of heart failure. Science (New York, NY). 1998;280:750-2.

30. Zeviani M, Gellera C, Antozzi C, et al. Maternally inherited myopathy and cardiomyopathy: association with mutation in mitochondrial DNA tRNA(Leu)(UUR). Lancet (London, England). 1991;338:143-7.

31. Kamisago $M$, Sharma SD, DePalma SR, et al. Mutations in sarcomere protein genes as a cause of dilated cardiomyopathy. $N$ Engl J Med. 2000;343:1688-96.

32. McNair WP, Ku L, Taylor MR, et al. SCN5A mutation associated with dilated cardiomyopathy, conduction disorder, and arrhythmia. Circulation. 2004;110:2163-7.

33. Brauch KM, Karst ML, Herron KJ, et al. Mutations in ribonucleic acid binding protein gene cause familial dilated cardiomyopathy. J Am Coll Cardiol. 2009;54:930-41.

34. Elliott P, O'Mahony C, Syrris P, et al. Prevalence of desmosomal protein gene mutations in patients with dilated cardiomyopathy. Circulation Cardiovascular genetics. 2010;3:31422.

35. Schmitt JP, Kamisago M, Asahi M, et al. Dilated cardiomyopathy and heart failure caused by a mutation in phospholamban. Science (New York, NY). 2003;299:1410-3.

36. Hassel D, Dahme T, Erdmann J, et al. Nexilin mutations destabilize cardiac Z-disks and lead to dilated cardiomyopathy. Nat Med. 2009;15:1281-8.

37. Zemljic-Harpf AE, Miller JC, Henderson SA, et al. Cardiac-myocyte-specific excision of the vinculin gene disrupts cellular junctions, causing sudden death or dilated cardiomyopathy. Molecular and cellular biology. 2007;27:7522-37.

38. Villard E, Perret C, Gary F, et al. A genome-wide association study identifies two loci associated with heart failure due to dilated cardiomyopathy. Eur Heart J. 2011;32:1065-76.

39. Herman DS, Lam L, Taylor MR, et al. Truncations of titin causing dilated cardiomyopathy. $N$ Engl J Med. 2012;366:619-28.

40. Gerull B, Gramlich M, Atherton J, et al. Mutations of TTN, encoding the giant muscle filament titin, cause familial dilated cardiomyopathy. Nature genetics. 2002;30:201-4.

41. Roberts AM, Ware JS, Herman DS, et al. Integrated allelic, transcriptional, and phenomic dissection of the cardiac effects of titin truncations in health and disease. Science translational medicine. 2015;7:270ra6.

42. Ortiz-Genga MF, Cuenca $S$, Dal Ferro $M$, et al. Truncating FLNC Mutations Are Associated With High-Risk Dilated and Arrhythmogenic Cardiomyopathies. J Am Coll Cardiol. 2016;68:2440-2451.

43. Dec GW and Arbustini E. Utilizing the MOGE(S) Classification for Predicting Prognosis in Dilated Cardiomyopathy. J Am Coll Cardiol. 2015;66:1324-6.

44. Codd MB, Sugrue DD, Gersh BJ, et al. Epidemiology of idiopathic dilated and hypertrophic cardiomyopathy. A populationbased study in Olmsted County, Minnesota, 1975-1984. Circulation. 1989;80:564-72.

45. Hershberger RE, Morales A and Siegfried JD. Clinical and genetic issues in dilated cardiomyopathy: a review for genetics professionals. Genetics in medicine : official journal of the American College of Medical Genetics. 2010;12:655-67.

46. Global, regional, and national incidence, prevalence, and years lived with disability for 310 diseases and injuries, 1990-2015: a systematic analysis for the Global Burden of Disease Study 2015. Lancet (London, England). 2016;388:15451602.

47. Maron BJ, Gardin JM, Flack JM, et al. Prevalence of hypertrophic cardiomyopathy in a general population of young adults. Echocardiographic analysis of 4111 subjects in the CARDIA Study. Coronary Artery Risk Development in (Young) Adults. Circulation. 1995;92:785-9.

48. Redfield MM, Jacobsen SJ, Burnett JC, Jr., et al. Burden of systolic and diastolic ventricular dysfunction in the community: appreciating the scope of the heart failure epidemic. Jama. 2003;289:194-202.

49. Wang TJ, Evans JC, Benjamin EJ, et al. Natural history of asymptomatic left ventricular systolic 
dysfunction in the community. Circulation. 2003;108:977-82.

50. Yeboah J, Rodriguez CJ, Stacey B, et al. Prognosis of individuals with asymptomatic left ventricular systolic dysfunction in the multi-ethnic study of atherosclerosis (MESA). Circulation. 2012;126:2713-9.

51. Global, regional, and national incidence, prevalence, and years lived with disability for 354 diseases and injuries for 195 countries and territories, 1990-2017: a systematic analysis for the Global Burden of Disease Study 2017. Lancet (London, England). 2018;392:1789-1858.

52. Castelli G, Fornaro A, Ciaccheri $M$, et al. Improving survival rates of patients with idiopathic dilated cardiomyopathy in Tuscany over 3 decades: impact of evidence-based management. Circulation Heart failure. 2013;6:913-21.

53. Schultheiss HP, Fairweather D, Caforio ALP, et al. Dilated cardiomyopathy. Nature reviews Disease primers. 2019;5:32.

54. Lund LH, Khush KK, Cherikh WS, et al. The Registry of the International Society for Heart and Lung Transplantation: Thirty-fourth Adult Heart Transplantation Report-2017; Focus Theme: Allograft ischemic time. The Journal of heart and lung transplantation : the official publication of the International Society for Heart Transplantation. 2017;36:1037-1046.

55. Al-Khatib SM, Stevenson WG, Ackerman MJ, et al. 2017 AHA/ACC/HRS guideline for management of patients with ventricular arrhythmias and the prevention of sudden cardiac death: Executive summary: A Report of the American College of Cardiology/American Heart Association Task Force on Clinical Practice Guidelines and the Heart Rhythm Society. Heart Rhythm. 2018;15:e190-e252.

56. Priori SG, Blomstrom-Lundqvist C, Mazzanti A, et al. 2015 ESC Guidelines for the management of patients with ventricular arrhythmias and the prevention of sudden cardiac death: The Task Force for the Management of Patients with Ventricular Arrhythmias and the Prevention of Sudden Cardiac Death of the European Society of Cardiology (ESC). Endorsed by: Association for European Paediatric and Congenital Cardiology (AEPC). Eur Heart J. 2015;36:2793-867.

57. Gollob $\mathrm{MH}$, Blier L, Brugada $\mathrm{R}$, et al. Recommendations for the use of genetic testing in the clinical evaluation of inherited cardiac arrhythmias associated with sudden cardiac death: Canadian Cardiovascular Society/Canadian Heart Rhythm Society joint position paper. Can J Cardiol. 2011;27:232-45.
58. Ackerman MJ, Priori SG, Willems S, et al. HRS/EHRA expert consensus statement on the state of genetic testing for the channelopathies and cardiomyopathies: this document was developed as a partnership between the Heart Rhythm Society (HRS) and the European Heart Rhythm Association (EHRA). Europace. 2011;13:1077-109.

59. Mahon NG, Murphy RT, MacRae CA, et al. Echocardiographic evaluation in asymptomatic relatives of patients with dilated cardiomyopathy reveals preclinical disease. Annals of internal medicine. 2005;143:108-15.

60. Halliday BP, Cleland JGF, Goldberger JJ, et al. Personalizing Risk Stratification for Sudden Death in Dilated Cardiomyopathy: The Past, Present, and Future. Circulation. 2017;136:215231.

61. Seferović PM, Polovina M, Bauersachs J, et al. Heart failure in cardiomyopathies: a position paper from the Heart Failure Association of the European Society of Cardiology. Eur J Heart Fail. 2019;21:553-576.

62. van Rijsingen IA, Arbustini E, Elliott PM, et al. Risk factors for malignant ventricular arrhythmias in lamin a/c mutation carriers a European cohort study. J Am Coll Cardiol. 2012;59:493-500.

63. van Berlo JH, de Voogt WG, van der Kooi AJ, et al. Meta-analysis of clinical characteristics of 299 carriers of LMNA gene mutations: do lamin A/C mutations portend a high risk of sudden death? Journal of molecular medicine (Berlin, Germany). 2005;83:79-83.

64. Pasotti M, Klersy C, Pilotto A, et al. Long-term outcome and risk stratification in dilated cardiolaminopathies. J Am Coll Cardiol. 2008;52:1250-60.

65. Anselme F, Moubarak G, Savouré A, et al. Implantable cardioverter-defibrillators in lamin A/C mutation carriers with cardiac conduction disorders. Heart Rhythm. 2013;10:1492-8.

66. Meune C, Van Berlo JH, Anselme F, et al. Primary prevention of sudden death in patients with lamin A/C gene mutations. $N$ Engl J Med. 2006;354:209-10.

67. Corden B, Jarman J, Whiffin N, et al. Association of Titin-Truncating Genetic Variants With Lifethreatening Cardiac Arrhythmias in Patients With Dilated Cardiomyopathy and Implanted Defibrillators. JAMA Netw Open. 2019;2:e196520.

68. Rapezzi C, Arbustini E, Caforio AL, et al. Diagnostic work-up in cardiomyopathies: bridging the gap between clinical phenotypes and final diagnosis. A position statement from the ESC Working Group on Myocardial and 
Pericardial Diseases. Eur Heart J. 2013;34:1448-

58.

69. Yancy CW, Jessup M, Bozkurt B, et al. 2017 ACC/AHA/HFSA Focused Update of the 2013 ACCF/AHA Guideline for the Management of Heart Failure: A Report of the American College of Cardiology/American Heart Association Task Force on Clinical Practice Guidelines and the Heart Failure Society of America. Circulation. 2017;136:e137-e161.

70. Merlo M, Pyxaras SA, Pinamonti B, et al. Prevalence and prognostic significance of left ventricular reverse remodeling in dilated cardiomyopathy receiving tailored medical treatment. J Am Coll Cardiol. 2011;57:1468-76.

71. Fatkin D, Huttner IG, Kovacic JC, et al. Precision Medicine in the Management of Dilated Cardiomyopathy: JACC State-of-the-Art Review. J Am Coll Cardiol. 2019;74:2921-2938.

72. Muchir A, Wu W, Choi JC, et al. Abnormal p38alpha mitogen-activated protein kinase signaling in dilated cardiomyopathy caused by lamin A/C gene mutation. Human molecular genetics. 2012;21:4325-33.

73. El Refaey M, Xu L, Gao Y, et al. In Vivo Genome Editing Restores Dystrophin Expression and Cardiac Function in Dystrophic Mice. Circulation research. 2017;121:923-929.

74. Amoasii L, Hildyard JCW, Li H, et al. Gene editing restores dystrophin expression in a canine model of Duchenne muscular dystrophy. Science (New York, NY). 2018;362:86-91. 
- 25 - 
-26 - 
Clinical consequences of genetics

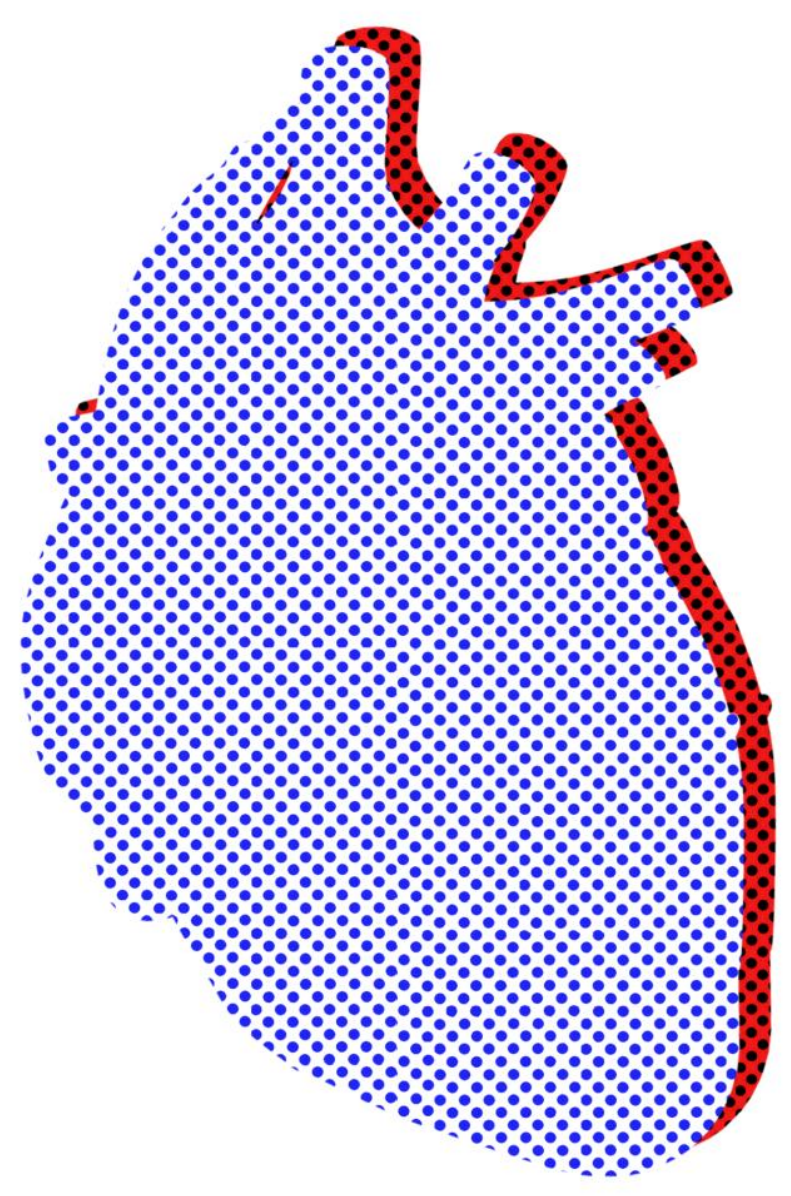




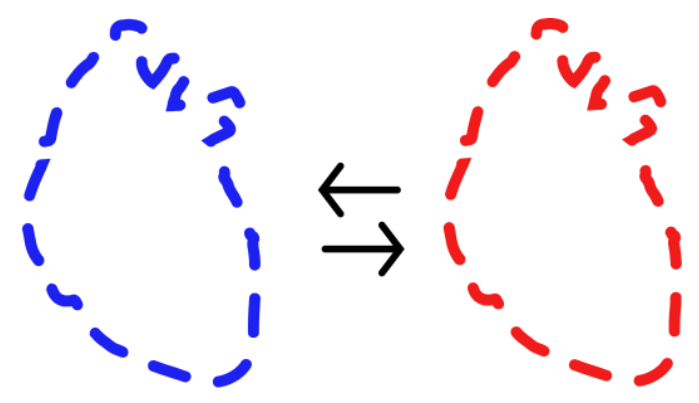




\title{
CHAPTER 2
}

\section{Implications of Genetic Testing in Dilated Cardiomyopathy}

\author{
Job A.J. Verdonschot, $M D, M S c^{1,2}$ \\ Mark R. Hazebroek, MD, $P h D^{1}$ \\ Ingrid P.C. Krapels, $M D, P h D^{2}$ \\ Michiel T.H.M. Henkens, $M D^{I}$ \\ Anne Raafs, $M D^{1}$ \\ Ping Wang, $P h D^{2}$ \\ Jort J. Merken, $M D^{1}$ \\ Godelieve R.F. Claes, $P h D^{2}$ \\ Els K. Vanhoutte, $M D, P h D^{2}$ \\ Arthur van den wijngaard, $P h D^{2}$ \\ Stephane R.B. Heymans, $M D, P h D^{1,3,4}$ \\ Han G. Brunner, $M D, P h D^{25,6}$
}
${ }^{1}$ Department of Cardiology, Cardiovascular Research Institute (CARIM), Maastricht University Medical Center, Maastricht, The Netherlands.
${ }^{2}$ Department of Clinical Genetics, Maastricht University Medical Center, Maastricht, The Netherlands. ${ }^{3}$ Department of Cardiovascular Sciences, Centre for Molecular and Vascular Biology,
KU Leuven, Belgium.
${ }^{4}$ The Netherlands Heart Institute, Nl-HI, Utrecht, The Netherlands.
${ }^{5}$ Radboud University Medical Center, Department of Human Genetics, Donders Institute for Brain, Cognition and Behaviour, Nijmegen.
${ }^{6}$ GROW Institute for Developmental Biology and Cancer, Maastricht University Medical Centre, Maastricht, The Netherlands.




\begin{abstract}
Background: Genetic analysis is a first-tier test in dilated cardiomyopathy (DCM). Electrical phenotypes are common in genetic DCM but their exact contribution to the clinical course and outcome is unknown. We determined the prevalence of pathogenic gene variants in a large unselected DCM population, and determined the role of electrical phenotypes in association with outcome.
\end{abstract}

Methods: This study included 689 DCM patients from the Maastricht Cardiomyopathy Registry, undergoing genetic evaluation using a 48 cardiomyopathy-associated gene-panel, echocardiography, endomyocardial biopsies and Holter monitoring. Upon detection of a pathogenic variant in a DCM patient, familial segregation was performed. Outcome was defined as cardiovascular death, heart transplantation, heart failure hospitalization and/or occurrence of life-threatening arrythmias.

Results: A (likely) pathogenic gene variant was found in $19 \%$ of patients, varying from $36 \%$ in familial to $13 \%$ in non-familial DCM. Family segregation analysis showed familial disease in $46 \%$ of DCM patients who were initially deemed non-familial by history. Overall, $18 \%$ of patients with a non-genetic risk factor had a pathogenic gene variant. Almost all pathogenic gene variants occurred in just 12 genes previously shown to have robust disease association with DCM. Genetic DCM was independently associated with electrical phenotypes such as atrial fibrillation (AF), non-sustained ventricular tachycardia (NSVT) and AV-block (AVB), and inversely correlated with the presence of a left bundle branch block $(p<0.01)$. After a median follow-up of 4 years, event-free survival was reduced in genetic versus non-genetic DCM patients $(p=0.01)$. This effect on outcome was mediated by the associated electrical phenotypes of genetic $\mathrm{DCM}(\mathrm{p}<0.001)$.

Conclusion: One in five patients with an established non-genetic risk factor or a non-familial disease still carries a pathogenic gene variant. Genetic DCM is characterized by a profile of electrical phenotypes (AF, NSVT and AVB), which carries increased risk for adverse outcomes. Based on these findings, we envisage a broader role for genetic testing in DCM. 


\section{INTRODUCTION}

Dilated cardiomyopathy (DCM) is often inherited and more than 60 genes have been associated with it in various studies ${ }^{1}$. Testing by gene panels is generally accepted in patients with familial DCM, in the absence of an environmental cause ${ }^{23}$. In patients with familial DCM, the genetic yield can be as high as $55 \%{ }^{4}$. Focusing genetic testing on familial cases only, is likely too restrictive. In fact, the yield of genetic testing in non-familial DCM varies from $11 \%$ to $26 \%$ in different studies ${ }^{56}$. Also, genetic and non-genetic risk factors are not mutually exclusive, and the extent to which these factors interact in DCM pathogenesis is not fully known. There is accumulating evidence for a more interactive model in which genetic variants increase susceptibility for non-genetic factors to trigger the phenotype ${ }^{78}$. For example, genetic variants are prevalent in DCM patients with specific triggers such as excess alcohol consumption, myocarditis or cardiotoxic chemotherapy 9-11. To accommodate such interactions between genetic and non-genetic risk factors, the World Heart Federation developed a new classification scheme, called MOGE(S), which quantifies disease modifiers in relation to the genetic background ${ }^{12}$. Electrical phenotypes are electrical disturbances that could be either cause or consequence of DCM. Atrial fibrillation (AF) and non-sustained ventricular tachycardia (NSVT) are examples of electrical phenotypes which are often associated with specific gene variants ${ }^{13}{ }^{14}$. Despite the association of these electrical phenotypes with both genetic variants and life-threatening arrhtyhmias and their clinical implications, these phenotypes are not considered in recent classification and definitions of cardiomyopathies 2812 . It remains to be determined whether the electrical phenotypes mediate the clinical course of (genetic) DCM.

This study aimed: 1 ) to determine the genetic yield in a large, well-phenotyped DCM cohort (both familial and non-familial; acquired and idiopathic) using a panel of 48 genes, and 2) to determine the electrical phenotype landscape in genetic and non-genetic DCM at the time of diagnosis, and their subsequent role in the clinical course of DCM.

\section{METHODS}

\section{Study population}

The study population consisted of 689 consecutive, unrelated, DCM patients (both familial and non-familial) prospectively included from the out-patient clinic between 2004 and 2019 in the Maastricht Cardiomyopathy Registry. Inclusion and exclusion criteria were as described previously ${ }^{14}$. In short, DCM patients were included in the absence of a (i) myocardial infarction and/or significant coronary artery disease; (ii) primary valvular disease; (iii) hypertensive or congenital heart disease; (iv) acute myocarditis; (v) arrhythmogenic right ventricular dysplasia; (vi) hypertrophic, restrictive or peripartum cardiomyopathy, in accordance with the latest ESC proposal ${ }^{2}$. As part of the protocol, patients were referred to the clinical genetics department of the Maastricht University Medical Center (MUMC, Maastricht, the Netherlands) for genetic counseling and DNA testing using a 47 cardiomyopathy-associated gene panel between 2012 and 2019. Since June 2018, FLNC was added to the gene panel.

All patients underwent a physical examination, blood sampling (including an auto-

immunity screening for auto-antibodies), 12-lead electrocardiogram, 24-hours Holter monitoring, a complete echocardiographic and Doppler evaluation and coronary angiography $(C A G)$ at baseline ${ }^{15}$. Endomyocardial biopsies (EMB) $(n=448,65 \%)$ were performed at the discretion of the treating physician and with consent of the patient. The main indication for EMB was a LVEF $<45 \%$ after 3 months of optimal medical treatment in accordance with the 
latest ESC position statement ${ }^{16}$. The study was performed according to the declaration of Helsinki and was approved by the institutional Medical Ethics Committee. All patients gave written informed consent.

\section{Genetic analysis}

Patients at the genetics outpatient clinic received genetic counseling and testing using our 47 cardiomyopathy-associated gene panel either with whole exome sequencing (WES) or single molecule Molecular Inversion Probes (smMIP) (Suppl. Table 1). FLNC was added as an additional gene starting June 2018, and this gene was sequenced in only the latest 172 included patients (a total of 48 genes). All detected variants have been confirmed with Sanger sequencing.

A family history of cardiac-related disease and sudden cardiac death was obtained by pedigree analysis at the initial visit of the patient. Familial disease was determined at baseline based on the family history provided by the patient. Familial inheritance was defined as recommended by the ESC: (i) two or more individuals (first or second-degree relatives) have DCM fulfilling diagnostic criteria for 'definite' disease OR (ii) in the presence of an index patient fulfilling diagnostic criteria for DCM and a first-degree relative with autopsy-proven DCM and sudden death at $<50$ years of age ${ }^{2}$. The family segregation strategy is described in the Supplemental Methods.

Genetic variants were carefully and stringently classified in 5 different classes: pathogenic, likely pathogenic, variant of unknown significance (VUS), likely benign or benign based on the criteria as proposed by the ACMG guidelines ${ }^{17}$. The ACMG framework for variant classification was adapted for DCM, as described in detail in the Supplemental Methods. Both pathogenic (class 5) and likely pathogenic (class 4) variants are reported here as pathogenic variants. All other variants were considered non-pathogenic. Importantly, only truncating mutations with a PSI score $>99 \%$ in TTN were classified as pathogenic ${ }^{18}$. Patients were classified as 'familial unknown-genetic' if they had a family history of DCM and DNA testing did not identify a pathogenic gene variant. Patients were classified as 'non-genetic' if they had neither a pathogenic gene variant, nor a family history of DCM (Suppl. Figure 1). For selected LMNA variants, additional functional evidence was sought by microscopic analysis of the nuclear lamina on patients' fibroblasts, as previously reported ${ }^{19}$.

Definitions of non-genetic risk factors, electrical phenotypes and contributing comorbidities In line with our diagnostic pathway and previous literature we defined the following nongenetic risk factors $\left.{ }^{815}: 1\right)$ cardiac inflammation as defined by the ESC ( $\geq 14$ CD 45 , including up to 4 CD68-infiltrating cells $\left./ \mathrm{mm}^{2}{ }^{16}\right) ; 2$ ) an auto-immune disease with proven cardiac involvement ${ }^{20}$; and 3 ) toxic (alcohol abuse (a history of alcohol consumption $>21 \mathrm{U} /$ week for men and $>14 \mathrm{U} /$ week for women; $1 \mathrm{U}$ of alcohol $=10 \mathrm{ml}$ or $8 \mathrm{~g}$ of pure alcohol ${ }^{11}$ ), hard drugs (a history of cocaine use) or chemotherapy (history of treatment with cardiotoxic chemotherapy ${ }^{21}$ ).

Electrical disturbances were considered as separate phenotypes as they could be either cause or consequence of DCM. These electrical phenotypes consisted of conduction disorders (left bundle branch block (LBBB); atrioventricular blocks (AVB) and arrhythmias (AF; NSVT; prevalent (>20\%) premature ventricular complexes (PVC)) ${ }^{22}{ }^{23}$. Definitions of the electrical disturbances can be found in the Supplemental Methods. Diabetes mellitus and hypertension when requiring therapy were treated as contributing comorbidities in our analyses. 


\section{Follow-up}

The median follow-up time was 51 months (interquartile range 27-96 months). Information about the occurrence of adverse events at follow-up was retrieved from the hospital medical records, the Dutch Personal Records Database and/or telephone contact with the patient or their general practitioners. See the Supplemental Methods for further information on collection and registration of adverse events. We collected information regarding four different adverse events: 1) death due to cardiovascular disease, 2) heart transplantation, 3) heart failure that required a non-elective hospitalization despite optimal heart failure therapy according to the ESC/ACC/AHA guidelines 2425 , and 4) life-threatening arrhythmias (LTA) defined as non-fatal ventricular fibrillation (with or without ICD-shock), and/or sustained ventricular tachycardia with appropriate ICD shock. The combined end-point is defined as the occurrence of at least one of the above-mentioned adverse events. No patients were lost to follow-up.

\section{Statistical analysis}

Categorical data were compared using Pearson's chi-square test or Fisher's exact test. For continuous variables, unpaired Student's t-tests or Mann-Whitney U-test were used. KaplanMeier survival curves were estimated and differences between groups were assessed by the log-rank test, using time at diagnosis as time zero. Univariable Cox regression analysis was used to determine the hazard ratio (HR) and subsequent 95\% confidence interval $(\mathrm{Cl})$.

$\mathrm{R}$ mediation package 4.5.0 was used to perform a causal mediation analysis ${ }^{26}$. The causal mechanism describes that a clinical variable affects outcome through an intermediate variable. This subdivides and quantifies the total effect into a direct (clinical determinant to outcome, called the average direct effect $(A D E)$ ) and indirect (clinical determinant to mediator to outcome, called the average causal mediation effect (ACME)) effect (Suppl. Figure 2). The mediator was modeled with a logistic regression model including the clinical determinant. The outcome model was a logistic regression including the mediator and the clinical determinant.

Multivariable binary logistic regression analysis was performed to find associations between clinical variables and pathogenic variants. All univariable associated factors were added in a backward selection fashion with $p<0.1$ and $p<0.05$ as the cut-off for entry and retention, respectively. Calculations were done using SPSS version 23.0 (SPSS Inc., Chicago, Illinois) or R version 3.6.1 (R Foundation for Statistical Computing, Vienna, Austria).

\section{RESULTS}

\section{Genetics of dilated cardiomyopathy}

Comprehensive genetic testing allowed us to categorize 689 DCM patients in three groups: 129 carried a pathogenic gene variant (29\%), 111 were familial but without an evident genetic cause $(16 \%)$ and 449 remained negative after careful family history and testing of the 48-gene panel (65\%) (Table 1; Suppl. Figure 1). Truncating TTN variants (TTNtv) were most prevalent ( $n=67 / 689 ; 9.7 \%)$, followed by $L M N A(n=21 / 689 ; 3 \%)$, truncating FLNC variants (FLNCtv; $\mathrm{n}=3 / 172 ; 1.7 \%)$ and $M Y H 7$ (n=78/689; 1\%; Figure 1; Suppl. Table 2 and 3). The overwhelming majority of the 132 detected pathogenic gene variants (89\%; $n=118$ ) occurred in 12 genes that have previously been shown to have robust evidence for DCM disease association ${ }^{27}$. We found pathogenic gene variants in RBM2O (n=5) and $F L N C(n=3)$, both two genes that were not evaluated by previous mentioned study, but carry convincing evidence for DCM pathogenicity. In addition, variants in TTR $(n=2), E M D(n=1), M Y L 2(n=1$, homozygous) and 
SCN5A ( $n=1)$ were present in a small number of patients and their pathogenicity was supported by additional clinical and/or functional evidence (Supplemental Table 3).

As expected, pathogenic variants were more frequent in familial than in non-familial DCM (36\% vs $13 \%$, respectively; $p<0.001)$. The number of VUSs was comparable between familial and non-familial DCM patients ( $38 \%$ vs 32\%; $p=0.16$; Suppl. Table 2 and 4). Although our series is relatively large, no pathogenic variant was detected in $65 \%$ (31/48) of the genes on our diagnostic panel. Fifty-four percent (26/48) of the genes on the panel yielded only VUSs (Figure 1).

\section{Genetic yield in dilated cardiomyopathy with non-genetic risk factors}

Thirty-three percent (228/689) of the patients had at least one non-genetic risk factor that could have contributed to the phenotype (Figure 2 ). In these DCM patients with a non-genetic risk factor, a pathogenic gene variant was found in $18 \%(41 / 228)$ (Figure 3A). This is comparable to patients without such a non-genetic risk factor $(19 \% ; 88 / 461 ; p=0.73)$. Pathogenic gene variants were detected in all subgroups of the cohort, whether familial or non-familial, and in the presence or absence of established non-genetic risk factors. The prevalence of pathogenic gene variants was similar for the various non-genetic risk factors, ranging from 15-19\% (Figure 3B). Diabetic and hypertensive patients (both 17\%) equally carried pathogenic gene variants.

Taken together, we find that as many as 1 in 5 of the DCM patients with a non-genetic risk factor or comorbidity carry a pathogenic gene variant and that the finding of a nongenetic risk factor does not preclude a genetic predisposition.

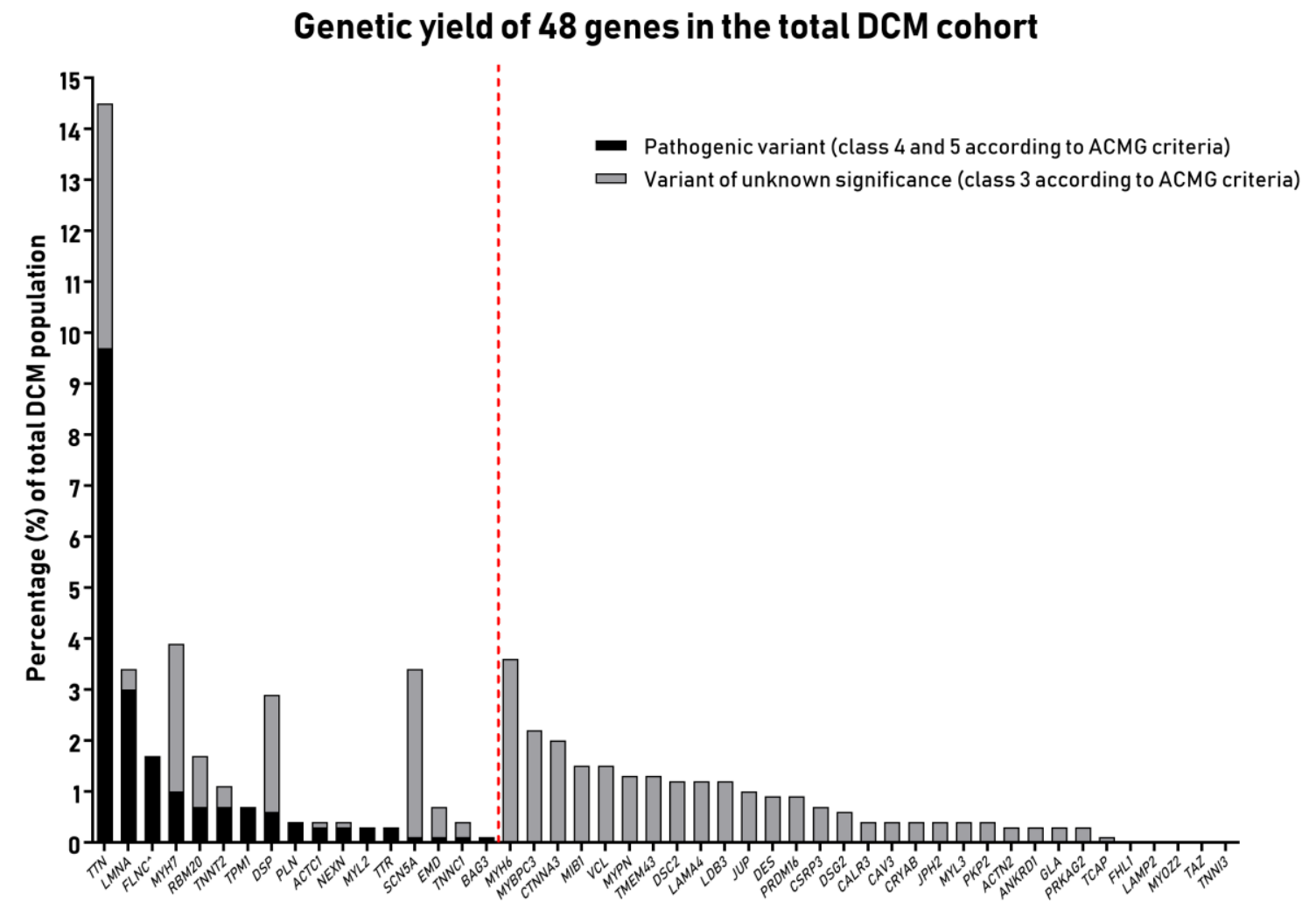

Figure 1. Genetic yield of the 48 cardiomyopathy-associated gene panel in 689 DCM patients. Genes are ordered according to prevalence of pathogenic variants. ^ $\mathrm{FLNC}$ was only sequenced in $172 \mathrm{DCM}$ patients. The prevalence is calculated within this subgroup. 
Table 1. Baseline characteristics and clinical features of 689 dilated cardiomyopathy patients

\begin{tabular}{|c|c|c|c|c|c|}
\hline & $\begin{array}{l}\text { Genetic } \\
(n=129)\end{array}$ & $\begin{array}{c}\text { Familial } \\
\text { Unknown- } \\
\text { Genetic } \\
(n=111)\end{array}$ & $\begin{array}{l}\text { Non-Genetic } \\
\quad(n=449)\end{array}$ & $\begin{array}{c}\text { Total } \\
(n=689)\end{array}$ & p-value \\
\hline \multicolumn{6}{|l|}{ Demographics } \\
\hline Age at diagnosis (years) & $52 \pm 12(22-80)$ & $51 \pm 13(18-78)$ & $54 \pm 13(18-90)$ & $53 \pm 13(18-90)$ & $0.042 \dagger$ \\
\hline Male & $90(70 \%)$ & 65 (59\%) & $284(63 \%)$ & 439 (64\%) & NS \\
\hline Hypertension & $45(35 \%)$ & 43 (39\%) & $175(39 \%)$ & $263(38 \%)$ & NS \\
\hline Diabetes Mellitus & $13(10 \%)$ & $14(13 \%)$ & 49 (11\%) & $76(11 \%)$ & NS \\
\hline Body Mass Index & $26.8 \pm 4(16-38)$ & $26.9 \pm 5(18-48)$ & $26.7 \pm 5(16-44)$ & $26.7 \pm 5(16-48)$ & NS \\
\hline Auto-immune Disease & $7(7 \%)$ & $11(10 \%)$ & $40(9 \%)$ & $60(9 \%)$ & NS \\
\hline Toxic trigger & $12(9 \%)$ & $6(5 \%)$ & 47 (11\%) & $65(9 \%)$ & NS \\
\hline \multicolumn{6}{|l|}{ Presentation } \\
\hline Family history of DCM & $63(49 \%)$ & $111(100 \%)$ & $0(0 \%)$ & $174(25 \%)$ & $<0.001 \ddagger \# \dagger$ \\
\hline NYHA class III or IV & $46(36 \%)$ & $36(32 \%)$ & 109 (24\%) & $191(28 \%)$ & $0.01 \ddagger$ \\
\hline $\begin{array}{c}\text { Out of hospital cardiac } \\
\text { arrest }\end{array}$ & $11(9 \%)$ & $7(6 \%)$ & $29(7 \%)$ & $47(7 \%)$ & NS \\
\hline \multicolumn{6}{|l|}{ Initial ECG / holter } \\
\hline Atrial Fibrillation & 47 (36\%) & $23(21 \%)$ & $97(22 \%)$ & $167(24 \%)$ & $\begin{array}{c}0.001 \ddagger / \\
0.008 \#\end{array}$ \\
\hline Atrioventricular Block & $28(22 \%)$ & $11(10 \%)$ & $45(10 \%)$ & $84(12 \%)$ & $\begin{array}{c}<0.001 \ddagger / \\
0.014 \#\end{array}$ \\
\hline $1^{\text {st }} / 2^{\text {nd }} / 3^{\text {rd }}$ degree AVB & $24 / 2 / 2$ & $10 / 1 / 0$ & $34 / 5 / 6$ & $68 / 8 / 8$ & \\
\hline LBBB & $27(21 \%)$ & $32(29 \%)$ & $131(29 \%)$ & $190(28 \%)$ & NS \\
\hline NSVT & $62(48 \%)$ & $29(26 \%)$ & $116(26 \%)$ & $207(30 \%)$ & $<0.001 \ddagger \#$ \\
\hline PVC $>20 \%$ & $3(2 \%)$ & $8(7 \%)$ & $39(9 \%)$ & $50(7 \%)$ & $0.014 \ddagger$ \\
\hline $\begin{array}{l}\text { Echocardiography } \\
\text { Left ventricular ejection } \\
\text { fraction }\end{array}$ & $30 \pm 11(10-49)$ & $32 \pm 10(13-49)$ & $32 \pm 11(8-49)$ & $31 \pm 11(8-49)$ & NS \\
\hline LVEF below $35 \%$ & $88(68 \%)$ & $70(63 \%)$ & $261(58 \%)$ & $419(61 \%)$ & $0.039 \ddagger$ \\
\hline $\begin{array}{l}\text { Indexed left ventricular } \\
\text { end-diastolic diameter }\end{array}$ & $30 \pm 4(18-47)$ & $32 \pm 5(21-53)$ & $31 \pm 5(20-51)$ & $31 \pm 5(18-53)$ & NS \\
\hline \multicolumn{6}{|l|}{ Endomyocardial Biopsy } \\
\hline Cardiac inflammation & $22(17 \%)$ & $21(19 \%)$ & 85 (19\%) & $128(19 \%)$ & NS \\
\hline $\begin{array}{c}\text { Significant viral load } \\
\text { present }\end{array}$ & $8(6 \%)$ & $5(5 \%)$ & $64(14 \%)$ & 77 (11\%) & $\begin{array}{c}0.015 \ddagger / \\
0.005 \dagger\end{array}$ \\
\hline \multicolumn{6}{|l|}{ Medication } \\
\hline Beta-blocker & $104(81 \%)$ & 93 (84\%) & $390(87 \%)$ & $587(85 \%)$ & NS \\
\hline ACE-inhibitors/ARB & $110(85 \%)$ & $92(83 \%)$ & $400(89 \%)$ & $602(87 \%)$ & NS \\
\hline $\begin{array}{l}\text { Mineralocorticoid } \\
\text { receptor antagonist }\end{array}$ & 73 (57\%) & 55 (50\%) & 210 (47\%) & 338 (49\%) & NS \\
\hline Diuretics & 67 (52\%) & 58 (52\%) & 214 (48\%) & 339 (49\%) & NS \\
\hline $\begin{array}{r}\text { Significance }<0.05 \text { us } \\
\text { + Familial } \\
\text { \# Genetic } \\
\text { \# Genetic } \\
\text { Abbreviations: DCM: } \\
\text { significance } \\
\text { Values are denicted }\end{array}$ & $\begin{array}{l}\text { g Student's T-test, o } \\
\text { innown-genetic vers } \\
\text { ersus Non-genetic } \\
\text { ersus Familial unkno } \\
\text { ilated Cardiomyopa }\end{array}$ & $\begin{array}{l}\chi^{2} \text {-test/Fisher Exact te } \\
\text { s Non-genetic } \\
\text { n-genetic } \\
\text { y; ECG: Electrocardio }\end{array}$ & st where appropriate & lar Ejection & S: no \\
\hline
\end{tabular}




\section{Prevalence of pathogenic variants in apparent non-familial dilated cardiomyopathy}

A pathogenic gene variant was found in $13 \%(n=66)$ of sporadic DCM patients, i.e. those without a familial history of DCM (Suppl. Table 2). Data on family segregation was available in 35 of these patients (53\%). In two families, the pathogenic variant in the index patient was proven to be de novo, as both parents tested negative for the variant. In 14 families, at least one relative carried the pathogenic variant and had cardiac abnormalities after cardiac examination. In two additional families in which only cardiac examination was performed, a relative with cardiac abnormalities was detected. Overall, 16 out of 35 (46\%) of the initially non-familial DCM pedigrees, turned out to be familial after segregation (Suppl. Table 2). Suppl. Figure 3 shows all initially 'non-familial' pedigrees in which segregation analysis could be performed.

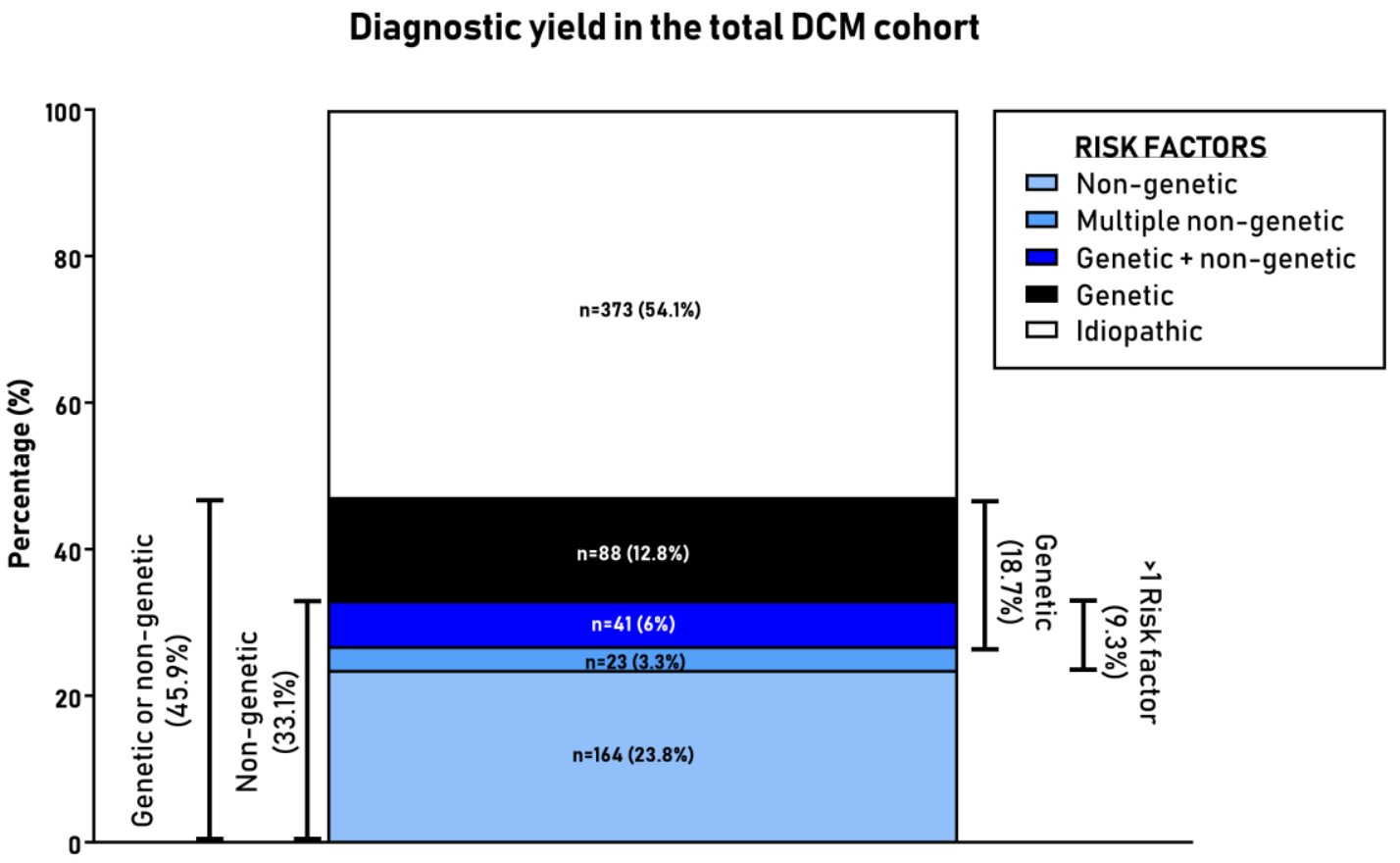

Figure 2. Diagnostic yield in 689 DCM patients after complete diagnostic work-up.

Genetic dilated cardiomyopathy is associated with electrical phenotypes and adverse events

After a median of 4-year follow-up, 20\% (143/689) of the cohort had experienced at least one serious adverse event. Adverse events occurred more frequently in those with genetic than those with non-genetic DCM (31\% vs 18\%; HR 1.6 [1.1-2.4]; log-rank $p=0.01$; Figure 4A; Table 2). Having a pathogenic variant and an additional non-genetic risk factor does not change outcome (log-rank $p=0.28$; Suppl. Figure 4). The adverse outcome of genetic DCM patients was mainly driven by LTA (HR 2.2 [1.3-3.7], $p=0.002$, Table 2). 


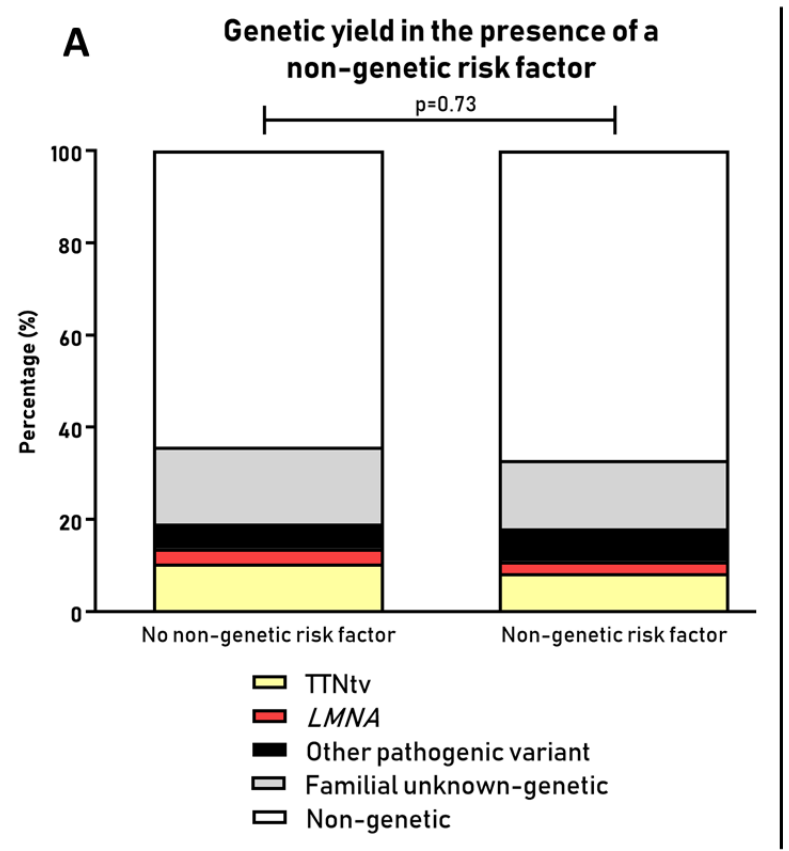

B Genetic yield per non-genetic risk factor

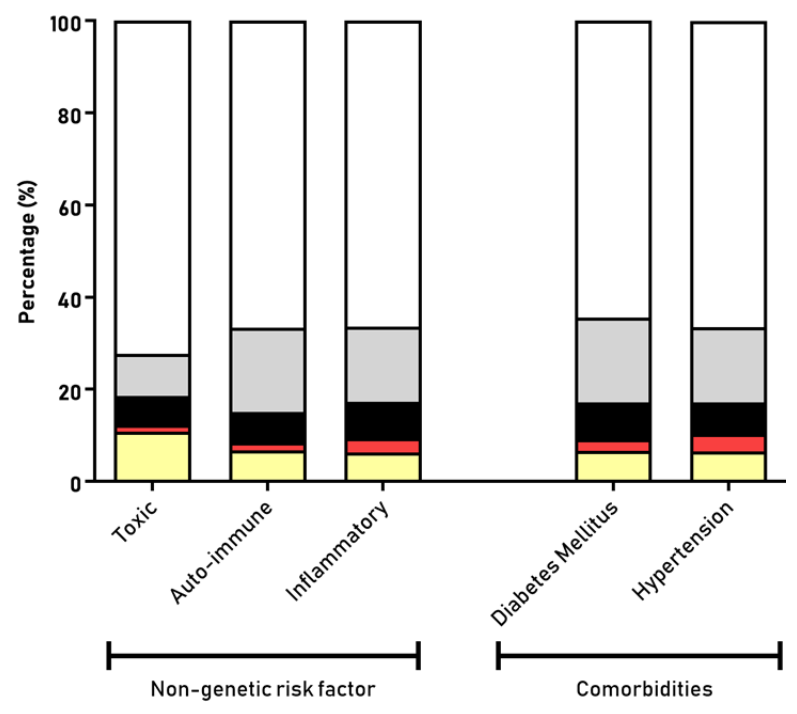

Figure 3. Genetic yield in DCM patients with and without a non-genetic risk factor $(A)$ and divided per subgroup of non-genetic risk factors and/or comorbidities (B). P-value is calculated using $\chi^{2}$-test comparing the prevalence of pathogenic gene variants between groups.

Electrical phenotypes are frequently observed in DCM patients. In our cohort, $A F$, NSVT and an AVB were independently associated with genetic DCM, while frequent PVCs and LBBB were associated with non-genetic DCM (Table 3; Figure 5). Familial disease and electrical phenotypes are the only distinguishing factor between genetic and non-genetic DCM (Table 3). Electrical phenotypes were also associated with an adverse outcome in DCM (Table 4, Figure 4). This was also mainly driven by the strong association between electrical phenotypes and LTA (HR 5.0 [2.2-11.6]; $p<0.001$; Suppl. Table 5). We performed a causal mediation analysis to quantify the contribution of electrical phenotypes to the prognosis of genetic DCM. Electrical phenotypes mediate the effect between genetic DCM and an adverse outcome (ACME $p<0.001$ ) but there was no direct effect (ADE $p=0.1$; Figure 6, Suppl. Figure 2). This shows that the adverse outcome of genetic DCM is partly due to the strong association with electrical phenotypes.

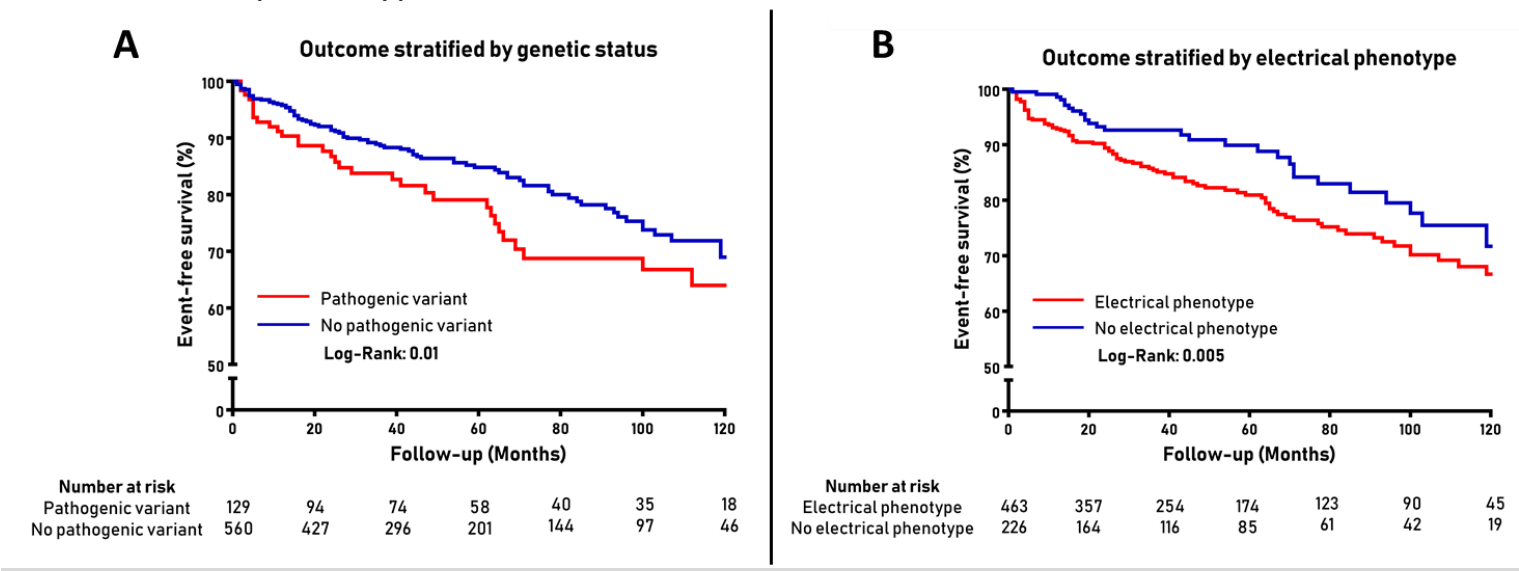

Figure 4. Survival curves show freedom from composite endpoint (cardiac death or transplantation, heart failure hospitalization or life-threatening arrhythmia) stratified on genetic status (A), and stratified on the presence of an electrical phenotype (B). Event-free survival is measured from time of diagnosis. Curves are compared using the log-rank test. 
Table 2. Association of genetic status with adverse outcomes on Cox proportional hazards analysis

\begin{tabular}{|c|c|c|c|c|}
\hline & $\begin{array}{l}\text { Pathogenic variant } \\
\qquad(\mathrm{n}=129)\end{array}$ & $\begin{array}{l}\text { No pathogenic variant } \\
\qquad(n=560)\end{array}$ & $\begin{array}{l}\text { Hazard Ratio } \\
{[95 \% \mathrm{Cl}]}\end{array}$ & p-value \\
\hline Death & $9(7 \%)$ & $37(7 \%)$ & - & NS \\
\hline Heart transplantation & $7(5 \%)$ & $6(1 \%)$ & $5.1[1.4-19.2]$ & 0.015 \\
\hline Heart failure hospitalization & $22(17 \%)$ & 59 (11\%) & - & NS \\
\hline Life-threatening arrhythmia & $23(18 \%)$ & $46(8 \%)$ & $2.2[1.3-3.7]$ & 0.002 \\
\hline Combined end-point & 40 (31\%) & $103(18 \%)$ & $1.6[1.1-2.4]$ & 0.011 \\
\hline $\begin{array}{l}\text { Combined end-point without } \\
\text { life-threatening arrhythmia }\end{array}$ & $27(21 \%)$ & $83(15 \%)$ & - & NS \\
\hline
\end{tabular}

Significance $<0.05$ using Cox regression analysis.

Abbreviations: Cl: Confidence Interval; NS: Not Significant.

Given the strong association between the presence of NSVT and worse outcome (HR 2.72 [1.95-3.78]; $p<0.001$; Table 4), we distinguished between specific adverse events. Baseline NSVT represents a strong predictor for an LTA (HR 6.1), and is also a marker of overall poor outcome (Suppl. Table 6). NVST is prevalent in genetic DCM and occurred in 62 (48\%) patients in this group (Table 1; Figure 5). In contrast, LBBB and frequent PVCs, are more common in non-genetic DCM, and these phenotypes are not associated with adverse events (Table 3). These data underline the prognostic relevance of the electrical phenotypes that are frequent in genetic DCM.

Electrical phenotyping in genetic and non-genetic DCM

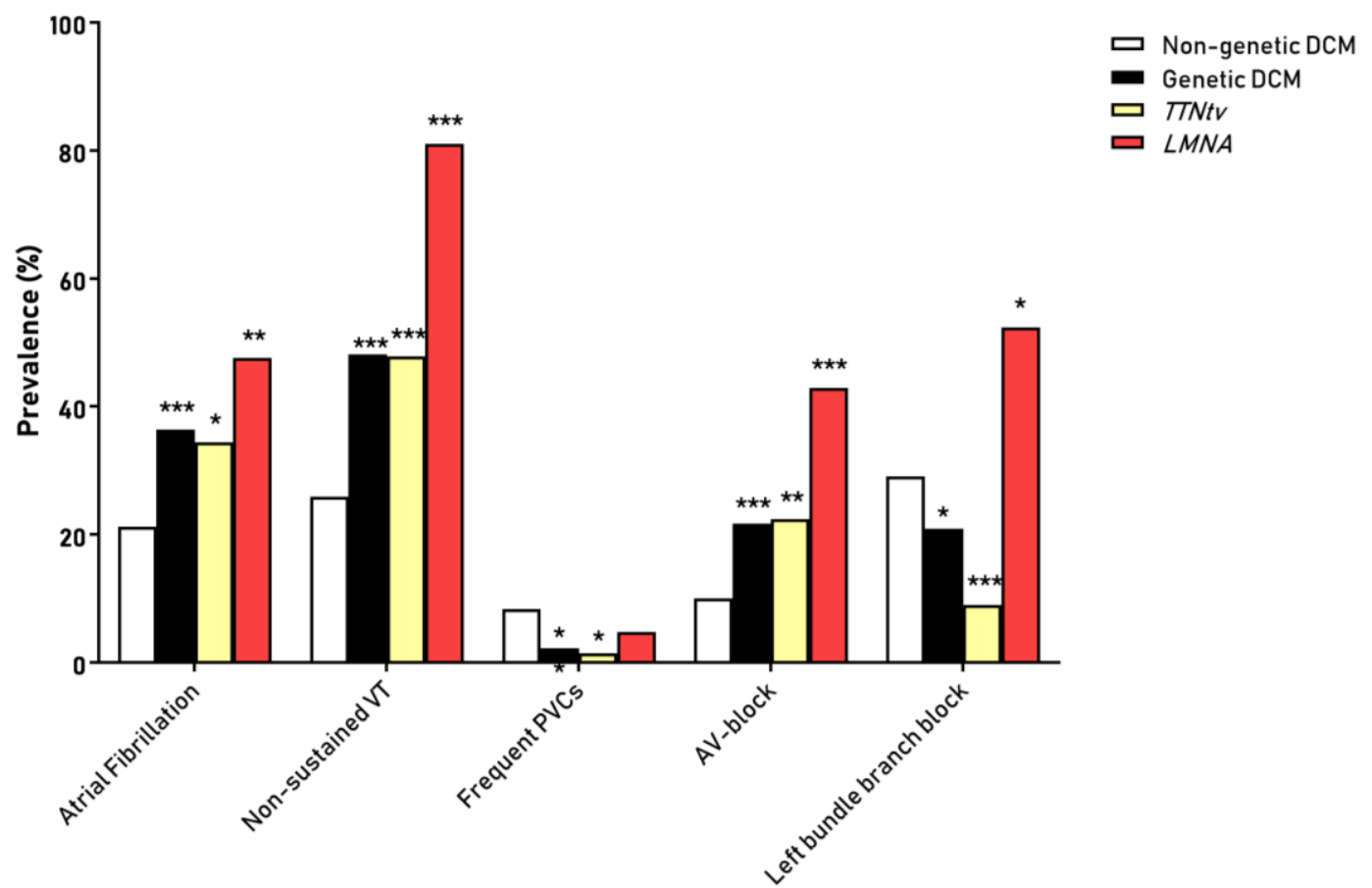

Figure 5. Prevalence of atrial fibrillation, non-sustained VTs, AV-block, left bundle branch block and prevalent premature ventricular complexes within genetic and non-genetic DCM. P-value is calculated using $\chi^{2}$-test comparing the prevalence with the non-genetic DCM group. ${ }^{*}=p<0.05 ;^{* *}=p<0.01 ; * * *=p<0.001$.

The genetic DCM group constitutes of a heterogeneous mixture of variants. The subgroup of patients with either a TTNtv or LMNA variant were large enough to investigate separately. The TTNtv patients had an electrical signature which closely resembles that of the total genetic DCM cohort: a strong association with AF, NSVT and AVB and a significant underrepresentation of LBBB (Figure 5). In contrast, LBBB is common in patients with a LMNA variant, in addition to AF, NSVT and AVB. 
Table 3. Uni- and multivariable model for predicting the likelihood of a pathogenic variant in dilated cardiomyopathy patients.

\begin{tabular}{ccccccc}
\hline & \multicolumn{3}{c}{ Univariable } & \multicolumn{3}{c|}{ Multivariable } \\
Variable & OR & $\mathbf{9 5 \%} \mathbf{~ C l}$ & p-value & OR & $\mathbf{9 5 \%} \mathbf{~ C l}$ & p-value \\
\hline Familial disease & 3.86 & $2.6-5.8$ & $<0.001$ & 3.72 & $2.4-5.7$ & $<0.001$ \\
Atrial fibrillation & 2.1 & $1.4-3.2$ & $<0.001$ & 1.68 & $1.1-2.6$ & 0.024 \\
Non-sustained VT & 2.65 & $1.8-3.9$ & $<0.001$ & 2.7 & $1.8-4.2$ & $<0.001$ \\
AV-block & 2.5 & $1.5-4.1$ & $<0.001$ & 2.5 & $1.4-4.4$ & 0.002 \\
Left bundle branch block & 0.65 & $0.4-1.0$ & 0.06 & 0.51 & $0.3-0.9$ & 0.01 \\
>20\% PVCs & 0.26 & $0.1-0.8$ & 0.03 & 0.19 & $0.1-0.6$ & 0.008 \\
NYHA $\geq$ III & 1.59 & $1.1-2.4$ & 0.03 & - & - & - \\
\hline
\end{tabular}

Abbreviations: OR: Odds Ratio; Cl: Confidence Interval; LVEDD: Left Ventricular End-Diastolic Diameter

Finally, we performed the analysis with the exclusion of $L M N A$ variants as they might drive the worse prognosis due to their known malignant arrhythmic profile. In general the results remained the same, showing AF, NSVT and AVB associated with genetic DCM (Suppl. Table 7). The adverse outcome was worse in genetic DCM compared to non-genetic DCM (Suppl. Figure 5), which was mainly driven by LTA (Suppl. Table 8). This worse outcome was strongly mediated by the association with electrical phenotypes (ADE $p=0.14 ; A C M E p<0.001$; Suppl. Figure 6), implying that the adverse outcome of non-LMNA genetic DCM relies strongly on the association with electrical phenotypes.

Table 4. Electrical phenotypes in association to adverse outcome in all patients.

\begin{tabular}{ccccc} 
& $\begin{array}{c}\text { Adverse } \\
\text { Outcome } \\
(n=143)\end{array}$ & $\begin{array}{c}\text { No Adverse } \\
\text { Outcome } \\
(n=546)\end{array}$ & $\begin{array}{c}\text { Hazard Ratio } \\
{[95 \% \text { Cl] }}\end{array}$ & p-value \\
\hline Electrical phenotype $(n=463)$ & $111(24 \%)$ & $352(76 \%)$ & $1.76[1.18-2.63]$ & 0.005 \\
No electrical phenotype $(n=226)$ & $32(14 \%)$ & $194(86 \%)$ & - & - \\
Atrial fibrillation $(n=167)^{*}$ & $48(29 \%)$ & $119(71 \%)$ & $1.64[1.16-2.33]$ & 0.005 \\
Non-sustained VT $(n=207)^{*}$ & $79(38 \%)$ & $128(62 \%)$ & $2.72[1.95-3.78]$ & $<0.001$ \\
AV-block $(n=84)^{*}$ & $24(29 \%)$ & $60(71 \%)$ & - & NS \\
Left bundle branch block $(n=190)$ & $34(18 \%)$ & $156(82 \%)$ & - & NS \\
$>20 \%$ PVCS $(n=50)$ & $13(26 \%)$ & $37(74 \%)$ & - & NS \\
\hline
\end{tabular}

An asterisk indicates the electrical phenotypes which are significantly associated with genetic DCM.

Abbreviations: CI: Confidence Interval; VT: Ventricular Tachycardia; PVC: Premature Ventricular Complex; NS: Not Significant

\section{DISCUSSION}

The overall genetic yield in unselected DCM is $19 \%$ using strict criteria to classify genetic variants. This study shows that patients with apparently non-familial DCM still carry a pathogenic gene variant in $13 \%$, and that after family segregation a clinically affected relative carrying the variant was found in $46 \%$ of what first appeared to be "non-familial" pedigrees. Furthermore, we find a pathogenic gene variant in $18 \%$ of the DCM patients with a nongenetic risk factor or comorbidity. Clearly, neither the absence of a family history, nor the presence of a non-genetic risk factor excludes the chance of finding genetic DCM. Importantly, genetic DCM carries an increased risk of adverse events which is partly mediated by electrical phenotypes such as NSVT, AF, and AVB (Figure 6). Based on these results, we propose that genetic testing is clinically relevant and warranted across a wide range of clinical situations.

\section{Diagnostic yield in dilated cardiomyopathy}

We detected at least one genetic or non-genetic risk factor in $46 \%$ of patients after a complete diagnostic work-up. This diagnostic yield is comparable to that in previous reports on large 
DCM populations studied before large-scale genetic testing became available ${ }^{28}$. A recent study in 100 DCM patients identified a cause in $86 \%$ of patients after EMB and CMR, but without genetic testing ${ }^{29}$. Inflammation-associated disease was reported in $49 \%$ of the DCM patients in that study. Such inflammation-associated disease was defined by suspected or previous myocarditis on MRI without confirmation by EMB. Such a scenario would not fit our diagnostic criteria for DCM. There is a continued need for standardized criteria for diagnosis and the definition of pertinent risk factors, in order to better understand DCM pathogenesis, as previously proposed by the WHF ${ }^{12}$. We decided to treat electrical disturbances as separate phenotypes, rather than as independent risk factors. Our data do not allow us to determine whether these electrical disturbances are cause or consequence of DCM.

\section{Genetic yield in non-familial and acquired dilated cardiomyopathy}

Genetic diagnostics is widely accepted in familial DCM, especially when there are no other specific triggers ${ }^{2}$. We find that limiting DNA-testing to familial DCM may not be the best strategy, as pathogenic variants were present in $13 \%$ of non-familial DCM patients in our cohort. Conversely, of those who tested positive for a pathogenic DCM gene variant in our study, almost half lacked a positive family history at baseline. Family history provided by patients is frequently incomplete, and some of these relatives will develop overt DCM at a later age ${ }^{30}$. Accurate family segregation revealed a pathogenic gene variant and clinically relevant DCM in a relative in $46 \%$ of the non-familial DCM pedigrees. There may be diagnostic as well as prognostic value to search for the genetic substrate in all DCM patients and subsequently in other family members.

Recent reports of an increased burden of genetic variants in DCM patients exposed to chemotherapy or alcohol, suggest that the clinical impact of a pathogenic gene variant may be amplified by exposure to such non-genetic risk factors ${ }^{7811} 18$. In one study, DCM patients with a TTNtv who consumed excess alcohol had a more severe clinical presentation compared with DCM with neither TTNtv nor excess alcohol consumption ${ }^{11}$. We detected pathogenic gene variants in an average of $18 \%$ of DCM patients across a range of non-genetic risk factors. Showing that the finding of a non-genetic risk factor does not exclude a genetic predisposition. Also, there was no detectable effect of having an additional non-genetic risk factor on prognosis in our cohort (Suppl. Figure 4).

\section{Composition of diagnostic gene panels for dilated cardiomyopathy}

Genetic testing aims to test the smallest number of DCM genes that maximizes the diagnostic yield, while simultaneously keeping the number of VUSs as low as possible. A VUS complicates the clinical translation of genetics to the patient and family, as the clinical consequences are unknown. The number of genes reported as disease-causing for DCM is constantly increasing, but many such genes currently lack reliable and robust evidence 2731 .

In our cohort, a genetic variant was present in $19 \%$ of DCM patients, which is slightly lower compared to most published studies using high-throughput genetic screening 453233 . One important difference is that familial DCM was less frequent in our cohort (25\%) compared to $\sim 50 \%$ in most published studies. Our study was a consecutive series of patients, not selected for familial occurrence. We used a diagnostic panel including $47 / 48$ genes in contrast to gene panels including up to 149 genes used for genetic screening in previous studies 532 . 


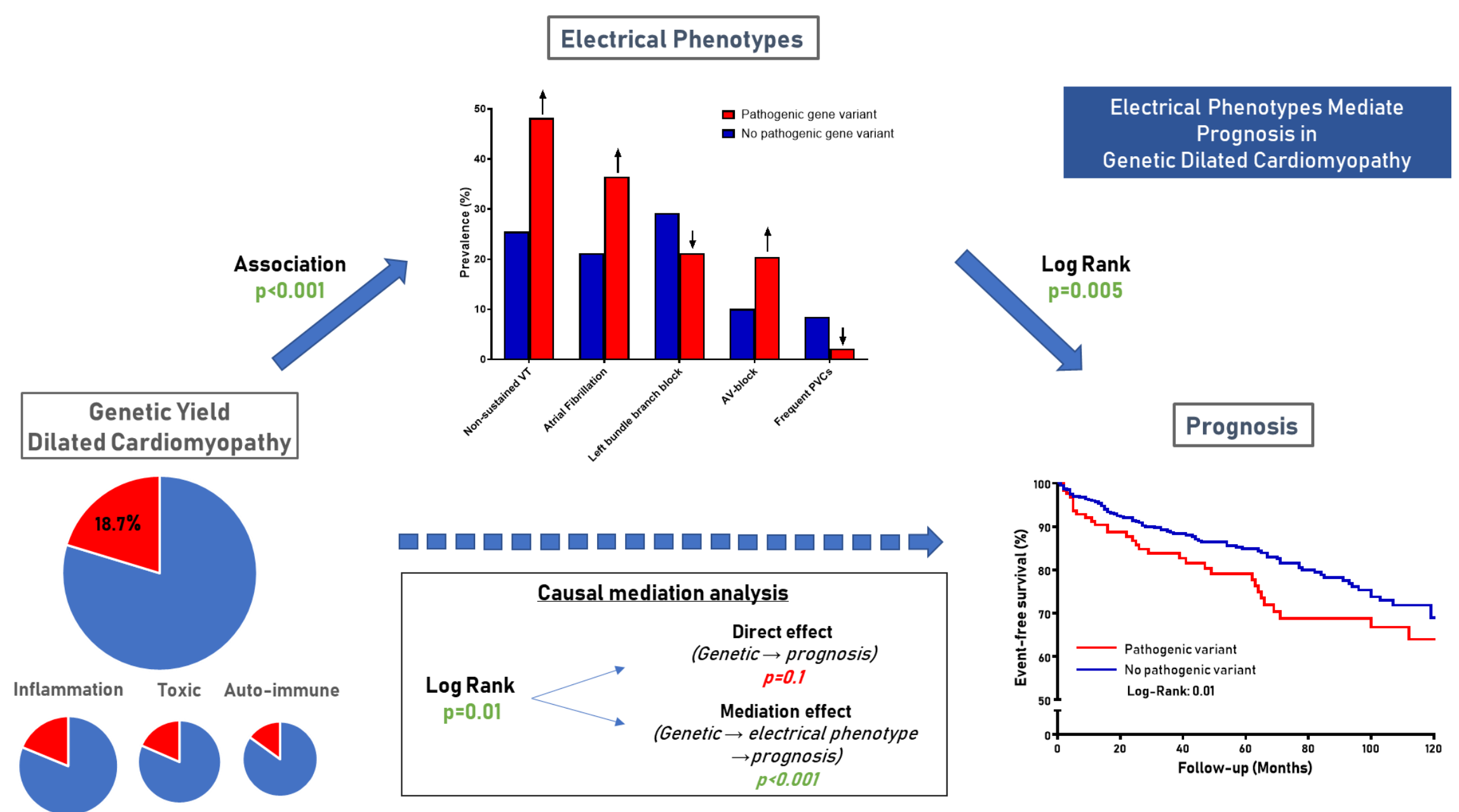

Figure 6. Genetic DCM has an electrical phenotype characterized by an increased prevalence of non-sustained ventricular tachycardia, atrial fibrillation and AV-block. This electrical phenotype mediates the worse prognosis in genetic versus non-genetic DCM. The direct effect is the contribution of genetics to outcome, the mediation effect is the contribution of electrical phenotypes to the association between genetics and outcome. 
Another important difference is the criteria used for interpreting pathogenicity of variants. We used the strict ACMG guidelines to systematically classify variants, and incorporated evidence per gene as suggested by Mazzarotto and colleagues ${ }^{27}$. Previous studies sometimes used less strict and uniform criteria. For example, a previous study reported 2 or more gene variants in up to $38 \%$ of DCM patients. This number greatly exceeds previous studies ${ }^{33}$. One possible explanation for this discrepancy is the inclusion of TTN missense variants, the majority of which are now considered to be likely benign ${ }^{34}$.

It has been argued that only 12 genes have sufficiently robust evidence for DCMrelated pathogenicity, and that the inclusion of more genes to a DCM screening panel is questionable 2731 . Classifying variants in further non-established DCM genes should be done with caution. In our study evaluating only the proposed established DCM genes would have detected the overwhelming majority of pathogenic gene variants (89\%; 118/132). Additional pathogenic variants were detected in RBM2O and FLNC that were not reviewed by Mazzarotto et al. ${ }^{27}$, in patients with multisystemic disease (EMD and TTR), an arrhythmogenic form of DCM (SCN5A), or in a rare homozygous form (MYL2). These genes collectively added $2 \%$ to the genetic yield, of which $1.2 \%$ attributed to FLNC and RBM20. This confirms that pathogenic gene variants mostly reside in the core DCM genes: TTN, LMNA, MYH7, TNNT2, TPM1, DSP, $V C L, B A G 3, T N N C 1, A C T C 1, N E X N, P L N$, with the addition of RBM20 and FLNC. Focusing genetic testing to solely these 14 genes would substantially reduce VUSs with $70 \%$. These VUSs were detected in these non-established DCM genes with questionable diagnostic utility in a clinical setting. We found three pathogenic hypertrophic cardiomyopathy (HCM) founder mutations in MYBPC3 (Supplemental Table 4), which are difficult to interpret when detected in a DCM patient. None of these patients appeared to have end-stage HCM. This is a remarkable finding, as these pathogenic variants are solely linked to HCM in the literature. That a gene can be associated with both HCM and DCM is not entirely without precedent. An example of this would be MYH7, which is both an established DCM and HCM gene ${ }^{27} 31$. We note that DCM founder mutations affecting specific genes occur in distinct geographical areas. Thus, the precise composition of a diagnostic gene panel should ideally include those genes that are more commonly involved locally.

\section{Genotype-phenotype associations in dilated cardiomyopathy}

The electrical signature of genetic DCM is characterized by AF, NSVT and AVB in the absence of LBBB and frequent PVCs. Previous studies described the arrhythmogenic potential in $L M N A$ and TTN-associated DCM, and related this to a higher degree of cardiac fibrosis ${ }^{13}{ }^{14}$. It is possible, that our risk stratification could be further improved using novel imaging techniques such as CMR to detect and quantify this cardiac fibrosis ${ }^{35}$. We showed that the electrical phenotype of genetic DCM may mediate a worse prognosis compared to non-genetic DCM (Figure 6). Genetic DCM was mainly related to an increased risk of LTA. The relation between LTAs and genetic DCM remained in our study, even after exclusion of $L M N A$ variants. This is in contrast to a recent study in which the association between LTAs and genetic DCM failed to reach significance ${ }^{36}$. A subgroup analysis in that study suggested significantly higher risk for LTAs for LMNA and desmosomal gene carriers only. Based on our findings, the arrhythmogenic phenotype that predisposes to LTAs would seem to be distributed over a broader range of DCM-associated genes. This may have implications for DCM management. The susceptibility for ventricular arrhythmias in LMNA as well as in non-LMNA genetic DCM 
argues for early preventive measures irrespective of left ventricular dysfunction. This should be further investigated in a more extensive study designed to answer this question.

The conduction disorder LBBB is rare in TTN-associated DCM, but is not uncommon in patients carrying a $L M N A$ variant, in line with previous literature ${ }^{37}$. We note that $L M N A$ variants are known to affect the septum which is important for electrical conduction ${ }^{38}$.

\section{Clinical Implications}

A key finding of our study is that an established acquired trigger in DCM does not exclude a pathogenic gene variant. Interactions between pathogenic gene variants and non-genetic risk factors may contribute to the incomplete penetrance and variability within families. Overall, our finding of $19 \%$ pathogenic variants across a range of non-genetic risk factors (Figure 3 ) suggests that it may be justifiable to perform genetic diagnostics in all DCM patients.

There will always be a trade-off between diagnostic sensitivity and specificity in DCM genetic testing. On the one hand, some families that tested negative in our study may have monogenic causes that are not covered by the 47 gene DCM panel, such as FLNC which was only tested in $25 \%$ of our cohort. On the other hand, decreasing the number of genes in our diagnostic gene panel to only the genes which have robust evidence to be DCM-associated (TTN, LMNA, MYH7, RBM20, TNNT2, TPM1, DSP, VCL, BAG3, TNNC1, ACTC1, NEXN, FLNC and $P L N)^{27} 3139$ would have detected $95 \%$ of all pathogenic gene variants found in this cohort (126/132). Patients with VUSs in the aforementioned established genes (excluding missense variants in TTN) showed a similar prognostic trend compared to patient with a classified (likely) pathogenic variant (Supplemental Figure 7). This suggests that at least some of these VUSs are likely pathogenic, but currently lack sufficient evidence to be classified as (likely) pathogenic with the ACMG guidelines. The diagnostic and prognostic value of VUSs in established DCM genes should be analyzed detail in larger cohorts, as previously performed for $\mathrm{HCM}^{4041}$. In addition, these VUSs could explain some of the familial occurrence in the 111 'familial unknown genetic' DCM patients in our cohort.

\section{Study Limitations}

This study represents a single-center, retrospective data analysis. Results of this study still need to be confirmed in an external cohort. There is an ongoing debate about the definition of risk factors that contribute to the cardiac phenotype. We tried to be as strict as possible using only previously defined quantifiable non-genetic risk factors, following previous guidelines and position statements. Regardless of whether such factors are truly causative, our main findings underscore the importance of genetic variants in patients with apparent non-genetic risk factors. The exclusion of coronary artery disease and valvular disease does not exclude the possibility that there could be a genetic predisposition in this DCM subgroup. In fact, DCM patient with non-genetic risk factors still carried pathogenic gene variants in a signicant percentage. Our cohort is mainly from Caucasian ancestry, and the genetic results should be interpreted and translated within this ancestral context.

\section{CONCLUSION}

One in five patients with an established non-genetic risk factor or non-familial disease still carries a pathogenic gene variant. Genetic DCM is characterized by a profile of electrical phenotypes (high prevalence of AF, NSVT and AVB), and carries increased risk for adverse outcomes. Based on these findings, we envisage a broader role for genetic testing in DCM. 


\section{REFERENCES}

1. Japp AG, Gulati A, Cook SA, et al. The Diagnosis and Evaluation of Dilated Cardiomyopathy. J Am Coll Cardiol 2016;67(25):2996-3010.

2. Pinto YM, Elliott PM, Arbustini E, et al. Proposal for a revised definition of dilated cardiomyopathy, hypokinetic non-dilated cardiomyopathy, and its implications for clinical practice: a position statement of the ESC working group on myocardial and pericardial diseases. Eur Heart J 2016;37(23):1850-8.

3. Bozkurt B, Colvin M, Cook J, et al. Current Diagnostic and Treatment Strategies for Specific Dilated Cardiomyopathies: A Scientific Statement From the American Heart Association. Circulation 2016;134(23):e579-e646.

4. Pugh TJ, Kelly MA, Gowrisankar S, et al. The landscape of genetic variation in dilated cardiomyopathy as surveyed by clinical DNA sequencing. Genetics in medicine : official journal of the American College of Medical Genetics 2014;16(8):601-8.

5. Akinrinade $\mathrm{O}$, Ollila $\mathrm{L}$, Vattulainen $\mathrm{S}$, et al. Genetics and genotype-phenotype correlations in Finnish patients with dilated cardiomyopathy. Eur Heart J 2015;36(34):2327-37.

6. Hazebroek MR, Krapels I, Verdonschot J, et al. Prevalence of Pathogenic Gene Mutations and Prognosis Do Not Differ in Isolated Left Ventricular Dysfunction Compared With Dilated Cardiomyopathy. Circulation Heart failure 2018;11(3):e004682.

7. Piran S, Liu P, Morales A, et al. Where genome meets phenome: rationale for integrating genetic and protein biomarkers in the diagnosis and management of dilated cardiomyopathy and heart failure. J Am Coll Cardiol 2012;60(4):283-9.

8. Hazebroek MR, Moors S, Dennert $R$, et al. Prognostic Relevance of Gene-Environment Interactions in Patients With Dilated Cardiomyopathy: Applying the MOGE(S) Classification. J Am Coll Cardiol 2015;66(12):1313-23.

9. Belkaya $S$, Kontorovich $A R$, Byun $M$, et al. Autosomal Recessive Cardiomyopathy Presenting as Acute Myocarditis. I Am Coll Cardiol 2017;69(13):1653-65.

10. Garcia-Pavia P, Kim Y, Restrepo-Cordoba MA, et al. Genetic Variants Associated With Cancer Therapy-Induced Cardiomyopathy. Circulation 2019;140(1):31-41.

11. Ware JS, Amor-Salamanca A, Tayal U, et al. Genetic Etiology for Alcohol-Induced Cardiac Toxicity. J Am Coll Cardiol 2018;71(20):2293-302.
12. Arbustini E, Narula N, Tavazzi L, et al. The MOGE(S) classification of cardiomyopathy for clinicians. J Am Coll Cardiol 2014;64(3):304-18.

13. Hasselberg NE, Haland TF, Saberniak J, et al. Lamin A/C cardiomyopathy: young onset, high penetrance, and frequent need for heart transplantation. Eur Heart J 2017

14. Verdonschot JAJ, Hazebroek MR, Derks KWJ, et al. Titin cardiomyopathy leads to altered mitochondrial energetics, increased fibrosis and long-term life-threatening arrhythmias. Eur Heart J 2018

15. Verdonschot JAJ, Hazebroek MR, Ware JS, et al. Role of Targeted Therapy in Dilated Cardiomyopathy: The Challenging Road Toward a Personalized Approach. Journal of the American Heart Association 2019;8(11):e012514.

16. Caforio AL, Pankuweit $\mathrm{S}$, Arbustini $\mathrm{E}$, et al. Current state of knowledge on aetiology, diagnosis, management, and therapy of myocarditis: a position statement of the European Society of Cardiology Working Group on Myocardial and Pericardial Diseases. Eur Heart J 2013;34(33):2636-48, 48a-48d.

17. Richards S, Aziz N, Bale S, et al. Standards and guidelines for the interpretation of sequence variants: a joint consensus recommendation of the American College of Medical Genetics and Genomics and the Association for Molecular Pathology. Genetics in medicine : official journal of the American College of Medical Genetics 2015;17(5):405-24.

18. Ware JS, Cook SA. Role of titin in cardiomyopathy: from DNA variants to patient stratification. Nature reviews Cardiology 2018;15(4):241-52.

19. van Tienen FHJ, Lindsey PJ, Kamps MAF, et al. Assessment of fibroblast nuclear morphology aids interpretation of LMNA variants. European journal of human genetics : EJHG 2019;27(3):389-99.

20. Caforio ALP, Adler Y, Agostini C, et al. Diagnosis and management of myocardial involvement in systemic immune-mediated diseases: a position statement of the European Society of Cardiology Working Group on Myocardial and Pericardial Disease. Eur Heart J 2017;38(35):2649-62.

21. Lenneman CG, Sawyer DB. Cardio-Oncology: An Update on Cardiotoxicity of Cancer-Related Treatment. Circulation research 2016;118(6):1008-20.

22. Gopinathannair R, Etheridge SP, Marchlinski FE, et al. Arrhythmia-Induced Cardiomyopathies: Mechanisms, Recognition, and Management. J Am Coll Cardiol 2015;66(15):1714-28.

23. Auffret V, Martins RP, Daubert C, et al. Idiopathic/latrogenic Left Bundle Branch Block- 
Induced Reversible Left Ventricle Dysfunction: JACC State-of-the-Art Review. J Am Coll Cardiol 2018;72(24):3177-88.

24. Ponikowski P, Voors AA, Anker SD, et al. 2016 ESC Guidelines for the diagnosis and treatment of acute and chronic heart failure: The Task Force for the diagnosis and treatment of acute and chronic heart failure of the European Society of Cardiology (ESC)Developed with the special contribution of the Heart Failure Association (HFA) of the ESC. Eur Heart J 2016;37(27):2129200.

25. Yancy CW, Jessup M, Bozkurt B, et al. 2017 ACC/AHA/HFSA Focused Update of the 2013 ACCF/AHA Guideline for the Management of Heart Failure: A Report of the American College of Cardiology/American Heart Association Task Force on Clinical Practice Guidelines and the Heart Failure Society of America. Circulation 2017;136(6):e137-e61.

26. Tingley D, Yamamoto $T$, Hirose $K$, et al. Mediation: $R$ package for causal mediation analysis. 2014

27. Mazzarotto F, Tayal U, Buchan RJ, et al. Reevaluating the Genetic Contribution of Monogenic Dilated Cardiomyopathy. Circulation 2020;141(5):387-98.

28. Felker GM, Thompson RE, Hare JM, et al. Underlying causes and long-term survival in patients with initially unexplained cardiomyopathy. N Engl J Med 2000;342(15):1077-84.

29. Sotiriou E, Heiner S, Jansen T, et al. Therapeutic implications of a combined diagnostic workup including endomyocardial biopsy in an all-comer population of patients with heart failure: a retrospective analysis. ESC heart failure 2018;5(4):630-41.

30. Hershberger RE, Givertz MM, Ho CY, et al. Genetic Evaluation of Cardiomyopathy-A Heart Failure Society of America Practice Guideline. J Card Fail 2018;24(5):281-302.

31. Walsh R, Thomson KL, Ware JS, et al. Reassessment of Mendelian gene pathogenicity using 7,855 cardiomyopathy cases and 60,706 reference samples. Genetics in medicine : official journal of the American College of Medical Genetics 2017;19(2):192-203.

32. Dal Ferro $M$, Stolfo $D$, Altinier $A$, et al. Association between mutation status and left ventricular reverse remodelling in dilated cardiomyopathy. Heart (British Cardiac Society) 2017

33. Haas J, Frese KS, Peil B, et al. Atlas of the clinical genetics of human dilated cardiomyopathy. Eur Heart J 2015;36(18):1123-35a.

34. Akinrinade $\mathrm{O}$, Helio $\mathrm{T}$, Lekanne Deprez $\mathrm{RH}$, et al. Relevance of Titin Missense and Non-
Frameshifting Insertions/Deletions Variants in Dilated Cardiomyopathy. Sci Rep 2019;9(1):4093.

35. Halliday BP, Baksi AJ, Gulati A, et al. Outcome in Dilated Cardiomyopathy Related to the Extent, Location, and Pattern of Late Gadolinium Enhancement. JACC Cardiovasc Imaging 2018

36. Gigli M, Merlo M, Graw SL, et al. Genetic Risk of Arrhythmic Phenotypes in Patients With Dilated Cardiomyopathy. J Am Coll Cardiol 2019;74(11):1480-90.

37. Corden B, Jarman J, Whiffin N, et al. Association of Titin-Truncating Genetic Variants With Lifethreatening Cardiac Arrhythmias in Patients With Dilated Cardiomyopathy and Implanted Defibrillators. JAMA Netw Open 2019;2(6):e196520.

38. Kumar S, Androulakis AF, Sellal JM, et al. Multicenter Experience With Catheter Ablation for Ventricular Tachycardia in Lamin A/C Cardiomyopathy. Circulation Arrhythmia and electrophysiology 2016;9(8)

39. Verdonschot JAJ, Vanhoutte EK, Claes GRF, et al. A mutation update for the FLNC gene in myopathies and cardiomyopathies. Hum Mutat 2020

40. Ho CY, Day SM, Ashley EA, et al. Genotype and Lifetime Burden of Disease in Hypertrophic Cardiomyopathy: Insights from the Sarcomeric Human Cardiomyopathy Registry (SHaRe). Circulation 2018;138(14):1387-98.

41. Ingles J, Goldstein J, Thaxton C, et al. Evaluating the Clinical Validity of Hypertrophic Cardiomyopathy Genes. Circulation Genomic and precision medicine 2019;12(2):e002460. 


\section{SUPPLEMENTARY METHODS}

\section{Classification according to the ACMG guidelines}

The ACMG guidelines forms a framework for variant classification and the implementation of the proposed criteria needs to be defined for the disease and genes on which it is applied. To make the ACMG criteria fit for our data, we defined the following criteria to "Dilated cardiomyopathy" and the 48 sequenced genes (Suppl. Table 1).

Note that the ACMG guidelines are already specified and adapted to variants in MYH7. These specific guidelines were used to classify all MYH7 variants in this study.

- PVS1: null variant in a gene where loss-of-function is a known mechanism related to DCM: TTN, FLNC, BAG3, DSP, TPM1, LMNA and VCL. Based on the Atlas of Cardiac Genetic Variation.

- The following (curated) databases were used to search for established pathogenic variants in a gene: Human Gene Mutation Database (HGMD), Leiden Open Variation Database (LOVD) and ClinVar. Pathogenic variants annotated in these database were used to score PS1, PM5 and PP5.

- The aforementioned database and additional searches on PubMed were performed to analyze available functional studies supporting pathogenicity of the variant (PS3). For selected $L M N A$ variants, additional functional evidence was sought by microscopic analysis of the nuclear lamina on patients' fibroblasts, as previously reported. Variants were scored with PS3 when the score on this validated nuclear morphology classifier was indicative of a laminopathy.

- The gnomAD database was used to analyze the prevalence of a detected variant in the population. PM2 was scored when the variant was absent from gnomAD or present $<0.004 \%$.

- If segregation data was available, PP1 was scored when the variant segregates $\geq 3$ meioses. As stated in the ACMG guideline, increased segregation data can increase the support for a variant to be pathogenic, although not further defined.

- PP2: missense variants in a gene that has a low rate of benign missense variation and in which missense variants are a common mechanism related to DCM: ACTC1, TNNT2 and TPM1.

- Four in-silico prediction tools were used to support a deleterious effect on the gene: Align GVGD, SIFT, MutationTaster and PolyPhen-2. PP3 was scored when $\geq 3$ of the insilico tools predicted a deleterious effect.

\section{Family segregation}

Family segregation analysis is performed according to hospital protocols to ensure standardization. The protocols are based on the Dutch law and recommendations from the Cardiogenetics workgroup of the Dutch Clinical Genetics Society (Vereniging Klinische Genetica Nederland (VKGN)). In short, the most important restrictions and possibilities for family segregation are:

1. Directly contacting or informing a relative of a patient is prohibited. All contact to relatives has to go via the index patient. To facilitate this, referral letters are created that the index patient can give to his/her relatives. This means that the index patient is responsible to actively inform his relatives, and the geneticist is expected to facilitate this. 
2. All relatives are advised to undergo cardiac examination. The results of these cardiac examinations are collected, except for a minority for whom cardiac examination is performed at another hospital.

3. In the case of a VUS (class 3 variant) in the index patient, relatives are advised to undergo cardiac examination. When cardiac abnormalities are detected, the relative can choose to undergo genetic testing for the specific VUS. When cardiac examination is normal, genetic testing is not performed.

4. In the case of a (likely) pathogenic variant (class 4 or 5 variant), relatives have the choice between genetic testing and/or cardiac examination.

5. If a relative already is known to have documented DCM, they are offered a full genetic evaluation including the complete panel of 47 genes.

\section{Registration of adverse events}

The Maastricht University Medical Center (MUMC) is connected to the Dutch Personal Records Database, which registers if a patient deceases. All included patients in the Maastricht Cardiomyopathy Registry gave informed consent to be contacted about any information regarding adverse events. Every 6-12 months, all included patients are contacted and questioned regarding the occurrence of adverse events:

- $\quad$ Did the patient receive a heart transplant or is on the waiting-list or is being considered for a heart transplantation?

- $\quad$ Did the patient receive a left ventricular assist device or is being considered for a left ventricular assist device implantation?

- Was the patient admitted to hospital? If yes, was this hospitalization for an elective procedure? Was this hospitalization cardiovascular/heart failurerelated?

If there is an indication that an adverse event took place, medical information is retrieved from the concerned hospital. If the hospitalization or hospital visit could indicate a lifethreatening arrhythmia (ventricular fibrillation (with or without ICD-shock), hemodynamic unstable sustained ventricular tachycardia, and/or sustained ventricular tachycardia with appropriate ICD shock), this specific information is requested.

\section{Definition of electrical phenotypes}

We use the term electrical phenotype to denote electrical disturbances that could be either cause or consequence of DCM. They contribute to the disease phenotype but are often associated with genetic and other etiologies of DCM. The five most common electrical disturbances were defined as an electrical phenotype, which are all measurable via electrical monitoring, and subjected to clear definitions:

- Conduction disorders

- Atrioventricular block: comprising first, second and third degree blocks

- First degree: PR-interval greater than $200 \mathrm{~ms}$. Every P-wave is followed by a QRS-complex.

- Second degree: both Mobitz I (increase in PR-duration with a skipping of a beat) and Mobitz II (not all P-waves are followed by a QRS-complex) are considered.

- Third degree: no relationship between P-waves and QRS complexes (loss of 1:1 ratio). 
- Left bundle branch block: defined using the Strauss criteria: QRS duration $\geq 140$ ms for men and $\geq 130$ ms for women, along with mid-QRS notching or slurring in $\geq 2$ contiguous leads.

- Arrhythmias

- Non-sustained ventricular tachycardia: three or more consecutive beats with a heart rate $>100$ beats per minute with a duration less than 30 seconds.

- Frequent premature ventricular complexes: $>20 \%$ of total heartbeats are PVCs on a 24-hour registration.

- Atrial fibrillation: absolutely irregular RR intervals and no discernible, distinct P-waves on ECG.

All above mentioned electrical phenotypes are diagnosed in patients using the ECG/Holter source documentation and medical history. 


\section{SUPPLEMENTARY FIGURES}

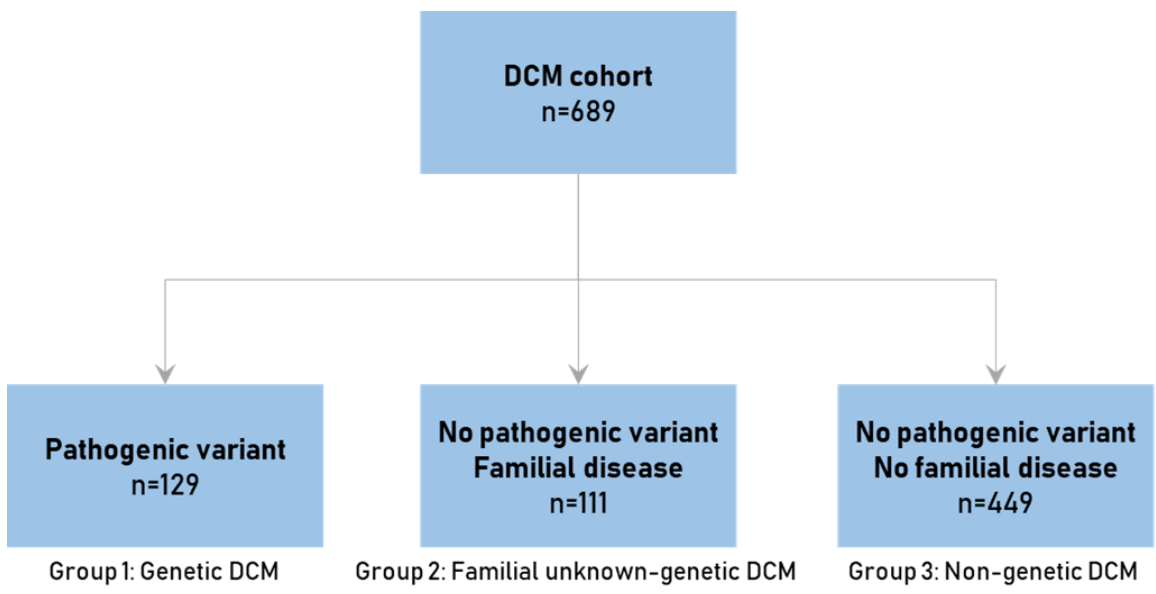

Supplemental Figure 1. Dilated cardiomyopathy (DCM) study population
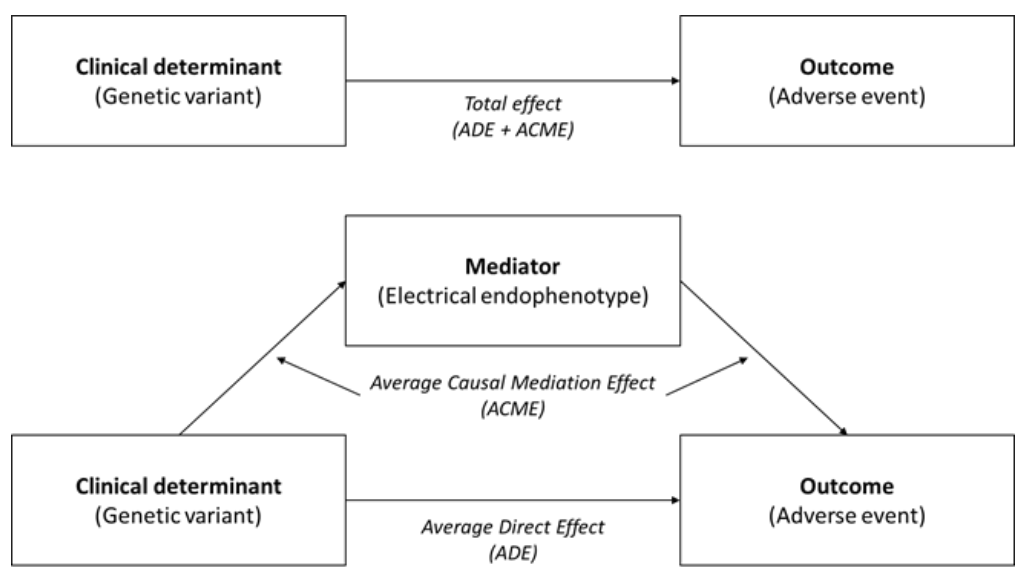

Causal Mediation Analysis

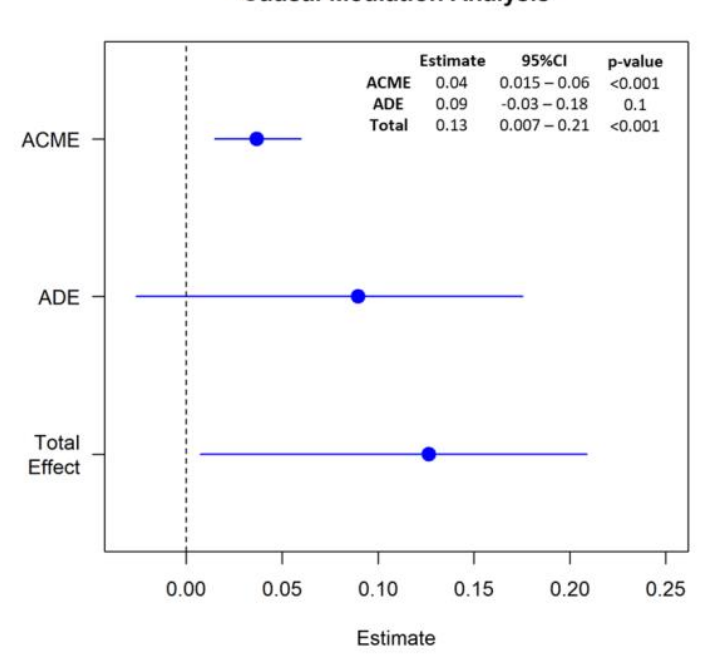

Supplemental Figure 2. Causal mediation analysis of the mediating effect of an electrical phenotype on the outcome of dilated cardiomyopathy (DCM) patients with a genetic variant. In total there is a significant effect of having a genetic variant with regards to outcome (total effect $p<0.001$ ), however this is mediated through the association with an electrical phenotype (ACME $p<0.001$ and ADE $p=0.1$ ). 


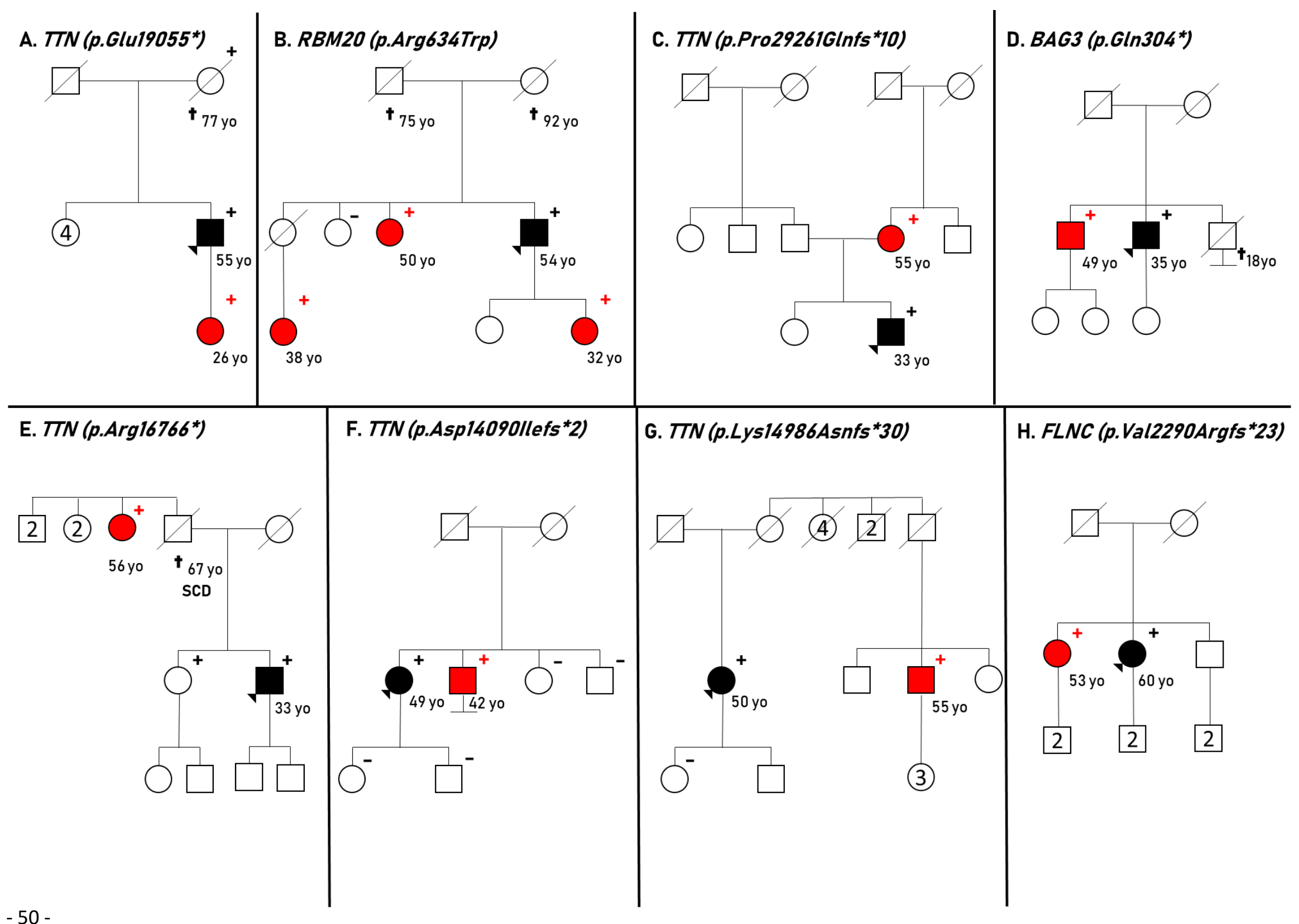




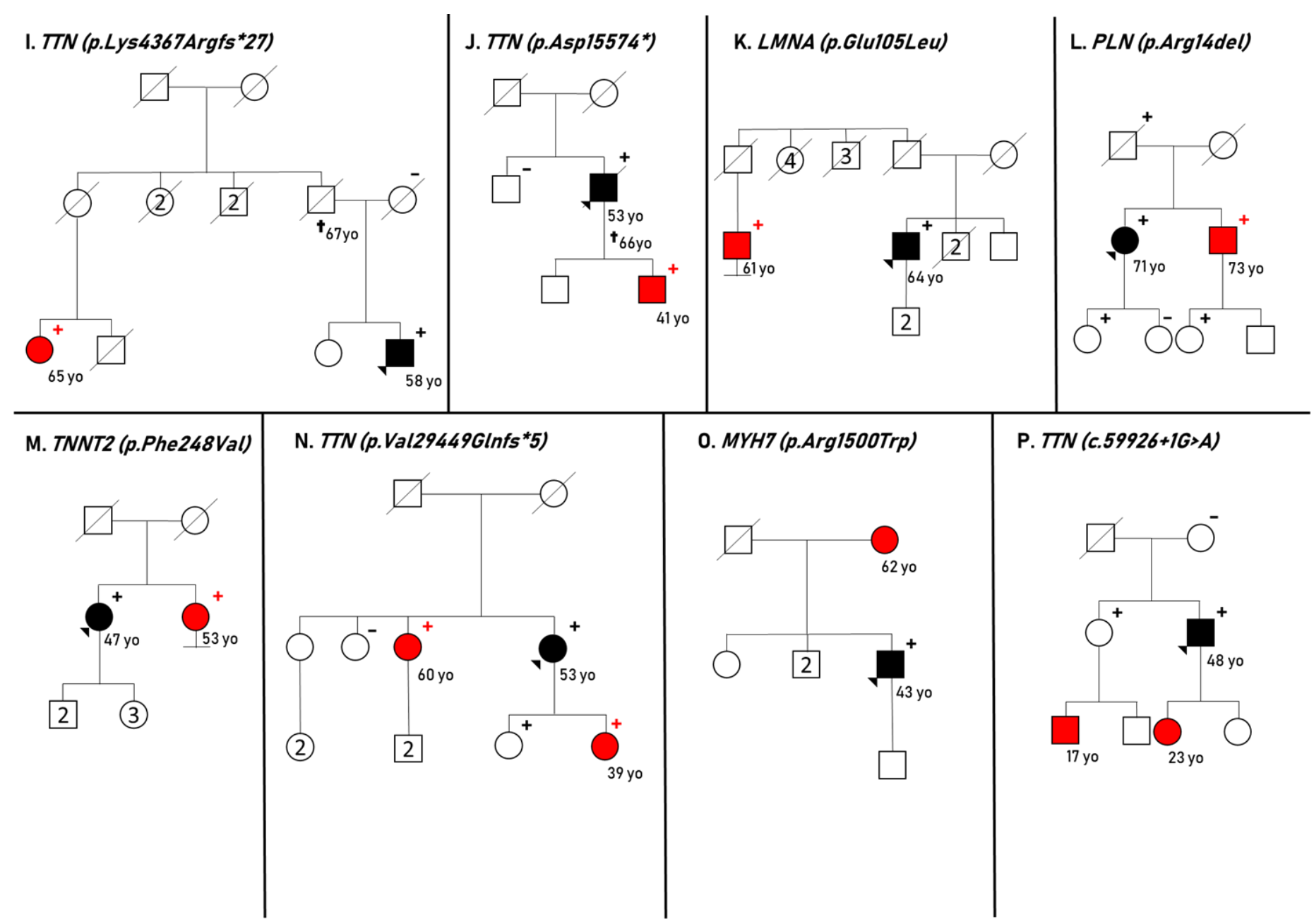

Supplemental Figure 3. Family pedigrees illustrating the importance of family segregating by cardiac and genetic screening although the DCM is initially classified as nonfamilial. Pedigree $\mathbf{A}-\mathbf{N}$ are of non-familial DCM families in which relatives have been detected with both a phenotype and the genetic variant. Pedigree $\mathbf{O}$ and $\mathbf{P}$ depict two pedigrees of non-familial DCM families in which relatives are diagnosed with DCM after cardiac examination. No genetic testing was performed in these relatives. $+=$ Carrier of familial variant; - = familial variant absent; arrow = index patient; red color = asymptomatic DCM, discovered after cardiac screening during family segregation; yo = years old; $S C D=$ sudden cardiac death. 


\section{Outcome stratified by non-genetic risk factors within the genetic DCM subgroup ( $n=129)$}

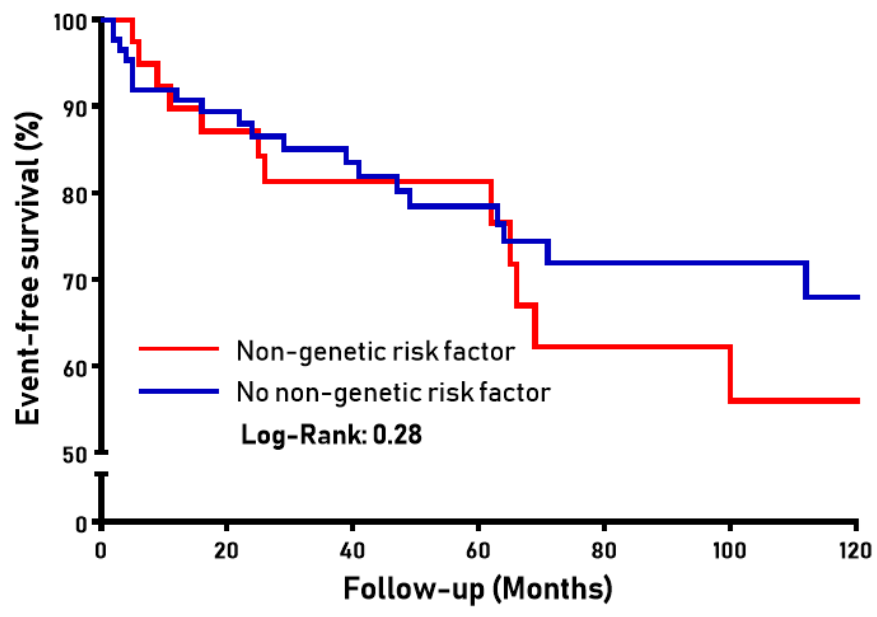

$\begin{array}{cccccccc}\begin{array}{c}\text { Number at risk } \\ \text { Non-genetic risk factor }\end{array} & 41 & 30 & 22 & 17 & 11 & 10 & 5 \\ \begin{array}{c}\text { No non-genetic risk } \\ \text { factor }\end{array} & 88 & 64 & 52 & 41 & 29 & 25 & 13\end{array}$

Supplemental Figure 4. Survival free of death, heart transplantation, heart failure hospitalization or lifethreatening arrhythmias stratified by the combination of a genetic and a non-genetic risk factor. Kaplan-Meier curves for the combined outcome.

Outcome stratified by genetic status without $\angle M N A$ variants

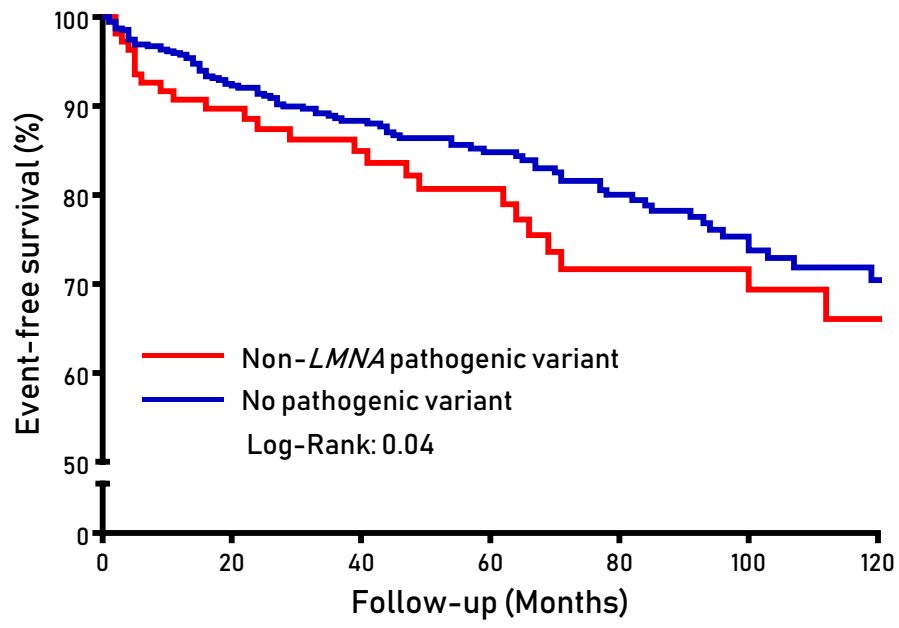

Number at risk

$\begin{array}{llllllll}\text { No pathogenic variant } & 560 & 427 & 296 & 201 & 144 & 97\end{array}$

Supplemental Figure 5. Survival free of death, heart transplantation, heart failure hospitalization or lifethreatening arrhythmias stratified by genetic status (with the exclusion of all pathogenic LMNA variants). KaplanMeier curves for the combined outcome. 


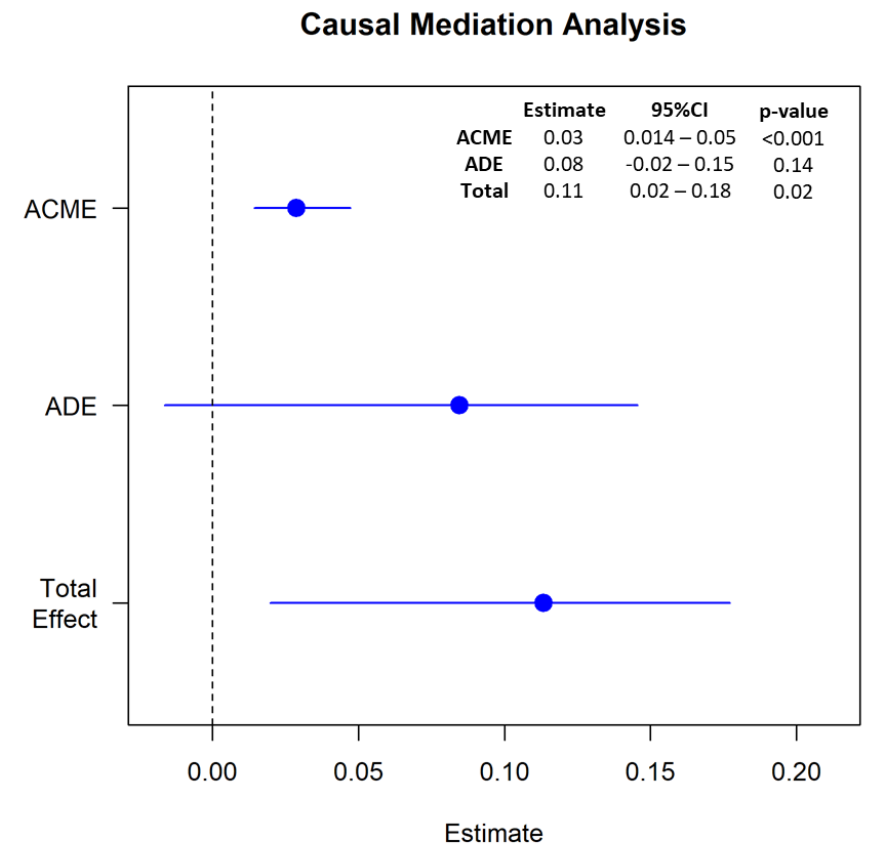

Supplemental Figure 6. Causal mediation analysis of the mediating effect of an electrical phenotype on the outcome of dilated cardiomyopathy (DCM) patients with a pathogenic gene variant (with the exclusion of all pathogenic LMNA variants). In total there is a significant effect of having a genetic variant with regards to outcome (total effect $p=0.02$ ), however this is mediated through the association with an electrical phenotype (ACME $p<0.001$ and ADE $p=0.14$ ).

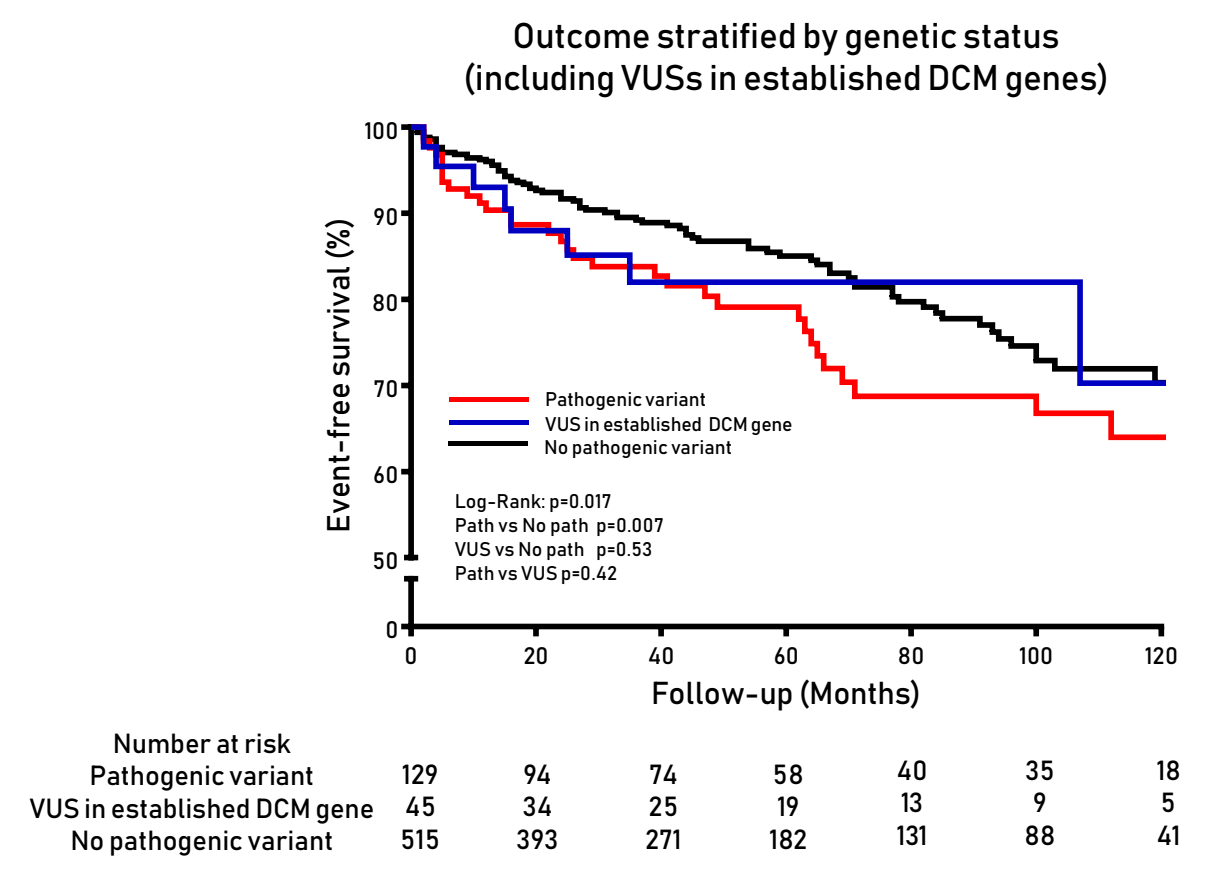

Supplemental Figure 7. Survival free of death, heart transplantation, heart failure hospitalization or lifethreatening arrhythmias stratified by genetic status. Kaplan-Meier curves for the combined outcome. 


\section{SUPPLEMENTARY TABLES}

Supplemental Table 1. Overview of all 48 genes used in the Maastricht Cardiomyopathy gene-panel HGNC.ID HGNC.symbol

\section{HGNC.Name}

\begin{tabular}{|c|c|c|c|}
\hline 1 & HGNC:143 & ACTC1 & Actin, alpha, cardiac muscle 1 \\
\hline 2 & HGNC:164 & ACTN2 & Actinin alpha 2 \\
\hline 3 & HGNC:15819 & $A N K R D 1$ & Ankyrin repeat domain 1 \\
\hline 4 & HGNC:939 & $B A G 3$ & BCL2 associated athanogene 3 \\
\hline 5 & HGNC:20407 & CALR3 & Calreticulin 3 \\
\hline 6 & HGNC:1529 & CAV3 & Caveolin 3 \\
\hline 7 & HGNC:2389 & CRYAB & Crystallin alpha B \\
\hline 8 & HGNC:2472 & CSRP3 & Cysteine and glycine rich protein 3 \\
\hline 9 & HGNC:2511 & CTNNA3 & Catenin alpha 3 \\
\hline 10 & HGNC:2770 & $D E S$ & Desmin \\
\hline 11 & HGNC:3036 & $D S C 2$ & Desmocollin 2 \\
\hline 12 & HGNC:3049 & $D S G 2$ & Desmoglein 2 \\
\hline 13 & HGNC:3052 & $D S P$ & Desmoplakin \\
\hline 14 & HGNC:3331 & $E M D$ & Emerin \\
\hline 15 & HGNC:3702 & FHL1 & Four and a half LIM domains 1 \\
\hline 16 & HGNC:4296 & GLA & Galactosidase alpha \\
\hline 17 & HGNC:14202 & $\mathrm{JPH} 2$ & Junctophilin 2 \\
\hline 18 & HGNC:6207 & $J U P$ & Junction plakoglobin \\
\hline 19 & HGNC:6484 & LAMA4 & Laminin subunit alpha 4 \\
\hline 20 & HGNC:6501 & LAMP2 & Lysosomal associated membrane protein 2 \\
\hline 21 & HGNC:15710 & $\angle D B 3$ & LIM domain binding 3 \\
\hline 22 & HGNC:6636 & LMNA & Lamin A/C \\
\hline 23 & HGNC:21086 & MIB1 & Mindbomb E3 ubiquitin protein ligase 1 \\
\hline 24 & HGNC:7551 & MYBPC3 & Myosin binding protein $\mathrm{C}$, cardiac \\
\hline 25 & HGNC:7576 & MYH6 & Myosin heavy chain 6 \\
\hline 26 & HGNC:7577 & MYH7 & Myosin heavy chain 7 \\
\hline 27 & HGNC:7583 & MYL2 & Myosin light chain 2 \\
\hline 28 & HGNC:7584 & MYL3 & Myosin light chain 3 \\
\hline 29 & HGNC:1330 & MYOZ2 & Myozenin 2 \\
\hline 30 & HGNC:23246 & MYPN & Myopalladin \\
\hline 31 & HGNC:29557 & NEXN & Nexilin F-actin binding protein \\
\hline 32 & HGNC:9024 & PKP2 & Plakophilin 2 \\
\hline 33 & HGNC:9080 & $P L N$ & Phospholamban \\
\hline 34 & HGNC:14000 & PRDM16 & PR/SET domain 16 \\
\hline 35 & HGNC:9386 & PRKAG2 & Protein kinase AMP-activated non-catalytic subunit gamma 2 \\
\hline 36 & HGNC:27424 & RBM20 & RNA binding motif protein 20 \\
\hline 37 & HGNC:10593 & SCN5A & Sodium voltage-gated channel alpha subunit 5 \\
\hline 38 & HGNC:11577 & $T A Z$ & Tafazzin \\
\hline 39 & HGNC:11610 & TCAP & Titin-cap \\
\hline 40 & HGNC:28472 & TMEM43 & Transmembrane protein 43 \\
\hline 41 & HGNC:11943 & TNNC1 & Troponin C1, slow skeletal and cardiac type \\
\hline 42 & HGNC:11947 & TNNI3 & Troponin 13 , cardiac type \\
\hline 43 & HGNC:11949 & TNNT2 & Troponin T2, cardiac type \\
\hline 44 & HGNC:12010 & TPM1 & Tropomyosin 1 \\
\hline
\end{tabular}




\begin{tabular}{cccc}
45 & HGNC:12403 & $T T N$ & Titin \\
46 & HGNC:12405 & $T T R$ & Transthyretin \\
47 & HGNC:12665 & VCL & Vinculin \\
48 & HGNC:3756 & FLNC & Filamin C \\
\hline
\end{tabular}

* FLNC was only sequenced in 172/689 (25\%) DCM patients, since the gene was added to the panel in June 2018.

Supplemental Table 2. Distribution of pathogenic and likely pathogenic variants among tested genes in patients with familial $(n=174)$ or non-familial $(n=515)$ dilated cardiomyopathy (DCM)

\begin{tabular}{|c|c|c|c|}
\hline Patients & $\begin{array}{l}\text { Total cohort } \\
\quad(n=689)\end{array}$ & Familial DCM ( $n=174)$ & Non-familial DCM (n=515) \\
\hline Pathogenic variant & $129 / 689$ (18.7\%) & $63 / 174(36.2 \%)$ & $66 / 515$ (12.8\%) \\
\hline$>1$ Pathogenic variant & $3 / 689(0.4 \%)$ & $2 / 174(1.1 \%)$ & $1 / 515(0.2 \%)$ \\
\hline$\geq 1$ VUS & $231 / 689$ (33.5\%) & $66 / 174$ (37.9\%) & $165 / 515(32 \%)$ \\
\hline Cytoskeleton & $\underline{70 / 689(10.1 \%)}$ & $\underline{36 / 174(20.6 \%)}$ & $\underline{34 / 515(6.6 \%)}$ \\
\hline TTN & $65 / 689$ (9.4\%) & $32 / 174(17.8 \%)$ & $33 / 515(6.4 \%)$ \\
\hline$N E X N$ & $2 / 689(0.3 \%)$ & $2 / 174(1.1 \%)$ & 0 \\
\hline FLNC & $3 / 172(1.7 \%)^{*}$ & $2 / 174(1.1 \%)$ & $1 / 515(0.2 \%)$ \\
\hline$\underline{\text { Sarcomere }}$ & $\underline{19 / 689(2.8 \%)}$ & $\underline{10 / 174(5.7 \%)}$ & $\underline{10 / 515(1.9 \%)}$ \\
\hline MYH7 & $7 / 689$ (1\%) & 0 & $7 / 515$ (1.4\%) \\
\hline TPM1 & $5 / 689(0.7 \%)$ & $5 / 174(2.9 \%)$ & 0 \\
\hline TNNT2 & $4 / 689(0.6 \%)$ & $2 / 174(1.1 \%)$ & $2 / 515(0.4 \%)$ \\
\hline TNNC1 & $1 / 689(0.1 \%)$ & $1 / 174(0.6 \%)$ & 0 \\
\hline ACTC1 & $2 / 689(0.3 \%)$ & $2 / 174(1.1 \%)$ & $1 / 515(0.2 \%)$ \\
\hline Desmosomes & $4 / 689(0.6 \%)$ & $\underline{1 / 174(0.6 \%)}$ & $\underline{3 / 515(0.6 \%)}$ \\
\hline$D S P$ & $4 / 689(0.6 \%)$ & $1 / 174(0.6 \%)$ & $3 / 515(0.6 \%)$ \\
\hline Nuclear Envelope & $\underline{21 / 689(3 \%)}$ & $11 / 174(6.3 \%)$ & $10 / 515(1.9 \%)$ \\
\hline LMNA & $20 / 689(2.9 \%)$ & $10 / 174(5.7 \%)$ & $10 / 515$ (1.9\%) \\
\hline$E M D$ & $1 / 689(0.1 \%)$ & $1 / 174(0.6 \%)$ & 0 \\
\hline Ion Channel & $1 / 689(0.1 \%)$ & $\underline{0}$ & $1 / 515(0.2 \%)$ \\
\hline SCN5A & $1 / 689(0.1 \%)$ & 0 & $1 / 515(0.2 \%)$ \\
\hline$\underline{\text { Nucleus }}$ & $\underline{6 / 6890.9 \%)}$ & $\underline{1 / 174(0.6 \%)}$ & $\underline{5 / 515(1 \%)}$ \\
\hline RBM20 & $5 / 689(0.7 \%)$ & $1 / 174(0.6 \%)$ & $4 / 515(0.8 \%)$ \\
\hline$B A G 3$ & $1 / 689(0.1 \%)$ & 0 & $1 / 515(0.2 \%)$ \\
\hline Sarcoplasmic Reticulum & $\underline{3 / 689(0.4 \%)}$ & $\underline{2 / 174(1.1 \%)}$ & $1 / 515(0.2 \%)$ \\
\hline$P L N$ & $3 / 689(0.4 \%)$ & $2 / 174(1.1 \%)$ & $1 / 515(0.2 \%)$ \\
\hline Other & $\underline{2 / 689(0.3 \%)}$ & $\underline{0}$ & $\underline{2 / 515(0.4 \%)}$ \\
\hline TTR & $2 / 689(0.3 \%)$ & 0 & $2 / 515(0.4 \%)$ \\
\hline \multicolumn{4}{|l|}{ Multiple Variants } \\
\hline$T T N+T N N T 2$ & $1 / 689(0.1 \%)$ & $1 / 174(0.6 \%)$ & 0 \\
\hline$T T N+L M N A$ & $1 / 689(0.1 \%)$ & $1 / 174(0.6 \%)$ & 0 \\
\hline Homozygous MYL2 & $1 / 689(0.1 \%)$ & 0 & $1 / 515(0.2 \%)$ \\
\hline
\end{tabular}

* FLNC was only sequenced in 172 DCM patients. 
Supplemental Table 3. Overview of the segregation and classification of all (likely) pathogenic gene variants

\begin{tabular}{|c|c|c|c|c|c|c|c|c|}
\hline Gene & Nucleotide & Amino Acid & $\begin{array}{c}\text { Index } \\
\text { patients }\end{array}$ & $\begin{array}{l}\text { gnoMAD } \\
\text { frequency }\end{array}$ & $\begin{array}{c}\text { ACMG } \\
\text { criteria^^}^{\wedge}\end{array}$ & Classification & Segregationt & $\begin{array}{l}\text { Additional information to } \\
\text { determine pathogenicity } \ddagger\end{array}$ \\
\hline ACTC1 & c. $813 \mathrm{G}>\mathrm{A}$ & p.Met271lle & 2 & $0 \%$ & $\begin{array}{c}\text { PM2; PM5; } \\
\text { PP1; PP2; } \\
\text { PP3 }\end{array}$ & LP & YES (2/2 families) & - \\
\hline$B A G 3$ & c. $910 \mathrm{C}>\mathrm{T}$ & p.Gln304* & 1 & $0 \%$ & $\begin{array}{l}\text { PVS1; PM2; } \\
\text { PP1; PP3; } \\
\text { PP5 }\end{array}$ & $P$ & YES & - \\
\hline$D S P$ & c.3383_3384del & Val1128Glyfs*5 & 1 & $0 \%$ & $\begin{array}{l}\text { PVS1; PM2; } \\
\text { PP1; PP3 }\end{array}$ & LP & YES & - \\
\hline$D S P$ & c.6393del & p.Gly2133Valfs*2 & 1 & $0 \%$ & $\begin{array}{l}\text { PVS1; PM2; } \\
\text { PP3; PP5 }\end{array}$ & $P$ & YES & - \\
\hline$D S P$ & c.7773_7776del & Ser2591Argfs*11 & 2 & $0.0012 \%$ & $\begin{array}{l}\text { PVS1; PM2; } \\
\text { PP3 }\end{array}$ & $P$ & $\begin{array}{l}\text { Not performed }(2 / 2 \\
\text { families) }\end{array}$ & - \\
\hline$E M D$ & c.110_112del & p.Lys37del & 1 & $0.0011 \%$ & $\begin{array}{l}\text { PM1; PM2; } \\
\text { PM4; PP3; } \\
\text { PP4; PP5 }\end{array}$ & LP & Not performed & $\begin{array}{l}\text { Patient with DCM and } \\
\text { muscle strength loss }\end{array}$ \\
\hline FLNC & c. $2838 \mathrm{~T}>\mathrm{A}$ & p.Tyr946* & 1 & $0 \%$ & $\begin{array}{l}\text { PVS1; PM2; } \\
\text { PP1; PP3; } \\
\text { PP4 }\end{array}$ & LP & YES & \\
\hline FLNC & c.6864_6867dup & p.Val2290Argfs*23 & 2 & $0 \%$ & $\begin{array}{l}\text { PVS1; PM2; } \\
\text { PP1; PP3; } \\
\text { PP4 }\end{array}$ & LP & YES (2/2 families) & \\
\hline LMNA & c. $1130 \mathrm{G}>\mathrm{T}$ & p.Arg377Leu & 1 & $0 \%$ & $\begin{array}{l}\text { PS3; PS4; } \\
\text { PM1; PM2; } \\
\text { PM5; PP1; } \\
\text { PP3; PP4; } \\
\text { PP5 }\end{array}$ & $P$ & YES & - \\
\hline LMNA & c. $1201 C>T$ & p.Arg401Cys & 1 & $0.0078 \%$ & $\begin{array}{l}\text { PS3; PP3; } \\
\text { PP4 }\end{array}$ & LP & Not performed & $\begin{array}{c}9.67 \% \text { abnormal nuclei: } \\
\text { laminopathy }\end{array}$ \\
\hline LMNA & c. $1300 \mathrm{G}>\mathrm{A}$ & p.Ala434Thr & 1 & $0.0012 \%$ & $\begin{array}{l}\text { PS3; PM2; } \\
\text { PP3; PP4 }\end{array}$ & LP & Not performed & $\begin{array}{c}\text { 19.33\% abnormal nuclei: } \\
\text { laminopathy }\end{array}$ \\
\hline LMNA & c. $1303 C>T$ & p.Arg435Cys & 1 & $0.0008 \%$ & $\begin{array}{l}\text { PS3; PM2; } \\
\text { PP3; PP4 }\end{array}$ & LP & Not performed & $\begin{array}{l}\text { 15\% abnormal nuclei: } \\
\text { laminopathy }\end{array}$ \\
\hline
\end{tabular}




\begin{tabular}{|c|c|c|c|c|c|c|c|c|}
\hline LMNA & c. $1517 A>C$ & p.His506Pro & 1 & $0.0032 \%$ & $\begin{array}{l}\text { PS3; PM1; } \\
\text { PM2; PP4 }\end{array}$ & $\mathrm{LP}$ & YES & $\begin{array}{c}\text { 11.3\% abnormal nuclei: } \\
\text { laminopathy }\end{array}$ \\
\hline LMNA & c. $208 \mathrm{G}>\mathrm{A}$ & p.Val70lle & 1 & $0 \%$ & $\begin{array}{l}\text { PS3; PM2; } \\
\text { PP3; PP4 }\end{array}$ & $\mathrm{LP}$ & YES & $\begin{array}{l}\text { 29\% abnormal nuclei: } \\
\text { laminopathy }\end{array}$ \\
\hline LMNA & c. $236 \mathrm{C}>\mathrm{A}$ & p.Ala79Asp & 1 & $0 \%$ & $\begin{array}{l}\text { PS3; PM2; } \\
\text { PP1; PP3; } \\
\text { PP4 }\end{array}$ & LP & YES & $\begin{array}{l}\text { 10.33\% abnormal nuclei: } \\
\text { laminopathy }\end{array}$ \\
\hline LMNA & c.313_314delinsTT & p.Glu105Leu & 3 & $0 \%$ & $\begin{array}{l}\text { PS3; PM1; } \\
\text { PM2; PP1; } \\
\text { PP3; PP4 }\end{array}$ & $P$ & $\begin{array}{l}\text { YES (2/3 families); } \\
\text { Not performed ( } 1 / 3 \\
\text { families) }\end{array}$ & $\begin{array}{l}\text { 18.33\% abnormal nuclei: } \\
\text { laminopathy }\end{array}$ \\
\hline LMNA & c. $357-2 A>G$ & p.? & 1 & $0 \%$ & $\begin{array}{l}\text { PS3; PM2; } \\
\text { PP1; PP3; } \\
\text { PP4; PP5 }\end{array}$ & $P$ & 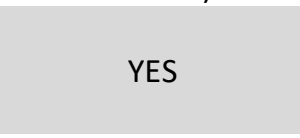 & $\begin{array}{l}\text { RNA-analysis validated } \\
\text { effect on splicing of this } \\
\text { variant }\end{array}$ \\
\hline LMNA & c. $481 G>A$ & p.Glu161Lys & 4 & $0 \%$ & $\begin{array}{l}\text { PS3; PM1; } \\
\text { PM2; PP1; } \\
\text { PP3; PP4; } \\
\text { PP5 }\end{array}$ & $\mathrm{P}$ & YES (4/4 families) & $\begin{array}{l}\text { 15\% abnormal nuclei: } \\
\text { laminopathy }\end{array}$ \\
\hline LMNA & c. $568 \mathrm{C}>\mathrm{T}$ & p.Arg190Trp & 1 & $0 \%$ & $\begin{array}{l}\text { PS3; PM1; } \\
\text { PM2; PP3; } \\
\text { PP4; PP5 }\end{array}$ & $\mathrm{P}$ & Not performed & - \\
\hline LMNA & c. $647 \mathrm{G}>\mathrm{A}$ & p.Arg216His & 1 & $0.0025 \%$ & $\begin{array}{l}\text { PS3; PM1; } \\
\text { PM2; PM5; } \\
\text { PP1; PP3; } \\
\text { PP4 }\end{array}$ & $\mathrm{P}$ & Not performed & $\begin{array}{l}15.67 \% \text { abnormal nuclei: } \\
\text { laminopathy }\end{array}$ \\
\hline LMNA & c. $658 \mathrm{C}>\mathrm{T}$ & p.Arg220Cys & 1 & $0.0024 \%$ & $\begin{array}{l}\text { PM1; PM2; } \\
\text { PM5; PP3; } \\
\text { PP4 }\end{array}$ & $\mathrm{LP}$ & YES & - \\
\hline LMNA & c. $810 G>A$ & p.Lys270Lys & 1 & 0 & $\begin{array}{l}\text { PS2; PS3; } \\
\text { PM2; PM4; } \\
\text { PP3; PP4 }\end{array}$ & $P$ & DE NOVO & $\begin{array}{l}\text { RNA-analysis in our lab } \\
\text { showed clear disturbed } \\
\text { splicing (last } 45 \mathrm{bp} \text { of exon } 4 \\
\text { are not present in the } \\
\text { mutant RNA allele). This } \\
\text { resulted in a shortened } \\
\text { protein: } \text { p.256_270del15. }\end{array}$ \\
\hline LMNA & c. $992 \mathrm{G}>\mathrm{A}$ & p.Arg331Gln & 1 & $0.0012 \%$ & $\begin{array}{l}\text { PS3; PM1; } \\
\text { PM2; PM5; }\end{array}$ & $P$ & YES & $\begin{array}{c}\text { 19.33\% abnormal nuclei: } \\
\text { laminopathy }\end{array}$ \\
\hline
\end{tabular}




\begin{tabular}{|c|c|c|c|c|c|c|c|c|}
\hline & & & & & $\begin{array}{l}\text { PP1; PP3; } \\
\text { PP4; PP5 }\end{array}$ & & & \\
\hline MYH7 & c. $1129 \mathrm{G}>\mathrm{C}$ & p.Gly377Arg & 1 & $0.0007 \%$ & $\begin{array}{l}\text { PM1; PM2; } \\
\text { PP3; } \\
\text { PS4_supp }\end{array}$ & $\mathrm{LP}$ & Not performed & $\begin{array}{c}\text { Adapted ACMG criteria for } \\
\text { MYH7 were used }\end{array}$ \\
\hline MYH7 & c. $1207 C>T$ & p.Arg403Trp & 2 & $0 \%$ & $\begin{array}{c}\text { PS4; } \\
\text { PP1_str; } \\
\text { PM1; PM2; } \\
\text { PM5; PP3; } \\
\text { PS4_supp }\end{array}$ & $P$ & $\begin{array}{l}\text { NO ( } 1 / 2 \text { families); } \\
\text { Not performed }(1 / 2 \\
\text { families) }\end{array}$ & $\begin{array}{c}\text { Adapted ACMG criteria for } \\
\text { MYH7 were used }\end{array}$ \\
\hline MYH7 & c. $2167 \mathrm{C}>\mathrm{T}$ & p.Arg723Cys & 1 & $0.0012 \%$ & $\begin{array}{c}\text { PS4; } \\
\text { PP1_str; } \\
\text { PM1; PM2; } \\
\text { PM5; PM6; } \\
\text { PP3 }\end{array}$ & $P$ & NO & $\begin{array}{c}\text { Adapted ACMG criteria for } \\
\text { MYH7 were used }\end{array}$ \\
\hline MYH7 & c. $2594 A>G$ & p.Lys865Arg & 1 & $0.0004 \%$ & $\begin{array}{l}\text { PM1; PM2; } \\
\text { PM5; } \\
\text { PS4_supp }\end{array}$ & LP & Not performed & $\begin{array}{c}\text { Adapted ACMG criteria for } \\
\text { MYH7 were used }\end{array}$ \\
\hline MYH7 & c. $4498 \mathrm{C}>\mathrm{T}$ & p.Arg1500Trp & 1 & $0 \%$ & $\begin{array}{l}\text { PS3; PM2; } \\
\text { PM5; PP1; } \\
\text { PP3; } \\
\text { PS4_supp }\end{array}$ & $P$ & Not performed & $\begin{array}{c}\text { Adapted ACMG criteria for } \\
\text { MYH7 were used }\end{array}$ \\
\hline MYH7 & c. $5774 \mathrm{G}>\mathrm{A}$ & p.Arg1925His & 1 & $0.0008 \%$ & $\begin{array}{l}\text { PM2; PM5; } \\
\text { PS4_mod; } \\
\text { PP3 }\end{array}$ & LP & NO & $\begin{array}{l}\text { Adapted ACMG criteria for } \\
\text { MYH7 were used; Dutch } \\
\text { founder mutation }\end{array}$ \\
\hline NEXN & c.1909_1912del & Tyr637Alafs*48 & 2 & $0.0011 \%$ & $\begin{array}{l}\text { PM2; PP1; } \\
\text { PP3; PP4; } \\
\text { PP5 }\end{array}$ & LP & $\begin{array}{l}\text { YES (1/2 families); } \\
\text { Not performed ( } 1 / 2 \\
\text { families) }\end{array}$ & - \\
\hline PLN & c.40_42del & p.Arg14del & 3 & $0.0007 \%$ & $\begin{array}{l}\text { PM1; PM2; } \\
\text { PM4; PP3; } \\
\text { PP5 }\end{array}$ & $\mathrm{LP}$ & YES (3/3 families) & Dutch founder mutation \\
\hline$R B M 20$ & c. $1528-1 \mathrm{G}>\mathrm{C}$ & p.? & 1 & $0 \%$ & $\begin{array}{l}\text { PVS1; PM2; } \\
\text { PP1; PP3; } \\
\text { PP4 }\end{array}$ & $\mathrm{LP}$ & YES & - \\
\hline$R B M 20$ & c. $1764 \mathrm{~T}>\mathrm{G}$ & p.lle588Met & 1 & $0 \%$ & $\begin{array}{l}\text { PM1; PM2; } \\
\text { PP3; PP4 }\end{array}$ & LP & Not performed & - \\
\hline
\end{tabular}




\begin{tabular}{|c|c|c|c|c|c|c|c|c|}
\hline$R B M 20$ & c. $1900 \mathrm{C}>\mathrm{T}$ & p.Arg634Trp & 2 & $0 \%$ & $\begin{array}{l}\text { PM1; PM2; } \\
\text { PM5; PP1; } \\
\text { PP3; PP4; } \\
\text { PP5 }\end{array}$ & LP & YES (2/2 families) & - \\
\hline$R B M 20$ & c.419del & p.Pro140Argfs*3 & 1 & $0 \%$ & $\begin{array}{l}\text { PVS1; PM2; } \\
\text { PP1; PP3; } \\
\text { PP4 }\end{array}$ & $P$ & YES & - \\
\hline SCN5A & c. $2482 C>T$ & p.Leu828Phe & 1 & $0 \%$ & $\begin{array}{l}\text { PS3; PM2; } \\
\text { PM5; PP2; } \\
\quad \text { PP3 }\end{array}$ & $P$ & NO & $\begin{array}{c}\text { Variant + functional } \\
\text { evidence published: ter } \\
\text { Bekke et al., HeartRhythm } \\
\text { Case Rep } 2018\end{array}$ \\
\hline TNNC1 & c. $317+1 G>A$ & p.? & 1 & $0 \%$ & $\begin{array}{l}\text { PS3; PM2; } \\
\text { PP1; PP3 }\end{array}$ & LP & YES & $\begin{array}{l}\text { RNA-analysis validates } \\
\text { effect on splicing: } \\
\text { r.203_317del, } \\
\text { p.Gly68Glufs*12 }\end{array}$ \\
\hline TNNT2 & c. $416 \mathrm{G}>\mathrm{A}$ & p.Arg139His & 1 & $0.0004 \%$ & $\begin{array}{l}\text { PS3; PM2; } \\
\text { PM5; PP1; } \\
\text { PP2; PP3 }\end{array}$ & $\mathrm{P}$ & YES & - \\
\hline TNNT2 & c. $742 \mathrm{~T}>\mathrm{G}$ & p.Phe248Val & 2 & $0 \%$ & $\begin{array}{l}\text { PM1; PM2; } \\
\text { PP1; PP2; } \\
\text { PP4; PP5 }\end{array}$ & LP & YES (2/2 families) & - \\
\hline TNNT2 & c. $442 C>T$ & p.Arg148Trp & 1 & $0 \%$ & $\begin{array}{l}\text { PM1; PM2; } \\
\text { PM5; PP2; } \\
\text { PP3 }\end{array}$ & LP & Not performed & - \\
\hline TPM1 & c. $184 \mathrm{G}>\mathrm{C}$ & p.Glu62Gln & 2 & $0 \%$ & $\begin{array}{l}\text { PM1; PM2; } \\
\text { PP1; PP2; } \\
\text { PP3 }\end{array}$ & LP & YES (2/2 families) & - \\
\hline TPM1 & c. $284 \mathrm{~T}>\mathrm{C}$ & p.Val95Ala & 1 & $0 \%$ & $\begin{array}{l}\text { PS3; PM2; } \\
\text { PP1; PP2; } \\
\text { PP3; PP5 }\end{array}$ & $\mathrm{P}$ & YES & - \\
\hline TPM1 & c. $829 \mathrm{G}>\mathrm{A}$ & p.Ala277Thr & 2 & $0.0028 \%$ & $\begin{array}{l}\text { PM1; PM5; } \\
\text { PP1; PP2 }\end{array}$ & LP & YES (2/2 families) & - \\
\hline$T T R$ & c. $148 \mathrm{G}>\mathrm{A}$ & p.Val50Met & 2 & $0.01 \%$ & $\begin{array}{l}\text { PS3; PM1; } \\
\text { PP3; PP5 }\end{array}$ & LP & $\begin{array}{l}\text { Not performed }(2 / 2 \\
\text { families) }\end{array}$ & $\begin{array}{c}\text { Founder mutation; both } \\
\text { patients had signs of cardiac } \\
\text { amyloidosis }\end{array}$ \\
\hline
\end{tabular}




\begin{tabular}{|c|c|c|c|c|c|c|c|c|}
\hline TTN & c.100217_100218del & p.Lys33406Argfs*13 & 1 & $0 \%$ & $\begin{array}{c}\text { PVS1; PM2; } \\
\text { PP3; PP4 }\end{array}$ & LP & Not performed & PSI $=1.0$ \\
\hline$T T N$ & c. $100825 C>T$ & p.Arg33609* & 1 & $0.0004 \%$ & $\begin{array}{c}\text { PVS1; PM2; } \\
\text { PP3; PP4 }\end{array}$ & LP & YES & $P S I=1.0$ \\
\hline TTN & c.102097_102101dup & p.Phe34034Leufs*23 & 1 & $0 \%$ & $\begin{array}{c}\text { PVS1; PM2; } \\
\text { PP3; PP4 }\end{array}$ & LP & NO & $P S I=1.0$ \\
\hline$T T N$ & c. $106792 C>T$ & p.Gln35598* & 1 & $0.0004 \%$ & $\begin{array}{l}\text { PVS1; PM2; } \\
\text { PP1; PP3; } \\
\text { PP4 }\end{array}$ & LP & YES & $\mathrm{PSI}=1.0$ \\
\hline TTN & c.13100del & p.Lys4367Argfs*27 & 7 & $0 \%$ & $\begin{array}{l}\text { PVS1; PM2; } \\
\text { PP1; PP3; } \\
\text { PP4 }\end{array}$ & LP & $\begin{array}{c}\text { YES (4/7 families); } \\
\text { NO (1/7 families); } \\
\text { Not performed ( } 2 / 7 \\
\text { families) }\end{array}$ & $P S I=1.0$ \\
\hline$T T N$ & c. $41641 C>T$ & p.Arg13881* & 1 & $0.0004 \%$ & $\begin{array}{l}\text { PVS1; PM2; } \\
\text { PP1; PP3; } \\
\text { PP4 }\end{array}$ & LP & YES & $P S I=1.0$ \\
\hline TTN & c. $42267 \mathrm{del}$ & p.Asp14090llefs*2 & 1 & $0 \%$ & $\begin{array}{l}\text { PVS1; PM2; } \\
\text { PP1; PP3; } \\
\text { PP4 }\end{array}$ & LP & YES & $P S I=1.0$ \\
\hline TTN & c.44958del & p.Lys14986Asnfs*30 & 1 & $0 \%$ & $\begin{array}{l}\text { PVS1; PM2; } \\
\text { PP1; PP3; } \\
\text { PP4 }\end{array}$ & $\mathrm{LP}$ & YES & $P S I=1.0$ \\
\hline TTN & c. $45189 \mathrm{G}>\mathrm{A}$ & p.Trp15063* & 1 & $0 \%$ & $\begin{array}{c}\text { PVS1; PM2; } \\
\text { PP3; PP4 }\end{array}$ & LP & Not performed & $P S I=1.0$ \\
\hline$T T N$ & c.46719dup & p.Asp15574* & 1 & $0 \%$ & $\begin{array}{l}\text { PVS1; PM2; } \\
\text { PP1; PP3; } \\
\text { PP4 }\end{array}$ & LP & YES & $P S I=1.0$ \\
\hline$T T N$ & c.47137_47138del & p.Ser15713Leufs*2 & 5 & $0 \%$ & $\begin{array}{l}\text { PVS1; PM2; } \\
\text { PP1; PP3; } \\
\text { PP4; PP5 }\end{array}$ & $P$ & YES (5/5 families) & $\mathrm{PSI}=1.0$ \\
\hline$T T N$ & c.50074_50075del & p.Asp16692Argfs*25 & 1 & $0 \%$ & $\begin{array}{c}\text { PVS1; PM2; } \\
\text { PP3; PP4 }\end{array}$ & $\mathrm{LP}$ & Not performed & $P S I=1.0$ \\
\hline TTN & c. $50296 C>T$ & p.Arg16766* & 1 & $0.0004 \%$ & $\begin{array}{l}\text { PVS1; PM2; } \\
\text { PP1; PP3; } \\
\text { PP4 }\end{array}$ & LP & YES & PSI $=1.0$ \\
\hline
\end{tabular}




\begin{tabular}{|c|c|c|c|c|c|c|c|c|}
\hline TTN & c.53918del & p.Gly17973Glufs*18 & 1 & $0.0004 \%$ & $\begin{array}{l}\text { PVS1; PM2; } \\
\text { PP3; PP4; } \\
\text { PP5 }\end{array}$ & LP & Not performed & $\begin{array}{c}\text { PSI = 1.0; Dutch founder } \\
\text { mutation }\end{array}$ \\
\hline TTN & c.54768del & p.Ser18258Valfs*34 & 2 & $0 \%$ & $\begin{array}{l}\text { PVS1; PM2; } \\
\text { PP3; PP4 }\end{array}$ & LP & $\begin{array}{l}\text { Not performed ( } 2 / 2 \\
\text { families) }\end{array}$ & $\mathrm{PSI}=1.0$ \\
\hline TTN & c. $57163 \mathrm{G}>\mathrm{T}$ & p.Glu19055* & 1 & $0 \%$ & $\begin{array}{l}\text { PVS1; PM2; } \\
\text { PP1; PP3; } \\
\text { PP4 }\end{array}$ & LP & YES & $\mathrm{PSI}=1.0$ \\
\hline TTN & c.58327del & p.Ala19443Leufs*2 & 3 & $0 \%$ & $\begin{array}{l}\text { PVS1; PM2; } \\
\text { PP1; PP3; } \\
\text { PP4 }\end{array}$ & LP & $\begin{array}{c}\text { YES ( } 1 / 3 \text { families); } \\
\text { Not performed ( } 2 / 3 \\
\text { families) }\end{array}$ & $\mathrm{PSI}=1.0$ \\
\hline TTN & c. $59926+1 G>A$ & p.? & 1 & $0.0004 \%$ & $\begin{array}{l}\text { PVS1; PM2; } \\
\text { PP3; PP4; } \\
\text { PP5 }\end{array}$ & LP & Not performed & Founder mutation \\
\hline TTN & c. $60931 \mathrm{C}>\mathrm{T}$ & p.Arg20311* & 2 & $0.0004 \%$ & $\begin{array}{l}\text { PVS1; PM2; } \\
\text { PP3; PP4; } \\
\text { PP5 }\end{array}$ & LP & $\begin{array}{l}\text { Not performed }(2 / 2 \\
\text { families) }\end{array}$ & PSI = 1.0 \\
\hline TTN & c. $61921 \mathrm{C}>\mathrm{T}$ & p.Arg20641* & 1 & $0 \%$ & $\begin{array}{l}\text { PVS1; PM2; } \\
\text { PP3; PP4 }\end{array}$ & LP & Not performed & $\mathrm{PSI}=1.0$ \\
\hline TTN & c. $63794-1 G>A$ & p.? & 4 & $0 \%$ & $\begin{array}{l}\text { PVS1; PM2; } \\
\text { PP1; PP3; } \\
\text { PP4; PP5 }\end{array}$ & $\mathrm{P}$ & $\begin{array}{c}\text { YES ( } 3 / 4 \text { families); } \\
\text { Not performed ( } 1 / 4 \\
\text { families) }\end{array}$ & - \\
\hline TTN & c.64688del & p.Pro21563Leufs*10 & 1 & $0.0004 \%$ & $\begin{array}{l}\text { PVS1; PM2; } \\
\text { PP3; PP4; } \\
\text { PP5 }\end{array}$ & LP & Not performed & PSI = 1.0 \\
\hline TTN & c. $64999 \mathrm{C}>\mathrm{T}$ & p.Arg21667* & 1 & $0 \%$ & $\begin{array}{l}\text { PVS1; PM2; } \\
\text { PP3; PP4; } \\
\text { PP5 }\end{array}$ & LP & NO & $\mathrm{PSI}=1.0$ \\
\hline TTN & c.66020_66021del & p.Ser22007Cysfs*18 & 1 & $0 \%$ & $\begin{array}{l}\text { PVS1; PM2; } \\
\text { PP1; PP3; } \\
\text { PP4 }\end{array}$ & LP & YES & $\mathrm{PSI}=1.0$ \\
\hline TTN & c.66296del & p.Ala22099Valfs*13 & 1 & $0 \%$ & $\begin{array}{l}\text { PVS1; PM2; } \\
\text { PP3; PP4 }\end{array}$ & LP & Not performed & PSI = 1.0 \\
\hline TTN & c. $66628 C>T$ & p.GIn22210* & 1 & $0 \%$ & $\begin{array}{l}\text { PVS1; PM2; } \\
\text { PP3; PP4; } \\
\text { PP5 }\end{array}$ & LP & Not performed & PSI = 1.0 \\
\hline
\end{tabular}




\begin{tabular}{|c|c|c|c|c|c|c|c|c|}
\hline TTN & c.67480_67484del & p.Glu22494* & 1 & $0 \%$ & $\begin{array}{c}\text { PVS1; PM2; } \\
\text { PP3; PP4 }\end{array}$ & LP & Not performed & PSI $=1.0$ \\
\hline$T T N$ & c.67667del & p.Pro22556Leufs*19 & 1 & $0 \%$ & $\begin{array}{c}\text { PVS1; PM2; } \\
\text { PP3; PP4 }\end{array}$ & LP & Not performed & $\mathrm{PSI}=1.0$ \\
\hline TTN & c. $67863 \mathrm{C}>\mathrm{G}$ & p.Tyr22621* & 1 & $0 \%$ & $\begin{array}{l}\text { PVS1; PM2; } \\
\text { PP1; PP3; } \\
\text { PP4 }\end{array}$ & LP & YES & PSI = 1.0 \\
\hline$T T N$ & c.71024_71027del & p.Lys23675Argfs*16 & 1 & $0 \%$ & $\begin{array}{c}\text { PVS1; PM2; } \\
\text { PP3; PP4 }\end{array}$ & LP & Not performed & $\mathrm{PSI}=1.0$ \\
\hline TTN & c. $71668 \mathrm{G}>\mathrm{T}$ & p.Gly23890* & 1 & $0 \%$ & $\begin{array}{c}\text { PVS1; PM2; } \\
\text { PP3; PP4 }\end{array}$ & LP & YES & $\mathrm{PSI}=1.0$ \\
\hline$T T N$ & c.72263del & p.Val24088Glufs*45 & 1 & $0 \%$ & $\begin{array}{c}\text { PVS1; PS2; } \\
\text { PM2; PP3; } \\
\text { PP4 }\end{array}$ & $P$ & DE NOVO & $\mathrm{PSI}=1.0$ \\
\hline$T T N$ & c. $73332 C>A$ & p.Cys24444* & 2 & $0 \%$ & $\begin{array}{l}\text { PVS1; PM2; } \\
\text { PP1; PP3; } \\
\text { PP4 }\end{array}$ & LP & YES (2/2 families) & $\mathrm{PSI}=1.0$ \\
\hline$T T N$ & c.78381dup & p.Arg26128Thrfs*2 & 1 & $0 \%$ & $\begin{array}{l}\text { PVS1; PM2; } \\
\text { PP1; PP3; } \\
\text { PP4 }\end{array}$ & LP & YES & $\mathrm{PSI}=1.0$ \\
\hline TTN & c.82313dup & p.Asn27438Lysfs*2 & 1 & $0 \%$ & $\begin{array}{l}\text { PVS1; PM2; } \\
\text { PP1; PP3; } \\
\text { PP4 }\end{array}$ & LP & YES & $\mathrm{PSI}=1.0$ \\
\hline$T T N$ & c. $87040 \mathrm{C}>\mathrm{T}$ & p.Arg29014* & 1 & $0.0008 \%$ & $\begin{array}{l}\text { PVS1; PM2; } \\
\text { PP1; PP3; } \\
\text { PP4; PP5 }\end{array}$ & $P$ & YES & PSI = 1.0; Founder mutation \\
\hline TTN & c. $87313 A>T$ & p.Lys29105* & 1 & $0 \%$ & $\begin{array}{c}\text { PVS1; PM2; } \\
\text { PP3; PP4 }\end{array}$ & LP & Not performed & $\mathrm{PSI}=1.0$ \\
\hline TTN & c.87782del & p.Pro29261Glnfs*10 & 5 & $0 \%$ & $\begin{array}{l}\text { PVS1; PM2; } \\
\text { PP1; PP3; } \\
\text { PP4 }\end{array}$ & LP & $\begin{array}{l}\text { YES ( } 2 / 5 \text { families); } \\
\text { Not performed ( } 3 / 5 \\
\text { families) }\end{array}$ & $\mathrm{PSI}=1.0$ \\
\hline TTN & c.88344_88359del & p.Val29449GInfs*5 & 1 & $0 \%$ & $\begin{array}{l}\text { PVS1; PM2; } \\
\text { PP1; PP3; } \\
\text { PP4 }\end{array}$ & LP & YES & $\mathrm{PSI}=1.0$ \\
\hline
\end{tabular}




\begin{tabular}{|c|c|c|c|c|c|c|c|c|}
\hline TTN & c.90370G >T & p.Glu30124* & 1 & $0 \%$ & $\begin{array}{l}\text { PVS1; PM2; } \\
\text { PP1; PP3; } \\
\text { PP4 }\end{array}$ & LP & YES & $\mathrm{PSI}=1.0$ \\
\hline TTN & c.90924_90925insT & p.SIn30309Serfs*31 & 1 & $0 \%$ & $\begin{array}{l}\text { PVS1; PM2; } \\
\text { PP1; PP3; } \\
\text { PP4 }\end{array}$ & LP & YES & $\mathrm{PSI}=1.0$ \\
\hline TTN & c.97427dup & p.Thr32477Asnfs*13 & 2 & $0 \%$ & $\begin{array}{l}\text { PVS1; PM2; } \\
\text { PP1; PP3; } \\
\text { PP4; PP5 }\end{array}$ & $\mathrm{P}$ & YES (2/2 families) & PSI $=1.0$ \\
\hline TTN & c. $65042 \mathrm{del}$ & p.Asp21681Alafs*15 & & $0 \%$ & $\begin{array}{l}\text { PVS1; PM2; } \\
\text { PP3; PP4 }\end{array}$ & LP & No data & $\mathrm{PSI}=1.0$ \\
\hline LMNA & c. $647 \mathrm{G}>\mathrm{A}$ & p.Arg216His & 1 & $0.0025 \%$ & $\begin{array}{c}\text { PS3; PM1; } \\
\text { PM2; PM5; } \\
\text { PP1; PP3; } \\
\text { PP4 }\end{array}$ & $\mathrm{P}$ & YES & $\begin{array}{c}15.67 \% \text { abnormal nuclei: } \\
\text { laminopathy }\end{array}$ \\
\hline TTN & c.97427dup & p.Thr32477Asnfs*13 & & $0 \%$ & $\begin{array}{l}\text { PVS1; PM2; } \\
\text { PP1; PP3; } \\
\text { PP4; PP5 }\end{array}$ & $\mathrm{P}$ & No data & $P S I=1.0$ \\
\hline TNNT2 & c. $742 T>G$ & p.Phe248Val & 1 & $0 \%$ & $\begin{array}{l}\text { PM1; PM2; } \\
\text { PP1; PP2; } \\
\text { PP4; PP5 }\end{array}$ & LP & YES & - \\
\hline MYL2 & c. $64 \mathrm{G}>\mathrm{A}+\mathrm{c} .64 \mathrm{G}>\mathrm{A}$ & $\begin{array}{c}\text { p.Glu22Lys + } \\
\text { p.Glu22Lys }\end{array}$ & 1 & $0.002 \%$ & $\begin{array}{l}\text { PS1; PP3; } \\
\text { PP4 }\end{array}$ & LP & Not performed & Dutch founder mutation \\
\hline
\end{tabular}


Supplemental Table 4. Overview of all variants of unknown significance

\begin{tabular}{|c|c|c|c|}
\hline Gene & Nucleotide & Amino Acid & $\begin{array}{c}\text { Index } \\
\text { patients }\end{array}$ \\
\hline ACTC1 & c. $623 G>A$ & Arg208His & 1 \\
\hline ACTN2 & c. $1907 A>G$ & p.Glu636Gly & 1 \\
\hline ACTN2 + MYH6 & c. $1484 \mathrm{C}>\mathrm{T}+\mathrm{c} .1558 \mathrm{G}>\mathrm{A}$ & p.Thr495Met + p.Ala520Thr & 1 \\
\hline$A N K R D 1$ & c. $234 \mathrm{~A}>\mathrm{T}$ & p.Arg78Ser & 1 \\
\hline$A N K R D 1$ & c. $347 C>T$ & p.Thr116Met & 1 \\
\hline CALR3 & c. $407 \mathrm{~T}>\mathrm{C}$ & p.lle136Thr & 3 \\
\hline CAV3 & c. $216 C>G$ & p.Cys72Trp & 1 \\
\hline CRYAB & c. $277 \mathrm{G}>\mathrm{T}$ & p.Val93Leu & 2 \\
\hline CRYAB & c. $487 \mathrm{C}>\mathrm{T}$ & p.Arg163Cys & 1 \\
\hline CSRP3 & c. $10 \mathrm{~T}>\mathrm{C}$ & p.Trp4Arg & 1 \\
\hline CSRP3 & c. $9 \mathrm{C}>\mathrm{G}$ & p.Asn3Lys & 1 \\
\hline$C S R P 3+S C N 5 A+M y B P C 3$ & $\begin{array}{c}\text { c. } 379 G>A+c .659 C>T+ \\
\text { c. } 644 G>A\end{array}$ & $\begin{array}{c}\text { p.Val127lle }+ \text { p.Thr220lle }+ \\
\text { p.Arg215His }\end{array}$ & 1 \\
\hline CTNNA3 & c. $1351 C>T$ & p.His451Tyr & 1 \\
\hline CTNNA3 & c. $1507 \mathrm{~A}>\mathrm{C}$ & p.lle503Leu & 1 \\
\hline CTNNA3 & c. $1647 C>A$ & p.His549GIn & 1 \\
\hline CTNNA3 & c. $1691 \mathrm{C}>\mathrm{T}$ & p.Thr564Met & 1 \\
\hline CTNNA3 & c. $2107 \mathrm{G}>\mathrm{T}$ & p.Val1703Phe & 1 \\
\hline CTNNA3 & c.2638dup & p.lle880Asnfs*9 & 1 \\
\hline CTNNA3 & c.324del & p.Phe108Leufs*25 & 1 \\
\hline CTNNA3 & c. $67 \mathrm{G}>\mathrm{A}$ & p.Val23Met & 5 \\
\hline CTNNA3 + SCN5A & $c .935 \mathrm{C}>\mathrm{T}+\mathrm{c} .4057 \mathrm{G}>\mathrm{A}$ & p.Ala312Val + Val1353Met & 1 \\
\hline CTNNA3 + MYL3 & c. $1603 C>T+$ c. $476 C>T$ & p.Arg535Cys + p.Thr159Met & 1 \\
\hline$D E S$ & c. $725 \mathrm{~T}>\mathrm{A}$ & Val242Glu & 1 \\
\hline$D E S$ & c.1310delG & p.Gly437Valfs*10 & 1 \\
\hline$D E S$ & c. $1333 A>G$ & p.Thr445Ala & 2 \\
\hline$D S C 2$ & c.21dup & p.Gly8Argfs*23 & 1 \\
\hline$D S C 2$ & c.2398del & p.Ala800Leufs*56 & 2 \\
\hline$D S C 2$ & c. $1321 \mathrm{G}>\mathrm{A}$ & p.Ala441Thr & 1 \\
\hline$D S C 2$ & c. $1721 \mathrm{G}>\mathrm{A}$ & p.Ser574Asn & 1 \\
\hline$D S C 2$ & c. $865 \mathrm{C}>\mathrm{T}$ & p.Pro289Ser & 1 \\
\hline$D S C 2+D S G 2$ & c. $2218 \mathrm{~T}>\mathrm{C}+\mathrm{c} .445 \mathrm{G}>\mathrm{A}$ & p.Ser740Pro + p.Val149lle & 1 \\
\hline$D S C 2+T M E M 43$ & $\begin{array}{c}\text { c.2686_2687dupGA + } \\
\text { c. } 947 G>C\end{array}$ & p.Ala897Lysfs*4 + p.Trp316Ser & 1 \\
\hline DSG2 & c. $862 \mathrm{G}>\mathrm{A}$ & p.Val288Ile & 1 \\
\hline$D S P$ & c. $3146 C>G$ & p.Ser1049Trp & 2 \\
\hline$D S P$ & c. $3863 A>G$ & p.Lys1288Arg & 1 \\
\hline$D S P$ & c. $5035 \mathrm{C}>\mathrm{T}$ & p.His1679Tyr & 1 \\
\hline$D S P$ & c. $6233 \mathrm{~T}>\mathrm{C}$ & p.lle2078Thr & 1 \\
\hline$D S P$ & c. $643 \mathrm{G}>\mathrm{A}$ & p.Glu215Lys & 1 \\
\hline$D S P$ & c. $695 \mathrm{G}>\mathrm{A}$ & p.Arg232His & 1 \\
\hline$D S P$ & c. $8392 \mathrm{~A}>\mathrm{T}$ & p.Thr2798Ser & 1 \\
\hline$D S P+M Y B P C 3$ & c. $8458 \mathrm{~T}>\mathrm{C}+\mathrm{c} .1696 \mathrm{~T}>\mathrm{C}$ & p.Ser2820Pro + p.Cys566Arg & 1 \\
\hline$D S P+J U P$ & c. $6059 \mathrm{G}>\mathrm{C}+$ c. $1982 \mathrm{G}>\mathrm{A}$ & p.Gly2020Ala + p.Arg661GIn & 1 \\
\hline
\end{tabular}




\begin{tabular}{|c|c|c|c|}
\hline$D S P+M Y H 6+M I B 1$ & $\begin{array}{c}\text { c. } 1163 C>T+\text { c. } 5696 G>A+ \\
\text { c. } 1371+1 G>A\end{array}$ & $\begin{array}{c}\text { p.Thr388lle }+ \text { p.Arg1899His }+ \\
\text { p.? }\end{array}$ & 1 \\
\hline$D S P+M Y H 7$ & c. $2933 T>A+$ c. $4717 G>A$ & p.lle978Lys + p.Glu1573Lys & 1 \\
\hline$D S P+S C N 5 A$ & $\begin{array}{c}\text { c.3609_3610delinsAG + } \\
\text { c.3308C>A }\end{array}$ & $\begin{array}{c}\text { p.Met1203_Ser1204delinslleGly } \\
\text { + p.Ser1103Tyr }\end{array}$ & 1 \\
\hline$E M D$ & c. $575 \mathrm{C}>\mathrm{T}$ & p.Ser192Phe & 1 \\
\hline$E M D+E M D$ & c. $148 \mathrm{C}>\mathrm{T}+\mathrm{c} .454 \mathrm{C}>\mathrm{T}$ & p.Pro50Ser + p.Arg152Cys & 1 \\
\hline$G L A$ & c. $427 \mathrm{G}>\mathrm{A}$ & p.Ala143Thr & 1 \\
\hline JPH2 & c. $1420 G>A$ & p.Glu474Lys & 1 \\
\hline JUP & c. $1219 G>A$ & p.Val407Ile & 1 \\
\hline JUP & c. $1982 \mathrm{G}>\mathrm{A}$ & p.Arg661Gln & 1 \\
\hline JUP & c. $521 \mathrm{C}>\mathrm{T}$ & p.Ala174Val & 1 \\
\hline JUP & c. $809 \mathrm{~T}>\mathrm{G}$ & p.Leu270Arg & 1 \\
\hline$J U P+R B M 20$ & c. $1730 \mathrm{G}>\mathrm{A}+\mathrm{c} .2147 \mathrm{G}>\mathrm{A}$ & p.Arg577His + p.Arg716Gln & 1 \\
\hline LAMA4 & c. $2599 \mathrm{C}>\mathrm{A}$ & p.Pro867Thr & 1 \\
\hline LAMA4 & c.4280del & p.Asn1427llefs*18 & 1 \\
\hline LAMA4 & c. $4446 \mathrm{~T}>\mathrm{G}$ & p.Phe1482Leu & 1 \\
\hline LAMA4 & c. $719-2 A>G$ & p.? & 1 \\
\hline$L A M A 4+M Y P N$ & c. $3506 C>T+c .3911 C>T$ & p.Pro1169Leu + p.Thr1304Met & 1 \\
\hline$L A M A 4+V C L$ & c. $4967 \mathrm{G}>\mathrm{T}+\mathrm{c} .2468 \mathrm{G}>\mathrm{A}$ & p.Gly1656Val + p.Arg823Gln & 1 \\
\hline$\angle D B 3$ & c. $1235 C>G$ & p.Pro412Arg & 1 \\
\hline$\angle D B 3$ & c. $160 G>A$ & p.Gly54Ser & 1 \\
\hline$\angle D B 3$ & c. $1675 C>T$ & p.Arg559Trp & 1 \\
\hline$\angle D B 3$ & c. $417 \mathrm{G}>\mathrm{T}$ & p.Glu139Asp & 1 \\
\hline$\angle D B 3$ & c. $608 \mathrm{C}>\mathrm{T}$ & p.Ser203Leu & 1 \\
\hline$\angle D B 3$ & c. $610 \mathrm{G}>\mathrm{A}$ & p.Ala204Thr & 1 \\
\hline$L D B 3+\angle A M A 4$ & c. $1675 C>T+$ c. $719-2 A>G$ & p.Arg559Trp + p.? & 1 \\
\hline LMNA & c. $328 C>A$ & p.Arg110Ser & 1 \\
\hline LMNA & c. $1001 \mathrm{G}>\mathrm{A}$ & p.Ser334Asn & 1 \\
\hline$L M N A+M Y H 7$ & c. $3690 \mathrm{C}>\mathrm{T}$ & p.Asp1230Asp & 1 \\
\hline MIB1 & c. $1771 \mathrm{~A}>\mathrm{T}$ & p.lle591Phe & 1 \\
\hline MIB1 & c. $2236 \mathrm{G}>\mathrm{C}$ & p.Gly746Arg & 1 \\
\hline MIB1 & c. $2273 T>C$ & p.Phe758Ser & 1 \\
\hline MIB1 & c. $476 \mathrm{G}>\mathrm{A}$ & p.Arg159GIn & 1 \\
\hline MIB1 & c. $4 A>G$ & p.Ser2Gly & 1 \\
\hline MIB1 & c. $2176 C>T$ & Arg906* & 1 \\
\hline MIB1 & c. $912 \mathrm{G}>\mathrm{A}$ & Trp304* & 1 \\
\hline$M y B P C 3+M I B 1$ & c. $1468 \mathrm{G}>\mathrm{A}+\mathrm{c} .632 \mathrm{del}$ & p.Gly490Arg + p.Gly211Alafs*5 & 1 \\
\hline MyBPC3 & c. $1468 \mathrm{G}>\mathrm{A}$ & p.Gly490Arg & 1 \\
\hline МуВРСЗ & c. $3673 \mathrm{G}>\mathrm{A}$ & p.Ala1225Thr & 1 \\
\hline МуВРСЗ & c. $529 C>T$ & p.Arg177Cys & 1 \\
\hline MyBPC3 & c. $644 \mathrm{G}>\mathrm{A}$ & p.Arg215His & 1 \\
\hline MyBPC3 & c. $713 \mathrm{G}>\mathrm{A}$ & p.Arg238His & 1 \\
\hline MYBPC3 & c. $3065 \mathrm{G}>\mathrm{C}$ & p.Arg1022Pro & 1 \\
\hline MYBPC3 + MYBPC3 & $c .977 G>A+c .2905 C>T$ & p.Arg326Gln + p.GIn969* & 1 \\
\hline MYBPC3 & c.2373dup & p.Trp792Valfs*41 & 1 \\
\hline MyBPC3 + DSG2 & c. $703 \mathrm{G}>\mathrm{A}+\mathrm{c} .3044 \mathrm{~T}>\mathrm{C}$ & p.Gly235Ser + p.Met1015Thr & 1 \\
\hline
\end{tabular}




\begin{tabular}{|c|c|c|}
\hline MyBPC3 + LAMA4 & c. $2938 \mathrm{C}>\mathrm{T}+\mathrm{c} .3161 \mathrm{G}>\mathrm{A}$ & p.Arg980Cys + p.Gly1054Asp \\
\hline MYHG & c. $1100 A>T$ & p.GIn367Leu \\
\hline MYH6 & c. $1891+10 C>T$ & p.? \\
\hline MYH6 & c. $2228 \mathrm{G}>\mathrm{T}$ & p.Gly743Val \\
\hline MYH6 & c. $2396 \mathrm{~T}>\mathrm{C}$ & p.Met799Thr \\
\hline MYH6 & c. $2614 C>T$ & p.Arg872Cys \\
\hline MYH6 & c. $3010 G>T$ & p.Arg1004Ser \\
\hline MYH6 & c. $3139 C>A$ & p.Arg1047Ser \\
\hline MYH6 & c. $3346 C>A$ & p.Arg1116Ser \\
\hline MYH6 & c. $3346 G>A$ & p.Glu1116Lys \\
\hline MYH6 & c. $4010 \mathrm{C}>\mathrm{T}$ & p.Ser1337Leu \\
\hline MYH6 & c. $4136 \mathrm{C}>\mathrm{T}$ & p.Thr1379Met \\
\hline MYH6 & c. $4328 \mathrm{C}>\mathrm{A}$ & p.Ala1443Asp \\
\hline MYH6 & c. $4602 \mathrm{G}>\mathrm{C}$ & p.GIn1534His \\
\hline MYH6 & c. $4685 \mathrm{G}>\mathrm{A}$ & p.Arg1562Gln \\
\hline MYH6 & c. $5140 \mathrm{C}>\mathrm{T}$ & p.Arg1714Trp \\
\hline MYH6 & c.5519A $>G$ & p.Lys1840Arg \\
\hline MYH6 & c.820del & p.Val274* \\
\hline MYH6 + CAV3 & $c .4136 \mathrm{C}>\mathrm{T}+\mathrm{c} .233 \mathrm{C}>\mathrm{T}$ & p.Thr1379Met + p.Thr78Met \\
\hline$M Y H 6+M Y L 3$ & $c .831 G>T+c .476 C>T$ & p.GIn277His + p.Thr159Met \\
\hline MYHG + TNNT2 & c. $4685 G>A+c .571-8 C>A$ & p.Arg1562GIn+ p.? \\
\hline MYH7 & c. $3169 \mathrm{G}>\mathrm{A}$ & p.Gly1057Ser \\
\hline MYH7 & c. $2890 \mathrm{G}>\mathrm{C}$ & p.Val964Leu \\
\hline MYH7 & c. $2945 \mathrm{~T}>\mathrm{C}$ & p.Met982Thr \\
\hline MYH7 & c. $3407 \mathrm{G}>\mathrm{A}$ & p.Arg1136His \\
\hline MYH7 & c. $4210 \mathrm{G}>\mathrm{A}$ & p.Val1404Met \\
\hline MYH7 & c. $5026 \mathrm{C}>\mathrm{T}$ & p.Arg1676Trp \\
\hline MYH7 & c. $5704 \mathrm{G}>\mathrm{A}$ & p.Glu1902Lys \\
\hline MYH7 & c. $625 \mathrm{C}>\mathrm{A}$ & p.Gln209Lys \\
\hline MYH7 & c. $4276 \mathrm{G}>\mathrm{A}$ & p.Glu1426Lys \\
\hline MYH7 & c.2201dupA & p.Phe735Valfs*3 \\
\hline MYH7 & c. $5507 \mathrm{C}>\mathrm{T}$ & p.Ser1836Leu \\
\hline$M Y H 7+G L A$ & c. $4536 \mathrm{G}>\mathrm{C}+\mathrm{c} .427 \mathrm{G}>\mathrm{A}$ & p.Leu1512Phe + p.Ala143Thr \\
\hline$M Y H 7+J P H 2+M I B 1$ & $\begin{array}{c}\text { c. } 2945 T>C+\text { c. } 1420 G>A+ \\
\text { c. } 2011 C>T\end{array}$ & $\begin{array}{c}\text { p.Met982Thr + p.Glu474Lys + } \\
\text { p.His671Tyr }\end{array}$ \\
\hline MYL3 & c. $476 \mathrm{C}>\mathrm{T}$ & Thr159Met \\
\hline MYPN & c. $59 A>G$ & p.Tyr20Cys \\
\hline MYPN & c. $2642 A>T$ & p.Asn881lle \\
\hline MYPN & c. $2726 \mathrm{~A}>\mathrm{C}$ & p.Glu909Ala \\
\hline MYPN & c. $3848 \mathrm{G}>\mathrm{A}$ & p.Arg1283His \\
\hline MYPN & c. $806 C>T$ & Pro269Leu \\
\hline$M Y P N+P R D M 16+V C L$ & $\begin{array}{c}\text { c. } 2642 A>T+c .3091 G>A+ \\
\text { c. } 1961 A>C\end{array}$ & $\begin{array}{c}\text { p.Asn881lle }+ \text { p.Glu1031Lys + } \\
\text { p.Asn654Thr }\end{array}$ \\
\hline PKP2 & c. $184 C>A$ & p.Gln62Lys \\
\hline PKP2 & c. $2077 \mathrm{~A}>\mathrm{G}$ & p.Ser693Gly \\
\hline PRDM16 & c. $2855 \mathrm{C}>\mathrm{A}$ & p.Thr952Lys \\
\hline PRDM16 & c. $2953 \mathrm{G}>\mathrm{A}$ & p.Asp985Asn \\
\hline
\end{tabular}




\begin{tabular}{|c|c|c|}
\hline PRDM16 & c.3221C>T & p.Ser1074Leu \\
\hline PRDM16 + CSRP3 & c. $692 \mathrm{G}>\mathrm{A}+\mathrm{c} .140 \mathrm{C}>\mathrm{T}$ & p.Arg231His + p.Thr47Met \\
\hline PRKAG2 & c. $500 \mathrm{C}>\mathrm{T}$ & p.Thr167lle \\
\hline PRKAG2 & c. $803 A>G$ & p.His268Arg \\
\hline$R B M 20$ & c. $2147 \mathrm{G}>\mathrm{A}$ & p.Arg716Gln \\
\hline RBM20 & c. $3578 A>G$ & p.Tyr1193Cys \\
\hline$R B M 20$ & c. $3584 C>A$ & p.Ser1195Tyr \\
\hline$R B M 20+J P H 2$ & c. $2201 \mathrm{G}>\mathrm{A}+\mathrm{c} .88 \mathrm{~A}>\mathrm{T}$ & p.Arg734Gln + p.Thr30Ser \\
\hline RBM20 + TMEM43 & c. $2147 \mathrm{G}>\mathrm{A}+\mathrm{c} .934 \mathrm{C}>\mathrm{T}$ & p.Arg716Gln + p.Arg312Trp \\
\hline$R B M 20+V C L$ & c. $3373 G>A+c .1237 G>A$ & p.Glu1125Lys + p.Ala413Thr \\
\hline SCN5A & c. $101 \mathrm{G}>\mathrm{A}$ & p.Arg34His \\
\hline SCN5A & c. $1298 \mathrm{G}>\mathrm{A}$ & p.Arg433His \\
\hline SCN5A & c. $1381 \mathrm{~T}>\mathrm{G}$ & p.Leu461Val \\
\hline SCN5A & c. $1417 \mathrm{G}>\mathrm{A}$ & p.Glu473Lys \\
\hline SCN5A & c. $1715 C>A$ & p.Ala572Asp \\
\hline SCN5A & c. $2944 T>C$ & p.Cys982Arg \\
\hline SCN5A & c. $3835 \mathrm{G}>\mathrm{A}$ & p.Val1279lle \\
\hline SCN5A & c. $5692 C>T$ & p.Arg1898Cys \\
\hline SCN5A & c. $5843 \mathrm{~T}>\mathrm{C}$ & p.lle1948Thr \\
\hline SCN5A & c. $6016 C>G$ & p.Pro2006Ala \\
\hline SCN5A & c. $647 \mathrm{C}>\mathrm{T}$ & p.Ser216Leu \\
\hline SCN5A & c. $2254 \mathrm{G}>\mathrm{A}$ & p.Gly752Arg \\
\hline SCN5A & c. $611 C>T$ & p.Ala204Val \\
\hline SCN5A & c. $311 \mathrm{G}>\mathrm{T}$ & p.Arg104Leu \\
\hline$S C N 5 A+E M D$ & c. $262 A>G+$ c. $469 C>T$ & p.Ser88Gly + p.Arg157Trp \\
\hline$S C N 5 A+M y B P C 3$ & c. $1571 C>A+c .1544 A>G$ & p.Ser524Tyr + p.Asn515Ser \\
\hline$S C N 5 A+M Y H 7$ & c. $6016 \mathrm{C}>\mathrm{G}+\mathrm{c} .3286 \mathrm{G}>\mathrm{T}$ & p.Pro2006Ala + p. Asp1096Tyr \\
\hline$S C N 5 A+P K P 2$ & c. $3412 G>A+c .1093 A>G$ & p.Glu1138Lys + p.Met365Val \\
\hline$T C A P$ & c. $208 C>T$ & p.Arg70Trp \\
\hline TMEM43 & c. $659 \mathrm{G}>\mathrm{A}$ & p.Arg22OHis \\
\hline TMEM43 & c.934C >T & p.Arg312Trp \\
\hline TMEM43 + CAV3 & c. $934 C>T+c .216 C>G$ & p.Arg312Trp + p.Cys72Trp \\
\hline TNNC1 & c. $202+5 G>C$ & p.? \\
\hline TNNC1 & c. $430 A>G$ & p.Asn144Asp \\
\hline TNNT2 & c. $662 \mathrm{~T}>\mathrm{C}$ & p.lle221Thr \\
\hline TNNT2 & c. $722 A>G$ & p.Tyr241Cys \\
\hline$T T N$ & c. $14309 A>G$ & p.Tyr4770Cys \\
\hline TTN & c. $20565 A>G$ & p.= \\
\hline TTN & c.2486_2491del & p.Gly829_Tyr830del \\
\hline$T T N$ & c. $25489 \mathrm{C}>\mathrm{T}$ & p.Arg8497Cys \\
\hline$T T N$ & c. $27676 T>A$ & p.Cys9226Ser \\
\hline$T T N$ & c. $51520 G>C$ & p.Asp17174His \\
\hline TTN & c. $5581 C>T$ & p.Arg1861Cys \\
\hline TTN & c. $63379 \mathrm{C}>\mathrm{T}$ & p.Arg21127Cys \\
\hline TTN & c. $64266 \mathrm{~A}>\mathrm{C}$ & p.Lys21422Asn \\
\hline$T T N$ & c.65389T>G & p.Cys21797Gly \\
\hline
\end{tabular}




\begin{tabular}{|c|c|c|c|}
\hline$T T N$ & c. $68604 C>G$ & p.Asp22868Glu & 1 \\
\hline TTN & c. $72487 C>A$ & p.Arg24163Ser & 1 \\
\hline TTN & c.76566_76568dup & p.Arg25523dup & 1 \\
\hline TTN & c. $80666 A>G$ & p.Tyr26889Cys & 1 \\
\hline$T T N$ & c. $83438 A>G$ & p.Tyr27813Cys & 1 \\
\hline TTN & c.87655_87656insAACCGCTTT & p.Gly29219delinsGluProLeuCys & 1 \\
\hline TTN & c. $93214 C>T$ & p.Arg31072Cys & 2 \\
\hline TTN & c. $97435 \mathrm{C}>\mathrm{T}$ & p.Arg32479Cys & 1 \\
\hline TTN & c. $98104 \mathrm{C}>\mathrm{T}$ & p.Pro32702Ser & 1 \\
\hline$T T N+C S R P 3$ & c. $5672 A>G+c .265 G>T$ & p.Tyr1891Cys + p.Gly89Cys & 1 \\
\hline$T T N+D E S$ & c. $14309 A>G+$ c. $1308 G>C$ & p.Tyr4470Cys + p.Arg436Ser & 1 \\
\hline$T T N+D E S+V C L$ & $\begin{array}{c}\text { c. } 100226 G>A+\text { c. } 635 G>A+ \\
\text { c. } 539 A>G\end{array}$ & $\begin{array}{c}\text { p.Cys33409Tyr + p.Arg212Gln + } \\
\text { p.Gln180Arg }\end{array}$ & 1 \\
\hline$T T N+D S G 2+J U P$ & $\begin{array}{c}\text { c. } 14309 A>G+\text { c. } 3146 G>T+ \\
\text { c. } 746 C>T\end{array}$ & $\begin{array}{c}\text { p.Tyr4770Cys + Arg1049lle + } \\
\text { p.Thr249Met }\end{array}$ & 1 \\
\hline$T T N+D S P$ & c. $21118 \mathrm{C}>\mathrm{T}+\mathrm{c} .2161 \mathrm{G}>\mathrm{A}$ & p.Arg7040* + p.Glu721Lys & 1 \\
\hline$T T N+D S P$ & c. $46133 A>G+c .6137 T>G$ & p.Asp15378Gly + p.Val2046Gly & 1 \\
\hline$T T N+D S P+L D B 3$ & $\begin{array}{c}\text { c. } 104845 C>T+\text { c. } 7994 C>T+ \\
\text { c. } 2174 T>A\end{array}$ & $\begin{array}{c}\text { p.Arg34949Cys + p.Thr2665Met } \\
+ \text { p.lle725Asn }\end{array}$ & 1 \\
\hline$T T N+M Y H 6$ & c. $29863 C>T+c .2113 C>T$ & p.Arg9955* + p.Arg705Cys & 1 \\
\hline$T T N+N E X N$ & $\begin{array}{c}\text { c.105514_105516del + } \\
\text { c.1174C>T }\end{array}$ & p.Ser35172del + p.Arg392* & 1 \\
\hline$T T N+S C N 5 A$ & c. $83062 C>T+$ c. $1858 C>T$ & p.Arg27688Cys + p.Arg620Cys & 1 \\
\hline$T T N+T T N$ & $\begin{array}{c}\text { c. } 45979 \mathrm{C}>\mathrm{T}+ \\
\text { c.105514_105516del }\end{array}$ & p.Arg15327Cys + p.Ser35172del & 1 \\
\hline$V C L$ & c. $1237 \mathrm{G}>\mathrm{A}$ & p.Ala413Thr & 1 \\
\hline$V C L$ & c. $1287 \mathrm{~T}>\mathrm{A}$ & p.Asp429Glu & 1 \\
\hline$V C L$ & c. $1558 \mathrm{C}>\mathrm{T}$ & p.Arg520Trp & 1 \\
\hline$V C L$ & c. $2444 A>G$ & p.Lys815Arg & 1 \\
\hline$V C L$ & c. $565 G>A$ & p.Val189Met & 1 \\
\hline$V C L$ & c. $2848 \mathrm{G}>\mathrm{A}$ & p.Glu950Lys & 1 \\
\hline
\end{tabular}

Supplemental Table 5. Association of electrical phenotype with adverse outcomes on Cox proportional hazards analysis

\begin{tabular}{cccc|c} 
& $\begin{array}{c}\text { Electrical } \\
\text { phenotype } \\
(\mathrm{n}=463)\end{array}$ & $\begin{array}{c}\text { No electrical } \\
\text { phenotype } \\
(\mathrm{n}=226)\end{array}$ & $\begin{array}{c}\text { Hazard Ratio } \\
{[95 \% \mathrm{Cl}]}\end{array}$ & p-value \\
\hline Death & $32(7 \%)$ & $14(6 \%)$ & - & NS \\
Heart transplantation & $11(2 \%)$ & $2(1 \%)$ & - & $\mathrm{NS}$ \\
Heart failure hospitalization & $64(14 \%)$ & $17(8 \%)$ & - & $\mathrm{NS}$ \\
$\begin{array}{c}\text { Life-threatening arrhythmia } \\
\text { Combined end-point }\end{array}$ & $63(14 \%)$ & $6(3 \%)$ & $5.0[2.2-11.6]$ & $<0.001$ \\
$\begin{array}{c}\text { Combined end-point without } \\
\text { life-threatening arrhythmia }\end{array}$ & $111(24 \%)$ & $32(14 \%)$ & $1.8[1.2-2.6]$ & 0.005 \\
\hline
\end{tabular}

Significance $<0.05$ using Cox regression analysis.

Abbreviations: Cl: Confidence Interval; NS: Not Significant. 
Supplemental Table 6. Association of non-sustained ventricular tachycardia with adverse outcomes on Cox proportional hazards analysis

\begin{tabular}{ccccc} 
& $\begin{array}{c}\text { Non-sustained } \\
\text { ventricular } \\
\text { tachycardia } \\
(\mathrm{n}=207)\end{array}$ & $\begin{array}{c}\text { No non-sustained } \\
\text { ventricular } \\
\text { tachycardia } \\
(\mathrm{n}=482)\end{array}$ & $\begin{array}{c}\text { Hazard Ratio } \\
{[95 \% \mathrm{Cl}]}\end{array}$ & p-value \\
\hline Death & $16(8 \%)$ & $30(6 \%)$ & - & NS \\
Heart transplantation & $10(5 \%)$ & $3(1 \%)$ & $5.1[1.4-18.7]$ & 0.015 \\
Heart failure hospitalization & $47(23 \%)$ & $34(7 \%)$ & $2.6[1.7-4.1]$ & $<0.001$ \\
Life-threatening arrhythmia & $51(25 \%)$ & $18(4 \%)$ & $6.1[3.6-10.5]$ & $<0.001$ \\
Combined end-point & $78(38 \%)$ & $65(13 \%)$ & $2.7[1.9-3.7]$ & $<0.001$ \\
Combined end-point without & $53(26 \%)$ & $57(12 \%)$ & $1.9[1.3-2.8]$ & 0.001 \\
life-threatening arrhythmia & 53 . & & & \\
\hline
\end{tabular}

Significance $<0.05$ using Cox regression analysis.

Abbreviations: Cl: Confidence Interval; NS: Not Significant.

Supplemental Table 7. Association of electrical phenotypes and a pathogenic gene variant (excluding $L M N A$ ).

\begin{tabular}{cccc} 
Outcome & $\begin{array}{c}\text { DCM with a pathogenic } \\
\text { variant (excl LMNA) } \\
(\mathbf{n = 1 0 8 )}\end{array}$ & $\begin{array}{c}\text { DCM without a } \\
\text { pathogenic variant } \\
(\mathbf{n = 5 6 0})\end{array}$ & p-value \\
Atrial fibrillation & $37(34 \%)$ & $120(21 \%)$ & 0.004 \\
Non-sustained VT & $45(42 \%)$ & $145(26 \%)$ & 0.001 \\
AV-block & $19(18 \%)$ & $56(10 \%)$ & 0.022 \\
Left bundle branch block & $16(15 \%)$ & $163(29 \%)$ & 0.002 \\
>20\% PVCs & $2(2 \%)$ & $47(8 \%)$ & 0.017 \\
\hline
\end{tabular}

Supplemental Table 8. Association of a pathogenic non-LMNA genetic variant with adverse outcomes on Cox proportional hazards analysis

\begin{tabular}{ccccc} 
& $\begin{array}{c}\text { Pathogenic non-LMNA } \\
\text { genetic variant } \\
(\mathrm{n}=108)\end{array}$ & $\begin{array}{c}\text { No pathogenic } \\
\text { gene variant } \\
(\mathrm{n}=560)\end{array}$ & $\begin{array}{c}\text { Hazard Ratio } \\
{[95 \% \text { Cl] }}\end{array}$ & p-value \\
\hline Death & $4(4 \%)$ & $37(7 \%)$ & - & NS \\
Heart transplantation & $3(3 \%)$ & $6(1 \%)$ & - & NS \\
Heart failure hospitalization & $13(12 \%)$ & $59(11 \%)$ & - & NS \\
Life-threatening arrhythmia & $16(15 \%)$ & $46(8 \%)$ & $2.1[1.2-3.7]$ & 0.007 \\
Combined end-point & $28(26 \%)$ & $102(18 \%)$ & $1.6[1.02-2.4]$ & 0.04 \\
Combined end-point without & $16(15 \%)$ & $83(15 \%)$ & - & NS \\
life-threatening arrhythmia & & & - & \\
\hline
\end{tabular}

Significance $<0.05$ using Cox regression analysis.

Abbreviations: Cl: Confidence Interval; NS: Not Significant. 


$$
\begin{aligned}
& \text { jôs } \\
& i_{0}^{1} i_{0}^{1} \stackrel{1}{1} i_{0}^{1}
\end{aligned}
$$




\title{
CHAPTER 3
}

\section{Titin cardiomyopathy leads to altered mitochondrial energetics, increased fibrosis and long-term life-threatening arrhythmias}

\author{
Job A.J. Verdonschot, $M D, M S c^{1,2}$ \\ Mark R. Hazebroek, $M D^{1}$ \\ Kasper W.J. Derks, PhD ${ }^{1,2}$ \\ Arantxa Barandiarán Aizpurua, $M D^{1}$ \\ Jort J. Merken, $M D^{1}$ \\ Ping Wang, $P h D^{2}$ \\ Jörgen Bierau, $P h D^{2}$ \\ Arthurvan den Wijngaard, $P h D^{2}$ \\ Simon M. Schalla, $M D, P h D^{1,3}$ \\ Myrurgia A. Abdul Hamid, $M D^{4}$ \\ Marc van Bilsen, $P h D^{1}$ \\ Vanessa P.M. van Empel, $M D, P h D^{l}$ \\ Christian Knackstedt, $M D, P h D^{1}$ \\ Hans-Peter Brunner-La Rocca, MD, PhD ${ }^{l}$ \\ Han G. Brunner, MD, $P h D^{25,6}$ \\ Ingrid P.C. Krapels, $M D, P h D^{2}$ \\ Stephane R.B. Heymans, MD, PhD $D^{1,7,8}$
}

${ }^{1}$ Department of Cardiology, Cardiovascular Research Institute (CARIM), Maastricht University Medical Center, Maastricht, The Netherlands.

${ }^{2}$ Department of Clinical Genetics, Maastricht University Medical Center, Maastricht, The Netherlands.

${ }^{3}$ Department of Radiology and Nuclear Medicine, Maastricht University Medical Center, Maastricht, The Netherlands.

${ }^{4}$ Department of Pathology, Maastricht University Medical Center, Maastricht, The Netherlands. ${ }^{5}$ Radboud University Medical Center, Department of Human Genetics, Donders Institute for Brain, Cognition and Behaviour, Nijmegen

${ }^{6}$ GROW Institute for Developmental Biology and Cancer, Maastricht University Medical Centre,

Maastricht, The Netherlands

${ }^{7}$ Department of Cardiovascular Sciences, Centre for Molecular and Vascular Biology,

KU Leuven, Belgium.

${ }^{8}$ The Netherlands Heart Institute, Nl-HI, Utrecht, The Netherlands. 


\begin{abstract}
Aims: Truncating titin variants (TTNtv) are the most prevalent genetic cause of dilated cardiomyopathy (DCM). We aim to study clinical parameters and long-term outcomes related to the TTNtv genotype, and determine the related molecular changes at tissue level in TTNtv DCM patients.
\end{abstract}

Methods and results: A total of 303 consecutive and extensively phenotyped DCM patients (including cardiac imaging, holter monitoring and endomyocardial biopsy) underwent DNA sequencing of 47 cardiomyopathy-associated genes including TTN, yielding 38 TTNtv positive (13\%) patients. At long-term follow-up (median of 45 months, up to 12 years), TTNtv DCM patients had increased ventricular arrhythmias compared to other DCM, but a similar survival. Arrhythmias are especially prominent in TTNtv patients with an additional environmental trigger (i.e. virus infection, cardiac inflammation, systemic disease, toxic exposure). Importantly, cardiac mass is reduced in TTNtv patients, despite similar cardiac function and dimensions at cardiac magnetic resonance. These enhanced life-threatening arrhythmias and decreased cardiac mass in TTNtv DCM patients go along with significant cardiac energetic and matrix alterations. All components of the mitochondrial electron transport chain are significantly upregulated in TTNtv hearts at RNA-sequencing. Also, interstitial fibrosis was augmented in TTNtv patients at histological and transcript level.

Conclusion: TTNtv lead to pronounced cardiac alterations in mitochondrial function, with increased interstitial fibrosis and reduced hypertrophy. Those structural and metabolic alterations in TTNtv hearts go along with increased ventricular arrhythmias at long-term follow-up, with a similar survival and overall cardiac function. 


\section{INTRODUCTION}

Dilated cardiomyopathy (DCM) has a prevalence of at least 1:250 and is an important cause of heart failure (HF) and transplantation in young adults ${ }^{12}$. The disease is familial in up to $45 \%$ of patients. Up to one quarter of DCM patients have a combined genetic and environmental etiology 134 . Approximately $15-20 \%$ of DCM patients have a truncating TTN variant (TTNtv), largely enriched in the titin A-band 56 . Furthermore, TTNtv located in constitutive exons (spliced in $>90 \%$ of transcripts) are strongly associated with DCM, irrespective of their position in $T T N^{5}$. As the pathogenicity of these TTNtv becomes increasingly evident, the non-genetic factors determining their penetrance remain largely unknown. Recently, chemotherapy was reported as a potential factor uncovering TTNtv effects ${ }^{7}$. Also, peripartum changes can be a factor uncovering the underlying genetic mutation as TTNtv are prevalent in patients with peripartum cardiomyopathy ${ }^{8}$. However, the overall involvement of non-genetic acquired factors is largely unclear.

Information regarding the clinical phenotypes and outcomes of DCM patients with TTNtv mutations at long-term is also sparse and often contradictory ${ }^{59}$. An increase in arrhythmias at early presentation, but a paradoxically more treatable phenotype at follow-up was reported in TTNtv patients ${ }^{910}$. However, long-term data on cardiac arrhythmias -and its relation to overall survival- are lacking.

Metabolic changes were reported in the TTNtv hearts of rats as compared to normal hearts ${ }^{6}$. Whether such metabolic changes reflect overall cardiac stress or are directly caused by the TTN mutation remains unknown. In ischemic etiologies of HF, metabolomics have provided valuable insights on the cardiometabolic disease state with additional prognostic value ${ }^{11}{ }^{12}$. By contrast, there is a paucity of data in non-ischemic DCM ${ }^{13}$. Data on cardiac structural and molecular changes in TTNtv patients are missing, but strongly needed to understand the pathophysiology of TTNtv.

Our aim in this study was to better understand and dissect the phenotypic and clinical significance of the TTNtv at long-term. We undertook deep phenotyping, longitudinal clinical follow-up and analysis of molecular pathways in endomyocardial biopsies of a large consecutive cohort of patients with TTNtv compared to TTNtv negative DCM patients.

\section{METHODS}

\section{Study design}

All clinical and follow-up data were collected from 2004 until July 2016 in the Maastricht Cardiomyopathy Registry ${ }^{3}$. Pedigree analysis and sequencing of a 47-cardiomyopathyassociated gene panel including TTN was performed in 303 consecutive, unrelated cardiomyopathy patients since 2012. The study was performed according to the declaration of Helsinki and was approved by the Medical Ethics Committee of Maastricht University Medical Centre. All patients gave written informed consent. All patients received treatment according to the latest ESC guidelines on acute and chronic HF therapy ${ }^{14}$.

\section{Patient recruitment}

Inclusion criteria for the inclusion of patients referred to our center with unexplained LV dysfunction were : 1) DCM defined as LVEF $<50 \%$ with an indexed left ventricular end diastolic diameter (LVEDDI) $>33 \mathrm{~mm} / \mathrm{m}^{2}$ (men) or $>32 \mathrm{~mm} / \mathrm{m}^{2}$ (women) measured by echocardiography; or a hypokinetic non-dilated cardiomyopathy (HNDC) defined as LVEF $<50 \%$ with an LVEDDI $\leq 33 \mathrm{~mm} / \mathrm{m}^{2}$ (men) or $\leq 32 \mathrm{~mm} / \mathrm{m}^{2}$ (women) measured by 
echocardiography ${ }^{15} 16$. This mixed population is further referred to DCM in this paper; 2) sequencing of $T T N ; 3$ ) age $\geq 18$ years; 4 ) written informed consent.

Exclusion criteria for the Maastricht Cardiomyopathy Registry included: 1) previous history of myocardial infarction and/or significant coronary artery disease (stenosis $>50 \%$ ) using coronary angiography sufficient to explain cardiac dysfunction; 2) primary valvular disease (mitral regurgitation grade $\geq 3$, aortic regurgitation grade $\geq 2$, or aortic stenosis with AVA $<1 \mathrm{~cm}^{2}$ ); 3) hypertensive heart disease; 4) congenital heart disease; 5) acute myocarditis; 6) arrhythmogenic right ventricular dysplasia; 7) hypertrophic or peripartum cardiomyopathy.

\section{Follow-up}

Patients were followed for at least 6 months after their first visit to our specialized outpatient clinic up to 12 years with a median follow-up of 45 months (interquartile range, 20-77 months). Follow-up data on (cardiac) death, heart transplantation (HTx), life-threatening ventricular arrhythmias (LTA), and unscheduled HF hospitalization were collected using medical records, municipal population register and/or telephone contact with general practitioners. End of follow-up was defined by July $1^{\text {st }} 2016$. No patients were lost to followup. The primary endpoint was the combination of cardiac death, HTX, LTAs and HF hospitalization. LTA is defined as non-fatal ventricular fibrillation (with or without ICD-shock), hemodynamic unstable sustained ventricular tachycardia and/or sustained ventricular tachycardia with appropriate ICD-shock.

\section{Transthoracic echocardiography}

Echocardiographic measurements were performed in the standard parasternal, apical and subxiphoidal views at baseline and after 1 year follow-up (median 10 months; interquartile range, 7 to 14 months). Follow-up echocardiography after 1 year was available in 260 (86\%) patients. Left ventricular reverse remodeling (LVRR) was characterized as an absolute increase of $\geq 10 \%$ or $L V E F \geq 50 \%$ with at least $5 \%$ improvement. Observers of echocardiography were blinded to clinical and genetic data.

\section{Magnetic resonance imaging}

Cine and late enhacement (LE) images were acquired on a 1.5-Tesla MRI system (Intera, Philips Medical Systems, Best, The Netherlands). LE imaging was performed 10 minutes after an intravenous bolus of $0.2 \mathrm{mmol} / \mathrm{kg}$ body weight gadolinium-diethylenetriaminepentaacetic acid (Gadovist, Bayer, Berlin, Germany). Two observers, blinded to genotyping data, analysed the cine and LE-images by using commercially available software (CAAS MRV3.0, Pie Medical Imaging, Maastricht, The Netherlands).

\section{Endomyocardial biopsy}

At least six endomyocardial biopsies (EMB) samples were taken from the right ventricular septum via the internal jugular vein or the femoral artery using a transcatheter bioptome. Biopsies were collected as part of routine diagnostics for DCM, to identify other triggers related to DCM such as viruses, inflammation, storage diseases and metabolic disorders. Biopsy specimens were used for the immunohistological analysis and for the detection of viral genomes (adenovirus, enterovirus, cytomegalovirus, parvovirus B19, human herpesvirus-6, and Epstein-Barr virus). Significant viral load was defined as $\geq 500$ copies/ $\mu g$ DNA ${ }^{317}$. A more detailed description of the methods is in the supplementals. Increased cardiac inflammation was defined as $\geq 14 \mathrm{CD} 45$, including up to $4 \mathrm{CD} 68$-infiltrating cells $/ \mathrm{mm}^{2}$ according to the 
current ESC guidelines ${ }^{18}$. Collagen fraction volume (CFV) was quantified as percentage tissue positive for Sirius red of the total myocardial area.

\section{Genetic evaluation}

All patients received genetic counseling and analysis of a 47-cardiomyopathy-associated gene-panel including TTN (Suppl. Table 1). The family history of cardiac related disease and/sudden cardiac death was obtained by pedigree analysis. Familial inheritance was defined as the presence of two or more affected non-ischemic DCM individuals in a single family ${ }^{16}$. The performance and interpretation of the genetic analysis is described in the supplemental methods.

\section{Metabolomic analysis}

Metabolomic analysis of acylcarnitines and amino acids was performed in plasma samples of all DCM patients included since February 2015 using electro-spray ionization ultraperformance liquid chromatography tandem mass spectrometry (UPLC-MS/MS) Acylcarnitine profiling and amino acid quantification was performed as described previously ${ }^{19}$.

\section{RNA-sequencing}

RNA was isolated from EMBs of selected DCM patients and checked for quality and integrity. The mRNA sequencing library was generated using TruSeq mRNA sample preparation kit (Illumina) and sequenced on the NextSeq 500 (Illumina). The bioinformatics tools Ingenuity ${ }^{\circledR}$ pathway analysis (IPA ${ }^{\circledast}$ ) and GAGE (with KEGG pathway enrichment) were used for molecular pathway analysis. Metabolic pathways were used from Wikipathways and Pathvisio software to visualize the expression changes found in the datasets. The RNA sequencing steps and bioinformatical analysis are described in more detail in the Supplemental Methods.

\section{Statistical analysis}

Variables are displayed as numbers (percentage), mean \pm standard deviation or median (interquartile range) as appropriate. Categorical variables were compared using $\chi 2$ test or Fisher's exact test. Continuous variables were compared using Student's t test or MannWhitney $U$ test. Kaplan-Meier method was used to calculate survival curves (comparison between groups by log-rank test). Univariable Cox proportional hazards regression analysis was performed to assess clinical and demographic covariates associated with event-free survival. Statistical significance was accepted at the 95\% confidence interval $(P<0.05)$. Statistical analysis was performed using SPSS 23.0 (IBM Corp., Armon, NY) software.

\section{RESULTS}

\section{Study population}

Thirty-eight out of 303 patients had a TTNtv (13\%) (Suppl. Figure 1; Suppl. Table 2). A pathogenic mutation in a different cardiomyopathy-associated gene was detected in 35 patients ( $n=12 \%$; Suppl. Table 3). None of these patients had a double pathogenic mutation. The majority of these patients were male (76\%). Significantly more patients with a TTNtv had a family history of DCM as compared to the TTNtv negative patients (Table 1; 55\% versus 15\%; $p<0.001)$. 


\section{Lower left ventricular mass in TTNtv patients}

LVEF and/or left ventricular end-diastolic/systolic diameter did not differ between the two groups at baseline (Table 1). However, indexed left ventricular mass (LVMi) was significantly reduced in TTNtv patients despite the absence of differences in left ventricular end diastolic volume (LVEDVi) (Table 1). As LVMi normally increases with increasing LVEDVi, the increase of LVMi per $1 \mathrm{ml} / \mathrm{m}^{2}$ LVEDVi is significantly reduced in TTNtv patients $\left(0.13 \mathrm{~g} / \mathrm{m}^{2}\right.$ in TTNtv versus $0.37 \mathrm{~g} / \mathrm{m}^{2} ; \mathrm{p}<0.001$ (Figure 1)).

\section{Enhanced interstitial myocardial fibrosis and arrhythmias at baseline in TTNtv patients} Interstitial myocardial fibrosis at EMB was significant higher in patients with a TTNtv (Figure 2; $12 \%$ versus $6.3 \%$; $p<0.001$ ), whereas mid-wall replacement fibrosis at CMR did not differ among the groups (Table 1). The interstitial fibrosis at EMBs had a homogeneous intramyocardial pattern, suggestive of reactive fibrosis (Figure 2; Suppl. Figure 2).

Increased interstitial myocardial fibrosis went along with a slightly higher incidence of non-sustained ventricular tachycardias (NSVT) at baseline (Suppl. Figure 3; $p=0.04$ ). Notably, TTNtv patients had more NSVT at early presentation (Table $1 ; p<0.001$ ). The latter was also reflected by a higher incidence of (pre)syncope and/or palpitations in TTNtv patients. This interstitial myocardial fibrosis at EMB did not correlate with the focal mid-wall fibrosis measured by CMR LE imaging $(p=0.11)$. In conclusion, these data revealed enhanced interstitial myocardial fibrosis at EMBs but decreased mass of TTNtv hearts, along with increased NSVTs and related symptoms at baseline.

\section{Indexed LV mass against indexed LV end-diastolic volume}

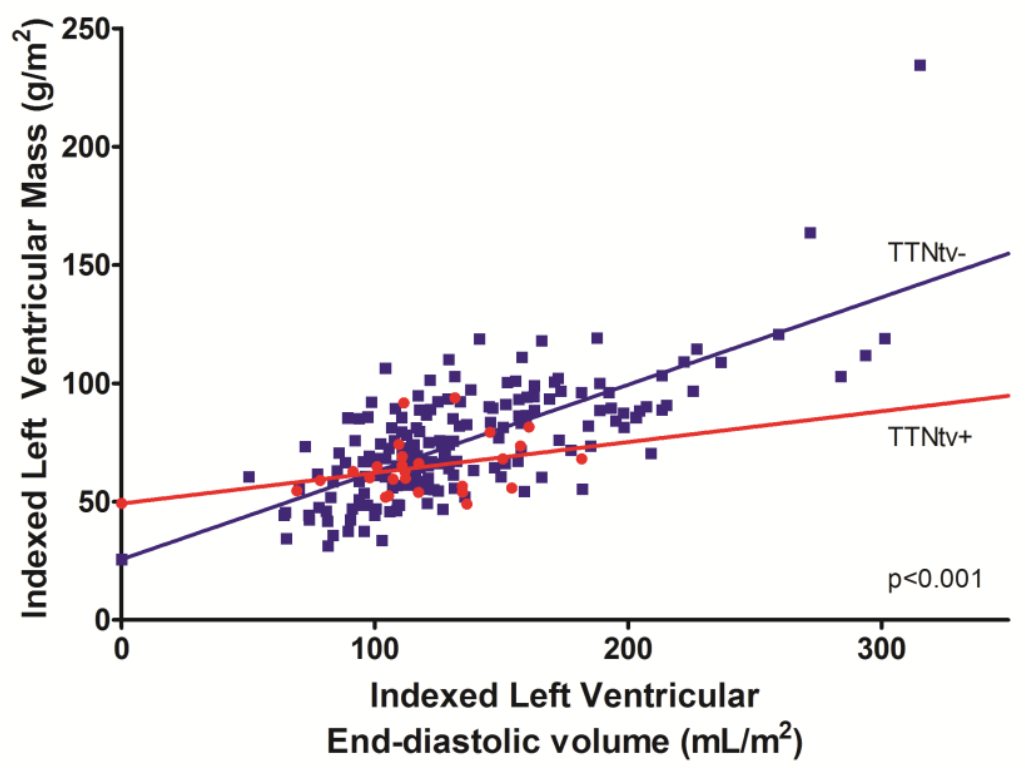

Figure 1. Indexed LV mass against indexed LV end-diastolic volume. Indexed LV mass (LVMi) is plotted against indexed LV end-diastolic volume (LVEDVi). There is a reduced increase in LVMi with increasing LVEDVi in TTNtV patients (regression equations: TTNtv+: LVMi=49.29+0.13*LVEDVi versus TTNtV-: LVMi= 26.62+0.37*LVEDVi; $\mathrm{p}<0.001)$. 
Table 1. Baseline characteristics of DCM patients with and without a TTNtv

\begin{tabular}{|c|c|c|c|}
\hline Baseline variables & $\begin{array}{l}\text { TTNtv + } \\
(n=38)\end{array}$ & $\begin{array}{l}\text { TTNtv - } \\
(n=265)\end{array}$ & P-value \\
\hline Male gender (\%) & $29(76)$ & $161(61)$ & 0.07 \\
\hline Age of diagnosis (years) & $51 \pm 10$ & $52 \pm 12$ & 0.65 \\
\hline Family history of DCM (\%) & $20(53)$ & $40(15)$ & $<0.001$ \\
\hline Hypertension (\%) & $13(34)$ & $88(33)$ & 0.90 \\
\hline Diabetes Mellitus (\%) & $6(16)$ & $27(10)$ & 0.28 \\
\hline Beta blocker (\%) & $31(82)$ & $217(82)$ & 0.96 \\
\hline ACE-I/ARB (\%) & $35(92)$ & $232(88)$ & 0.67 \\
\hline MRA (\%) & $16(42)$ & $92(35)$ & 0.37 \\
\hline NYHA $\geq 3(\%)$ & $10(26)$ & $59(22)$ & 0.58 \\
\hline Symptom duration (month) & $5[2-62]$ & $3[0-21]$ & 0.09 \\
\hline \multicolumn{4}{|l|}{ Genetic analysis } \\
\hline Pathogenic mutation (\%) & $38(100)$ & $35(13)$ & $<0.001$ \\
\hline \multicolumn{4}{|l|}{ Baseline ECG/Holter } \\
\hline cLBBB (\%) & $3(8)$ & $81(31)$ & 0.004 \\
\hline AV block (\%) & $6(16)$ & $19(7)$ & 0.16 \\
\hline Atrial fibrillation (\%) & $11(29)$ & $48(18)$ & 0.12 \\
\hline NSVT (\%) & $15(39)$ & $22(8)$ & $<0.001$ \\
\hline \multicolumn{4}{|l|}{ Echocardiography } \\
\hline LVEF (\%) & $32 \pm 10$ & $33 \pm 11$ & 0.55 \\
\hline LVEF $\leq 35 \%(\%)$ & $23(61)$ & $134(51)$ & 0.25 \\
\hline LVEDD (mm) & $60 \pm 7$ & $60 \pm 9$ & 0.99 \\
\hline LVESD $(\mathrm{mm})$ & $50 \pm 9$ & $50 \pm 11$ & 0.91 \\
\hline LVEDDi $\left(\mathrm{mm} / \mathrm{m}^{2}\right)$ & $30 \pm 4$ & $30 \pm 5$ & 0.54 \\
\hline \multicolumn{4}{|l|}{ Cardiac MRI } \\
\hline Performed (\%) & $31(82)$ & $198(75)$ & 0.36 \\
\hline LV Mi & $65 \pm 12$ & $75 \pm 24$ & 0.001 \\
\hline LV EDVi & $122 \pm 32$ & $133 \pm 47$ & 0.20 \\
\hline LV ESVi & $85 \pm 34$ & $91 \pm 49$ & 0.58 \\
\hline LV SVi & $39 \pm 10$ & $43 \pm 11$ & 0.06 \\
\hline LV EF & $32 \pm 10$ & $35 \pm 13$ & 0.17 \\
\hline Midwall fibrosis (\%) & $15(48)$ & $85(43)$ & 0.62 \\
\hline \multicolumn{4}{|l|}{ Endomyocardial Biopsy } \\
\hline Performed (\%) & $26(68)$ & $215(81)$ & 0.09 \\
\hline Cardiac Inflammation (\%) & $4(15)$ & $53(25)$ & 0.40 \\
\hline CD3+ (cells/m²) & $5.99[3.1-8.3]$ & $5[2.8-8.5]$ & 0.51 \\
\hline CD45+ (cells $\left./ \mathrm{mm}^{2}\right)$ & $8[6-10.8]$ & $9[5.5-13.2]$ & 0.31 \\
\hline CD68+ (cells/mm²) & $2[0.9-5.9]$ & $2[1.2-4.7]$ & 0.75 \\
\hline Myocardial Fibrosis & $12.14[7-16.5]$ & $6.34[3.8-9.9]$ & $<0.001$ \\
\hline \multicolumn{4}{|l|}{ Significant viral load } \\
\hline PVB19 (\%) & $2(8)$ & $28(13)$ & 0.75 \\
\hline HHV6 (\%) & $0(0)$ & $7(3)$ & 0.99 \\
\hline
\end{tabular}

Values are mean $\pm S D, n, n(\%)$, or median (interquartile range)

Abbreviations, ACE-I/ARB, angiotensin converting enzyme-inhibitor/angiotensin II receptor blocker; MRA. Mineralocorticoid receptor antagonist; cLBBB, complete left bundle branch block; AV-block, atrioventricular block; NSVT, non-sustained ventricular tachycardia; $L V E F$, left ventricular ejection fraction; LVEDD, left ventricular end-diastolic diameter; LVESD, left ventricular end-systolic diameter; LVEDDI, indexed LVEDD; LV Mi, indexed left ventricular mass; LV EDVi, indexed left ventricular end-diastolic volume; LV ESVi, indexed left ventricular end-systolic volume; LV SVi, indexed left ventricular stroke volume; PVB19, parvovirus B19; HHV6, human herpesvirus 6. 


\section{More life threatening arrhythmias but similar survival at long-term follow-up}

The rate of LVRR did not significantly differ between the groups at one-year echocardiographic follow up (Figure 3 ). A total of 51 patients $(17 \%)$ reached the primary endpoint during a median follow-up of 45 months (interquartile range, 20-77 months): cardiac death $(n=12), H t x(n=2)$, LTA $(n=28)$, or HF hospitalization $(n=18)$. Short- and long-term outcome, survival and the combined primary endpoints did not significantly differ between the groups (Table 2; Figure 4A). However, TTNtv patients had a significantly higher risk of a LTA at long-term follow-up, mainly sustained VT (9 (24\%) versus 19 (7\%); HR 2.78; confidence interval 1.23-6.29; $p=0.014 ;$ Table 2, Figure 4B). The occurrence of a LTA significantly correlated with the presence of NSVT at baseline ( $p<0.001 ;$ HR 5.27, confidence interval 2.4611.29).

\section{Gene-environmental interactions in TTNtv patients}

Patients were classified according to their possible contributing environmental factors to score the gene-environmental interactions in TTNtv patients as described before (Suppl. Table 4) ${ }^{3}$. Seventeen TTNtv patients (45\%) had an environmental factor possible contributing to their phenotype (i.e. viral, immune-mediated, toxic). TTNtv patients with an additional environmental factor have a significantly higher risk of developing LTA at long-term (log-rank = 0.047; Figure 5). However, due to the low number of TTNtv patients, these results should be considered as hypothesis-generating.

Table 2. Primary end-point and hazard ratios

\begin{tabular}{ccccc}
\hline & $\begin{array}{c}\text { TTNtv + } \\
(\mathbf{n = 3 8 )}\end{array}$ & $\begin{array}{c}\text { TTNtv - } \\
(\mathbf{n = 2 6 5 )}\end{array}$ & P-value & $\begin{array}{c}\text { Gender-adjusted } \\
\text { Hazard Ratio [95\% } \mathbf{C l}]\end{array}$ \\
\hline Follow-up duration (months) & $39[16-87]$ & $46[22-76]$ & 0.84 & - \\
Death & $0(0)$ & $12(5)$ & 0.37 & - \\
Heart transplantation & $0(0)$ & $2(1)$ & 0.99 & - \\
HF hospitalisation & $3(8)$ & $15(6)$ & 0.48 & $1.20[0.34-4.25]$ \\
Life-threatening Arrhythmia & $9(24)$ & $19(7)$ & 0.004 & $2.78[1.23-6.29]$ \\
Combined end-point & $10(26)$ & $41(15)$ & 0.10 & $1.52[0.75-3.08]$ \\
\hline
\end{tabular}

Values are $n, n(\%)$, or median (interquartile range). Abbreviations, $H F$, heart failure; $\mathrm{Cl}$, confidence interval.

\section{Alteration of metabolic and structural pathways in the heart of TTNtv patients}

To gain better insight into the cardiac pathophysiological changes in response to TTNtv, genome-wide transcriptome analysis (RNA-sequencing of EMB) was performed in a representative group of patients with similar HF severity, as defined by LVEF and NYHA class ( $n=21,13$ TTNtv+, 8 TTNtv- DCM; Suppl. Table 5; Suppl. Figure 4). In general, RNA sequencing showed comparable TTN transcript levels in patients with or without TTNtv (Suppl. Table 6). Subsequent gene set enrichment analysis with Kyoto Encyclopedia of Genes and Genomes (KEGG) terms using the GAGE bioinformatics tool, revealed significant alterations of different molecular pathways in TTNtv patients compared to the other HF group (Figure 6A). Many of the significantly enriched pathways were related to cardiac energy metabolism. Differential expressed genes were analyzed with the IPA ${ }^{\circledR}$ software to further validate these findings. Here, mitochondrial dysfunction was most affected (Table 3). Notably, closer visualization on the mitochondrial oxidative phosphorylation pathway by means of Pathvisio and Wikipathways revealed increased expression of genes across all the electron transport chain complexes (ETC), involving complex I to IV as well as the ATP synthase (complex V) (Figure 6B). 

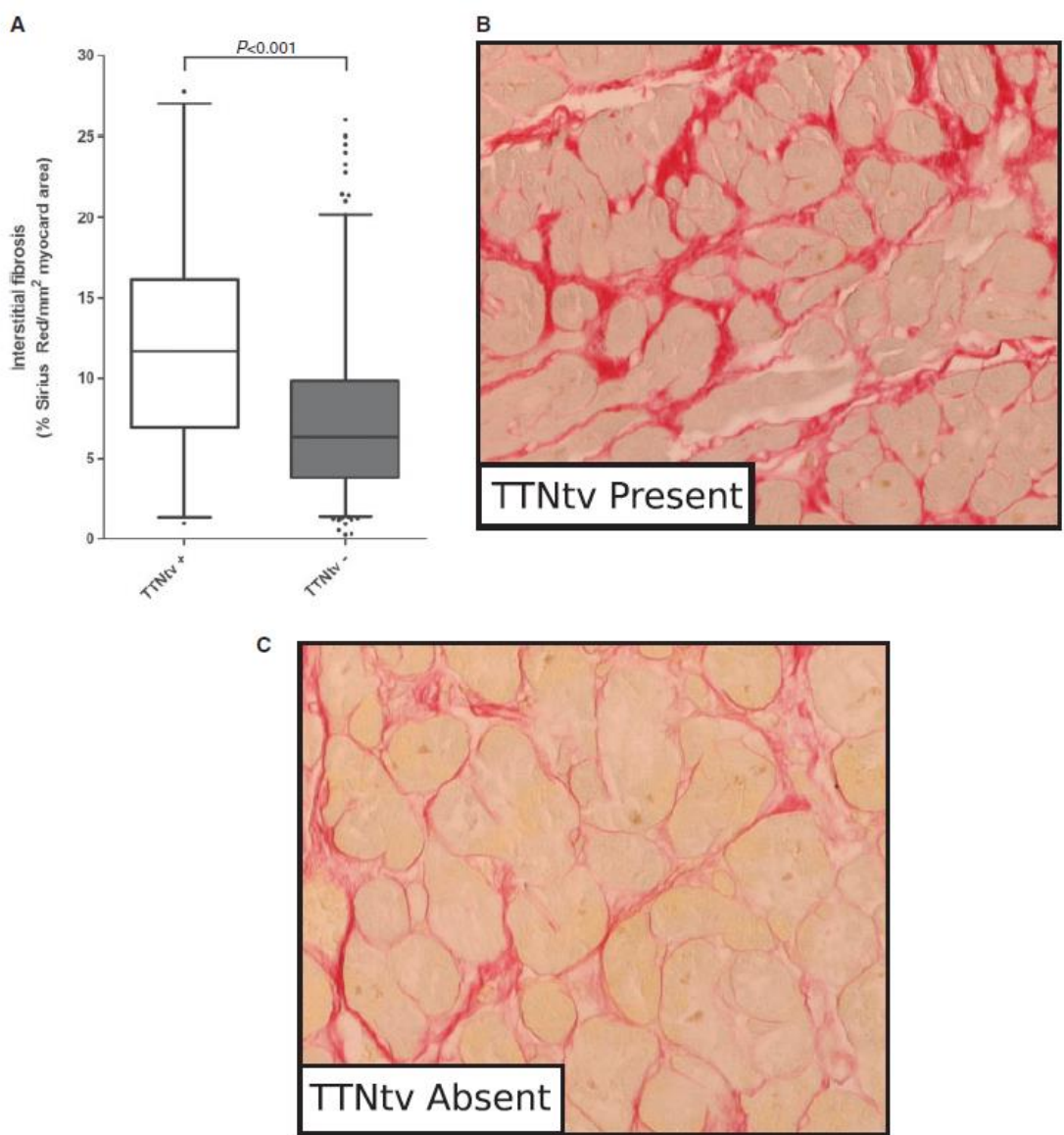

Figure 2. Interstitial myocardial fibrosis in DCM. Interstitial fibrosis quantified in the EMBs of patients with and without a TTNtv (A). Boxes represent the median and interquartile range, whiskers are 5-95\% range, dots represent the outliers. Representative pictures of Sirius red stainings on EMBs of a TTNtv (B) and a patient without TTNtv (C). Magnification 200x.

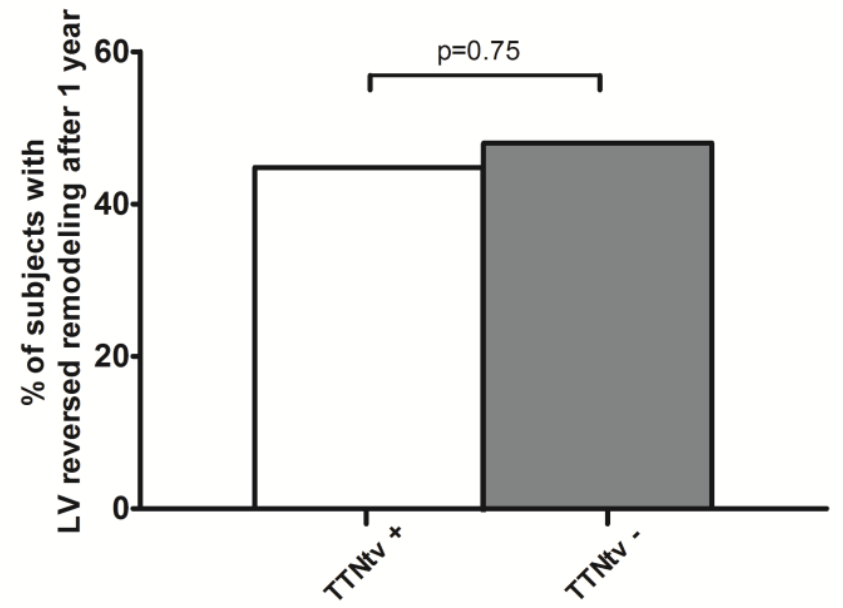

Figure 3. Left ventricular reverse remodeling in DCM. Percentage of patients who improved or/and recovered after 1 year measured using echocardiographic follow-up data. Improvement is defined as an absolute increase of $\geq 10 \%$ or a $L V E F \geq 50 \%$ with at least $5 \%$ improvement. 
A

Death, cardiac transplant, heart failure hospitalisation or life-threatening arrhythmia

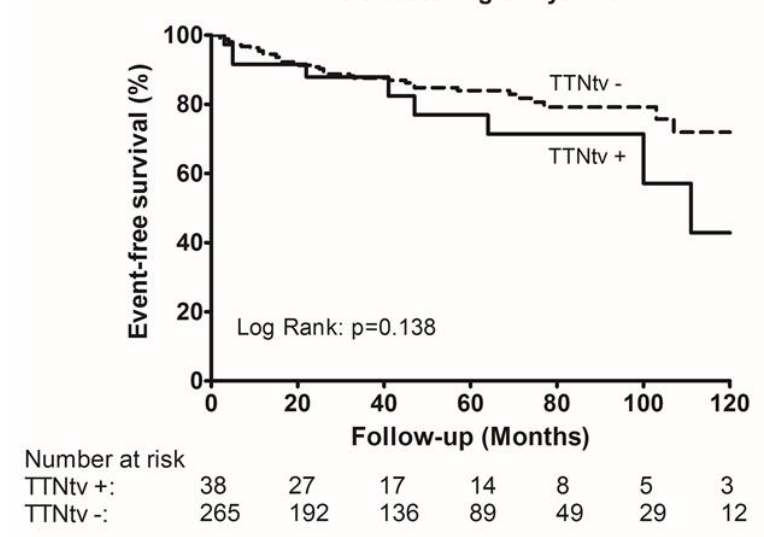

B

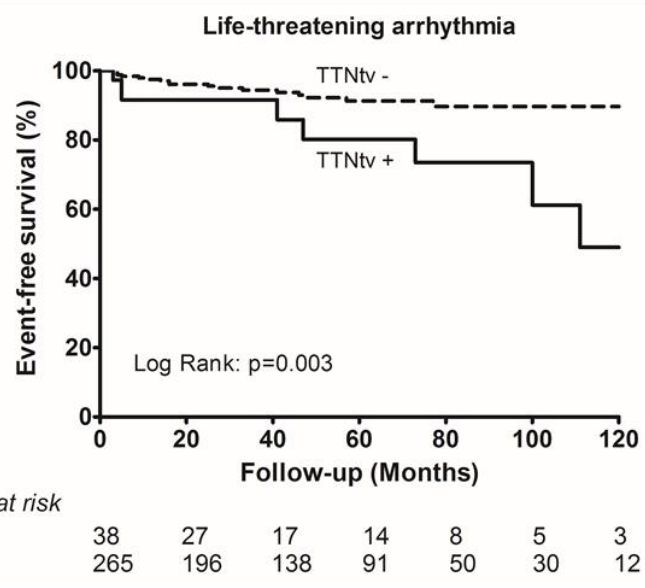

Figure 4. TTNtv and long-term outcome in DCM. Primary outcomes in TTNtv+ and TTNtv- DCM patients (A). Primary end-point after enrollment (cardiac death, Htx, HF hospitalization or LTA) (B). Occurrence of a LTA after enrollment. Event-free survival regarding LTA is reduced in TTNtv patients.

Remarkably, the marked changes in ETC were not associated with concurrent changes in the expression of enzymes involved in the citric acid cycle, fatty acid oxidation, or glycolysis (Suppl. Figures 5-7). Next to alterations in the ETC, perturbations in cardiac hypertrophy, oxidative stress and fibrosis in the TTNtv heart are suggested by gene set enrichment analysis showing overlapping KEGG and IPA ${ }^{\circledR}$ terms (Figure 6A; Table 3).

To further investigate the metabolic consequence of altered gene expression in TTNtv patients; amino acids, acylcarnitines and very-long-chain fatty acids were measured in plasma (Suppl. Table 7). Relative to the other group, short- and medium-chain acyl-carnitines and very-long-chain fatty acids were elevated in the plasma of TTNtv patients (Suppl. Figure 8; Suppl. Table 8). The principal long-chain acylcarnitine (C16:0, C18:0, C18:1 and C18:2) did not differ. Also, plasma branched-chain amino acids (BCAA) were not elevated in TTNtv patients (Suppl. Figure 9). All metabolic analyses were corrected for gender, as all TTNtv patients were male (Suppl. Table 7).

\section{DISCUSSION}

This study examined the phenotypic characteristics and clinical outcomes at baseline and after long-term follow-up of TTNtv DCM patients, and unveiled the underlying cardiac molecular changes. In TTNtv DCM hearts, pronounced cardiac alterations in mitochondrial function, increased interstitial fibrosis and reduced ventricular mass was found. Those structural and metabolic alterations related to TTNtv went along with an increased propensity to (life-threatening) ventricular arrhythmias at long-term follow-up.

In our cohort, the prevalence of TTNtv mutations was 13\% (33\% in familial DCM and $7 \%$ in sporadic cases) in line with previous studies ${ }^{42021}$. Structural analysis of EMB from living TTNtv patients revealed enhanced myocardial interstitial fibrosis as compared to TTNtv negative DCM patients. Fibrosis is known to be a substrate for arrhythmogenesis in DCM patients ${ }^{22}$, and may therefore in part explain the ventricular arrhythmogenic burden in TTNtv patients at long-term follow-up found here, and by others at baseline ${ }^{5910}$. The degree of late enhancement at CMR did not differ in TTNtv compared to TTNtv negative DCM patients, as 
observed previously ${ }^{5}$. This paradox between histological fibrosis and CMR late enhancement is in line with the rather reactive diffuse interstitial fibrosis at EMB upon pathogenic TTN gene mutation, which cannot be detected with late enhancement CMR, the latter only sensitive for extensive replacement fibrosis ${ }^{23}$. Despite the increased interstitial fibrosis, TTNtv patients seem to respond well to therapy, possibly due to the reactive character of the fibrosis in the absence of cardiomyocyte death. Whether T1 mapping -not routinely performed in our center before 2016- may be helpful to quantify this diffuse interstitial fibrosis requires further investigation.

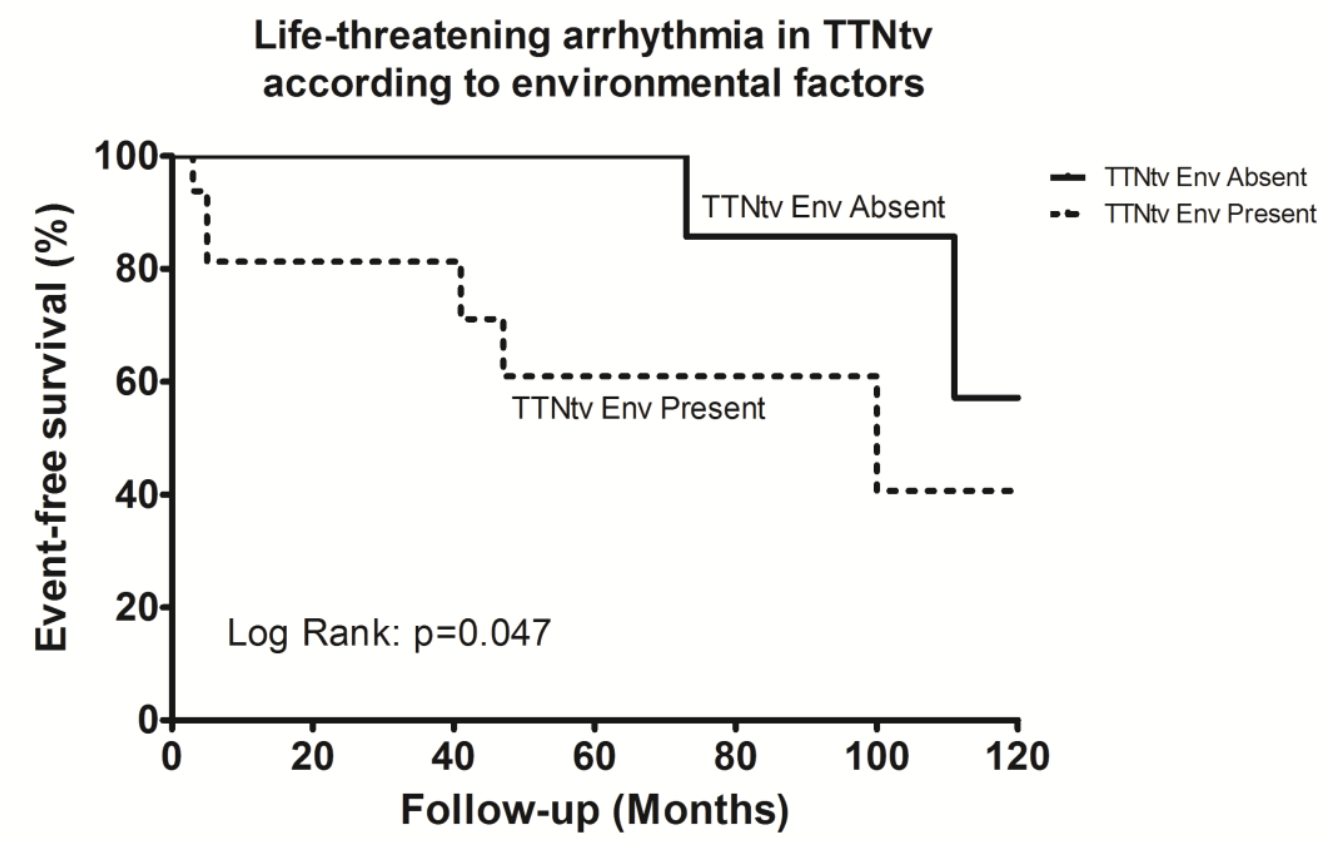

Number at risk

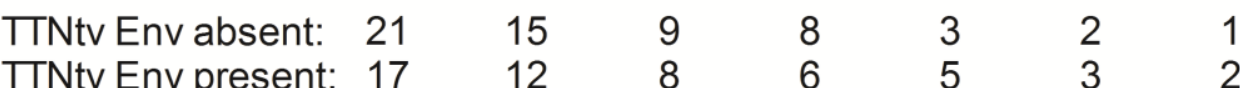

Figure 5. Life-threatening arrhythmia in TTNtv according to environmental factors . The occurrence of a lifethreatening arrhythmia after enrollment. There is significant reduction in event-free survival regarding LTA in TTNtv patients ( $p=0.047)$. TTNtv Env absent= absence of an environmental factor; Env Present= presence of an environmental factor.

\section{More life-threatening arrhythmias but a similar prognosis in TTNtv DCM patients}

Our patients with a TTNtv had more ventricular arrhythmias at baseline and LTAs at longterm, mainly in those with additional environmental factors, suggesting a more severe clinical course when a second (environmental) trigger is present on top of the TTNtv. This first insight into the importance of gene-environmental interaction in the LTA of our well-phenotyped TTNtv patients, is in line with similar observations with the phenotypic and prognostic impact of gene-environmental interaction in other DCM cohorts ${ }^{324}$.

Despite the arrhythmogenic burden, we did not observe a significant impact on prognosis (i.e. death, heart transplantation), potentially due to effective device therapy (ICD). Also, almost $50 \%$ of our TTNtv patients have significant left ventricular reverse remodeling after 1-year HF medication. TTNtv DCM thus seems to respond well to HF therapy in line with previous observations ${ }^{9}$, but still are more prone to ventricular arrhythmias at long-term. In the latest ESC guidelines, LMNA mutations are the only mutations for which preventive ICD 
therapy should be considered in DCM ${ }^{25}$. However, the current and other studies indicate that TTNtv should also be considered as an additional genetic risk for ventricular arrhythmias ${ }^{510}$.

A

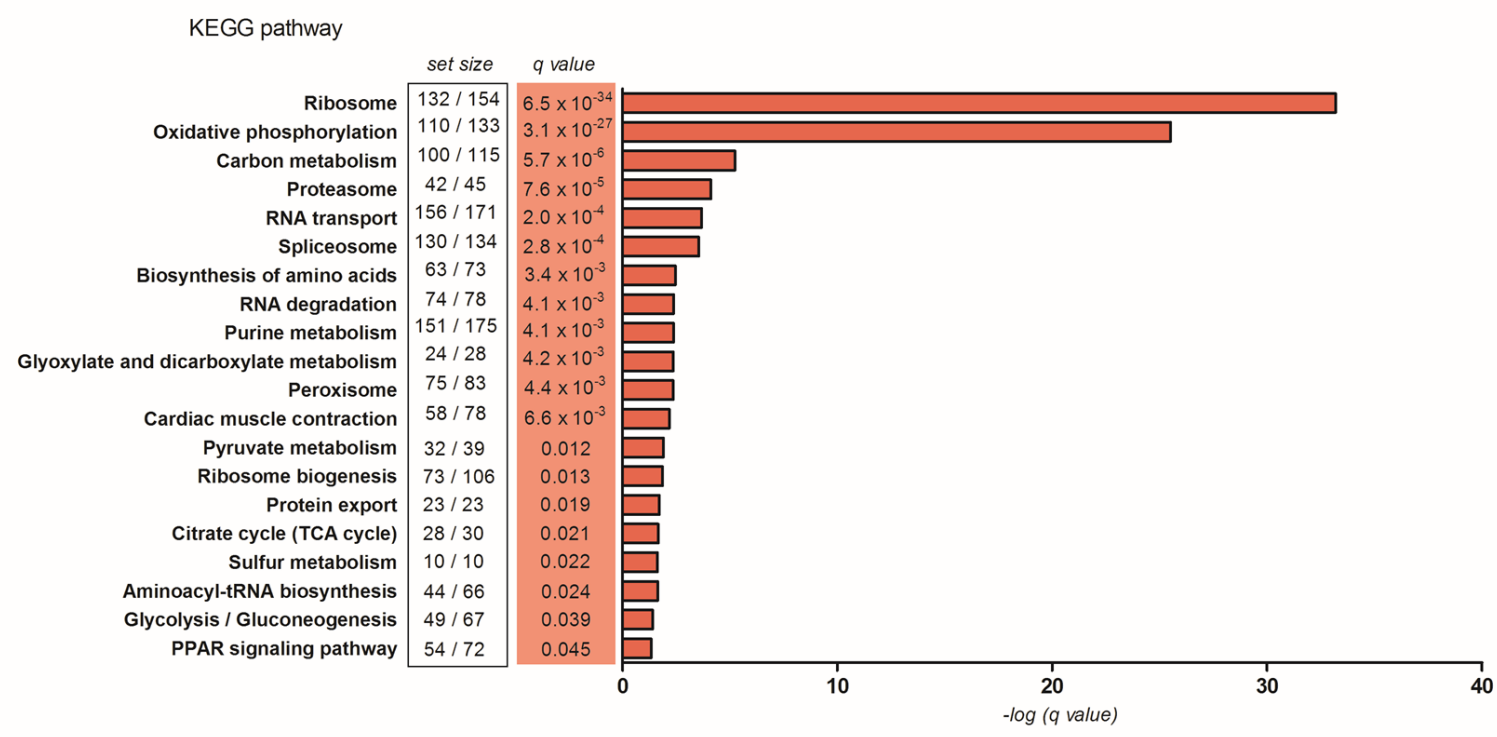

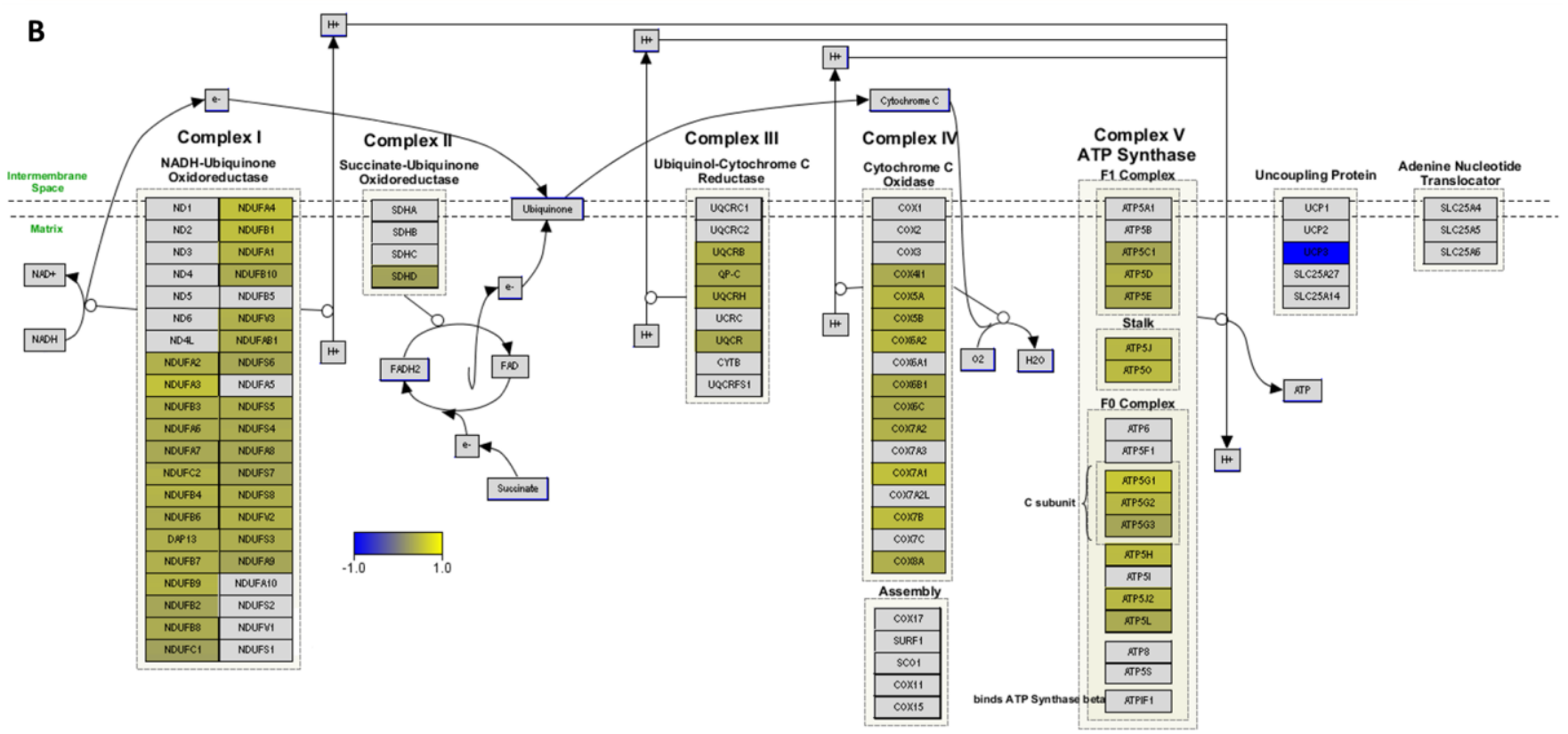

Figure 6. Molecular pathway analysis in EMB of TTNtv DCM patients. Pathway analysis based on RNA-seq data of EMBs from DCM patients show altered metabolism and structural integrity in TTNtv patients (A). Significantly enriched pathways are highlighted in red ( $q$ value $<0.05$ ). Set size is the number of found genes in the RNA-seq data in relation to the total genes in a KEGG pathway. The top enriched oxidative phosphorylation pathway is graphically depicted using Wikipathways and Pathvisio (B). Yellow shows significant upregulation of a gene. 
Table 3. Top five differentially expressed biological pathways using Ingenuity pathway analysis (IPA) between TTNtv+ and TTNtv- patients.

\begin{tabular}{ccc} 
Ingenuity Toxicity List* & P value & \% genest \\
\hline TTNtv+ versus TTNtv- & & \\
Mitochondrial Dysfunction & $1.58 \mathrm{E}-21$ & $35 \%(62 / 176)$ \\
Cardiac Hypertrophy & $4.57 \mathrm{E}-03$ & $13 \%(44 / 342)$ \\
Increases Renal Nephritis & 0.02 & $19 \%(11 / 58)$ \\
NRF2-mediated Oxidative Stress Response & 0.02 & $13 \%(27 / 216)$ \\
Cardiac Fibrosis & 0.03 & $13 \%(27 / 207)$ \\
\hline
\end{tabular}

* 1.796 significantly differentially expressed transcripts were considered for pathway analysis

+ Percentage of pathway-related genes that are differentially expressed between the datasets followed by the absolute number

\section{Pronounced mitochondrial remodeling in TTNtv DCM patients}

The consistently higher transcript levels of all mitochondrial electron transport chain (ETC) complexes in TTNtv hearts is remarkable. Especially while other building blocks of cardiac energy metabolism (citric acid cycle, fatty acid oxidation, glycolysis) are relatively unaffected. These findings suggest an increased efficiency of mitochondrial ATP production trying to counterbalance a TTNtv-related sarcomeric defect. It may thus represent an attempt to compensate for the limited contractile and reserve capacity in TTNtv mutations previously observed in vitro ${ }^{26}$. This finding supports the suggestion that TTNtv hearts are in a compensated state inflexible to further stress as suggested by others ${ }^{6}$.

Our cardiac metabolic data are in line with the metabolic remodeling found in TTNtv mutated rat hearts compared to control hearts recently reported ${ }^{6}$. Similar to our patients, TTNtv mutated rat hearts had an enrichment of mitochondrial pathways and increased transcript levels of respiratory chain constituents (Atp1a3, Atp6ap1). Importantly, these TTNtv rats were compared to healthy wild type hearts. The latter suggests that the human cardiac changes we observe in TTNtv patients reflect true upregulation of mitochondrial energetics and not less downregulation compared to TTNtv negative DCM patients. The direct causal links by which these TTN gene mutations lead to mitochondrial alterations require further investigation e.g. in vitro, animal or humanized cell models, to understand the significance from a clinical and therapeutic perspective.

In $\mathrm{HF}$, it is generally acknowledged that mitochondrial oxidative energy metabolism becomes impaired and is compensated by an increased anaerobic glycolysis ${ }^{11}$. This translates in elevated long-chain acylcarnitines levels in the circulation of these patients. Also, BCAA are often elevated in HF patients, although reasons for this increase are unknown. However, plasma levels of small- and medium chain acylcarnitines were increased in our TTNtv patients, but circulating levels of long-chain acylcarnitines were unaltered. Elevated blood long-chain acylcarnitines have been independently associated with arrhythmias and adverse clinical outcomes ${ }^{12}$. The unaltered level of long-chain acylcarnitines does not support the idea that cardiac mitochondrial function is severely compromised in TTNtv patients ${ }^{11}$. Instead, the massive upregulation of respiratory chain transcripts may indeed point to a compensatory increase in mitochondrial function. Therefore, the increased propensity for LTAs in TTNtV patients is not driven by an accumulation of noxious lipid intermediates in blood.

Increased cardiac fibrosis, pronounced energetic alterations and increased ventricular arrhythmias at long-term were associated with a decreased LV cardiac mass in TTNtv patients, whereas cardiac systolic function or dimensions did not differ. Although this decreased cardiac mass is not directly clinically relevant, it offers insight in the pathomechanism of TTNtv DCM. Whether this reduction in cardiac mass relates to a lower ability for hypertrophic 
compensation to (mechanical) stress (as titin is important for mechanotransduction at the sarcomere), or whether less cardiomyocyte hypertrophy is needed to compensate for this gene deficit compared to others, requires further investigations.

\section{Limitations}

The current study population was enrolled in a single tertiary referral center, thus imposing a possible selection bias. Because of the relative low number of TTNtv patients in our cohort, the current study had insufficient power to perform multivariable modeling. Although the results are clear and unidirectional, sub-analyses (such as the gene-environmental analysis) lacks adequate power and should be considered as hypothesis-generating. Therefore, these results should be interpreted with caution and need confirmation in larger cohorts.

\section{CONCLUSION}

In summary, DCM patients with a TTNtv are prone to ventricular arrhythmias at long-term, which is aggravated in combination with an additional environmental factor. These arrhythmias go hand in hand with increased cardiac interstitial fibrosis and pronounced changes in mitochondrial function.
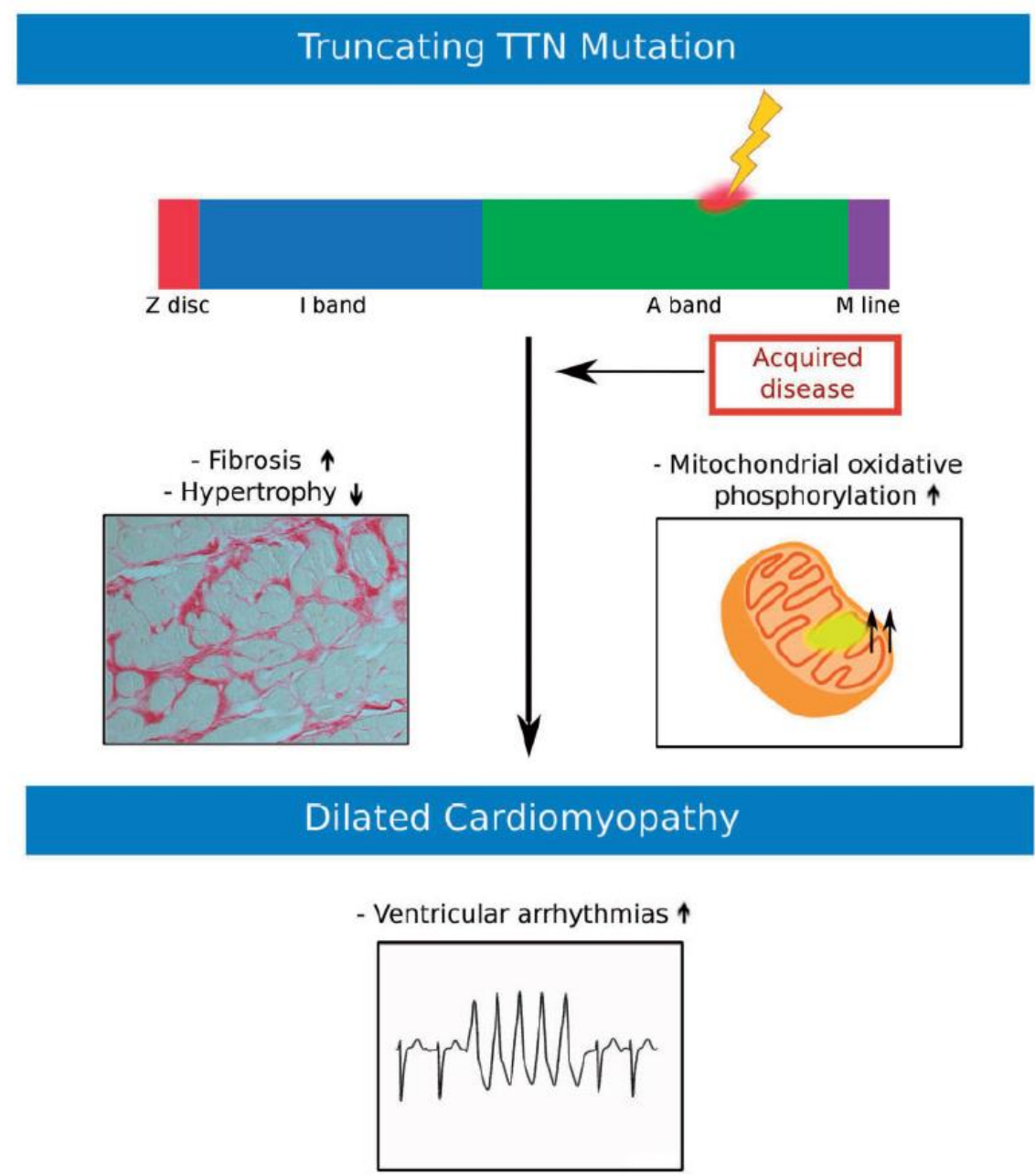

Take home Figure. Truncating TTN mutations and Dilated Cardiomyopathy. Truncating mutations in the TTN gene can lead to DCM associated with ventricular arrhythmias, increased interstitial fibrosis, lower ventricular mass and alterations of the mitochondrial energy pathways. 


\section{REFERENCES}

1. Hershberger RE, Hedges DJ, Morales A. Dilated cardiomyopathy: the complexity of a diverse genetic architecture. Nature reviews Cardiology 2013;10(9):531-47.

2. Merlo M, Pivetta A, Pinamonti B, et al. Long-term prognostic impact of therapeutic strategies in patients with idiopathic dilated cardiomyopathy: changing mortality over the last 30 years. Eur J Heart Fail 2014;16(3):317-24.

3. Hazebroek MR, Moors S, Dennert $R$, et al. Prognostic Relevance of Gene-Environment Interactions in Patients With Dilated Cardiomyopathy: Applying the MOGE(S) Classification. J Am Coll Cardiol 2015;66(12):1313-23.

4. Haas J, Frese KS, Peil B, et al. Atlas of the clinical genetics of human dilated cardiomyopathy. Eur Heart J 2015;36(18):1123-35a.

5. Roberts AM, Ware JS, Herman DS, et al. Integrated allelic, transcriptional, and phenomic dissection of the cardiac effects of titin truncations in health and disease. Science translational medicine 2015;7(270):270ra6.

6. Schafer S, de Marvao A, Adami E, et al. Titintruncating variants affect heart function in disease cohorts and the general population. Nature genetics 2017;49(1):46-53.

7. Linschoten M, Teske AJ, Baas AF, et al. Truncating Titin (TTN) Variants in Chemotherapy-Induced Cardiomyopathy. J Card Fail 2017;23(6):476-79.

8. van Spaendonck-Zwarts $K Y$, Posafalvi $A$, van den Berg MP, et al. Titin gene mutations are common in families with both peripartum cardiomyopathy and dilated cardiomyopathy. Eur Heart J 2014;35(32):2165-73.

9. Jansweijer JA, Nieuwhof K, Russo $F$, et al. Truncating titin mutations are associated with a mild and treatable form of dilated cardiomyopathy. Eur J Heart Fail 2016;19(4):51221.

10. Tayal U, Newsome S, Buchan R, et al. Truncating Variants in Titin Independently Predict Early Arrhythmias in Patients With Dilated Cardiomyopathy. J Am Coll Cardiol 2017;69(19):2466-68.

11. Ussher JR, Elmariah S, Gerszten RE, et al. The Emerging Role of Metabolomics in the Diagnosis and Prognosis of Cardiovascular Disease. J Am Coll Cardiol 2016;68(25):2850-70.

12. Ahmad T, Kelly JP, McGarrah RW, et al. Prognostic Implications of Long-Chain Acylcarnitines in Heart Failure and Reversibility With Mechanical Circulatory Support. J Am Coll Cardiol 2016;67(3):291-9.
13. Alexander D, Lombardi R, Rodriguez $G$, et al. Metabolomic distinction and insights into the pathogenesis of human primary dilated cardiomyopathy. Eur J Clin Invest 2011;41(5):527-38

14. Ponikowski P, Voors AA, Anker SD, et al. 2016 ESC Guidelines for the diagnosis and treatment of acute and chronic heart failure: The Task Force for the diagnosis and treatment of acute and chronic heart failure of the European Society of Cardiology (ESC)Developed with the special contribution of the Heart Failure Association (HFA) of the ESC. Eur Heart J 2016;37(27):2129200.

15. Merlo M, Pyxaras SA, Pinamonti $B$, et al. Prevalence and prognostic significance of left ventricular reverse remodeling in dilated cardiomyopathy receiving tailored medical treatment. J Am Coll Cardiol 2011;57(13):146876.

16. Pinto YM, Elliott PM, Arbustini E, et al. Proposal for a revised definition of dilated cardiomyopathy, hypokinetic non-dilated cardiomyopathy, and its implications for clinical practice: a position statement of the ESC working group on myocardial and pericardial diseases. Eur Heart J 2016;37(23):1850-8.

17. Verdonschot J, Hazebroek M, Merken J, et al. Relevance of cardiac parvovirus B19 in myocarditis and dilated cardiomyopathy: review of the literature. Eur J Heart Fail 2016;18(12):1430-41.

18. Caforio AL, Pankuweit S, Arbustini $E$, et al. Current state of knowledge on aetiology, diagnosis, management, and therapy of myocarditis: a position statement of the European Society of Cardiology Working Group on Myocardial and Pericardial Diseases. Eur Heart J 2013;34(33):2636-48, 48a-48d.

19. Waterval WA, Scheijen $J L$, Ortmans-Ploemen $M M$, et al. Quantitative UPLC-MS/MS analysis of underivatised amino acids in body fluids is a reliable tool for the diagnosis and follow-up of patients with inborn errors of metabolism. Clinica chimica acta; international journal of clinical chemistry 2009;407(1-2):36-42.

20. Akinrinade $O$, Ollila $L$, Vattulainen $S$, et al. Genetics and genotype-phenotype correlations in Finnish patients with dilated cardiomyopathy. Eur Heart J 2015;36(34):2327-37.

21. Herman DS, Lam L, Taylor MR, et al. Truncations of titin causing dilated cardiomyopathy. $N$ Engl J Med 2012;366(7):619-28.

22. Gulati A, Jabbour A, Ismail TF, et al. Association of fibrosis with mortality and sudden cardiac death in patients with nonischemic dilated cardiomyopathy. JAMA 2013;309(9):896-908. 
23. Mewton $\mathrm{N}$, Liu CY, Croisille $\mathrm{P}$, et al. Assessment of myocardial fibrosis with cardiovascular magnetic resonance. J Am Coll Cardiol 2011;57(8):891-903.

24. Ware JS, Li J, Mazaika E, et al. Shared Genetic Predisposition in Peripartum and Dilated Cardiomyopathies. $N$ Engl J Med 2016;374(3):233-41.

25. Priori SG, Blomstrom-Lundqvist C, Mazzanti A, et al. 2015 ESC Guidelines for the management of patients with ventricular arrhythmias and the prevention of sudden cardiac death: The Task Force for the Management of Patients with Ventricular Arrhythmias and the Prevention of Sudden Cardiac Death of the European Society of Cardiology (ESC). Endorsed by: Association for European Paediatric and Congenital Cardiology (AEPC). Eur Heart J 2015;36(41):2793-867.

26. Hinson JT, Chopra A, Nafissi N, et al. HEART DISEASE. Titin mutations in iPS cells define sarcomere insufficiency as a cause of dilated cardiomyopathy. Science (New York, NY) 2015;349(6251):982-6. 


\section{SUPPLEMENTARY METHODS \\ Endomyocardial biopsy}

At least six endomyocardial biopsies (EMB) samples were taken from the right ventricular septum via the internal jugular vein or the femoral artery using a transcatheter bioptome (Cordis, Miami, FL, USA). Biopsies were collected as part of routine diagnostics for DCM, to identify other triggers related to DCM such as viruses, inflammation, storage diseases and metabolic disorders. Two specimens were used for immunohistological analysis on four $\mu \mathrm{m}$-thick tissue sections from formalin-fixed, paraffinembedded EMBs. Four specimens were used for the detection of viral genomes by using polymerase chain reaction (PCR) and reverse transcriptase PCR analysis ${ }^{1}$. Deoxyribonucleic acid (DNA) and ribonucleic acid (RNA) were isolated separately, so that remaining RNA could be used for further analysis. Six primer pairs were used to detect cardiotropic DNA and RNA viruses, including adenovirus, enterovirus, cytomegalovirus, parvovirus B19, human herpesvirus-6, and Epstein-Barr virus ${ }^{1}$. Significant viral load was defined as $\geq 500$ copies/ $\mu$ gNA ${ }^{23}$. Increased cardiac inflammation was defined as $\geq 14 \mathrm{CD} 45$, including up to 4 CD68-infiltrating cells $/ \mathrm{mm}^{2}$ according to the current ESC guidelines ${ }^{4}$. Collagen fraction volume (CFV) was quantified as percentage tissue positive for Sirius red of the total myocardial area. Perivascular and direct sub-endomyocardial collagen were not included in the quantification, as cutting artifacts may influence those.

\section{Genetic analysis}

All DCM-related genes were analyzed using NGS, either with whole exome sequencing (WES) or single molecule Molecular Inversion Probe (smMIP). NGS testing includes either the custom made smMIP panel containing the 47 DCM-related genes, followed by multiplexed analysis on a NextSeq 500 System (illumina) or WES with the Illumina Hiseq2000 (Illumina) sequencer after enrichment with SureSelectXT Human All Exon V4+UTRs ${ }^{56}$. In these methods, the same 47 DCM-associated genes were systematically analyzed. All found variants were validated with Sanger sequencing. Variants were classified in 5 different classes: pathogenic, likely pathogenic, variant of clinical unknown significance (VUS), likely benign or benign. Classification of variants was based on the score of in silico prediction software scores (SIFT, MutationTaster, PolyPhen-2, PhyloP, Align-GVGD), the frequency in reference population databases (ExAC, gnomAD, 1000 genomes, ESP projects) and previously published variations in NCBI's ClinVar and HGMD. Both pathogenic and likely pathogenic mutations were classified as pathogenic mutations. All others were considered as non-pathogenic based on the current knowledge. Titin mutations were only considered pathogenic in case of truncating variants in the late I-band or A-band region with percentage spliced in (PSI) $>99 \%{ }^{7}$.

\section{Total RNA isolation}

Total RNA was isolated using mirVana (Ambion), according to the manufacturer's protocol. The integrity of the RNA from each sample was scored on the Agilent 2100 Bioanalyzer (Agilent) via 2100 an Expert Eukaryote Total RNA Pico chip according manufacturer's protocol. Samples that had a RIN of over 6 were subsequently used for the generation of the mRNA sequence library.

\section{mRNA sequencing library generation}

The mRNA sequencing library was generated using TruSeq mRNA sample preparation kit (Illumina) according to manufacturer's protocol apart from two adjustments due to the lower amount of total RNA than normally used. In short, mRNA was enriched using magnetic beads coated with poly-dT, followed by fragmentation. The fragmented mRNA enriched samples were subjected to cDNA synthesis by reverse transcriptase, followed by $\mathrm{dA}$-tailing and ligation of specific double-stranded barcoded adapters. For the subsequence clean-up we used an ampure beads to sample volume ratio of 0.8:1 to eliminate accidentally generated adaptor dimers. Next a library amplification for 19 cycles was performed to compensate for the lower input amount and after cleanup the sizes of the libraries were determined on an Agilent 2100 Bioanalyzer (Agilent) via an DNA 1000 chip according manufacturer's protocol. 


\section{mRNA library sequencing}

Pooled libraries consisting of equal molar samples were sequenced on a high-output $75 \mathrm{bp}$ single read on the NextSeq500 (Illumina).

\section{Total RNA analysis pipeline}

The analyses of sequencing datasets were performed as earlier described ${ }^{8}$. In short, reads were aligned to the human hg38 reference genome using TopHat ${ }^{9}$ and exonic reads were summed per transcript and transcripts were referred to as being expressed when at least five aligned reads were present in all samples of either of the groups. Statistical analysis and log fold-changes were generated using the edge R bioconductor package ${ }^{10}$.

\section{Pathway analysis}

The molecular landscape of the heart was investigated on a genome-wide scale for the DCM patients, i.e. with TTNtv or no identified TTNtv, for over-represented pathways among differentially transcribed genes. We used KEGG ${ }^{11}$ pathway enrichment analysis with GAGE ${ }^{12}$, Ingenuity ${ }^{\circledR}$ pathway analysis $\left(\right.$ IPA $\left.^{\oplus}\right)$ software and pathvisio ${ }^{13}$ with Wikipathway tools ${ }^{14}$. For the GAGE analysis we used normalized count reads, i.e. counts per million and log2 transformed.

res_noM_TTN <-gage(entrez.data, gsets=kegg.gs, ref=1:8, samp=9:21, compare="unpaired")

columns 1:8 represents the DCM patients with no TTNtv, columns 9:21 represents the DCM with a TTNtv.Refseq id's were converted to Entrez Ids using id2eg from the pathview package. For the IPA ${ }^{\circledR}$ software we used the $p$-values and fold-changes generated with edgeR for analysis, with a cut-off of smaller then 0.05 on the $p$-value. For visualization with the pathvisio ${ }^{13}$ and wikipathways ${ }^{14}$ tools we imported the fold-changes of the significant genes ( $p$-value $<0.05$ ) and pathways, i.e. electron transport chain (WP111_89727), fatty acid beta oxidation (WP143_79783), Glycolosis en gluconeogenesis (WP_534_90450) and TCA Cycle (WP78_90661) .

\section{SUPPLEMENTARY REFERENCES}

1. Dennert R, Velthuis $S$, Schalla $S$, et al. Intravenous immunoglobulin therapy for patients with idiopathic cardiomyopathy and endomyocardial biopsy-proven high PVB19 viral load. Antivir Ther 2010;15(2):193201.

2. Hazebroek MR, Moors S, Dennert R, et al. Prognostic Relevance of Gene-Environment Interactions in Patients With Dilated Cardiomyopathy: Applying the MOGE(S) Classification. J Am Coll Cardiol 2015;66(12):1313-23.

3. Verdonschot J, Hazebroek M, Merken J, et al. Relevance of cardiac parvovirus B19 in myocarditis and dilated cardiomyopathy: review of the literature. Eur J Heart Fail 2016;18(12):1430-41.

4. Caforio AL, Pankuweit S, Arbustini E, et al. Current state of knowledge on aetiology, diagnosis, management, and therapy of myocarditis: a position statement of the European Society of Cardiology Working Group on Myocardial and Pericardial Diseases. Eur Heart J 2013;34(33):2636-48, 48a-48d.

5. Claes GR, van Tienen FH, Lindsey $\mathrm{P}$, et al. Hypertrophic remodelling in cardiac regulatory myosin light chain (MYL2) founder mutation carriers. Eur Heart J 2016;37(23):1815-22.

6. O'Roak BJ, Vives L, Fu W, et al. Multiplex targeted sequencing identifies recurrently mutated genes in autism spectrum disorders. Science (New York, NY) 2012;338(6114):1619-22.

7. Roberts AM, Ware JS, Herman DS, et al. Integrated allelic, transcriptional, and phenomic dissection of the cardiac effects of titin truncations in health and disease. Science translational medicine 2015;7(270):270ra6.

8. Derks KW, Misovic $\mathrm{B}$, van den Hout MC, et al. Deciphering the RNA landscape by RNAome sequencing. RNA Biol 2015;12(1):30-42.

9. Kim D, Pertea G, Trapnell C, et al. TopHat2: accurate alignment of transcriptomes in the presence of insertions, deletions and gene fusions. Genome biology 2013;14(4):R36.

10. Robinson MD, McCarthy DJ, Smyth GK. edgeR: a Bioconductor package for differential expression analysis of digital gene expression data. Bioinformatics (Oxford, England) 2010;26(1):139-40.

11. Kanehisa M, Goto S. KEGG: kyoto encyclopedia of genes and genomes. Nucleic Acids Res 2000;28(1):27-30. 
12. Luo W, Friedman MS, Shedden K, et al. GAGE: generally applicable gene set enrichment for pathway analysis. BMC Bioinformatics 2009;10:161.

13. Kutmon M, van lersel MP, Bohler A, et al. PathVisio 3: an extendable pathway analysis toolbox. PLoS Comput Biol 2015;11(2):e1004085.

14. Kutmon M, Riutta A, Nunes N, et al. WikiPathways: capturing the full diversity of pathway knowledge. Nucleic Acids Res 2016;44(D1):D488-94.

\section{SUPPLEMENTARY FIGURES}

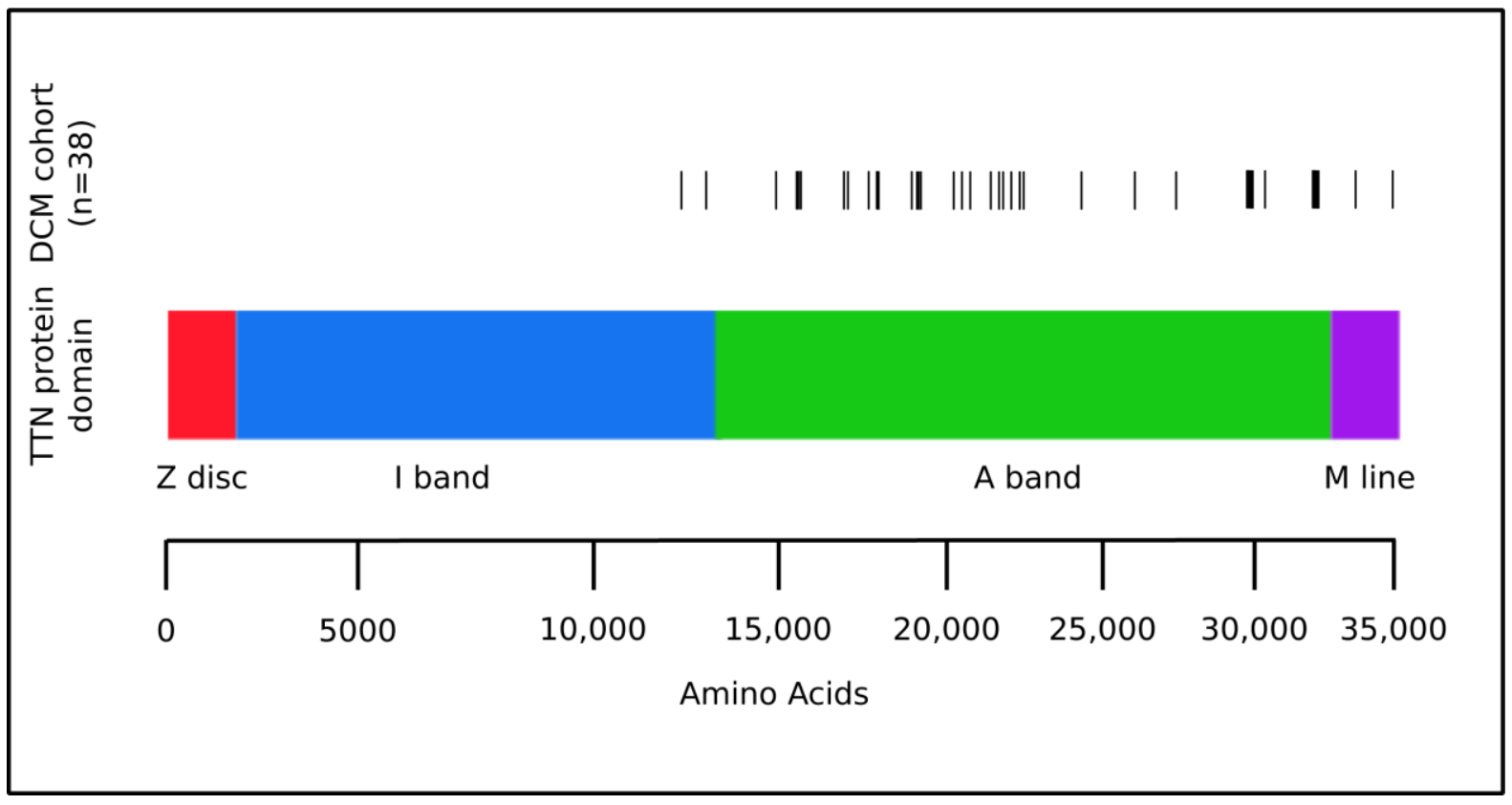

\section{Supplemental Figure 1. Position of TTNtv mutations}

Each individual bar indicates a truncated mutation in a DCM patient. The different mutations are sorted according to the amino acid position in the TTN protein.

\section{TTNtv- patients $(n=8)$}
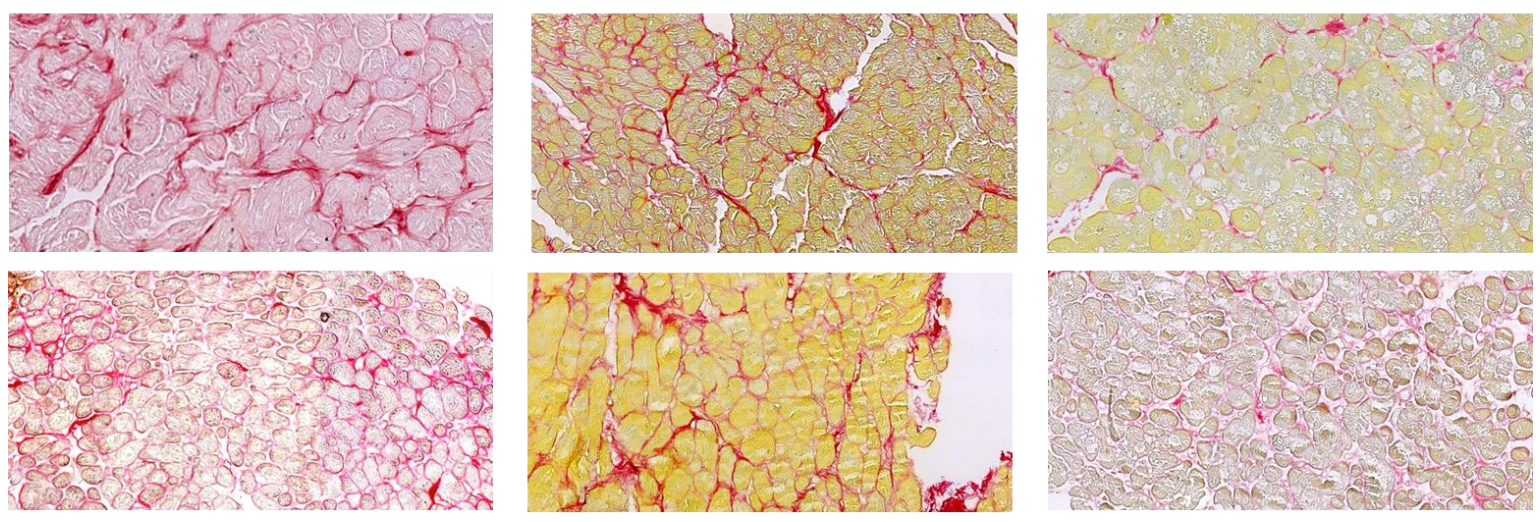
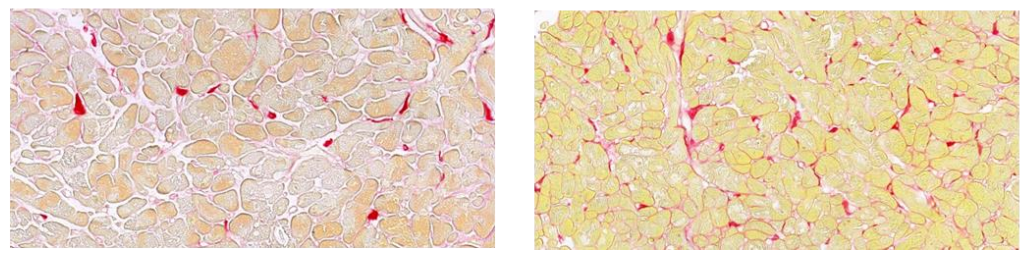


\section{TTNtv+ patients $(n=13)$}
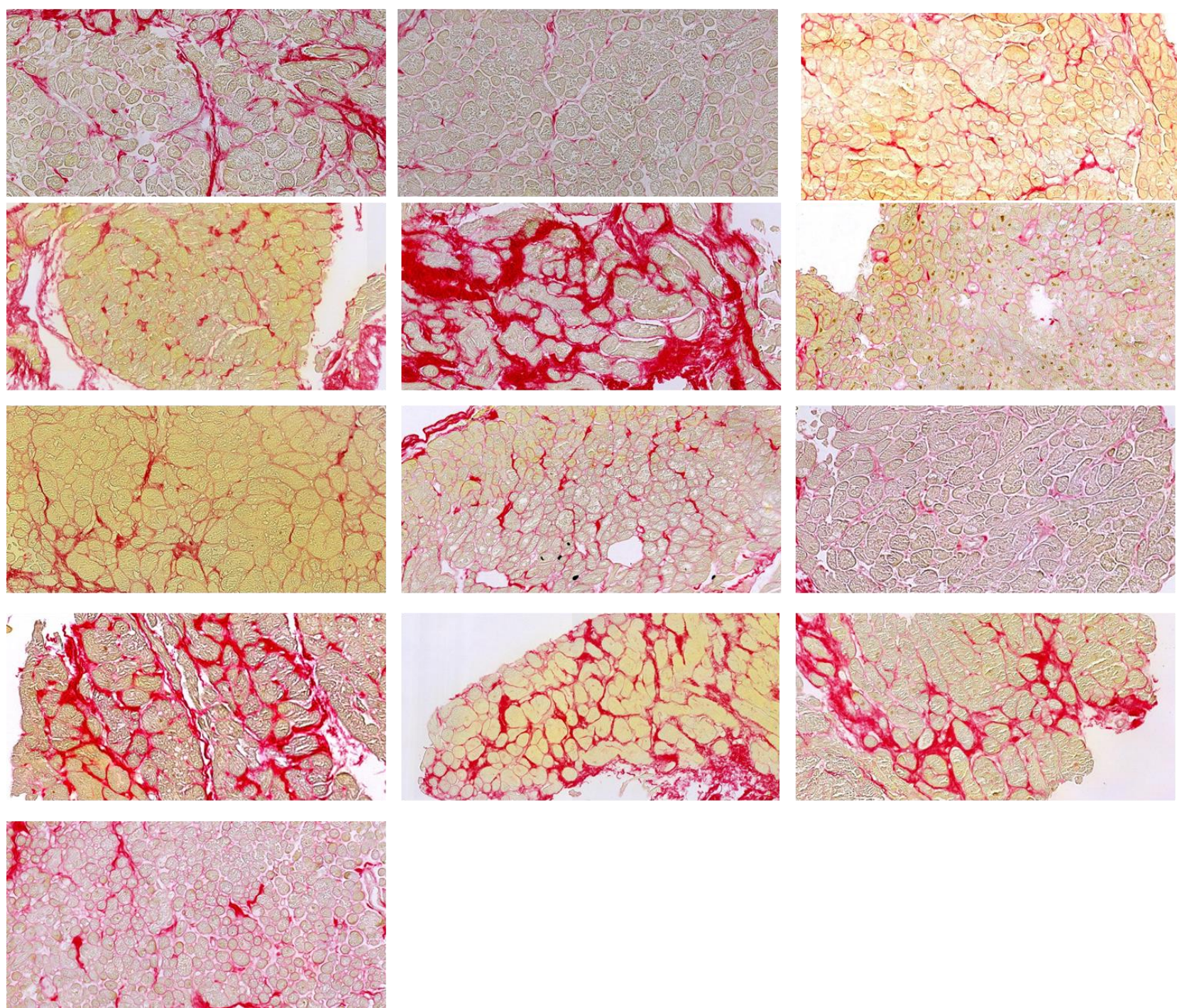

\section{Supplemental Figure 2. Sirius Red staining of endomyocardial biopsies}

Interstitial fibrosis was visualized in endomyocardial biopsies (EMB) using Sirius Red staining. Pictures were shown of patients used for RNA-sequencing (TTNtv- $n=8 ; T T N t v+n=13$ ), showing the variation in interstitial fibrosis among the patients. Magnification 100x. 


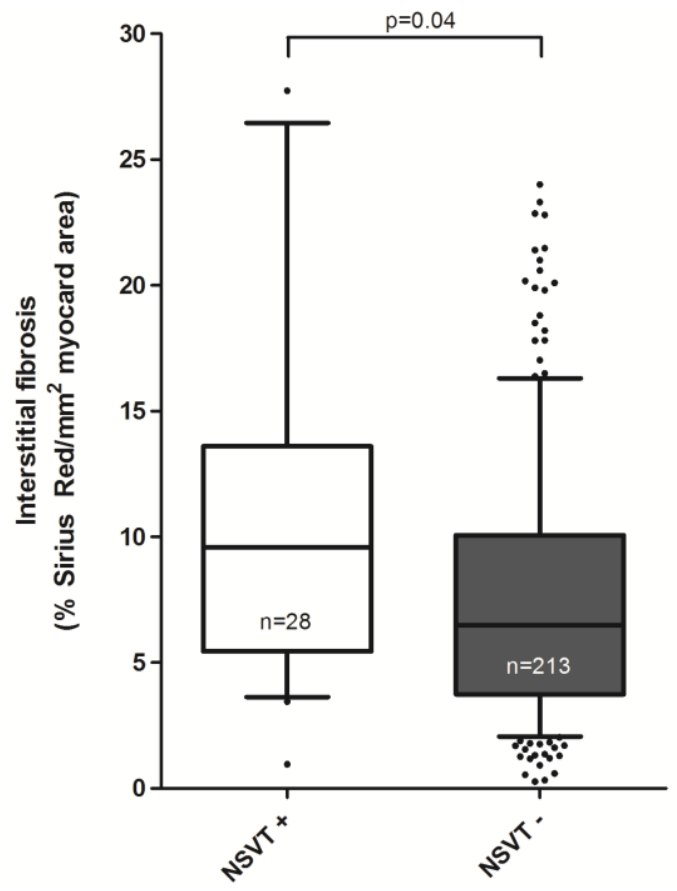

Supplemental Figure 3. Increased interstitial fibrosis in DCM patients with NSVT

Interstitial fibrosis quantified in the endomyocardial biopsies of patients with $(n=28)$ and without non-sustained ventricular tachycardias (NSVT) $(n=213)$. Boxes represent the median and interquartile range, whiskers are 10$90 \%$ quartiles, dots represent the outliers.

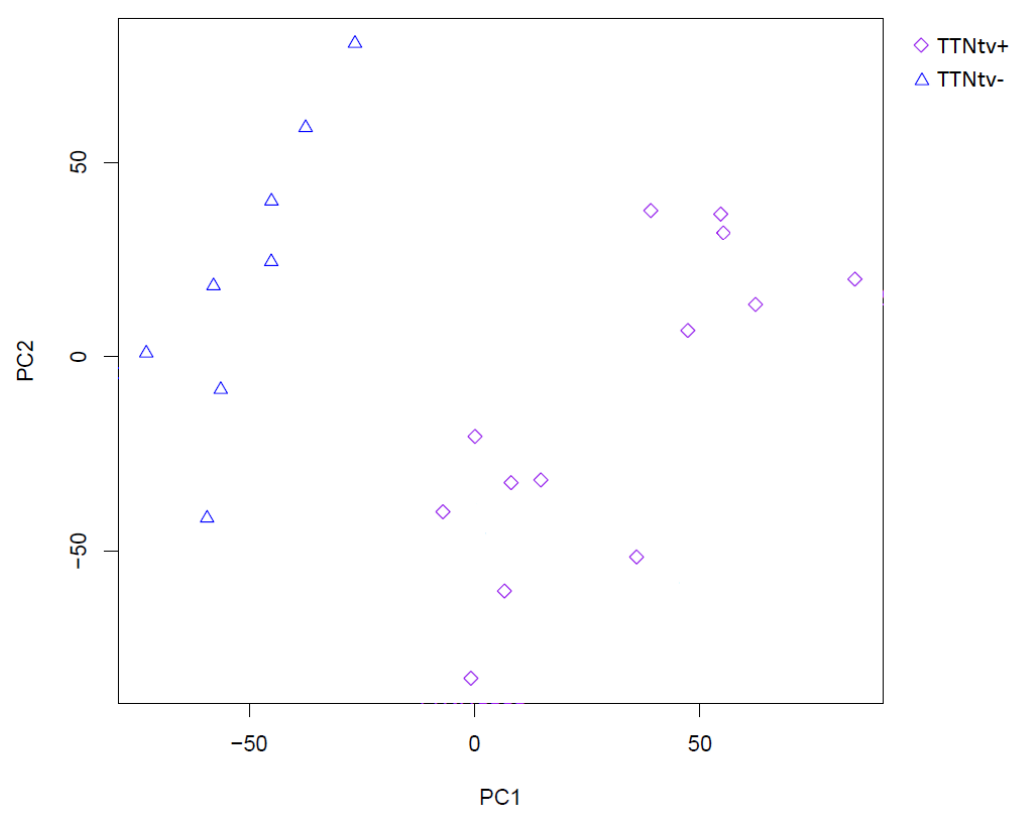

\section{Supplemental Figure 4. Principal Component Analysis (PCA)}

Principal component analysis using RNA-sequencing data from the endomyocardial biopsies. PCA component 1 can separate the TTNtv group from the non-TTNtv group. 


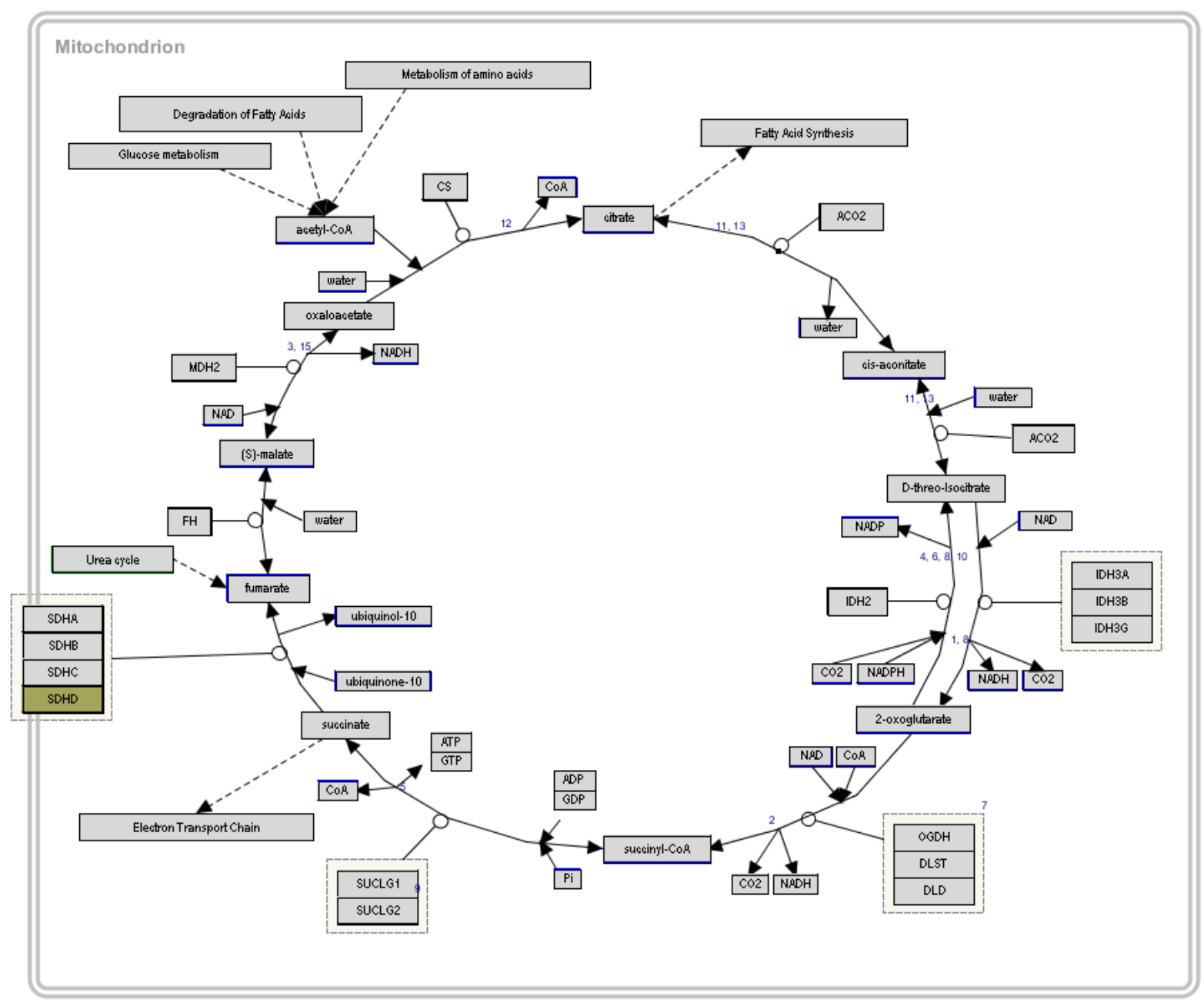

Supplemental Figure 5. Pathway analysis of the TCA cycle

Graphic representation of the TCA cycle using Wikipathways and Pathvisio. Yellow blocks indicate upregulated mRNA levels. Blocks with a blue line represent metabolites instead of genes. 

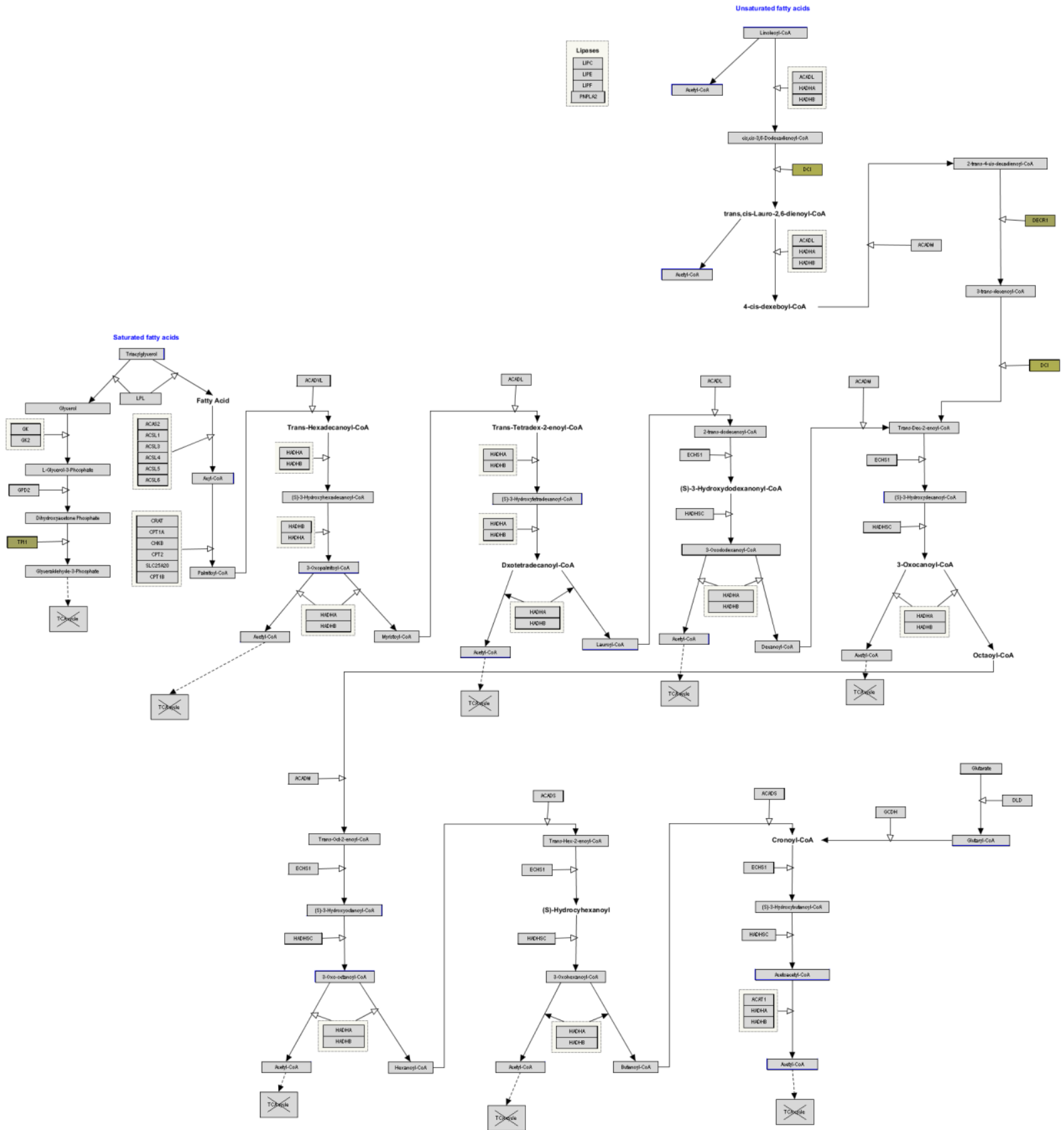

\section{Supplemental Figure 6. Pathway analysis of fatty acid $\beta$-oxidation}

Graphic representation of the fatty acid $\beta$-oxidation using Wikipathways and Pathvisio. Yellow blocks indicate upregulated mRNA levels. Blocks with a blue line represent metabolites instead of genes. 


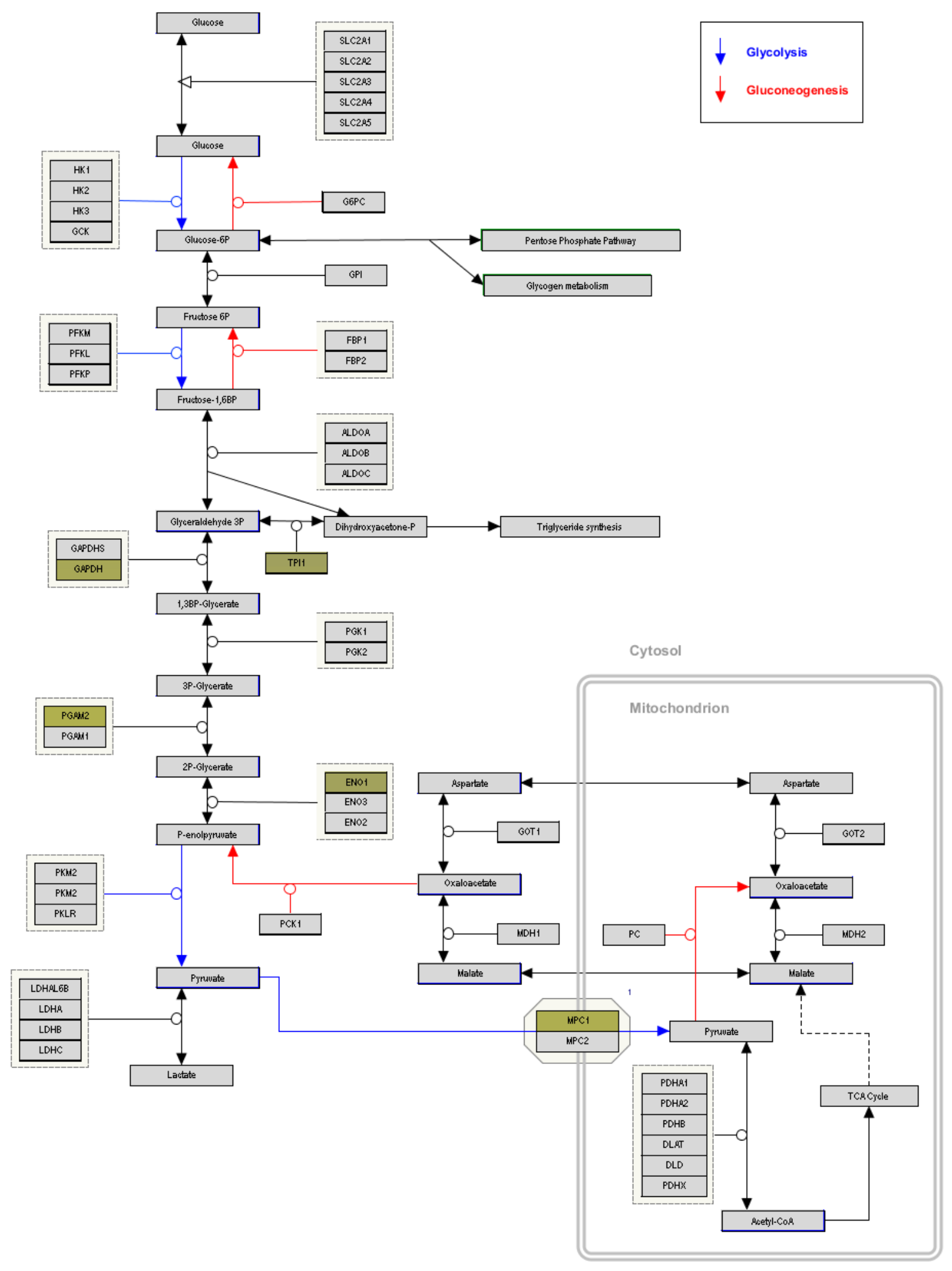

Supplemental Figure 7. Pathway analysis of glycolysis

Graphic representation of the glycolysis pathways using Wikipathways and Pathvisio. Yellow blocks indicates upregulated mRNA levels. Blocks with a blue line represent metabolites instead of genes. 


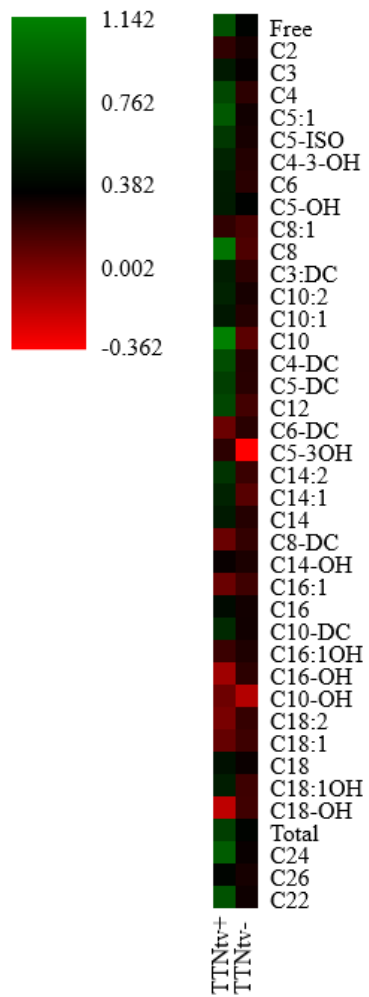

\section{Supplemental Figure 8. Heat map of plasma acylcarnitines}

Plasma acylcarnitines and very-long-chain fatty acids were measured in DCM patients with and without a TTNtv. The measured values of each metabolite were subtracted by the mean then divided by the standard deviation to get $z$-scores. The mean z-scores of each group is illustrated in the heatmap.
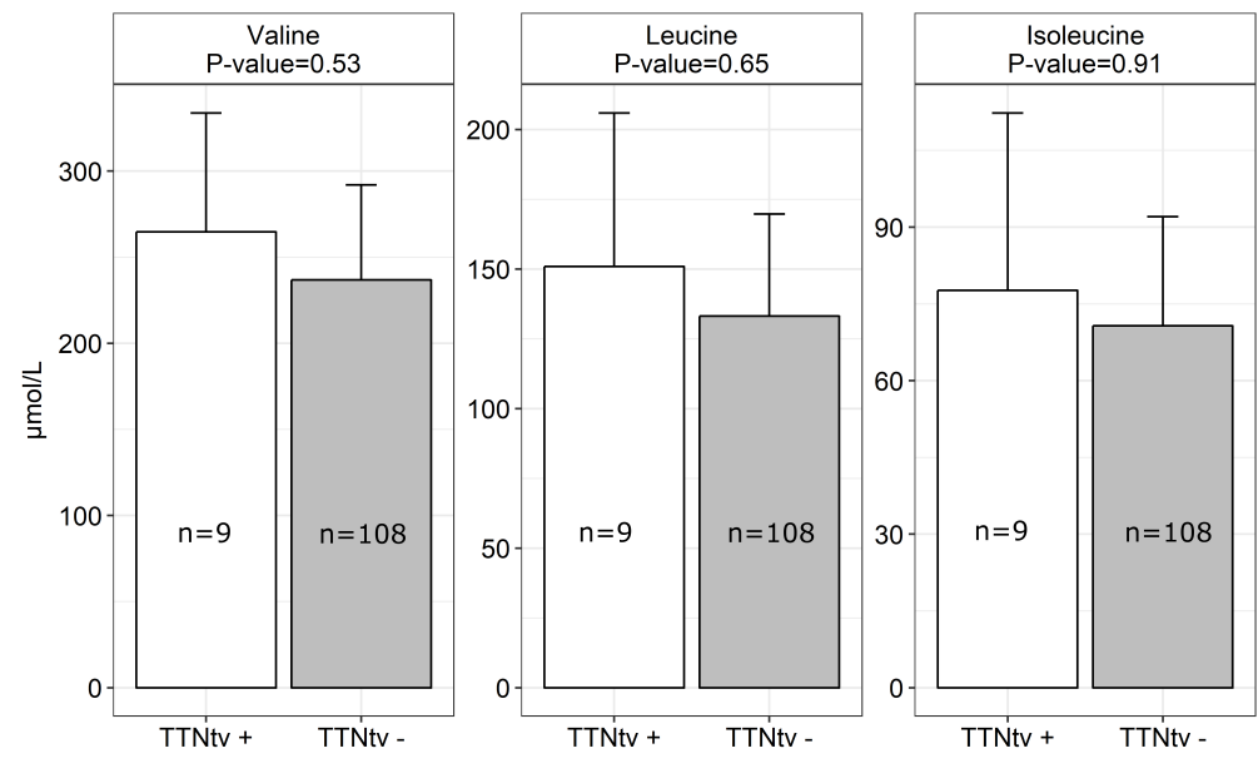

Supplemental Figure 9. Plasma branched-chain amino acids

Values of plasma valine, leucine and isoleucine of DCM patients with $(n=9)$ and without a TTNtv $(n=108)$. Data are shown as means + SD; analysis was adjusted for gender. 


\section{SUPPLEMENTARY TABLES}

Supplemental Table 1. Overview of all 47 genes used in the Maastricht Cardiomyopathy gene-panel.

\begin{tabular}{|c|c|c|c|}
\hline & HGNC.ID & HGNC.symbol & HGNC.Name \\
\hline 1 & HGNC:143 & ACTC1 & Actin, alpha, cardiac muscle 1 \\
\hline 2 & HGNC:164 & ACTN2 & Actinin alpha 2 \\
\hline 3 & HGNC:15819 & ANKRD1 & Ankyrin repeat domain 1 \\
\hline 4 & HGNC:939 & $B A G 3$ & BCL2 associated athanogene 3 \\
\hline 5 & HGNC:20407 & CALR3 & Calreticulin 3 \\
\hline 6 & HGNC:1529 & CAV3 & Caveolin 3 \\
\hline 7 & HGNC:2389 & CRYAB & Crystallin alpha B \\
\hline 8 & HGNC:2472 & CSRP3 & Cysteine and glycine rich protein 3 \\
\hline 9 & HGNC:2511 & CTNNA3 & Catenin alpha 3 \\
\hline 10 & HGNC:2770 & $D E S$ & Desmin \\
\hline 11 & HGNC:3036 & DSC2 & Desmocollin 2 \\
\hline 12 & HGNC:3049 & DSG2 & Desmoglein 2 \\
\hline 13 & HGNC:3052 & $D S P$ & Desmoplakin \\
\hline 14 & HGNC:3331 & $E M D$ & Emerin \\
\hline 15 & HGNC:3702 & FHL1 & Four and a half LIM domains 1 \\
\hline 16 & HGNC:4296 & $G L A$ & Galactosidase alpha \\
\hline 17 & HGNC:14202 & JPH2 & Junctophilin 2 \\
\hline 18 & HGNC:6207 & $J U P$ & Junction plakoglobin \\
\hline 19 & HGNC:6484 & LAMA4 & Laminin subunit alpha 4 \\
\hline 20 & HGNC:6501 & LAMP2 & Lysosomal associated membrane protein 2 \\
\hline 21 & HGNC:15710 & $\angle D B 3$ & LIM domain binding 3 \\
\hline 22 & HGNC:6636 & LMNA & Lamin A/C \\
\hline 23 & HGNC:21086 & MIB1 & Mindbomb E3 ubiquitin protein ligase 1 \\
\hline 24 & HGNC:7551 & MYBPC3 & Myosin binding protein $\mathrm{C}$, cardiac \\
\hline 25 & HGNC:7576 & MYH6 & Myosin heavy chain 6 \\
\hline 26 & HGNC:7577 & MYH7 & Myosin heavy chain 7 \\
\hline 27 & HGNC:7583 & MYL2 & Myosin light chain 2 \\
\hline 28 & HGNC:7584 & $M Y L 3$ & Myosin light chain 3 \\
\hline 29 & HGNC:1330 & MYOZ2 & Myozenin 2 \\
\hline 30 & HGNC:23246 & MYPN & Myopalladin \\
\hline 31 & HGNC:29557 & NEXN & Nexilin F-actin binding protein \\
\hline 32 & HGNC:9024 & PKP2 & Plakophilin 2 \\
\hline 33 & HGNC:9080 & PLN & Phospholamban \\
\hline 34 & HGNC:14000 & PRDM16 & PR/SET domain 16 \\
\hline 35 & HGNC:9386 & PRKAG2 & Protein kinase AMP-activated non-catalytic subunit gamma 2 \\
\hline 36 & HGNC:27424 & RBM20 & RNA binding motif protein 20 \\
\hline 37 & HGNC:10593 & SCN5A & Sodium voltage-gated channel alpha subunit 5 \\
\hline 38 & HGNC:11577 & $T A Z$ & Tafazzin \\
\hline 39 & HGNC:11610 & TCAP & Titin-cap \\
\hline 40 & HGNC:28472 & TMEM43 & Transmembrane protein 43 \\
\hline 41 & HGNC:11943 & TNNC1 & Troponin C1, slow skeletal and cardiac type \\
\hline 42 & HGNC:11947 & TNNI3 & Troponin 13, cardiac type \\
\hline 43 & HGNC:11949 & TNNT2 & Troponin T2, cardiac type \\
\hline 44 & HGNC:12010 & TPM1 & Tropomyosin 1 \\
\hline
\end{tabular}




\begin{tabular}{llcc}
45 & HGNC:12403 & $T T N$ & Titin \\
46 & HGNC:12405 & $T T R$ & Transthyretin \\
47 & HGNC:12665 & $V C L$ & Vinculin \\
\hline
\end{tabular}

Supplemental table 3. Overview of all identified pathogenic mutations with the used (46)-gene panel in 303 DCM patients

\begin{tabular}{|c|c|c|c|c|c|}
\hline & cDNA variant & Variant type & Amino acid change & $\begin{array}{l}\text { No. of index } \\
\text { patients }\end{array}$ & $\begin{array}{c}\text { ExAC Count } \\
\text { (percentage) }\end{array}$ \\
\hline ACTC & c. $623 G>A$ & Missense & p.(Arg208His) & 1 & 5 (4.12E-05) \\
\hline$B A G 3$ & c. $910 \mathrm{C}>\mathrm{T}$ & Nonsense & p. $\left(\mathrm{G} \ln 304^{*}\right)$ & 1 & 0 \\
\hline$D E S$ & c. $725 \mathrm{~T}>\mathrm{A}$ & Missense & p.(Val242Glu) & 1 & 0 \\
\hline$D S P$ & c.7773_7776del & Frameshift & p.(Ser2591Argfs*11) & 1 & $1(8.24 \mathrm{E}-06)$ \\
\hline $\mathrm{JPH} 2$ & c. $88 \mathrm{~A}>\overline{\mathrm{T}}$ & Missense & p.(Thr30Ser) & 1 & 0 \\
\hline LAMA4 & c. $3161 \mathrm{G}>\mathrm{A}$ & Missense & p.(Gly1054Asp) & 1 & 1 (8.24E-06) \\
\hline \multirow[t]{5}{*}{ LMNA } & c.313_314delinsTT & Missense & p.(Glu105Leu) & 1 & 0 \\
\hline & c. $208 \mathrm{G}>\mathrm{A}$ & Missense & p.(Val70lle) & 1 & 0 \\
\hline & c. $586 \mathrm{C}>\mathrm{T}$ & Missense & p.(Arg190Trp) & 1 & 0 \\
\hline & c. $481 G>A$ & Missense & p.(Glu161Lys) & 2 & 0 \\
\hline & c. $357-2 A>G$ & Essential splice site & p.(?) & 1 & 0 \\
\hline \multirow[t]{3}{*}{ MIB1 } & c. $912 \mathrm{G}>\mathrm{A}$ & Nonsense & p. $\left(\operatorname{Trp} 304^{*}\right)$ & 1 & 0 \\
\hline & c. $1371+1 \mathrm{G}>\mathrm{A}$ & Essential splice site & p.(?) & 1 & 0 \\
\hline & c. $2176 \mathrm{C}>\mathrm{T}$ & Nonsense & p.(Arg906*) & 1 & $8(6.6 \mathrm{E}-05)$ \\
\hline \multirow[t]{2}{*}{ МуВРСЗ } & c. $3065 \mathrm{G}>\mathrm{C}$ & Missense & p.(Arg1022Pro) & 1 & $3(2.5 E-05)$ \\
\hline & c. $1468 \mathrm{G}>\mathrm{A}$ & Missense & p.(Gly490Arg) & 1 & 25 (2.1E-04) \\
\hline \multirow[t]{2}{*}{ MYH7 } & c. $2167 C>T$ & Missense & p.(Arg723Cys) & 1 & $3(2.5 \mathrm{E}-05)$ \\
\hline & c.2201dupA & Frameshift & p.(Phe735Valfs*3) & 1 & 0 \\
\hline$M Y L 3$ & c. $476 C>T$ & Missense & p.(Thr159Met) & 2 & 3 (2.5E-05) \\
\hline MYPN & c. $806 \mathrm{C}>\mathrm{T}$ & Missense & p.(Pro269Leu) & 1 & 0 \\
\hline$N E X N$ & c.1909_1912del & Frameshift & p.(Tyr637Alafs*48) & 2 & $1(8.3 E-06)$ \\
\hline PKP2 & c. $2077 \bar{A}>G$ & Missense & p.(Ser693Gly) & 1 & 2 (1.6E-05) \\
\hline PLN & c.40_42del & Deletion & p.(Arg14del) & 1 & 0 \\
\hline \multirow[t]{5}{*}{ RBM20 } & c.419del & Frameshift & p.(Pro140Argfs*3) & 1 & 0 \\
\hline & c. $1900 C>T$ & Missense & p.(Arg634Trp) & 2 & 0 \\
\hline & c. $2147 \mathrm{G}>\mathrm{A}$ & Missense & p. $(\operatorname{Arg} 716 \mathrm{Gln})$ & 1 & $6(3.0 \mathrm{E}-04)$ \\
\hline & c. $1528-1 G>C$ & Essential splice site & p.(?) & 1 & 0 \\
\hline & c. $1764 \mathrm{~T}>\mathrm{G}$ & Missense & p.(Ile588Met) & 1 & 0 \\
\hline SCN5A & c. $4057 \mathrm{G}>\mathrm{A}$ & Missense & p.(Val1353Met) & 1 & 6 (4.9E-05) \\
\hline TNNC1 & c. $317+1 G>A$ & Essential splice site & p.(?) & 1 & 0 \\
\hline$V C L$ & c. $2848 \mathrm{G}>\mathrm{A}$ & Missense & p.(Glu950Lys) & 1 & 0 \\
\hline
\end{tabular}


Supplemental table 2. Overview of all identified pathogenic TTNtv mutations in 303 DCM patients

\begin{tabular}{|c|c|c|c|c|c|c|c|}
\hline cDNA variant & Variant type & Amino acid change & $\begin{array}{c}\text { No. of index } \\
\text { patients }\end{array}$ & $\begin{array}{l}\text { ExAC Count } \\
\text { (percentage) }\end{array}$ & $\begin{array}{l}\text { TTN } \\
\text { exon }\end{array}$ & $\begin{array}{l}\text { TTN } \\
\text { band }\end{array}$ & Co-segregation in family* \\
\hline c.41641C>T & Nonsense & p.(Arg13381*) & 1 & 1 (3.24E-05) & 227 & I-band & Not known \\
\hline c. $42267 \mathrm{del}$ & Frameshift & p.(Asp14090llefs*2) & 1 & 0 & 230 & I-band & Not known \\
\hline c.44958del & Frameshift & p.(Lys14986Asnfs*30) & 1 & 0 & 244 & I-band & Not known \\
\hline c.47137_47138del & Frameshift & p.(Ser15713Leufs*2) & 3 & 0 & 252 & A-band & Yes ( 1 of 3 families) \\
\hline c.50074_50075del & Frameshift & p.(Asp16692Argfs*25) & 1 & 0 & 266 & A-band & Not known \\
\hline c. $50296 \bar{C}>T$ & Nonsense & p. $\left(\operatorname{Arg} 16766^{*}\right)$ & 1 & $1(4.1 \mathrm{E}-06)$ & 267 & A-band & Not known \\
\hline c.53918del & Frameshift & p.(Gly17973Glufs*18) & 1 & $1(4.24 \mathrm{E}-06)$ & 280 & A-band & Not known \\
\hline c.54768del & Frameshift & p.(Ser18258Valfs*34) & 2 & 0 & 282 & A-band & Not known \\
\hline c. $57163 \mathrm{G}>\mathrm{T}$ & Nonsense & p.(Glu19055*) & 1 & 0 & 293 & A-band & Yes \\
\hline c.58327del & Frameshift & p.(Ala19443Leufs*2) & 3 & 0 & 297 & A-band & Not known \\
\hline c. $60931 C>T$ & Nonsense & p. $\left(\operatorname{Arg} 20311^{*}\right)$ & 1 & 1 (4.09E-06) & 304 & A-band & Not known \\
\hline c. $61921 C>T$ & Nonsense & p. $\left(\operatorname{Arg} 20641^{*}\right)$ & 1 & 0 & 304 & A-band & Not known \\
\hline c. $63794-1 G>A$ & Essential splice site & p.(?) & 1 & 0 & 306 & A-band & Yes \\
\hline c.64688del & Frameshift & p.(Pro21563Leufs*10) & 1 & 0 & 310 & A-band & Not known \\
\hline c.65042del & Frameshift & p.(Asp21681Alafs*15) & 1 & 0 & 311 & A-band & Not known \\
\hline c.66020_66021del & Frameshift & p.(Ser22007Cysfs*18) & 1 & 0 & 314 & A-band & Yes \\
\hline c.66296del & Frameshift & p.(Ala22099Valfs*13) & 1 & 0 & 315 & A-band & Not known \\
\hline c. $66628 \mathrm{C}>\mathrm{T}$ & Nonsense & p. $\left(G \ln 22210^{*}\right)$ & 1 & 0 & 316 & A-band & Not known \\
\hline c.67480_67484del & Nonsense & p.(Glu22494*) & 1 & 0 & 319 & A-band & Not known \\
\hline c. $67863 \mathrm{C}>\mathrm{G}$ & Nonsense & p.(Tyr22621*) & 1 & 0 & 320 & A-band & Yes \\
\hline c. $73332 C>A$ & Nonsense & p.(Cys24444*) & 1 & 0 & 326 & A-band & Not known \\
\hline c.78381dup & Frameshift & p.(Arg26128Thrfs*2) & 1 & 0 & 326 & A-band & Not known \\
\hline c.82313dup & Frameshift & p.(Asn27438Lysfs*2) & 1 & 0 & 326 & A-band & Not known \\
\hline c.87782del & Frameshift & p.(Pro29261Glnfs*10) & 4 & 0 & 329 & A-band & Yes (1 of 4 families) \\
\hline c. $90370 \mathrm{G}>\mathrm{T}$ & Nonsense & p.(Glu30124*) & 1 & 0 & 335 & A-band & Yes \\
\hline c.97427dup & Frameshift & p.(Thr32477Asnfs*13) & 3 & 0 & 349 & A-band & Yes ( 1 of 3 families) \\
\hline c. $100825 C>T$ & Nonsense & p. $(\operatorname{Arg} 33609 *)$ & 1 & 0 & 358 & A-band & Not known \\
\hline c. $106792 C>T$ & Nonsense & p. $\left(G \ln 35598^{*}\right)$ & 1 & 1 (4.07E-06) & 360 & M-band & Not known \\
\hline
\end{tabular}

* In 8 families, the mutation segregated with the DCM phenotype in the family 
Supplemental Table 4. Possible etiologies identified in DCM patients

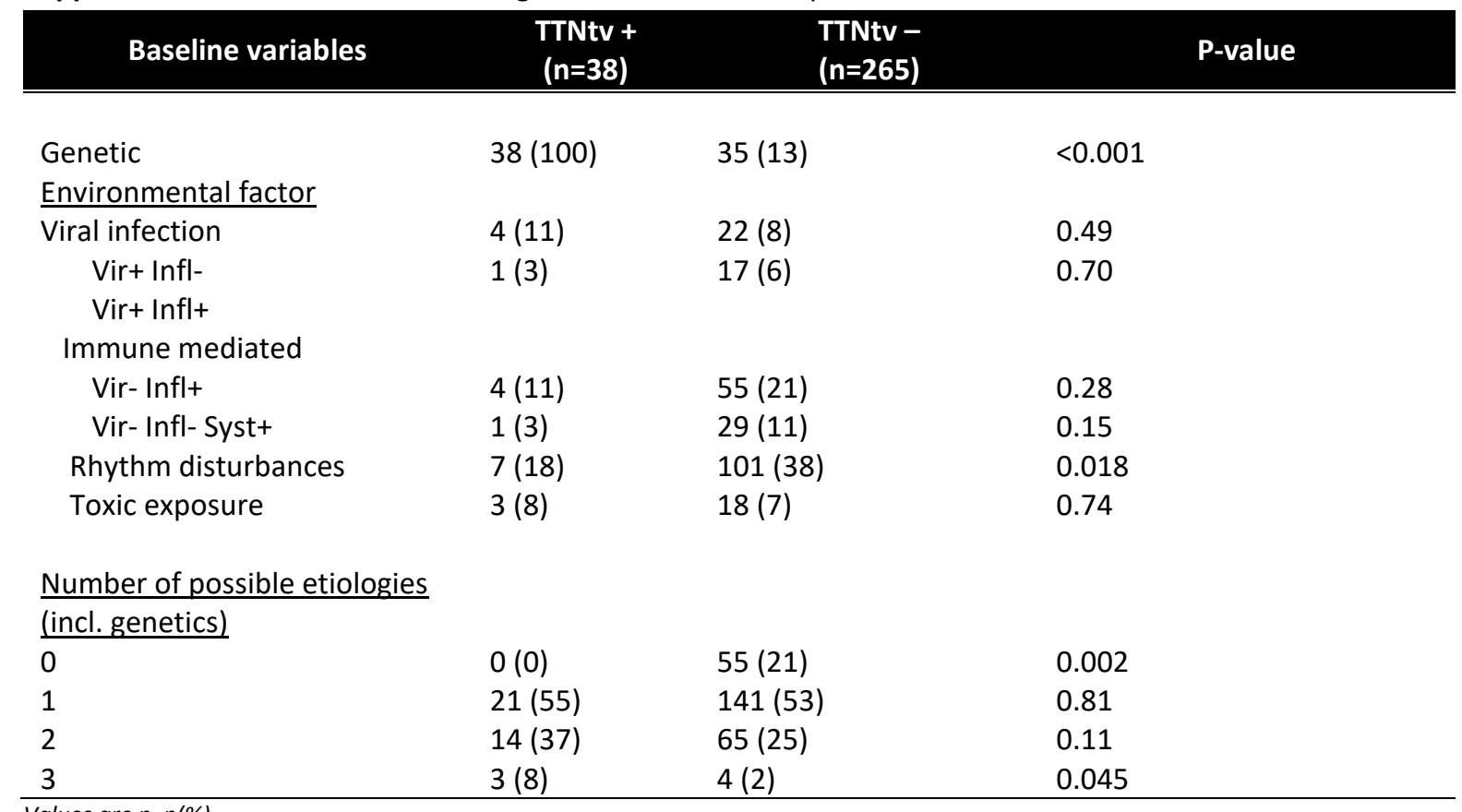

Values are $n, n(\%)$

Abbreviations, Virt, viral load >500 c/ug DNA in heart biopsy; Vir-, no virus presence or viral load <500 c/ug DNA; Infl+, significant cardiac inflammation according to the ESC guidelines; Infl-, no significant cardiac inflammation; Syst+, presence of a systemic disease

Supplemental Table 5. Baseline characteristics of DCM patients in the RNA-sequencing cohort

\begin{tabular}{|c|c|c|c|}
\hline Baseline variables & $\begin{array}{l}\text { TTNtv+ } \\
(n=13)\end{array}$ & $\begin{array}{l}\text { TTNtv- } \\
(n=8)\end{array}$ & p-value \\
\hline Male gender (\%) & $8(62)$ & $5(63)$ & 0.99 \\
\hline Age of diagnosis (years) & $51 \pm 12$ & $54 \pm 12$ & 0.28 \\
\hline Family history of DCM/SCD & $7(54)$ & $1(13)$ & 0.06 \\
\hline NYHA $\geq 3(\%)$ & $5(39)$ & $3(38)$ & 0.99 \\
\hline Symptom duration (month) & $6[3-96]$ & 2 [0-39] & 0.12 \\
\hline \multicolumn{4}{|l|}{ Genetic analysis } \\
\hline Pathogenic mutation (\%) & $13(100)$ & $0(0)$ & $<0.001$ \\
\hline \multicolumn{4}{|l|}{ ECG/Holter } \\
\hline cLBBB (\%) & $1(8)$ & $2(25)$ & 0.53 \\
\hline AV block (\%) & $0(0)$ & $0(0)$ & 0.99 \\
\hline $\begin{array}{c}\text { Atrial fibrillation (\%) } \\
\text { NSVT (\%) }\end{array}$ & $\begin{array}{l}3(23) \\
5(39)\end{array}$ & $\begin{array}{l}1(13) \\
1(13)\end{array}$ & $\begin{array}{l}0.99 \\
0.34\end{array}$ \\
\hline \multicolumn{4}{|l|}{ Echocardiography } \\
\hline LVEF (\%) & $29 \pm 11$ & $30 \pm 12$ & 0.74 \\
\hline LVEF $\leq 35 \%(\%)$ & $8(62)$ & $5(63)$ & 0.99 \\
\hline LVEDD (mm) & $62 \pm 6$ & $65 \pm 15$ & 0.65 \\
\hline LVESD (mm) & $54 \pm 8$ & $55 \pm 17$ & 0.87 \\
\hline LVEDDI $\left(\mathrm{mm} / \mathrm{m}^{2}\right)$ & $32 \pm 8$ & $33 \pm 3$ & 0.68 \\
\hline \multicolumn{4}{|l|}{ Cardiac MRI } \\
\hline LV Mi & $68 \pm 11$ & $96 \pm 43$ & $<0.001$ \\
\hline LV EDVi & $129 \pm 33$ & $179 \pm 62$ & 0.002 \\
\hline LV ESVi & $94 \pm 30$ & $137 \pm 63$ & 0.002 \\
\hline LV SVi & $38 \pm 9$ & $42 \pm 10$ & 0.58 \\
\hline
\end{tabular}




$\begin{array}{cccr}\text { LV EF } & 29 \pm 9 & 25 \pm 10 & 0.10 \\ \text { Midwall fibrosis (\%) } & 7(54) & 2(50) & 0.99 \\ \text { Endomyocardial Biopsy } & & & \\ \text { Cardiac Inflammation (\%) } & 2(15) & 2(25) & 0.62 \\ \text { CD3+ (cells/mm²) } & 5.94[4.23-9] & 4.88[1.02-10.4] & 0.62 \\ \text { CD45+ (cells/mm }) & 8.46[6.6-10.6] & 10.1[6.08-11.8] & 0.41 \\ \text { CD68+ (cells/mm } \mathrm{mm}^{2} \text { ) } & 3.8[1.9-6.3] & 3.69[2-20.5] & 0.60 \\ \text { Myocardial Fibrosis } & 12.1[8.62-16.1] & 6.33[5.66-7.3] & <0.001 \\ \text { Significant viral load } & & & \\ \text { PVB19 (\%) } & 1(8) & 0(0) & 0.53 \\ \text { HHV6 (\%) } & 0(0) & 0(0) & 0.99\end{array}$

Values are mean $\pm S D, n, n(\%)$, or median (interquartile range).

Abbreviations, CLBBB, complete left bundle branch block; AV-block, atrioventricular block; NSVT, non-sustained ventricular tachycardia; LVEF, left ventricular ejection fraction; LVEDD, left ventricular end-diastolic diameter; LVESD, left ventricular end-systolic diameter; LVEDDI, indexed LVEDD; LV Mi, indexed left ventricular mass; LV EDVi, indexed left ventricular end-diastolic volume; LV ESVi, indexed left ventricular endsystolic volume; LV SVi, indexed left ventricular stroke volume; PVB19, parvovirus B19; HHV6, human herpesvirus 6.

Supplemental Table 6. TTN mRNA expression in endomyocardial biopsies from DCM patients with and without TTNtv

\begin{tabular}{cccc}
\hline Transcript & Fold-change TTNtv+/TTNtv- & P value & FDR \\
\hline TTN N2BA & 0.62 & 0.13 & 0.66 \\
TTN N2B & 0.62 & 0.13 & 0.66 \\
TTN N2A & 0.62 & 0.13 & 0.66 \\
TTN 1C & 0.62 & 0.13 & 0.66 \\
TTN Novex-1 & 0.62 & 0.13 & 0.66 \\
TTN Novex-2 & 0.62 & 0.13 & 0.66 \\
TTN Novex-3 & 0.39 & 0.017 & 0.25 \\
\hline FDR = False discovery rate & & &
\end{tabular}

Supplemental Table 7. Baseline characteristics of DCM patients in the metabolomics cohort

\begin{tabular}{cccc} 
Baseline variables & $\begin{array}{c}\text { TTNtv }+ \\
\text { (n=9) }\end{array}$ & $\begin{array}{c}\text { TTNtv - } \\
\text { (n=108) }\end{array}$ & P-value \\
\hline Male gender (\%) & $9(100)$ & $63(58)$ & 0.012 \\
Age of diagnosis (years) & $51 \pm 10$ & $52 \pm 18$ & 0.77 \\
Family history of DCM/SCD & $4(44)$ & $14(13)$ & 0.031 \\
NYHA $\geq 3$ (\%) & $1(11)$ & $19(18)$ & 0.99 \\
Symptom duration (month) & $3[0-8]$ & $3[0-21]$ & 0.72 \\
Genetic analysis & & & \\
Pathogenic mutation (\%) & $9(100)$ & $14(13)$ & $<0.001$ \\
& & & \\
ECG/Holter & $1(11)$ & $34(31)$ & 0.28 \\
CLBBB (\%) & $2(22)$ & $5(5)$ & 0.08 \\
AV block (\%) & $3(33)$ & $18(17)$ & 0.20 \\
Atrial fibrillation (\%) & $3(33)$ & $13(12)$ & 0.07 \\
NSVT (\%) & & & \\
Echocardiography & $33 \pm 13$ & $33 \pm 10$ & 0.96 \\
LVEF (\%) & $4(44)$ & $50(46)$ & 0.99 \\
LVEF $\leq 35 \%(\%)$ & $60 \pm 10$ & $59 \pm 9$ & 0.70 \\
LVEDD (mm) & & &
\end{tabular}




\begin{tabular}{cccc} 
LVESD $(\mathrm{mm})$ & $52 \pm 12$ & $49 \pm 10$ & 0.53 \\
LVEDDI (mm/m²) & $31 \pm 5$ & $30 \pm 5$ & 0.65 \\
Cardiac MRI & & & \\
\hline LV Mi & $63 \pm 4$ & $74 \pm 20$ & $<0.001$ \\
LV EDVi & $124 \pm 39$ & $134 \pm 46$ & 0.61 \\
LV ESVi & $91 \pm 37$ & $91 \pm 48$ & 0.97 \\
LV SVi & $40 \pm 6$ & $44 \pm 11$ & 0.44 \\
LV EF & $31 \pm 12$ & $36 \pm 13$ & 0.39 \\
Midwall fibrosis (\%) & $5(71)$ & $27(25)$ & 0.1 \\
& & & \\
Endomyocardial Biopsy & & $19(18)$ & 0.99 \\
Cardiac Inflammation (\%) & $1(20)$ & $5[2.8-8.5]$ & 0.60 \\
CD3+ (cells/mm²) & $5.9[4.5-7]$ & $8.9[5.5-13.2]$ & 0.82 \\
CD45+ (cells/mm $\left./ \mathrm{mm}^{2}\right)$ & $10[6.8-11.6]$ & $2.3[1.2-4.7]$ & 0.65 \\
CD68+ (cells/mm $\left./ \mathrm{mm}^{2}\right)$ & $2[2-3.9]$ & $6.21[3.7-9.7]$ & 0.05 \\
Myocardial Fibrosis & $15.48[6.5-21.7]$ & & \\
Significant viral load & & $16(15)$ & 0.59 \\
PVB19 (\%) & $0(0)$ & $3(3)$ & 0.99 \\
HHV6 (\%) & $0(0)$ & &
\end{tabular}

Values are mean $\pm S D, n, n(\%)$, or median (interquartile range).

Abbreviations, CLBBB, complete left bundle branch block; AV-block, atrioventricular block; NSVT, non-sustained ventricular tachycardia; LVEF, left ventricular ejection fraction; LVEDD, left ventricular end-diastolic diameter; LVESD, left ventricular end-systolic diameter; LVEDDI, indexed LVEDD; LV Mi, indexed left ventricular mass; LV EDVi, indexed left ventricular end-diastolic volume; LV ESVi, indexed left ventricular endsystolic volume; LV SVi, indexed left ventricular stroke volume; PVB19, parvovirus B19; HHV6, human herpesvirus 6.

Supplemental table 8. Plasma acylcarnitines and very long chain fatty acids from TTNtv+ and TTNtv- DCM patients in $\mu \mathrm{mol} / \mathrm{L}$.

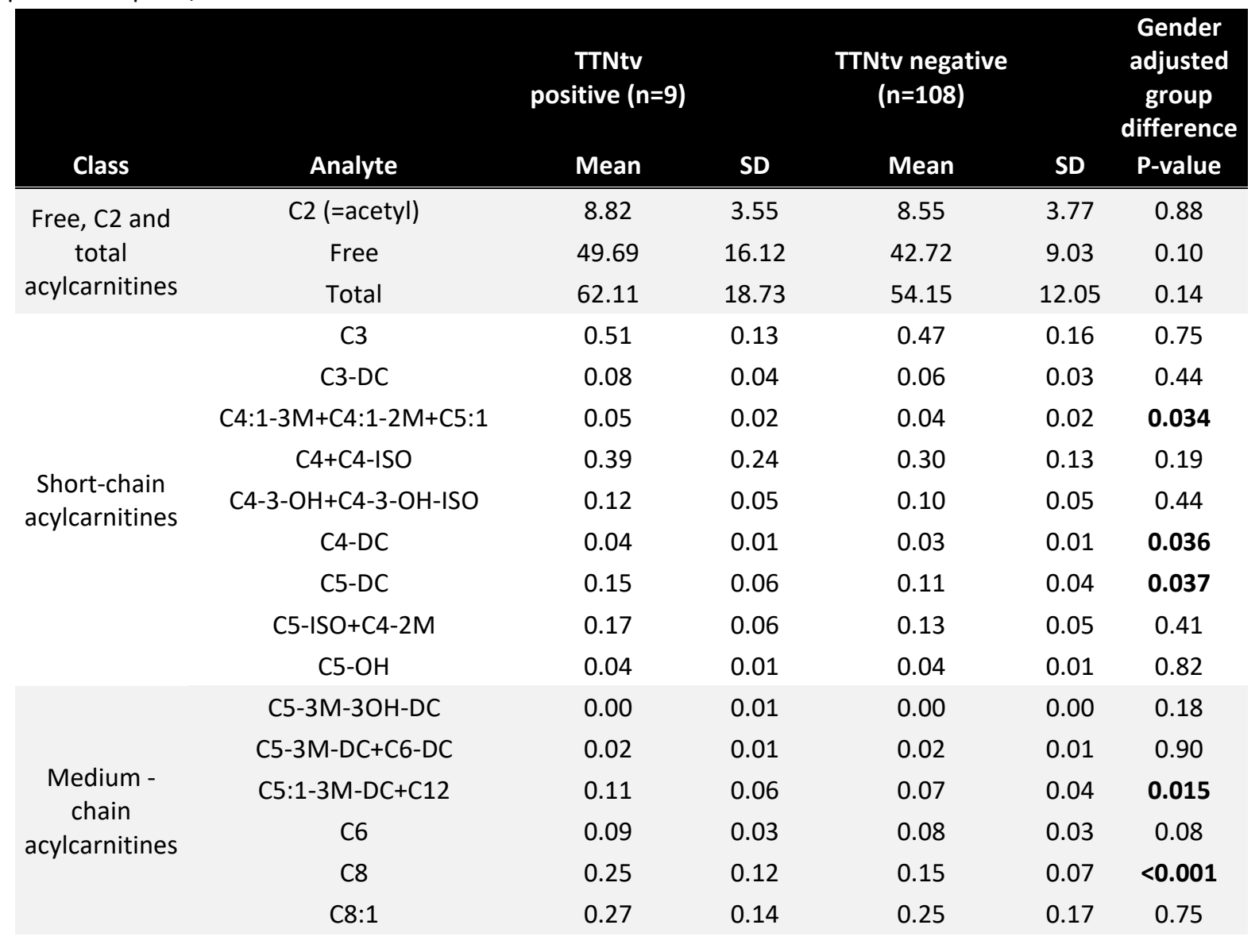




\begin{tabular}{ccccccc} 
& C8-DC & 0.02 & 0.01 & 0.02 & 0.01 & 0.79 \\
& C10 & 0.38 & 0.21 & 0.20 & 0.11 & 0.001 \\
& $\mathrm{C} 10: 1$ & 0.15 & 0.06 & 0.12 & 0.05 & 0.18 \\
& $\mathrm{C} 10: 2$ & 0.04 & 0.01 & 0.03 & 0.01 & 0.16 \\
& $\mathrm{C} 10-\mathrm{DC}$ & 0.02 & 0.01 & 0.02 & 0.01 & 0.27 \\
& $\mathrm{C} 10-\mathrm{OH}$ & 0.00 & 0.01 & 0.00 & 0.00 & 0.23 \\
& $\mathrm{C} 14: 1$ & 0.11 & 0.07 & 0.08 & 0.05 & 0.06 \\
& $\mathrm{C} 14: 2$ & 0.06 & 0.03 & 0.04 & 0.02 & 0.06 \\
& $\mathrm{C} 14+\mathrm{C} 8: 1-\mathrm{DC}$ & 0.05 & 0.01 & 0.04 & 0.02 & 0.06 \\
Long-chain & $\mathrm{C} 14-\mathrm{OH}$ & 0.01 & 0.01 & 0.01 & 0.01 & 0.49 \\
acylcarnitines & $\mathrm{C} 16: 1$ & 0.03 & 0.02 & 0.04 & 0.02 & 0.93 \\
& $\mathrm{C} 16: 1-\mathrm{OH}$ & 0.01 & 0.01 & 0.01 & 0.01 & 0.91 \\
& $\mathrm{C} 16+\mathrm{C} 10: 1-\mathrm{DC}$ & 0.14 & 0.03 & 0.12 & 0.04 & 0.27 \\
& $\mathrm{C} 16-\mathrm{OH}$ & 0.01 & 0.01 & 0.01 & 0.00 & 0.25 \\
& $\mathrm{C} 18$ & 0.07 & 0.02 & 0.06 & 0.02 & 0.36 \\
& $\mathrm{C} 18: 1$ & 0.14 & 0.04 & 0.15 & 0.10 & 0.99 \\
Very long & $\mathrm{C} 18: 1-\mathrm{OH}$ & 0.01 & 0.01 & 0.01 & 0.00 & 0.30 \\
chain fatty & $\mathrm{C} 18: 2$ & 0.06 & 0.02 & 0.07 & 0.04 & 0.71 \\
acids & $\mathrm{C} 18-\mathrm{OH}$ & 0.01 & 0.01 & 0.01 & 0.00 & 0.45 \\
\hline & $\mathrm{C} 22: 0$ & 77.20 & 18.93 & 64.31 & 14.57 & $\mathbf{0 . 0 1 6}$ \\
& $\mathrm{C} 24: 0$ & 72.60 & 17.43 & 60.72 & 14.86 & $\mathbf{0} 0.038$ \\
& $\mathrm{C} 26: 0$ & 0.80 & 0.36 & 0.72 & 0.30 & 0.47 \\
\hline
\end{tabular}


- 103 - 


\title{
EDITORIAL COMMENT
}

Titin cardiomyopathy: why we need to go big to understand the giant

\author{
Upasana Tayal, MD, $P h D^{1}$ \\ Sanjay K. Prasad, $M D^{2}$ \\ ${ }^{1}$ National Heart Lung Institute, Imperial College London, UK. \\ ${ }^{2}$ Cardiovascular Research Centre, Royal Brompton Hospital, London, UK.
}

EUROPEAN HEART JOURNAL.

2018. MAR 7;39(10):874-875. 
Truncating variants in the titin gene (TTNtv) are the most common genetic contributor to dilated cardiomyopathy (DCM), found in $\sim 15 \%$ of unselected DCM patients ${ }^{1}$ and up to $27 \%$ of end-stage DCM patients ${ }^{2}$. As such, identification of reproducible and clinically informative genotype-phenotype correlations offers potential for precision genotype-guided management of DCM. Titin is the largest human protein. It is expressed in cardiac and skeletal muscle, and is integral to sarcomere structure and function. In cardiomyocytes, one titin molecule spans the hemi-sarcomere from the $Z$ disc to the $M$ line. Titin has roles in maintaining sarcomere integrity, force transmission, stretch sensing, and signaling. It is composed of four modular domains ( $Z$ disc, I band, A band, and $M$ line), with the extensible I band contributing to sarcomere relaxation and contraction. Dilated cardiomyopathy has been shown to have a familial basis in $\sim 20-30 \%$ of cases ${ }^{3}$. A genetic basis to DCM has been proposed in $\sim 40 \%$ of cases ${ }^{4}$, although with a more critical evaluation of the genes linked to DCM the true percentage is likely to be smaller. The identification of TTNtv in DCM, however, has substantially increased the number of informative genetic tests. In the clinic, these data are particularly useful for informing cascade screening. The odds ratios for TTNtv in constitutively expressed exons (spliced into the final transcript with an efficiency of $>90 \%$ ) in the I (19-32) or A (49.8) bands are high ${ }^{5}$. Such a variant in the affected proband that is absent in an unaffected relative ought to facilitate the discharge of the relative from ongoing clinical follow-up; cost-effective for the healthcare system and importantly reassuring for the individual. There are a number of unanswered questions, and what remains unclear is how current data can be used to guide clinical management in an affected individual. What is the genotype-phenotype interaction and what does having a TTNtv mean with respect to longer term clinical outcomes-particularly compared with other forms of DCM? What are the mechanisms underpinning any differences in disease expression and how responsive is TTNtv DCM compared with other causes of DCM to usual therapy?

The higher prevalence of TTNtv in patients with end-stage heart failure in earlier studies has previously led to the conclusion that TTNtv are associated with a more severe phenotype and could inform prognosis. There have now been a number of TTNtv DCM phenotype studies ${ }^{126-8}$, including our own recently published study of 83 TTNtv DCM patients amongst a cohort of $>700$ DCM patients. Common to these studies so far is the finding that there are no clear phenotypic differences in biventricular function or distinctly different clinical outcomes between DCM patients with and without TTNtv. To date, TTNtv DCM is associated with early ventricular and atrial arrhythmias ${ }^{910}$, but studies have not consistently shown an association with arrhythmias occurring later in the disease course. In addition, it has been postulated that environmental factors could interact with TTNtv to modify the DCM phenotype ${ }^{511}$. This has not yet been robustly evaluated.

The elegant study by Verdonschot et al. in this issue of the journal ${ }^{12}$ seeks to address a number of these issues. The authors evaluate the phenotype of 38 patients with TTNtv, taken from a mixed DCM and hypokinetic non-dilated cardiomyopathy cohort of 303 patients. All patients underwent detailed evaluation including echocardiography, sequencing, as well as metabolic profiling. A high proportion had cardiovascular magnetic resonance for fibrosis detection and an endomyocardial biopsy to facilitate RNA sequencing, including evaluation of molecular pathways. A number of important observations are made. At baseline, the study replicates recent findings of a limited hypertrophic response in TTNtv DCM ${ }^{1}$ and confirms that there are no other clear phenotypic differences between positive and negative TTNtv groups. Patients were followed up for a median of $\mathbf{4 5}$ months for the primary composite endpoint of death, 
cardiac transplant, heart failure hospitalization, and major ventricular arrhythmias. In line with our recent study, the authors did not detect a difference in short- and long-term outcome survival between TTNtv-positive and TTNtv-negative DCM patients. TTNtv in DCM certainly do not have the adverse effect size on mortality associated with LMNA cardiomyopathy, the only currently clinically actionable genetic variant in DCM. There may be a more moderate effect, and this will need to be analysed in larger cohort studies. On subgroup analysis, TTNtv DCM patients appeared to have more life-threatening ventricular arrhythmias. This is therefore the first study to demonstrate a long-term arrhythmic phenotype of TTNtv DCM, albeit one which is not associated with adverse survival. As there were only 28 arrhythmic events across the cohort, including 9 in patients with TTNtv, multivariable analysis of the predictors of an arrhythmic phenotype was not possible and so the current findings will need replication and validation in a larger cohort of patients before any potential mainstream clinical application. Mechanistically, subset endomyocardial biopsy data identified increased interstitial fibrosis in TTNtv-positive patients compared with TTNtvnegative patients, and this may partly account for the relatively increased arrhythmic burden though, as interstitial fibrosis is a reactive and dynamic process, it is not yet clear if this is a TTNtv-specific finding or reflects other circumstances such as stage of disease, concurrent medication, or environmental factors.

Biopsy data also enabled the authors to perform an exploratory analysis of possible environmental modifiers of the TTNtv DCM phenotype such as viral load and inflammation, as well as systemic disease and toxin exposure. They found that the presence of an additional environmental risk factor in a patient with TTNtv DCM (present in 17 patients with TTNtv $D C M)$ was associated with a trend to an increased risk of long-term arrhythmias $(P=0.047)$. Unfortunately, conclusive analysis of the role of environmental modifiers was not possible with the sample size and event rate in this study. It is still highly plausible that the phenotype and prognosis of TTNtv DCM will be altered in the presence of an environmental modifier; this could also account for some of the differences in expressivity seen in families. This remains an interesting and active area of research, but one that will need larger, multicentre studies of outcome in TTNtv DCM. Insights from myocardial RNA sequencing demonstrated up-regulation of the mitochondrial electron transport chain in TTNtv hearts. This is a striking finding, mirroring recent findings in a pre-clinical model ${ }^{5}$. Further work will be important to elucidate the mechanistic link between increased mitochondrial activity and TTNtv.

So how does one harness this information in a clinical setting. Clinically, the study by Verdonschot et al. ${ }^{12}$ makes an important contribution, adding to our understanding of the clinical phenotype of TTNtv DCM, that will help guide further study of DCM pathobiology, but does not yet support a change to current guidelines or clinical practice. The novel association with long-term arrhythmias identified in this study is interesting and will require replication in a larger cohort. Future studies should also focus on defining the subpopulations of DCM with a higher risk of arrhythmia to elucidate more fully any potential interaction. For example, TTNtv may have a role to play in risk stratification within a higher risk subset of DCM patients, such as those with low left ventricular ejection fraction and mid-wall fibrosis. It is notable in this study that patients with TTNtv DCM had more severely impaired ventricular function than found in previous studies.

Finally, a major unmet need in DCM risk stratification is in the identification of the relative importance of different risk variables across demographic, clinical, imaging, and 
genetic domains. A collective effort is now needed for multicentre collaboration to evaluate multiparametric risk stratification in DCM. 


\section{REFERENCES}

1. Tayal U, Newsome S, Buchan R, et al. Phenotype and Clinical Outcomes of Titin Cardiomyopathy. $J$ Am Coll Cardiol 2017;70(18):2264-74.

2. Herman DS, Lam L, Taylor MR, et al. Truncations of titin causing dilated cardiomyopathy. $N$ Engl J Med 2012;366(7):619-28.

3. Petretta $M$, Pirozzi $F$, Sasso $L$, et al. Review and metaanalysis of the frequency of familial dilated cardiomyopathy. Am J Cardiol 2011;108(8):11716.

4. Hershberger RE, Hedges DJ, Morales A. Dilated cardiomyopathy: the complexity of a diverse genetic architecture. Nature reviews Cardiology 2013;10(9):531-47.

5. Schafer S, de Marvao A, Adami E, et al. Titintruncating variants affect heart function in disease cohorts and the general population. Nature genetics 2017;49(1):46-53.

6. Franaszczyk M, Chmielewski P, Truszkowska G, et al. Titin Truncating Variants in Dilated Cardiomyopathy - Prevalence and GenotypePhenotype Correlations. PLoS One 2017;12(1):e0169007.

7. Jansweijer JA, Nieuwhof $K$, Russo $F$, et al. Truncating titin mutations are associated with a mild and treatable form of dilated cardiomyopathy. Eur J Heart Fail 2016;19(4):51221.

8. Roberts AM, Ware JS, Herman DS, et al. Integrated allelic, transcriptional, and phenomic dissection of the cardiac effects of titin truncations in health and disease. Science translational medicine 2015;7(270):270ra6.

9. Hoorntje ET, van Spaendonck-Zwarts KY, Te Rijdt $W P$, et al. The first titin (c.59926 + 1G > A) founder mutation associated with dilated cardiomyopathy. Eur J Heart Fail 2018;20(4):80306.

10. Tayal U, Newsome S, Buchan R, et al. Truncating Variants in Titin Independently Predict Early Arrhythmias in Patients With Dilated Cardiomyopathy. J Am Coll Cardiol 2017;69(19):2466-68.

11. Linschoten $M$, Teske AJ, Baas AF, et al. Truncating Titin (TTN) Variants in Chemotherapy-Induced Cardiomyopathy. J Card Fail 2017;23(6):476-79.

12. Verdonschot JAJ, Hazebroek MR, Derks KWJ, et al. Titin cardiomyopathy leads to altered mitochondrial energetics, increased fibrosis and long-term life-threatening arrhythmias. Eur Heart J 2018;39(10):864-73. 
- 109 - 


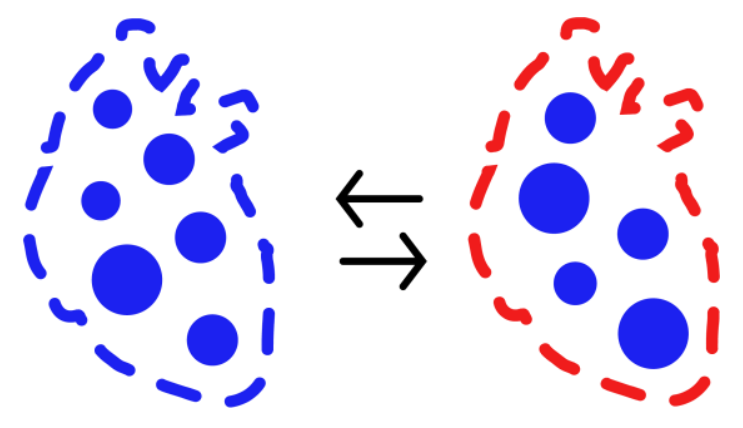

-110 - 


\section{CHAPTER 4}

\section{A Mutation Update for the FLNC gene in Myopathies and Cardiomyopathies}

\author{
Job A.J. Verdonschot ${ }^{1,2 *}$ \\ Els K. Vanhoutte ${ }^{l^{*}}$ \\ Godelieve R.F. Claes ${ }^{1}$ \\ Apollonia T.J.M. Helderman-van den Enden ${ }^{2}$ \\ Janneke G.J. Hoeijmakers ${ }^{3}$ \\ Debby M.E.I. Hellebrekers ${ }^{l}$ \\ Amber de Haan ${ }^{1}$ \\ Imke Christiaans ${ }^{4,7}$ \\ Ronald H. Lekanne Depriz \\ Hanne M. Boen ${ }^{5}$ \\ Emeline M. van Craenenbroeck \\ Bart L. Loeys ${ }^{6}$ \\ Yvonne M. Hoedemaekers ${ }^{7,8}$
}

\author{
Carlo Marcelis ${ }^{8}$ \\ Marlies Kempers ${ }^{8}$ \\ Esther Brusse ${ }^{9}$ \\ Jaap I. van Waning ${ }^{10,11}$ \\ Annette F. Baas ${ }^{12}$ \\ Dennis Dooijes ${ }^{12}$ \\ Folkert W. Asselbergs ${ }^{13}$ \\ Daniela Q.C.M. Barge-Schaapveld ${ }^{14}$ \\ Pieter Koopman ${ }^{15}$ \\ Arthur van den Wijngaard ${ }^{I}$ \\ Stephane R.B. Heymans ${ }^{2,16,17}$ \\ Ingrid P.C. Krapels ${ }^{1}$ \\ Han G. Brunner ${ }^{1,, 18}$
}

\footnotetext{
* Contributed equally

${ }^{1}$ Department of Clinical Genetics, Maastricht University Medical Center, Maastricht, The Netherlands. ${ }^{2}$ Department of Cardiology, Cardiovascular Research Institute (CARIM), Maastricht University Medical Center, Maastricht, The Netherlands.

${ }^{3}$ Department of Neurology, Maastricht University Medical Center, Maastricht, The Netherlands. ${ }^{4}$ Department of Clinical Genetics, Amsterdam University Medical Center, Amsterdam, The Netherlands. ${ }^{5}$ Department of Cardiology, Antwerp University Hospital, University of Antwerp, Antwerp, Belgium. ${ }^{6}$ Center of Medical Genetics, Antwerp University Hospital, University of Antwerp, Antwerp, Belgium. ${ }^{7}$ Department of Clinical Genetics, University Medical Centre Groningen, Groningen, The Netherlands. ${ }^{8}$ Department of Clinical Genetics, Radboud University Medical Centre, Nijmegen, The Netherlands. ${ }^{9}$ Department of Neurology, Erasmus MC University Medical Centre, Rotterdam, The Netherlands. ${ }^{10}$ Department of Clinical Genetics, Erasmus MEdical Center, Rotterdam, The Netherlands.

${ }^{11}$ Department of Cardiology, Radboud University Medical Centre, Nijmegen, The Netherlands.

${ }^{12}$ Department of Genetics, University Medical Center Utrecht, Utrecht, The Netherlands.

${ }^{13}$ Department of Cardiology, University Medical Center Utrecht, Utrecht, The Netherlands.

${ }^{14}$ Department of Clinical Genetics, Leiden University Medical Center, The Netherlands. ${ }^{15}$ Department of Cardiology, Heart Center Hasselt, Belgium

${ }^{16}$ Department of Cardiovascular Sciences, Centre for Molecular and Vascular Biology, KU Leuven, Belgium. ${ }^{17}$ The Netherlands Heart Institute,, Nl-HI, Utrecht, The Netherlands.

${ }^{18}$ GROW Institute for Developmental Biology and Cancer, Maastricht University Medical Centre, Maastricht, The Netherlands.
}

HUMAN MUTATION.

2020. JUN;41(6):1091-1111 


\section{ABSTRACT}

Filamin C (FLNC) variants are associated with cardiac and muscular phenotypes. Originally, FLNC variants were described in myofibrillar myopathy (MFM) patients. Later, highthroughput screening in cardiomyopathy cohorts determined a prominent role for FLNC in isolated hypertrophic and dilated cardiomyopathies (HCM and DCM). FLNC variants are now among the more prevalent causes of genetic DCM. FLNC-associated DCM is associated with a malignant clinical course and a high risk of sudden cardiac death. The clinical spectrum of FLNC suggests different pathomechanisms related to variant types and their location in the gene. Proper functioning of FLNC is crucial for structural integrity and cell signaling of the sarcomere. The secondary protein structure of FLNC is critical to ensure this function. Truncating variants with subsequent haploinsufficiency are associated with DCM and cardiac arrhythmias. Interference with the dimerization and folding of the protein leads to aggregate formation detrimental for muscle function, as found in HCM and MFM. Variants associated with $\mathrm{HCM}$ are predominantly missense variants which cluster in the ROD2 domain. This domain is important for binding to the sarcomere and ensure proper cell signaling. We here review FLNC genotype-phenotype correlations based on available evidence. 


\section{BACKGROUND}

Similar to other filamins, Filamin C (FLNC) is a structural protein which has an actin-binding domain (ABD) composed of two calponin homology (CH) domains, 24 immunoglobulin (Ig) domains divided into a ROD1 and ROD2 sub-domain and a C-terminal dimerization domain ${ }^{1}$. Dimerization of two identical filamin proteins is necessary for proper function, and occurs via Ig-like domain $24^{2}$. In contrast to filamin A and B, filamin C expression is restricted to striated muscles and localizes around the Z-disc, the sarcolemma, the myotendinous junction and the intercalated discs ${ }^{3}$. Its main role is maintaining the structural integrity of the sarcomere. This is through crosslinking actin filaments and the anchoring of sarcolemmal proteins to the cytoskeleton. The main interactors of FLNC are either part of the Z-disc (myotilin, myozenin, myopodin, calsarcins), signaling molecules ${ }^{4}$ or sarcolemma-associated proteins (integrin $\beta 1$, sarcoglycan delta) ${ }^{5-7}$. Proteases such as calpain can regulate the interaction between FLNC and the sarcoglycans by cleaving the corresponding binding domains of FLNC ${ }^{8}$. In addition, FLNC interacts with the Xin actin-binding repeat-containing proteins (XIRP) and aciculin to fulfill a function in muscle maintenance ${ }^{9-11}$. This interaction is mediated via a unique insertion in Ig-like domain 20, which is absent in the other filamin paralogs. The ROD1 domain (Ig 1-15) is more stretched and lacks interdomain interactions in contrast to the ROD2 domain (Ig 1623) which is more compact globularly arranged by domain pairs. These organizational differences between the domains explain why certain ligands bind exclusively to ROD1 or ROD2.

The FLNC gene maps to chromosome $7 \mathrm{q} 32-35$ and has two main transcripts, NM_001127487.2 and NM_001458.4. It comprises $\sim 29.5 \mathrm{~kb}$ of genomic DNA and is composed of 49 coding exons ${ }^{12}$. The difference between the two transcripts is the presence or absence of exon 31 encoding the hinge region between Ig-like domains 15 and $16{ }^{13}$. The longest transcript, NM_001458.4, encodes a protein with a molecular mass of $291 \mathrm{kDa}$ and 2.725 amino acids, whereas the shorter transcript NM_001127487.2 encodes a slightly shorter protein ( $287 \mathrm{kDa}, 2.692$ amino acids) that is assumed to be less flexible. The exact roles of these two isoforms are unknown, but the long FLNC isoform is more abundantly expressed during cardiac stress while almost absent in the normal situation ${ }^{14}$. This could potentially alter the integrity and function of the key sarcomeric structures in order to cope with increased cell stress. The short isoform is mainly expressed in the normal situation and is 3.5 times higher expressed in skeletal compared to cardiac muscle.

Variants in FLNC are traditionally associated with myofibrillar myopathy (MFM; MIM\# 609524), but subsequently also with isolated cardiomyopathies (MIM\# 617047). To date, most available basic research on FLNC has focused on myopathies. Paradoxically, most genetic variants are described in cardiomyopathy patients, as this clinical entity is more prevalent and studies using high-throughput sequencing of $F L N C$ are more frequent in cardiomyopathy cohorts. This overview highlights known and novel FLNC variants and focuses on specific pathomechanisms important for distinct cardiac or muscular phenotypes.

\section{VARIANTS}

All FLNC variants are described according to current Human Genome Variation Society (HGVS) mutation nomenclature guidelines based on Genbank accession number NM_001458.4 (longest transcript). Previously reported and novel variants are interpreted and classified using ACMG classification recommendations ${ }^{15}$.

A total of 285 unique variants could be retrieved from the international peer-reviewed literature, the Human Gene Mutation Database (HGMD) and the Leiden Open Variation 
Database (LOVD; Figure 1). We added 40 novel unique variants, leading to a total of 325 unique variants. All variants were submitted to the LOVD. A clear description of the phenotype associated with the variant was a requirement for this overview. One-hundred variants were excluded from the main analysis as no clinical information was available (Suppl. Table 1). Most FLNC variants have been reported over the last 5 years, from the time that FLNC was recognized as a disease-associated gene in the field of cardiomyopathies. Variant interpretation remains challenging, as the available evidence for pathogenicity is still limited for most type of variants. In general, truncating variants were classified as (likely) pathogenic, and missense variants as a variant of unknown significance (VUS) unless additional evidence from segregation and/or functional experiments was available.

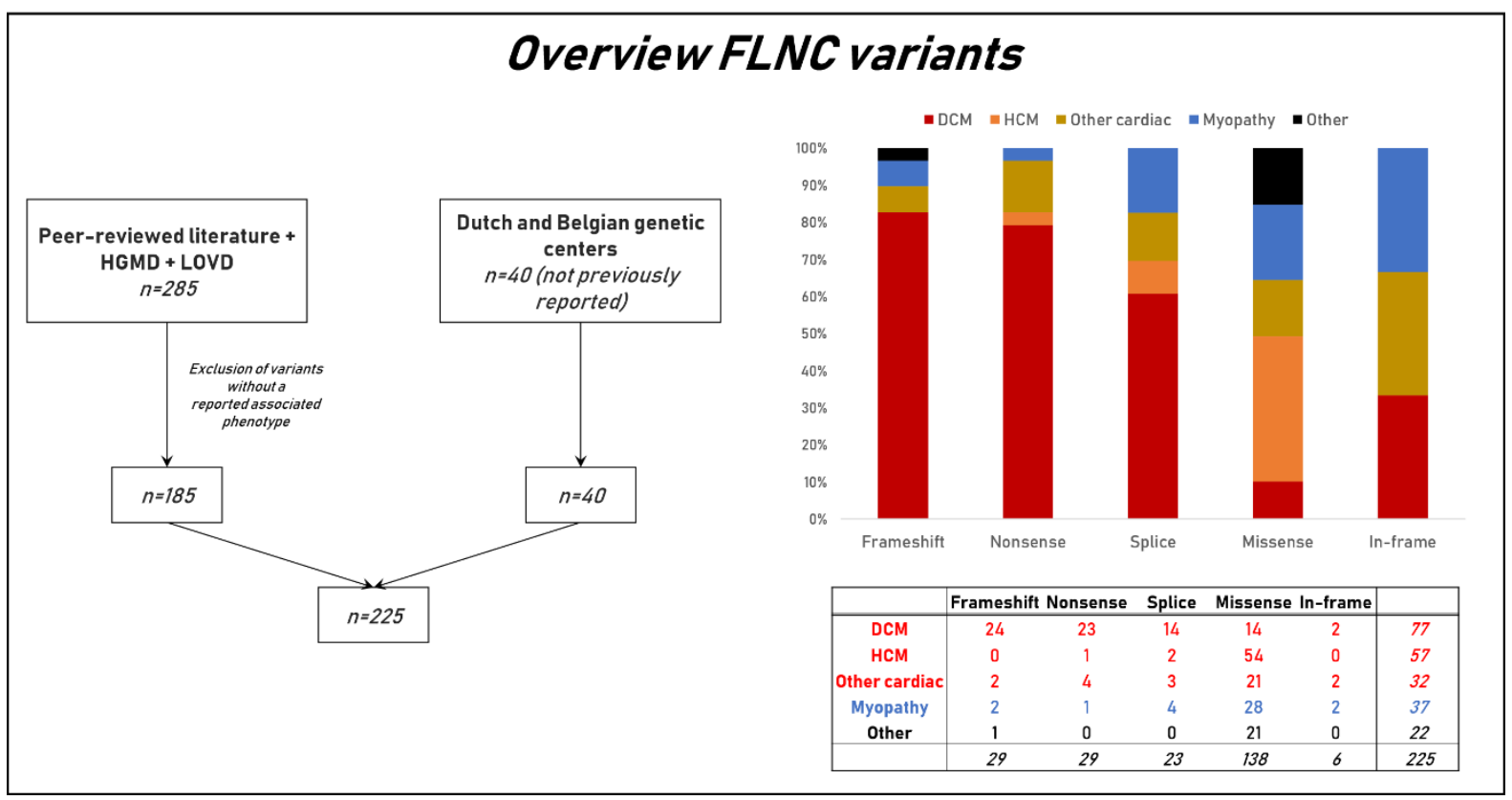

Figure 1. Variant selection of all FLNC variants and the overview of all variants in association with their phenotype. DCM indicates Dilated Cardiomyopathy; HCM, Hypertrophic Cardiomyopathy; HGMD, Human Gene Mutation Database; LOVD, Leiden Open Variation Database.

\subsection{Cardiomyopathies}

Cardiomyopathies are a heterogeneous group of myocardial diseases associated with mechanical or electrical dysfunction that exhibit inappropriate ventricular hypertrophy or dilatation ${ }^{16} 17$. In this review, we distinguish between:

- Dilated cardiomyopathy (DCM): characterized by the presence of left ventricular dilatation and contractile dysfunction, in the absence of abnormal loading condition and severe coronary artery disease ${ }^{16} 17$.

- Hypertrophic cardiomyopathy (HCM):the presence of increased left ventricular wall thickness that is not solely explained by abnormal loading conditions ${ }^{1617}$.

- Other cardiac diseases which do not fulfill these criteria for DCM or HCM.

\subsubsection{Dilated Cardiomyopathy}

Variants predicted to result in a premature stopcodon are strongly enriched in DCM, and are classified as (likely) pathogenic, since FLNC is highly intolerant for loss-of-function variants ( $p L I-s c o r e=1$ ). (Figure 1 and 2). The prevalence of FLNC variants in DCM patients ranges from 
1 to 4.5\% (Ader et al., 2019; Begay et al., 2018; Janin et al., 2017; Ortiz-Genga et al., 2016) (Table 1).

Three missense variants have been classified as likely pathogenic: p.Phe106Leu, p.Ala123Met and p.Gly2070Ser. The p.Phe106Leu missense variant occurred on the opposite allele of a nonsense variant (p.Arg991*) in a neonatal DCM patient ${ }^{18}$. Compound heterozygosity for these FLNC variants led to an early-onset phenotype. Heterozygous carriers had not developed DCM by age 40 years. Protein levels were decreased for the p.Phe106Leu variant, and the p.Arg991* was not detectable. The p.Val123Met variant reported in the current study is classified as likely pathogenic, due to the well-investigated p.Val123Ala variant at the same codon in $\mathrm{HCM}^{19}$. The p.Gly2070Ser variant is predicted to alter a canonical splice site, although RNA analysis was not performed ${ }^{20}$. All other missense variants are classified as VUS and are not included in Figure 2, but are listed in Table 1.

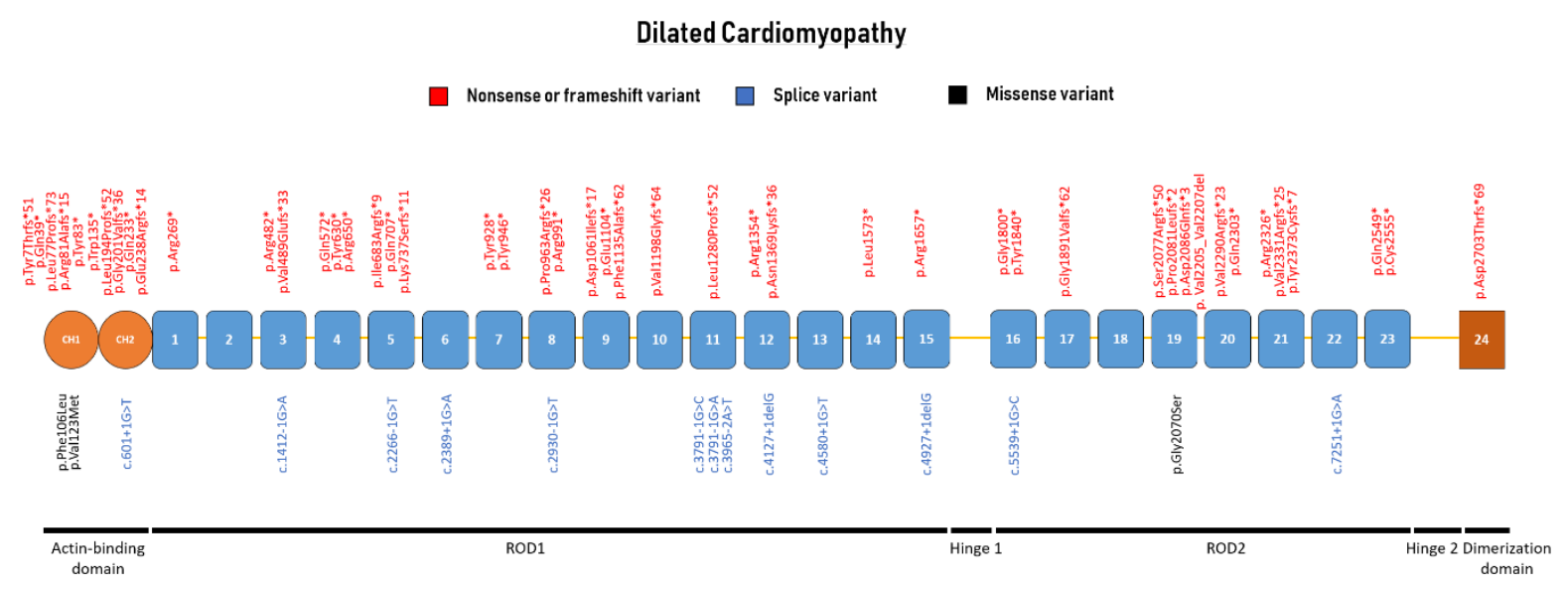

Figure 2. Schematic representation of the FLNC gene with their protein coding domains. Numbers inside the boxes refer to the Ig-like domains of filamin C. Above and below the schematic are all unique variants associated with dilated cardiomyopathy. Variants are annotated at the protein level.

\subsubsection{Hypertrophic Cardiomyopathy}

Missense variants are mainly associated with $\mathrm{HCM}$ with a varying prevalence from 1.3 to $8.7 \%$ in HCM cohorts (Figure 1; Table 2) ${ }^{19} 21-23$. Two studies did not detect an excess of rare missense variants between HCM patients and controls, questioning the importance of FLNC missense variants in $\mathrm{HCM}^{2124}$. Only 13 of the 54 missense variants are supported to be (likely) pathogenic by additional evidence such as functional studies $(n=4)$ and/or segregation $(n=13)$ 19 21-23. Based on current diagnostic classification criteria, all other missense variants would individually be classified as VUS (Table 2). There is a strong clustering of missense variants in the ROD2 domain of the FLNC, which is an important domain for cell signaling (Figure 3 ). Thus, collectively, missense variants in the ROD2 domain carry increased likelihood of being pathogenic for HCM. 
Table 1. FLNC variants found in individuals with Dilated Cardiomyopathy (DCM) previously reported and from this study

\begin{tabular}{|c|c|c|c|c|c|c|c|}
\hline Exon & c-Notation & p-Notation & $\begin{array}{c}\text { Variant } \\
\text { type }\end{array}$ & Domain & Location & Reference & Effect \\
\hline 1 & c.19del & p.Tyr7Thrfs*51 & Frameshift & $A B D$ & $\mathrm{CH} 1$ & (Ader et al., 2019) & Likely pathogenic \\
\hline 1 & c. $115 C>T$ & p.Gln39* & Nonsense & $A B D$ & $\mathrm{CH} 1$ & (Janin et al., 2017) & Likely pathogenic \\
\hline 1 & c.230_234delTCAGC & p.Leu77Profs*73 & Frameshift & $A B D$ & $\mathrm{CH} 1$ & (Janin et al., 2017) & Likely pathogenic \\
\hline 1 & c.241delC & p.Arg81Alafs*15 & Frameshift & $A B D$ & $\mathrm{CH} 1$ & (Ortiz-Genga et al., 2016) & Likely pathogenic \\
\hline 1 & c. $248 \_265$ dup & $\begin{array}{c}\text { p.Pro88_Arg89insHisArg } \\
\text { LysPheHisPro }\end{array}$ & In-frame & $A B D$ & $\mathrm{CH} 1$ & Current study & VUS \\
\hline 1 & c. $249 C>G$ & p.Tyr83* & Nonsense & $A B D$ & $\mathrm{CH} 1$ & (Ortiz-Genga et al., 2016) & Likely pathogenic \\
\hline 1 & c. $318 \mathrm{C}>\mathrm{G}$ & p.Phe106Leu & Missense & $A B D$ & $\mathrm{CH} 1$ & (Reinstein et al., 2016) & Likely pathogenic \\
\hline 1 & c. $328 \mathrm{G}>\mathrm{A}$ & p.Glu110Lys & Missense & $A B D$ & $\mathrm{CH} 1$ & Current study & VUS \\
\hline 2 & c. $367 \mathrm{G}>\mathrm{A}$ & p.Val123Met & Missense & $A B D$ & $\mathrm{CH} 1$ & Current study & Likely pathogenic \\
\hline 2 & c. $404 G>A$ & p.Trp135* & Nonsense & $A B D$ & $\mathrm{CH} 1$ & LOVD & Likely pathogenic \\
\hline 2 & c.581_599del19 & p.Leu194Profs*52 & Frameshift & $A B D$ & $\mathrm{CH} 2$ & (Ortiz-Genga et al., 2016) & Likely pathogenic \\
\hline 2 & c. $601+1 \mathrm{G}>\mathrm{T}$ & p.? & Splice & $A B D$ & $\mathrm{CH} 2$ & (Janin et al., 2017) & Likely pathogenic \\
\hline 3 & $\begin{array}{c}\text { c.602-716_1010delins } \\
\text { TGCCCCGGGAGGGGTGC } \\
\text { CTCAGTCTCCC } \\
\text { TGTCCCTCTG }\end{array}$ & p.Gly201Valfs*36 & Frameshift & $A B D$ & $\mathrm{CH} 2$ & (Ortiz-Genga et al., 2016) & Likely pathogenic \\
\hline 3 & c. $697 \mathrm{C}>\mathrm{T}$ & p.Gln233* & Nonsense & $A B D$ & $\mathrm{CH} 2$ & Current study & Likely pathogenic \\
\hline 4 & c.711del & p.Glu238Argfs*14 & Frameshift & $A B D$ & $\mathrm{CH} 2$ & (Ader et al., 2019) & Likely pathogenic \\
\hline 4 & c. $805 \mathrm{C}>\mathrm{T}$ & p.Arg269* & Nonsense & ROD1 & Ig-like 1 & (Begay et al., 2018) & Likely pathogenic \\
\hline 6 & c. $970-4 A>G$ & p.? & Splice & ROD1 & Ig-like 1 & Current study & VUS \\
\hline 9 & c. $1412-1 \mathrm{G}>\mathrm{A}$ & p.? & Splice & ROD1 & Ig-like 3 & (Ader et al., 2019) & Likely pathogenic \\
\hline 9 & c. $1444 C>T$ & p.Arg $482 *$ & Nonsense & ROD1 & Ig-like 3 & (Tobita et al., 2017) & Likely pathogenic \\
\hline 9 & c.1466_1472del & p.Val489Glyfs*33 & Frameshift & ROD1 & Ig-like 3 & Current study & Likely pathogenic \\
\hline 9 & c.1466_1473delinsA & p.Val489Glufs*33 & Frameshift & ROD1 & Ig-like 3 & LOVD & Likely pathogenic \\
\hline 11 & c. $1714 C>T$ & p.Gln572* & Nonsense & ROD1 & Ig-like 4 & (Ortiz-Genga et al., 2016) & Likely pathogenic \\
\hline 12 & c. $1890 \mathrm{C}>\mathrm{A}$ & p.Tyr630* & Nonsense & ROD1 & Ig-like 4 & (Janin et al., 2017) & Likely pathogenic \\
\hline 12 & c. $1948 C>T$ & p.Arg650* & Nonsense & ROD1 & Ig-like 4 & LOVD & Likely pathogenic \\
\hline
\end{tabular}




\begin{tabular}{|c|c|c|c|c|c|c|c|}
\hline 13 & c.2041_2047dup & p.lle683Argfs*9 & Frameshift & ROD1 & Ig-like 5 & (Ader et al., 2019) & Likely pathogenic \\
\hline 13 & c. $2119 C>T$ & p.G $\ln 707^{*}$ & Nonsense & ROD1 & Ig-like 5 & (Begay et al., 2018) & Likely pathogenic \\
\hline 14 & c.2208delT & p.Lys737Serfs*11 & Frameshift & ROD1 & Ig-like 5 & (Ortiz-Genga et al., 2016) & Likely pathogenic \\
\hline 15 & c. $2266-1 \mathrm{G}>\mathrm{T}$ & p.? & Splice & ROD1 & Ig-like 5 & (Janin et al., 2017) & Likely pathogenic \\
\hline 15 & c. $2389+1 G>A$ & p.? & Splice & ROD1 & Ig-like 6 & (Nozari et al., 2018) & Likely pathogenic \\
\hline 16 & c. $2425 \mathrm{G}>\mathrm{A}$ & p.Val809Met & Missense & ROD1 & Ig-like 6 & (Janin et al., 2017) & VUS \\
\hline 18 & c. $2784 C>G$ & p.Tyr928* & Nonsense & ROD1 & Ig-like 7 & (Ader et al., 2019) & Likely pathogenic \\
\hline 19 & c. $2838 \mathrm{~T}>\mathrm{A}$ & p.Tyr946* & Nonsense & ROD1 & Ig-like 7 & LOVD & Likely pathogenic \\
\hline 19 & c.2888delC & p.Pro963Argfs*26 & Frameshift & ROD1 & Ig-like 8 & (Ortiz-Genga et al., 2016) & Likely pathogenic \\
\hline 20 & c. $2930-1 \mathrm{G}>\mathrm{T}$ & p.? & Splice & ROD1 & Ig-like 8 & (Begay et al., 2018) & Likely pathogenic \\
\hline 20 & c. $2971 C>T$ & p.Arg991* & Nonsense & ROD1 & Ig-like 8 & (Reinstein et al., 2016) & Likely pathogenic \\
\hline 20 & c.3180del & p.Asp1061llefs*17 & Frameshift & ROD1 & Ig-like 9 & Current study & Likely pathogenic \\
\hline 21 & c. $3310 \mathrm{G}>\mathrm{T}$ & p.Glu1104* & Nonsense & ROD1 & Ig-like 9 & (Janin et al., 2017) & Likely pathogenic \\
\hline 21 & c.3380_3402dup23 & p.Phe1135Alafs*62 & Frameshift & ROD1 & Ig-like 9 & (Ortiz-Genga et al., 2016) & Likely pathogenic \\
\hline 21 & c.3592dup & p.Val1198Glyfs*64 & Frameshift & ROD1 & Ig-like 10 & (Ader et al., 2019) & Likely pathogenic \\
\hline 22 & c. $3791-1 \mathrm{G}>\mathrm{A}$ & p.? & Splice & ROD1 & Ig-like 11 & (Ortiz-Genga et al., 2016) & Likely pathogenic \\
\hline 22 & c. $3791-1 G>C$ & p.? & Splice & ROD1 & Ig-like 11 & (Deo et al., 2014) & Likely pathogenic \\
\hline 22 & c.3838dup & p.Leu1280Profs $* 52$ & Frameshift & ROD1 & Ig-like 11 & Current study & Likely pathogenic \\
\hline 23 & c. $3965-2 A>T$ & p.? & Splice & ROD1 & Ig-like 11 & (Ortiz-Genga et al., 2016) & Likely pathogenic \\
\hline 23 & c. $4060 \mathrm{C}>\mathrm{T}$ & p.Arg1354* & Nonsense & ROD1 & Ig-like 12 & (Janin et al., 2017) & Likely pathogenic \\
\hline 23 & c.4106dupA & p.Asn1369Lysfs*36 & Frameshift & ROD1 & Ig-like 12 & (Cuenca et al., 2016) & Likely pathogenic \\
\hline 23 & c. $4127+1$ delG & p.? & Splice & ROD1 & Ig-like 12 & (Ortiz-Genga et al., 2016) & Likely pathogenic \\
\hline 26 & c. $4580+1 G>T$ & p.? & Splice & ROD1 & Ig-like 13 & (Ortiz-Genga et al., 2016) & Likely pathogenic \\
\hline 27 & c. $4700 \mathrm{G}>\mathrm{A}$ & p.Arg1567Gln & Missense & ROD1 & Ig-like 14 & (Esslinger et al., 2017) & VUS \\
\hline 27 & c. $4718 \mathrm{~T}>\mathrm{A}$ & p.Leu1573* & Nonsense & ROD1 & Ig-like 14 & (Augusto et al., 2019) & Likely pathogenic \\
\hline 28 & c. $4927+1$ delG & p.? & Splice & ROD1 & Ig-like 15 & (Ortiz-Genga et al., 2016) & Likely pathogenic \\
\hline 30 & c. $4969 C>T$ & p.Arg1657* & Nonsense & ROD1 & Ig-like 15 & LOVD & Likely pathogenic \\
\hline 30 & c. $5036 \mathrm{C}>\mathrm{A}$ & p.Thr1679Lys & Missense & ROD1 & Ig-like 15 & Current study & VUS \\
\hline 32 & c.5398G $>\mathrm{T}$ & p.Gly1800* & Nonsense & ROD2 & Ig-like 16 & (Ortiz-Genga et al., 2016) & Likely pathogenic \\
\hline
\end{tabular}




\begin{tabular}{|c|c|c|c|c|c|c|c|}
\hline 33 & c.5520T>A & p.Tyr1840* & Nonsense & ROD2 & Ig-like 16 & $\begin{array}{l}\text { (Chanavat, Janin, \& Millat, } \\
\text { 2016) }\end{array}$ & Likely pathogenic \\
\hline 33 & c. $5539+1 G>C$ & p.? & Splice & ROD2 & Ig-like 16 & (Ortiz-Genga et al., 2016) & Likely pathogenic \\
\hline 35 & c.5672delG & p.Gly1891Valfs*62 & Frameshift & ROD2 & Ig-like 17 & (Begay et al., 2018) & Likely pathogenic \\
\hline 35 & c. $5791 \mathrm{C}>\mathrm{T}$ & p.Arg1931Cys & Missense & ROD2 & Ig-like 17 & Current study & VUS \\
\hline 37 & c. $6100 \mathrm{G}>\mathrm{C}$ & p.Gly2034Arg & Missense & ROD2 & Ig-like 18 & Current study & VUS \\
\hline 37 & c. $6208 \mathrm{G}>\mathrm{A}$ & p.Gly2070Ser & Missense & ROD2 & Ig-like 19 & (Ortiz-Genga et al., 2016) & Likely pathogenic \\
\hline 38 & c.6231delT & p.Ser2077Argfs*50 & Frameshift & ROD2 & Ig-like 19 & (Cuenca et al., 2016) & Likely pathogenic \\
\hline 38 & c.6240_6259del & p.Pro2081Leufs*2 & Frameshift & ROD2 & Ig-like 19 & (Ortiz-Genga et al., 2016) & Likely pathogenic \\
\hline 38 & c.6255_6256del & p.lle2086Glnfs*3 & Frameshift & ROD2 & Ig-like 19 & Current study & Likely pathogenic \\
\hline 40 & c. $6518 \mathrm{G}>\mathrm{A}$ & p.Arg $2173 \mathrm{His}$ & Missense & ROD2 & Intradomain & Current study & VUS \\
\hline 40 & c.6614_6622del & p.Val2205_Val2207del & In-frame & ROD2 & Intradomain & Current study & Likely pathogenic \\
\hline 41 & c. $6790 \mathrm{G}>\mathrm{A}$ & p.Ala2264Thr & Missense & ROD2 & Ig-like 20 & Current study & VUS \\
\hline 41 & c.6864_6867dup & p.Val2290Argfs $* 23$ & Frameshift & ROD2 & Ig-like 20 & Current study & Likely pathogenic \\
\hline 41 & c. $6877 \mathrm{C}>\mathrm{T}$ & p.Arg2293Cys & Missense & ROD2 & Ig-like 20 & Current study & VUS \\
\hline 41 & c. $6907 \mathrm{C}>\mathrm{T}$ & p.GIn2303* & Nonsense & ROD2 & Ig-like 20 & Current study & Likely pathogenic \\
\hline 41 & c. $6976 \mathrm{C}>\mathrm{T}$ & p.Arg2326* & Nonsense & ROD2 & Ig-like 21 & (Ortiz-Genga et al., 2016) & Likely pathogenic \\
\hline 41 & c.6989dupG & p.Val2331Argfs*25 & Frameshift & ROD2 & Ig-like 21 & (Janin et al., 2017) & Likely pathogenic \\
\hline 42 & c.7118_7119del & p.Tyr2373Cysfs*7 & Frameshift & ROD2 & Ig-like 21 & (Ader et al., 2019) & Likely pathogenic \\
\hline 43 & c. $7251+1 \mathrm{G}>\mathrm{A}$ & p.? & Splice & ROD2 & Ig-like 22 & (Ortiz-Genga et al., 2016) & Likely pathogenic \\
\hline 45 & c. $7450 \mathrm{G}>\mathrm{A}$ & p.Gly2484Ser & Missense & ROD2 & Ig-like 22 & Current study & VUS \\
\hline 46 & c. $7645 C>T$ & p.Gln2549* & Nonsense & ROD2 & Ig-like 23 & (Ader et al., 2019) & Likely pathogenic \\
\hline 46 & c. $7652 A>G$ & p.Asp2551Gly & Missense & ROD2 & Ig-like 23 & Current study & VUS \\
\hline 46 & c. $7665 T>A$ & p.Cys $2555^{*}$ & Nonsense & ROD2 & Ig-like 23 & (Ader et al., 2019) & Likely pathogenic \\
\hline 48 & c.8107delG & p.Asp2703Thrfs*69 & Frameshift & Dimerization & Ig-like 24 & (Ortiz-Genga et al., 2016) & Likely pathogenic \\
\hline
\end{tabular}




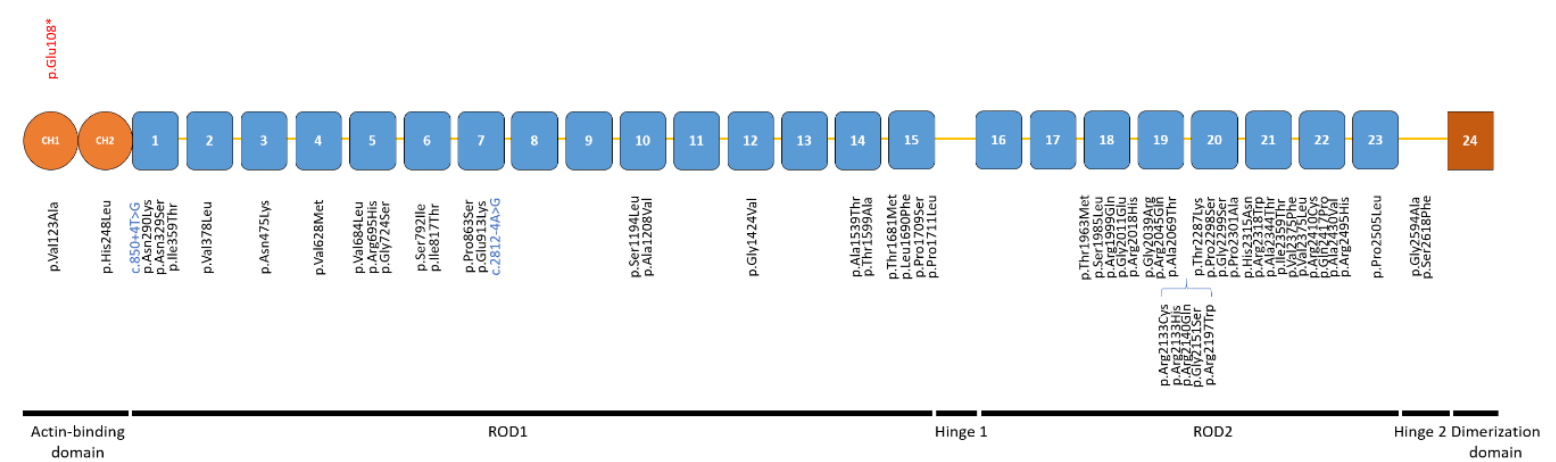

Figure 3. Schematic representation of the FLNC gene with their protein coding domains. Numbers inside the boxes refer to the Ig-like domains of filamin C. Above and below the schematic are all unique variants associated with hypertrophic cardiomyopathy. Variants are annotated at the protein level.

\subsubsection{Other cardiac phenotypes}

FLNC variants have been associated with other cardiac phenotypes such as: arrhythmias without detectable structural abnormalities, congenital heart disease (CHD), restrictive (RCM) and non-compaction (NCCM) cardiomyopathies (Figure 4; Table 3). The association of FLNC with a broad spectrum of cardiac phenotypes shows an important gap in knowledge. Hence, not all reported variants have proven to be causal. There can also be large overlap between phenotypes: cardiac non-compaction can be a trait observed in other cardiomyopathies and healthy hearts ${ }^{25}$.

One patient with arrhythmogenic bileaflet mitral valve prolapse syndrome (ABiMVPS) was recently reported in association with a truncating variant (p.Trp34*) identified in whole exome sequencing data ${ }^{26}$. It was speculated that FLNC haploinsufficiency was the underlying arrhythmogenic substrate which was exacerbated by the mitral valve prolapse. Another recent report described familial sudden cardiac death without signs of cardiomyopathy in association with a truncating variant in FLNC (p.Pro2513Glufs*12) ${ }^{27}$. Both cases highlight the arrhythmogenic potential associated with FLNC truncating variants. Although it remains unknown if alternative diagnostic tools such as global longitudinal strain analysis, could detect subtle changes in cardiac function. In addition, arrhythmias can also be accompanied by a cardiomyopathy phenotype with right, left, or biventricular involvement, called arrhythmogenic cardiomyopathy (ACM). When there is prominent left ventricular involvement, it is difficult to clinically distinguish it from DCM. A recent cohort study in ACM patients found 4 truncating variants (3.3\%; Table 3$)^{28}$.

FLNC variants in RCM and NCCM are less prevalent compared to DCM and HCM, making it difficult to draw any conclusions on the role of FLNC in these cardiomyopathies. One truncating variant has been described in association with NCCM (p.Gln1024*) ${ }^{29}$. Unfortunately, little clinical information was available to assess the arrhythmogenic potential of this individual. In addition, this patient also carried a pathogenic $R Y R 2$ variant. 
Table 2. FLNC variants found in individuals with Hypertrophic Cardiomyopathy (HCM) previously reported and from this study

\begin{tabular}{|c|c|c|c|c|c|c|c|}
\hline Exon & c-Notation & p-Notation & Variant type & Domain & Location & Reference & Effect \\
\hline 1 & c. $322 \mathrm{G}>\mathrm{T}$ & p.Glu108* & Nonsense & $A B D$ & $\mathrm{CH} 1$ & (Valdes-Mas et al., 2014) & Likely pathogenic \\
\hline 2 & c. $368 \mathrm{~T}>\mathrm{C}$ & p.Val123Ala & Missense & $A B D$ & $\mathrm{CH} 1$ & (Valdes-Mas et al., 2014) & Likely pathogenic \\
\hline 4 & c. $743 \mathrm{~A}>\mathrm{T}$ & p.His248Leu & Missense & $A B D$ & $\mathrm{CH} 2$ & Current study & VUS \\
\hline 4 & $c .850+4 \mathrm{~T}>\mathrm{G}$ & p.? & Splice & ROD1 & Ig-like 1 & (Cui et al., 2018) & VUS \\
\hline 5 & c. $870 \mathrm{C}>\mathrm{A}$ & p.Asn290Lys & Missense & ROD1 & Ig-like 1 & (Valdes-Mas et al., 2014) & VUS \\
\hline 6 & c. $986 A>G$ & p.Asn329Ser & Missense & ROD1 & Ig-like 1 & (Alejandra Restrepo-Cordoba et al., 2017) & VUS \\
\hline 7 & c. $1076 \mathrm{~T}>\mathrm{C}$ & p.lle359Thr & Missense & ROD1 & Ig-like 1 & (Cui et al., 2018) & VUS \\
\hline 7 & c. $1132 \mathrm{G}>\mathrm{T}$ & p.Val378Leu & Missense & ROD1 & Ig-like 2 & (Cui et al., 2018) & VUS \\
\hline 9 & c. $1425 C>A$ & p.Asn475Lys & Missense & ROD1 & Ig-like 3 & Current study & VUS \\
\hline 12 & c. $1882 \mathrm{G}>\mathrm{A}$ & p.Val628Met & Missense & ROD1 & Ig-like 4 & (Cui et al., 2018) & VUS \\
\hline 13 & c. $2050 \mathrm{G}>\mathrm{C}$ & p.Val684Leu & Missense & ROD1 & Ig-like 5 & (Cui et al., 2018) & VUS \\
\hline 13 & c. $2084 G>A$ & p.Arg695His & Missense & ROD1 & Ig-like 5 & Current study & VUS \\
\hline 14 & c. $2170 \mathrm{G}>\mathrm{A}$ & p.Gly724Ser & Missense & ROD1 & Ig-like 5 & (Cui et al., 2018) & VUS \\
\hline 15 & c. $2375 \mathrm{G}>\mathrm{T}$ & p.Ser792Ile & Missense & ROD1 & Ig-like 6 & (Jaafar et al., 2016) & VUS \\
\hline 16 & c. $2450 \mathrm{~T}>\mathrm{C}$ & p.lle817Thr & Missense & ROD1 & Ig-like 6 & (Cirino et al., 2017) & VUS \\
\hline 17 & c. $2587 \mathrm{C}>\mathrm{T}$ & p.Pro863Ser & Missense & ROD1 & Ig-like 7 & (Cui et al., 2018) & VUS \\
\hline 18 & c. $2737 \mathrm{G}>\mathrm{A}$ & p.Glu913Lys & Missense & ROD1 & Ig-like 7 & (Cui et al., 2018) & VUS \\
\hline 19 & c. $2812-4 A>G$ & p.? & Splice & ROD1 & Ig-like 7 & (Cui et al., 2018) & VUS \\
\hline 21 & c. $3581 C>T$ & p.Ser1194Leu & Missense & ROD1 & Ig-like 10 & (Ader et al., 2019) & VUS \\
\hline 21 & c. $3623 C>T$ & p.Ala1208Val & Missense & ROD1 & Ig-like 10 & (Cui et al., 2018) & VUS \\
\hline 24 & c. $4271 \mathrm{G}>\mathrm{T}$ & p.Gly1424Val & Missense & ROD1 & Ig-like 12 & (Ader et al., 2019) & Likely pathogenic \\
\hline 27 & c. $4615 G>A$ & p.Ala1539Thr & Missense & ROD1 & Ig-like 14 & (Valdes-Mas et al., 2014) & Likely pathogenic \\
\hline 28 & c. $4795 A>G$ & p.Thr1599Ala & Missense & ROD1 & Ig-like 14 & (Gomez et al., 2017) & VUS \\
\hline 30 & c. $5042 C>T$ & p.Thr1681Met & Missense & ROD1 & Ig-like 15 & (Gomez et al., 2017) & VUS \\
\hline 30 & c. $5068 \mathrm{C}>\mathrm{T}$ & p.Leu1690Phe & Missense & ROD1 & Ig-like 15 & (Gomez et al., 2017) & VUS \\
\hline 30 & c. $5125 C>T$ & p.Pro1709Ser & Missense & ROD1 & Ig-like 15 & (Cui et al., 2018) & VUS \\
\hline 30 & c. $5132 C>T$ & p.Pro1711Leu & Missense & ROD1 & Ig-like 15 & (Cui et al., 2018) & VUS \\
\hline
\end{tabular}




\begin{tabular}{|c|c|c|c|c|c|c|c|}
\hline 36 & c. $5888 \mathrm{C}>\mathrm{T}$ & p.Thr1963Met & Missense & ROD2 & Ig-like 18 & (Cui et al., 2018) & VUS \\
\hline 36 & c. $5954 C>T$ & p.Ser1985Leu & Missense & ROD2 & Ig-like 18 & Current study & VUS \\
\hline 36 & c.5996G $>A$ & p.Arg1999GIn & Missense & ROD2 & Ig-like 18 & (Jaafar et al., 2016) & VUS \\
\hline 37 & c. $6032 \mathrm{G}>\mathrm{A}$ & p.Gly2011Glu & Missense & ROD2 & Ig-like 18 & (Ader et al., 2019) & VUS \\
\hline 37 & c. $6053 \mathrm{G}>\mathrm{A}$ & p.Arg2018His & Missense & ROD2 & Ig-like 18 & (Chanavat et al., 2016) & VUS \\
\hline 37 & c. $6115 \mathrm{G}>\mathrm{A}$ & p.Gly2039Arg & Missense & ROD2 & Ig-like 19 & (Ader et al., 2019) & Likely pathogenic \\
\hline 37 & c. $6134 \mathrm{G}>\mathrm{A}$ & p.Arg2045Gln & Missense & ROD2 & Ig-like 19 & (Chanavat et al., 2016) & VUS \\
\hline 37 & c. $6205 \mathrm{G}>\mathrm{A}$ & p.Ala2069Thr & Missense & ROD2 & Ig-like 19 & Current study & VUS \\
\hline 39 & c. $6398 \mathrm{G}>\mathrm{A}$ & p.Arg2133His & Missense & ROD2 & Intradomain & (Valdes-Mas et al., 2014) & Likely pathogenic \\
\hline 39 & c.6397C >T & p.Arg2133Cys & Missense & ROD2 & Intradomain & (Cui et al., 2018) & Likely pathogenic \\
\hline 39 & c.6419G $>A$ & p.Arg2140Gln & Missense & ROD2 & Intradomain & (Gomez et al., 2017) & Likely pathogenic \\
\hline 39 & c. $6451 \mathrm{G}>\mathrm{A}$ & p.Gly2151Ser & Missense & ROD2 & Intradomain & (Valdes-Mas et al., 2014) & VUS \\
\hline 40 & c.6589C $>\mathrm{T}$ & p.Arg2197Trp & Missense & ROD2 & Intradomain & (Alejandra Restrepo-Cordoba et al., 2017) & VUS \\
\hline 41 & c. $6860 \mathrm{C}>\mathrm{A}$ & p.Thr2287Lys & Missense & ROD2 & Ig-like 20 & Current study & VUS \\
\hline 41 & c. $6892 \mathrm{C}>\mathrm{T}$ & p.Pro2298Ser & Missense & ROD2 & Ig-like 20 & (Gomez et al., 2017) & Likely pathogenic \\
\hline 41 & c. $6895 \mathrm{G}>\mathrm{A}$ & p.Gly2299Ser & Missense & ROD2 & Ig-like 20 & (Ader et al., 2019) & VUS \\
\hline 41 & c. $6901 \mathrm{C}>\mathrm{G}$ & p.Pro2301Ala & Missense & ROD2 & Ig-like 20 & (Gomez et al., 2017) & Likely pathogenic \\
\hline 41 & c. $6943 C>A$ & p.His2315Asn & Missense & ROD2 & Ig-like 21 & (Valdes-Mas et al., 2014) & Likely pathogenic \\
\hline 41 & c. $6952 C>T$ & p.Arg2318Trp & Missense & ROD2 & Ig-like 21 & (Gomez et al., 2017) & VUS \\
\hline 42 & c. $7030 \mathrm{G}>\mathrm{A}$ & p.Ala2344Thr & Missense & ROD2 & Ig-like 21 & (Cui et al., 2018) & VUS \\
\hline 42 & c. $7076 \mathrm{~T}>\mathrm{C}$ & p.lle2359Thr & Missense & ROD2 & Ig-like 21 & (Ader et al., 2019) & VUS \\
\hline 42 & c. $7123 \mathrm{G}>\mathrm{T}$ & p.Val2375Phe & Missense & ROD2 & Ig-like 21 & (Gomez et al., 2017) & VUS \\
\hline 42 & c. $7123 \mathrm{G}>\mathrm{C}$ & p.Val2375Leu & Missense & ROD2 & Ig-like 21 & (Ader et al., 2019) & Likely pathogenic \\
\hline 43 & c. $7228 \mathrm{C}>\mathrm{T}$ & p.Arg2410Cys & Missense & ROD2 & Ig-like 22 & (Ader et al., 2019) & VUS \\
\hline 43 & c. $7250 A>C$ & p.GIn2417Pro & Missense & ROD2 & Ig-like 22 & (Ader et al., 2019) & VUS \\
\hline 44 & c. $7289 \mathrm{C}>\mathrm{T}$ & p.Ala2430Val & Missense & ROD2 & Ig-like 22 & (Valdes-Mas et al., 2014) & Likely pathogenic \\
\hline 45 & c.7484G $>A$ & p.Arg2495His & Missense & ROD2 & Ig-like 22 & (Ader et al., 2019) & Likely pathogenic \\
\hline 45 & c. $7514 C>T$ & p.Pro2505Leu & Missense & ROD2 & Ig-like 23 & (Cui et al., 2018) & VUS \\
\hline 47 & c. $7781 \mathrm{G}>\mathrm{C}$ & p.Gly2594Ala & Missense & ROD2 & Hinge 2 & (Chanavat et al., 2016) & VUS \\
\hline 47 & c. $7853 \mathrm{C}>\mathrm{T}$ & p.Ser2618Phe & Missense & ROD2 & Hinge 2 & Current study & VUS \\
\hline
\end{tabular}




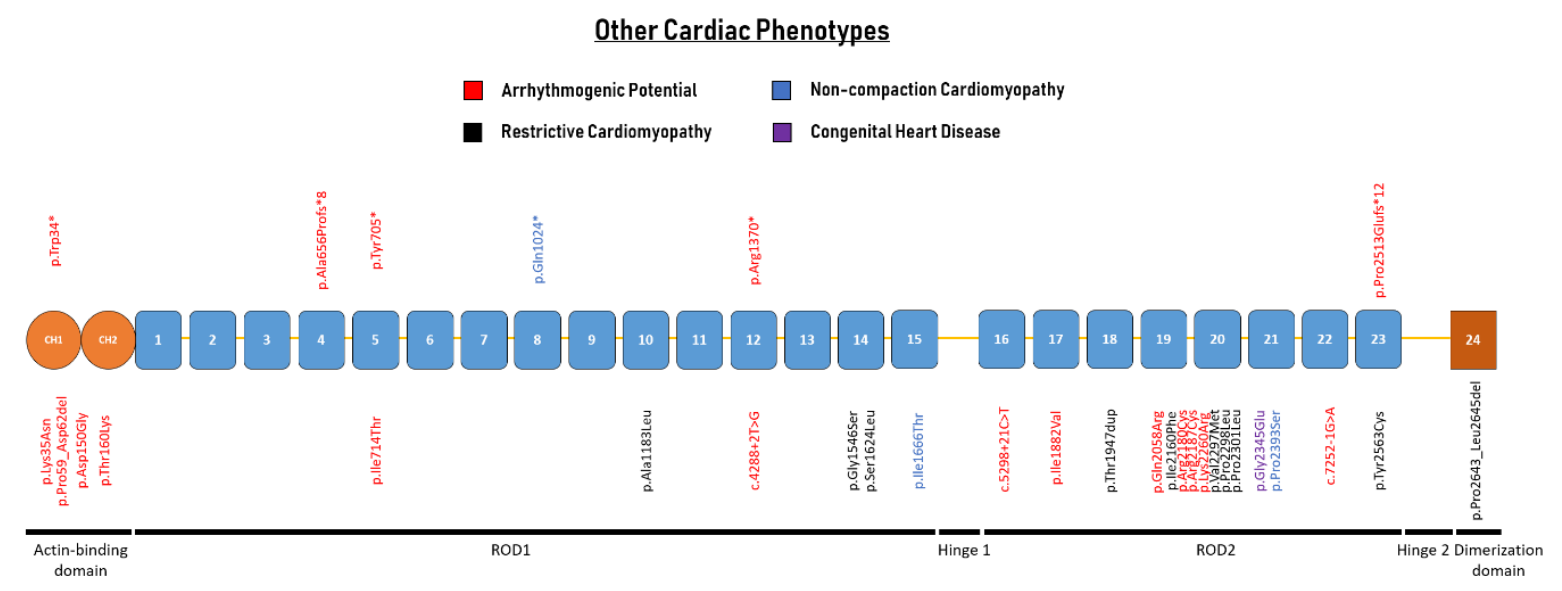

Figure 4. Schematic representation of the FLNC gene with their protein coding domains. Numbers inside the boxes refer to the Ig-like domains of filamin C. Above and below the schematic are all unique variants associated with different cardiac phenotypes. Variants are annotated at the protein level.

\subsection{Myopathies}

Classification of myopathies can be based on either clinical presentation, cause or pathology. To enhance clinical utility and prevent miscommunication, we suggest the classification based on clinical presentation, such as distal and/or proximal myopathy. The more specific diagnosis MFM requires finding protein aggregates in muscle.

The first description of a human phenotype related to FLNC was in 2005, when a nonsense variant (p.Trp2710*) was described in a German family with a novel type of autosomal dominant MFM ${ }^{30}$. The variant was later described in cohorts of varying ethnicity, suggesting codon 2710 to be a mutational hotspot ${ }^{31}$. FLNC variants are mostly associated with a proximal myopathy, with occasional distal involvement (Figure 5; Table 4) ${ }^{32}$. There are few associations between variant type or location and corresponding myopathy phenotype or special features such as cardiac involvement. We observed a cluster of missense variants in Ig-like domain 10, a domain which is rarely involved in cardiomyopathies. There was no genotype-phenotype association between variant location, and features of MFM, including tissue protein aggregate formation. (Figure 5). Missense, in-frame and nonsense variants are all associated with protein aggregate formation in muscle tissue 303334 . Only two truncating variants have been reported to cause myopathy. Both are in Ig-like 15, and associated with a form of isolated distal myopathy.

\subsection{Other non-striated muscle diseases}

Twenty-two unique FLNC variants have been described in association with non-cardiac or muscular phenotypes. These variants are listed in the Supplementals as they are not the main focus of this overview (Suppl. Table 2). There is a single large study which performed highthroughput sequencing in patients with frontotemporal dementia (FTD) and found a rare FLNC missense variant in $3.6 \%$ of the patients ${ }^{35}$. 
Table 3. FLNC variants found in individuals with a variety of cardiac phenotypes as previously reported and from this study

\begin{tabular}{|c|c|c|c|c|c|c|c|c|}
\hline Exon & c-Notation & p-Notation & Variant type & Domain & Location & Phenotype & Reference & Effect \\
\hline 1 & c. $102 \mathrm{G}>\mathrm{A}$ & p.Trp34* & Nonsense & $A B D$ & $\mathrm{CH} 1$ & ABiMVPS & (Bains et al., 2019) & Likely pathogenic \\
\hline 1 & c. $105 \mathrm{G}>\mathrm{C}$ & p.Lys35Asn & Missense & $A B D$ & $\mathrm{CH} 1$ & $\mathrm{ACM} / \mathrm{SCD}$ & (Hall et al., 2019) & VUS \\
\hline 1 & c.174_185del & p.Pro59_Asp62del & In-frame & $A B D$ & $\mathrm{CH} 1$ & ACM & (Hall et al., 2019) & VUS \\
\hline 2 & c. $449 A>G$ & p.Asp150Gly & Missense & $A B D$ & $\mathrm{CH} 1$ & ACM & Current study & VUS \\
\hline 2 & c. $479 \mathrm{C}>\mathrm{A}$ & p.Thr160Lys & Missense & $A B D$ & $\mathrm{CH} 2$ & $\mathrm{ACM} / \mathrm{SCD}$ & (Hall et al., 2019) & VUS \\
\hline 12 & c.1965_1966delTG & p.Ala656Profs*8 & Frameshift & ROD1 & Ig-like 4 & $\mathrm{ACM} / \mathrm{SCD}$ & (Hall et al., 2019) & Likely pathogenic \\
\hline 13 & c.2115_2120delTGCCCA & p.Tyr705* & Nonsense & ROD1 & Ig-like 5 & $\mathrm{ACM} / \mathrm{SCD}$ & (Hall et al., 2019) & Likely pathogenic \\
\hline 14 & c. $2141 \mathrm{~T}>\mathrm{C}$ & p.Ile714Thr & Missense & ROD1 & Ig-like 5 & $\mathrm{ACM}$ & (Hall et al., 2019) & VUS \\
\hline 20 & c. $3070 \mathrm{C}>\mathrm{T}$ & p.G $\ln 1024 *$ & Nonsense & ROD1 & Ig-like 8 & NCCM & $\begin{array}{c}\text { (Miszalski-Jamka et } \\
\text { al., 2017) }\end{array}$ & Likely pathogenic \\
\hline 21 & c.3547_3548delinsCT & p.Ala1183Leu & Missense & ROD1 & Ig-like 10 & $\mathrm{RCM}$ & (Kiselev et al., 2018) & VUS \\
\hline 23 & c. $4108 \mathrm{C}>\mathrm{T}$ & p.Arg1370* & Nonsense & ROD1 & Ig-like 12 & $\mathrm{ACM} / \mathrm{SCD}$ & (Hall et al., 2019) & Likely pathogenic \\
\hline 24 & c. $4288+2 T>G$ & p.? & Splice & ROD1 & Ig-like 12 & $\mathrm{ACM} / \mathrm{SCD}$ & (Hall et al., 2019) & Likely pathogenic \\
\hline 27 & c. $4636 \mathrm{G}>\mathrm{A}$ & p.Gly1546Ser & Missense & ROD1 & Ig-like 14 & $\mathrm{RCM}$ & $\begin{array}{c}\text { (Sanoja, Li, Fricker, } \\
\text { Kingsmore, \& } \\
\text { Wallace, 2018) }\end{array}$ & VUS \\
\hline 28 & c. $4871 \mathrm{C}>\mathrm{T}$ & p.Ser1624Leu & Missense & ROD1 & Ig-like 14 & $\mathrm{RCM}$ & (Brodehl et al., 2016) & Likely pathogenic \\
\hline 30 & c. $4997 \mathrm{~T}>\mathrm{C}$ & p.lle1666Thr & Missense & ROD1 & Ig-like 15 & NCCM & (Ader et al., 2019) & VUS \\
\hline 31 & c. $5298+21 C>T$ & p.? & Splice & ROD2 & Ig-like 16 & $\mathrm{ACM} / \mathrm{SCD}$ & (Hall et al., 2019) & VUS \\
\hline 34 & c. $5644 A>G$ & p.lle1882 Val & Missense & ROD2 & Ig-like 17 & ACM & (Hall et al., 2019) & VUS \\
\hline 35 & c.5839_5841dup & p.Thr1947dup & In-frame & ROD2 & Ig-like 18 & $\mathrm{RCM}$ & (Ader et al., 2019) & VUS \\
\hline 37 & c. $6173 \mathrm{~A}>\mathrm{G}$ & p.Gln2058Arg & Missense & ROD2 & Ig-like 19 & ACM & (Hall et al., 2019) & VUS \\
\hline 39 & c. $6478 \mathrm{~A}>\mathrm{T}$ & p.lle2160Phe & Missense & ROD2 & Intradomain & $\mathrm{RCM}$ & (Brodehl et al., 2016) & Likely pathogenic \\
\hline 40 & c. $6538 \mathrm{C}>\mathrm{T}$ & p.Arg2180Cys & Missense & ROD2 & Intradomain & ACM & Current study & VUS \\
\hline 40 & c.6559C>T & p.Arg2187Cys & Missense & ROD2 & Intradomain & ACM & Current study & VUS \\
\hline 41 & c.6779A>G & p.Lys2260Arg & Missense & ROD2 & Ig-like 20 & $\mathrm{ACM}$ & (Hall et al., 2019) & VUS \\
\hline 41 & c. $6889 \mathrm{G}>\mathrm{A}$ & p.Val2297Met & Missense & ROD2 & Ig-like 20 & RCM & (Tucker et al., 2017) & Likely pathogenic \\
\hline
\end{tabular}




\begin{tabular}{|c|c|c|c|c|c|c|c|c|}
\hline 41 & c. $6893 C>T$ & p.Pro2298Leu & Missense & ROD2 & Ig-like 20 & $\mathrm{RCM}$ & $\begin{array}{c}\text { (Schubert et al., } \\
\text { 2018) }\end{array}$ & Likely pathogenic \\
\hline 41 & c. $6902 C>T$ & p.Pro2301Leu & Missense & ROD2 & Ig-like 20 & $\mathrm{RCM}$ & $\begin{array}{c}\text { (Roldan-Sevilla et al., } \\
\text { 2019) }\end{array}$ & VUS \\
\hline 42 & c. $7034 \mathrm{G}>\mathrm{A}$ & p.Gly2345Glu & Missense & ROD2 & Ig-like 21 & $\begin{array}{c}\text { Congenital heart } \\
\text { disease }\end{array}$ & $\begin{array}{c}\text { (Kosmicki et al., } \\
\text { 2017) }\end{array}$ & VUS \\
\hline 43 & c. $7177 \mathrm{C}>\mathrm{T}$ & p.Pro2393Ser & Missense & ROD2 & Ig-like 21 & NCCM & Current study & Likely pathogenic \\
\hline 44 & c. $7252-1 \mathrm{G}>\mathrm{A}$ & p.? & Splice & ROD2 & Ig-like 22 & $\mathrm{ACM}$ & (Hall et al., 2019) & Likely pathogenic \\
\hline 45 & c.7536_7548del13 & p.Pro2513Glufs*12 & Frameshift & ROD2 & Ig-like 23 & $\begin{array}{c}\text { Cardiac } \\
\text { arrhythmia }\end{array}$ & $\begin{array}{c}\text { (Mangum \& Ferns, } \\
\text { 2019) }\end{array}$ & Likely pathogenic \\
\hline 46 & c. $7688 \mathrm{~A}>\mathrm{G}$ & p.Tyr2563Cys & Missense & ROD2 & Ig-like 23 & $\mathrm{RCM}$ & $\begin{array}{c}\text { (Schubert et al., } \\
\text { 2018) }\end{array}$ & Likely pathogenic \\
\hline 47 & c.7927_7935del & p.Pro2643_Leu2645del & In-frame & ROD2 & Ig-like 24 & $\mathrm{RCM}$ & (Ader et al., 2019) & VUS \\
\hline
\end{tabular}




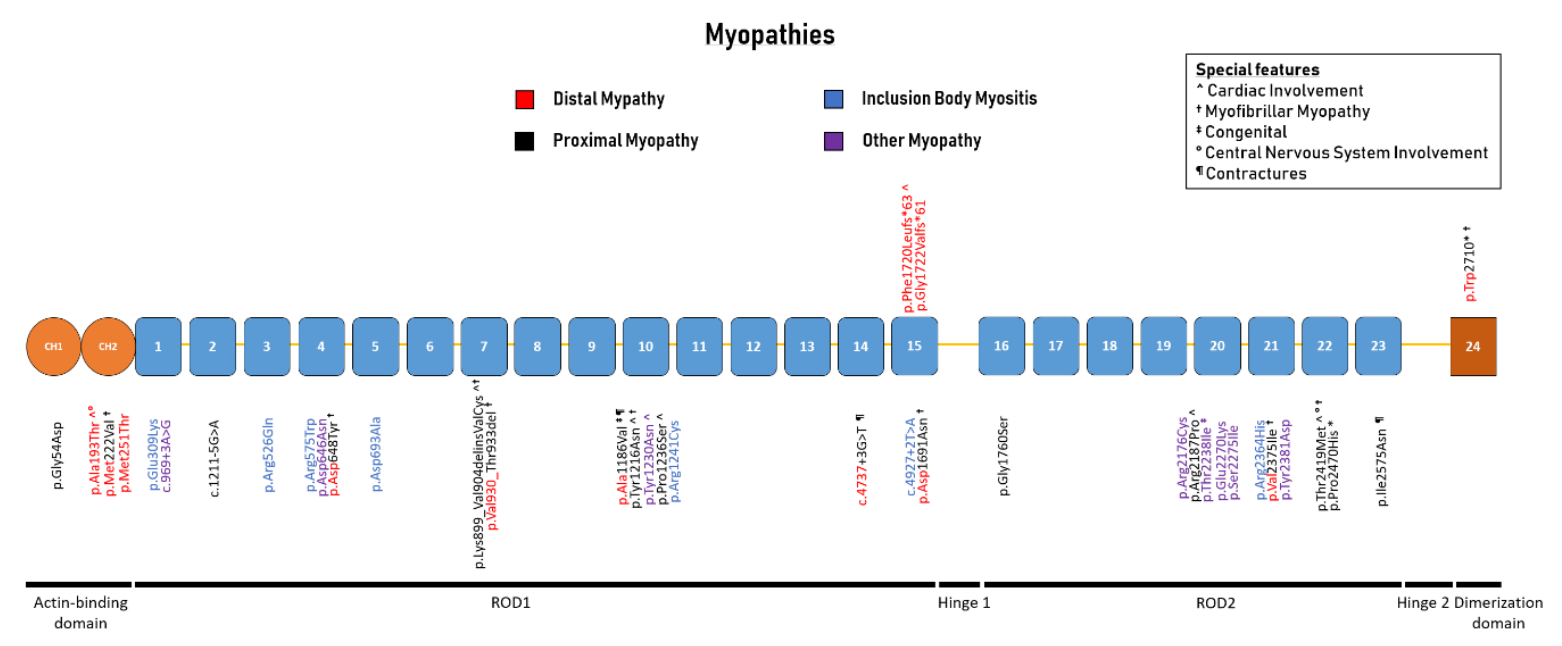

Figure 5. Schematic representation of the FLNC gene with their protein coding domains. Numbers inside the boxes refer to the Ig-like domains of filamin C. Above and below the schematic are all unique variants associated with myopathies. Variants are annotated at the protein level.

\section{BIOLOGICAL RELEVANCE}

FLNC variants are associated with a spectrum of cardiac and muscular phenotypes, suggesting that specific variants fall into three pathomechanisms, as previously suggested ${ }^{5}$ :

1. Variants that are predicted to lead to expression of misfolded proteins which saturate the proteasome and autophagy pathways;

2. Variants which give a toxic gain of function by altering ligand binding properties;

3. Variants causing a premature stop codon and concomitant nonsense-mediated decay (NMD), resulting in haploinsufficiency.

\subsection{Pathomechanisms of FLNC variants in cardiomyopathy phenotypes}

The landmark paper regarding FLNC variants in DCM showed the strong association between truncating variants and this disease ${ }^{20}$. NMD and subsequent haploinsufficiency was validated for a number of truncating variants as the pathomechanism in FLNC-associated DCM. Immunohistochemical analysis showed normal FLNC protein in the intercalated discs of DCM patients. Abnormal FLNC protein aggregates in the cytoplasm were not detectable ${ }^{20}$. The absence of aggregates in cardiac tissue of patients with truncating FLNC variants in the ROD2 domain indicates the lack of an abnormal FLNC protein. In addition, Western blot analysis in zebrafish models and rat cardiac myoblasts showed the absence of a truncated protein in the truncating variant models ${ }^{18} 36$. Haploinsufficiency affects force transduction of striated muscle, specifically in tissues dependent on high-force generation, such as the myocardium. Both HCM and RCM patients have an enrichment of missense variants causing changes in the secondary protein structure resulting in an abnormal protein. Missense variants are strongly clustered in the short ROD2 domain of the protein in HCM (Figure 3). This region of the protein is important for the interaction between FLNC and the Z-disc. Five missense variants were reported in the intradomain insert (between Ig-like domain 19 and 20), which mediates the specific targeting to the Z-disc. Abnormal protein has been observed within aggregates in the tissue of FLNC-associated HCM and RCM patients in association with marked sarcomeric abnormalities ${ }^{19}$ 37. The progressive accumulation of protein aggregates in the cardiac muscle eventually leads to sarcomeric disarray. Functional studies including transfection of missense variants in rat cardiac myoblasts confirmed the formation of insoluble filamin C aggregates ${ }^{19}$, 
Table 4. FLNC variants found in individuals with a muscular phenotype as previously reported and from this study

\begin{tabular}{|c|c|c|c|c|c|c|c|c|}
\hline Exon & c-Notation & p-Notation & $\begin{array}{c}\text { Variant } \\
\text { type }\end{array}$ & Domain & Location & Phenotype & Reference & Effect \\
\hline 1 & c.161G>A & p.Gly54Asp & Missense & ABD & $\mathrm{CH} 1$ & PM & (Fichna et al., 2018) & VUS \\
\hline 2 & c. $577 \mathrm{G}>\mathrm{A}$ & p.Ala193Thr & Missense & $A B D$ & $\mathrm{CH} 2$ & DM; PM; Car; CNS & (Duff et al., 2011) & Likely pathogenic \\
\hline 3 & c. $664 A>G$ & p.Met222Val & Missense & $A B D$ & $\mathrm{CH} 2$ & DM; PM; MFM & (Gemelli et al., 2019) & Likely pathogenic \\
\hline 4 & c. $752 \mathrm{~T}>\mathrm{C}$ & p.Met251Thr & Missense & $A B D$ & $\mathrm{CH} 2$ & DM; PM & (Duff et al., 2011) & Likely pathogenic \\
\hline 5 & c. $925 G>A$ & p.Glu309Lys & Missense & ROD1 & Ig-like 1 & IBM & (Weihl et al., 2015) & VUS \\
\hline 5 & c. $969+3 A>G$ & p.? & Splice & ROD1 & Ig-like 1 & OM & (Dai et al., 2015) & VUS \\
\hline 8 & c. $1211-5 G>A$ & p.? & Splice & ROD1 & Ig-like 2 & PM & Current study & VUS \\
\hline 10 & c. $1577 G>A$ & p.Arg526Gln & Missense & ROD1 & Ig-like 3 & IBM & (Weihl et al., 2015) & VUS \\
\hline 11 & c. $1723 \mathrm{C}>\mathrm{T}$ & p.Arg575Trp & Missense & ROD1 & Ig-like 4 & IBM & (Weihl et al., 2015) & VUS \\
\hline 12 & c. $1936 G>A$ & p.Asp646Asn & Missense & ROD1 & Ig-like 4 & OM & Current study & VUS \\
\hline 12 & & p.Asp648Tyr & Missense & ROD1 & Ig-like 4 & PM; DM; MFM & (Y. T. Zhang et al., 2018) & Likely pathogenic \\
\hline 13 & c. $2078 \mathrm{~A}>\mathrm{C}$ & p.Asp693Ala & Missense & ROD1 & Ig-like 5 & IBM & (Weihl et al., 2015) & VUS \\
\hline 18 & c.2695_2712del18insGTTTGT & p.Lys899_Val904delinsValCys & In-frame & ROD1 & Ig-like 7 & PM; Car; MFM & (Luan et al., 2010) & Likely pathogenic \\
\hline 18 & c.2789_2800del12 & p.Val930_Thr933del & In-frame & ROD1 & Ig-like 7 & PM; DM; MFM & (Shatunov et al., 2009) & Likely pathogenic \\
\hline 21 & c. $3557 \mathrm{C}>\mathrm{T}$ & p.Ala1186Val & Missense & ROD1 & Ig-like 10 & PM; DM; CM; Con & (Ghaoui et al., 2015) & Likely pathogenic \\
\hline 21 & $c .3646 \mathrm{~T}>\mathrm{A}$ & p.Tyr1216Asn & Missense & ROD1 & Ig-like 10 & PM; Car; MFM & $\begin{array}{l}\text { (Avila-Smirnow et al., } \\
\text { 2010) }\end{array}$ & Likely pathogenic \\
\hline 21 & c. $3688 \mathrm{~T}>\mathrm{A}$ & p.Tyr1230Asn & Missense & ROD1 & Ig-like 10 & OM; Car & (Vill et al., 2017) & VUS \\
\hline 21 & c. $3706 C>T$ & p.Pro1236Ser & Missense & ROD1 & Ig-like 10 & PM; Car & (Yu et al., 2017) & VUS \\
\hline 21 & $c .3721 \mathrm{C}>\mathrm{T}$ & p.Arg1241Cys & Missense & ROD1 & Ig-like 10 & IBM & (Weihl et al., 2015) & VUS \\
\hline 27 & c. $4737+3 G>T$ & p.? & Splice & ROD1 & Ig-like 14 & PM; DM; Con & Current study & VUS \\
\hline 28 & c. $4927+2 T>A$ & p.? & Splice & ROD1 & Ig-like 15 & OM & (Zenagui et al., 2018) & VUS \\
\hline 30 & c. $5071 \mathrm{G}>\mathrm{A}$ & p.Asp1691Asn & Missense & ROD1 & Ig-like 15 & PM; DM; MFM & (Y. T. Zhang et al., 2018) & Likely pathogenic \\
\hline 30 & c.5160delC & p.Phe1720Leufs*63 & Frameshift & ROD1 & Ig-like 15 & DM; Car & $\begin{array}{l}\text { (Guergueltcheva et al., } \\
\text { 2011) }\end{array}$ & Likely pathogenic \\
\hline 30 & c.5165delG & p.Gly1722Valfs*61 & Frameshift & ROD1 & Ig-like 15 & DM & (Rossi et al., 2017) & Likely pathogenic \\
\hline 31 & c. $5278 \mathrm{G}>\mathrm{A}$ & p.Gly1760Ser & Missense & ROD2 & Ig-like 16 & PM & (Yu et al., 2017) & VUS \\
\hline
\end{tabular}




\begin{tabular}{|c|c|c|c|c|c|c|c|c|}
\hline 40 & c. $6526 C>T$ & p.Arg2176Cys & Missense & ROD2 & Intradomain & IBM & (Cerino et al., 2017) & VUS \\
\hline 40 & c. $6560 \mathrm{G}>\mathrm{C}$ & p.Arg2187Pro & Missense & ROD2 & Intradomain & PM; Car & Current study & VUS \\
\hline 40 & c. $6713 \mathrm{C}>\mathrm{T}$ & p.Thr2238Ile & Missense & ROD2 & Intradomain & $\mathrm{OM} ; \mathrm{CM}$ & Current study & VUS \\
\hline 41 & c. $6808 \mathrm{G}>\mathrm{A}$ & p.Glu2270Lys & Missense & ROD2 & Ig-like 20 & OM & Current study & VUS \\
\hline 41 & c. $6824 G>T$ & p.Ser2275Ile & Missense & ROD2 & Ig-like 20 & OM & Current study & VUS \\
\hline 42 & c. $7091 \mathrm{G}>\mathrm{A}$ & p.Arg2364His & Missense & ROD2 & Ig-like 21 & IBM & (Weihl et al., 2015) & VUS \\
\hline 42 & c. $7123 \mathrm{G}>\mathrm{A}$ & p.Val2375Ile & Missense & ROD2 & Ig-like 21 & PM; DM; MFM & (Chen et al., 2019) & VUS \\
\hline 43 & c. $7141 \mathrm{~T}>\mathrm{G}$ & p.Tyr2381Asp & Missense & ROD2 & Ig-like 21 & OM & Current study & VUS \\
\hline 44 & c. $7256 C>T$ & p.Thr2419Met & Missense & ROD2 & Ig-like 22 & $\begin{array}{c}\text { PM; Car; CNS; } \\
\text { MFM }\end{array}$ & (Tasca et al., 2012) & VUS \\
\hline 45 & c. $7409 C>A$ & p.Pro2470His & Missense & ROD2 & Ig-like 22 & PM; Car & (Reddy et al., 2017) & VUS \\
\hline 46 & c. $7724 \mathrm{~T}>\mathrm{A}$ & p.lle2575Asn & Missense & ROD2 & Ig-like 23 & PM; Con & Current study & Likely pathogenic \\
\hline 48 & c. $8130 \mathrm{G}>\mathrm{A}$ & p.Trp2710* & Nonsense & Dimerization & Ig-like 24 & PM; DM; MFM & (Vorgerd et al., 2005) & Likely pathogenic \\
\hline
\end{tabular}


although there were differences in size of aggregates and signal strength on Western blots per variant. The overall histopathology of FLNC-associated HCM constituted of large nuclei and large fiber diameters, as comparable to established non-FLNC HCM.

\subsection{Pathomechanisms of FLNC variants in myopathy phenotypes with and without protein aggregate formation}

The p.Trp2710* variant leads to truncation of Ig-domain 24 which is needed for the formation of FLNC dimers ${ }^{30}$. The mutant mRNA is stable and not subject to degradation by NMD, probably because the variant is in the last exon of the gene. Instead, the nonsense variant leads to the formation of protein aggregates of mutated filamin fragments, other known MFM associated proteins and a number of filamin C binding partners in the skeletal muscle ${ }^{38} 39$. Interestingly, there is one truncating variant in the last exon described in association with DCM (p.Asp2703Thrfs*69), which is subject to NMD ${ }^{20}$. This shows that not only the variant type in the last exon is of importance but also the pathomechanism for the subsequent phenotype.

The autophagy pathways which clear protein aggregates in MFM (mainly the 'chaperone-assisted selective autophagy' (CASA) pathway) are activated but unable to clear the aggregates, preventing recovery of homeostasis ${ }^{40} 41$. More proteins are being associated with the regulation of FLNC proteostasis via autophagy, such as Hspb7 ${ }^{42}$. Consequently, many of the FLNC binding partners are dispersed which disturbs the stability between the cytoskeleton and the membrane protein complexes, affecting cell signaling. The formation of protein aggregates in combination with FLNC depletion causes myofiber disintegration and muscle weakness, which is aggravated by muscle activity ${ }^{31} 43$.

Two missense variants (p.Ala193Thr, p.Met251Thr) in the actin-binding domain (ABD) are associated with a form of isolated distal myopathy without protein aggregate formation ${ }^{44}$. In contrast to all other reported missense variants in the $A B D$, these variants are predicted to change from a hydrophobic to an uncharged amino acid. They are predicted to alter intradomain interactions, thereby increasing the binding affinity of filamin $\mathrm{C}$ to actin .

A frameshift variant (p.Phe1720Leufs*63) has also been associated with an isolated distal myopathy without protein aggregates ${ }^{45}$. In contrast to $p$. $\operatorname{Trp} 2710^{*}$, this variant occurs in Ig-like domain 15 and activates NMD: analysis of RNA and protein in patients' muscle biopsies showed a $50 \%$ decrease in FLNC mRNA and protein, implicating haploinsufficiency ${ }^{45}$. When compared to the truncating variants in DCM, this is the only reported frameshift variant in Ig-like domain 15, which is the domain before hinge 1 . This hinge is only present in the long isoform of FLNC which incorporates exon $31^{13}$, and might be important in the isoform switch during cell stress ${ }^{14}$. There is no comparable frameshift variant encompassing exon 31 in DCM. The different impact of the frameshift on the two isoforms could be a mechanistic explanation for the clinical variation, although this hypothesis needs further testing to accurately determine the importance of the isoform shift.

\section{ANIMAL MODELS}

Animal studies of FLNC are performed in (zebra)fish, mice and Drosophila (Figure 6). Shortly after the description of FLNC variants in MFM, the first mouse model was developed by deletion of the last 8 exons of FLNC ${ }^{46}$. Homozygous mice died shortly after birth due to respiratory failure. Heterozygous 
mice had less muscle mass and a decreased number of primary muscle fibers. Their muscles also showed excessive fiber size variation, centrally located nuclei and disorganized muscle structure. This suggests a key role for FLNC in myogenesis as well as in myofiber structure maintenance.

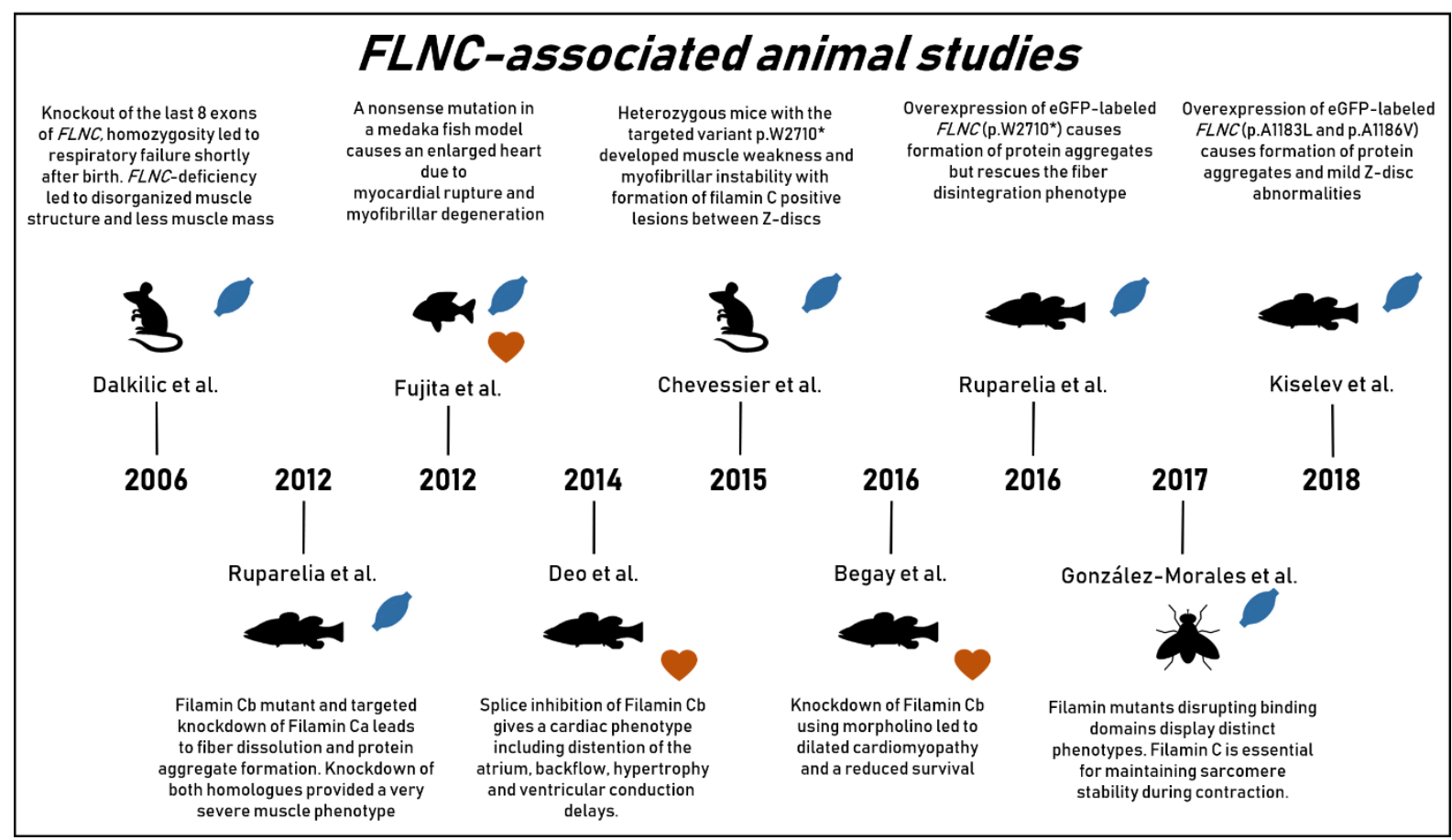

Figure 6. A timeline representation of animal models generated to study FLNC variants. eGFP indicates enhanced green fluorescent protein.

A Medaka fish (Orzyias latipes) model was developed to investigate the cardiac and muscular phenotype of $F L N C$ variants ${ }^{9}$. It contained a nonsense variant resulting in truncation at Ig-15. Despite this variant, these fish had normal myogenesis. However, the myofibrils gradually degenerated and became disorganized eventually leading to myocardial rupture. This suggests that $F L N C$ is mainly involved in muscle structure maintenance instead of myogenesis, partly by affording protection against mechanical stress related to muscle contraction. Fiber dissolution and protein aggregate formation was not described in this model. These characteristics of MFM were observed in a zebrafish (Danio rerio) model in which the filamin $\mathrm{C}-\mathrm{b}$ homologue $(\mathrm{fIncb})$ contains a nonsense variant in exon $30^{47}$. A knockdown of the filamin $\mathrm{C}$-a homologue ( $\mathrm{flnca}$ ) yielded the same phenotype. However, loss of both homologues leads to major failure of the muscle fibers. Also here, it was shown that FLNC was mainly involved in fiber protection and maintenance rather than fiber specification and myogenesis. Investigation of the cardiac phenotype in fish with a flncb knockdown showed atrium distention and backflow upon contraction ${ }^{48}$. Optical mapping showed a decrease in ventricular conduction velocity suggesting alterations in junctional remodeling and cell-cell coupling. Later studies also showed sarcomere and Z-disc disorganization ${ }^{36}$. A study using Drosophila showed filamin $C$ as an important cohesive element within the Z-disc, where it acts as a bridge between thin filaments and the elastic scaffold protein titin ${ }^{49}$. The Z-disc requires filamin $\mathrm{C}$ to withstand the strong contractile forces acting on the sarcomere.

Other animal models were created to investigate targeted variants by knock-in experiments in mice or overexpression in zebrafish 374043 . Two models were created to investigate the hotspot variant p.Trp2710*40 43. Heterozygous mice developed muscle 
weakness and myofibrillar instability ${ }^{43}$. In addition to the classical protein aggregates, they also developed filamin $\mathrm{C}$ positive lesions between the Z-discs appearing upon physical exercise. Overexpression of the variant in zebrafish led to the formation of protein aggregates 40. In this model, mutant FLNC was localized around the Z-disc and is able to rescue the disintegration phenotype. This led to the hypothesis that it was mainly the aggregates and the sequestration of FLNC away from the Z-disc that cause myofibrillar disintegration. The study further showed that the CASA pathway is impaired, making the cell unable to clear the protein aggregates.

\section{CLINICAL AND DIAGNOSTIC RELEVANCE}

Cardiac involvement is a common clinical manifestation in hereditary muscular dystrophies

${ }^{50}$. Conversely, muscular problems in cardiomyopathy patients have also been described ${ }^{51}$, showing the strong molecular link between hereditary muscular dystrophies and cardiomyopathies. In line with this, cardiac involvement is not uncommon in FLNC-associated myopathy (Figure 5), but muscle involvement has not been described (yet) at the moment of diagnosis in FLNC-associated cardiomyopathy ${ }^{22}$. A single DCM patient developed distal myopathy during follow-up ${ }^{20}$.

\subsection{Specific clinical characteristics of FLNC-associated cardiomyopathies}

Compared to the average clinical course of DCM, FLNC-associated DCM is more malignant characterized by ventricular arrhythmias, myocardial fibrosis and a high risk of sudden cardiac death ${ }^{20}$. The average age of onset is $39.7 \pm 14.5$. Considering all available literature at the moment, filaminopathy has a distinct cardiac phenotype. In contrast to arrhythmogenic cardiomyopathies, FLNC-associated DCM is left-dominant in the absence of right ventricular involvement 2052 . Diagnostic clues can be inferolateral negative $T$ waves on the electrocardiogram, mild to moderate left ventricular dysfunction and regional dyskinesia. It also has a characteristic ring-like scar pattern in the left ventricle as detected by cardiovascular magnetic resonance (CMR) imaging ${ }^{52}$. The combination of increased myocardial fibrosis, ventricular arrhythmias and sudden cardiac death are also found in laminopathies, desminopathies and desmosomal variants ${ }^{53} 54$. In contrast to these forms of genetic DCM, cardiac conduction abnormalities such as an atrioventricular block are uncommon in FLNC-associated DCM while they are common in laminopathies and desminopathies. In addition, desmosomal variants are strongly correlated to isolated or predominant right ventricular involvement. For patients with DCM, the finding of a truncating FLNC is likely causative and relatives should be screened for this variant ${ }^{55}$. The finding of a truncating FLNC variant in otherwise healthy subjects outside of a familial context is much less clear at the moment, as there is not enough knowledge regarding penetrance, expression and clinical correlation. Although the prevalence of truncating FLNC variants is very low in the general population $(<0.01 \%$ in gnomAD).

The mean age of onset of HCM in FLNC carriers is $35.9 \pm 14.8$. In a previous cohort of $\mathrm{HCM}$ patients it was reported that $34 \%$ of the FLNC variant carriers had elevated creatine kinase (CK) levels ${ }^{19}$, also in RCM there were some patients with mildly elevated CK levels ${ }^{56}$. However, this finding is not consistent across different patient cohorts ${ }^{22}$.

\subsection{Specific clinical characteristics of FLNC-associated myopathies}

The two classic muscular phenotypes are MFM and distal myopathy (with and without protein aggregates respectively) ${ }^{5}$. Other genes have been associated with these phenotypes, such as 
$D E S, \angle D B 3$ and $B A G 3^{50}$. Besides the muscular phenotype, these genes are also associated with isolated cardiomyopathies. Characteristic features of FLNC-associated MFM is the symmetrical involvement of proximal muscles in the lower extremities, respiratory weakness during the disease course and a specific set of imaging characteristics for muscle involvement 31. About one-third of the FLNC-MFM showed cardiac involvement. Distal myopathies due to FLNC variants are characterized by weakness in the hand and calf muscles with an onset in early adulthood ${ }^{5}$.

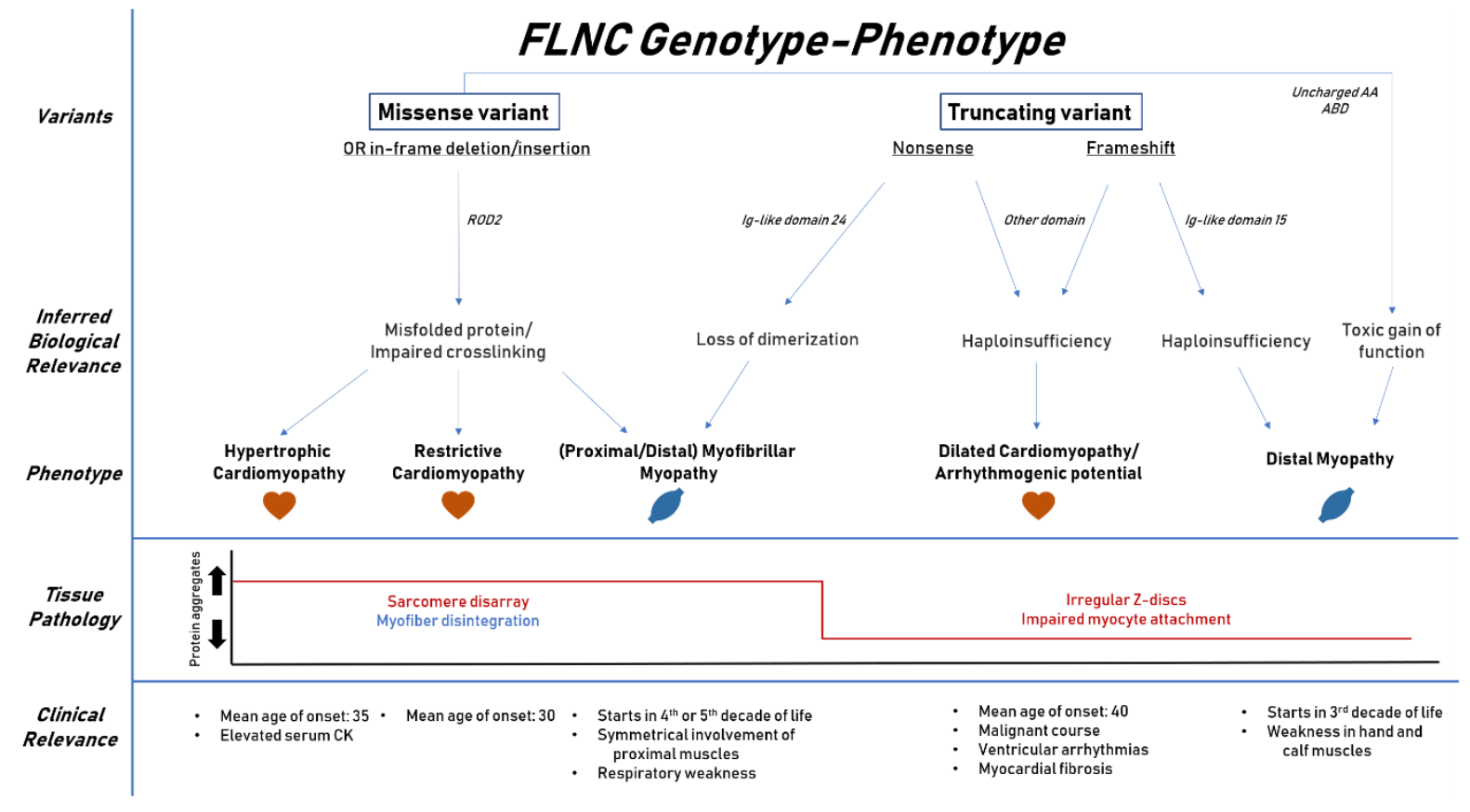

Figure 7. A summary how different variants in FLNC lead to a variety of disease mechanisms eventually giving a spectrum of clinical entities with corresponding structural histological changes. These FLNC-associated diseases contain specific clinical characteristics compared to other forms of the corresponding disease.

\section{GENOTYPE/PHENOTYPE CORRELATIONS}

Genotype-phenotype correlations are currently incomplete, but some patterns are starting to emerge (Figure 7).

There is no clear clustering of DCM variants in any specific region of the gene, partly because most truncating variants are predicted to result in NMD. Truncating FLNC variants are strongly associated with DCM and arrhythmogenic potential. Just 3 out of 55 truncating variants are associated with a muscular phenotype. Two are a frameshift in lg-like 15 spanning hinge 1 and one is a nonsense variant in Ig-like 24 of the dimerization domain. The different pathomechanism underlying missense and truncating variants partly explains the structural changes at the histological level and the corresponding clinical phenotype. Missense variants are mainly found in ROD2, which is essential for filamin C dimerization and Z-disc interaction. These variants interfere with the secondary protein structure, leading to sarcomere disarray and aggregate formation. These histopathological changes are associated with HCM and RCM phenotypes.

- Truncating variants are found throughout the whole gene, and are expected to invoke haploinsufficiency via NMD. This will lead to Z-disc disarray and weakened cell-cell adhesion with subsequent impaired mechanotransduction. These structural alterations make the heart prone for developing DCM with arrhythmogenesis, and promote fibrogenesis contributing to the arrhythmogenesis. 
- Frameshift variants spanning hinge 1 (exon 31) potentially interfere with flexibility for isoform switching. Both described frameshift variants spanning exon 31 are associated with distal myopathy.

- Missense variants in the ABD that create an uncharged amino acid give a toxic gainof-function with a stronger actin binding activity. These variants have been associated with a distal myopathy.

- A nonsense variant in the dimerization domain interferes with the ability to form FLNC homodimers, although proteins are still translated. These proteins form aggregates in the skeletal muscle leading to MFM.

\section{FUTURE PROSPECTS}

As FLNC is now included in many genetic screening panels for muscular and cardiac diseases, the number of variants will increase in the coming years. Better understanding of the molecular alterations due to FLNC variants can shed light on potential treatment targets. It can help us in understanding and predicting genotype-phenotype correlations. Proper functioning of FLNC depends on multiple interactions with other proteins ${ }^{42}$. These interactions could contribute to the specific phenotypes. The ROD2 domain encompasses binding sites for the majority of FLNC interactors, and is necessary for mechanosensing and muscle maintenance functions. Chaperones such as HspB1 need to bind to FLNC to ensure these functions ${ }^{57}$. The inability of HspB1 to bind to FLNC can lead to cardiac dysfunction. This is one example how variants in a specific domain could affect protein interactions and contribute to a specific phenotypes. A field which should be further explored. As an example, we formulated the following research questions:

- Which factors explain the clinical variety among truncating variants in the FLNC gene. For example: why does p. Phe1720Leufs*63 lead to a distal myopathy while p.Asn1369Lysfs*36 leads to DCM? Does flexibility in isoform switching play a role in this?

- How does a nonsense variant in Ig-like 24 (p.Trp2710*) lead to a MFM and a frameshift variant in the same region (p.Asp2703Thrfs*69) lead to arrhythmogenic DCM?

- What are the exact structural protein changes related to missense variants in the ABD and how do they explain the clinical difference between HCM and distal myopathy?

- Are truncating variants in certain parts of the gene better tolerated clinically and therefore less penetrant?

- Which molecular pathways are differentially activated due to FLNC variants and can these pathways serve as potential therapeutic targets?

- What is the clinical relevance and outcome of (truncating) FLNC variants in the general population?

\section{CONCLUSION}

Variants in FLNC can lead to myopathies and cardiomyopathies. Difference in phenotypes can be partly explained by the pathomechanism associated with the variant type and location within the gene. As a general rule, interference with the dimerization and folding of the protein leads to aggregate formation, as found in HCM or MFM. Truncating variants with subsequent haploinsufficiency lead to weakened structural adhesion mainly associated with DCM and cardiac arrhythmias. 


\section{REFERENCES}

1. van der Flier A, Sonnenberg A. Structural and functional aspects of filamins. Biochimica et biophysica acta 2001;1538(2-3):99-117.

2. Himmel M, Van Der Ven PF, Stocklein $W$, et al. The limits of promiscuity: isoform-specific dimerization of filamins. Biochemistry 2003;42(2):430-9.

3. Thompson TG, Chan YM, Hack AA, et al. Filamin 2 (FLN2): A muscle-specific sarcoglycan interacting protein. The Journal of cell biology 2000;148(1):115-26.

4. Zhang $M$, Liu J, Cheng $A$, et al. Identification of CAP as a costameric protein that interacts with filamin C. Mol Biol Cell 2007;18(12):4731-40.

5. Furst DO, Goldfarb LG, Kley RA, et al. Filamin Crelated myopathies: pathology and mechanisms. Acta neuropathologica 2013;125(1):33-46.

6. Takada F, Vander Woude DL, Tong HQ, et al. Myozenin: an alpha-actinin- and gamma-filaminbinding protein of skeletal muscle $Z$ lines. Proceedings of the National Academy of Sciences of the United States of America 2001;98(4):1595600.

7. Anastasi G, Cutroneo G, Trimarchi F, et al. Evaluation of sarcoglycans, vinculin-talin-integrin system and filamin2 in alpha- and gammasarcoglycanopathy: an immunohistochemical study. Int J Mol Med 2004;14(6):989-99.

8. Guyon JR, Kudryashova E, Potts A, et al. Calpain 3 cleaves filamin $C$ and regulates its ability to interact with gamma- and delta-sarcoglycans. Muscle \& nerve 2003;28(4):472-83.

9. Fujita $\mathrm{M}$, Mitsuhashi $\mathrm{H}$, Isogai $\mathrm{S}$, et al. Filamin $\mathrm{C}$ plays an essential role in the maintenance of the structural integrity of cardiac and skeletal muscles, revealed by the medaka mutant zacro. Developmental biology 2012;361(1):79-89.

10. Leber $Y$, Ruparelia AA, Kirfel G, et al. Filamin C is a highly dynamic protein associated with fast repair of myofibrillar microdamage. Human molecular genetics 2016;25(13):2776-88.

11. Molt S, Bührdel JB, Yakovlev $S$, et al. Aciculin interacts with filamin $C$ and $X$ in and is essential for myofibril assembly, remodeling and maintenance. J Cell Sci 2014;127(Pt 16):3578-92.

12. Chakarova C, Wehnert MS, Uhl K, et al. Genomic structure and fine mapping of the two human filamin gene paralogues FLNB and FLNC and comparative analysis of the filamin gene family. Human genetics 2000;107(6):597-611.

13. Xie Z, Xu W, Davie EW, et al. Molecular cloning of human $A B P L$, an actin-binding protein homologue. Biochemical and biophysical research communications 1998;251(3):914-9.
14. Kong SW, Hu YW, Ho JW, et al. Heart failureassociated changes in RNA splicing of sarcomere genes. Circulation Cardiovascular genetics 2010;3(2):138-46.

15. Richards S, Aziz N, Bale S, et al. Standards and guidelines for the interpretation of sequence variants: a joint consensus recommendation of the American College of Medical Genetics and Genomics and the Association for Molecular Pathology. Genetics in medicine : official journal of the American College of Medical Genetics 2015;17(5):405-24.

16. Elliott $\mathrm{P}$, Andersson $\mathrm{B}$, Arbustini $\mathrm{E}$, et al. Classification of the cardiomyopathies: a position statement from the European Society of Cardiology Working Group on Myocardial and Pericardial Diseases. Eur Heart J 2008;29(2):2706.

17. Maron BJ, Towbin JA, Thiene G, et al. Contemporary definitions and classification of the cardiomyopathies: an American Heart Association Scientific Statement from the Council on Clinical Cardiology, Heart Failure and Transplantation Committee; Quality of Care and Outcomes Research and Functional Genomics and Translational Biology Interdisciplinary Working Groups; and Council on Epidemiology and Prevention. Circulation 2006;113(14):180716.

18. Reinstein E, Gutierrez-Fernandez A, Tzur S, et al. Congenital dilated cardiomyopathy caused by biallelic mutations in Filamin C. European journal of human genetics : EJHG 2016;24(12):1792-96.

19. Valdes-Mas R, Gutierrez-Fernandez A, Gomez J, et al. Mutations in filamin $C$ cause a new form of familial hypertrophic cardiomyopathy. Nature communications 2014;5:5326.

20. Ortiz-Genga MF, Cuenca $S$, Dal Ferro $M$, et al. Truncating FLNC Mutations Are Associated With High-Risk Dilated and Arrhythmogenic Cardiomyopathies. J Am Coll Cardiol 2016;68(22):2440-51.

21. Cui H, Wang J, Zhang C, et al. Mutation profile of FLNC gene and its prognostic relevance in patients with hypertrophic cardiomyopathy. Molecular genetics \& genomic medicine 2018;6(6):1104-13.

22. Ader F, De Groote $P$, Reant $P$, et al. FLNC pathogenic variants in patients with cardiomyopathies: Prevalence and genotypephenotype correlations. Clinical genetics 2019;96(4):317-29.

23. Gomez J, Lorca R, Reguero JR, et al. Screening of the Filamin C Gene in a Large Cohort of Hypertrophic Cardiomyopathy Patients. Circulation Cardiovascular genetics 2017;10(2) 
24. Walsh R, Mazzarotto F, Whiffin N, et al. Quantitative approaches to variant classification increase the yield and precision of genetic testing in Mendelian diseases: the case of hypertrophic cardiomyopathy. Genome Med 2019;11(1):5.

25. Hershberger RE, Givertz MM, Ho CY, et al. Genetic Evaluation of Cardiomyopathy-A Heart Failure Society of America Practice Guideline. J Card Fail 2018;24(5):281-302.

26. Bains S, Tester DJ, Asirvatham SJ, et al. A Novel Truncating Variant in FLNC-Encoded Filamin C May Serve as a Proarrhythmic Genetic Substrate for Arrhythmogenic Bileaflet Mitral Valve Prolapse Syndrome. Mayo Clinic proceedings 2019;94(5):906-13.

27. Mangum KD, Ferns SJ. A novel familial truncating mutation in the filamin $C$ gene associated with cardiac arrhythmias. European journal of medical genetics 2019;62(4):282-85.

28. Hall CL, Akhtar MM, Sabater-Molina M, et al. Filamin $C$ variants are associated with a distinctive clinical and immunohistochemical arrhythmogenic cardiomyopathy phenotype. Int J Cardiol 2019

29. Miszalski-Jamka K, Jefferies JL, Mazur W, et al. Novel Genetic Triggers and Genotype-Phenotype Correlations in Patients With Left Ventricular Noncompaction. Circulation Cardiovascular genetics 2017;10(4)

30. Vorgerd $M$, van der Ven PF, Bruchertseifer $V$, et al. A mutation in the dimerization domain of filamin c causes a novel type of autosomal dominant myofibrillar myopathy. American journal of human genetics 2005;77(2):297-304.

31. Kley RA, Serdaroglu-Oflazer P, Leber $Y$, et al. Pathophysiology of protein aggregation and extended phenotyping in filaminopathy. Brain : $a$ journal of neurology 2012;135(Pt 9):2642-60.

32. van den Bogaart FJ, Claeys KG, Kley RA, et al. Widening the spectrum of filamin-C myopathy: Predominantly proximal myopathy due to the p.A193T mutation in the actin-binding domain of FLNC. Neuromuscular disorders : NMD 2017;27(1):73-77.

33. Avila-Smirnow D, Béhin A, Gueneau L, et al. P2. 18 A novel missense FLNC mutation causes arrhythmia and late onset myofibrillar myopathy with particular histopathology features. 2010;20(9):623-24.

34. Luan X, Hong D, Zhang W, et al. A novel heterozygous deletion-insertion mutation (26952712 del/GTTTGT ins) in exon 18 of the filamin C gene causes filaminopathy in a large Chinese family. Neuromuscular disorders : NMD 2010;20(6):390-6.

35. Janssens J, Philtjens $S$, Kleinberger $G$, et al. Investigating the role of filamin $\mathrm{C}$ in Belgian patients with frontotemporal dementia linked to GRN deficiency in FTLD-TDP brains. Acta neuropathologica communications 2015;3:68.

36. Begay RL, Tharp CA, Martin A, et al. FLNC Gene Splice Mutations Cause Dilated Cardiomyopathy. JACC Basic to translational science 2016;1(5):344-59.

37. Kiselev A, Vaz R, Knyazeva A, et al. De novo mutations in FLNC leading to early-onset restrictive cardiomyopathy and congenital myopathy. Hum Mutat 2018;39(9):1161-72.

38. Lowe T, Kley RA, van der Ven PF, et al. The pathomechanism of filaminopathy: altered biochemical properties explain the cellular phenotype of a protein aggregation myopathy. Human molecular genetics 2007;16(11):1351-8.

39. Kley RA, Maerkens A, Leber $Y$, et al. A combined laser microdissection and mass spectrometry approach reveals new disease relevant proteins accumulating in aggregates of filaminopathy patients. Mol Cell Proteomics 2013;12(1):215-27.

40. Ruparelia AA, Oorschot V, Ramm G, et al. FLNC myofibrillar myopathy results from impaired autophagy and protein insufficiency. Human molecular genetics 2016;25(11):2131-42.

41. Kley RA, van der Ven PF, Olive $M$, et al. Impairment of protein degradation in myofibrillar myopathy caused by FLNC/filamin C mutations. Autophagy 2013;9(3):422-3.

42. Mercer EJ, Lin YF, Cohen-Gould L, et al. Hspb7 is a cardioprotective chaperone facilitating sarcomeric proteostasis. Developmental biology 2018;435(1):41-55.

43. Chevessier F, Schuld J, Orfanos Z, et al. Myofibrillar instability exacerbated by acute exercise in filaminopathy. Human molecular genetics 2015;24(25):7207-20.

44. Duff RM, Tay V, Hackman P, et al. Mutations in the $\mathrm{N}$-terminal actin-binding domain of filamin $\mathrm{C}$ cause a distal myopathy. American journal of human genetics 2011;88(6):729-40.

45. Guergueltcheva V, Peeters K, Baets J, et al. Distal myopathy with upper limb predominance caused by filamin C haploinsufficiency. Neurology 2011;77(24):2105-14.

46. Dalkilic I, Schienda J, Thompson TG, et al. Loss of FilaminC (FLNc) results in severe defects in myogenesis and myotube structure. Molecular and cellular biology 2006;26(17):6522-34.

47. Ruparelia AA, Zhao $M$, Currie PD, et al. Characterization and investigation of zebrafish models of filamin-related myofibrillar myopathy. Human molecular genetics 2012;21(18):4073-83.

48. Deo RC, Musso G, Tasan M, et al. Prioritizing causal disease genes using unbiased genomic features. Genome biology 2014;15(12):534. 
49. Gonzalez-Morales N, Holenka TK, Schock F. Filamin actin-binding and titin-binding fulfill distinct functions in Z-disc cohesion. PLOS genetics 2017;13(7):e1006880.

50. Hermans MC, Pinto YM, Merkies IS, et al. Hereditary muscular dystrophies and the heart. Neuromuscular disorders : NMD 2010;20(8):47992.

51. Limongelli G, D'Alessandro R, Maddaloni V, et al. Skeletal muscle involvement in cardiomyopathies. Journal of cardiovascular medicine (Hagerstown, Md) 2013;14(12):837-61.

52. Augusto JB, Eiros R, Nakou E, et al. Dilated cardiomyopathy and arrhythmogenic left ventricular cardiomyopathy: a comprehensive genotype-imaging phenotype study. European heart journal cardiovascular Imaging 2019

53. Hasselberg NE, Haland TF, Saberniak J, et al. Lamin A/C cardiomyopathy: young onset, high penetrance, and frequent need for heart transplantation. Eur Heart J 2017

54. Lopez-Ayala JM, Gomez-Milanes I, Sanchez Munoz JJ, et al. Desmoplakin truncations and arrhythmogenic left ventricular cardiomyopathy: characterizing a phenotype. Europace 2014;16(12):1838-46.

55. Tayal U, Cook SA. Truncating Variants in Filamin C: The Challenges of Genotype-Phenotype Correlations in Cardiomyopathies. J Am Coll Cardiol 2016;68(22):2452-53.

56. Brodehl A, Ferrier RA, Hamilton SJ, et al. Mutations in FLNC are Associated with Familial Restrictive Cardiomyopathy. Hum Mutat 2016;37(3):269-79.

57. Collier MP, Alderson TR, de Villiers $C P$, et al. HspB1 phosphorylation regulates its intramolecular dynamics and mechanosensitive molecular chaperone interaction with filamin C. Science advances 2019;5(5):eaav8421. 


\section{SUPPLEMENTARY TABLES}

Supplemental Table 1. FLNC variants from the Leiden Open Variation Database (LOVD) without additional phenotypical information.

\section{c-Notation}

c.2791_2805del

c.5306_5314del

c. $1081 \mathrm{C}>\mathrm{T}$

c. $1094 \mathrm{~A}>\mathrm{G}$

C. $1132 \mathrm{G}>\mathrm{A}$

C.1142G $>A$

c.1196A $>\mathrm{T}$

C. $1225 \mathrm{G}>\mathrm{A}$

c. $1258 \mathrm{C}>\mathrm{T}$

c. $1348 \mathrm{G}>\mathrm{A}$

c. $1382 \mathrm{G}>\mathrm{A}$

c. $1471 \mathrm{G}>\mathrm{A}$

c. $1474 A>G$

c. $1510 \mathrm{G}>\mathrm{A}$

c. $1513 \mathrm{G}>\mathrm{A}$

c. $1600 \mathrm{G}>\mathrm{A}$

c. $1645 A>G$

c. $1673 \mathrm{G}>\mathrm{A}$

c. $1885 \mathrm{C}>\mathrm{T}$

c. $1886 \mathrm{G}>\mathrm{A}$

c. $1895 C>A$

c.1924G $>A$

c. $1928 \mathrm{~T}>\mathrm{A}$

c. $2065 \mathrm{G}>\mathrm{A}$

c. $2068 \mathrm{~T}>\mathrm{C}$

c. $2075 \mathrm{~T}>\mathrm{C}$

c. $2120 A>G$

c. $2164 \mathrm{G}>\mathrm{A}$

c. $2627 \mathrm{G}>\mathrm{T}$

c. $2642 \mathrm{G}>\mathrm{T}$

c. $2983 \mathrm{G}>\mathrm{A}$

c. 2993A $>T$

c. $3004 \mathrm{C}>\mathrm{T}$

c. $3022 \mathrm{C}>\mathrm{T}$

c. $3076 \mathrm{G}>\mathrm{A}$

c. $3149 G>A$

c.3259A $>C$

c. $3304 \mathrm{C}>\mathrm{T}$

c. $3382 \mathrm{G}>\mathrm{A}$

c. $3506 \mathrm{~A}>\mathrm{G}$

c. $3658 \mathrm{~T}>\mathrm{C}$

\section{p-Notation}

p.Lys931_Val935del

p.Glu1769_Pro1771del

p.Arg361Cys

p.Glu365Gly

p.Val378Met

p.Arg381His

p.Asp399Val

p.Asp409Asn

p.Arg420Trp

p.Val450Met

p.Arg461His

p.Val491Met

p.Lys492Glu

p.Ala504Thr

p.Gly505Ser

p.Glu534Lys

p.lle549Val

p.Arg558His

p.Arg629Trp

p.Arg629GIn

p.Pro632His

p.Val642Ile

p.lle643Asn

p.Glu689Lys

p.Phe690Leu

p.lle692Thr

p.GIn707Arg

p.Gly722Ser

p.Gly876Val

p.Gly881Val

p.Gly995Ser

p.GIn998Leu

p.Arg1002Trp

p.Arg1008Cys

p.Val1026Met

p.Ser1050Asn

p.Thr1087Pro

p.Pro1102Ser

p.Glu1128Lys

p.Lys1169Arg

p.Phe1220Leu

\section{Variant type}

In-frame

In-frame

Missense

Missense

Missense

Missense

Missense

Missense

Missense

Missense

Missense

Missense

Missense

Missense

Missense

Missense

Missense

Missense

Missense

Missense

Missense

Missense

Missense

Missense

Missense

Missense

Missense

Missense

Missense

Missense

Missense

Missense

Missense

Missense

Missense

Missense

Missense

Missense

Missense

Missense

Missense

\section{Reference}

LOVD

LOVD

LOVD

LOVD

LOVD

LOVD

LOVD

LOVD

LOVD

LOVD

LOVD

LOVD

LOVD

LOVD

LOVD

LOVD

LOVD

LOVD

LOVD

LOVD

LOVD

LOVD

LOVD

LOVD

LOVD

LOVD

LOVD

LOVD

LOVD

LOVD

LOVD

LOVD

LOVD

LOVD

LOVD

LOVD

LOVD

LOVD

LOVD

LOVD

LOVD 


\begin{tabular}{|c|c|c|c|}
\hline c. $3800 \mathrm{G}>\mathrm{A}$ & p.Arg1267Gln & Missense & LOVD \\
\hline c. $3853 \mathrm{G}>\mathrm{A}$ & p.Gly1285Ser & Missense & LOVD \\
\hline c. $3881 \mathrm{~A}>\mathrm{G}$ & p.Asn1294Ser & Missense & LOVD \\
\hline c. $3967 \mathrm{G}>\mathrm{A}$ & p.Val1323Met & Missense & LOVD \\
\hline c. $4009 A>C$ & p.Lys1337Gln & Missense & LOVD \\
\hline c. $4054 C>T$ & p.Arg1352Cys & Missense & LOVD \\
\hline c. $420 \mathrm{C}>\mathrm{A}$ & p.His140Gln & Missense & LOVD \\
\hline c. $4270 \mathrm{G}>\mathrm{A}$ & p.Gly1424Arg & Missense & LOVD \\
\hline c. $439 A>G$ & p.Met147Val & Missense & LOVD \\
\hline c. $4413 \mathrm{~A}>\mathrm{T}$ & p.Gln $1471 \mathrm{His}$ & Missense & LOVD \\
\hline c. $4418 \mathrm{G}>\mathrm{A}$ & p.Gly1473Asp & Missense & LOVD \\
\hline c. $4481 \mathrm{G}>\mathrm{A}$ & p.Arg1494GIn & Missense & LOVD \\
\hline c. $4553 \mathrm{~A}>\mathrm{G}$ & p.Lys1518Arg & Missense & LOVD \\
\hline c. $4556 A>G$ & p.Tyr1519Cys & Missense & LOVD \\
\hline c. $4583 C>G$ & p.Pro1528Arg & Missense & LOVD \\
\hline c. $4705 \mathrm{G}>\mathrm{A}$ & p.Ala1569Thr & Missense & LOVD \\
\hline c. $4741 C>A$ & p.Pro1581Thr & Missense & LOVD \\
\hline c. $4763 C>G$ & p.Ala1588Gly & Missense & LOVD \\
\hline c. $4811 C>T$ & p.Pro1604Leu & Missense & LOVD \\
\hline c. $4826 \mathrm{G}>\mathrm{A}$ & p.Arg1609GIn & Missense & LOVD \\
\hline c. $4852 \mathrm{G}>\mathrm{A}$ & p.Gly1618Ser & Missense & LOVD \\
\hline c. $4880 \mathrm{G}>\mathrm{T}$ & p.Arg1627Leu & Missense & LOVD \\
\hline c. $4925 C>G$ & p.Thr1642Arg & Missense & LOVD \\
\hline c. $4991 C>T$ & p.Thr1664Met & Missense & LOVD \\
\hline c. $5020 \mathrm{G}>\mathrm{A}$ & p.Gly1674Ser & Missense & LOVD \\
\hline c. $5128 \mathrm{G}>\mathrm{A}$ & p.Glu1710Lys & Missense & LOVD \\
\hline c. $5311 \mathrm{C}>\mathrm{G}$ & p.Pro1771Ala & Missense & LOVD \\
\hline c.5363T >G & p.Val1788Gly & Missense & LOVD \\
\hline$c .5375 C>T$ & p.Ala1792Val & Missense & LOVD \\
\hline c. $5468 \mathrm{C}>\mathrm{T}$ & p.Thr1823Met & Missense & LOVD \\
\hline c. $5797 \mathrm{G}>\mathrm{A}$ & p.Asp1933Asn & Missense & LOVD \\
\hline c. $5903 \mathrm{~A}>\mathrm{C}$ & p.Lys1968Thr & Missense & LOVD \\
\hline c. $5945 \mathrm{G}>\mathrm{A}$ & p.Arg1982His & Missense & LOVD \\
\hline c. $6094 \mathrm{C}>\mathrm{G}$ & p.Leu2032Val & Missense & LOVD \\
\hline c. $6133 C>T$ & p.Arg2045Trp & Missense & LOVD \\
\hline c. $61 A>G$ & p.Met21Val & Missense & LOVD \\
\hline c. $6497 A>G$ & p.Gln2166Arg & Missense & LOVD \\
\hline c. $6716 \mathrm{G}>\mathrm{T}$ & p.Arg2239Leu & Missense & LOVD \\
\hline c. $6825 C>A$ & p.Ser2275Arg & Missense & LOVD \\
\hline c. $6988 \mathrm{G}>\mathrm{A}$ & p.Gly2330Ser & Missense & LOVD \\
\hline c. $7067 A>T$ & p.Lys2356Ile & Missense & LOVD \\
\hline c. $7091 \mathrm{G}>\mathrm{C}$ & p.Arg2364Pro & Missense & LOVD \\
\hline c.7334A>G & p.His2445Arg & Missense & LOVD \\
\hline c. $7399 \mathrm{C}>\mathrm{T}$ & p.Arg2467Cys & Missense & LOVD \\
\hline c. $7423 \mathrm{G}>\mathrm{A}$ & p.Val2475Ile & Missense & LOVD \\
\hline c. $7582 \mathrm{G}>\mathrm{A}$ & p.Val2528Met & Missense & LOVD \\
\hline
\end{tabular}




\begin{tabular}{cccc} 
c. $7678 G>A$ & p.Val2560Met & Missense & LOVD \\
c.7814A $>$ G & p.Glu2605Gly & Missense & LOVD \\
c. $7862 G>A$ & p.Arg2621Gln & Missense & LOVD \\
c.8003T>C & p.Met2668Thr & Missense & LOVD \\
c.8017C $>T$ & p.His2673Tyr & Missense & LOVD \\
c. $856 G>A$ & p.Glu286Lys & Missense & LOVD \\
c. $977 T>C$ & p.Val326Ala & Missense & LOVD \\
c. $2265+5 G>A$ & p.? & Splice & LOVD \\
c. $2930-5 C>G$ & p.? & Splice & LOVD \\
c. $700-2 A>G$ & p.? & Splice & LOVD \\
c. $6807 C>T$ & p.Gly2269 $=$ & Synonymous & LOVD \\
c. $7284 C>T$ & p.Ser2428 $=$ & Synonymous & LOVD \\
c. $7554 C>T$ & p.Gly2518 $=$ & Synonymous & LOVD \\
\hline
\end{tabular}


Supplemental Table 2. FLNC variants found in individuals with non-striated muscle diseases.

\begin{tabular}{|c|c|c|c|c|c|c|c|c|}
\hline Exon & c-Notation & p-Notation & $\begin{array}{c}\text { Variant } \\
\text { type }\end{array}$ & Domain & Location & Phenotype & Reference & Effect \\
\hline 1 & c. $241 C>T$ & p.Arg81Cys & Missense & $\mathrm{CH} 1$ & $A B D$ & Frontotemporal dementia & (Janssens et al., 2015) & VUS \\
\hline 5 & c. $968 \mathrm{~A}>\mathrm{T}$ & p.Glu323Val & Missense & Ig-like 1 & ROD1 & Frontotemporal dementia & (Janssens et al., 2015) & VUS \\
\hline 7 & c.1141C>T & p.Arg381Cys & Missense & Ig-like 2 & ROD1 & Breast cancer & (Aloraifi et al., 2015) & VUS \\
\hline 7 & c. $1171 \mathrm{G}>\mathrm{T}$ & p.Val391Leu & Missense & Ig-like 2 & ROD1 & Autism spectrum disorder & (Takata et al., 2018) & VUS \\
\hline 10 & c. $1571 A>G$ & p.Lys524Arg & Missense & Ig-like 3 & ROD1 & Frontotemporal dementia & (Janssens et al., 2015) & VUS \\
\hline 14 & c. $2128 \mathrm{G}>\mathrm{A}$ & p.Asp710Asn & Missense & Ig-like 5 & ROD1 & Frontotemporal dementia & (Janssens et al., 2015) & VUS \\
\hline 16 & c. $2416 \mathrm{G}>\mathrm{A}$ & p.Ala806Thr & Missense & Ig-like 6 & ROD1 & Progressive supranuclear palsy & (Janssens et al., 2015) & VUS \\
\hline 16 & c. $2491 \mathrm{G}>\mathrm{A}$ & p.Val831lle & Missense & Ig-like 6 & ROD1 & Pick's disease & (Janssens et al., 2015) & VUS \\
\hline 20 & c.3092delC & p.Pro1031Argfs*47 & Frameshift & Ig-like 8 & ROD1 & Autism spectrum disorder & (Kosmicki et al., 2017) & VUS \\
\hline 20 & c. $3139 \mathrm{G}>\mathrm{C}$ & p.Val1047Leu & Missense & Ig-like 8 & ROD1 & Frontotemporal dementia & (Janssens et al., 2015) & VUS \\
\hline 21 & c. $3488 \mathrm{C}>\mathrm{G}$ & p.Pro1163Arg & Missense & Ig-like 10 & ROD1 & Frontotemporal dementia & (Janssens et al., 2015) & VUS \\
\hline 23 & c. $4003 \mathrm{G}>\mathrm{A}$ & p.Val1335Met & Missense & Ig-like 11 & ROD1 & Frontotemporal dementia & (Janssens et al., 2015) & VUS \\
\hline 23 & c. $4092 \mathrm{G}>\mathrm{C}$ & p.Leu1364Phe & Missense & Ig-like 12 & ROD1 & Progressive supranuclear palsy & (Janssens et al., 2015) & VUS \\
\hline 23 & c. $4109 \mathrm{G}>\mathrm{A}$ & p.Arg1370GIn & Missense & Ig-like 12 & ROD1 & Frontotemporal dementia & (Janssens et al., 2015) & VUS \\
\hline 27 & c. $4651 \mathrm{G}>\mathrm{A}$ & p.Ala1551Thr & Missense & Ig-like 14 & ROD1 & Frontotemporal dementia & (Janssens et al., 2015) & VUS \\
\hline 27 & c. $4711 \mathrm{G}>\mathrm{A}$ & p.Glu1571Lys & Missense & Ig-like 14 & ROD1 & Frontotemporal dementia & (Janssens et al., 2015) & VUS \\
\hline 31 & c. $5272 C>T$ & p.Arg1758Trp & Missense & Hinge 1 & Hinge 1 & Frontotemporal dementia & (Janssens et al., 2015) & VUS \\
\hline 34 & c. $5578 \mathrm{C}>\mathrm{T}$ & p.Arg1860Cys & Missense & Ig-like 17 & ROD2 & Inflammatory bowel disease & (Ostrowski et al., 2016) & VUS \\
\hline 37 & c. $6041 \mathrm{~T}>\mathrm{C}$ & p.Val2014Ala & Missense & Ig-like 18 & ROD2 & Progressive nonfluent aphasia & (Janssens et al., 2015) & VUS \\
\hline 37 & c. $6074 \mathrm{C}>\mathrm{T}$ & p.Thr2025lle & Missense & Ig-like 18 & ROD2 & Frontotemporal dementia & (Janssens et al., 2015) & VUS \\
\hline 41 & c. $6953 \mathrm{G}>\mathrm{A}$ & p.Arg2318Gln & Missense & Ig-like 21 & ROD2 & Progressive nonfluent aphasia & (Janssens et al., 2015) & VUS \\
\hline 44 & c. $7382 \mathrm{G}>\mathrm{A}$ & p.Ser2461Asn & Missense & Ig-like 22 & ROD2 & Frontotemporal dementia & (Janssens et al., 2015) & VUS \\
\hline
\end{tabular}




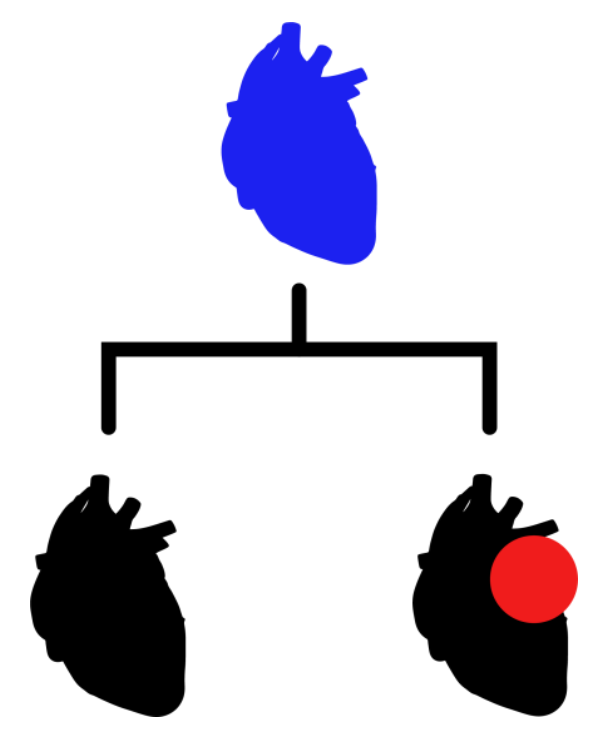

-140 - 


\title{
CHAPTER 5
}

\section{Mutations in PDLIM5 are Rare in Dilated Cardiomyopathy but are Emerging as Potential Disease Modifiers}

\author{
Job A.J. Verdonschot, $M D, M S c^{1,2}$ \\ Emma Louise Robinson, $P h D^{2}$ \\ Kiely N. James, $P h D^{3}$ \\ Mohamed W. Mohamed, $M D^{4,5}$ \\ Godelieve R.F. Claes, $P h D^{2}$ \\ Kari Casas, $M D^{4,5}$ \\ Els K. Vanhoutte, $M D, P h D^{2}$ \\ Mark R. Hazebroek, $M D^{1}$ \\ Gabriel Kringlen, MS, CGC ${ }^{4}$ \\ Michele M. Pasierb, $M D^{4}$ \\ Arthur van den Wijngaard, $P h D^{2}$ \\ Jan F.C. Glatz, $P h D^{2}$ \\ Stephane R.B. Heymans, MD, PhD1,6,7 \\ Ingrid P.C. Krapels, $M D, P h D^{2}$ \\ Shareef Nahas, PhD, FACMG \\ Han G. Brunner, $M D, P h D^{2,8,9}$ \\ Radek Szklarczyk, $P h D^{2}$
}

${ }^{1}$ Department of Cardiology, Cardiovascular Research Institute (CARIM), Maastricht University Medical Center, Maastricht, The Netherlands.

${ }^{2}$ Department of Clinical Genetics, Maastricht University Medical Center, Maastricht, The Netherlands.

${ }^{3}$ Rady Children's Institute for Genomic Medicine, San Diego, CA, USA.

${ }^{4}$ Sanford Children's Hospital, Fargo, ND, USA.

${ }^{5}$ North Dakota University, Fargo, ND, USA.

${ }^{6}$ Department of Cardiovascular Sciences, Centre for Molecular and Vascular Biology,

KU Leuven, Belgium.

${ }^{7}$ The Netherlands Heart Institute, Nl-HI, Utrecht, The Netherlands.

${ }^{8}$ Department of Human Genetics, and Donders Center for Neuroscience, Radboudumc, Nijmegen, The Netherlands.

${ }^{9} \mathrm{GROW}$ Institute for Developmental Biology and Cancer, Maastricht University Medical Center, Maastricht, The Netherlands. 


\begin{abstract}
Background: A causal genetic mutation is found in $40 \%$ of families with dilated cardiomyopathy (DCM), leaving a large percentage of families genetically unsolved. This prevents adequate counseling and clear recommendations in these families. We aim to identify novel genes or modifiers associated with DCM.

Methods: We performed computational ranking of human genes based on co-expression with a pre-defined set of genes known to be associated with DCM, which allowed us to prioritize gene candidates for their likelihood of being involved in DCM. Top candidates will be checked for variants in the available whole exome sequencing data of 142 DCM patients. RNA was isolated from cardiac biopsies to investigate gene expression.
\end{abstract}

Results: PDLIM5 was classified as the top candidate. An interesting heterozygous variant (189_190delinsGG) was found in a DCM patient with a known pathogenic truncating TTNvariant. The PDLIM5 loss-of-function (LoF) variant affected all cardiac-specific isoforms of PDLIM5 and no LoF variants were detected in the same region in a control cohort of 26.000 individuals. RNA expression of PDLIM5 and its direct interactors (MYOT, LDB3 and MYOZ2) was increased in cardiac tissue of this patient, indicating a possible compensatory mechanism. The PDLIM5 variant co-segregated with the TTN-variant and the phenotype, leading to a high disease penetrance in this family. A second patient was an infant with a homozygous $10 \mathrm{~kb}-$ deletion of exon 2 in PDLIM5 resulting in early-onset cardiac disease, showing the importance of PDLIM5 in cardiac function.

Conclusion: Heterozygous PDLIM5 variants are rare and therefore will not have a major contribution in DCM. Although they likely play a role in disease development as this gene plays a major role in contracting cardiomyocytes and homozygous variants lead to early-onset cardiac disease. Other environmental and/or genetic factors are probably necessary to unveil the cardiac phenotype in PDLIM5 mutation carriers. 


\section{INTRODUCTION}

Dilated cardiomyopathy (DCM) is a leading cause of heart failure (HF) and the most frequent cause for cardiac transplantation ${ }^{1}$. This condition is a complex final phenotype resulting from genetic and environmental triggers ${ }^{2}$. Increasing diagnostic possibilities such as advanced imaging and genetic testing allow better assessment of etiologies contributing to this phenotype in individual patients as a first step towards personalized medicine.

The genetic basis of DCM is an area of great interest as it is likely to explain the observed differences in disease susceptibility between individuals exposed to the same environmental triggers. Currently, over 60 causal genes are described in DCM of which truncating mutations in TTN (OMIM: $\left.{ }^{*} 188840\right)$ are the most prevalent ${ }^{1}$. Clinical utility of cardio-genetics is still limited due to incomplete disease penetrance and clinical variability in genetic DCM families. Also, the currently used monogenetic model only explains only up to $\sim 40 \%$ in familial cases ${ }^{3}$. Therefore, a large percentage of DCM families remain currently unsolved. Interaction between genetic variants can modify the disease, illustrated by a family with a more severe phenotype in the relatives having a mutation in both TTN and LMNA (OMIM: *150330) ${ }^{4}$. This 'double hit' phenomenon illustrates the potential of current unknown DCM-associated genes to modify the disease in patients, explaining the phenotypic variability within families. Large genome-wide association studies (GWAS) in DCM try to provide further insight in identifying modifying variants without panel restriction. Several interesting single nucleotide polymorphisms (SNP) have been identified in a large variety of genes, potentially modifying the penetrance and susceptibility of $\mathrm{DCM}^{56}$. However, the large variation among genes with rare variants and their unknown interactions make interpretation and implementation difficult. We therefore believe that 'double hit' approach can help us to unravel the gene-disease interaction network.

This study puts forward new DCM-associated genes in whole exome data using weighted ranking for co-expression with known DCM-causing genes and careful classification of found variants.

\section{METHODS}

\section{DCM cohort}

This study consisted of 142 unrelated familial and non-familial DCM patients from the Maastricht Cardiomyopathy Registry with inclusion and exclusion criteria as described previously ${ }^{7}$. In short, both DCM or hypokinetic non-dilated cardiomyopathy (HNDC; also called isolated LV dysfunction) according to the latest European Society of Cardiology (ESC) proposal were included (DCM defined as left ventricular ejection fraction (LVEF) $<50 \%$ with an indexed left ventricular end diastolic diameter (LVEDDi) $>33 \mathrm{~mm} / \mathrm{m} 2$ (men) or $>32 \mathrm{~mm} / \mathrm{m} 2$ (women) measured by echocardiography; and HNDC defined as LVEF $<50 \%$ with an LVEDDi $\leq 33 \mathrm{~mm} / \mathrm{m} 2$ (men) or $\leq 32 \mathrm{~mm} / \mathrm{m} 2$ (women) measured by echocardiography) in the absence of a (i) history of myocardial infarction and/or significant coronary artery disease; (ii) primary valvular disease; (iii) hypertensive or congenital heart disease; (iv) acute myocarditis; (v) arrhythmogenic right ventricular dysplasia; (vi) hypertrophic or restrictive cardiomyopathy ${ }^{8}$. As part of the protocol, patients were referred to the clinical cardio-genetics department of the Maastricht University Medical Center (MUMC, Maastricht, the Netherlands) for genetic counseling and DNA testing (with informed consent) between 2012 and 2018. Genetic analysis was performed using whole exome sequencing (WES) in all included patients.

All patients underwent a physical examination, blood sampling, 12-lead electrocardiogram, 24-h Holter monitoring, a complete echocardiographic and Doppler 
evaluation and coronary angiography (CAG) at baseline. Endomyocardial biopsies (EMB) were performed upon discretion of the treating physician and consent of the patient. The study was performed according to the declaration of Helsinki and was approved by the Medical Ethics Committee of Maastricht University Medical Centre. All patients gave written informed consent.

\section{Genetic analysis}

Patients at the cardio-genetics outpatient clinic in Maastricht received genetic counseling and testing using our 47-cardiomyopathy gene panel with WES (Suppl. Table 1). Exome sequencing was performed on a Illumina HiSeq machine with a minimum coverage of 20x. A family history of cardiac-related disease and sudden cardiac death was obtained by pedigree analysis. Familial inheritance was defined as recommended by the ESC: (i) two or more individuals (first or second degree relatives) have DCM or HNDC fulfilling diagnostic criteria for 'definite' disease OR (ii) in the presence of an index patient fulfilling diagnostic criteria for DCM/HNDC and a first-degree relative with autopsy-proven DCM and sudden death at $<50$ years of age ${ }^{8}$.

Genetic variants were carefully and stringently classified in 5 different classes: pathogenic, likely pathogenic, variant of clinical unknown significance (VUS), likely benign or benign based on the criteria as proposed by the ACMG guidelines ${ }^{9}$. Classification of variants was based on the score of in silico prediction software scores (SIFT, MutationTaster, PolyPhen-2, PhyloP, Align-GVGD), the frequency in reference population databases (gnomAD, 1000 genomes, ESP projects), functional studies and previously published variations in NCBI's ClinVar and HGMD. Both pathogenic and likely pathogenic mutations are reported here as pathogenic mutations. All others were considered non-pathogenic. Only truncating mutations with a PSI score $>99 \%$ in TTN were classified as pathogenic ${ }^{10}$.

The infant carrying a homozygous deletion of exon 2 of PDLIM5 (OMIM: *605904) was treated at Sanford Medical Center Fargo (North Dakota, USA) and referred to Rady Children's Institute for Genomic Medicine (RCIGM, San Diego, California, USA) for whole genome sequencing and variant analysis. Sequencing was performed on a NovaSeq6000 (Illumina, San Diego, CA) to 50X coverage, and rapid alignment and nucleotide variant calling was performed using the Dragen (Edico Genome) hardware and software. Structural variants were identified with Manta and CNVnator, then filtered to retain those affecting coding regions of known disease genes and with allele frequencies $<2 \%$ in the RCIGM database. Multiplex ligation-dependent probe amplification was used to orthogonally confirm the deletion in the proband (homozygous state) and both parents (heterozygous state). The research groups used GeneMatcher to identify genotypic and phenotypic overlap of their patients ${ }^{11}$.

\section{Control cohort}

To compare the frequencies of the genetic variants in candidate genes we collected variant data from ExAC and GnomAD database ${ }^{12}$. We additionally collected truncating variants from our internal cohort of patients with intellectual disabilities (ID) and their parents ( $n=26.000$ ), of whom to a large extend represent similar genetic background as our 142 patients. No cardiomyopathy was reported among ID patients and their parents with a truncating PDLIM5 mutation. 


\section{Network ranking}

To support the association of gene candidates with DCM in mammals, we investigated the coexpression of the genes with a pre-defined set of genes known to be associated with DCM: Zdisk or cardiac sarcomere cellular component and associated genes i.e. genes that might be essential for the initial assembly, stabilization and functional integrity of the titin filament, but also ion channels, enzymes, nuclear and desmosomal genes (Suppl. Table 1). We used expression datasets from total $\sim 30.000$ expression measurements in $\sim 500$ murine and $~ 500$ human gene expression datasets collected from the Gene Expression Omnibus (GEO) ${ }^{13}$. We selected expression datasets that co-regulate with a DCM gene set (query genes) ${ }^{14}$. Ranking the co-expression of human genes allowed us to prioritize gene candidates for their likelihood of being involved in DCM. In short, the transcriptome measurements (from GEO) are converted into a correlation matrix. The average correlation with the query set (sgene) is used for gene ranking and the dataset weight calculation. Gene scores sgene from all datasets are combined taking into account the precomputed weights. Subsequently different transcriptome platforms and species data are integrated to arrive at the final ranking. The process is repeated after excluding each query gene to construct a receiver operating characteristic (ROC) curve that visualizes predictive power of the method for a specific query set of genes. All these steps are further elucidated in the methods paper ${ }^{14}$.

After the network ranking and prioritization based on known DCM-associated genes, all previously described genes were excluded, leaving only unreported gene candidates for human DCM. Selection of top gene candidates was based on the combined position on the Zdisk and sarcomere ranking list. As most top genes were already reported in human DCM, even genes ranked $>100$ were selected. The created shortlist of genes was further prioritized based on: (1) type of mutation (missense or loss-of-function (LoF; which is defined as any variant which leads to truncation or loss of a protein); (2) location and function of the corresponding protein (potential for DCM development); (3) tissue expression (GTEx: heart/skeletal muscle); and (4) animal models (gene knockout and cardiac disease development). The final list of gene candidates was checked for variants in the WES data of the patients and subsequent variants were analyzed according to the ACMG diagnostic guidelines for variant classification as specified before.

\section{Gene expression analysis by RT-PCR}

RNA extraction was performed using the miRVana ${ }^{\mathrm{TM}}$ miRNA isolation kit (Ambion Life Technologies \#AM1560) following the manufacturer's instructions for total RNA isolation. Specifically, EMBs were thawed on ice in $1 \mathrm{~mL}$ Lysis/Binding buffer followed by homogenization using the Tissuelyser II (Qiagen \#85300) using the setting 50 oscillations/second for 5 minutes. The RNA was eluted in $50 \mu$ nuclease free water that had been pre-heated to $95{ }^{\circ} \mathrm{C}$. cDNA synthesis was performed from 100 ng RNA per sample using the Superscript II reverse transcription kit (Thermo Scientific ${ }^{\mathrm{TM}}$ \#18064014) following the manufacturer's instructions and using a T100 ${ }^{\mathrm{TM}}$ Thermal Cycler (BIO-RAD, \#1861096). The final cDNA synthesis reaction was diluted 1:10 with nuclease free water prior to qPCR. Primers were ordered from Eurogentec with purification by desalting and designed to measure mature mRNA by spanning exon-exon boundaries, with a melting temperature (Tm) of between $58-62{ }^{\circ} \mathrm{C}$ and the maximum Tm difference of the primer pair being $0.5^{\circ} \mathrm{C}$, a GC content of the primers between $45-61 \%$ and a product size between $90-150 \mathrm{bp}$. In addition, the absence of primer secondary structure and predicted primer dimers was verified in silico. Furthermore, in silico PCR was performed to confirm absence of non-specific DNA 
amplification using the web-based UCSC In-Silico PCR programme (http://genome.ucsc.edu/cgi-bin/hgPcr). Each RT-qPCR reaction was performed in technical triplicate, with the mean $\mathrm{Ct}$ value of the triplicates taken forward into subsequent analysis when the values are all within 0.6 Ct. QPCR was performed using a CFX96 Touch $^{\text {TM }}$ Real-Time PCR Detection System (BIO-RAD \#1855195), iQ ${ }^{\text {TM }}$ Supermix (BIO-RAD \#1708860), a final primer concentration of $200 \mathrm{nM}$ and PCR programme: $95^{\circ} \mathrm{C} 3 \mathrm{mins}$, (95 $\left.{ }^{\circ} \mathrm{C} 15 \mathrm{sec}, 60^{\circ} \mathrm{C} 1 \mathrm{~min}\right) \times 45$ cycles. Expression data of targets were normalized to the geometric mean of three reference genes, GAPDH, SDHA and TBP, which demonstrated stability between samples from the same amount of starting material.

\section{Statistical analysis}

Statistical analysis was performed in GraphPad Prism v7 by means of a one-way ANOVA with post-hoc Tukey analysis for multiple comparisons for each target gene analyzed. Changes in expression were accepted to be significant when the $p$-value was below 0.05 unless otherwise stated. Statistical stars on data figures represent $* p \leq=0.05, * * p=\leq 0.01, * * * p$ $=\leq 0.001$.

\section{RESULTS}

\section{Patient population}

The baseline characteristics of our DCM cohort are shown in Table 1. Only patients who had genetic analysis using WES were included, irrespective of the presence of a pathogenic mutation or other etiology. Twenty-six percent (37/142) of patients had a pathogenic mutation of which $46 \%$ (17/37) had at least one relative with DCM. Fifteen percent (22/142) of the total population had familial DCM without a proven mutation.

\section{Ranking of newly discovered candidate genes}

After the network ranking and prioritization based on known DCM-associated genes, we selected 21 genes which were not previously associated with DCM in patients (Table 2). The 5 most promising genes were selected based on the ranking of co-expression with DCM genes, type of mutation, biological context (location and function), tissue expression of the gene, and potential evidence from animal knockout models (Table 2 and 3). All variants in these 5 genes were analyzed according to the ACMG diagnostic guidelines for variant classification, resulting in a list of interesting variants ( $A C M G$ class $2 / 3$ or 3): PDLIM5 ( $n=2$ variants), CMYA5 ( $\mathrm{n}=2$ variants), SPEG ( $\mathrm{n}=3$ variants), SYNM ( $\mathrm{n}=2$ variants) and $A L P K 3$ ( $\mathrm{n}=1$ variant) (Table 3 ). PDLIM5 was chosen as top candidate considering all collected data (Table $\mathbf{2}$ and $\mathbf{3}$ ), and an additional case of an infant with a homozygous PDLIM5 deletion.

\section{Cardiac phenotype in an infant with a homozygous PDLIM5 deletion}

In a family from native American origin, the firstborn child of healthy consanguineous parents was born at 37 weeks gestation (Figure 1B). The newborn fed poorly. By 3 days old, he was lethargic, cyanotic, and in respiratory distress. Echocardiography revealed a large patent ductus arteriosus, small ventricular septal defect with left to right shunt, small atrial septal defect with bidirectional flow, and cardiac dilatation (initially right-sided, with subsequent dilatation of all four chambers) with systolic dysfunction. The infant was poorly responsive to treatment with dopamine, ACE inhibitor, and diuretics. He underwent patent ductus arteriosus ligation at 6 weeks old. The cardiac dilatation resolved, and he was weaned off all medications by 4 months old. However, he then experienced growth failure. Family history 
was negative for sudden death, cardiomyopathies or congenital heart disease. Echocardiography of the mother revealed normal structural findings.

Table 1. Baseline characteristics of the included DCM patients

\begin{tabular}{|c|c|}
\hline Parameter & Total cohort $(n=142)$ \\
\hline Female gender & $45(32 \%)$ \\
\hline Age & $51 \pm 12$ \\
\hline \multicolumn{2}{|c|}{ Etiologies and phenotype } \\
\hline Familial disease & $39(27 \%)$ \\
\hline Muscle involvement & $8(6 \%)$ \\
\hline Genetic mutation & 37 (26\%) \\
\hline Electrical disease & $47(33 \%)$ \\
\hline Toxic trigger & $8(6 \%)$ \\
\hline Peripartum & $3(2 \%)$ \\
\hline Systemic & $12(8 \%)$ \\
\hline Inflammation & $37(26 \%)$ \\
\hline Viral & $11(8 \%)$ \\
\hline Viral-positive inflammation & $8(6 \%)$ \\
\hline \multicolumn{2}{|c|}{ Arrhythmias } \\
\hline AV block & $18(13 \%)$ \\
\hline Left bundle branch block & $45(32 \%)$ \\
\hline Non-sustained VT & $44(31 \%)$ \\
\hline Atrial fibrillation & 37 (26\%) \\
\hline \multicolumn{2}{|c|}{ Outcome } \\
\hline Pacemaker & $7(5 \%)$ \\
\hline ICD & $23(16 \%)$ \\
\hline CRT-D & 39 (27\%) \\
\hline HF rehospitalization & $13(9 \%)$ \\
\hline Death & $11(8 \%)$ \\
\hline Heart transplantation & $2(1 \%)$ \\
\hline Life-threatening arrhythmia & $23(16 \%)$ \\
\hline \multicolumn{2}{|c|}{ Baseline Echocardiography } \\
\hline LVEF & $30 \pm 11$ \\
\hline LVEDD & $62 \pm 9$ \\
\hline LVESD & $53 \pm 11$ \\
\hline IVS & $9 \pm 2$ \\
\hline PW & $9 \pm 1$ \\
\hline EA ratio & $1.2 \pm 0.6$ \\
\hline
\end{tabular}

Abbreviations: $A V=$ atrioventricular; $V T=$ ventricular tachycardia; $I C D=$ implantable cardiac defibrillator; $C R T$ - $D=$ cardiac resynchronization therapy device; $H F=$ heart failure; $L V E F=$ left ventricular ejection fraction; $L V E D D=$ left ventricular end-diastolic diameter; $L V E S D=$ left ventricular end-systolic diameter; IVS = interventricular septum; $P W=$ posterior wall thickness 
Table 2. Information of the top 20 selected genes after ranking all genes based on co-expression with DCM-associated genes

\begin{tabular}{|c|c|c|c|c|c|c|c|c|c|c|c|}
\hline \multirow[b]{2}{*}{ Gene } & \multicolumn{2}{|c|}{ Ranking } & \multirow{2}{*}{$\begin{array}{l}\text { Number } \\
\text { of } \\
\text { variants }\end{array}$} & \multirow[b]{2}{*}{$\begin{array}{c}\text { Type of } \\
\text { mutations }\end{array}$} & \multirow[b]{2}{*}{ Protein } & \multirow[b]{2}{*}{$\begin{array}{c}\text { Protein } \\
\text { location/function }\end{array}$} & \multicolumn{2}{|c|}{ Expression } & \multirow[b]{2}{*}{ Animal studies } & \multicolumn{2}{|c|}{ Gene tolerance } \\
\hline & Sarcomere & Z-disc & & & & & Heart & $\begin{array}{l}\text { Skeletal } \\
\text { muscle }\end{array}$ & & pLi & Z-score \\
\hline PDLIM5 & 25 & 9 & 5 & 2/5 LoF & $\begin{array}{l}\text { PDZ and LIM domain } \\
\text { protein } 5\end{array}$ & Z-disc & High & High & KO mouse: DCM & 0.01 & -0.47 \\
\hline MYOM1 & 16 & 37 & 8 & All missense & Myomesin-1 & Sarcomere & High & High & - & 0 & -0.35 \\
\hline CKM & 28 & 31 & 4 & 2/4 LoF & Creatine kinase, muscle & Cytoplasm & Medium & High & - & 0.01 & 0.95 \\
\hline CMYA5 & 30 & 23 & 14 & 1/14 LoF & $\begin{array}{c}\text { Cardiomyopathy } \\
\text { Associated protein } 5\end{array}$ & Anchor protein for PKA & Medium & High & - & 0 & -2.73 \\
\hline PDE4DIP & 32 & 33 & 27 & 1/27 LoF & $\begin{array}{l}\text { Phosphodiesterase 4D } \\
\text { Interacting Protein }\end{array}$ & $\begin{array}{c}\text { Anchor protein for } \\
\text { CAMP-dependent } \\
\text { pathway }\end{array}$ & Medium & High & - & - & - \\
\hline$T X L N B$ & 33 & 16 & 5 & All missense & Beta-taxilin & $\begin{array}{l}\text { Promotes motor nerve } \\
\text { regeneration }\end{array}$ & Medium & High & - & 0 & -0.91 \\
\hline$M Y L K 3$ & 43 & 92 & 5 & All missense & $\begin{array}{c}\text { Myosin light chain kinase } \\
3\end{array}$ & $\begin{array}{c}\text { Kinase that } \\
\text { phosphorolates MYL2 }\end{array}$ & High & Medium & KO mouse: DCM & 0.14 & -0.25 \\
\hline LMOD3 & 48 & 26 & 6 & All missense & Leiomodin-3 & Sarcomere & Low & Low & $\begin{array}{l}\text { Only for LMOD2: } \\
\text { DCM }\end{array}$ & 0.01 & -2.05 \\
\hline MYH11 & 72 & 190 & 7 & All missense & Myosin-11 & Sarcomere & Absent & Absent & $\begin{array}{l}\text { KO mouse: no cardiac } \\
\text { phenotype }\end{array}$ & 1 & 3.15 \\
\hline$X I R P 1$ & 73 & 89 & 10 & All missense & $\begin{array}{c}\text { Xin Actin Binding Repeat } \\
\text { Containing } 1\end{array}$ & Sarcomere & Low & Low & KO mouse: myopathy & 0 & -1.7 \\
\hline$D M P K$ & 75 & 131 & 6 & All missense & $\begin{array}{l}\text { Dystrophia myotonica } \\
\text { protein kinase }\end{array}$ & $\begin{array}{l}\text { Kinase necessary } \\
\text { maintaining muscle } \\
\text { structure }\end{array}$ & Medium & Medium & $\begin{array}{l}\text { KO mouse: decreased } \\
\text { contractility }\end{array}$ & 0.03 & 1.47 \\
\hline$A D C K 3$ & 84 & 74 & 4 & 1/4 LoF & $\begin{array}{l}\text { aarF domain containing } \\
\text { kinase } 3\end{array}$ & $\begin{array}{l}\text { Mitochondrial } \\
\text { respiratory chain }\end{array}$ & Low & High & - & 0 & -1.6 \\
\hline SORBS1 & 87 & 143 & 7 & All missense & $\begin{array}{l}\text { Sorbin and SH3 Domain } \\
\text { Containing } 1\end{array}$ & $\begin{array}{l}\text { Involved in cytoskeletal } \\
\text { formation }\end{array}$ & Low & Low & - & 0 & 0.3 \\
\hline$T N X B$ & 92 & 469 & 34 & 1/34 LoF & Tenascin XB & Extracellular matrix & Low & Low & - & 0.77 & 3 \\
\hline ALPK3 & 92 & 51 & 12 & All missense & Alpha kinase 3 & $\begin{array}{l}\text { Cardiomyocyte } \\
\text { differentiation }\end{array}$ & Medium & High & - & 0 & 0.31 \\
\hline OBSCN & 105 & 395 & 200 & 4/200 LoF & Obscurin & Z-disc & Medium & High & - & -1 & 0 \\
\hline
\end{tabular}




\begin{tabular}{|c|c|c|c|c|c|c|c|c|c|c|c|}
\hline SORBS2 & 109 & 795 & 4 & All missense & $\begin{array}{c}\text { Sorbin and SH3 Domain } \\
\text { Containing } 2\end{array}$ & $\begin{array}{l}\text { Involved in cytoskeletal } \\
\text { formation }\end{array}$ & Medium & Low & - & 0.09 & 0.38 \\
\hline SPEG & 111 & 355 & 22 & All missense & $\begin{array}{c}\text { Striated Muscle Enriched } \\
\text { Protein Kinase }\end{array}$ & $\begin{array}{c}\text { Myocyte cytoskeletal } \\
\text { development }\end{array}$ & Low & Medium & KO mouse: HF & 1 & 6 \\
\hline SYNM & 116 & 84 & 14 & All missense & Synemin & $\begin{array}{l}\text { Intermediate filament } \\
\text { within the cytoskelet }\end{array}$ & Low & Medium & KO mouse: HF & 0 & -0.15 \\
\hline$X I R P 2$ & 117 & 128 & 20 & 4/20 LoF & $\begin{array}{c}\text { Xin Actin Binding Repeat } \\
\text { Containing } 2\end{array}$ & Sarcomere & Low & High & - & 0 & -5 \\
\hline
\end{tabular}


Table 3. All class 3 variants in the top 5 DCM-candidate genes

\begin{tabular}{cccccccc} 
Gene & Nucleotide change & $\begin{array}{c}\text { Amino acid } \\
\text { change }\end{array}$ & PhyloP & CADD & $\begin{array}{c}\text { Grantham } \\
\text { Score }\end{array}$ & gnoMAD & Class* \\
\hline ALPK3 & c.4447C>T & p.Arg1483Trp & 0.104 & 18.9 & 101 & $7 / 246240$ & 3 \\
CMYA5 & c.8161A>T & p.Lys2721* & 2.513 & 49 & 1000 & $2 / 276328$ & 3 \\
CMYA5 & c.8885T>G & p.lle2962Ser & 6.939 & 19.61 & 142 & 0 & $2 / 3$ \\
PDLIM55 & c.189_190delinsGG & p.Leu64Glyfs*15 & 1.665 & 19.85 & 1000 & 0 & 3 \\
PDLIM5 & c.1333C>T & p.Arg445* & 1.874 & 40 & 1000 & $1 / 30906$ & 3 \\
SPEG & c.437G>C & p.Arg146Pro & 2.477 & 17.55 & 103 & 0 & 3 \\
SPEG & c.7442C>T & p.Ser2481Leu & 3.364 & 10.74 & 145 & $1 / 30942$ & $2 / 3$ \\
SPEG & c.7442C>T & p.Ser2481Leu & 3.364 & 10.74 & 145 & $1 / 30942$ & $2 / 3$ \\
SYNM & c.1929G $>$ A & p.Val643Ile & 4.667 & 24.9 & 0 & $8 / 277020$ & $2 / 3$ \\
SYNM & c.2838C>T & p.Arg946Trp & 1.89 & 19.32 & 0 & $19 / 266622$ & $2 / 3$ \\
\hline${ }^{*}$ Classification according to the $A C M G$ quidelines & & & & &
\end{tabular}

Whole genome sequencing revealed a homozygous chromosomal deletion of $10.16 \mathrm{~kb}$ at the start of PDLIM5 (Hg19: chr4:95,376,436 - chr4:95,386,599) which was heterozygous present in both healthy parents. The deletion starts in the second exon and includes nucleotides of the start codon for the heart-specific isoform (Figure 2). It leaves only the first 39 nucleotides of the exon intact and encompasses deletion of the remaining 259 nucleotides of the second exon, including the start codon. In-silico analysis predicts loss of the start codon of almost all isoforms. The effect of the homozygous mutation is most likely devastating for the PDLIM5 protein as both the start codon and the donor splice site of the second exon essential for the cardiac expression of the gene are deleted. This loss of PDLIM5 and earlyonset cardiac disease, is a first proof of principle for a connection between the gene and DCM.

\section{PDLIM5 variants in a DCM cohort}

The next step was to investigate the heterozygous PDLIM5 variants identified in the DCM cohort. In total, we detected 5 variants in PDLIM5: 1 frameshift, 1 nonsense, and 3 nonsynonymous missense variants (Suppl. Table 2). Both LoF variants were extremely rare in reference database, located in functional domains of the protein and predicted to have functional consequences, and therefore classified as class 3 . In contrast, the missense mutations were less rare in the gnomAD database.

The human PDLIM5 gene constitutes of 625 amino acids and is divided in 29 exons. The gene is highly complex as it undergoes extensive splicing; currently 23 splice variants have been described that have tissue-specific expression. Using the available expression data of the PDLIM5 isoforms in the GTEx portal, we could identify ENST00000508216.1 and ENST00000380180.3 as almost exclusively expressed in the heart and therefore representing the cardiac isoforms (Figure 2). After mapping of our variants to the cardiac isoforms, 1 LoF and 1 missense variant were in an exon included in the cardiac isoform (Figure 2). Since the other 3 found variants are absent or only very low expressed in the heart, they are probably not contributing to the cardiac phenotype. The clinical characteristics of both patients who carry a variant in the cardiac isoform can be found in Supplemental Table 3. 
A

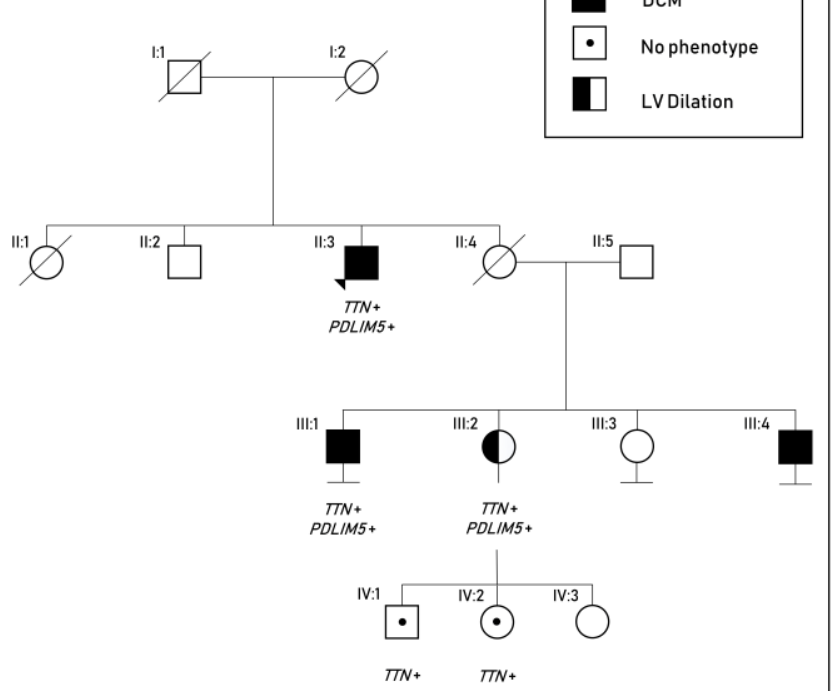

B

Patent ductus arteriosus, ventricular and atrial septal defect and cardi

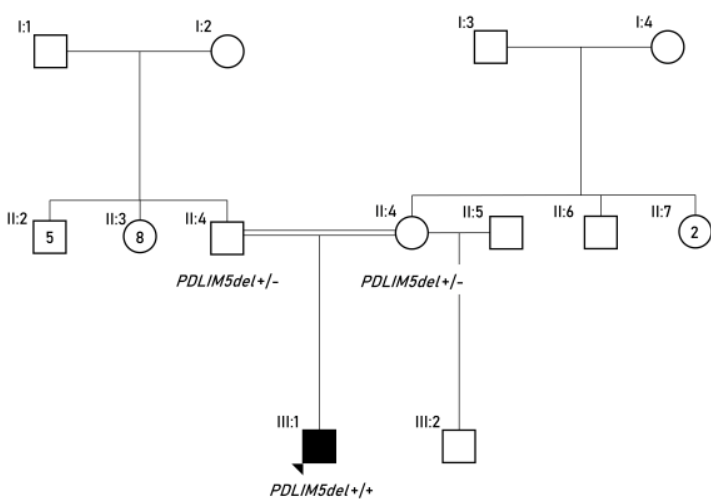

Figure 1. (A) Segregation of the PDLIM5 frameshift variant (p.Leu64Glyfs*15) in a DCM family with also a truncating TTN variant (p.Thr32477Asnfs*). The index patient (II:3; arrow) has DCM and carries both mutations, just like his cousin (III:1). His niece (III:2) also carries both mutations, but only has left ventricular dilation without a decreased ejection fraction. Both her children had no phenotype at cardiac screening but were only tested for the TTN variant. The other cousin (III:4) is known with DCM but did not consent for genetic analysis.

\section{Burden of PDLIM5 loss-of-function variants in a control cohort}

PDLIM5 has a pLi-score of 0.01 , suggesting its tolerance for LoF variants. However, the pLiscore does not make a distinction among different isoforms. We used available WES data of our control cohort of 26.000 individuals to extract a list of LoF variants in PDLIM5. We identified 12 truncating variants (10 unique variants; prevalence of $0.05 \%$ ) and mapped them along the gene. All the LoF variants identified were localized in the $3^{\prime}$ ending of the gene and not expressed in the cardiac isoforms near the PDZ-domain at the 5' ending (Figure 2). This highlights the scarcity of LoF mutations, and in particular the variants in cardiac isoforms we detected in our DCM cohort (2/142; prevalence of 1.4\%), depicting the frameshift in the PDZdomain of PDLIM5 (p.Leu64Glyfs*15).

\section{Clinical characteristics associated with a heterozygous frameshift PDLIM5 variant}

We focused on the family with the most interesting frameshift mutation (p.Leu64Glyfs*15), as the other LoF variant was not in the cardiac isoform and the missense variant in the cardiac isoform was too prevalent in gnomAD (Suppl. Table 2). The frameshift variant was found in a male patient of 67 years old who presented with acute heart failure (LVEF 10\%) and atrial fibrillation (AF). He rapidly normalized after initial medical treatment with standard heart failure therapy (LVEF 53\%). Diagnostic work-up revealed a pathogenic truncating TTN mutation (c.97427dup; p.Thr32477Asnfs*13) which segregated with the phenotype among the relatives (Figure 1A; Table 4). In all tested affected family members the PDLIM5 variant was detected (c.189_190delinsGG; p.Leu64Glyfs*15). 


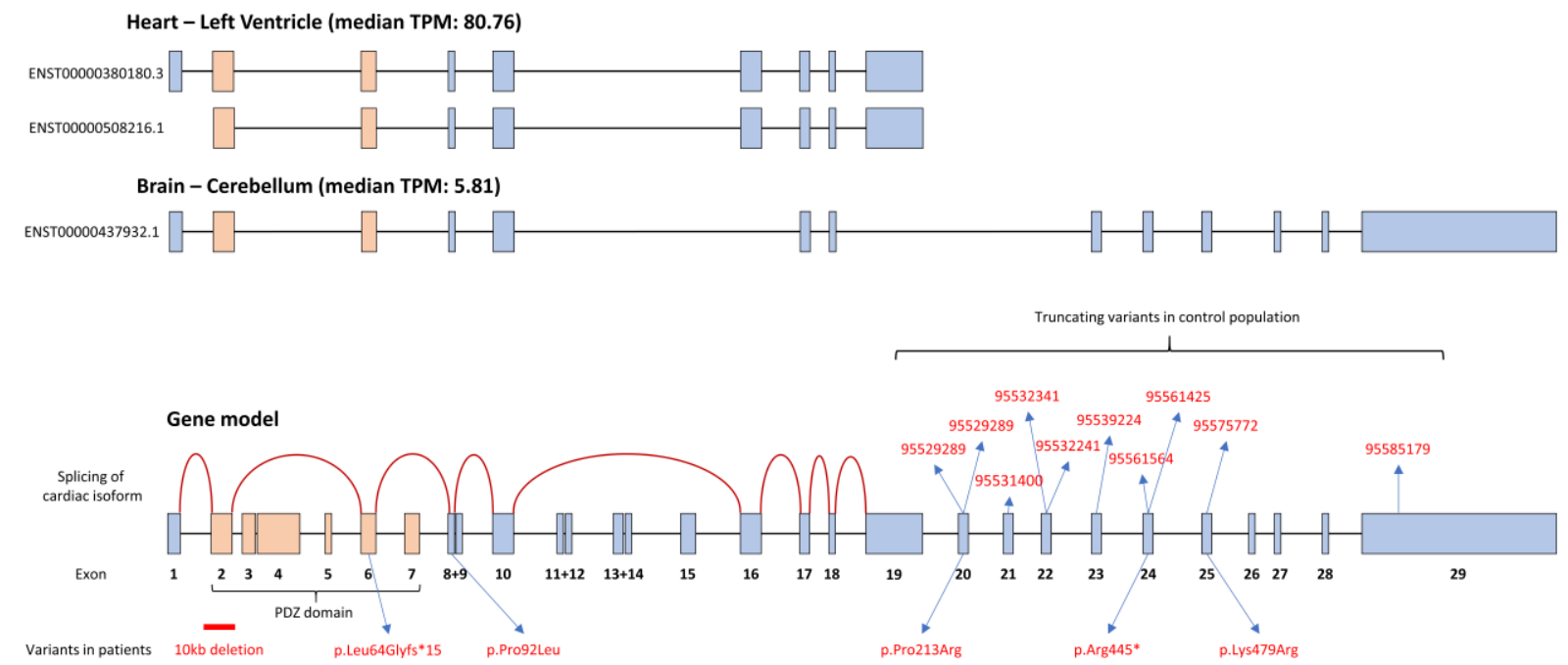

Figure 2. Cardiac isoforms of PDLIM5 in comparison with the longer isoform in the brain. Note the low expression of the transcript in the brain compared to the heart (TPM = transcripts per million). Exon skipping of the cardiac isoform is shown by the red line in the gene model. Position of truncating variants in the control population correspond to their g.position.

Table 4. Clinical features of affected patients with PDLIM5 mutations

\begin{tabular}{|c|c|c|c|c|c|c|}
\hline Individual & Sex & Origin & $\begin{array}{c}\text { Age at } \\
\text { Diagnosis }\end{array}$ & $\begin{array}{l}\text { Presenting } \\
\text { Symptoms }\end{array}$ & Outcome & Genotype \\
\hline A-II:3 & M & Dutch & 67 & Acute heart failure & $\begin{array}{c}\text { Normalized } \\
\text { LVEF }\end{array}$ & $\begin{array}{c}\text { Heterozygous TTN: } \\
\text { p.Thr32477Asnfs* + } \\
\text { heterozygous PDLIM5: } \\
\text { p.Leu64Glyfs*15 }\end{array}$ \\
\hline A-III:1 & M & Dutch & 26 & $\begin{array}{c}\text { Severe } \\
\text { biventricular DCM }\end{array}$ & $\begin{array}{c}\text { Normalized } \\
\text { LVEF }\end{array}$ & $\begin{array}{c}\text { Heterozygous TTN: } \\
\text { p.Thr32477Asnfs* + } \\
\text { heterozygous PDLIM5: } \\
\text { p.Leu64Glyfs*15 }\end{array}$ \\
\hline A-III:2 & $\mathrm{F}$ & Dutch & 48 & $\begin{array}{l}\text { LV dilatation at } \\
\text { echocardiography }\end{array}$ & $\begin{array}{c}\text { Stable } \\
\text { cardiac } \\
\text { function }\end{array}$ & $\begin{array}{c}\text { Heterozygous TTN: } \\
\text { p.Thr32477Asnfs* }+ \\
\text { heterozygous PDLIM5: } \\
\text { p.Leu64Glyfs*15 }\end{array}$ \\
\hline A-III:4 & $\mathrm{M}$ & Dutch & 26 & $\begin{array}{c}\text { Severe DCM with } \\
\text { NSVTs }\end{array}$ & $\begin{array}{l}\text { LVEF } 30 \% \text {, } \\
\text { multiple } \\
\text { appropriate } \\
\text { ICD shocks }\end{array}$ & NA \\
\hline B-III:1 & $M$ & $\begin{array}{c}\text { Native } \\
\text { American }\end{array}$ & At birth & $\begin{array}{l}\text { Patent ductus } \\
\text { arteriosus, } \\
\text { ventricular and } \\
\text { atrial septal defect } \\
\text { and cardiac } \\
\text { dilation }\end{array}$ & $\begin{array}{l}\text { Normalized } \\
\text { cardiac } \\
\text { dimensions }\end{array}$ & $\begin{array}{c}\text { Homozygous PDLIM5 } \\
\text { deletion of } 10.16 \mathrm{~kb}(\mathrm{Hg} 19 \\
\text { chr4:95,376,436- } \\
\text { chr4:95,386,599) }\end{array}$ \\
\hline
\end{tabular}


A

Association between PDLIM5 expression and LVEF

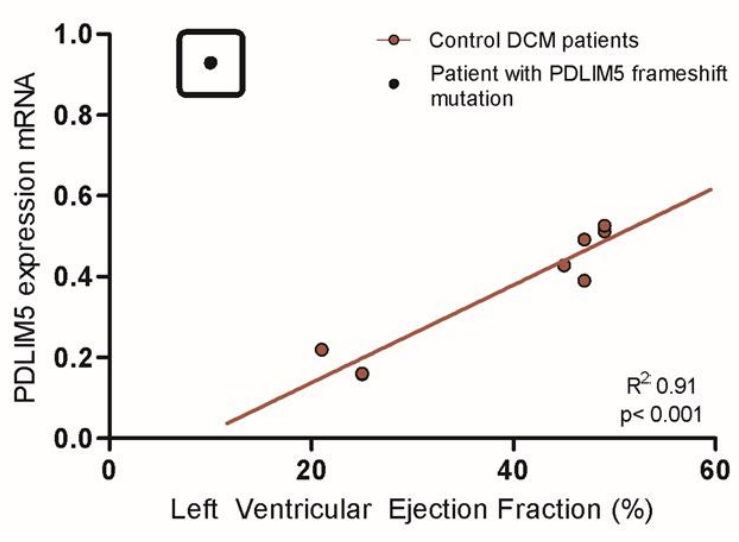

B

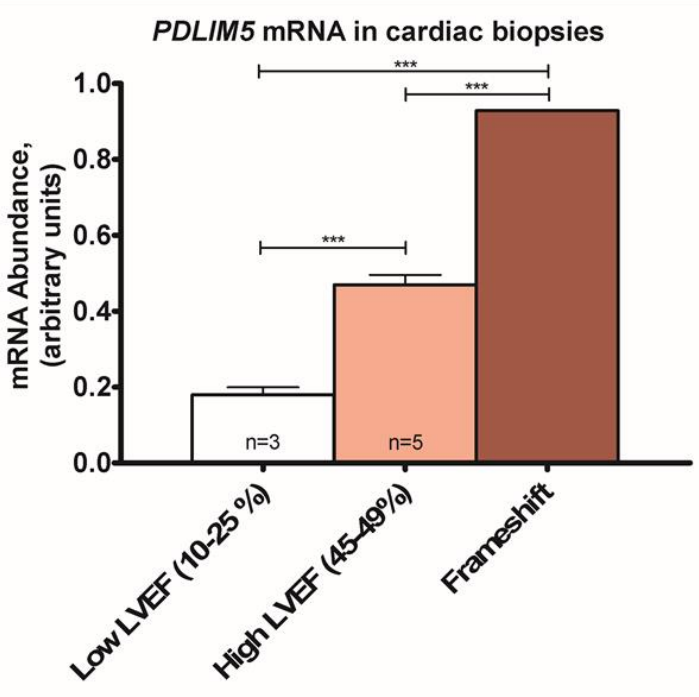

Figure 3. (A) Association between PDLIM5 expression in the heart and left ventricular ejection fraction (LVEF) in the patient with the PDLIM5 frameshift mutation and 8 control DCM patients. (B) Expression of PDLIM5 mRNA in the cardiac biopsy of the patient with the PDLIM5 frameshift mutation and two DCM control control groups with high and low left ventricular ejection fraction (LVEF) respectively.

\section{Cardiac PDLIM5 expression in DCM patients}

RNA was isolated from cardiac tissue of the patient with the PDLIM5 frameshift variant and 8 control DCM patients. The short isoform of PDLIM5 was detected in all cardiac tissues, in contrast to the longer isoforms which could not be detected. Instead, the longer isoform could be detected in cDNA of a mixed HEK/HeLa cell line, confirming tissue-specificity of the different isoforms of PDLIM5.

PDLIM5 expression is correlated to LVEF: a higher expression was observed in the patients with a higher LVEF ( $p<0.001, R^{2}$ : 0.91; Figure 3A). In contrast, PDLIM5 RNA was highly expressed in the heart of the patient with the PDLIM5 frameshift variants although he had a LVEF of $10 \%$ (Figure 3B). In line with previous research regarding a PDLIM5-knockout mouse developing $\mathrm{DCM}^{15}$, we investigated the most important interactors of PDLIM5 which showed transcriptional upregulation after loss of PDLIM5: LDB3 and MYOZ2 which form a complex with PDLIM5 at the Z-line, in close interaction with MYOT (Figure 4 and 5). All the PDLIM5interactors were also upregulated in the myocardium of our patient (Figure 4).

\section{DISCUSSION}

This study used an innovative method to discover new DCM-associated genes in WES data. We ranked variants in PDLIM5, CMYA5, SPEG, SYNM and ALPK3 as the most promising disease candidate genes using computational ranking based on co-expression followed by strict variant selection. The interesting complexity and tissue-specificity of PDLIM5 isoforms called for further investigation of this gene, although the other genes also require additional research. Based on the computational, literature and molecular indications we show altered RNA expression of PDLIM5 in a patient carrying a heterozygous frameshift mutation in the short cardiac isoform. Moreover, the patient also harbors a pathogenic TTN mutation that segregates with the disease in the family. All affected family members that were tested also harbored the PDLIM5 variant. Heterozygous mutations in PDLIM5 do not seem to play a major role in DCM, but it can be speculated that myocardial PDLIM5 dysfunction leads to increased 
susceptibility of the heart to develop DCM. The familial segregation of both TTN and PDLIM5 and high disease penetrance is suggestive for an additive effect of both mutations in the development of DCM. In addition, a neonatal cardiac patient carrying a homozygous PDLIM5 deletion strongly suggests the association between PDLIM5 dysfunction and cardiac failure.

\section{Interactors of PDLIM5, mRNA cardiac biopsies}

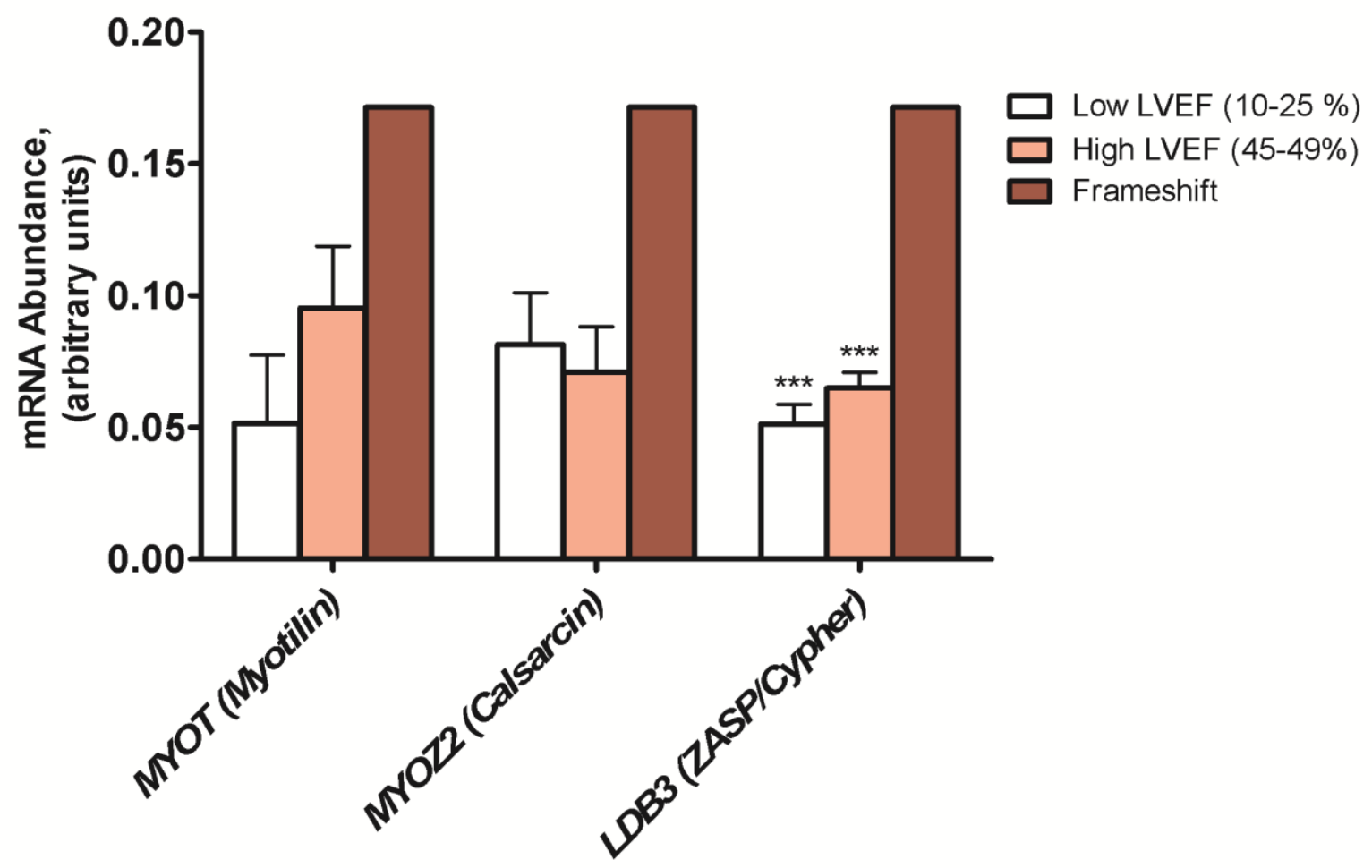

Figure 4. Expression of known interactors with PDLIM5 in the cardiac biopsy of the patient with the PDLIM5 frameshift mutation and two DCM control control groups with high and low left ventricular ejection fraction (LVEF) respectively.

\section{Genetic complexity of PDLIM5}

Four major isoforms of PDLIM5 have been identified: one long isoform (ENH1) expressed in all tissues, and three shorter isoforms (ENH2-4) that are mainly expressed in cardiac and skeletal tissues ${ }^{16}$. ENH1 appears to be the embryonic isoform in the heart, whereas ENH2-4 are found in the adult heart ${ }^{17}$. Subsequent research showed that PDLIM5 undergoes even more extensive splicing and that there are multiple isoforms in the mouse, and 23 transcripts in the human; although only 14 are shown to be protein coding ${ }^{15}{ }^{18}$. We showed that only the short isoform could be found in the human myocardium, and that the longer isoforms were undetectable. This strongly indicates tissue specificity of the short form of PDLIM5 in the heart. The heterozygous frameshift mutation we found in exon 6 is present in almost all isoforms of PDLIM5. Remarkably, the LoF mutations detected in a large control cohort were all located in exons not present in the cardiac isoforms of PDLIM5. In addition, the homozygous deletion started in exon 2 including the start codon which probably prevents the transcription of PDLIM5 including the cardiac isoform. Therefore, it is unlikely that variants occurring in exons not included in the cardiac isoform will have any effect on cardiac function. 


\section{Homology within the PDLIM protein family}

All PDLIM proteins can be divided into two subfamilies: the ALP and Enigma family ${ }^{19}$. PDLIM5 is located at the Z-line of the sarcomere, in a complex formed by the Enigma family ${ }^{20}$. All members of the Enigma family interact directly with $\alpha$-actinin-2, and constitute of PDLIM7 (also called LMP), LDB3 (also called ZASP; and PDLIM6 in the past), and PDLIM5 (also called $E N H)^{15}$. They contain a N-terminal PDZ domain and $3 \mathrm{C}$-terminal LIM-domains which are used for binding to $\alpha$-actinin and multiple signaling proteins. Variants in LDB3 are well-known and investigated as cause for dilated and hypertrophic cardiomyopathies in human and mice ${ }^{2122}$. Variants in PDLIM7 have not been described in humans. However, a PDLIM7 knockout mouse developed mild cardiac dysfunction mainly due to structural aberrations of the cardiac valves, despite the main tissue expression of human PDLIM7 is in the vascular and gastrointestinal system ${ }^{23}$. This contrasts with PDLIM5 and $L D B 3$ which are mainly expressed in the heart.

Next to the Enigma-family of PDLIM-proteins, the ALP-family constituting of PDLIM1 4, all contain $1 \mathrm{C}$-terminal LIM-domain ${ }^{19}$. Although all members of the Enigma-family have been linked to a cardiac phenotype in animal models, only a PDLIM3 knockout mouse is related to isolated right ventricular DCM ${ }^{24}$. Selective genotyping of PDLIM3 in 185 DCM patients yielded one suspicious variant in a 33-year old woman who developed DCM during her pregnancy ${ }^{25}$. Transfection experiments with the specific variant showed no exogeneous PDLIM3 expression. They speculated that myocardial PDLIM3 deficiency could have increased the susceptibility of the maladaptive responses to the hemodynamic and hormonal burdens of pregnancy. Variants in PDLIM3 do not seem to play a causal role in DCM, however, due to their multiple significant interactions at the Z-line, they may play a role in the disease pathogenesis by unknown mechanisms. In line with this observation, the same might be true for PDLIM5 in DCM, suggesting a contributing role for PDLIM-variants in the pathogenesis of DCM.

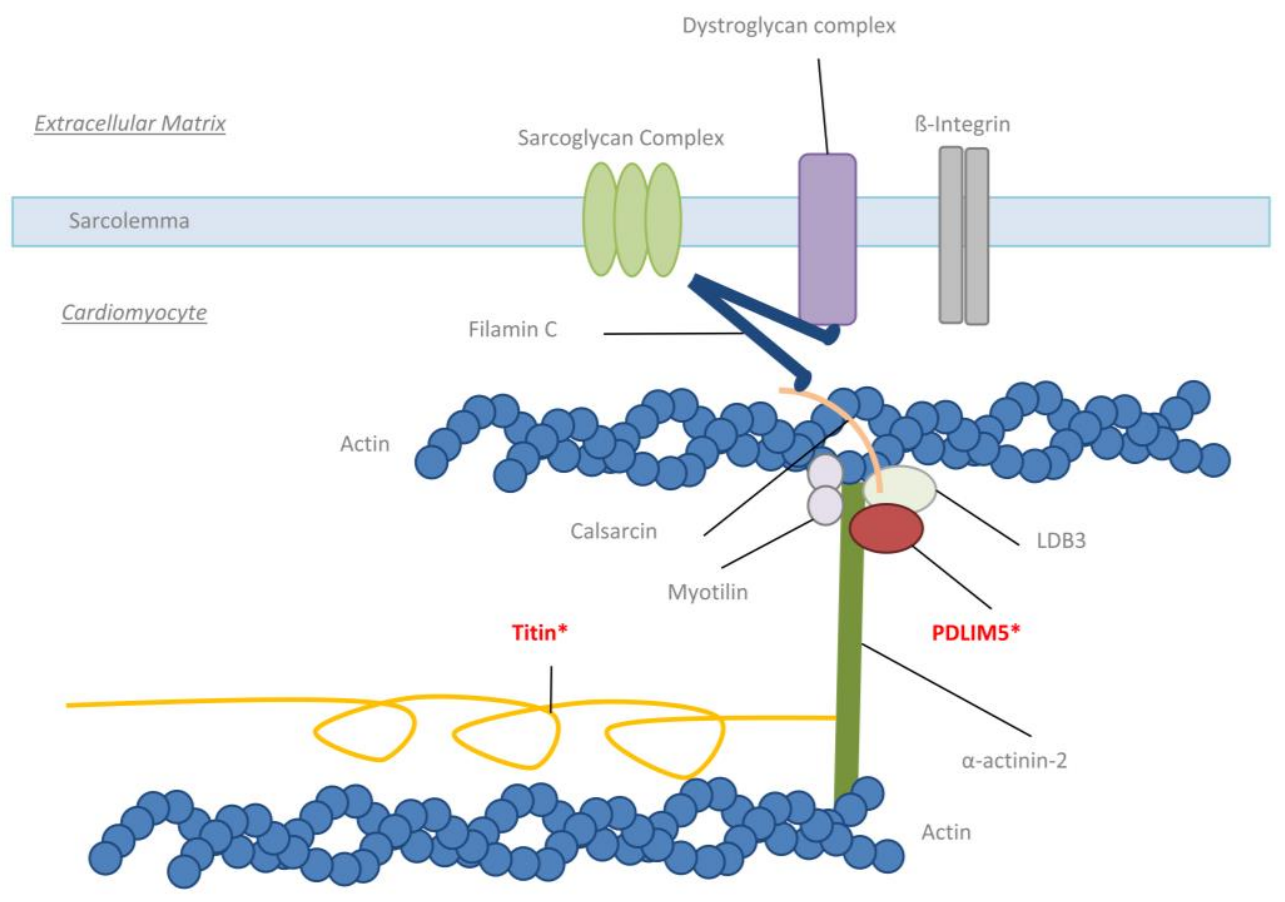

Figure 5. Schematic figure of the Z-line in the cardiomyocyte. PDLIM5 has a direct interaction with LDB3 and forms a complex together with calsarcin. They bind to $\alpha$-actinin-2 on one side and to the filamin $\mathrm{C}$-complex at the other side. The large protein titin also binds to $\alpha$-actinin-2. The patient described in our manuscript has a loss of function mutation in both TTN and PDLIM5 (as indicated by an asterisk). 


\section{PDLIM5 and its interactors in cardiac disease}

The finding of $\angle D B 3$ variants $D C M$ is a strong indication that additional members of the Enigma subfamily could be involved in DCM, corroborated by the presence of cardiac-specific isoforms of human PDLIM5. Indeed, a cardiac-specific ENH (PDLIM5) knockout mouse model developed DCM within 3 months with progressive deterioration ${ }^{15}$. Recently, it was shown that novel polymorphisms in PDLIM5 are associated with an increased risk for developing DCM in a Chinese Han population ${ }^{26}$. In addition, the congenital heart disease in an infant with a homozygous PDLIM5 deletion strengthens the association between PDLIM5 and cardiac (dys)function. We observed a correlation between LVEF and PDLIM5 expression, with a decrease in PDLIM5 expression with decreasing LVEF in patients without a PDLIM5 LoF variant. In contrast, our patient with a frameshift mutation had an increased expression of PDLIM5 despite of a low LVEF of $10 \%$. This could indicate a strong compensatory effect by the wild-type allele due to the frameshift mutation in PDLIM5, irrespective of the functional status of the cardiomyocyte. However, this finding needs to be validated and investigated in more detail.

It is important to realize the complexity of the Z-line which constitutes of many different protein complexes involved in the signal transduction, myocyte stability and force transmission ${ }^{20}$. Besides the Enigma family, myotilin (MYOT) and calsarcin (MYOZ2) also bind to $\alpha$-actinin-2, and a previous pull-down assay showed complex formation between PDLIM5, LDB3 and MYOZ2 ${ }^{15}$. The cardiac-specific knockout of PDLIM5 subsequently led to a significant decrease of MYOZ2 and LDB3 and a strong increase in MYOT protein expression over time, probably to compensate for the loss of the PDLIM5-containing complex. This was accompanied by an increase in the RNA expression of MYOZ2 and LDB3, suggesting a posttranslational mechanism for the decreased protein expression and not due to decreased mRNA synthesis. In our experiments, we also observed an increase of $\angle D B 3$, MYOZ2 and MYOT RNA expression in our patient, although we could not verify their expression at protein level. MYOZ2 has been shown to interact with filamin C (FLNC), which directly interacts with $\beta 1$ integrin, and is associated with the sarcoglycan and dystroglycan complexes at the sarcolemma ${ }^{27}$. In the knockout model, these filamin C-associated complexes were upregulated, suggesting a compensatory mechanism to strengthen the disruption of the Zline stability to the extracellular matrix ${ }^{15}$.

\section{Potential link between PDLIM5 and TTN}

Besides these complexes, titin (TTN) is also an important protein which binds directly to $\alpha$ actinin- ${ }^{10}$. The fact that both PDLIM5 and TTN bind to the same protein in the Z-line, makes our patient with a LoF mutation in both genes very interesting. Evidence is accumulating that truncating variants in TTN (TTNtv) are likely to be susceptibility variants needing a second hit to develop a pronounced phenotype ${ }^{10}$. In the general population, $\sim 0.4 \%$ carries a TTNtv with an estimated penetrance of $12 \%{ }^{10}$. This would mean that only a minority of the TTNtv carriers will develop a phenotype. Apparently, the heart can compensate adequately for the physiological consequences of TTN haploinsufficiency ${ }^{728}$. Multiple studies have shown an increased burden of TTNtv in populations with acquired causes for DCM, such as chemotherapy, pregnancy and alcohol ${ }^{29-31}$. It is likely that, besides acquired factors, also multiple genetic modifiers can influence the penetrance of TTNtv. PDLIM5 is a good candidate to be such modifier as it is physically involved in the Z-line in approximation of titin attached to $\alpha$-actinin-2. Two patients with a pronounced DCM and one with LV dilation in our family all carried the mutation in PDLIM5 and TTN. This high penetrance of disease could be due to the 
combination of both mutations, as disease penetrance in TTN-associated DCM is relatively low, however further investigations are necessary to better dissect this protein-protein interaction. The concept of mutations as susceptibility factor was elegantly shown in hypertrophic cardiomyopathy (HCM) patients with a MYL2 mutation ${ }^{32}$. Carriers of the mutation only developed HCM when hypertension was present, clearly showing a geneenvironment interaction for disease development.

\section{Limitations}

This study describes only two families; therefore, the results should be interpreted with caution. However, data from our families and the previous animal studies suggest that PDLIM5 is an interesting candidate to investigate further in relation to DCM. Functional studies are necessary to estimate any consequences related to the specific described heterozygous mutations, in absence and presence of any additional mutation in the Z-line proteins. As cardiac tissue obtained during life is quite limited, we did not have enough tissue from the patient with the frameshift mutation to study PDLIM5 at the protein level. Therefore, we were limited to the data from RNA expression. Also, as cardiac tissue is gathered during a diagnostic procedure, we don't have any available tissue from healthy control subjects for measuring RNA expression. Some individuals that tested mutationnegative in our study may have other monogenic causes that are not covered by the diagnostic 47 gene DCM panel. As an extension of this, in an ideal situation we would have the WES data of a DCM cohort constituting of familial disease without proven pathogenic mutation in the absence of an underlying, as this will increase the likelihood of identifying novel genetic variants. However, due to our extensive diagnostic trajectory we already identified a contributing etiology in most DCM patients. Although this does not necessary exclude a genetic predisposition.

\section{CONCLUSION}

Computational ranking based on co-expression is a valid tool to create a shortlist of interesting DCM-associated candidate genes. Our study suggests a role for PDLIM5 in DCM, although heterozygous mutations are not a major cause of DCM in contrast to a homozygous LoF mutation which leads to early onset cardiac disease. However, as PDLIM5 plays a major role in contracting cardiomyocytes, a heterozygous LoF is likely to play a role in disease development. Other environmental and/or genetic factors are probably necessary to unveil the cardiac phenotype in heterozygous PDLIM5 mutation carriers. 


\section{REFERENCES}

1. Japp AG, Gulati A, Cook SA, et al. The Diagnosis and Evaluation of Dilated Cardiomyopathy. J Am Coll Cardiol 2016;67(25):2996-3010.

2. Verdonschot Job AJ, Hazebroek Mark R, Ware James $S$, et al. Role of Targeted Therapy in Dilated Cardiomyopathy: The Challenging Road Toward a Personalized Approach. Journal of the American Heart Association 2019;8(11):e012514.

3. Hershberger RE, Hedges DJ, Morales A. Dilated cardiomyopathy: the complexity of a diverse genetic architecture. Nature reviews Cardiology 2013;10(9):531-47.

4. Roncarati R, Viviani Anselmi C, Krawitz P, et al. Doubly heterozygous LMNA and TTN mutations revealed by exome sequencing in a severe form of dilated cardiomyopathy. European journal of human genetics : EJHG 2013;21(10):1105-11.

5. Meder B, Ruhle F, Weis T, et al. A genome-wide association study identifies $6 \mathrm{p} 21$ as novel risk locus for dilated cardiomyopathy. Eur Heart $J$ 2014;35(16):1069-77.

6. Villard E, Perret C, Gary F, et al. A genome-wide association study identifies two loci associated with heart failure due to dilated cardiomyopathy. Eur Heart J 2011;32(9):106576.

7. Verdonschot JAJ, Hazebroek MR, Derks KWJ, et al. Titin cardiomyopathy leads to altered mitochondrial energetics, increased fibrosis and long-term life-threatening arrhythmias. Eur Heart J 2018

8. Pinto YM, Elliott PM, Arbustini E, et al. Proposal for a revised definition of dilated cardiomyopathy, hypokinetic non-dilated cardiomyopathy, and its implications for clinical practice: a position statement of the ESC working group on myocardial and pericardial diseases. Eur Heart J 2016;37(23):1850-8.

9. Richards S, Aziz N, Bale $S$, et al. Standards and guidelines for the interpretation of sequence variants: a joint consensus recommendation of the American College of Medical Genetics and Genomics and the Association for Molecular Pathology. Genetics in medicine : official journal of the American College of Medical Genetics 2015;17(5):405-24.

10. Ware JS, Cook SA. Role of titin in cardiomyopathy: from DNA variants to patient stratification. Nature reviews Cardiology 2018;15(4):241-52.

11. Sobreira N, Schiettecatte F, Valle $D$, et al. GeneMatcher: a matching tool for connecting investigators with an interest in the same gene. Hum Mutat 2015;36(10):928-30.
12. Lek M, Karczewski KJ, Minikel EV, et al. Analysis of protein-coding genetic variation in 60,706 humans. Nature 2016;536(7616):285-91.

13. Barrett T, Wilhite SE, Ledoux $P$, et al. NCBI GEO: archive for functional genomics data sets-update. Nucleic Acids Res 2013;41(Database issue):D991-5.

14. Szklarczyk R, Megchelenbrink W, Cizek P, et al. WeGET: predicting new genes for molecular systems by weighted co-expression. Nucleic Acids Res 2016;44(D1):D567-73.

15. Cheng $\mathrm{H}$, Kimura K, Peter AK, et al. Loss of enigma homolog protein results in dilated cardiomyopathy. Circulation research 2010;107(3):348-56.

16. Niederlander N, Fayein NA, Auffray $C$, et al. Characterization of a new human isoform of the enigma homolog family specifically expressed in skeletal muscle. Biochemical and biophysical research communications 2004;325(4):1304-11.

17. Yamazaki T, Walchli S, Fujita $T$, et al. Splice variants of enigma homolog, differentially expressed during heart development, promote or prevent hypertrophy. Cardiovasc Res 2010;86(3):374-82.

18. Consortium GT. The Genotype-Tissue Expression (GTEx) project. Nature genetics 2013;45(6):5805.

19. Zheng $\mathrm{M}$, Cheng $\mathrm{H}$, Banerjee $\mathrm{I}$, et al. ALP/Enigma PDZ-LIM domain proteins in the heart. $J \mathrm{Mol}$ Cell Biol 2010;2(2):96-102.

20. Sequeira V, Nijenkamp LL, Regan JA, et al. The physiological role of cardiac cytoskeleton and its alterations in heart failure. Biochimica et biophysica acta 2014;1838(2):700-22.

21. Zheng $\mathrm{M}$, Cheng $\mathrm{H}$, Li $\mathrm{X}$, et al. Cardiac-specific ablation of Cypher leads to a severe form of dilated cardiomyopathy with premature death. Human molecular genetics 2009;18(4):701-13.

22. Vatta $M$, Mohapatra B, Jimenez $S$, et al. Mutations in Cypher/ZASP in patients with dilated cardiomyopathy and left ventricular noncompaction. J Am Coll Cardiol 2003;42(11):201427.

23. Krcmery J, Gupta R, Sadleir RW, et al. Loss of the cytoskeletal protein Pdlim7 predisposes mice to heart defects and hemostatic dysfunction. PLoS One 2013;8(11):e80809.

24. Pashmforoush $\mathrm{M}$, Pomies $\mathrm{P}$, Peterson $\mathrm{KL}$, et al. Adult mice deficient in actinin-associated LIMdomain protein reveal a developmental pathway for right ventricular cardiomyopathy. Nat Med 2001;7(5):591-7.

25. Arola AM, Sanchez X, Murphy RT, et al. Mutations in PDLIM3 and MYOZ1 encoding myocyte $Z$ line proteins are infrequently found in 
idiopathic dilated cardiomyopathy. Mol Genet Metab 2007;90(4):435-40.

26. Wang D, Fang J, Lv J, et al. Novel polymorphisms in PDLIM3 and PDLIM5 gene encoding Z-line proteins increase risk of idiopathic dilated cardiomyopathy. Journal of cellular and molecular medicine 2019

27. Gontier $Y$, Taivainen A, Fontao L, et al. The Z-disc proteins myotilin and FATZ-1 interact with each other and are connected to the sarcolemma via muscle-specific filamins. J Cell Sci 2005;118(Pt 16):3739-49.

28. Schafer S, de Marvao A, Adami E, et al. Titintruncating variants affect heart function in disease cohorts and the general population. Nature genetics 2017;49(1):46-53 .

29. Linschoten $M$, Teske AJ, Baas AF, et al. Truncating Titin (TTN) Variants in Chemotherapy-Induced Cardiomyopathy. J Card Fail 2017;23(6):476-79.

30. Ware JS, Amor-Salamanca A, Tayal U, et al. Genetic Etiology for Alcohol-Induced Cardiac Toxicity. J Am Coll Cardiol 2018;71(20):2293-302.

31. Ware JS, Li J, Mazaika E, et al. Shared Genetic Predisposition in Peripartum and Dilated Cardiomyopathies. N Engl J Med 2016;374(3):233-41.

32. Claes GR, van Tienen FH, Lindsey $P$, et al. Hypertrophic remodelling in cardiac regulatory myosin light chain (MYL2) founder mutation carriers. Eur Heart J 2016;37(23):1815-22. 


\section{SUPPLEMENTARY TABLES}

Supplemental Table 1. Overview of all 47 genes used in the Maastricht Cardiomyopathy gene-panel.

\begin{tabular}{|c|c|c|c|}
\hline & HGNC.ID & HGNC.symbol & HGNC.Name \\
\hline 1 & HGNC:143 & ACTC1 & Actin, alpha, cardiac muscle 1 \\
\hline 2 & HGNC:164 & ACTN2 & Actinin alpha 2 \\
\hline 3 & HGNC:15819 & $A N K R D 1$ & Ankyrin repeat domain 1 \\
\hline 4 & HGNC:939 & $B A G 3$ & BCL2 associated athanogene 3 \\
\hline 5 & HGNC:20407 & CALR3 & Calreticulin 3 \\
\hline 6 & HGNC:1529 & CAV3 & Caveolin 3 \\
\hline 7 & HGNC:2389 & CRYAB & Crystallin alpha B \\
\hline 8 & HGNC:2472 & CSRP3 & Cysteine and glycine rich protein 3 \\
\hline 9 & HGNC:2511 & CTNNA3 & Catenin alpha 3 \\
\hline 10 & HGNC:2770 & $D E S$ & Desmin \\
\hline 11 & HGNC:3036 & DSC2 & Desmocollin 2 \\
\hline 12 & HGNC:3049 & DSG2 & Desmoglein 2 \\
\hline 13 & HGNC:3052 & $D S P$ & Desmoplakin \\
\hline 14 & HGNC:3331 & $E M D$ & Emerin \\
\hline 15 & HGNC:3702 & FHL1 & Four and a half LIM domains 1 \\
\hline 16 & HGNC:4296 & GLA & Galactosidase alpha \\
\hline 17 & HGNC:14202 & JPH2 & Junctophilin 2 \\
\hline 18 & HGNC:6207 & $J U P$ & Junction plakoglobin \\
\hline 19 & HGNC:6484 & LAMA4 & Laminin subunit alpha 4 \\
\hline 20 & HGNC:6501 & $L A M P 2$ & Lysosomal associated membrane protein 2 \\
\hline 21 & HGNC:15710 & LDB3 & LIM domain binding 3 \\
\hline 22 & HGNC:6636 & LMNA & Lamin A/C \\
\hline 23 & HGNC:21086 & MIB1 & Mindbomb E3 ubiquitin protein ligase 1 \\
\hline 24 & HGNC:7551 & MYBPC3 & Myosin binding protein $\mathrm{C}$, cardiac \\
\hline 25 & HGNC:7576 & MYH6 & Myosin heavy chain 6 \\
\hline 26 & HGNC:7577 & MYH7 & Myosin heavy chain 7 \\
\hline 27 & HGNC:7583 & MYL2 & Myosin light chain 2 \\
\hline 28 & HGNC:7584 & MYL3 & Myosin light chain 3 \\
\hline 29 & HGNC:1330 & MYOZ2 & Myozenin 2 \\
\hline 30 & HGNC:23246 & MYPN & Myopalladin \\
\hline 31 & HGNC:29557 & NEXN & Nexilin F-actin binding protein \\
\hline 32 & HGNC:9024 & PKP2 & Plakophilin 2 \\
\hline 33 & HGNC:9080 & PLN & Phospholamban \\
\hline 34 & HGNC:14000 & PRDM16 & PR/SET domain 16 \\
\hline 35 & HGNC:9386 & PRKAG2 & Protein kinase AMP-activated non-catalytic subunit gamma 2 \\
\hline 36 & HGNC:27424 & RBM20 & RNA binding motif protein 20 \\
\hline 37 & HGNC:10593 & SCN5A & Sodium voltage-gated channel alpha subunit 5 \\
\hline 38 & HGNC:11577 & $T A Z$ & Tafazzin \\
\hline 39 & HGNC:11610 & TCAP & Titin-cap \\
\hline 40 & HGNC:28472 & TMEM43 & Transmembrane protein 43 \\
\hline 41 & HGNC:11943 & TNNC1 & Troponin C1, slow skeletal and cardiac type \\
\hline 42 & HGNC:11947 & TNNI3 & Troponin 13, cardiac type \\
\hline 43 & HGNC:11949 & TNNT2 & Troponin T2, cardiac type \\
\hline
\end{tabular}




\begin{tabular}{llcc}
44 & HGNC:12010 & TPM1 & Tropomyosin 1 \\
45 & HGNC:12403 & TTN & Titin \\
46 & HGNC:12405 & TTR & Transthyretin \\
47 & HGNC:12665 & VCL & Vinculin \\
\hline
\end{tabular}

Supplemental Table 2. All class 2 and 3 variants found in PDLIM5

\begin{tabular}{cccccccccc} 
Nucleotide change & $\begin{array}{c}\text { Amino acid } \\
\text { change }\end{array}$ & PhyloP & CADD & $\begin{array}{c}\text { Grantham } \\
\text { Score }\end{array}$ & gnoMAD & Domain & Class* \\
\hline c.189_190delinsGG & p.Leu64Glyfs*15 & 1.665 & 19.85 & 1000 & 0 & $\begin{array}{c}\text { PDZ- } \\
\text { domain }\end{array}$ & 3 \\
c.275C>T & p.Pro92Leu & 2.813 & 12.55 & 98 & $33 / 277024$ & - & 2 \\
c.638C>G & p.Pro213Arg & -1.587 & 7.888 & 103 & $453 / 160532$ & - & 2 \\
c.1333C>T & p.Arg445* & 1.874 & 40 & 1000 & $1 / 30906$ & LIM zinc- & 3 \\
binding 1 & LIM zinc- & 2 \\
\hline
\end{tabular}

* Classification according to the ACMG guidelines

Supplemental Table 3. Clinical characteristics of the patients with a PDLIM5 variant in a cardiac isoform

\begin{tabular}{|c|c|c|}
\hline Family & 7098 & 17411 \\
\hline Individual & III:3 & III:4 \\
\hline Gender & Male & Male \\
\hline Age at diagnosis, yrs & 67 & 51 \\
\hline Variation & Frameshift & Missense \\
\hline Nucleotide change & c.189insGG & c. $275 \mathrm{C}>\mathrm{T}$ \\
\hline AA change & p.Leu64Glyfs*15 & p.Pro92Leu \\
\hline Secondary mutation & TTN: c.97427dup; p.Thr32477Asnfs*13 & TTR: c.148G>A; p.Val50Met \\
\hline NYHA functional class & II & III \\
\hline Symptoms & Dyspnea, palpitations, dizziness & Dyspnea \\
\hline Arrhythmias & $\mathrm{AF}$ & NSVTs, atrial flutter \\
\hline ECG & Normal & AVB1, iLBBB \\
\hline LVEDD, cm & 5.9 & 6.6 \\
\hline LVEF, \% & 10 & 20 \\
\hline $\mathrm{CK}, \mathrm{U} / \mathrm{I}$ & 59 & 186 \\
\hline Right Ventricle & Dilated and dysfunction & Dilated and mild dysfunction \\
\hline Endomyocardial Biopsy & $\begin{array}{l}\text { Slight hypertrophy and increased } \\
\text { interstitial fibrosis }\end{array}$ & Mild fibrosis and inflammation \\
\hline Cardiac MRI & $\begin{array}{c}\text { RV and LV apicolateral strong } \\
\text { trabecularisation. Midmyocardial, } \\
\text { midseptal fibrosis }\end{array}$ & $\begin{array}{c}\text { Basal-septal midmyocardial fibrosis. } \\
\text { Basal anterior and basal inferior- } \\
\text { inferolateral epicardial fibrosis }\end{array}$ \\
\hline Outcome & Normalised LVEF 53\% & LVEF 40\%; CRTD implantation \\
\hline Follow-up, yrs & 6 & 9 \\
\hline
\end{tabular}




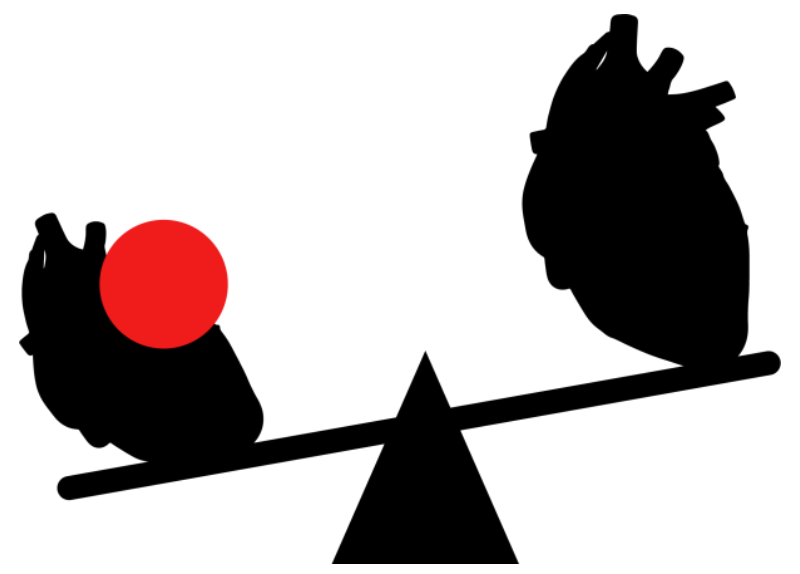

- 162 - 


\title{
CHAPTER 6
}

\section{Clinical phenotype and genotype associations with improvement in left ventricular function in dilated cardiomyopathy}

\author{
Job A.J. Verdonschot, $M D, M S c^{1,2}$ \\ Mark R. Hazebroek, MD, $P h D^{l}$ \\ Ping Wang, $P h D^{2}$ \\ Sandra Sanders - Van Wijk, MD, PhD \\ Jort J. Merken, $M D^{1}$ \\ Yvonne A. Adriaansen, $B S c^{2}$ \\ Arthurvan den Wijngaard, $P h D^{2}$ \\ Ingrid P.C. Krapels, $M D, P h D^{2}$ \\ Hans-Peter Brunner-La Rocca MD, PhD ${ }^{l}$ \\ Han G. Brunner, MD, $P h D^{2,3,4}$ \\ Stephane R.B. Heymans, MD, PhD $D^{1,5,6}$
}
${ }^{1}$ Department of Cardiology, Cardiovascular Research Institute (CARIM), Maastricht University Medical Center, Maastricht, The Netherlands.
${ }^{2}$ Department of Clinical Genetics, Maastricht University Medical Center, Maastricht, The Netherlands. ${ }^{3}$ Department of Human Genetics, and Donders Center for Neuroscience, Radboudumc, Nijmegen, The Netherlands.
${ }^{4}$ GROW Institute for Developmental Biology and Cancer, Maastricht University Medical Center, Maastricht, The Netherlands.
${ }^{5}$ Department of Cardiovascular Sciences, Centre for Molecular and Vascular Biology,
KU Leuven, Belgium.
${ }^{6}$ The Netherlands Heart Institute, Nl-HI, Utrecht, The Netherlands.




\begin{abstract}
Background: Improvement of left ventricular (LV) function (also called reverse remodeling (LVRR)) is an important treatment goal in patients with dilated and hypokinetic non-dilated cardiomyopathy (DCM/HNDC), and prognostically favorable. We tested whether genetic DCM mutations impact LVRR independent from clinical parameters.
\end{abstract}

Methods and results: DCM and HNDC patients ( $n=346$; mean LV ejection fraction (LVEF) $30 \%$ ) underwent genotyping for $47 \mathrm{DCM}$-associated genes in addition to extensive phenotyping. LVRR was defined as improvement of LVEF $>50 \%$ or $\geq 10 \%$ absolute increase, with cardiac dimensions (LV end-diastolic diameter) $\leq 33 \mathrm{~mm} / \mathrm{m}^{2}$ or $\geq 10 \%$ relative decrease. LVRR occurred in 180 (52\%) patients after a median follow-up of 12 months optimal medical treatment. Low baseline LVEF, a HNDC phenotype, high systolic blood pressure, absence of a family history of $D C M$, female gender, absence of AV block and treatment with beta-blockers were all independent positive clinical predictors of LVRR. With the exception of TTN, genetic mutations were strongly associated with a lower rate of LVRR (OR 0.19; [0.09-0.42]; $\mathrm{p}<0.0001$ ). TTN and LMNA were independently associated with LVRR (OR: 2.49 [1.09-6.20]; $p=0.038$ and 0.11 [0.01-0.99]; $p=0.049$ respectively). Adding mutation status significantly improved discrimination (C-statistics) and reclassification (IDI/NRI) of the clinical model predicting LVRR. Furthermore, the risk for HF hospitalization and cardiovascular death is lower in the LVRR patients on the long-term (HR 0.47 [0.24-0.91]; $p=0.009$ and 0.18 [0.040.82 ]; $p=0.007$ respectively) and LVRR is an independent predictor for event-free survival.

Conclusion: The genetic substrate is associated with the clinical course and long-term prognosis of DCM/HNDC patients. 


\section{INTRODUCTION}

Dilated cardiomyopathy (DCM) is an etiologically heterogeneous disease, characterized by systolic dysfunction and dilation of the left and/or right ventricle. Despite therapeutic improvements, the 5 -year mortality rate in DCM patients remains $20 \%{ }^{1}$. Reverse remodeling of the left ventricle (LVRR) is an important therapeutic goal, as absence of LVRR upon treatment is an early predictor of mortality ${ }^{2}$. Persistent systolic dysfunction often requires preventive device therapy such as an ICD implantation ${ }^{3}$. LVRR is characterized by a significant decrease in LV dimension and an increase in left ventricular ejection fraction (LVEF). Previous studies indicated that left bundle branch block (LBBB) and lower systolic blood pressure (SBP) are negative clinical predictors of LVRR in DCM ${ }^{2}$. Hypokinetic non-dilated cardiomyopathy (HNDC) was recently introduced as a new class of cardiomyopathies, suggested to be a preclinical state of DCM ${ }^{4}$. Knowledge regarding predictors of LVRR in HDNC are still lacking.

Genetic mutations are increasingly recognized as an important non-ischemic cause of DCM ${ }^{5}$. Over 60 causal cardiomyopathy genes were identified in the last decade, for a combined diagnostic yield of over $30 \%{ }^{5}$. However, this number seems to be a fair overestimation as new analytical approaches show that many implicated genes lack reliable validation ${ }^{6}$. Patients with genetic mutations are expected to respond less well to treatment as the cause is irreversible, compared to DCM patients with reversible causes such as viral infection, or inflammation ${ }^{7}$. However, within the genetic DCM group, treatability varies, since patients with TTN mutations respond better to therapy compared to patients with LMNA and Z-disk mutations ${ }^{8-10}$. Whether genetic profiles, add predictive information to clinical parameters for the prediction of LVRR is currently unknown. Previous studies focused solely on either genetic or clinical parameters, neglecting their potential interaction with medication. Knowing which patient may respond favorably to pharmacological treatment by LVRR may guide clinical decision-making, notably the timing of device therapy.

This study aimed to improve patient stratification by determining i) how phenotype (DCM; HNDC) influences the remodeling process, and ii) whether genotype in addition to clinical parameters improves the prediction of LVRR in a well-phenotyped cohort.

\section{METHODS}

\section{Study population}

A total of 346 patients with non-ischemic, non-valvular DCM who had genetic evaluation between 2012 and 2016 were included in this study from the Maastricht Cardiomyopathy Registry, with inclusion and exclusion criteria as described previously ${ }^{11}$. In short, both DCM or hypokinetic non-dilated cardiomyopathy (HNDC; also called isolated LV dysfunction) according to the latest ESC proposal ${ }^{4}$ were included (DCM defined as LVEF $<50 \%$ with an indexed left ventricular end diastolic diameter (LVEDDi) $>33 \mathrm{~mm} / \mathrm{m}^{2}$ (men) or $>32 \mathrm{~mm} / \mathrm{m}^{2}$ (women) measured by echocardiography; and HNDC defined as LVEF $<50 \%$ with an LVEDDi $\leq 33 \mathrm{~mm} / \mathrm{m}^{2}$ (men) or $\leq 32 \mathrm{~mm} / \mathrm{m}^{2}$ (women) measured by echocardiography) in the absence of a (i) history of myocardial infarction and/or significant coronary artery disease; (ii) primary valvular disease; (iii) hypertensive or congenital heart disease; (iv) acute myocarditis; (v) arrhythmogenic right ventricular dysplasia; (vi) hypertrophic, restrictive or peripartum cardiomyopathy.

All patients underwent a physical examination, blood sampling, 12-lead electrocardiogram, 24-h Holter monitoring, a complete echocardiographic and Doppler evaluation and coronary angiography (CAG) at baseline. Endomyocardial biopsies (EMB) $(n=292,84.4 \%)$ were performed upon discretion of the treating physician and consent of the 
patient. The main indication for EMB was a LVEF $<45 \%$ after 6 months of optimal medical treatment. Additional information regarding the EMB procedure is in the supplemental methods. The study was performed according to the declaration of Helsinki and was approved by the Medical Ethics Committee of Maastricht University Medical Centre. All patients gave written informed consent. The data that support the findings of this study are available from the corresponding author on reasonable request.

\section{Genetic evaluation}

Patients at the cardio-genetics outpatient clinic received genetic counseling and testing using our 47 cardiomyopathy gene panel either with whole exome sequencing (WES) or single molecule Molecular Inversion Probe (smMIP) (Suppl. Table 1). A family history of cardiacrelated disease and sudden cardiac death was obtained by pedigree analysis. Familial inheritance was defined as recommended by the ESC: (i) two or more individuals (first or second degree relatives) have DCM or HNDC fulfilling diagnostic criteria for 'definite' disease OR (ii) in the presence of an index patient fulfilling diagnostic criteria for DCM/HNDC and a first-degree relative with autopsy-proven DCM and sudden death at $<50$ years of age 4 .

Genetic variants were carefully and stringently classified in 5 different classes: pathogenic, likely pathogenic, variant of clinical unknown significance (VUS), likely benign or benign based on the criteria as proposed by the ACMG guideline ${ }^{12}$. Classification of variants was based on the score of in silico prediction software scores (SIFT, MutationTaster, PolyPhen-2, PhyloP, Align-GVGD), the frequency in reference population databases (gnomAD, 1000 genomes, ESP projects), functional studies and previously published variations in NCBI's ClinVar and HGMD. Both pathogenic and likely pathogenic mutations are reported here as pathogenic mutations. All others were considered non-pathogenic. Only truncating mutations with a PSI score $>99 \%$ in $T T N$ were classified as pathogenic ${ }^{13}$.

\section{Genotype-based classification and gene clusters}

To create coherent genotype-based classification for sub-analyses, patients sharing mutations in genes belonging to the same subcellular compartment were clustered together: (i) titin (TTN), (ii) lamin A/C (LMNA), (iii) structural cytoskeleton/Z disk, (iv) desmosomal, (v) motor sarcomeric and (vi) miscellaneous other gene mutations, as described ${ }^{10}$.

\section{LVRR definition}

Significant LVRR was defined as the combined presence at follow-up of: (i) an increase in LVEF of at least 10 points or a follow-up LVEF $\geq 50 \%$ with a minimal increase of $5 \%$; and (ii) a decrease in indexed left ventricular end-diastolic diameter of at least $10 \%$ or an indexed left ventricular end-diastolic diameter $\leq 33 \mathrm{~mm} / \mathrm{m}^{2}$ as described ${ }^{2}$.

\section{Follow-up}

Patients were systematically evaluated at the cardiology department for follow-up with echocardiography around 12 months after baseline (median 12, interquartile range 10 to 16 months) and received medical treatment according to the latest guidelines ${ }^{3}$.

Follow-up lasted for at least 9 months after their first visit to our specialized outpatient clinic, up to 13 years, with a median follow-up of 50 months (interquartile range 28 to 83 months). Follow-up data on (cardiac) death, heart transplantation (HTx), life-threatening ventricular arrhythmias (LTA), and unscheduled heart failure (HF) hospitalization, were collected using medical records, municipal population registers and/or telephone contact 
with general practitioners. The endpoint regarding the survival analysis was the combination of cardiac death, HTx, LTAs and HF hospitalization. LTA is defined as non-fatal ventricular fibrillation (with or without ICD-shock), hemodynamic unstable sustained ventricular tachycardia and/or sustained ventricular tachycardia with appropriate ICD-shock.

\section{Statistical analysis}

Data are presented as frequencies, mean \pm SD, median (interquartile range). Comparisons between groups were performed using chi-square tests (or Fisher exact test where necessary) for categorical data and 1-way analysis of variance or Kruskal-Wallis $\mathrm{H}$ test for continuous data, as appropriate. Analysis of continuous variables within patients at different time points was performed using paired Wilcoxon signed rank test. Two-way ANOVA type-III sums of squares analysis was used to test the significance of interactions (eg. Phenotype and $\triangle$ LVEF/LVEDDi).

Logistic regression analysis was performed to test the association between clinical and genetic parameters and LVRR. The univariate analysis was adjusted for time between baseline and follow-up echo and phenotype (DCM/HNDC). For multivariate analysis, we first included clinical variables that were associated with LVRR (cut-off for entry $p<0.2$ ) in the univariate analysis, together with time between echoes and phenotype. Then we performed predictor selection stepwise in both backward and forward directions guided by Akaike information criteria (AIC). The final clinical model only included predictors with $p<0.05$. In a second step, mutation status was tested individually with adjustment for the clinical model. Afterwards, the significant $(p<0.05)$ genetic clusters were added to the clinical model creating a genetic model.

Model accuracy, calibration, discrimination and reclassification were evaluated for the clinical (model 1) and the genetic model (model 2): 1) C-statistic, a measure of the area under the curve (AUC) and validation of AUC, sensitivity and specificity by 5 -fold-20-times cross validation, the comparison between models used t-test for cross-validation generated data points ( $n=100)$ and DeLong test for C-statistic; 2 ) the Hosmer-Lemeshow statistic, AIC, BIC, McFadden score, and the Brier score. The global goodness of fit of the models was evaluated by likelihood ratio tests; 3 ) integrated discrimination improvement (IDI) and (continuous) net reclassification index (NRI). Kaplan-Meier method was used to calculate survival curves and comparison between those curves was done by using log-rank test. Univariate Cox proportional-hazard regression analysis was performed in order to assess clinical and demographic covariates associated with event-free survival. Multivariate Cox proportionalhazards regression analysis was performed using all covariates associated with event-free survival. Variables were added in a stepwise fashion with $\mathrm{P}<0.1$ and $\mathrm{P}<0.05$ as the cut-off for entry or retention, respectively. The level of significance was $p<0.05$ and tests were 2-sided. Calculations were done using SPSS version 23.0 (SPSS Inc., Chicago, Illinois), and $\mathrm{R}$ environment version 3.4 (R Foundation for Statistical Computing, Vienna, Austria).

\section{RESULTS}

\section{Patient population}

Demographic, clinical and imaging baseline data are displayed in Table 1. Male gender predominated $(62 \%)$ and the mean age at diagnosis was $54 \pm 12$ years. The majority of the patients belonged to NYHA class I or II (69\%). Overall, mean LVEF was $30 \pm 11 \%$ and the LVEDDi $31 \pm 5 \mathrm{~mm} / \mathrm{m}^{2}, 54 \%(\mathrm{n}=188)$ were classified as HNDC patients. More than half of the HNDC 
patients showed LVRR ( $n=101,54 \%$ ), slightly higher compared to the DCM patients $(n=79$, $50 \%)$.

The influence of dilation at baseline on LVRR was further characterized by dividing the population on phenotype (DCM/HNDC) and outcome (LVRR+ or -). LVEDDi significantly decreased at follow-up in the LVRR+ patients, irrespective of the phenotype ( $p$-value $<0.0001$ in both groups; Figure 1A, Suppl. Table 2). In contrast, LVRR- HNDC patients did not show a decrease of LVEDDi ( $p$-value 0.15; Figure 1A, Suppl. Table 2). Also, the relation between the increase of LVEF and the decrease of LVEDDi is comparable between HNDC and DCM patients in respect to the strength (the slope of linear correlation), implying that both phenotypes followed the same pattern of remodeling (Figure 1B). This reflects the remodeling process including decrease of dilation in DCM as well as HNDC patients. Nonetheless, in contrast to the improvement of LVEF, the absolute decrease in LVEDDi significantly depends on phenotype as expected, showing the importance of adjusting for phenotype in further analysis (Suppl. Table 3).

\section{Clinical predictors for LVRR}

One hundred eighty patients (52\%) achieved LVRR after 12 months of treatment (Table 1). Due to the variance in the duration between the two echoes (IQR: 10-16 months), the analysis was corrected for duration between echoes, in addition to the phenotype (DCM/HNDC).

The final predictive model for LVRR of solely clinical parameters included high baseline LVEF and LVESD, a positive family history for DCM, the presence of an AV block and female gender to be associated with a lower odds for LVRR. In contrast, medical treatment with betablockers, high systolic blood pressure (SBP), longer duration between echoes and HNDC phenotype were positively associated with LVRR in multivariate logistic regression (Table 2; Suppl. Table 4 and Suppl. Figure 1). All parameters which are univariate tested can be found in Supplemental Table 4.

Table 2. Significant LVRR associated baseline clinical variables at univariate $(p<0.2)$ and multivariate logistic regression analysis $(p<0.05)$

\begin{tabular}{|c|c|c|c|c|c|c|}
\hline \multirow[b]{2}{*}{ Baseline variables } & \multicolumn{3}{|c|}{ Univariate analysis* } & \multicolumn{3}{|c|}{ Multivariate analysis } \\
\hline & OR & $95 \% \mathrm{Cl}$ & P Value & OR & $95 \% \mathrm{Cl}$ & P Value \\
\hline LVEF, per point \% increase & 0.93 & $0.91-0.95$ & $<0.0001$ & 0.90 & $0.87-0.93$ & $<0.0001$ \\
\hline Diuretics & 1.91 & $1.23-2.96$ & 0.004 & --- & --- & --- \\
\hline Family history of DCM & 0.46 & $0.27-0.79$ & 0.005 & 0.48 & $0.27-0.88$ & 0.017 \\
\hline MRA & 1.99 & $1.22-3.25$ & 0.006 & --- & --- & --- \\
\hline Beta-blocker & 3.02 & $1.34-6.83$ & 0.008 & 3.17 & $1.27-8.01$ & 0.015 \\
\hline ACE-i/ARB & 2.49 & $1.20-5.17$ & 0.014 & --- & --- & --- \\
\hline NYHA IV & 2.75 & $1.22-6.20$ & 0.015 & --- & --- & --- \\
\hline AV block & 0.42 & $0.20-0.90$ & 0.026 & 0.35 & $0.15-0.81$ & 0.015 \\
\hline LVESD, per $\mathrm{mm}$ increase & 1.03 & $1.00-1.06$ & 0.030 & 0.95 & $0.91-0.99$ & 0.009 \\
\hline Systemic disease & 0.40 & $0.17-0.94$ & 0.036 & --- & --- & --- \\
\hline Heart rate, per BPM increase & 1.01 & $1.00-1.02$ & 0.076 & --- & --- & --- \\
\hline Female gender & 0.68 & $0.43-1.06$ & 0.086 & 0.51 & $0.30-0.86$ & 0.011 \\
\hline Diabetes Mellitus & 0.61 & $0.32-1.17$ & 0.136 & --- & --- & --- \\
\hline Systolic BP, per $\mathrm{mmHg}$ increase & 1.01 & $1.00-1.02$ & 0.181 & 1.02 & $1.00-1.03$ & 0.009 \\
\hline Phenotype & & Fixed & & 2.16 & $1.16-4.01$ & 0.014 \\
\hline Duration between echoes, per mo & & Fixed & & 1.04 & $1.01-1.09$ & 0.036 \\
\hline
\end{tabular}


Table 1. Baseline characteristics of the total study population $(n=346)$

\begin{tabular}{|c|c|c|c|}
\hline Baseline variables & $\begin{array}{c}\text { LVRR + } \\
(n=180)\end{array}$ & $\begin{array}{l}\text { LVRR - } \\
(n=166)\end{array}$ & p-value \\
\hline Age of diagnosis (years) & $54 \pm 12$ & $54 \pm 12$ & 0.80 \\
\hline Family history of DCM (\%) & $28(16)$ & $46(28)$ & 0.006 \\
\hline Hypertension (\%) & 70 (39) & $62(37)$ & 0.77 \\
\hline $\mathrm{SBP}, \mathrm{mm} \mathrm{Hg}$ & $135 \pm 23$ & $131 \pm 20$ & 0.12 \\
\hline $\mathrm{DBP}, \mathrm{mm} \mathrm{Hg}$ & $82 \pm 15$ & $80 \pm 12$ & 0.16 \\
\hline Heart rate, BPM & $79 \pm 18$ & $73 \pm 15$ & 0.08 \\
\hline Chronic kidney disease (\%) & $14(8)$ & $16(10)$ & 0.54 \\
\hline Liver damage (\%) & $5(3)$ & $6(4)$ & 0.66 \\
\hline Diabetes Mellitus (\%) & $19(11)$ & $26(16)$ & 0.16 \\
\hline Systemic disease (\%) & $9(5)$ & $18(11)$ & 0.043 \\
\hline Cardiotoxic chemo (\%) & $8(4)$ & $8(5)$ & 0.87 \\
\hline NYHA III-IV (\%) & $63(35)$ & $43(26)$ & 0.07 \\
\hline Genetic Diagnostic Yield & $32(18)$ & $44(27)$ & 0.05 \\
\hline Titin $(T T N)$ & $23(13)$ & $10(6)$ & 0.033 \\
\hline Lamin $\mathrm{A} / \mathrm{C}(L M N A)$ & $1(1)$ & $8(5)$ & 0.016 \\
\hline Structural cytoskeleton Z-disc & $3(2)$ & $4(2)$ & 0.71 \\
\hline Desmosomal genes & $1(1)$ & $2(1)$ & 0.61 \\
\hline Motor Sarcomeric & $3(2)$ & $10(6)$ & 0.033 \\
\hline Miscellaneous genes & $1(1)$ & $10(6)$ & 0.004 \\
\hline \multicolumn{4}{|l|}{ Medication } \\
\hline Beta blocker (\%) & $171(95)$ & $144(87)$ & 0.007 \\
\hline ACE-I/ARB (\%) & $168(93)$ & $141(85)$ & 0.012 \\
\hline MRA (\%) & $63(35)$ & $26(16)$ & 0.024 \\
\hline Diuretics (\%) & $99(56)$ & $69(42)$ & 0.009 \\
\hline \multicolumn{4}{|l|}{ Baseline ECG/Holter } \\
\hline cLBBB (\%) & $47(26)$ & $47(28)$ & 0.65 \\
\hline AV block (\%) & $11(6)$ & $22(13)$ & 0.024 \\
\hline Atrial fibrillation (\%) & $46(26)$ & $36(22)$ & 0.40 \\
\hline NSVT (\%) & $40(22)$ & $48(29)$ & 0.15 \\
\hline \multicolumn{4}{|l|}{ Echocardiography } \\
\hline LVEF (\%) & $27 \pm 11$ & $32 \pm 10$ & $<0.0001$ \\
\hline $\mathrm{LA}(\mathrm{mm})$ & $45 \pm 9$ & $45 \pm 12$ & 0.68 \\
\hline LVESD (mm) & $52 \pm 11$ & $51 \pm 10$ & 0.40 \\
\hline LVEDDI $\left(\mathrm{mm} / \mathrm{m}^{2}\right)$ & $31 \pm 5$ & $32 \pm 5$ & 011 \\
\hline HNDC & $101(56)$ & $87(52)$ & 0.13 \\
\hline \multicolumn{4}{|l|}{ Endomyocardial Biopsy } \\
\hline Cardiac Inflammation (\%) & $51(33)$ & $41(30)$ & 0.70 \\
\hline $\mathrm{CD} 3+\left(\right.$ cells $/ \mathrm{mm}^{2}$ ) & $5.2[3.3-8.2]$ & $4.5[2-8.2]$ & 0.17 \\
\hline CD45+ (cells $\left./ \mathrm{mm}^{2}\right)$ & $8.9[6.1-12.7]$ & $7.9[4.5-13.1]$ & 0.26 \\
\hline CD68+ (cells $\left./ \mathrm{mm}^{2}\right)$ & $2.8[1.5-4.8]$ & $2.3[1.1-4.9]$ & 0.38 \\
\hline Myocardial Fibrosis & $7.3[3.8-11.4]$ & $6.2[3.1-12.4]$ & 0.34 \\
\hline \multicolumn{4}{|l|}{ Significant viral load } \\
\hline PVB19 (\%) & $25(16)$ & $21(16)$ & 0.93 \\
\hline HHV6 (\%) & $1(1)$ & $1(1)$ & 0.99 \\
\hline
\end{tabular}

Values are mean $\pm S D, n, n(\%)$, or median (interquartile range).

Abbreviations, ACE-I/ARB, angiotensin converting enzyme-inhibitor/angiotensin II receptor blocker; MRA. Mineralocorticoid receptor antagonist; $C L B B B$, complete left bundle branch block; AV-block, atrioventricular block; NSVT, non-sustained ventricular tachycardia; $L V E F$, left ventricular ejection fraction; LVEDD, left ventricular end-diastolic diameter; LVESD, left ventricular end-systolic diameter; LVEDDI, indexed LVEDD; PVB19, parvovirus B19; HHV6, human herpesvirus 6. 
A

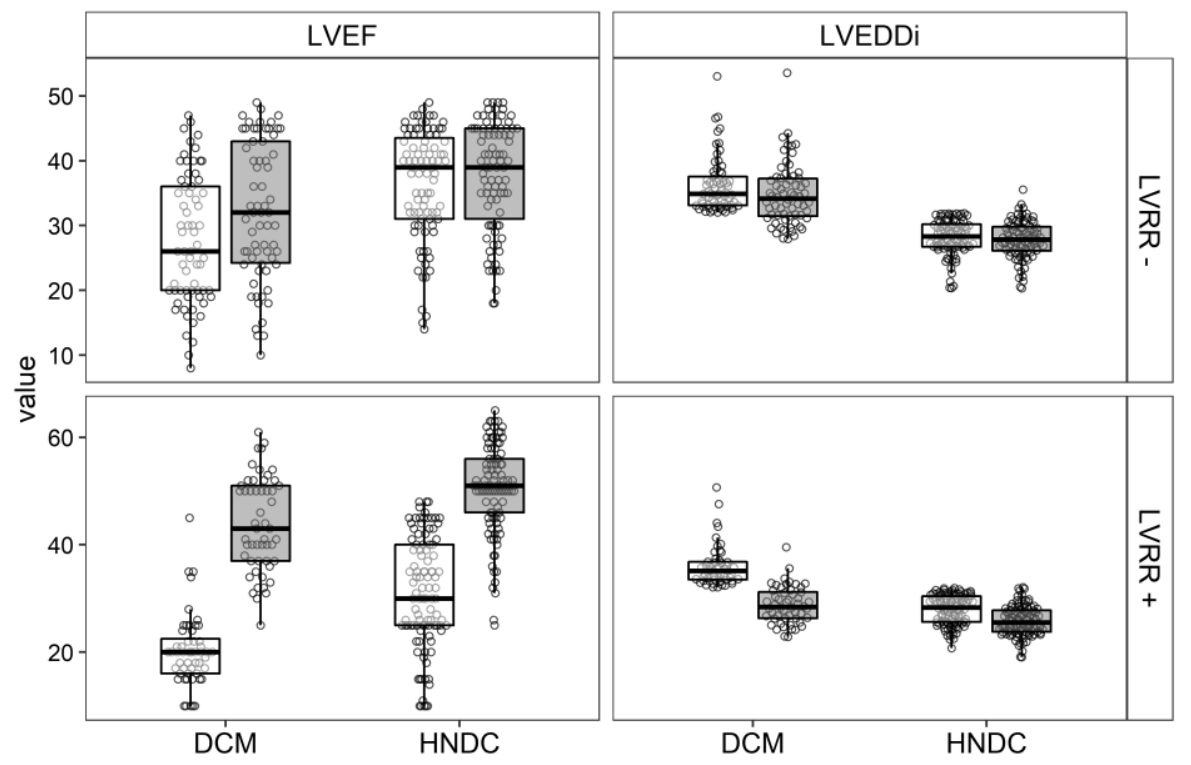

审 baseline 审 follow-up

B

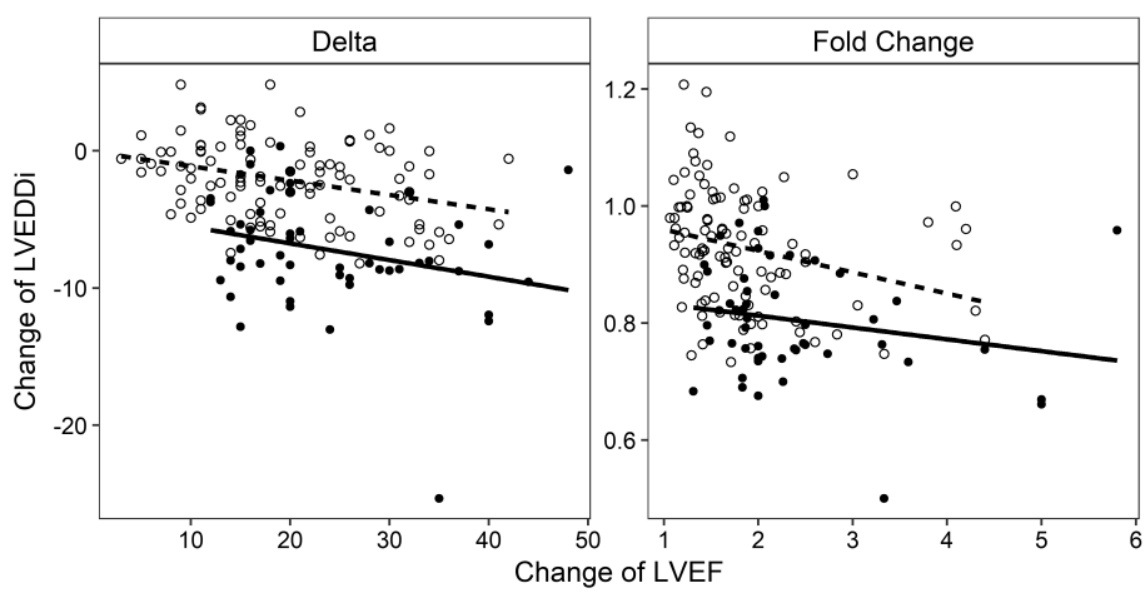

$\rightarrow$ DCM $\rightarrow$ HNDC

Figure 1. Changes of LVEF and LVEDDi show the similar LVRR alterations in DCM and HNDC patients

Change of LVEF and LVEDDi from baseline to follow-up in DCM and HNDC patients who showed or did not show LVRR (A). The relation between the change of LVEF and the change in LVEDDi, in absolute delta $(-0.10$ and -0.12 change of LVEDDi with each point of LVEF increase for HNDC and DCM, respectively; $p$-value $=0.79$ ) or fold change (-0.036 and -0.02 change of LVEDDi with each point of LVEF increase for HNDC and DCM, respectively; $p$ value $=0.41$ ), showed similar slope in DCM and HNDC patients with LVRR (B).

\section{Mutation status and LVRR}

The genetic diagnostic yield for pathogenic gene mutations in this population was $22 \%$ ( $n=78$ unrelated patients) with the majority of mutations found in $\operatorname{TTN}(n=33,9.5 \%)$, followed by LMNA ( $n=9,2.6 \%$; Suppl. Table 5 and 6 ). None of the included patients had multiple pathogenic variants. Overall, patients who don't favorably remodel are more likely to have variants in genes such as LMNA and those who do are more likely to have a TTN mutation. (Suppl. Figure 2). In univariate analysis, TTN mutations were associated with a significant 
higher rate of LVRR (Figure 2; Suppl. Table 7). Overall, the genetic substrate is associated with the clinical course of a DCM patient (Figure 2; Suppl. Table 7).

\section{Value of adding mutation status to clinical variables}

A genetic mutation in TTN is the strongest positive predictor for LVRR after adjustment for the clinical model (Figure 2; Suppl. Table 7). Mutations in LMNA and miscellaneous gene mutations remained negative predictors for LVRR. However, only TTN and LMNA gene mutations remained independent predictors for LVRR when added to the initial clinical predictive model, showing an enrichment of TTN mutations in patients who favorably remodel and $L M N A$ mutations in patients with more severe disease (Table 3 ).

The performance metrics of the clinical predictive model without (model 1 ) versus with (model 2) mutations are summarized in Table 4. In terms of discrimination - indicating the model's ability to correctly distinguish 2 classes of outcome (LVRR+/LVRR-) - mutation status significantly improved the $\mathrm{C}$-statistic $(\mathrm{p}=0.03)$ and was validated by cross-validation. In terms of calibration - depicting the goodness-of-fit for a model (predicted and observed values agree for any reasonable grouping of the observation) - both models performed well but most calibration metrics showed a significant improvement of goodness-of-fit of the model after including mutation status. In terms of reclassification - analyzing how well a new predictive model correctly classifies outcome of patients compared to the older model- the model including mutation status did significantly better compare to the model with only clinical parameters (IDI 3.87\%; $p=0.018$; continuous NRI 27\%; $p<0.001$ ), indicating better patient stratification when mutation status is taken into account.

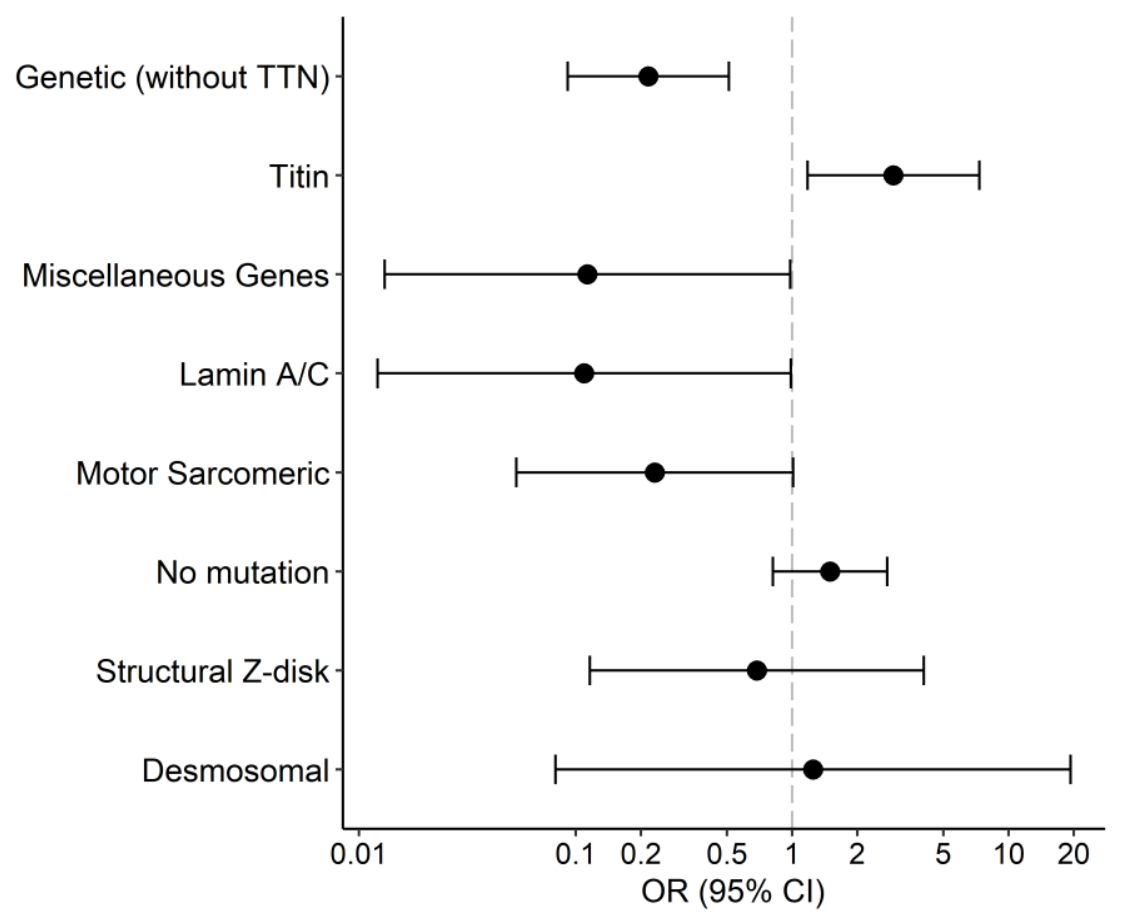

Figure 2. Association between mutation status and left ventricular reverse remodelling adjusted for the clinical prediction model.

Lines depict odss ratio (OR) and 95\% confidence interval for the association with LVRR after adjustment for the clinical prediction model. A higher OR reflect a higher chance for achieving LVRR. 


\section{Long-term follow-up in LVRR}

Long-term prognosis was better in patients with LVRR ( $p=0.027$; Figure 3; Suppl. Table 8). In particular, heart failure re-hospitalization (HR 0.47; confidence interval $0.24-0.91 ; p=0.026$ ) and cardiovascular death (HR 0.18; confidence interval $0.04-0.82 ; p=0.026$ ) was lower in patients with LVRR.

Table 3. The final model of baseline predictors of LVRR including mutation status

\begin{tabular}{cccc}
\hline Baseline variables & OR & $\mathbf{9 5 \%}$ Cl & P Value \\
\hline LVEF, per point \% increase & 0.90 & $0.87-0.94$ & $<0.0001$ \\
HNDC phenotype & 2.32 & $1.23-4.38$ & 0.009 \\
Systolic BP, per mmHg increase & 1.02 & $1.01-1.03$ & 0.009 \\
LVESD, per mm increase & 0.95 & $0.91-0.99$ & 0.018 \\
Female gender & 0.48 & $0.28-0.83$ & 0.008 \\
Family history of DCM & 0.47 & $0.25-0.83$ & 0.025 \\
AV block & 0.33 & $0.14-0.78$ & 0.012 \\
Beta-blocker & 3.07 & $1.17-8.09$ & 0.022 \\
Duration between echoes, per month & 1.04 & $1.00-1.08$ & 0.049 \\
Titin (TTN) & 2.49 & $1.09-6.20$ & 0.038 \\
Lamin A/C (LMNA) & 0.11 & $0.01-0.99$ & 0.049 \\
\hline
\end{tabular}

Abbreviations, LVEF, left ventricular ejection fraction; HNDC, hypokinetic nondilated cardiomyopathy; BP, blood pressure; LVESD, left ventricular end-systolic diameter; DCM, dilated cardiomyopathy; AV block, atrioventricular block.

Table 4. Comparison of the model performance metrics between the clinical and combined predictive model for LVRR

\begin{tabular}{|c|c|c|}
\hline & $\begin{array}{l}\text { Model } 1 \\
\text { (Clinical) }\end{array}$ & $\begin{array}{c}\text { Model } 2 \\
\text { (Combined clinical and genetics) }\end{array}$ \\
\hline \multicolumn{3}{|l|}{ Discrimination } \\
\hline \multirow{2}{*}{ C-statistic } & 0.767 & 0.789 \\
\hline & Reference & $p=0.03$ \\
\hline \multicolumn{3}{|l|}{ Cross-validation (mean $\pm S D$ ) } \\
\hline \multirow[t]{2}{*}{ AUC } & $0.742 \pm 0.05$ & $0.760 \pm 0.05$ \\
\hline & Reference & $p=0.008$ \\
\hline \multirow[t]{2}{*}{ Sensitivity } & $0.712 \pm 0.07$ & $0.738 \pm 0.06$ \\
\hline & Reference & $p=0.004$ \\
\hline \multirow[t]{2}{*}{ Specificity } & $0.653 \pm 0.08$ & $0.653 \pm 0.07$ \\
\hline & Reference & $p=0.951$ \\
\hline \multicolumn{3}{|l|}{ Calibration } \\
\hline Hosmer-Lemeshow test & $p=0.129$ & $p=0.387$ \\
\hline Brier score & 0.196 & 0.186 \\
\hline Pseudo $\mathrm{R}^{2}$ (McFadden) & 0.167 & 0.201 \\
\hline AIC & 419.06 & 408.78 \\
\hline $\mathrm{BIC}$ & 457.52 & 455.79 \\
\hline Likelihood ratio & Reference & $p=0.001$ \\
\hline \multicolumn{3}{|l|}{ Reclassification } \\
\hline \multirow[t]{2}{*}{ IDI (\%) } & Reference & $3.87 \%$ \\
\hline & & $p=0.018$ \\
\hline \multirow[t]{2}{*}{ NRI - all (\%) } & Reference & $27 \%$ \\
\hline & & $p<0.001$ \\
\hline
\end{tabular}

Abbreviations, SD, standard deviation; AUC, area under the curve; AIC, Akaike information criteria; BIC, Bayesian information criterion; IDI, integrated discrimination improvement; NRI, net reclassification index. 
In the univariate analysis, absence of LVRR, high NYHA-score, a positive family history of DCM, non-sustained ventricular tachycardia (NSVT) and LMNA mutations were significant predictors of a worse prognosis (Suppl. Table 9). In the multivariate model, LVRR, NSVT, LMNA mutations and baseline EF remained independent predictors for long-term outcome (Table 5). Thus, LVRR remained a strong predictor of long-term outcome, independently from other clinical and genetic parameters (HR 0.56; confidence interval $0.33-0.95 ; p=0.033$ ).

\section{DISCUSSION}

This is the largest study combining clinical, genetic and medication parameters for the prediction of LVRR in subjects with DCM and the first in subjects with HNDC. LVRR occurs in approximately half of our mixed patient population receiving tailored medical therapy and is predictive for long-term outcome. We find that in addition to the clinical phenotype, genetic mutation status is an independent predictor of LVRR, and improves patient stratification. This reflects the need for alternative treatment strategies for genetic cardiomyopathies.

\section{Left ventricular reverse remodeling in HNDC and DCM}

About half of the patients in this study achieved LVRR after 12-months follow-up on conventional HF medication, which is slightly higher compared to earlier reports stating that LVRR occurred in 37 to $46 \%$ of patients after 24 months ${ }^{2}{ }^{14}$. Our heterogeneous patient population with multiple (reversible) etiologies could have influenced the high rate of LVRR. Also, the mixed population of DCM/HNDC probably may have increased the percentage of patients who remodeled after 12 months. HNDC had the same remodeling signature as DCM patients (increase in EF and decrease in LVEDD), but the absolute decrease in cardiac diastolic dimensions was less when HNDC patients reached LVRR. The HNDC phenotype was an

Death, cardiac transplant, heart failure hospitalisation or life-threatening arrhythmia

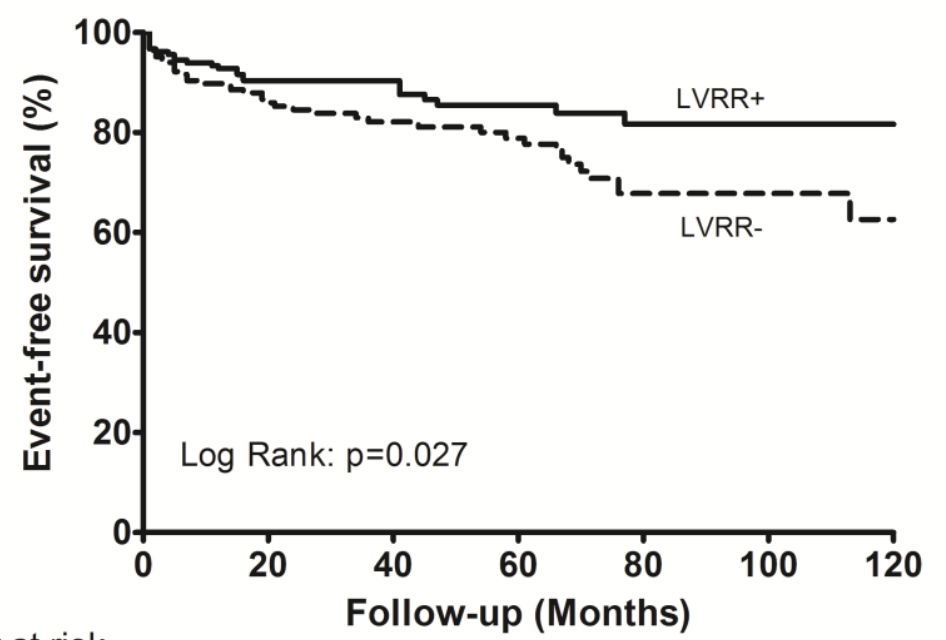

Number at risk

$\begin{array}{llllllll}\text { LVRR+: } & 180 & 143 & 97 & 57 & 33 & 17 & 8 \\ \text { LVRR-: } & 166 & 127 & 86 & 66 & 41 & 18 & 9\end{array}$

Figure 3. Long-term outcome of LVRR in non-ischemic DCM patients ( $n=346)$.

Comparison of long-term event-free survival (cardiovascular death, Htx, HF hospitalization and LTA) between LVRR and non-LVRR patients. 
independent predictor for LVRR, probably as they have no dilation at baseline. Taken together these data provide further support that HNDC represent an earlier stage of DCM.

In line with previous literature, LVRR is associated with a better event-free survival in DCM patients ${ }^{2}$. Especially, the risk for HF hospitalization and cardiovascular death is lower in LVRR patients. LVRR remained an independent predictor for event-free survival after adjustment for clinical and genetic parameters.

\section{Genetic mutations predict LVRR in DCM and HNDC}

Genetic mutations are important predictors for LVRR, when added to accepted clinical parameters. This information may enhance tailoring therapy towards a personalized approach. One previous study similarly revealed less LVRR in patients with a rare variant in structural cytoskeleton Z-disk genes (ie. DES, FLNC, NEXN, MYPN) ${ }^{10}$. This study focused on genetics only, without correcting for clinical phenotyping. Further, the very high diagnostic yield in this study (57\%) contrasts with the lower genetic yield in the current study and that of others ${ }^{15}$ (22 and $30 \%$ resp.), possibly reflecting differences in composition of the cohort, molecular testing strategies or a combination of these. We note that in the Italian study, the malignant FLNC predominated, whereas our group of patients with a Z-disk mutation was small and had a different gene constitution, as FLNC is not in our panel ${ }^{10,16 .}$

A family history of DCM was an independent predictor of a lower rate of LVRR in our study. Familial DCM is likely to reflect a genetic predisposition, although a family history of DCM was not associated with a detectable mutation in our cohort. Indeed, it is possible that novel DCM genes not present in our current cardiomyopathy panel of 47 genes, await discovery. Our study is the first to show TTN as an independent predictor of LVRR after taking clinical parameters into account. In fact, mutation in TTN was the strongest independent positive predictor for LVRR, therefore patients may be more likely to respond to standard HF medical management, in line with previous studies ${ }^{917}{ }^{18}$. It has been suggested, that the TTNmutated heart has the ability to adapt to the heterozygous loss of TTN, but will decompensate upon additional hemodynamical, toxic or hormonal stress to the heart muscle ${ }^{131819}{ }^{1}$. This is consistent with the high incidence of TTN mutations in peripartum cardiomyopathy patients, suggesting a disease-modifying role for TTN mutations where overt disease is triggered by a second inciting hit ${ }^{20}$. Mechanical unloading with device therapy leads to recovery in patients with truncating TTN mutations ${ }^{821}$. The high potential for remodeling in our study reflects improved recovery after unloading the heart, further underscoring the stress-dependent phenotype of titinopathies. Besides that titin is a key molecule of the sarcomere, it contains also important signaling properties, involved in stress signaling ${ }^{1322}$. Metabolic and energetic compensation have shown to be an important adaptation to cope with the signaling failure and sarcomere insufficiency resulted from a TTN mutation ${ }^{131823}$. It seems likely that the heart is inflexible to further compensate when exposed to additional hemodynamic stress. When the stressor is removed the adaptation mechanisms are able to restore the balance in the heart. However, other times TTN mutations are truly pathogenic and can have severe outcomes, showing the complexity of unraveling the pathogenicity of truncating TTN variants.

Most genetic clusters in our study were associated with a decreased capability for remodeling. This is in line with the well-known worse outcome described in DCM patients with mutations in PLN, RBM2O and SCN5A ${ }^{24}$. Mutations in LMNA remained borderline independently negatively associated with LVRR. A cardiolaminopathy is associated with severe disease that often proves refractory to guideline-directed medical therapy (GDMT). Therefore, close follow-up and aggressive management is indicated ${ }^{24}{ }^{25}$. This also highlights 
the need for mechanistically-based therapy. Treatment with a p38 inhibitor (ARRY-371797) prevented left ventricular dilation and deterioration of EF in LMNA-mutated mice ${ }^{26}$. There is even a double-blinded, randomized, placebo-controlled study investigating the benefit of ARRY-371797 in patients with symptomatic DCM due to a LMNA mutation (ClinicalTrials.gov identifier: NCT03439514).

Table 5. Independent predictors for the combined end-point (death, heart transplantation, HF hospitalisation and/or life-threatening arrhythmias)

\begin{tabular}{cccc} 
Clinical variable & HR & 95\% Cl & P-value \\
\hline Non-sustained VT & 2.25 & $1.36-3.74$ & 0.002 \\
LVEF, per point \% increase & 0.97 & $0.95-0.99$ & 0.021 \\
LVRR & 0.56 & $0.33-0.95$ & 0.033 \\
LMNA variant & 3.68 & $1.40-9.69$ & 0.008 \\
\hline
\end{tabular}

Abbreviations: VT: ventricular tachycardia, LVEF: left ventricular ejection fraction, LVRR: left ventricular reverse remodeling

\section{Clinical Implications}

Clinical decision making regarding preventive device therapy currently depends largely on LVEF ${ }^{27}$. We argue that the threshold and timing for preventive interventions, such as device therapy, may need to be reduced for gene mutations with lower chance of cardiac improvement such as $L M N A$, and higher for those more likely to recover upon HF medication. However, increased risk for (life-threatening) arrhythmias independent of cardiac function as seen in specific gene mutations (eg. LMNA) - must also be taken into account ${ }^{2829}$. Of note, a higher LVRR rate is present in patients with TTN mutations. Paradoxically, TTN mutated patients are more prone to arrhythmias ${ }^{18} 2130$. Therefore, not only cardiac function but also the underlying pathological mechanism is relevant to determine one's risk of arrhythmias in $\mathrm{DCM}^{31}$.

\section{Limitations}

We included DCM and HNDC patients in the cohort for this study. The HNDC patients had no dilation at baseline, possibly leading to an overestimation of the number of patients that remodeled. This is the first study that characterizes the potential of remodeling in the relative new HNDC class of cardiomyopathies.

All DCM/HNDC patients were included according to our inclusion criteria involving different etiologies of DCM. This reflects the heterogeneous patient population seen in the clinics with both potential reversible and irreversible causes.

Different criteria for LVRR have been proposed in the literature. We used the most commonly used, which includes significant improvement of LVEF and normalization of cardiac dimensions ${ }^{2}$. Still, the absolute cut-off values may feel arbitrary. Due to the relatively low genetic yield among DCM patients, only LMNA and TTN could be analyzed separately. All other gene mutations were clustered, which makes it difficult to draw final conclusions on LVRR, but the potential for clinical decision making is high. Future larger studies may evaluate the prognostic relevance of mutations in further DCM genes. Only rare truncated variants in TTN (TTNtv) in exons that are constitutively expressed in the heart were classified as pathogenic in this study, to achieve the highest probability of true pathogenic mutations ${ }^{23}$. However, determining pathogenicity in such complicated molecule remains challenging. 


\section{CONCLUSION}

Genetic substrate is associated with the clinical course of a DCM/HNDC patient. Adding mutation status to clinical parameters in DCM and HNDC improves patient stratification in terms of LVRR and long-term outcome. 


\section{REFERENCES}

1. Kober L, Thune JJ, Nielsen JC, et al. Defibrillator Implantation in Patients with Nonischemic Systolic Heart Failure. $N$ Engl J Med 2016;375(13):1221-30.

2. Merlo M, Pyxaras SA, Pinamonti $B$, et al. Prevalence and prognostic significance of left ventricular reverse remodeling in dilated cardiomyopathy receiving tailored medical treatment. J Am Coll Cardiol 2011;57(13):146876.

3. Writing Committee $\mathrm{M}$, Yancy $\mathrm{CW}$, Jessup $\mathrm{M}$, et al. 2013 ACCF/AHA guideline for the management of heart failure: a report of the American College of Cardiology Foundation/American Heart Association Task Force on practice guidelines. Circulation 2013;128(16):e240-327.

4. Pinto YM, Elliott PM, Arbustini E, et al. Proposal for a revised definition of dilated cardiomyopathy, hypokinetic non-dilated cardiomyopathy, and its implications for clinical practice: a position statement of the ESC working group on myocardial and pericardial diseases. Eur Heart J 2016;37(23):1850-8.

5. Japp AG, Gulati A, Cook SA, et al. The Diagnosis and Evaluation of Dilated Cardiomyopathy. J Am Coll Cardiol 2016;67(25):2996-3010.

6. Walsh R, Thomson KL, Ware JS, et al. Reassessment of Mendelian gene pathogenicity using 7,855 cardiomyopathy cases and 60,706 reference samples. Genetics in medicine : official journal of the American College of Medical Genetics 2017;19(2):192-203.

7. Mann DL, Barger PM, Burkhoff D. Myocardial recovery and the failing heart: myth, magic, or molecular target? J Am Coll Cardiol 2012;60(24):2465-72.

8. Felkin LE, Walsh R, Ware JS, et al. Recovery of Cardiac Function in Cardiomyopathy Caused by Titin Truncation. JAMA Cardiol 2016;1(2):234-5.

9. Jansweijer JA, Nieuwhof K, Russo $F$, et al. Truncating titin mutations are associated with a mild and treatable form of dilated cardiomyopathy. Eur J Heart Fail 2016;19(4):51221.

10. Dal Ferro M, Stolfo D, Altinier A, et al. Association between mutation status and left ventricular reverse remodelling in dilated cardiomyopathy. Heart (British Cardiac Society) 2017

11. Hazebroek MR, Moors S, Dennert R, et al. Prognostic Relevance of Gene-Environment Interactions in Patients With Dilated Cardiomyopathy: Applying the MOGE(S) Classification. J Am Coll Cardiol 2015;66(12):1313-23.
12. Richards S, Aziz N, Bale S, et al. Standards and guidelines for the interpretation of sequence variants: a joint consensus recommendation of the American College of Medical Genetics and Genomics and the Association for Molecular Pathology. Genetics in medicine : official journal of the American College of Medical Genetics 2015;17(5):405-24.

13. Schafer S, de Marvao A, Adami E, et al. Titintruncating variants affect heart function in disease cohorts and the general population. Nature genetics 2017;49(1):46-53.

14. Kubanek M, Sramko M, Maluskova J, et al. Novel predictors of left ventricular reverse remodeling in individuals with recent-onset dilated cardiomyopathy. J Am Coll Cardiol 2013;61(1):54-63.

15. Akinrinade $\mathrm{O}$, Ollila $\mathrm{L}$, Vattulainen $\mathrm{S}$, et al. Genetics and genotype-phenotype correlations in Finnish patients with dilated cardiomyopathy. Eur Heart J 2015;36(34):2327-37.

16. Ortiz-Genga MF, Cuenca $S$, Dal Ferro $M$, et al. Truncating FLNC Mutations Are Associated With High-Risk Dilated and Arrhythmogenic Cardiomyopathies. J Am Coll Cardiol 2016;68(22):2440-51.

17. Luk K, Bakhsh A, Giannetti N, et al. Recovery in Patients With Dilated Cardiomyopathy With Loss-of-Function Mutations in the Titin Gene. JAMA Cardiol 2017;2(6):700-02.

18. Verdonschot JAJ, Hazebroek MR, Derks KWJ, et al. Titin cardiomyopathy leads to altered mitochondrial energetics, increased fibrosis and long-term life-threatening arrhythmias. Eur Heart J 2018

19. Linschoten $M$, Teske AJ, Baas AF, et al. Truncating Titin (TTN) Variants in Chemotherapy-Induced Cardiomyopathy. J Card Fail 2017;23(6):476-79. doi: 10.1016/j.cardfail.2017.03.003

20. Ware JS, Li J, Mazaika E, et al. Shared Genetic Predisposition in Peripartum and Dilated Cardiomyopathies. N Engl J Med 2016;374(3):233-41.

21. Roberts AM, Ware JS, Herman DS, et al. Integrated allelic, transcriptional, and phenomic dissection of the cardiac effects of titin truncations in health and disease. Science translational medicine 2015;7(270):270ra6.

22. Tabish AM, Azzimato V, Alexiadis A, et al. Genetic epidemiology of titin-truncating variants in the etiology of dilated cardiomyopathy. Biophys Rev 2017;9(3):207-23.

23. Ware JS, Cook SA. Role of titin in cardiomyopathy: from DNA variants to patient stratification. Nature reviews Cardiology 2018;15(4):241-52.

24. Kayvanpour E, Sedaghat-Hamedani F, Amr A, et al. Genotype-phenotype associations in dilated 
cardiomyopathy: meta-analysis on more than 8000 individuals. Clin Res Cardiol 2016

25. van Rijsingen IA, Arbustini E, Elliott PM, et al. Risk factors for malignant ventricular arrhythmias in lamin a/c mutation carriers a European cohort study. J Am Coll Cardiol 2012;59(5):493-500.

26. Muchir A, Wu W, Choi JC, et al. Abnormal p38alpha mitogen-activated protein kinase signaling in dilated cardiomyopathy caused by lamin A/C gene mutation. Human molecular genetics 2012;21(19):4325-33.

27. Ponikowski P, Voors AA, Anker SD, et al. 2016 ESC Guidelines for the diagnosis and treatment of acute and chronic heart failure: The Task Force for the diagnosis and treatment of acute and chronic heart failure of the European Society of Cardiology (ESC)Developed with the special contribution of the Heart Failure Association (HFA) of the ESC. Eur Heart J 2016;37(27):2129200.

28. Hasselberg NE, Haland TF, Saberniak J, et al. Lamin A/C cardiomyopathy: young onset, high penetrance, and frequent need for heart transplantation. Eur Heart J 2017

29. Priori SG, Blomstrom-Lundqvist C, Mazzanti A, et al. 2015 ESC Guidelines for the management of patients with ventricular arrhythmias and the prevention of sudden cardiac death: The Task Force for the Management of Patients with Ventricular Arrhythmias and the Prevention of Sudden Cardiac Death of the European Society of Cardiology (ESC). Endorsed by: Association for European Paediatric and Congenital Cardiology (AEPC). Eur Heart J 2015;36(41):2793-867.

30. Tayal U, Newsome S, Buchan R, et al. Truncating Variants in Titin Independently Predict Early Arrhythmias in Patients With Dilated Cardiomyopathy. J Am Coll Cardiol 2017;69(19):2466-68.

31. Halliday BP, Cleland JGF, Goldberger JJ, et al. Personalizing Risk Stratification for Sudden Death in Dilated Cardiomyopathy: The Past, Present, and Future. Circulation 2017;136(2):215-31. 


\section{SUPPLEMENTARY METHODS}

\section{Endomyocardial biopsy}

At least six endomyocardial biopsies (EMB) samples were taken from the right ventricular septum via the internal jugular vein using a transcatheter bioptome (Cordis, Miami, FL, USA). Biopsies were collected as part of routine diagnostics for DCM, to identify other triggers related to DCM such as viruses, inflammation, storage diseases and metabolic disorders. Two specimens were used for immunohistological analysis on four $\mu \mathrm{m}$-thick tissue sections from formalin-fixed, paraffin-embedded EMBs. Four specimens were used for the detection of viral genomes by using polymerase chain reaction (PCR) and reverse transcriptase PCR analysis. Deoxyribonucleic acid (DNA) and ribonucleic acid (RNA) were isolated separately, so that remaining RNA could be used for further analysis. Six primer pairs were used to detect cardiotropic DNA and RNA viruses, including adenovirus, enterovirus, cytomegalovirus, parvovirus B19, human herpesvirus-6, and Epstein-Barr virus(1). Significant viral load was defined as $\geq 500$ copies/ $\mu$ g DNA. Increased cardiac inflammation was defined as $\geq 14$ CD45, including up to 4 CD68-infiltrating cells $/ \mathrm{mm}^{2}$ according to the current ESC guidelines. Collagen fraction volume (CFV) was quantified as percentage tissue positive for Sirius red of the total myocardial area. Perivascular and direct sub-endomyocardial collagen were not included in the quantification, as cutting artifacts may influence those.

\section{SUPPLEMENTARY FIGURES}

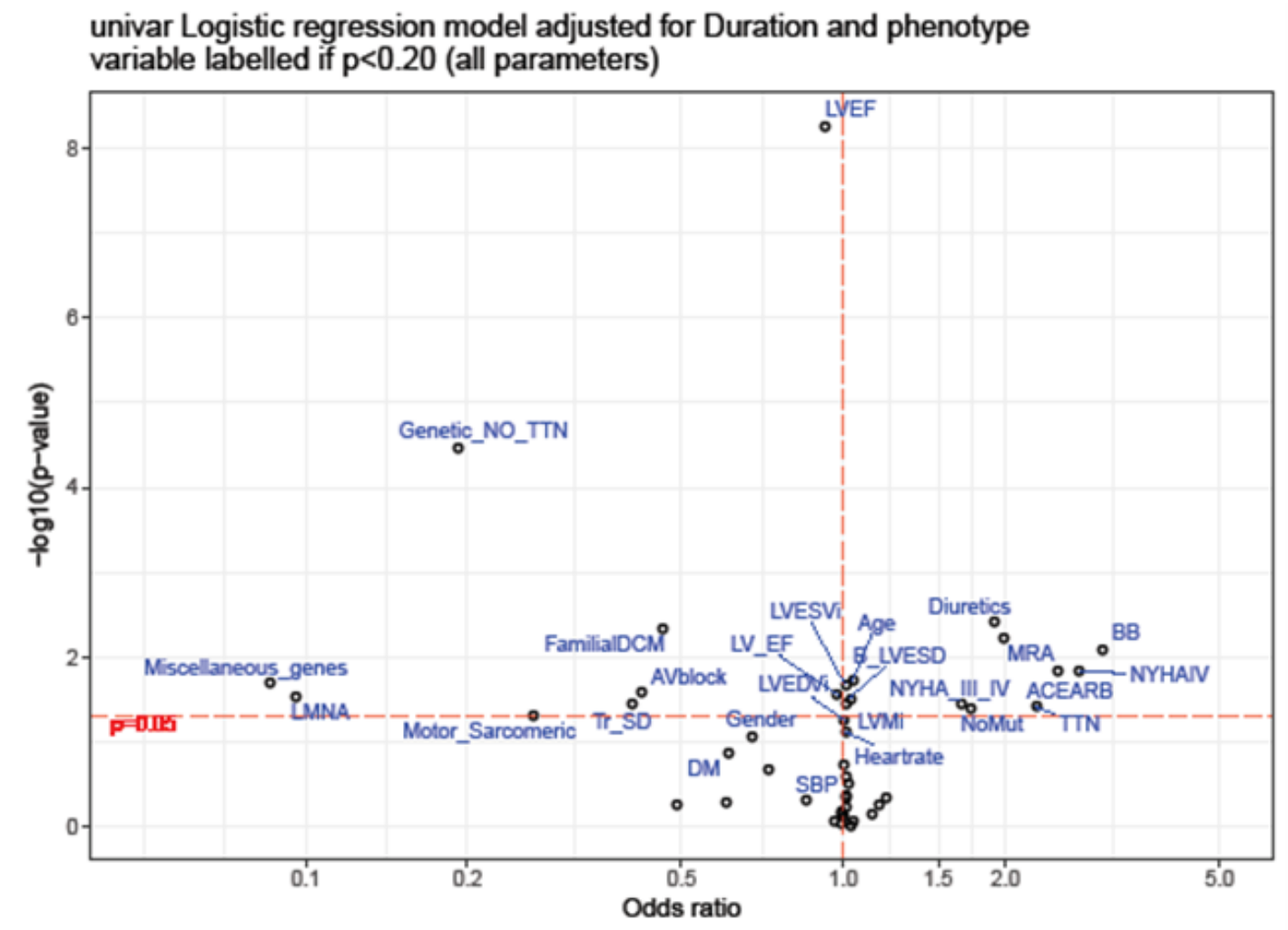

Supplemental Figure 1. Volcano plot of univariate logistic regression analysis corrected for phenotype and duration between echoes. 


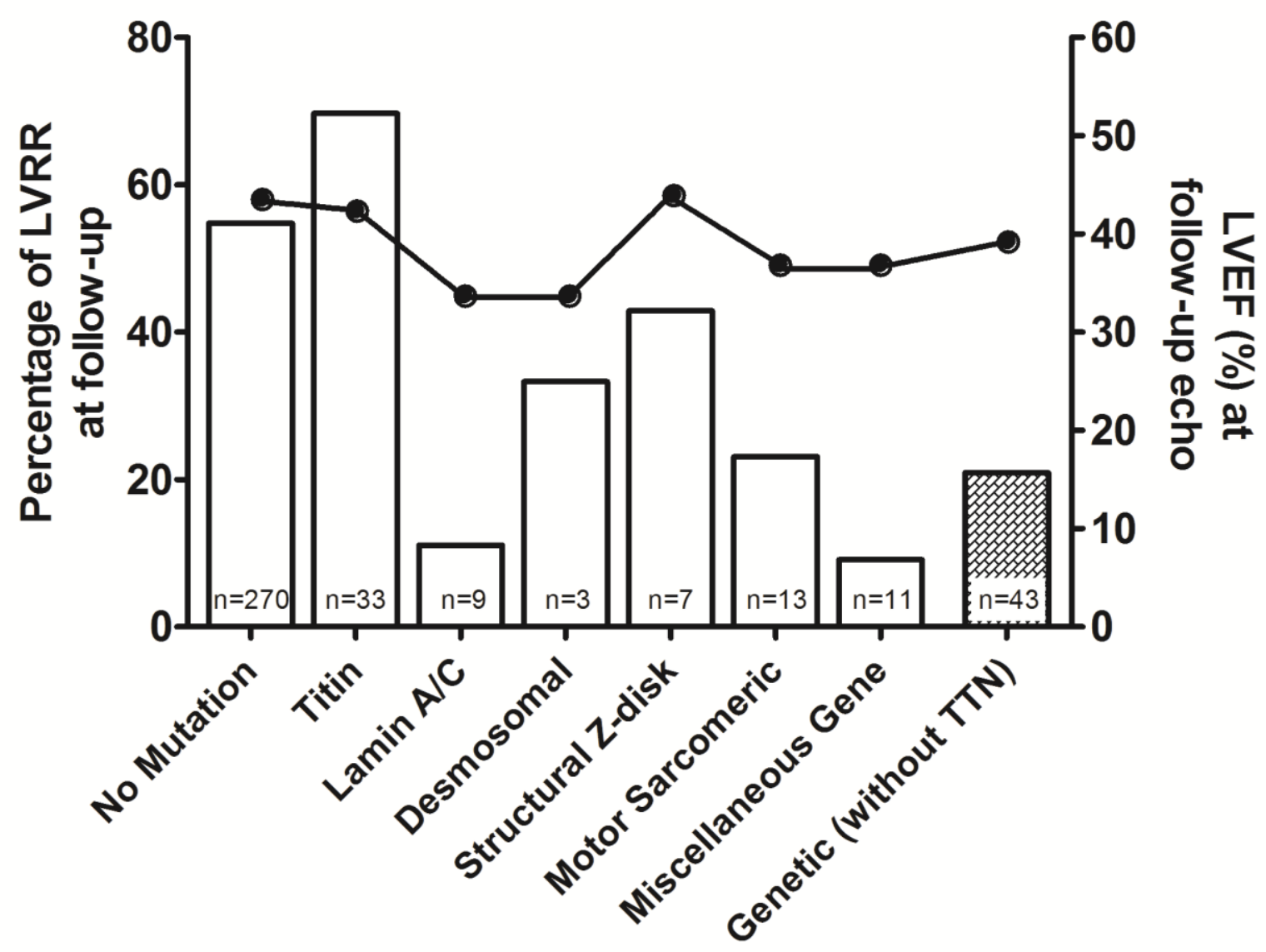

Supplemental figure 2. Rate of left ventricular reverse remodelling per genetic cluster in DCM patients.

Columns depict the percentage of LVRR at follow-up. The dots reflect the mean left ventricular ejection fraction (LVEF) at the follow-up echo.

\section{SUPPLEMENTAL TABLES}

Supplemental Table 1. Overview of all 47 genes used in the Maastricht Cardiomyopathy gene-panel.

\begin{tabular}{cccc} 
& HGNC.ID & HGNC.symbol & HGNC.Name \\
\hline 1 & HGNC:143 & ACTC1 & Actin, alpha, cardiac muscle 1 \\
2 & HGNC:164 & ACTN2 & Actinin alpha 2 \\
3 & HGNC:15819 & ANKRD1 & Ankyrin repeat domain 1 \\
4 & HGNC:939 & BAG3 & BCL2 associated athanogene 3 \\
5 & HGNC:20407 & CALR3 & Calreticulin 3 \\
6 & HGNC:1529 & CAV3 & Caveolin 3 \\
7 & HGNC:2389 & CRYAB & Crystallin alpha B \\
8 & HGNC:2472 & CSRP3 & Cysteine and glycine rich protein 3 \\
9 & HGNC:2511 & CTNNA3 & Catenin alpha 3 \\
10 & HGNC:2770 & DES & Desmin \\
11 & HGNC:3036 & DSC2 & Desmocollin 2 \\
12 & HGNC:3049 & DSG2 & Desmoglein 2 \\
13 & HGNC:3052 & DSP & Desmoplakin \\
\hline
\end{tabular}




\begin{tabular}{|c|c|c|c|}
\hline 14 & HGNC:3331 & $E M D$ & Emerin \\
\hline 15 & HGNC:3702 & FHL1 & Four and a half LIM domains 1 \\
\hline 16 & HGNC:4296 & GLA & Galactosidase alpha \\
\hline 17 & HGNC:14202 & JPH2 & Junctophilin 2 \\
\hline 18 & HGNC:6207 & JUP & Junction plakoglobin \\
\hline 19 & HGNC:6484 & LAMA4 & Laminin subunit alpha 4 \\
\hline 20 & HGNC:6501 & LAMP2 & Lysosomal associated membrane protein 2 \\
\hline 21 & HGNC:15710 & $\angle D B 3$ & LIM domain binding 3 \\
\hline 22 & HGNC:6636 & LMNA & Lamin A/C \\
\hline 23 & HGNC:21086 & MIB1 & Mindbomb E3 ubiquitin protein ligase 1 \\
\hline 24 & HGNC:7551 & МYВРCЗ & Myosin binding protein $\mathrm{C}$, cardiac \\
\hline 25 & HGNC:7576 & MYH6 & Myosin heavy chain 6 \\
\hline 26 & HGNC:7577 & MYH7 & Myosin heavy chain 7 \\
\hline 27 & HGNC:7583 & MYL2 & Myosin light chain 2 \\
\hline 28 & HGNC:7584 & MYL3 & Myosin light chain 3 \\
\hline 29 & HGNC:1330 & MYOZ2 & Myozenin 2 \\
\hline 30 & HGNC:23246 & MYPN & Myopalladin \\
\hline 31 & HGNC:29557 & NEXN & Nexilin F-actin binding protein \\
\hline 32 & HGNC:9024 & PKP2 & Plakophilin 2 \\
\hline 33 & HGNC:9080 & PLN & Phospholamban \\
\hline 34 & HGNC:14000 & PRDM16 & PR/SET domain 16 \\
\hline 35 & HGNC:9386 & PRKAG2 & Protein kinase AMP-activated non-catalytic subunit gamma 2 \\
\hline 36 & HGNC:27424 & $R B M 20$ & RNA binding motif protein 20 \\
\hline 37 & HGNC:10593 & SCN5A & Sodium voltage-gated channel alpha subunit 5 \\
\hline 38 & HGNC:11577 & $T A Z$ & Tafazzin \\
\hline 39 & HGNC:11610 & TCAP & Titin-cap \\
\hline 40 & HGNC:28472 & TMEM43 & Transmembrane protein 43 \\
\hline 41 & HGNC:11943 & TNNC1 & Troponin C1, slow skeletal and cardiac type \\
\hline 42 & HGNC:11947 & TNNI3 & Troponin 13 , cardiac type \\
\hline 43 & HGNC:11949 & TNNT2 & Troponin T2, cardiac type \\
\hline 44 & HGNC:12010 & TPM1 & Tropomyosin 1 \\
\hline 45 & HGNC:12403 & TTN & Titin \\
\hline 46 & HGNC:12405 & $T T R$ & Transthyretin \\
\hline 47 & HGNC:12665 & $V C L$ & Vinculin \\
\hline
\end{tabular}

Supplemental Table 2. Change of LVEF and LVEDDi after follow-up in DCM and HNDC patients with and without LVRR.

\begin{tabular}{cccccccc} 
Variable & LVRR & Phenotype & $\begin{array}{c}\text { Fold } \\
\text { change }\end{array}$ & SD & $\begin{array}{c}\text { Absolute } \\
\text { change }\end{array}$ & SD & $\begin{array}{c}\text { Paired Wilcoxon } \\
\text { signed Rank p-value }\end{array}$ \\
\hline \multirow{3}{*}{ LVEF } & $0-$ & DCM & 1.20 & 0.29 & 4.5 & 6.5 & $1.7 \times 10^{-6}$ \\
& \multirow{2}{*}{$1+$} & HNDC & 1.06 & 0.18 & 1.3 & 5.4 & 0.006 \\
& & DCM & 2.37 & 0.95 & 23.7 & 9.2 & $3.5 \times 10^{-10}$ \\
& \multirow{2}{*}{0} & HNDC & 1.83 & 0.74 & 19.4 & 9.0 & $2.6 \times 10^{-18}$ \\
LVEDDi & & DCM & 0.96 & 0.07 & -1.5 & 2.7 & $5.3 \times 10^{-5}$ \\
& \multirow{2}{*}{$1+$} & HNDC & $\mathbf{0 . 9 9}$ & $\mathbf{0 . 0 8}$ & $-\mathbf{0 . 3}$ & $\mathbf{2 . 3}$ & $\mathbf{0 . 1 5}$ \\
& & HCM & 0.81 & 0.10 & -7.2 & 4.2 & $5.6 \times 10^{-10}$ \\
& & HNDC & 0.93 & 0.10 & -2.1 & 2.9 & $1.8 \times 10^{-9}$ \\
\hline
\end{tabular}


Supplemental Table 3. Interaction analysis between phenotype (DCM/HNDC) and LVRR in respect to changes in LVEF and LVEDDi

\begin{tabular}{ccc} 
& $\begin{array}{c}\Delta \text { LVEF } \\
\text { (p-value) }\end{array}$ & $\begin{array}{c}\Delta \text { LVEDDi } \\
\text { (p-value) }\end{array}$ \\
\hline LVRR & $<2.2 \times 10^{-16}$ & $<2.2 \times 10^{-16}$ \\
Phenotype & 0.010 & 0.017 \\
LVRR*Phenotype & 0.585 & $6.0 \times 10^{-08}$ \\
\hline
\end{tabular}

Supplemental Table 4. Univariable regression analysis adjusted for phenotype and duration between echoes

\begin{tabular}{|c|c|c|c|c|}
\hline Parameter & Label in figure & OR & $95 \% \mathrm{Cl}$ for $\mathrm{OR}$ & P-value \\
\hline Baseline LVEF & LVEF & 0.93 & $0.91-0.95$ & $5.4 \mathrm{E}-09$ \\
\hline Diuretics & Diuretics & 1.91 & $1.23-2.96$ & 0.004 \\
\hline Family history of DCM & FamilialDCM & 0.46 & $0.27-0.79$ & 0.005 \\
\hline MRA & MRA & 1.99 & $1.22-3.25$ & 0.006 \\
\hline Beta-blocker & BB & 3.02 & $1.34-6.83$ & 0.008 \\
\hline ACE-I / ARB & ACEARB & 2.49 & $1.20-5.17$ & 0.014 \\
\hline NYHA IV & NYHAIV & 2.75 & $1.22-6.20$ & 0.015 \\
\hline Age of diagnosis & Age & 1.04 & $1.01-1.08$ & 0.019 \\
\hline AV block & AVblock & 0.42 & $0.20-0.90$ & 0.026 \\
\hline LV end-systolic diameter & B_LVESD & 1.03 & $1.00-1.06$ & 0.030 \\
\hline Systemic disease & Tr_SD & 0.4 & $0.17-0.94$ & 0.036 \\
\hline Heart rate & Heartrate & 1.01 & $1.00-1.02$ & 0.076 \\
\hline Female Gender & Gender & 0.68 & $0.43-1.06$ & 0.086 \\
\hline Diabetes Mellitus & DM & 0.61 & $0.32-1.17$ & 0.136 \\
\hline Systolic blood pressure & SBP & 1.01 & $1.00-1.02$ & 0.181 \\
\hline Non-sustained VT & & 0.73 & $0.45-1.19$ & 0.207 \\
\hline Diastolic blood pressure & & 1.01 & $0.99-1.02$ & 0.262 \\
\hline CD3 cardiac biopsy & & 1.02 & $0.98-1.06$ & 0.305 \\
\hline CD45 cardiac biopsy & & 1.01 & $0.99-1.03$ & 0.413 \\
\hline Cardiac Inflammation & & 1.2 & $0.74-1.95$ & 0.453 \\
\hline Collagen fraction volume & & 1.01 & $0.98-1.04$ & 0.457 \\
\hline Atrial fibrillation & & 1.17 & $0.70-1.93$ & 0.552 \\
\hline $\mathrm{BMI}$ & & 1.01 & $0.97-1.06$ & 0.604 \\
\hline Left atrial diameter & & 1 & $0.97-1.02$ & 0.685 \\
\hline Viral presence & & 1.13 & $0.60-2.10$ & 0.711 \\
\hline CD68 cardiac biopsy & & 1.01 & $0.97-1.05$ & 0.776 \\
\hline Hypertension & & 1.05 & $0.67-1.62$ & 0.839 \\
\hline Left bundle branch block & & 0.97 & $0.60-1.56$ & 0.887 \\
\hline LVEDDi & & 1 & $0.93-1.07$ & 0.925 \\
\hline Cardiotoxic chemo & & 1.04 & $0.37-2.87$ & 0.947 \\
\hline
\end{tabular}




\begin{tabular}{|c|c|c|c|c|c|c|c|}
\hline Baseline variables & $\begin{array}{c}\text { No mutation } \\
(n=270)\end{array}$ & Titin (n=33) & $\begin{array}{c}\operatorname{Lamin} A / C \\
(n=9)\end{array}$ & Desmosomal (n=3) & $\begin{array}{c}\text { Motor Sarcomeric } \\
(n=13)\end{array}$ & $\begin{array}{c}\text { Structural Z-disk } \\
(n=7)\end{array}$ & $\begin{array}{l}\text { Miscellaneous Gene } \\
(\mathrm{n}=11)\end{array}$ \\
\hline Male gender (\%) & $167(62)$ & $22(67)$ & $8(89)$ & $0(0)$ & $6(46)$ & $5(71)$ & $7(64)$ \\
\hline Age of diagnosis (years) & $54 \pm 12$ & $50 \pm 9$ & $54 \pm 10$ & $57 \pm 5$ & $56 \pm 9$ & $53 \pm 14$ & $51 \pm 14$ \\
\hline Family history of DCM (\%) & $41(15)$ & $16(49)$ & $4(44)$ & $1(33)$ & $3(23)$ & $3(43)$ & $6(55)$ \\
\hline Hypertension (\%) & $98(36)$ & $13(39)$ & $5(56)$ & $2(67)$ & $5(39)$ & $5(71)$ & $4(36)$ \\
\hline $\mathrm{SBP}, \mathrm{mm} \mathrm{Hg}$ & $133 \pm 23$ & $129 \pm 16$ & $135 \pm 13$ & $115 \pm 27$ & $126 \pm 17$ & $150 \pm 20$ & $132 \pm 15$ \\
\hline $\mathrm{DBP}, \mathrm{mm} \mathrm{Hg}$ & $80 \pm 14$ & $82 \pm 14$ & $92 \pm 9$ & $72 \pm 21$ & $80 \pm 14$ & $92 \pm 10$ & $77 \pm 10$ \\
\hline Heart rate, BPM & $77 \pm 17$ & $77 \pm 16$ & $70 \pm 12$ & $63 \pm 9$ & $75 \pm 17$ & $73 \pm 18$ & $76 \pm 17$ \\
\hline Diabetes Mellitus (\%) & $35(13)$ & $5(15)$ & $1(11)$ & $0(0)$ & $2(15)$ & $1(14)$ & $1(9)$ \\
\hline Systemic disease (\%) & $19(7)$ & $5(15)$ & $1(11)$ & $0(0)$ & $1(8)$ & $0(0)$ & $1(9)$ \\
\hline Cardiotoxic chemo (\%) & $14(5)$ & $1(3)$ & $0(0)$ & $0(0)$ & $1(8)$ & $0(0)$ & $0(0)$ \\
\hline NYHA III-IV (\%) & $80(30)$ & $10(30)$ & $4(44)$ & $1(33)$ & $5(39)$ & $2(29)$ & $4(36)$ \\
\hline$\frac{\text { Medication }}{\text { Beta blocker (\%) }}$ & $237(88)$ & $32(97)$ & $7(78)$ & $3(100)$ & $11(85)$ & $4(57)$ & $8(73)$ \\
\hline Beta blocker (\%) & 241 (89) & $30(91)$ & $7(78)$ & $3(100)$ & $12(92)$ & $5(71)$ & $11(100)$ \\
\hline ACE-I/ARB (\%) & $83(31) 124$ & $10(30)$ & $2(22)$ & $0(0)$ & $5(39)$ & $0(0)$ & $3(27)$ \\
\hline $\begin{array}{c}\text { MRA (\%) } \\
\text { Diuretics (\%) }\end{array}$ & (46) & $21(66)$ & $4(44)$ & $2(67)$ & $10(77)$ & $1(14)$ & $6(55)$ \\
\hline \multicolumn{8}{|l|}{ Baseline ECG/Holter } \\
\hline cLBBB (\%) & $79(29)$ & $4(12)$ & $5(56)$ & $0(0)$ & $2(15)$ & $2(29)$ & $2(18)$ \\
\hline AV block (\%) & $23(9)$ & $5(15)$ & $1(11)$ & $1(33)$ & $2(15)$ & $0(0)$ & $1(9)$ \\
\hline Atrial fibrillation (\%) & $60(22)$ & $9(27)$ & $4(44)$ & $0(0)$ & $4(31)$ & $2(29)$ & $3(27)$ \\
\hline NSVT (\%) & $58(22)$ & $15(46)$ & $7(78)$ & $1(33)$ & $3(23)$ & $0(0)$ & $4(36)$ \\
\hline \multicolumn{8}{|l|}{ Echocardiography } \\
\hline LVEF (\%) & $30 \pm 11$ & $27 \pm 11$ & $35 \pm 9$ & $32 \pm 9$ & $29 \pm 10$ & $35 \pm 10$ & $33 \pm 12$ \\
\hline $\mathrm{LA}(\mathrm{mm})$ & $45 \pm 8$ & $48 \pm 16$ & $57 \pm 25$ & $40 \pm 1$ & $47 \pm 7$ & $41 \pm 3$ & $37 \pm 15$ \\
\hline LVESD (mm) & $52 \pm 10$ & $53 \pm 9$ & $49 \pm 11$ & $49 \pm 2$ & $49 \pm 10$ & $47 \pm 18$ & $51 \pm 11$ \\
\hline LVEDDI $\left(\mathrm{mm} / \mathrm{m}^{2}\right)$ & $31 \pm 5$ & $31 \pm 3$ & $27 \pm 3$ & $31 \pm 2$ & $31 \pm 8$ & $31 \pm 3$ & $31 \pm 4$ \\
\hline \multicolumn{8}{|l|}{ Endomyocardial Biopsy } \\
\hline Cardiac Inflammation (\%) & $76(34)$ & $6(19)$ & $1(20)$ & $1(33)$ & $3(25)$ & $1(17)$ & $4(44)$ \\
\hline $\mathrm{CD} 3+\left(\right.$ cells $\left./ \mathrm{mm}^{2}\right)$ & $5[3-8]$ & $6[3-8]$ & $4[3-11]$ & 4 & $5[2-8]$ & $3[2-8]$ & $5[1-9]$ \\
\hline CD45+ (cells $\left./ \mathrm{mm}^{2}\right)$ & $9[5-14]$ & $8[4-11]$ & $6[4-23]$ & 8 & $7[6-11]$ & $8[3-12]$ & $9[4-17]$ \\
\hline
\end{tabular}




\begin{tabular}{|c|c|c|c|c|c|c|c|}
\hline CD68+ (cells/mm²) & $3[4-11]$ & $2[1-6]$ & $2[2-2]$ & 4 & $3[1-5]$ & $2[1-4]$ & $2[0-7]$ \\
\hline Myocardial Fibrosis & $7[4-11]$ & $10[6-17]$ & $5[2-23]$ & 12 & $8[4-13]$ & $12[5-29]$ & $6[4-9]$ \\
\hline \multicolumn{8}{|l|}{ Significant viral load } \\
\hline PVB19 (\%) & $36(16)$ & $4(13)$ & $0(0)$ & $0(0)$ & $2(17)$ & $3(50)$ & $1(11)$ \\
\hline HHV6 (\%) & $2(1)$ & $0(0)$ & $0(0)$ & $0(0)$ & $0(0)$ & $0(0)$ & $0(0)$ \\
\hline
\end{tabular}

block; NSVT, non-sustained ventricular tachycardia; LVEF, left ventricular ejection fraction; LVEDD, left ventricular end-diastolic diameter; LVESD, left ventricular end-systolic diameter; LVEDDI, indexed LVEDD;; PVB19, parvovirus B19; HHV6, human herpesvirus 6 .

Supplemental Table 6. Genetic mutations and corresponding clusters found in DCM patients ( $n=346$ )

\begin{tabular}{|c|c|c|c|c|c|c|c|}
\hline Gene & cDNA variant & Variant type & Amino acid change & $\begin{array}{l}\text { No. of } \\
\text { index } \\
\text { patients }\end{array}$ & $\begin{array}{l}\text { ExAC Count } \\
\text { (percentage) }\end{array}$ & Gene cluster & $\begin{array}{c}\text { Variant } \\
\text { segregation } \\
\text { in family* }\end{array}$ \\
\hline TTN & c.13100del & Frameshift & p.(Lys4367Argfs*27) & 1 & 0 & Titin & Yes \\
\hline TTN & c.42267del & Frameshift & p.(Asp14090llefs*2) & 1 & 0 & Titin & Not known \\
\hline TTN & c.44958del & Frameshift & p.(Lys14986Asnfs*30) & 1 & 0 & Titin & Not known \\
\hline TTN & c.46179dup & Nonsense & p. $\left(\right.$ Asp $\left.15574^{*}\right)$ & 1 & 0 & Titin & Not known \\
\hline TTN & c. $47137 \_47138 \mathrm{del}$ & Frameshift & p.(Ser15713Leufs*2) & 3 & 0 & Titin & Yes (1/3 families) \\
\hline TTN & c. $50296 \mathrm{C}>\mathrm{T}$ & Nonsense & p. $\left(\operatorname{Arg} 16766^{*}\right)$ & 1 & $1(4.1 \mathrm{E}-06)$ & Titin & Not known \\
\hline TTN & c.53918del & Frameshift & p.(Gly17973Glufs*18) & 1 & $1(4.24 \mathrm{E}-06)$ & Titin & Not known \\
\hline TTN & c.54768del & Frameshift & p.(Ser18258Valfs*34) & 1 & 0 & Titin & Not known \\
\hline TTN & c. $58327 \mathrm{del}$ & Frameshift & p.(Ala19443Leufs*2) & 2 & 0 & Titin & Not known \\
\hline TTN & c. $60931 \mathrm{C}>\mathrm{T}$ & Nonsense & p. $\left(\operatorname{Arg} 20311^{*}\right)$ & 1 & 1 (4.09E-06) & Titin & Not known \\
\hline TTN & c. $61921 C>T$ & Nonsense & p. $\left(\operatorname{Arg} 20641^{*}\right)$ & 1 & 0 & Titin & Not known \\
\hline TTN & c. $63794-1 G>A$ & Essential splice site & p.(?) & 1 & 0 & Titin & Yes \\
\hline TTN & c.64688del & Frameshift & p.(Pro21563Leufs*10) & 1 & 0 & Titin & Not known \\
\hline TTN & c.65042del & Frameshift & p.(Asp21681Alafs*15) & 1 & 0 & Titin & Not known \\
\hline TTN & c.66020_66021del & Frameshift & p.(Ser22007Cysfs*18) & 1 & 0 & Titin & Yes \\
\hline TTN & c.66296del & Frameshift & p.(Ala22099Valfs*13) & 1 & 0 & Titin & Not known \\
\hline TTN & c.67480_67484del & Nonsense & p.(Glu22494*) & 1 & 0 & Titin & Not known \\
\hline TTN & c. $67863 \mathrm{C}>\mathrm{G}$ & Nonsense & p.(Tyr22621*) & 1 & 0 & Titin & Yes \\
\hline TTN & c. $73332 C>A$ & Nonsense & p.(Cys24444*) & 1 & 0 & Titin & Not known \\
\hline TTN & c. $87782 \mathrm{del}$ & Frameshift & p.(Pro29261Glnfs*10) & 3 & 0 & Titin & Yes (1/3 families) \\
\hline$T T N$ & c.90370G >T & Nonsense & p.(Glu30124*) & 1 & 0 & Titin & Yes \\
\hline TTN & c.97427dup & Frameshift & p.(Thr32477Asnfs*13) & 4 & 0 & Titin & Yes \\
\hline
\end{tabular}




\begin{tabular}{|c|c|c|c|c|c|c|c|}
\hline TTN & c. $100825 C>T$ & Nonsense & p.(Arg33609*) & 1 & 0 & Titin & Not known \\
\hline TTN & c.102097_102101dup & Frameshift & p.(Phe34034Leufs*23) & 1 & 0 & Titin & Not known \\
\hline$T T N$ & c. $106792 C>T$ & Nonsense & p.(Gln35598*) & 1 & 1 (4.07E-06) & Titin & Not known \\
\hline LMNA & c. $481 \mathrm{G}>\mathrm{A}$ & Missense & p.(Glu161Lys) & 2 & 0 & Lamin A/C & Yes (2/2 families) \\
\hline LMNA & c. $357-2 A>G$ & Essential splice site & p.(?) & 1 & 0 & Lamin A/C & Yes \\
\hline LMNA & c.313_314delinsTT & Missense & p.(Glu105Leu) & 2 & 0 & Lamin A/C & Not known \\
\hline LMNA & c. $236 C>A$ & Missense & p.(Ala79Asp) & 1 & 0 & Lamin A/C & Not known \\
\hline LMNA & c. $208 \mathrm{G}>\mathrm{A}$ & Missense & p.(Val70lle) & 1 & 0 & Lamin A/C & Not known \\
\hline LMNA & c. $1300 \mathrm{G}>\mathrm{A}$ & Missense & p.(Ala434Thr) & 1 & 0 & Lamin A/C & Yes \\
\hline LMNA & c. $1201 C>T$ & Missense & p.(Arg401Cys) & 1 & 0 & Lamin A/C & Not known \\
\hline$D S P$ & c.7773_7776del & Frameshift & p.(Ser2591Argfs*11) & 1 & $1(8.24 \mathrm{E}-06)$ & Desmosomal & Not known \\
\hline$D S P$ & c.6393del & Frameshift & p.(Gly2133Valfs*2) & 1 & 0 & Desmosomal & Not known \\
\hline$D S P$ & c.3383_3384del & Frameshift & p.(Val1128Glyfs*5) & 1 & 0 & Desmosomal & Yes \\
\hline TPM1 & c. $829 \mathrm{G}>\mathrm{A}$ & Missense & p.(Ala277Thr) & 1 & $4(3.46 \mathrm{E}-05)$ & Motor Sarcomeric & Yes \\
\hline TNNC1 & c. $317+1 G>A$ & Essential splice site & p.(?) & 1 & 0 & Motor Sarcomeric & Yes \\
\hline MYL3 & c. $476 \mathrm{C}>\mathrm{T}$ & Missense & p.(Thr159Met) & 2 & $3(2.5 \mathrm{E}-05)$ & Motor Sarcomeric & Not known \\
\hline MYL2 & c. $64 \mathrm{G}>\mathrm{A}$ & Missense & p.(Glu22Lys) & 1 & $1(8.3 \mathrm{E}-06)$ & Motor Sarcomeric & Yes \\
\hline MYH7 & c. $2594 A>G$ & Missense & p.(Lys865Arg) & 1 & $1(8.3 \mathrm{E}-06)$ & Motor Sarcomeric & Not known \\
\hline MYH7 & c.2201dupA & Frameshift & p.(Phe735Valfs*3) & 1 & 0 & Motor Sarcomeric & Yes \\
\hline MYH7 & c. $2167 \mathrm{C}>\mathrm{T}$ & Missense & p.(Arg723Cys) & 1 & $3(2.5 \mathrm{E}-05)$ & Motor Sarcomeric & Not known \\
\hline МYBPC3 & c. $2938 C>T$ & Missense & p.(Arg980Cys) & 1 & $2(6.2 \mathrm{E}-05)$ & Motor Sarcomeric & Yes \\
\hline МYBPCЗ & c. $3065 \mathrm{G}>\mathrm{C}$ & Missense & p.(Arg1022Pro) & 1 & $3(2.5 \mathrm{E}-05)$ & Motor Sarcomeric & Not known \\
\hline МYBPC3 & c. $1696 \mathrm{~T}>\mathrm{C}$ & Missense & p.(Cys566Arg) & 1 & 0 & Motor Sarcomeric & Not known \\
\hline МYBPCЗ & c. $1468 \mathrm{G}>\mathrm{A}$ & Missense & p.(Gly490Arg) & 1 & 25 (2.1E-04) & Motor Sarcomeric & Yes \\
\hline МYBPC3 & c.2373dup & Frameshift & p.(Trp792Valfs*41) & 1 & 1 (3.7E-05) & Motor Sarcomeric & Not known \\
\hline$N E X N$ & c.1909_1912del & Frameshift & p.(Tyr637Alafs*48) & 2 & $1(8.3 \mathrm{E}-06)$ & Structural Z-disk & Yes ( $2 / 2$ families) \\
\hline NEXN & c. $11 \overline{7} 4 \mathrm{C}>\mathrm{T}$ & Nonsense & p. $\left(\operatorname{Arg} 392^{*}\right)$ & 1 & $1(8.6 \mathrm{E}-06)$ & Structural Z-disk & Yes \\
\hline MYPN & c. $806 C>T$ & Missense & p.(Pro269Leu) & 1 & 0 & Structural Z-disk & Yes \\
\hline MYPN & c. $59 A>G$ & Missense & p.(Tyr20Cys) & 1 & $111(0.2 E-04)$ & Structural Z-disk & Not known \\
\hline MYOT & c. $179 \mathrm{C}>\mathrm{T}$ & Missense & p.(Ser60Phe) & 1 & $5(4.1 \mathrm{E}-05)$ & Structural Z-disk & Not known \\
\hline$D E S$ & c. $725 \mathrm{~T}>\mathrm{A}$ & Missense & p.(Val242Glu) & 1 & 0 & Structural Z-disk & Not known \\
\hline SCN5A & c. $2254 \mathrm{G}>\mathrm{A}$ & Missense & p.(Gly752Arg) & 1 & 0 & Miscellaneous gene & Not known \\
\hline$R B M 20$ & c. $1900 C>T$ & Missense & p.(Arg634Trp) & 2 & 0 & Miscellaneous gene & Yes (2/2 families) \\
\hline$R B M 20$ & c. $1764 \mathrm{~T}>\mathrm{G}$ & Missense & p.(Ile588Met) & 1 & 0 & Miscellaneous gene & Not known \\
\hline RBM20 & C. $1528-1 G>C$ & Essential splice site & p.(?) & 1 & 0 & Miscellaneous gene & Not known \\
\hline PLN & c. $40 \_42$ del & Deletion & p.(Arg14del) & 2 & 0 & Miscellaneous gene & Yes ( $1 / 2$ families) \\
\hline
\end{tabular}




\begin{tabular}{|c|c|c|c|c|c|c|c|}
\hline MIB1 & c. $912 \mathrm{G}>\mathrm{A}$ & Nonsense & p. $\left(\operatorname{Trp} 304^{*}\right)$ & 1 & 0 & Miscellaneous gene & Yes \\
\hline MIB1 & c. $2176 \mathrm{C}>\mathrm{T}$ & Nonsense & p. $\left(\operatorname{Arg} 906^{*}\right)$ & 1 & $8(6.6 \mathrm{E}-05)$ & Miscellaneous gene & Not known \\
\hline $\mathrm{JPH} 2$ & $\mathrm{c} .88 \mathrm{~A}>\mathrm{T}$ & Missense & p.(Thr30Ser) & 1 & 0 & Miscellaneous gene & Not known \\
\hline$B A G 3$ & c. $910 \mathrm{C}>\mathrm{T}$ & Nonsense & p. $\left(\mathrm{G} \ln 304^{*}\right)$ & 1 & 0 & Miscellaneous gene & Not known \\
\hline
\end{tabular}

* Yes indicates families in which the mutation segregated with the DCM phenotype in the family. Not known indicates that there is no information regarding segregation in the family.

Supplemental Table 7. Association between mutation status and LVRR by logistic regression analysis

\begin{tabular}{|c|c|c|c|c|c|c|}
\hline \multirow[b]{2}{*}{ Genetic status } & \multicolumn{3}{|c|}{ Univariate analysis* } & \multicolumn{3}{|c|}{ Adjusted for clinical model** } \\
\hline & OR & $95 \% \mathrm{Cl}$ & P Value & OR & $95 \% \mathrm{Cl}$ & P Value \\
\hline No mutation & 1.73 & $1.03-2.91$ & 0.039 & 1.49 & $0.82-2.76$ & 0.192 \\
\hline Genetic mutation (Titin excluded) & 0.19 & $0.09-0.42$ & $<0.0001$ & 0.22 & $0.09-0.51$ & 0.0004 \\
\hline Titin & 2.28 & $1.04-4.97$ & 0.039 & 2.94 & $1.18-7.32$ & 0.021 \\
\hline Lamin A/C & 0.10 & $0.01-0.78$ & 0.029 & 0.11 & $0.01-0.95$ & 0.048 \\
\hline Structural cytoskeleton Z-disk & 0.61 & $0.13-2.79$ & 0.521 & 0.69 & $0.12-4.07$ & 0.68 \\
\hline Desmosomal & 0.49 & $0.04-5.53$ & 0.565 & 1.25 & $0.08-19.3$ & 0.873 \\
\hline Motor Sarcomeric & 0.26 & $0.07-0.99$ & 0.048 & 0.23 & $0.05-1.01$ & 0.052 \\
\hline Miscellaneous genes*** & 0.09 & $0.01-0.68$ & 0.020 & 0.11 & $0.01-0.98$ & 0.048 \\
\hline
\end{tabular}

*Adjustment for phenotype (DCM/HNDC) and time between echoes

** Clinical model is LVEF, family history of DCM, beta-blocker usage, age (interaction with HNDC), AV block presence, LVESD, female gender, systolic BP, phenotype (DCM/HNDC) and duration between echoes

*** This cluster constitutes of various genes: PLN, RBM20, SCN5A, BAG3, MIB1, JPH2 
Supplemental Table 8. Primary end-point and hazard ratios

\begin{tabular}{ccccc} 
& $\begin{array}{c}\text { LVRR+ } \\
(\mathbf{n = 1 8 0})\end{array}$ & $\begin{array}{c}\text { LVRR- } \\
(\mathbf{n = 1 6 6 )}\end{array}$ & P-value & Hazard Ratio [95\%Cl] \\
\hline Follow-up duration (months) & $48[27-78]$ & $52[28-85]$ & 0.42 & - \\
CV Death & $2(1)$ & $11(7)$ & 0.007 & $0.18[0.04-0.82]$ \\
Heart transplantation & $0(0)$ & $3(2)$ & 0.11 & - \\
HF hospitalisation & $13(7)$ & $27(16)$ & 0.009 & $0.47[0.24-0.91]$ \\
Life-threatening Arrhythmia & $13(7)$ & $20(12)$ & 0.13 & $0.62[0.31-1.25]$ \\
Combined end-point & $24(13)$ & $40(24)$ & 0.010 & $0.57[0.34-0.95]$ \\
\hline
\end{tabular}

Supplemental Table 9: Association of clinical variables with combined endpoint (death, heart transplantation, HF-hospitalization, life-threatening arrhythmia)

\begin{tabular}{|c|c|c|c|c|c|c|}
\hline \multirow[b]{2}{*}{ Clinical variables } & \multicolumn{3}{|c|}{ Univariate analysis* } & \multicolumn{3}{|c|}{ Multivariate analysis } \\
\hline & HR & $95 \% \mathrm{Cl}$ & P Value & HR & $95 \% \mathrm{Cl}$ & P Value \\
\hline Age (per 1 yr) & 1.01 & $0.98-1.03$ & 0.65 & - & - & - \\
\hline Female gender & 0.74 & $0.44-1.25$ & 0.26 & - & - & - \\
\hline NYHA III-IV & 1.68 & $1.02-2.77$ & $0.042 *$ & - & - & - \\
\hline Hypertension & 0.73 & $0.43-1.25$ & 0.25 & - & - & - \\
\hline Atrial fibrillation & 1.60 & $0.94-2.71$ & $0.084^{*}$ & - & - & - \\
\hline Complete LBBB & 0.85 & $0.48-1.2$ & 0.59 & - & - & - \\
\hline Diabetes mellitus & 1.61 & $0.86-3.01$ & 0.14 & - & - & - \\
\hline Family history of DCM & 1.93 & $1.14-3.5$ & $0.014^{*}$ & - & - & - \\
\hline Non-sustained VT & 2.74 & $1.68-4.48$ & $<0.001 * \dagger$ & 2.25 & $1.36-3.74$ & 0.002 \\
\hline AV-block & 1.14 & $0.52-2.50$ & 0.74 & - & - & - \\
\hline LVEF, per \% increase & 0.98 & $0.96-1.00$ & $0.058 *+$ & 0.97 & $0.95-0.99$ & 0.021 \\
\hline LVEDDi, per $\mathrm{mm}$ increase & 1.02 & 0.97-1.07 & 0.40 & - & - & - \\
\hline LVRR & 0.57 & $0.34-0.95$ & $0.01 *+$ & 0.56 & $0.33-0.95$ & 0.033 \\
\hline HNDC phenotype & 1.02 & $0.60-1.73$ & 0.95 & - & - & - \\
\hline No mutation & 0.49 & $0.30-0.82$ & $0.007^{*}$ & - & - & - \\
\hline$T T N$ & 1.82 & $0.92-3.57$ & $0.084^{*}$ & - & - & - \\
\hline LMNA & 4.82 & $1.92-12.1$ & $0.001 * \dagger$ & 3.68 & $1.40-9.69$ & 0.008 \\
\hline Motor sarcomeric & 1.78 & $0.65-4.89$ & 0.27 & - & - & - \\
\hline Structural cytoskeletion Z-disk & 1.34 & $0.33-5.48$ & 0.69 & - & - & - \\
\hline Desmosomal genes & 0.05 & $0.00-1016$ & 0.55 & - & - & - \\
\hline Miscellaneous genes & 0.91 & $0.22-3.70$ & 0.89 & - & - & - \\
\hline
\end{tabular}

*: Variables entered into multivariable analysis (cutoff for entry $p<0.1$ ).

+ Variables that remained significant in multivariate analysis

Abbreviations: yr: year, LBBB: left bundle branch block, DCM: Dilated cardiomyopathy, VT: ventricular tachycardia, LVEF: left ventricular ejection fraction, LVEDDi: indexed left ventricular end-diastolic diameter, LVRR: left ventricular reverse remodeling, HNDC: hypokinetic nondilated cardiomyopathy 


\section{EDITORIAL COMMENT}

Linking clinical parameters and genotype in dilated cardiomyopathy

Jared M. Churko, MD, $P h D^{1}$

${ }^{1}$ Department of Cellular and Molecular Medicine, University of Arizona, Tucson, USA.

CIRCULATION: HEART FAILURE.

2018. NOV;11(11):e005459. 
Establishing an accurate diagnosis, assessing prognosis, and managing treatment are fundamental to patient care. Clinical parameters, such as left ventricular (LV) ejection fraction and LV end-diastolic diameter, are critical to initially establish a diagnosis of dilated cardiomyopathy (DCM) and hypokinetic non-DCM. These clinical parameters are also used to monitor disease progression and treatment response. However, use of sequencing/genotyping technologies within the clinic is becoming more common in the era of personalized medicine. Efforts to sequence patients and classify the impact of mutations in cardiomyopathy-associated genes are helping to identify key diagnostic subgroups.

In this issue of Circulation: Heart Failure, Verdonschot et $a l^{1}$ studied the association between DCM and hypokinetic non-DCM patients' genotype with improvement in reverse remodeling of the LV in response to medical therapy. The study defined reverse remodeling of the LV as an increase in LV ejection fraction and a decrease in LV end-diastolic diameter for $\approx 1$ year of follow-up. Patients were genotyped using a custom panel of 47 genes associated with cardiomyopathy. Over $52 \%$ of the 346 patients studied had reverse remodeling of the LV after 12 months of treatment. Pathogenic gene mutations were found in $22 \%$ of patients $(n=78)$. The most prevalent genes involved in these patients were titin (TTN, 9.5\%) followed by lamin A/C (LMNA; 2.6\%).

The findings of Verdonschot et al ${ }^{1}$ confirm and enhance the published literature ${ }^{2-6}$, indicating that disease caused by mutations in LMNA are associated with a particularly severe form of DCM, with high arrhythmic burden, progression to end-stage heart failure, and here, lower likelihood of LV recovery. In contrast, disease caused by truncation mutations in titin (TTNtv) is associated with more mild disease that seems more responsive to unloading therapy ${ }^{7-9}$, and thus more likely to favorably remodel and recover with standard medical therapy for heart failure. Furthermore, because of the large size of titin and the presence of multiple isoforms, the location of variants within the gene also plays a role in determining DCM disease burden. TTNtv localized towards the $\mathrm{N}$ terminus, affecting certain TTN isoforms (N2B and N2BA), and within exons commonly removed during splicing have been associated with a more mild or no pathology ${ }^{10}$, whereas variants located in commonly expressed exons (and thus, translated into the final protein product) are predicted to be pathogenic ${ }^{9}$. These findings underscore that genotype can influence the clinical decision-making process and that differentiating clinically relevant subgroups of DCM, guided by genetic cause, may play an important role in diagnosis, prognosis, and management. From an academic perspective, gaining greater understanding in gene- and variant-specific mechanisms will undoubtedly lead to advances in our understanding of how protein-protein domain perturbations ${ }^{11}$, changes in mRNA splicing ${ }^{12}$, and protein functional changes ${ }^{13}$ contribute to in disease pathogenesis. This information will be critical to identify molecular mechanisms leading to DCM and to design targeted drug therapies to treat distinct genetic subclasses of DCM pathology.

The genetic basis of DCM/hypokinetic non-DCM also has important clinical implications. Receipt of genetic results is a reminder to think about the patient's family in addition to the individual presenting for care in clinic ${ }^{14}$. If there is no clear explanation for why cardiomyopathy developed, a detailed family history should be obtained, and firstdegree relatives should be screened to evaluate for familial disease ${ }^{15}$. Although the genetic cause of DCM is diverse and incompletely resolved and the yield of clinical genetic testing is currently relatively modest (a genetic cause can be detected $\approx 20 \%$ of the time) ${ }^{15} 16$, identifying a pathogenic mutation in a patient provides unique and valuable information to 
guide management of the patient and their family. At-risk relatives can be definitively identified and followed appropriately. Relatives not at risk can be reassured. Affected individuals can be given more precise prognostic forecasts, and more aggressive therapy can be steered towards those predicted to have more aggressive disease, based on genetic substrate. The work of Verdonschot et al1 highlight that adding genetic information, in addition to clinical parameters, may better predict reverse remodeling of the LV than clinical parameters alone. Linking the degree of disease burden to variants (and properly defining variants of unknown significance) is still necessary. Efforts in linking disease burden to variants will ultimately lead to improvements in patient care and in our understanding of cardiomyopathies. 


\section{REFERENCES}

1. Verdonschot JAJ, Hazebroek MR, Wang $P$, et al. Clinical Phenotype and Genotype Associations With Improvement in Left Ventricular Function in Dilated Cardiomyopathy. Circulation Heart failure 2018;11(11):e005220.

2. Taylor MR, Fain PR, Sinagra G, et al. Natural history of dilated cardiomyopathy due to lamin $\mathrm{A} / \mathrm{C}$ gene mutations. J Am Coll Cardiol 2003;41(5):771-80.

3. van Tintelen JP, Hofstra RM, Katerberg $\mathrm{H}$, et al. High yield of LMNA mutations in patients with dilated cardiomyopathy and/or conduction disease referred to cardiogenetics outpatient clinics. Am Heart J 2007;154(6):1130-9.

4. van Rijsingen IA, Arbustini E, Elliott PM, et al. Risk factors for malignant ventricular arrhythmias in lamin a/c mutation carriers a European cohort study. J Am Coll Cardiol 2012;59(5):493-500.

5. Pasotti $M$, Klersy $C$, Pilotto $A$, et al. Long-term outcome and risk stratification in dilated cardiolaminopathies. J Am Coll Cardiol 2008;52(15):1250-60.

6. Kumar S, Baldinger SH, Gandjbakhch E, et al. Long-Term Arrhythmic and Nonarrhythmic Outcomes of Lamin A/C Mutation Carriers. J Am Coll Cardiol 2016;68(21):2299-307.

7. Felkin LE, Walsh R, Ware JS, et al. Recovery of Cardiac Function in Cardiomyopathy Caused by Titin Truncation. JAMA Cardiol 2016;1(2):234-5.

8. Jansweijer JA, Nieuwhof K, Russo $F$, et al. Truncating titin mutations are associated with a mild and treatable form of dilated cardiomyopathy. Eur J Heart Fail 2016;19(4):51221.

9. Schafer S, de Marvao A, Adami E, et al. Titintruncating variants affect heart function in disease cohorts and the general population. Nature genetics 2017;49(1):46-53.

10. Roberts AM, Ware JS, Herman DS, et al. Integrated allelic, transcriptional, and phenomic dissection of the cardiac effects of titin truncations in health and disease. Science translational medicine 2015;7(270):270ra6.

11. Laddach A, Ng JC, Chung SS, et al. Genetic variants and protein-protein interactions: a multidimensional network-centric view. Curr Opin Struct Biol 2018;50:82-90.

12. Caminsky N, Mucaki EJ, Rogan PK. Interpretation of mRNA splicing mutations in genetic disease: review of the literature and guidelines for information-theoretical analysis. F1000Res 2014;3:282.
13. Mahlich $Y$, Reeb J, Hecht $M$, et al. Common sequence variants affect molecular function more than rare variants? Sci Rep 2017;7(1):1608.

14. Cirino AL, Harris S, Lakdawala NK, et al. Role of Genetic Testing in Inherited Cardiovascular Disease: A Review. JAMA Cardiol 2017;2(10):1153-60.

15. Hershberger RE, Givertz MM, Ho CY, et al. Genetic Evaluation of Cardiomyopathy-A Heart Failure Society of America Practice Guideline. J Card Fail 2018;24(5):281-302.

16. Mogensen J, van Tintelen JP, Fokstuen S, et al. The current role of next-generation DNA sequencing in routine care of patients with hereditary cardiovascular conditions: a viewpoint paper of the European Society of Cardiology working group on myocardial and pericardial diseases and members of the European Society of Human Genetics. Eur Heart J 2015;36(22):1367-70. 
- 192 - 


\section{Part II}

Redefining disease diagnostics and therapeutics

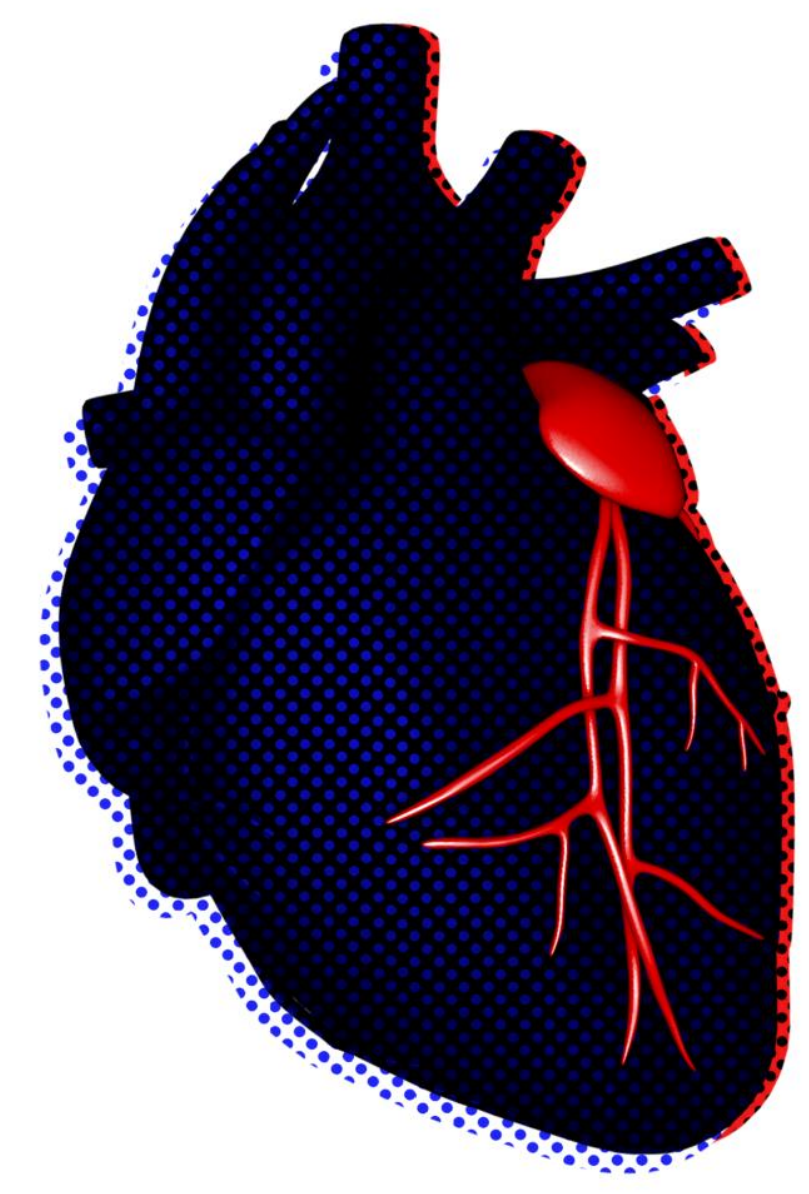




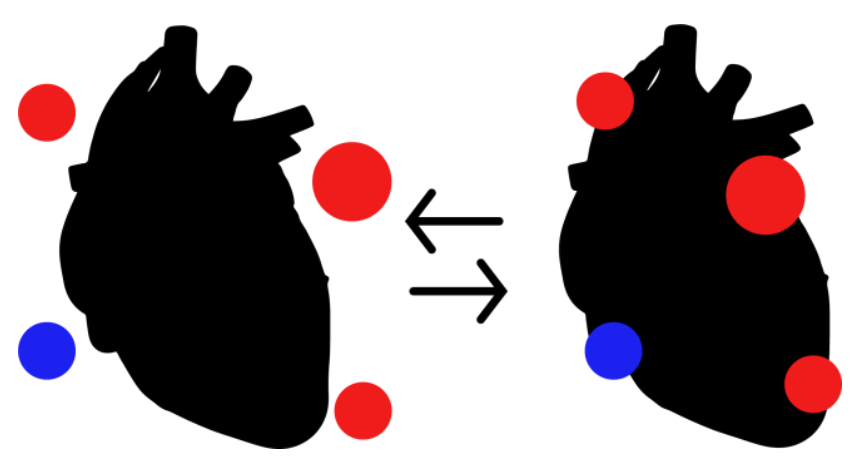

- 194 - 


\title{
CHAPTER 7
}

\section{Distinct Cardiac Transcriptomic Clustering in Titin and Lamin A/C-associated Dilated Cardiomyopathy Patients}

\author{
Job A.J. Verdonschot, $M D, M S c^{1,2}$ \\ Kasper W.J. Derks, $P h D^{2}$ \\ Mark R. Hazebroek, $M D, P h D^{l}$ \\ Ping Wang, $P h D^{2}$ \\ Emma Louise Robinson, $P h D^{2}$ \\ Michiel E. Adriaens, $P h D^{3}$ \\ Ingrid P.C. Krapels, $M D, P h D^{2}$ \\ Arthur van den Wijngaard, $P h D^{2}$ \\ Han G. Brunner, MD, $P h D^{2,4,5}$ \\ Stephane R.B. Heymans, $M D, P h D^{1,6,7}$
}

${ }^{1}$ Department of Cardiology, Cardiovascular Research Institute (CARIM), Maastricht University Medical Center, Maastricht, The Netherlands.

${ }^{2}$ Department of Clinical Genetics, Maastricht University Medical Center, Maastricht, The Netherlands. ${ }^{3}$ Maastricht Centre for Systems Biology, Maastricht University, Maastricht, The Netherlands.

${ }^{4}$ Radboud University Medical Center, Department of Human Genetics, Donders Institute for Brain, Cognition and Behaviour, Nijmegen.

${ }^{5}$ GROW Institute for Developmental Biology and Cancer, Maastricht University Medical Centre, Maastricht, The Netherlands.

${ }^{6}$ Department of Cardiovascular Sciences, Centre for Molecular and Vascular Biology,

KU Leuven, Belgium.

${ }^{7}$ The Netherlands Heart Institute, Nl-HI, Utrecht, The Netherlands. 
Dilated cardiomyopathy (DCM) caused by a genetic mutation has a low rate of left ventricular reverse remodeling (LVRR) in response to treatment. Still, the rate of LVRR varies according to the specific gene mutated, indicating different cardiac molecular consequences ${ }^{1}$. E.g., patients carrying a $L M N A$ variant respond less favorably to pharmacological treatment. Up to $80 \%$ of patients with a truncating TTN variant (TTNtv) recovered upon treatment, but the other $20 \%$ still had a more malignant clinical course, with persistent dysfunction and lifethreatening arrhythmias ${ }^{12}$. Understanding the molecular consequences of specific genetic variants on cardiac pathophysiological pathways will help us to enhance genotype-phenotype correlations. This knowledge can improve the selection of patients who will benefit the most from specific treatments. RNA-sequencing makes it possible to quantify the activity of specific molecular pathways in genetic DCM patients. Previously, cardiac RNA profiling in TTNtv revealed a strong upregulation of the oxidative phosphorylation pathway, both in human ${ }^{2}$ and rat $^{3}$ hearts. This reflects a distinct transcriptomic signature in titin cardiomyopathy, which could be translated towards more specific treatment ${ }^{2}$. Here, we related cardiac transcriptomic profiles of 29 genetic DCM patients to in-depth phenotyping.

We performed RNA-sequencing on right septal biopsies gathered during diagnostic work-up in 29 DCM patients carrying a pathogenic genetic variant (13 TTNtv, 7 LMNA, 5 RBM20, 4 MYH7), as part of the Maastricht Cardiomyopathy Registry ${ }^{12}$. None of the patients had end-stage disease. Included patients were selected based on the availability of spare biopsies for research, with a prerequisite of minimal three patients per gene subgroup. Only patients with a pathogenic variant in one of the earlier mentioned genes fulfilled these criteria. The study was performed according to the declaration of Helsinki and was approved by the institutional Medical Ethics Committee. All patients gave written informed consent.

The transcriptome profile of TTNtv and LMNA-carriers clustered distinctly from the other genetic DCM patients, as revealed by a principal component analysis (PCA) (Figure 1, left). In addition, two distinct subgroups were present within the TTNtv patients, further denoted as $\operatorname{TTNtv1}(n=6)$ and TTNtv2(n=7). There was no clear pattern of the location of the truncating variant in the gene between these subgroups (Figure 1, bottom). Subsequent Ingenuity Pathway Analysis (IPA ${ }^{\circledR}$ ) indicated an upregulation of NFKB signaling, cardiac necrosis and fibrosis, and downregulation of the hypertrophy pathways in TTNtv2 subgroup (Figure 1, right), the latter in line with a decreased cardiac mass in TTNtV-DCM versus other DCM patients ${ }^{2}$. Discriminant analysis on the phenotypical data revealed a distinct malignant phenotype behind the TTNtv2 subgroup: a lower ejection fraction (EF), thinner interventricular septum, increased cardiac fibrosis, more arrhythmias, higher concentration of blood inflammation markers and NT-proBNP (Figure 1, middle). The transcriptomic profile of the malignant TTNtv2 clustered close to LMNA patients, with similarities in the clinical phenotype. IPA ${ }^{\circledast}$ showed increased cardiac fibrosis and necrosis, and decreased hypertrophy and proliferation as most distinctive pathways between LMNA and TTNtv1 (Figure 1, right). NFKB signaling and oxidative stress response were the only differences between LMNA and TTNtv2. Interestingly, the transcriptomic profiles of $R B M 2 O$ and $M Y H 7$, did not cluster together with $L M N A$ and TTNtv, although having clinical similarities. The separate clustering of TTNtv and LMNA from the others indicates a specific transcriptomic profile irrespective of solely cardiac function and treatment duration.

Current guidelines recommend standard heart failure (HF) medication regardless of the heterogeneous nature of the DCM pheno- and genotype. Still $50 \%$ of DCM patients do not functionally improve, despite improved diagnostics of the underlying causes ${ }^{1}$. A post-hoc genetic analysis in the PercutaneOus StEm Cell Injection Delivery Effects On Neomyogenesis 
in Dilated CardioMyopathy (POSEIDON-DCM) trial showed that the genotype contributes to the response to treatment ${ }^{4}$. This emphasized the need for a better understanding of the individual pathomechanisms and associated likelihood to respond to treatment, for which RNA profiling can provide a first insight. Here, we reveal distinct RNA profiles not only in LMNA-DCM, but also 2 distinct RNA profiles in TTNtv-DCM. These findings challenges the concept that TTNtv-DCM is completely benign: not only over $20 \%$ do not functionally improve upon standard HF therapy, also the malignant TTNtv subgroup presents with a distinct molecular profile. Current dogma tries to classify patients in specific etiologies, leading to recommendations which are difficult to translate to the general DCM population. Improved DCM stratification will require unsupervised phenomapping -as recently done in HF with preserved $\mathrm{EF}^{5}$-, in combination with in-depth molecular analysis of heart tissue for effective precision medicine in DCM. Our findings show the feasibility of stratifying patients based on their pathophysiological processes, which eventually can uncover novel pharmacological targets. In conclusion, RNA profiles strongly differ in TTNtv and LMNA-mutated DCM patients, despite clinical similarities as other pathogenic variant carriers such as RBM2O and MYH7, suggesting a specific genetic effect on the cardiac transcriptome in addition to the effect of the clinical component. The understanding of these different profiles is a first step towards precision medicine. 

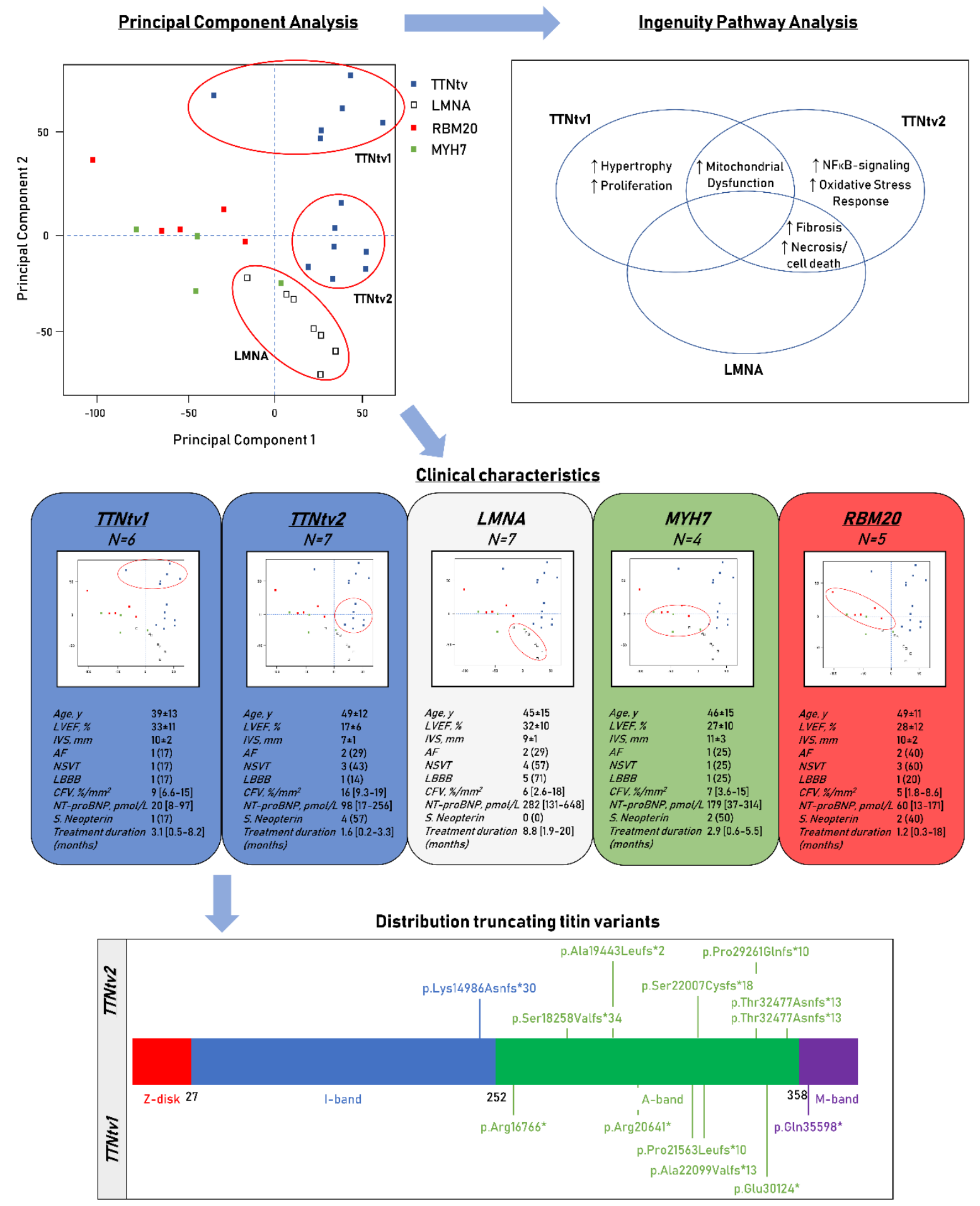

Figure 1. Transcriptomic results based on genotype and phenotype of the patients. Principal component analysis of the transcriptomic profile isolated from the hearts of dilated cardiomyopathy patients with a TTNtv, LMNA, RBM20 or MYH7 variant (left). Venn diagram of the distinctive processes per genotype (right). Analysis was performed with IPA ${ }^{\circledast}$. An overview of the clinical characteristics per genotype (middle). Values are displayed as $\mathrm{n}(\%)$, mean $\pm S D$ or median [interquartile range]. Schematic representation of the TTN gene with all included truncating variants mapped across the gene (bottom). The numbers indicate the exon at the boundary of the specified gene region.

Abbreviations: LVEF indicates left ventricular ejection fraction; IVS, interventricular septum; $A F$, atrial fibrillation; NSVT, non-sustained ventricular tachycardia; $L B B B$, left bundle-branch block; CFV, collagen volume fraction; $S$. neopterin, significant concentrations of serum neopterin. 


\section{REFERENCES}

1. Verdonschot JAJ, Hazebroek MR, Wang P, et al. Clinical Phenotype and Genotype Associations With Improvement in Left Ventricular Function in Dilated Cardiomyopathy. Circulation Heart failure 2018;11(11):e005220.

2. Verdonschot JAJ, Hazebroek MR, Derks KWJ, et al. Titin cardiomyopathy leads to altered mitochondrial energetics, increased fibrosis and long-term life-threatening arrhythmias. Eur Heart J 2018;39(10):864-873.

3. Schafer S, de Marvao A, Adami E, et al. Titintruncating variants affect heart function in disease cohorts and the general population. Nature genetics 2017;49(1):46-53.

4. Rieger AC, Myerburg RJ, Florea V, et al. Genetic determinants of responsiveness to mesenchymal stem cell injections in non-ischemic dilated cardiomyopathy. EBioMedicine 2019;48:377-85.

5. Shah SJ, Katz DH, Selvaraj S, et al. Phenomapping for novel classification of heart failure with preserved ejection fraction. Circulation 2015;131(3):269-79. 


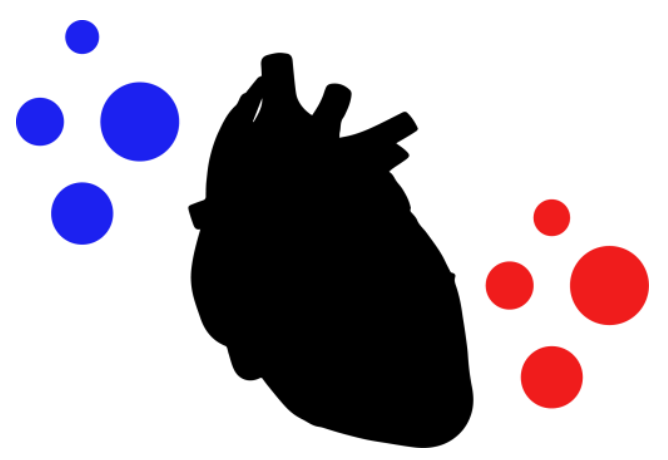

- 200 - 


\title{
CHAPTER 8
}

\section{Phenotypic clustering of dilated cardiomyopathy patients highlights important etiological differences}

\author{
Job A.J. Verdonschot, $M D, M S c^{1,2}$ \\ Marco Merlo, $M D^{3}$ \\ Fernando Dominguez, $M D, P h D^{4,5}$ \\ Ping Wang, $P h D^{2}$ \\ Michiel T.H.M. Henkens, $M D^{l}$ \\ Michiel E. Adriaens, $P h D^{6}$ \\ Mark R. Hazebroek, $M D, P h D^{1}$ \\ Marco Masè, $M D^{3}$ \\ Luis E. Escobar, $M D^{4,5}$ \\ Kasper W.J. Derks, PhD \\ Arthur van den Wijngaard, $P h D^{2}$ \\ Ingrid P.C. Krapels, $M D, P h D^{2}$ \\ Han G. Brunner, $M D, P h D^{2,7,8}$ \\ Gianfranco Sinagra, $M D, P h D^{3}$ \\ Pablo Garcia-Pavia, MD, PhD ${ }^{4,5,9}$ \\ Stephane R.B. Heymans, MD, $P h D^{3,10,11}$
}

${ }^{1}$ Department of Cardiology, Cardiovascular Research Institute (CARIM), Maastricht University Medical Center, Maastricht, The Netherlands.

${ }^{2}$ Department of Clinical Genetics, Maastricht University Medical Center, Maastricht, The Netherlands. ${ }^{3}$ Cardiovascular Department, Azienda Sanitaria Universitaria Giuliano Isontina (ASUGI), University of Trieste, Italy. ${ }^{4}$ Department of Cardiology, Hospital Universitario Puerta de Hierro, Madrid, Spain.

${ }^{5}$ Centro de Investigación Biomédica en Red Enfermedades in Cardiovascular Diseases (CIBERCV), Madrid, Spain.

${ }^{6}$ Maastricht Centre for Systems Biology, Maastricht University, Maastricht, The Netherlands.

${ }^{7}$ Radboud University Medical Center, Department of Human Genetics, Donders Institute for Brain, Cognition and Behavior, Nijmegen.

${ }^{8}$ GROW Institute for Developmental Biology and Cancer, Maastricht University Medical Centre, Maastricht, The Netherlands.

${ }^{9}$ Universidad Francisco de Vitoria (UFV), Pozuelo de Alarcon, Spain.

${ }^{10}$ Department of Cardiovascular Sciences, Centre for Molecular and Vascular Biology, KU Leuven, Belgium.

${ }^{11}$ The Netherlands Heart Institute, Nl-HI, Utrecht, The Netherlands. 


\begin{abstract}
Aims: The dilated cardiomyopathy (DCM) phenotype is the result of combined genetic and acquired triggers. Until now, clinical decision-making in DCM has mainly been based on ejection fraction and NYHA classification, not taking into account the DCM heterogenicity. The present study aimed to identify patient subgroups by phenotypic clustering integrating etiologies, comorbidities and cardiac function, which may better reflect the heterogeneity of DCM.
\end{abstract}

Methods and results: We used 795 consecutive DCM patients from the Maastricht Cardiomyopathy Registry who underwent in-depth phenotyping, including imaging, genetics and endomyocardial biopsies. Four mutually exclusive and clinically distinct phenogroups were identified based upon unsupervised hierarchical clustering of principal components. These phenogroups integrate cardiac function, etiologies and comorbidities of DCM, and highlight auto-immune disease and genetics as important etiological contributors. Autoimmune disease, atrial fibrillation, ejection fraction and serum creatinine are the key parameters able to place patients in one of these four, as identified by supervised decision tree modelling. Event-free survival differed among the four phenogroups independent of age, gender, NT-proBNP or ejection fraction. As a validation, DCM patients from two independent cohorts (Spain, $n=352$ and Italy, $n=437$ ) could be successfully placed in the identified phenogroups, and also associated with outcome.

Conclusion: The present study identified four different DCM phenogroups associated with significant differences in clinical presentation and adverse outcome in three independent European DCM cohorts. These phenogroups highlight the importance of etiology as contributing factor to the DCM phenotype, and pave the way towards a more personalized approach. 


\section{INTRODUCTION}

Non-ischemic, non-valvular, dilated cardiomyopathy (DCM) represents a heterogeneous group of patients, as it results from a variety of genetic and acquired triggers ${ }^{12}$. The heterogeneity makes it difficult to classify and define DCM with great precision to guide clinical decision-making. Outcome and response to therapy differ pending on the underlying etiology ${ }^{34}$. Overall, the success rate of optimal heart failure therapy in DCM is only $50 \%$ after 12-24 months of treatment ${ }^{5-7}$. Until now, clinical decision-making in DCM has mainly been based on ejection fraction and NYHA classification ${ }^{8}{ }^{9}$, which does not recapitulate the complexity of the interactions with comorbidities and underlying etiologies in the development and progression of $\mathrm{DCM}^{10}{ }^{11}$. Classification which integrates etiological heterogeneity with cardiac function and comorbidities, may reflect the biological differences between patients with greater precision, and can allow better outcome prediction and guidance for targeted therapy ${ }^{6}$. In an ideal situation, patients with corresponding pathophysiology would be classified together, creating more homogeneous patient populations.

Phenomapping based upon unsupervised clustering of clinical data may help to create homogeneous DCM subgroups, called phenogroups ${ }^{12}$. Machine learning aids in detecting patterns between variables which explain the heterogeneity in a dataset ${ }^{13}$. The process in patient cohorts can be grossly divided into two steps: [1] unsupervised learning to detect sets of variables, which cluster patients with comparable phenotypes integrating cardiac function, comorbidities, genetic and acquired etiologies; and subsequent [2] supervised learning to translate these novel subgroups to clinical utility ${ }^{12-14}$. The methodology has previously helped to create clinically valid phenogroups in heart failure with preserved ejection fraction (HFpEF), which were associated with differences in long-term outcome ${ }^{12}$. So far, studies applying machine learning to create phenogroups in patients with DCM are missing. Here, we identified four distinct phenogroups in DCM patients, highlighting the etiological diversity. The subsequent supervised model was applicable in three independent European DCM registries.

\section{METHODS}

\section{Study population and clinical information}

The DCM diagnosis was defined according to the World Health Organization criteria and the latest ESC proposal ${ }^{7} 15$ 16. Enrolled patients presented with a left ventricular (LV) ejection fraction $<50 \%$ at baseline echocardiographic evaluation in the absence of any of the following conditions: obstruction $>50 \%$ of a major coronary artery branch (at coronary angiography), pericardial diseases, congenital heart diseases, cor pulmonale, and active myocarditis. Patients, when not contraindicated, received guideline-directed medical therapy titrated to the maximal tolerated dose as well as device therapy (ICD and CRT-D implantation) according to the latest ESC guidelnies ${ }^{79}$. Three European DCM cohorts were analyzed. The study was performed according to the declaration of Helsinki, and approved by all the local Institutional Review Boards. All patients gave written informed consent.

Index cohort - The index cohort was derived from the prospective Maastricht Cardiomyopathy Registry of the Maastricht University Medical Center (MUMC, Maastricht, the Netherlands) between 2012 and 2019. As part of the diagnostic protocol, all patients received genetic counseling and DNA testing using a 47 DCM-associated gene panel (Supplemental Table 1), and underwent a physical examination, blood sampling, 12-lead electrocardiogram, 24-h Holter monitoring, a complete echocardiographic and Doppler 
evaluation and coronary angiography (CAG) at baseline. See the Supplemental Methods for a detailed overview of the in/exclusion criteria, definitions of non-genetic disease modifiers and performed genetic analysis. Endomyocardial biopsies (EMB) and cardiovascular magnetic resonance $(C M R)$ imaging were performed if patient consented and was able to undergo these procedures. Table 1 demonstrates the phenotypic domains covering all of the collected clinical variables.

Validation cohorts - The validation cohort from Italy was derived from the prospective Heart Muscle Registry of Trieste Registry ${ }^{17}$. Patients were enrolled between 2006 and 2019. All patients underwent physical examination, blood sampling, 12-lead electrocardiogram, 24hours ECG Holter monitoring, a complete echocardiographic and Doppler evaluation at baseline and during structured, systematic follow-up ${ }^{11}$. Genetic testing was performed using a 23 DCM-associated gene panel. In order to exclude ischemic heart disease, a CAG was systematically performed in patients $\geq 35$ years, with cardiovascular risk factors and/or without familial history for DCM.

The validation cohort from Madrid included DCM patients followed in the Hospital Puerta de Hierro's Inherited Cardiac Diseases Unit from 2004-2019 ${ }^{18}$. All patients underwent a physical examination, blood sampling, genetic testing, a 12-lead electrocardiogram, echocardiogram and systematic follow-up.

Table 1. Phenotype domains and corresponding clinical variables

\section{Phenotypic Domain}

Demographics

Disease modifiers

Physical Characteristics

Echocardiography

Laboratory

ECG/Holter

Magnetic Resonance

Endomyocardial Biopsy

\section{Clinical Variables}

Age*, gender*

Genetic mutation, genetic group*, familial disease*, toxic trigger*, auto-immune disease*, cardiac inflammation*, significant viral load*, electrical trigger

NYHA $\geq I I *$, hypertension*, body mass index*, diabetes*, heart rate*, systolic blood pressure*, diastolic blood pressure

LV ejection fraction, LV end-diastolic diameter index, LV end-systolic diameter, left atrial volume index, LV mass index, E/A ratio, E/e' ratio, posterior wall thickness, intraventricular septum thickness

NT-pro B-type natriuretic peptide*, high-sensitive troponin T, creatinine*, soluble interleukin-2 receptor, neopterin, cardiac auto-antibodies, c-reactive protein*, aspartate transaminase*, alanine transaminase*

Atrial fibrillation*, non-sustained ventricular tachycardia*, left bundle branch block*, out of hospital cardiac arrest*, atrioventricular block*

LV mass index*, LV end-diastolic volume index*, LV end-systolic volume index, LV stroke volume index, LV ejection fraction*, late gadolinium enhancement*

Collagen volume fraction

NYHA indicates New York Heart Association classification of severity of Heart Failure; LV, left ventricular.

* Clinical variables used in the model-based clustering analyses after filtering to remove correlated variables $(r>0.6)$.

\section{Follow-up}

The median follow-up time of the Maastricht cohort was 53 months (interquartile range 2898 months), 85 months (interquartile range 34-145 months) for Madrid and 40 months (interquartile range 13-77 months) for Trieste. Information about the occurrence of adverse events at follow-up was retrieved from the hospital medical records, the Personal Records Database, and/or telephone contact with the patient or their general practitioners in the three cohorts. We collected information regarding the following composite outcome measures: 1) death due to cardiovascular (CV) disease (heart failure or thrombo-embolic events), 2) heart transplantation (Htx), 3) left ventricular assist device (LVAD) implantation, 
and 4) life-threatening arrhythmias (LTA) defined as non-fatal ventricular fibrillation (with or without ICD-shock), and/or sustained ventricular tachycardia treated with appropriate ICD shock. The combined end-point is defined as the first occurrence of at least one of the abovementioned adverse events: CV-related death, Htx, LVAD implantation or an LTA.

\section{Correlation between phenotypic variables and missing data imputation}

Figure 1 shows the study design including all data processing steps, performed statistical analysis and validation steps. The detailed description of these steps and used methods can be found in the Supplemental Methods. Table 1 includes all 47 clinical parameters which were measured in the DCM patients, see Supplemental Table 2 for an overview of the variable selection process. All 47 variables were used for data imputation using proximity from Random Forest for mixed-type data. CMR parameters were imputed based on their equivalent echocardiographic parameters (Suppl. Figure 1). The correlation matrix among variables is comparable between the imputed and non-imputed data, depicting high similarity (Suppl. Figure 2). 14 variables with more than 25\% missing data in the unimputed raw data were excluded from further analysis (Suppl. Figure 3). Only late gadolinium enhancement (LGE) was kept in the analysis despite $29.8 \%$ of missing data, due to the established clinical importance of this variable ${ }^{19}$. To prevent the inclusion of redundant variables, a correlation matrix of the clinical variables was created (Suppl. Figure 4). Five variables were excluded due to strong correlation (correlation coefficient $>0.6$ as proposed before, keeping the variable that was most informative and had the least missingness; Suppl. Table 2) ${ }^{12}$. This left 28 variables for the clustering analysis.

\section{Phenotypic clustering}

Phenotypic clusters were defined based on 28 clinical parameters without high multicollinearity using unsupervised hierarchical clustering of principal components (HCPC) approach, in which principle components of the clinical data were obtained with Factor Analysis for Mixed Data (FAMD), ensuring the balance of the influence between continuous and categorical variables. The optimal number of clusters was determined based on the gain in within-inertia (inside group variance) and clinical relevance. The over- or underrepresentation of character variables in each cluster were analyzed by v-test based on the hypergeometric distribution. The clustering was performed with FactoMineR package v1.41 in R. The patient groups created by the clustering were used as input for decision tree modeling to identify the key parameters able to distinguish the created groups. For this, conditional interference tree methods was used, a recursive binary partitioning with less overfitting and variable selection problems using the party package v1.3-3 in R. A detailed description of the used methods can be found in the Supplementals.

\section{Statistical analysis}

The link between clinical variables and the created clusters were analysed by ANOVA for numeric and $\chi^{2}$-test for categorical variables. The over- or underrepresentation of a variable within a cluster was analysed by v-test within the HCPC function, based on the hypergeometric distribution. A $p$-value $<0.05$ was considered as statistically significant.

For outcome analyses, we used unadjusted and multivariable adjusted Cox proportional hazards models to determine the independent association between patient groups and outcome. Primary outcome was the combined end-point of cardiovascular death, Htx, LVAD implantation, and/or a life-threatening arrhythmia. Covariates included in the 
multivariable model included variables known to be predictive of outcomes in DCM such as NT-proBNP, ejection fraction, age and gender. Kaplan-Meier survival curves were estimated, and differences between groups were assessed by the log-rank test, using time at diagnosis as time zero. Calculations were done using SPSS version 23.0 (SPSS Inc., Chicago, Illinois).

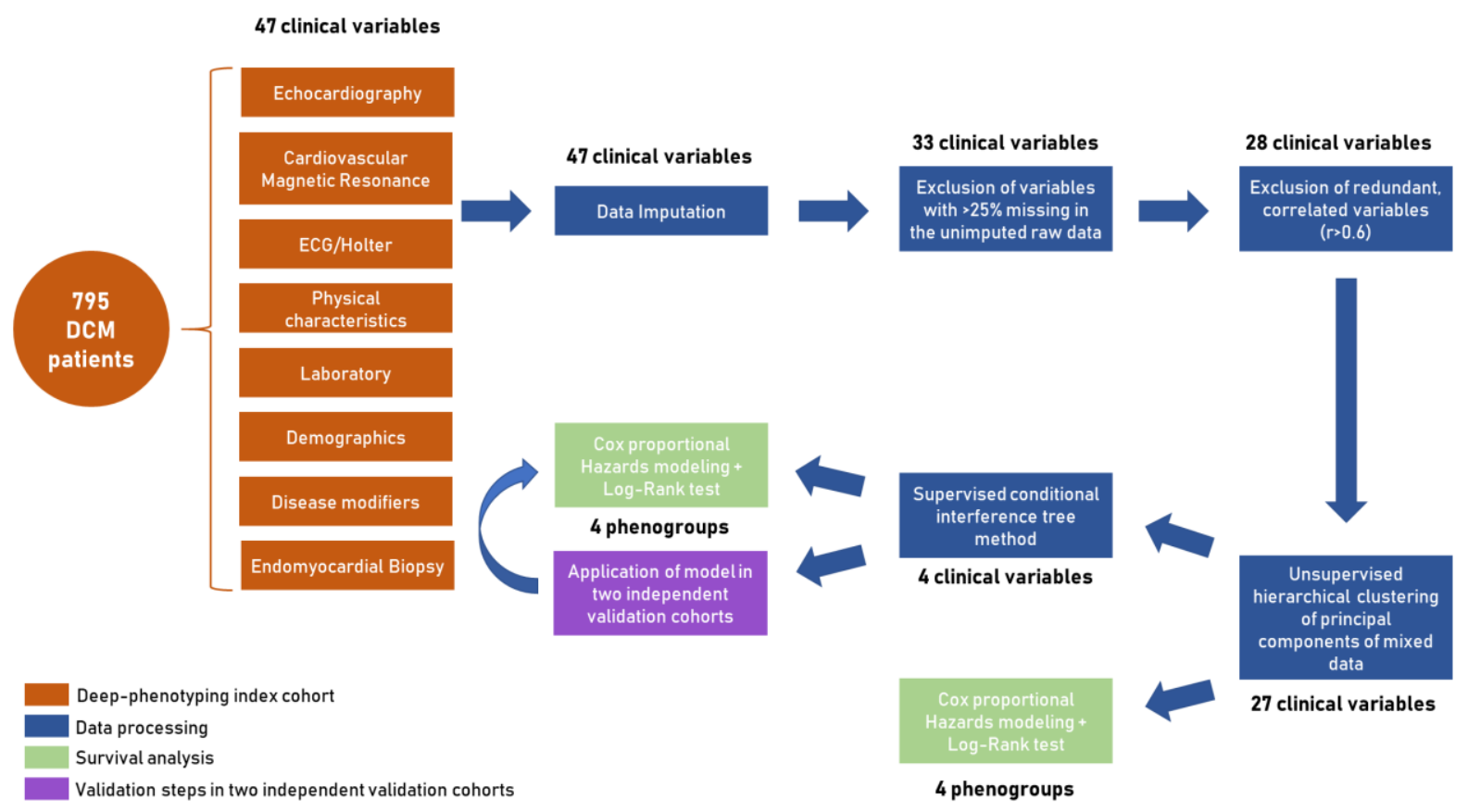

Figure 1. Summary of the study design including data processing steps, survival analysis and validation steps.

\section{RESULTS}

\section{Comparison of clinical characteristics among phenogroups}

Figure 1 summarizes the data processing of the clinical variables, selecting 28 variables as input for the cluster analysis (Figure 1; Table 1; Suppl. Table 2). Four was the minimal number of clusters which could accurately reflect the phenotypic variation in the index cohort (Figure 2). Twenty-seven of the 28 clinical input variables had a contribution in defining the patient clusters. Only the cardiac viral load in EMBs did not contribute (Figure 3; Suppl. Table 2). This led to the identification of four phenogroups with significant differences in their clinical characteristics (Table 2; Figure 3).

Phenogroup 1 ( $n=331 ; 42 \%)$ had a moderate reduction in left ventricular ejection fraction (LVEF; 43 $\pm 9 \%$ ) and overall smallest cardiac dimensions, lower NT-proBNP (16 [7-42] $\mathrm{pmol} / \mathrm{L})$ and NYHA class I and II (90\%) compared to the other patients. Phenogroup $4(\mathrm{n}=216$; 27\%) appears to be the opposite pole of severity, consisting of patients with a low LVEF $(23 \pm 8 \%)$, and the largest cardiac dimensions. More than half of the patients in this subgroup had NYHA III or IV (53\%).

Patients in phenogroup 2 and 3 more reflect the etiological diversity, irrespective of cardiac function and clinical presentation. Patients in phenogroup $2(n=83 ; 10 \%)$ consisted primarily of patients with an auto-immune disease (69\%), females (59\%) and chronic kidney disease (creatinine: 113 [87-149] $\mu \mathrm{mol} / \mathrm{L}$ ). In contrast, phenogroup 3 ( $\mathrm{n}=165 ; 21 \%)$ consisted mainly of males (88\%) with a high prevalence of cardiac arrhythmias (mainly AF $(61 \%)$ and 
NSVT (62\%)), pathogenic gene variants (57\%) and LGE at MRI (59\%). Two-third of the gene variants were pathogenic TTNtv and LMNA variants (Table 2 ).

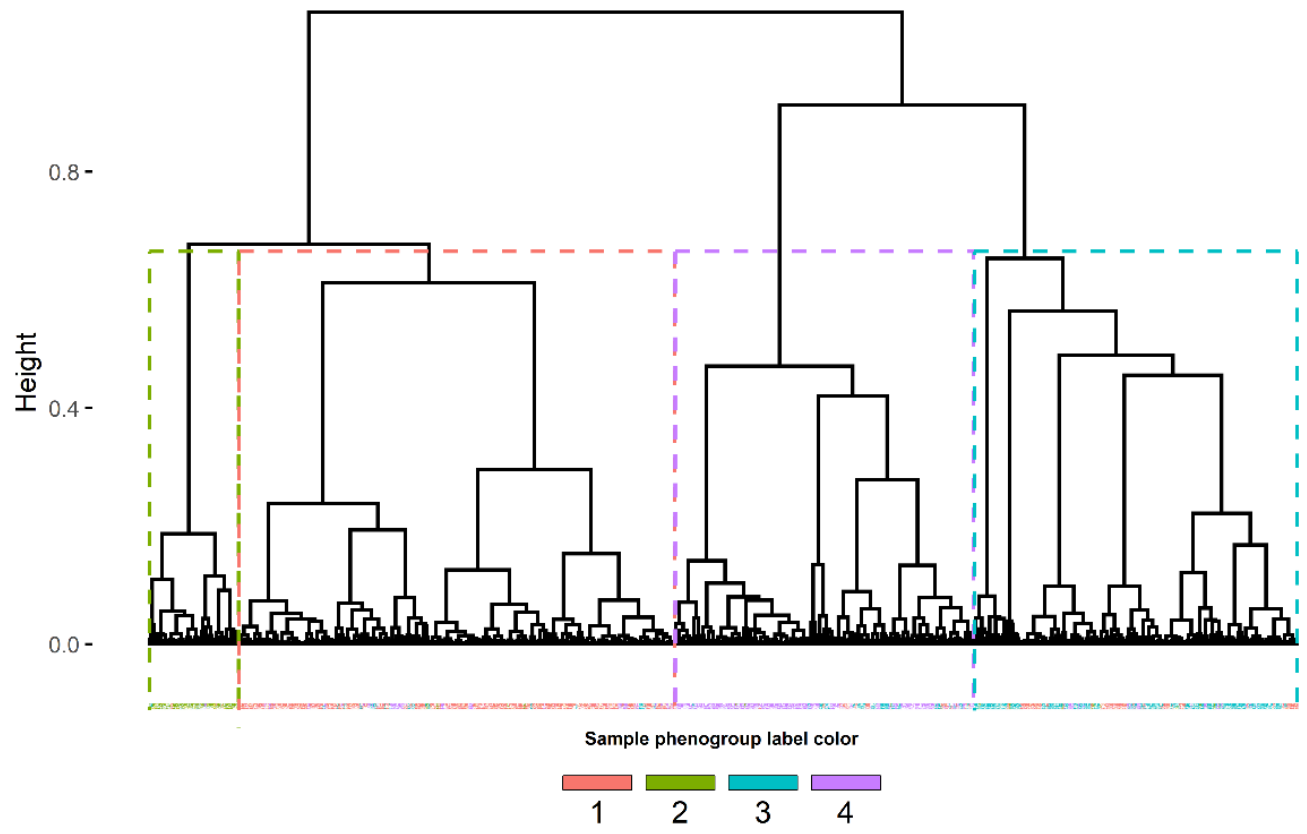

Figure 2. Hierarchical clustering for the identification of the optimal number of phenogroups. Four clusters yielded the highest gain in inertia (inside group variance).

Table 2. Clinical characteristics stratified by phenogroup

\begin{tabular}{|c|c|c|c|c|c|}
\hline Clinical Characteristic & $\begin{array}{l}\text { Group } 1 \\
(n=331)\end{array}$ & $\begin{array}{c}\text { Group } 2 \\
(n=83)\end{array}$ & $\begin{array}{l}\text { Group } 3 \\
(n=165)\end{array}$ & $\begin{array}{l}\text { Group } 4 \\
(n=216)\end{array}$ & P-value \\
\hline Age, $y$ & $50.5 \pm 13.1$ & $52.5 \pm 13.8$ & $57.9 \pm 11.5$ & $56.3 \pm 11.4$ & $<0.001$ \\
\hline Female, $n(\%)$ & $150(45)$ & 49 (59) & $20(12)$ & $77(36)$ & $<0.001$ \\
\hline \multicolumn{6}{|l|}{ Disease modifiers, $n(\%)$} \\
\hline Genetic mutation & 45 (14) & $9(11)$ & $94(57)$ & $27(13)$ & $<0.001$ \\
\hline TTNtv & $14(4)$ & $6(7)$ & $43(26)$ & $9(4)$ & $<0.001$ \\
\hline LMNA & $0(0)$ & $1(1)$ & $20(12)$ & $1(0)$ & $<0.001$ \\
\hline Other & $30(9)$ & $1(1)$ & $24(15)$ & $17(8)$ & $<0.001$ \\
\hline Multiple & $1(0)$ & $1(1)$ & $7(4)$ & $0(0)$ & $<0.001$ \\
\hline Familial disease & $59(18)$ & $15(20)$ & $78(41)$ & $48(23)$ & $<0.001$ \\
\hline Toxic trigger & $31(9)$ & $7(8)$ & $9(5)$ & $36(17)$ & 0.004 \\
\hline Auto-immune disease & $7(2)$ & $57(69)$ & $2(1)$ & $3(1)$ & $<0.001$ \\
\hline Cardiac inflammation & $50(15)$ & $16(19)$ & $20(12)$ & $44(20)$ & 0.14 \\
\hline Significant viral load & $31(9)$ & $11(13)$ & $15(9)$ & $25(12)$ & 0.63 \\
\hline Electrical trigger & $137(41)$ & $12(14)$ & $73(44)$ & $99(46)$ & $<0.001$ \\
\hline NYHA functional class $\geq I I I, n(\%)$ & $33(10)$ & $26(31)$ & $49(30)$ & $114(53)$ & $<0.001$ \\
\hline Hypertension, $n$ (\%) & $117(35)$ & $36(43)$ & $72(44)$ & $72(33)$ & 0.12 \\
\hline Body mass index, $\mathrm{kg} / \mathrm{m}^{2}$ & $26.8 \pm 5.2$ & $24.4 \pm 5.1$ & $28.3 \pm 4.4$ & $26.4 \pm 4.7$ & $<0.001$ \\
\hline Diabetes mellitus, $n$ (\%) & $22(7)$ & $14(17)$ & $21(13)$ & $29(13)$ & 0.012 \\
\hline Heart rate, bpm & $72.3 \pm 14.6$ & $80.1 \pm 18.1$ & $73.3 \pm 16.6$ & $77.7 \pm 17.2$ & $<0.001$ \\
\hline Systolic blood pressure, $\mathrm{mm} \mathrm{Hg}$ & $134.6 \pm 19.5$ & $136.9 \pm 26.3$ & $135.8 \pm 19.6$ & $128 \pm 19.6$ & $<0.001$ \\
\hline Diastolic blood pressure, $\mathrm{mm} \mathrm{Hg}$ & $78.6 \pm 12$ & $79.9 \pm 13.7$ & $80.3 \pm 12.6$ & $78.4 \pm 12.5$ & 0.39 \\
\hline \multicolumn{6}{|l|}{ Echocardiography } \\
\hline LV Ejection fraction, \% & $37.6 \pm 8.6$ & $33.4 \pm 10.2$ & $32.7 \pm 9.7$ & $21.8 \pm 7.2$ & $<0.001$ \\
\hline LVEDD index, $\mathrm{mm} / \mathrm{m}^{2}$ & $29 \pm 3.7$ & $30.9 \pm 5.3$ & $28.9 \pm 3.8$ & $34.7 \pm 5.2$ & $<0.001$ \\
\hline LVESD, $\mathrm{mm}$ & $45 \pm 7.3$ & $46 \pm 9.5$ & $49 \pm 8.9$ & $60 \pm 8.8$ & $<0.001$ \\
\hline
\end{tabular}




\begin{tabular}{|c|c|c|c|c|c|}
\hline LA Volume index, $\mathrm{mL} / \mathrm{m}^{2}$ & $39.2 \pm 13.8$ & $43.6 \pm 16.8$ & $53.2 \pm 16.5$ & $50.3 \pm 19.1$ & $<0.001$ \\
\hline LV Mass index, $\mathrm{g} / \mathrm{m}^{2}$ & $95.4 \pm 22.9$ & $108.1 \pm 32$ & $107 \pm 25$ & $140.2 \pm 36$ & $<0.001$ \\
\hline$E / A$ ratio & $1.1 \pm 0.5$ & $1.1 \pm 0.5$ & $1.4 \pm 0.9$ & $1.2 \pm 0.8$ & 0.39 \\
\hline$E / e^{\prime}$ ratio & $7.5 \pm 2.7$ & $11.6 \pm 5.2$ & $10.8 \pm 5.1$ & $13.1 \pm 4.3$ & $<0.001$ \\
\hline Posterior WT, $\mathrm{mm}$ & $8.6 \pm 1.3$ & $9.1 \pm 1.8$ & $9.1 \pm 1.6$ & $9.4 \pm 1.6$ & $<0.001$ \\
\hline Intraventricular ST, $\mathrm{mm}$ & $8.7 \pm 1.5$ & $9.1 \pm 2.2$ & $9.6 \pm 2$ & $9.2 \pm 1.7$ & $<0.001$ \\
\hline \multicolumn{6}{|l|}{ Laboratory data } \\
\hline NT-pro BNP, pmol/L & $\begin{array}{c}16 \\
{[7-42]}\end{array}$ & $\begin{array}{c}102 \\
{[39-520]}\end{array}$ & $\begin{array}{c}94 \\
{[39-230]}\end{array}$ & $\begin{array}{c}60 \\
{[19-229]}\end{array}$ & $<0.001$ \\
\hline Hs-Troponin T, ng/L & $8[4-12]$ & 19 [9-48] & 18 [10-32] & 17 [10-36] & $<0.001$ \\
\hline Creatinine, $\mu \mathrm{mol} / \mathrm{L}$ & $\begin{array}{c}83 \\
{[73-94]}\end{array}$ & $\begin{array}{c}113 \\
{[87-149]}\end{array}$ & $\begin{array}{c}103 \\
{[89-120]}\end{array}$ & $\begin{array}{c}97 \\
{[84-113]}\end{array}$ & $<0.001$ \\
\hline sIL-2R elevation, $n(\%)$ [488] & 39 (19) & $38(60)$ & $17(20)$ & $40(29)$ & $<0.001$ \\
\hline Neopterin elevation, $n$ (\%) [485] & $46(22)$ & $38(62)$ & $29(35)$ & $52(39)$ & $<0.001$ \\
\hline Cardiac auto-antibodies, $n$ (\%) [505] & $4(2)$ & $8(12)$ & $4(4)$ & $5(4)$ & 0.007 \\
\hline C-reactive protein, $\mathrm{mg} / \mathrm{L}$ & $2[1-5]$ & $5[2-11]$ & $2[1-5]$ & $3[1-11]$ & $<0.001$ \\
\hline$A S A T, U / L$ & 23 [19-28] & $24[19-28]$ & $28[22-36]$ & 26 [21-33] & $<0.001$ \\
\hline$A L A T, U / L$ & 24 [19-34] & 20 [15-27] & $32[24-42]$ & 27 [19-40] & $<0.001$ \\
\hline \multicolumn{6}{|l|}{ ECG/Holter, $n(\%)$} \\
\hline Atrial fibrillation & $43(13)$ & $17(20)$ & $100(61)$ & $31(14)$ & $<0.001$ \\
\hline Non-sustained VT & $49(15)$ & $16(19)$ & $103(62)$ & $60(28)$ & $<0.001$ \\
\hline Left bundle branch block & $94(28)$ & $8(10)$ & $40(24)$ & $83(38)$ & $<0.001$ \\
\hline Atrioventricular block & $26(8)$ & $7(8)$ & $42(25)$ & $21(10)$ & $<0.001$ \\
\hline Out of hospital cardiac arrest & $12(4)$ & $2(2)$ & $24(15)$ & $13(6)$ & $<0.001$ \\
\hline \multicolumn{6}{|l|}{ Cardiovascular $M R(n=558)$} \\
\hline LV Mass index, $\mathrm{g} / \mathrm{m}^{2}$ & $62.7 \pm 15.4$ & $66.8 \pm 24.3$ & $66.4 \pm 14.8$ & $94.2 \pm 27.4$ & $<0.001$ \\
\hline LV EDV index, $\mathrm{mL} / \mathrm{m}^{2}$ & $110.1 \pm 24.6$ & $113.5 \pm 31$ & $115.1 \pm 28.8$ & $178.1 \pm 52.5$ & $<0.001$ \\
\hline LVESV index, $\mathrm{mL} / \mathrm{m}^{2}$ & $63.4 \pm 21$ & $73.8 \pm 31.5$ & $75.1 \pm 27.4$ & $138.7 \pm 51.6$ & $<0.001$ \\
\hline LV Stroke volume index, $\mathrm{mL} / \mathrm{m}^{2}$ & $46.8 \pm 11$ & $40.1 \pm 11$ & $40.3 \pm 12.5$ & $39 \pm 12.8$ & $<0.001$ \\
\hline LV Ejection fraction, \% & $42.9 \pm 8.8$ & $37.4 \pm 12.5$ & $36.1 \pm 11.1$ & $22.8 \pm 8.2$ & $<0.001$ \\
\hline Late gadolinium enhancement, $n$ (\%) & $41(17)$ & $33(52)$ & $68(59)$ & $42(29)$ & $<0.001$ \\
\hline \multicolumn{6}{|l|}{ Endomyocardial biopsy } \\
\hline Collagen volume fraction, \%/area & $4.7[2-7]$ & $6[3-10]$ & $6[3-11]$ & $6.2[3-10]$ & 0.006 \\
\hline
\end{tabular}

Categorical variables are presented as counts and percentages; continuous variables are presented as mean $\pm S D$; and right-skewed variables are presented as median $\left(25^{\text {th }}-75^{\text {th }}\right.$ percentile). TTNtv indicates truncating titin variants; LMNA, lamin A/C; NYHA, New York Heart Association; $L V$, left ventricular; EDD, end-diastolic diameter; ESD, end-systolic diameter; LA, left atrial; WT, wall thickness; ST, septum thickness; sIL-2R, soluble interleukin-2 receptor; ASAT, aspartate transaminase; ALAT, alanine transaminase; VT, ventricular tachycardia; MR, magnetic resonance; EDV, end-diastolic volume; ESV, end-systolic volume.Association of phenogroups with prognostic outcome

We tested whether the four different phenogroups were associated with the combined outcome measure as defined by cardiovascular death, Htx, LVAD implantation or the occurrence of an LTA. Phenogroup 1 has the lowest, and phenogroup 3 the highest risk for an adverse event with a 5 -year risk of 8 and $32 \%$ respectively ( $p<0.001$; Figure 4; Table 3; Suppl. Figure 5). Both phenogroups 2 and 4 shared a high-risk profile with a 5-years risk of 24 and $29 \%$, respectively. Phenogroup 3 remained a high-risk subgroup, even after correction of established prognostic factors such as NT-proBNP, age, gender and LVEF (Table 3). Overall, the prognosis of the phenogroups 2, 3 and 4 were comparable (Figure 4), reflecting the influence of etiological fraction (group 2: auto-immune disease group 3: genetics) on prognosis irrespective of cardiac function (group 4). However, patients classified in phenogroup 3 had specifically an increased risk for LTAs compared to the other phenogroups (5-years risk of $27 \%$ versus $6 \%, 14 \%$ and $14 \%$ for phenogroups 1,2 and 4 respectively; Table 3, Suppl. Figure 6). 
Table 3. Association of phenogroups with adverse outcomes on cox proportional hazards analysis

\begin{tabular}{|c|c|c|c|c|c|}
\hline & $\begin{array}{l}\text { Group } 1 \\
(n=331) \\
\end{array}$ & $\begin{array}{c}\text { Group } 2 \\
(n=83)\end{array}$ & $\begin{array}{l}\text { Group } 3 \\
(n=165) \\
\end{array}$ & $\begin{array}{l}\text { Group } 4 \\
(n=216) \\
\end{array}$ & P-value \\
\hline \multicolumn{6}{|l|}{ Outcome, $n(\%)$} \\
\hline $\begin{array}{l}\text { Life-threatening } \\
\text { arrhythmias }\end{array}$ & $13(4)$ & $9(11)$ & 35 (21) & $20(9)$ & $<0.001$ \\
\hline CV Death/Htx/LVAD & $6(2)$ & $14(17)$ & $28(17)$ & $26(12)$ & $<0.001$ \\
\hline Combined end point & $19(6)$ & $19(23)$ & $54(33)$ & 42 (19) & $<0.001$ \\
\hline \multicolumn{6}{|l|}{ Unadjusted HR (95\% Cl) } \\
\hline $\begin{array}{l}\text { Life-threatening } \\
\text { arrhythmias }\end{array}$ & 1.0 & $2.9(1.2-6.7)^{*}$ & $5.5(2.9-10.4) \ddagger$ & $2.4(1.2-4.9)^{*}$ & $\cdots$ \\
\hline CV Death/Htx/LVAD & 1.0 & $9.0(3.4-23.3) \ddagger$ & $8.7(3.6-21) \ddagger$ & $7.4(3.1-18.1) \ddagger$ & $\ldots$ \\
\hline Combined end point & 1.0 & $4.0(2.1-7.5) \ddagger$ & $6.0(3.6-10.1) \ddagger$ & $3.9(2.3-6.7) \ddagger$ & $\ldots$ \\
\hline \multicolumn{6}{|l|}{ Adjusted HR $(95 \% \mathrm{Cl})$} \\
\hline $\begin{array}{l}\text { Life-threatening } \\
\text { arrhythmias }\end{array}$ & 1.0 & $2.9(1.1-7.1)^{*}$ & 6.0 (2.9-12.5)‡ & $2.4(1.1-5.9)^{*}$ & $\cdots$ \\
\hline CV Death/Htx/LVAD & 1.0 & $5.8(1.9-17.2)^{\dagger}$ & $6.9(2.5-19.2) \ddagger$ & $5.6(1.9-16.3)^{\dagger}$ & $\ldots$ \\
\hline Combined end point & 1.0 & $3.1(1.5-6.4)^{\dagger}$ & $5.5(3.0-9.9) \ddagger$ & $3.4(1.8-6.7) \ddagger$ & $\ldots$ \\
\hline
\end{tabular}

Adjusted analysis include the phenogroups + NT-proBNP + age + gender + ejection fraction.

$\mathrm{Cl}$ indicates confidence interval; $C V$, cardiovascular; $H t x$, heart transplantation; LVAD, left ventricular assist device; and HR, hazard ratio. ${ }^{*} P<0.05 ;+P<0.01 ; \neq P<0.001$.

\section{Supervised decision tree modelling to enhance clinical utility}

We applied supervised decision tree modelling to identify the key clinical variables of the created phenogroups. Narrowing down the needed number of clinical variables to the key parameters enhances the clinical applicability to other DCM cohorts. The presence of an autoimmune disease in the clinical history, LVEF, AF and creatinine were the most important four variables characteristic for these phenogroups with a combined accuracy of $71 \%$ (Suppl. Figure 7; Suppl. Table 3). However, placing patients in phenogroup 2 had an accuracy of 95\%, mostly by the presence of an auto-immune disease (Suppl. Table 3). The decision tree has the most difficulty in recognizing patients from phenogroup 3, mainly due to the placement is completely independent of LVEF (node 5 and 9 in Suppl. Figure 7; accuracy of $80 \%$ Suppl. Table 3).

\section{Validation of the Phenomapping analysis}

To validate the clinical utility of the supervised model, we applied the algorithm constituting of 4 clinical parameters on the index and two external, independent DCM cohorts (Table 4). The Spanish validation cohort was younger and constituted mainly of males, compared to the other cohorts. Cardiac function was the highest in the cohort of Maastricht, but was overall comparable among the cohorts. The decision tree model was able to successfully match every patient in one of the four phenogroups in all three cohorts (Table 4). The event rate of LTA was comparable over the cohorts $(p=0.49)$, but the two validation cohorts had a higher rate of cardiovascular death/Htx/LVAD ( $p<0.001)$.

The phenogrouping was associated with event-free survival in all cohorts $(p<0.001$; Figure 5). Phenogroup 1 was associated with the lowest event rate, in line with the unsupervised model (Figure 4). The survival curve of the Trieste cohort strongly resemble the curve of the unsupervised model (Figure 4), with phenogroup 3 having the worst outcome and phenogroup 2 and 4 as an intermediate group. The survival curves of the supervised model in Maastricht and Madrid are very similar, showing a high event rate in phenogroups 2, 3 and 4 (Figure 5). 


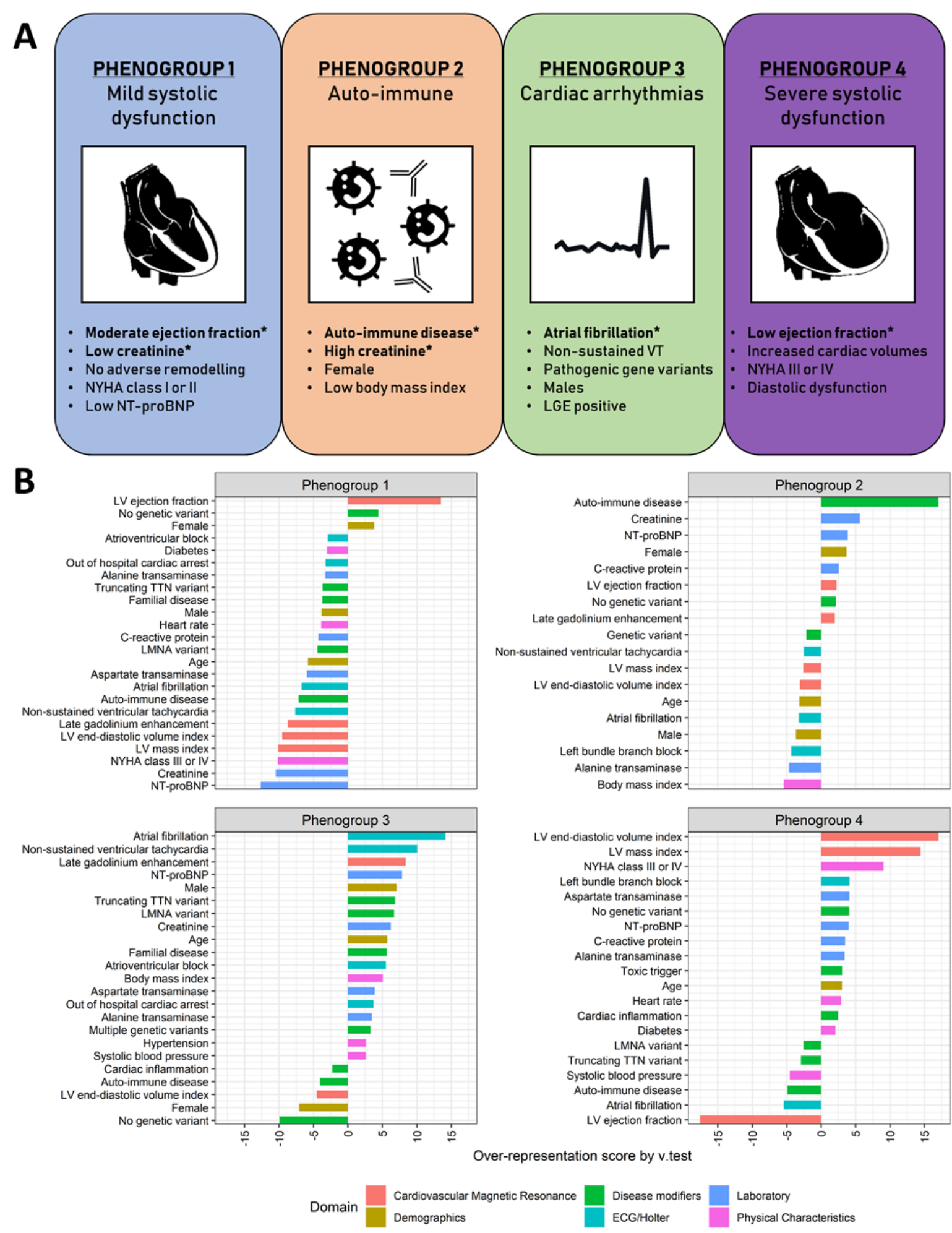

Figure 3. Four mutually exclusive phenogroups as determined by HCPC using phenotypical information as input. The most distinct clinical characteristics are listed per phenogroup. Variables with an asterisk are key parameters to distinguish the phenogroups, as selected by supervised decision tree modeling (A). Characteristic plots of the four proposed phenogroups including their most representative clinical variables. The over- or underrepresentation of a variable within a cluster was analyzed by v-test within the HCPC function, based on the hypergeometric distribution. A positive value indicates overrepresentation of this variable in the applicable phenogroup, a negative value indicates underrepresentation of the corresponding variable (B). 


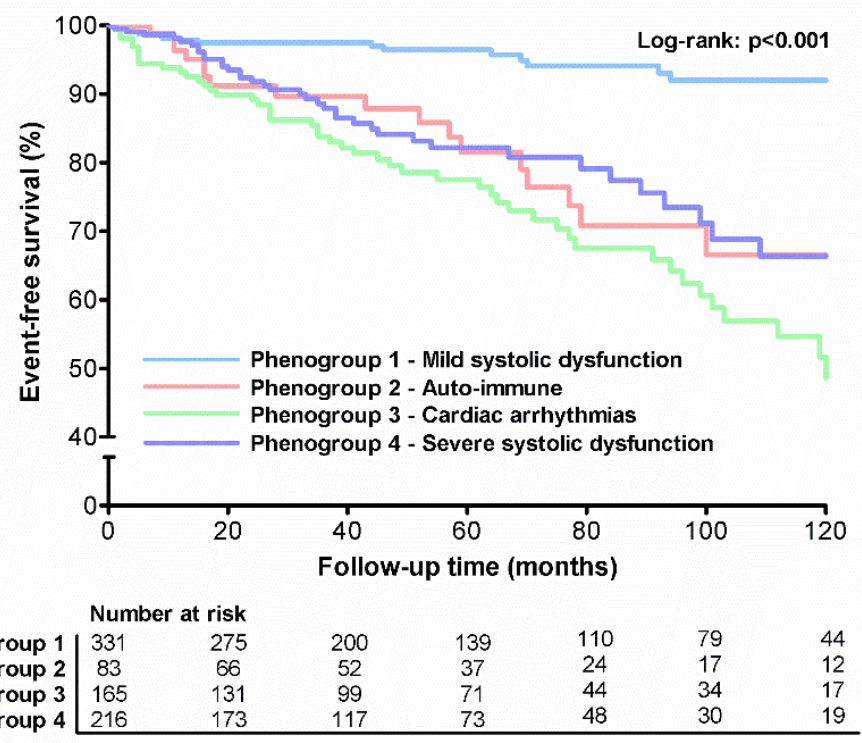

Figure 4. Event-free survival stratified by phenogroup. Kaplan-Meier curves for the combined outcome of lifethreatening arrhythmias, cardiovascular death, heart transplantation, or left ventricular assist device implantation stratified by phenogroup.

Table 4. Overview of the clinical characteristics of the supervised decision tree model, and outcome in the three DCM patient cohorts.

\begin{tabular}{ccccc} 
& $\begin{array}{c}\text { Index cohort, } \\
\text { Maastricht } \\
(\mathrm{n}=795)\end{array}$ & $\begin{array}{c}\text { Validation cohort, } \\
\text { Madrid } \\
(\mathrm{n}=352)\end{array}$ & $\begin{array}{c}\text { Validation cohort, } \\
\text { Trieste } \\
(\mathrm{n}=437)\end{array}$ & P-value \\
\hline Cohort demographics & & & & \\
Age, $y$ & $53.3 \pm 12.8$ & $49.2 \pm 25.6$ & $53.1 \pm 14.5$ & 0.0036 \\
Female, $n(\%)$ & $297(37)$ & $78(22)$ & $148(34)$ & $<0.001$ \\
Atrial fibrillation & $192(24)$ & $110(31)$ & $105(24)$ & 0.025 \\
Auto-immune disease & $69(9)$ & $32(9)$ & $29(7)$ & 0.36 \\
CMR, left ventricular ejection & $35.8 \pm 12.7$ & $32.7 \pm 11.7$ & $33.6 \pm 10.9$ & $<0.001$ \\
fraction & $92[79-110]$ & $88[71-106]$ & $85[72-99]$ & $<0.001$ \\
Creatinine, Hmol/L & & & & $174(40)$ \\
Decision tree algorithm & $305(38)$ & $125(36)$ & $29(7)$ & 0.46 \\
Phenogroup 1 & $69(9)$ & $32(9)$ & $112(26)$ & 0.36 \\
Phenogroup 2 & $211(27)$ & $108(31)$ & $122(28)$ & 0.59 \\
Phenogroup 3 & $210(26)$ & $87(25)$ & $35(8)$ & 0.49 \\
Phenogroup 4 & & & $89(20)$ & $<0.001$ \\
Outcome & $77(10)$ & $28(8)$ & $109(25)$ & $<0.001$ \\
\hline CV Death/Htx/LVAD & $74(9)$ & $68(19)$ & $89(25)$ &
\end{tabular}

Categorical variables are presented as counts and percentages; continuous variables are presented as mean $\pm S D$; and right-skewed variables are presented as median $\left(25^{\text {th }}-75^{\text {th }}\right.$ percentile).

CMR indicates cardiac magnetic resonance imaging; $C V$, cardiovascular; Htx, heart transplantation; LVAD, left ventricular assist device 


\section{Simplified adaptation of the decision tree from Supplemental Figure 7}

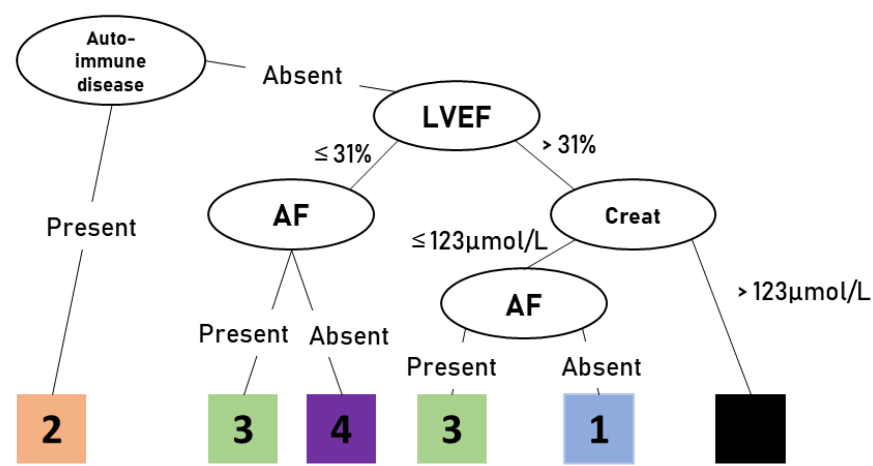

Survival curves after applying the decision tree model on the cohort
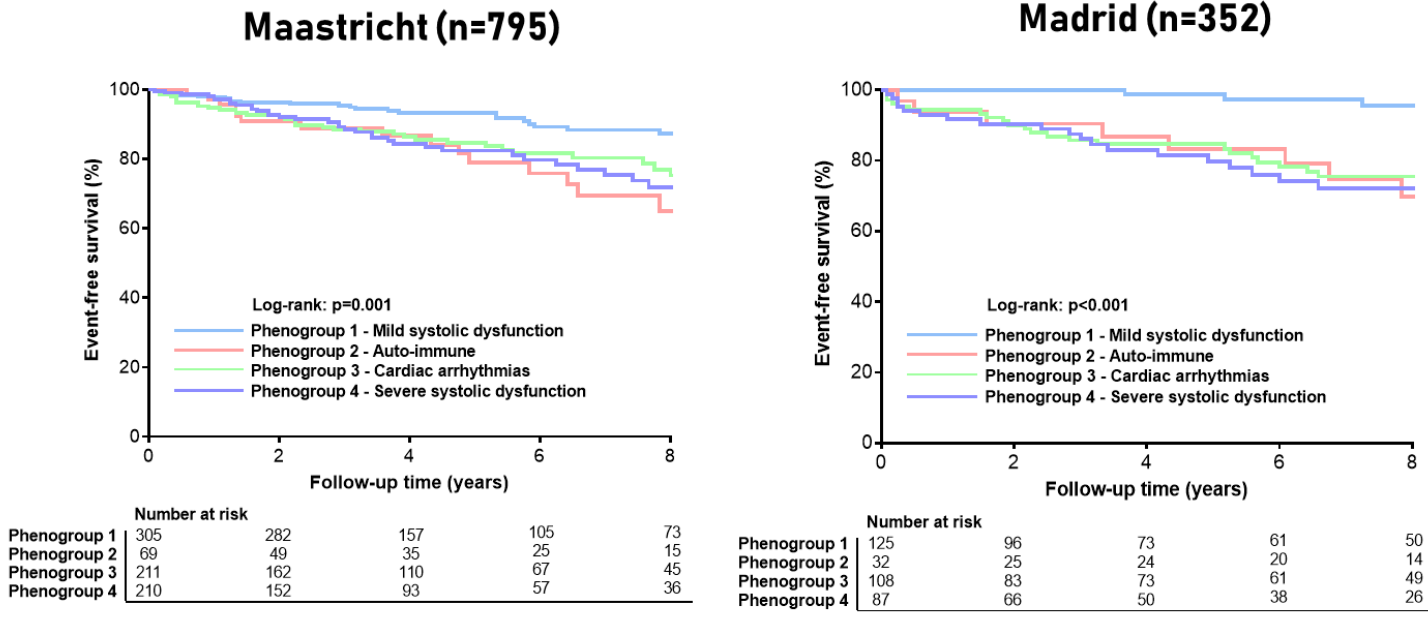

\section{Trieste $(n=437)$}

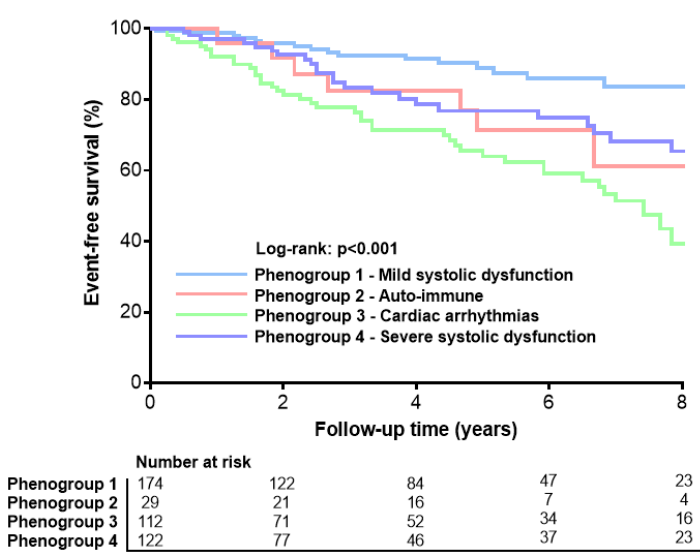

Figure 5. A simplified adaptation of the decision tree model shows the clinical parameters which are the core of the phenogroups. The black box indicates that the model cannot accurately place the patient in one of the four phenogroups (see Supplemental Figure 7 for exact accuracy) (Top). Event-free survival stratified by phenogroup as determined by the supervised decision tree model. Kaplan-Meier curves for the combined outcome of lifethreatening arrhythmias, cardiovascular death, heart transplantation, or left ventricular assist device implantation stratified by phenogroup in the index cohort (Maastricht) and both validation cohorts (Madrid and Trieste) (Bottom). 


\section{DISCUSSION}

This study addressed the feasibility and clinical validity of clustering methods in a cohort of 795 deep-phenotyped heterogenous DCM patients, with subsequent validation in two independent DCM cohorts. Clustering resulted in novel patient groups with unique clinical characteristics, which reflect the integration of cardiac function, comorbidities and underlying etiology. Two subgroups have a strong etiological contribution (auto-immune disease and genetics), showing the importance of etiology on the clinical course in DCM.

\section{Identified phenogroups in a heterogeneous DCM dataset}

The road to a more targeted approach in DCM, starts with clustering patients together who are likely to have a shared biological pathophysiology ${ }^{1}$. This is emphasized by a recent posthoc analysis of the POSEIDON-DCM trial, which showed a genetic etiology in a large percentage of non-responders ${ }^{20}$. Subsequently, a proposed model combining etiology, comorbidities and cardiac function, should be applicable in practice, thus having a low number of variables.

We identified four mutually exclusive phenogroups: [1] younger patients with mild systolic dysfunction, small cardiac dimensions and relatively few symptoms; [2] young females with a clinical history of auto-immune disease with cardiac and renal involvement; [3] males with a genetic mutation and prevalent ventricular and supraventricular arrhythmias; and [4] patients with severe systolic dysfunction, large cardiac dimensions, moderate diastolic dysfunction and pronounced symptoms. Interestingly, these phenogroups were associated with different clinical trajectories and adverse outcomes, irrespective of classic risk factors such as age, gender, NT-proBNP and ejection fraction. Furthermore, supervised decision tree modelling identified four parameters that are sufficient to adequately cluster patients: ejection fraction (1 and 4), auto-immune disease and creatinine (2), and atrial fibrillation (3). It is important to note that application of the algorithm on the index and both validation cohorts rendered comparable prognostic implications for the patients in the phenogroups. This implies that cardiac function is insufficient to predict patient outcome, as patients are placed in phenogroup 2 and 3 independent of their LVEF.

\section{Clinical validity of created phenogroups}

The present study puts forward a novel approach of DCM patient classification based on integrating extensive, but well-accessible diagnostic tools. The variety of underlying disease mechanisms in DCM could be an important reason for the variation in treatment efficacy in clinical trials patients ${ }^{120}$. The main goal of the present study was to find patterns in easily accessible clinical data of DCM patients to create valid patient subgroups, possibly reflecting a shared pathophysiology.

Phenogroup 1 and 4 represent two opposites of DCM severity, which is mainly reflected in their LVEF. Phenogroup 2 and 3 seem distinct disease subgroups independent of LVEF. Cardiac involvement in patients with a clinical history of auto-immune disease was associated with a relatively high-risk profile (phenogroup 2). Irreversible cardiac injury due to chronic or past myocardial inflammation, as well as late recognition and under-treatment, may explain this worse outcome ${ }^{21}$. Our findings emphasize immune-mediated DCM as a separate entity, underscoring the need for systematic cardiac screening in patients with a systemic disease, and the need of intense immunomodulatory therapy if cardiac involvement is present ${ }^{21}$. Immune-mediated disease comprises multiple diseases varying from complete autoimmunity to autoinflammatory as described in detail in the position statement of the ESC 
21. The broad spectrum of diseases could explain differences in outcome within phenogroup

2. For example, the autoimmune disease rheumatoid arthritis will affect the heart in a different way compared to sarcoidosis, an autoinflammatory disease.

Electrical phenotypes such as AF are often associated with genetic variants, forming the basis of phenogroup $3{ }^{2}$. Finding the gene variant which causes the electrical endophenotype may help to provide more targeted therapy in otherwise HF-therapy resistant patients. This is exemplified in DCM patients with a pathogenic lamin A/C (LMNA) variant, in which hyperactivation of the p38 $\alpha$ signalling pathway plays a central pathophysiological role ${ }^{22}$. ARRY-797, an oral, selective p38 inhibitor is currently under investigation in DCM patients with a $L M N A$ variant in a randomized, double-blind, placebo-controlled phase 3 trial (NCT03439514). In line, post-hoc genetic analysis in a recent DCM trial showed that the genetic profile predicted lack of treatment responsiveness, with genetic-positive DCM less likely to benefit from treatment ${ }^{20}$. Patients in phenogroup 3 had the highest risk profile, for LTA as well as CV death or heart transplantation. The annual risk for an LTA was $5 \%$ for patients in phenogroup 3, which is five times higher compared to DCM patients in phenogroup 1 despite having comparable LVEF. It is not unexpected that this phenogroup had the highest prevalence of $L M N A$ variants and NSVTs, both known risk factors for LTA ${ }^{23}$. This could indicate that patients in this specific phenogroup could benefit the most from prophylactic ICD therapy before advanced LV dysfunction, shedding new light on the outcome of the DANISH trial $^{924}$.

\section{Future directions of dilated cardiomyopathy patient care}

Although there was much emphasis on defining and classifying DCM to enhance clinical utility, the effect on clinical decision-making remains elusive. We acknowledge that the current approach is a major departure from the classical studies, but it is a first attempt to a more personalized approach of the DCM patient in everyday clinical practice. The clinically homogeneous phenogroups created in this study, can form the basis to investigate their shared pathobiology. Additional multi-level molecular profiling such as proteomics, metabolomics and epigenomics in cardiac samples or blood, could provide a more-value in DCM clinical and risk stratification. The addition of this molecular information to enrich phenomapping should be further explored in DCM, as previously performed in other diseases ${ }^{25}$. Application of patient clustering as a tool to guide clinical decision-making, should be further explored in prospective studies. Besides a refined DCM clustering and ensuing targeted therapies, an additional goal would be the development of a comprehensive DCM risk score for sudden cardiac death and LTA, as already available for hypertrophic cardiomyopathy ${ }^{26}$.

\section{Limitations and perspectives}

In this study, we chose to start with a complete unsupervised approach with hierarchical kmeans clustering of principal components to look in an unbiased way for underlying clinical patterns. Although, HC is sensitive to the input data, the HCPC method allows us to use principal components instead of individual variables and maximize robustness by an additional k-means consolidation step. This is a different strategy from model-based clustering methods, which are semi-supervised. The analysis of the registries are of a retrospective nature, although patients are included prospectively. The results and clinical implications of this study needs therefore to be validated in a prospective manner, not only 
for predicting outcome, but also for its more value in predicting the response to existing or novel HF therapies.

The definition of systemic immune-mediated diseases (based on the recent position statement of the $\mathrm{ESC}^{21}$ ), includes a broad spectrum of immune-mediated diseases with diverse implication in cardiac disease development. Larger registries studying this specific immune-mediated subset of DCM patients are needed to be able to look at the specific immune-mediated diseases and their impact on long-term outcome.

\section{CONCLUSIONS}

The present study identified four different DCM phenogroups associated with significant differences in clinical presentation and adverse outcome in three independent European DCM cohorts. The phenogroups highlight the etiological contribution to the phenotype, and pave the way towards a more personalized approach. 


\section{REFERENCES}

1. Verdonschot JAJ, Hazebroek MR, Ware JS, et al. Role of Targeted Therapy in Dilated Cardiomyopathy: The Challenging Road Toward a Personalized Approach. Journal of the American Heart Association 2019;8(11):e012514.

2. Sinagra G, Elliott PM, Merlo M. Dilated cardiomyopathy: so many cardiomyopathies! Eur Heart J 2019

3. Merlo M, Cannata A, Gobbo M, et al. Evolving concepts in dilated cardiomyopathy. Eur J Heart Fail 2018;20(2):228-39.

4. Dal Ferro M, Stolfo D, Altinier A, et al. Association between mutation status and left ventricular reverse remodelling in dilated cardiomyopathy. Heart (British Cardiac Society) 2017

5. Merlo M, Pyxaras SA, Pinamonti B, et al. Prevalence and prognostic significance of left ventricular reverse remodeling in dilated cardiomyopathy receiving tailored medical treatment. J Am Coll Cardiol 2011;57(13):146876.

6. Verdonschot JAJ, Hazebroek MR, Wang P, et al. Clinical Phenotype and Genotype Associations With Improvement in Left Ventricular Function in Dilated Cardiomyopathy. Circulation Heart failure 2018;11(11):e005220.

7. Bozkurt B, Colvin M, Cook J, et al. Current Diagnostic and Treatment Strategies for Specific Dilated Cardiomyopathies: A Scientific Statement From the American Heart Association. Circulation 2016;134(23):e579-e646.

8. Priori SG, Blomstrom-Lundqvist C, Mazzanti A, et al. 2015 ESC Guidelines for the management of patients with ventricular arrhythmias and the prevention of sudden cardiac death: The Task Force for the Management of Patients with Ventricular Arrhythmias and the Prevention of Sudden Cardiac Death of the European Society of Cardiology (ESC). Endorsed by: Association for European Paediatric and Congenital Cardiology (AEPC). Eur Heart J 2015;36(41):2793-867.

9. Ponikowski P, Voors AA, Anker SD, et al. 2016 ESC Guidelines for the diagnosis and treatment of acute and chronic heart failure: The Task Force for the diagnosis and treatment of acute and chronic heart failure of the European Society of Cardiology (ESC)Developed with the special contribution of the Heart Failure Association (HFA) of the ESC. Eur Heart J 2016;37(27):2129200.

10. Verdonschot JAJ, Hazebroek MR, Derks KWJ, et al. Titin cardiomyopathy leads to altered mitochondrial energetics, increased fibrosis and long-term life-threatening arrhythmias. Eur Heart J 2018

11. Gigli M, Merlo M, Graw SL, et al. Genetic Risk of Arrhythmic Phenotypes in Patients With Dilated Cardiomyopathy. J Am Coll Cardiol 2019;74(11):1480-90.

12. Shah SJ, Katz DH, Selvaraj S, et al. Phenomapping for novel classification of heart failure with preserved ejection fraction. Circulation 2015;131(3):269-79.

13. Hinton GE, Salakhutdinov RR. Reducing the dimensionality of data with neural networks. Science (New York, NY) 2006;313(5786):504-7.

14. Cheng WY, Ou Yang TH, Anastassiou D. Development of a prognostic model for breast cancer survival in an open challenge environment. Science translational medicine 2013;5(181):181ra50.

15. Richardson $\mathrm{P}$, McKenna W, Bristow $M$, et al. Report of the 1995 World Health Organization/International Society and Federation of Cardiology Task Force on the Definition and Classification of cardiomyopathies. Circulation 1996;93(5):84142.

16. Pinto YM, Elliott PM, Arbustini E, et al. Proposal for a revised definition of dilated cardiomyopathy, hypokinetic non-dilated cardiomyopathy, and its implications for clinical practice: a position statement of the ESC working group on myocardial and pericardial diseases. Eur Heart J 2016;37(23):1850-8.

17. Merlo $M$, Pivetta A, Pinamonti B, et al. Long-term prognostic impact of therapeutic strategies in patients with idiopathic dilated cardiomyopathy: changing mortality over the last 30 years. Eur J Heart Fail 2014;16(3):317-24.

18. Ware JS, Amor-Salamanca A, Tayal U, et al. Genetic Etiology for Alcohol-Induced Cardiac Toxicity. J Am Coll Cardiol 2018;71(20):2293-302.

19. Becker MAJ, Cornel JH, van de Ven PM, et al. The Prognostic Value of Late Gadolinium-Enhanced Cardiac Magnetic Resonance Imaging in Nonischemic Dilated Cardiomyopathy: A Review and Meta-Analysis. JACC Cardiovascular imaging 2018;11(9):1274-84.

20. Rieger AC, Myerburg RJ, Florea V, et al. Genetic determinants of responsiveness to mesenchymal stem cell injections in non-ischemic dilated cardiomyopathy. EBioMedicine 2019;48:377-85.

21. Caforio ALP, Adler $Y$, Agostini $C$, et al. Diagnosis and management of myocardial involvement in systemic immune-mediated diseases: a position statement of the European Society of Cardiology Working Group on Myocardial and Pericardial Disease. Eur Heart J 2017;38(35):2649-62. 
22. Muchir A, Wu W, Choi JC, et al. Abnormal p38alpha mitogen-activated protein kinase signaling in dilated cardiomyopathy caused by lamin A/C gene mutation. Human molecular genetics 2012;21(19):4325-33.

23. Hasselberg NE, Haland TF, Saberniak J, et al. Lamin A/C cardiomyopathy: young onset, high penetrance, and frequent need for heart transplantation. Eur Heart $J 2017$

24. Kober L, Thune JJ, Nielsen JC, et al. Defibrillator Implantation in Patients with Nonischemic Systolic Heart Failure. N Engl J Med 2016;375(13):1221-30.

25. Ottoboni L, Keenan BT, Tamayo P, et al. An RNA profile identifies two subsets of multiple sclerosis patients differing in disease activity. Science translational medicine 2012;4(153):153ra31.

26. O'Mahony $C$, Jichi $F$, Pavlou $M$, et al. A novel clinical risk prediction model for sudden cardiac death in hypertrophic cardiomyopathy (HCM risk-SCD). Eur Heart J 2014;35(30):2010-20. 


\section{SUPPLEMENTARY METHODS \\ Maastricht Cardiomyopathy Registry}

DCM patients were included in the absence of a (i) myocardial infarction and/or significant coronary artery disease; (ii) primary valvular disease; (iii) hypertensive or congenital heart disease; (iv) acute myocarditis; (v) arrhythmogenic right ventricular dysplasia; (vi) hypertrophic, restrictive or peripartum cardiomyopathy, in accordance with the latest ESC proposal. As part of the protocol, patients were referred to the clinical genetics department of the Maastricht University Medical Center (MUMC, Maastricht, the Netherlands) for genetic counseling and DNA testing using a 47 cardiomyopathy-associated gene panel between 2012 and 2019.

\section{Genetic analysis in Maastricht}

Patients at the genetics outpatient clinic received genetic counseling and testing using our 47 cardiomyopathy-associated gene panel either with whole exome sequencing (WES) or single molecule Molecular Inversion Probes (smMIP). All detected variants have been confirmed with Sanger sequencing.

A family history of cardiac-related disease and sudden cardiac death was obtained by pedigree analysis. Familial inheritance was defined as recommended by the ESC: (i) two or more individuals (first or second-degree relatives) have DCM fulfilling diagnostic criteria for 'definite' disease OR (ii) in the presence of an index patient fulfilling diagnostic criteria for DCM and a first-degree relative with autopsy-proven DCM and sudden death at $<50$ years of age. Genetic variants were carefully and stringently classified in 5 different classes: pathogenic, likely pathogenic, variant of unknown significance (VUS), likely benign or benign based on the criteria as proposed by the ACMG guidelines. Classification of variants was based on the score of in silico prediction software scores (SIFT, MutationTaster, PolyPhen-2, PhyloP, Align-GVGD), the frequency in reference population databases (gnomAD, 1000 genomes, ESP projects), functional studies and previously published variations in NCBI's ClinVar and HGMD. Both pathogenic (class 5) and likely pathogenic (class 4) variants are reported here as pathogenic variants. All other variants were considered non-pathogenic. Importantly, only truncating mutations with a PSI score $>99 \%$ in TTN were classified as pathogenic.

\section{Definitions of included non-genetic disease modifiers}

In line with our diagnostic pathway and previous literature we defined the following non-genetic disease modifiers:

1) cardiac inflammation as defined by the ESC ( $\geq 14$ CD45, including up to 4 CD68-infiltrating cells $/ \mathrm{mm}^{2}$;

2) significant cardiac viral load defined as $>500$ copies/ $\mu \mathrm{g} D N A$;

3) systemic immune-mediated diseases including auto-immune and auto-inflammatory diseases with proven cardiac involvement as defined and classified by the ESC.

4) toxic (alcohol abuse (a history of alcohol consumption $>21 \mathrm{U} /$ week for men and $>14 \mathrm{U} /$ week for women; $1 \mathrm{U}$ of alcohol $=10 \mathrm{ml}$ or $8 \mathrm{~g}$ of pure alcohol), hard drugs (a history of cocaine use) or chemotherapy (history of treatment with cardiotoxic chemotherapy); and

5) electrical disorders were defined as: a tachycardiomyopathy, $>20 \%$ of total heartbeats are premature ventricular complexes on a 24-hour registration, and left bundle branch block defined using the Strauss criteria: QRS duration $\geq 140 \mathrm{~ms}$ for men and $\geq 130 \mathrm{~ms}$ for women, along with mid-QRS notching or slurring in $\geq 2$ contiguous leads.

\section{Data processing}

All 47 variables were used for data imputation using proximity from Random Forest for mixed-type data with missForest package v1.4 in R. CMR parameters were imputed based on their equivalent echocardiographic parameters by regression modelling (Suppl. Figure 1). Eventually, this created 2 datasets which we refer to as the unimputed raw data and the imputed data. The correlation matrix among variables is comparable between the two datasets, depicting high similarity of the datasets 
(Suppl. Figure 2). Next, 14 variables with more than $25 \%$ missing data in the unimputed raw data were excluded from further analysis (Suppl. Figure 3). Only late gadolinium enhancement (LGE) was kept in the analysis despite $29.8 \%$ of missing data, due to the established clinical importance of this variable. To prevent the inclusion of redundant variables, a correlation matrix of the clinical variables was created using modified cor2 function for a dataframe of mixed-type data (Suppl. Figure 4). Five variables were excluded due to strong correlation (correlation coefficient $>0.6$ as proposed before, keeping the variable that was most informative and had the least missingness; Suppl. Table 2). This left 28 variables for the clustering analysis.

\section{Unsupervised machine learning methods to define phenotypic clusters}

Phenotypic clusters were defined using an unsupervised hierarchical clustering of principal components (HCPC) approach. As we had a mixed-type dataset as input (continuous and categorical variables), the principal components of the data were obtained with the factor analysis for mixed data (FAMD) method, in which numeric variables were first log2 transformed and then scaled to unit variance, and the categorical variables are transformed into a disjunctive data table (crisp coding) and then scaled using the specific scaling. This method ensures the balance between the influence of both continuous and categorical variables in the analysis. The first 11 principal components of FAMD with an eigenvalue $>1$, which accumulatively counted for $55.8 \%$ variance of the dataset were used as input for the HCPC method. The optimal number of clusters was determined based on the gain in withininertia (inside group variance), the shape of the dendrogram tree and clinical relevance. After cutting the tree to the desired number of clusters, a k-means consolidation was performed to increase the robustness of the created clusters, which is included in the HCPC method by default. The FAMD and HCPC were performed using the corresponding functions of the FactoMineR package v1.41 in R.

\section{Supervised clustering modelling to simplify clustering by creating a decision tree}

The patient groups created by unsupervised HCPC-FAMD were used as input for decision tree modeling to identify the key parameters necessary to distinguish among the patient groups. The conditional interference tree method was used, a recursive binary partitioning method which can deal with overfitting and variable selection problems using the ctree function of party package v1.3-3 in R. Afterwards, sensitivity, specificity and accuracy of the decision tree model per phenogroup were calculated.

\section{SUPPLEMENTARY FIGURES}

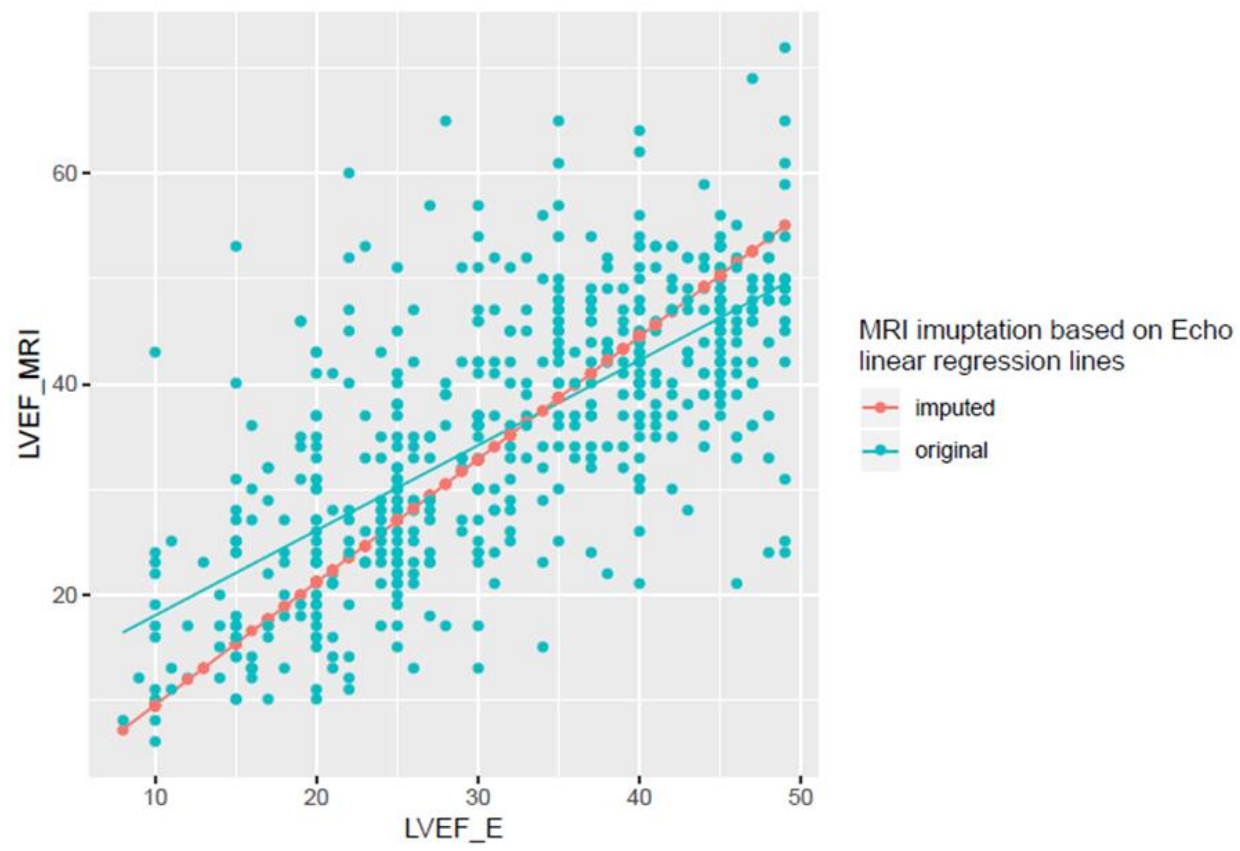



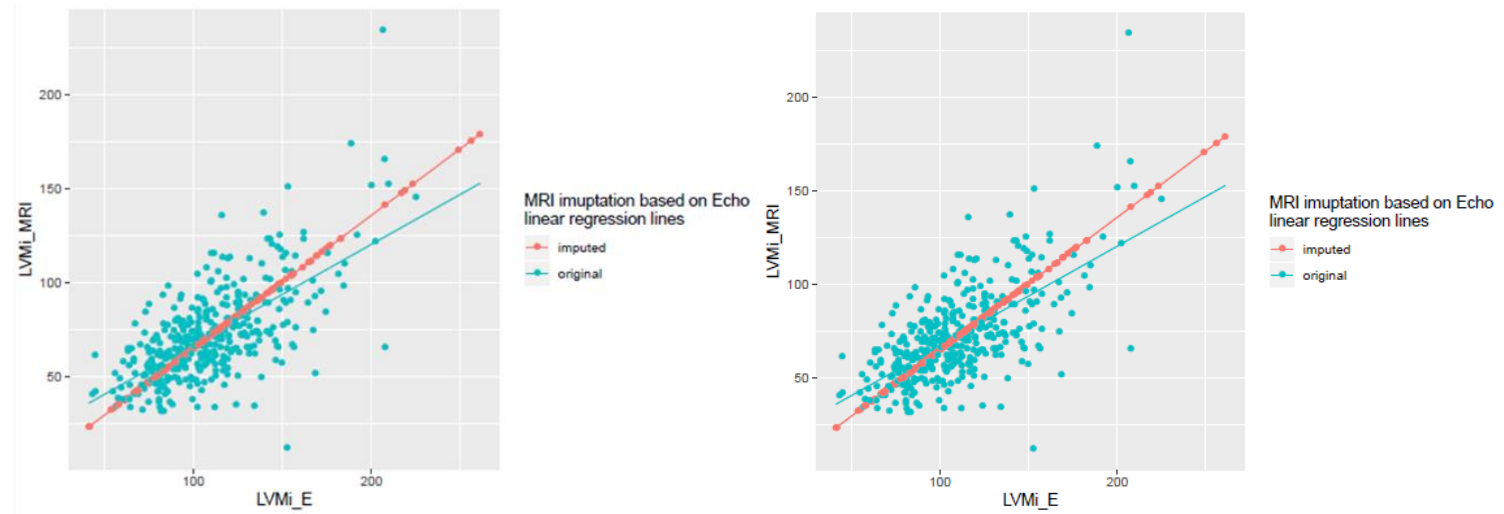

Supplemental Figure 1. The missing MRI parameters (LVEF, LVMi and LVEDVi) in the index cohort were imputed based on their equivalent echo parameters by Passing-Bablok (LVEF and LVMi) or normal linear regression (LVEDVi).

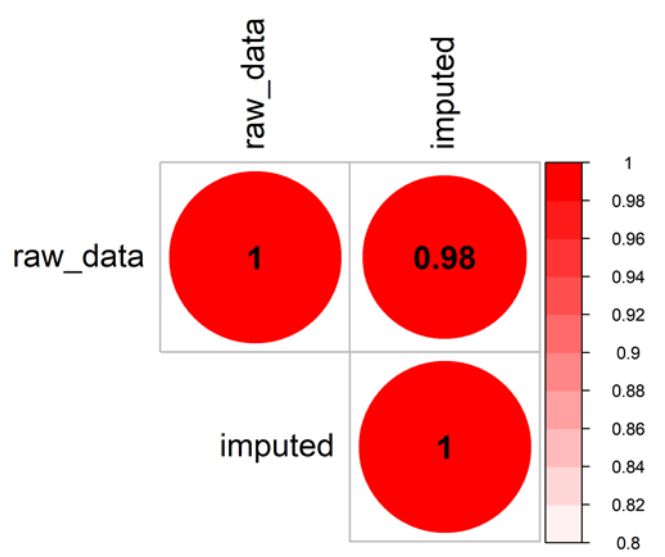

Supplemental Figure 2. The similarity of the raw (non-imputed) and imputed dataset is $98 \%$, depicting high similarity between the datasets.

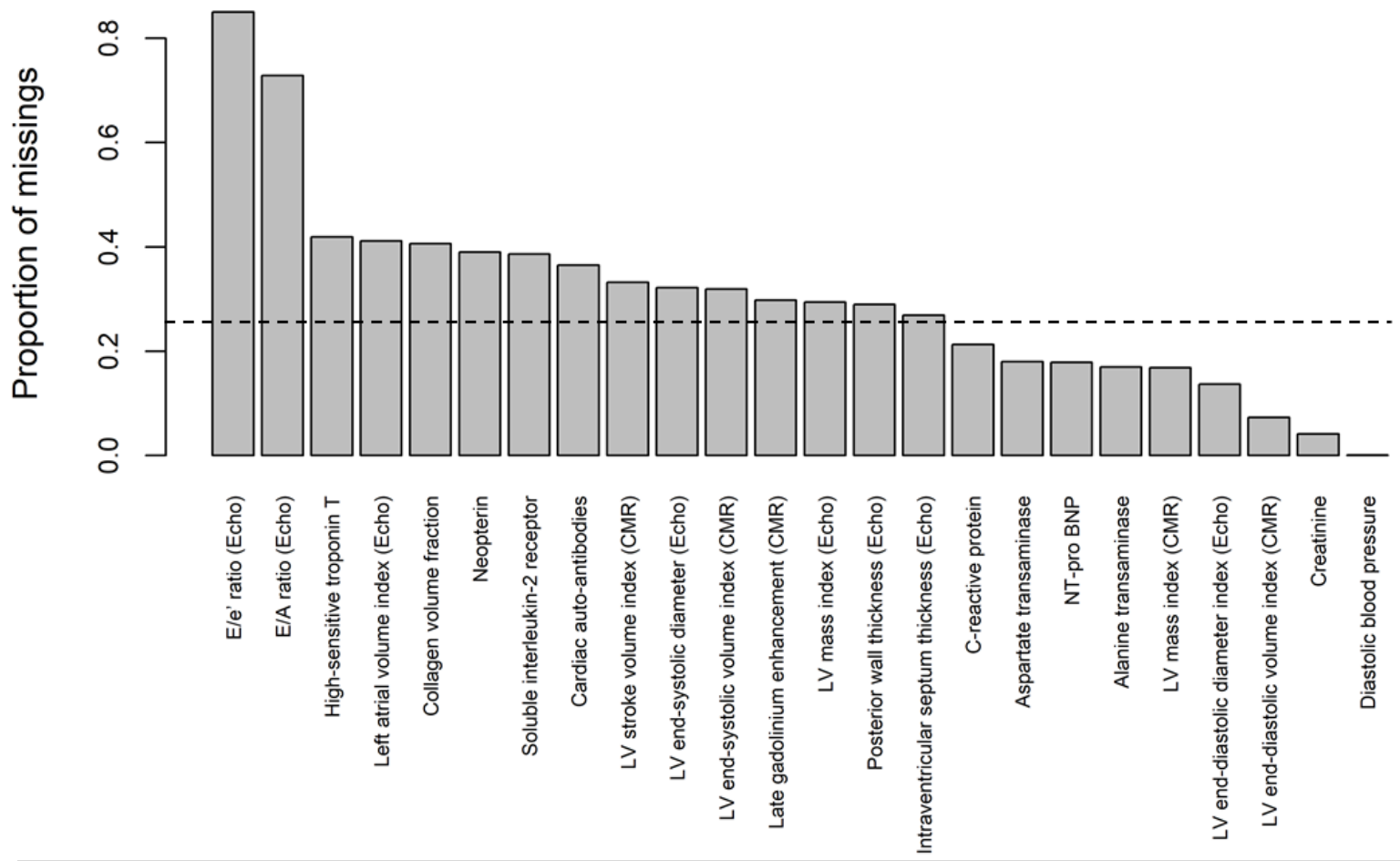

Supplemental Figure 3. All clinical variables with missing data. Clinical variables with missing data $>25 \%$ (proportion of 0.25 ) were excluded as input for the cluster analysis. 


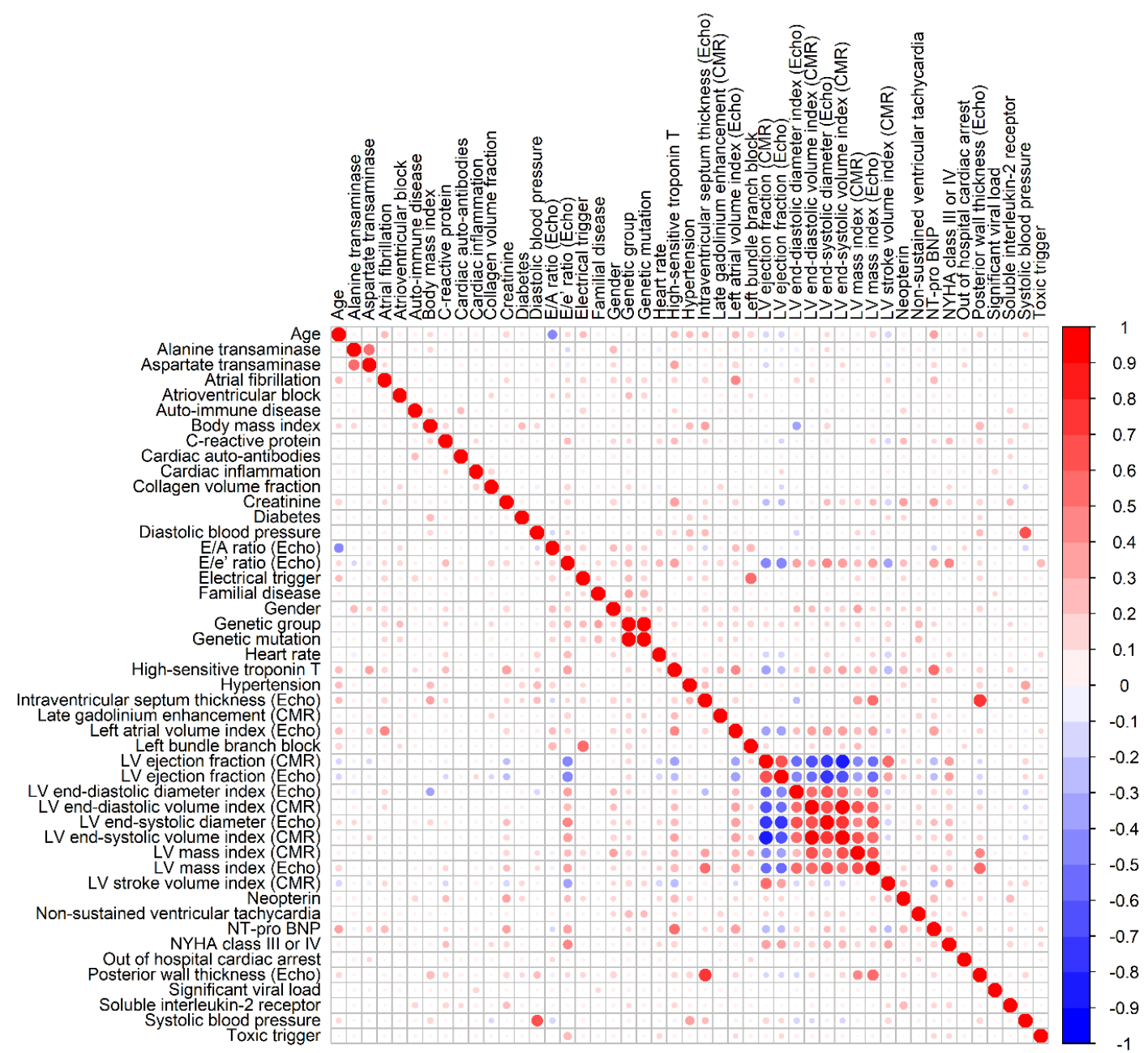

Supplemental Figure 4. A correlation matrix of the clinical variables. Red indicates high correlation, and blue indicates an inverse correlation between variables.

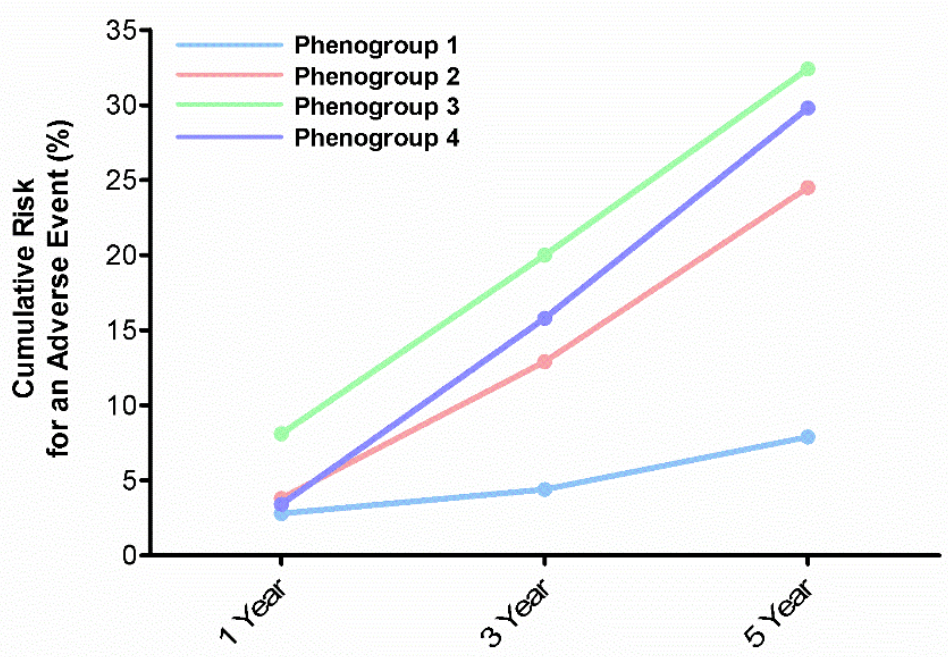

Supplemental Figure 5. Cumulative risk for an adverse event, defined as one of the following: life-threatening arrhythmias, death, heart transplantation, or left ventricular assist device implantation, stratified by phenogroup. Cumulative risk indicates the risk that an adverse event will occur within the mentioned time period. 


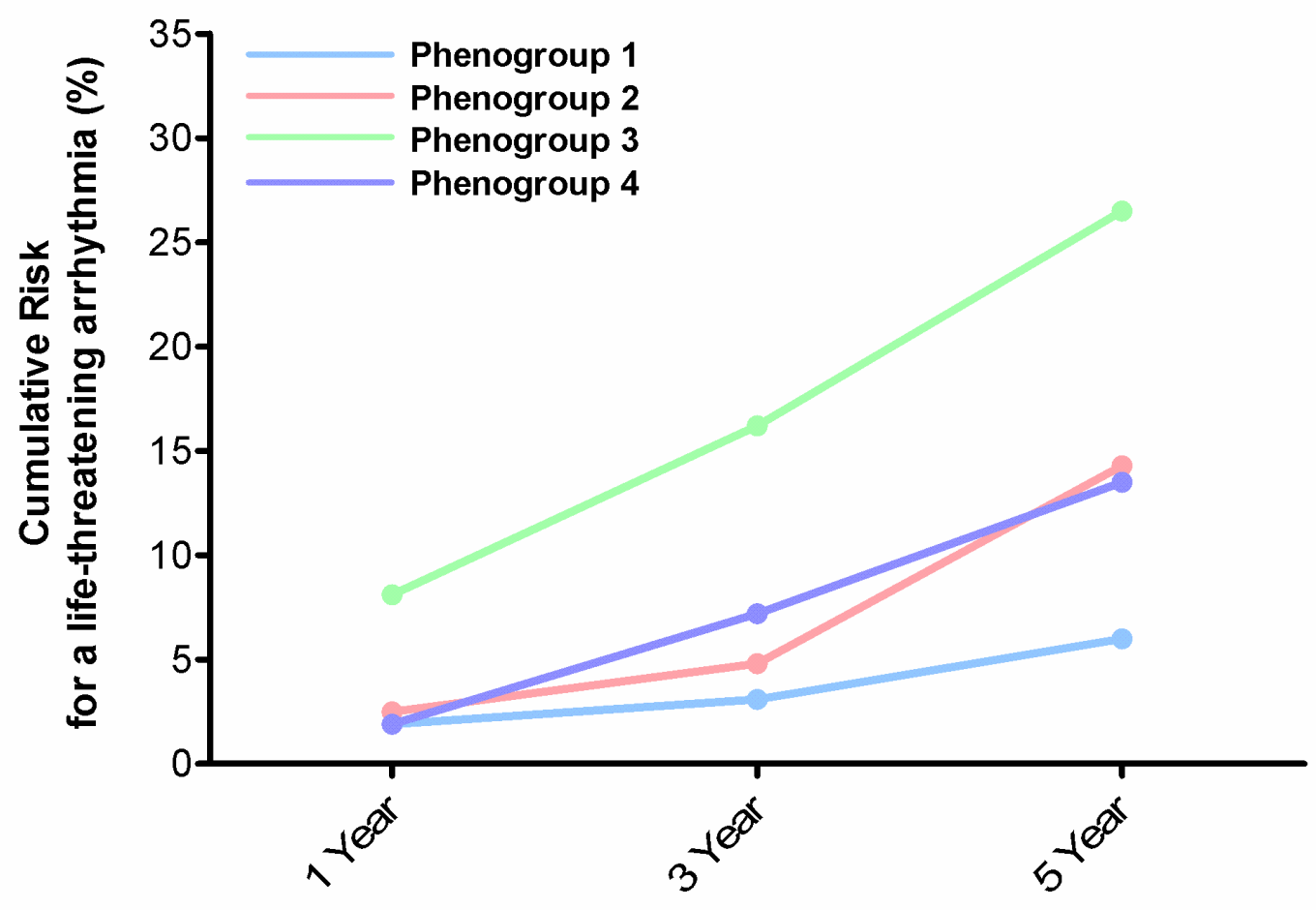

Supplemental Figure 6. Cumulative risk for a life-threatening arrhythmia, stratified by phenogroup. Cumulative risk indicates the risk that an adverse event will occur within the mentioned time period.

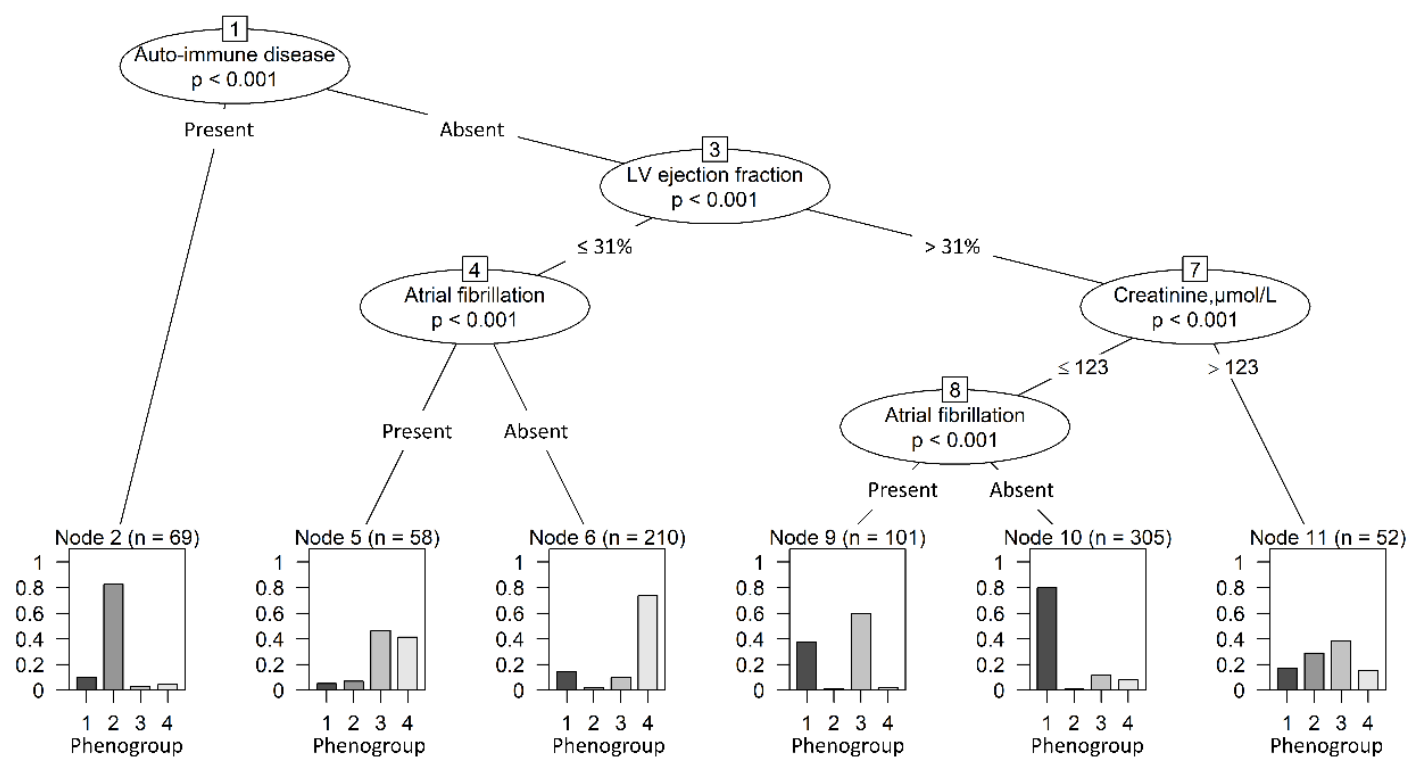

Supplemental Figure 7. Supervised decision tree modeling highlighted auto-immune disease, ejection fraction, atrial fibrillation and creatinine as the key parameters to distinguish the four phenogroups. 


\section{SUPPLEMENTARY TABLES}

Supplemental Table 1. Overview of all 47 genes used in the Maastricht Cardiomyopathy gene-panel.

\begin{tabular}{|c|c|c|c|}
\hline & HGNC.ID & HGNC.symbol & HGNC.Name \\
\hline 1 & HGNC:143 & ACTC1 & Actin, alpha, cardiac muscle 1 \\
\hline 2 & HGNC:164 & ACTN2 & Actinin alpha 2 \\
\hline 3 & HGNC:15819 & $A N K R D 1$ & Ankyrin repeat domain 1 \\
\hline 4 & HGNC:939 & $B A G 3$ & BCL2 associated athanogene 3 \\
\hline 5 & HGNC:20407 & CALR3 & Calreticulin 3 \\
\hline 6 & HGNC:1529 & CAV3 & Caveolin 3 \\
\hline 7 & HGNC:2389 & CRYAB & Crystallin alpha B \\
\hline 8 & HGNC:2472 & CSRP3 & Cysteine and glycine rich protein 3 \\
\hline 9 & HGNC:2511 & CTNNA3 & Catenin alpha 3 \\
\hline 10 & HGNC:2770 & $D E S$ & Desmin \\
\hline 11 & HGNC:3036 & DSC2 & Desmocollin 2 \\
\hline 12 & HGNC:3049 & $D S G 2$ & Desmoglein 2 \\
\hline 13 & HGNC:3052 & $D S P$ & Desmoplakin \\
\hline 14 & HGNC:3331 & $E M D$ & Emerin \\
\hline 15 & HGNC:3702 & FHL1 & Four and a half LIM domains 1 \\
\hline 16 & HGNC:4296 & GLA & Galactosidase alpha \\
\hline 17 & HGNC:14202 & JPH2 & Junctophilin 2 \\
\hline 18 & HGNC:6207 & JUP & Junction plakoglobin \\
\hline 19 & HGNC:6484 & LAMA4 & Laminin subunit alpha 4 \\
\hline 20 & HGNC:6501 & LAMP2 & Lysosomal associated membrane protein 2 \\
\hline 21 & HGNC:15710 & $\angle D B 3$ & LIM domain binding 3 \\
\hline 22 & HGNC:6636 & LMNA & Lamin A/C \\
\hline 23 & HGNC:21086 & MIB1 & Mindbomb E3 ubiquitin protein ligase 1 \\
\hline 24 & HGNC:7551 & MYBPC3 & Myosin binding protein $\mathrm{C}$, cardiac \\
\hline 25 & HGNC:7576 & MYH6 & Myosin heavy chain 6 \\
\hline 26 & HGNC:7577 & MYH7 & Myosin heavy chain 7 \\
\hline 27 & HGNC:7583 & MYL2 & Myosin light chain 2 \\
\hline 28 & HGNC:7584 & MYL3 & Myosin light chain 3 \\
\hline 29 & HGNC:1330 & MYOZ2 & Myozenin 2 \\
\hline 30 & HGNC:23246 & $M Y P N$ & Myopalladin \\
\hline 31 & HGNC:29557 & NEXN & Nexilin F-actin binding protein \\
\hline 32 & HGNC:9024 & PKP2 & Plakophilin 2 \\
\hline 33 & HGNC:9080 & PLN & Phospholamban \\
\hline 34 & HGNC:14000 & PRDM16 & PR/SET domain 16 \\
\hline 35 & HGNC:9386 & PRKAG2 & Protein kinase AMP-activated non-catalytic subunit gamma 2 \\
\hline 36 & HGNC:27424 & RBM20 & RNA binding motif protein 20 \\
\hline 37 & HGNC:10593 & SCN5A & Sodium voltage-gated channel alpha subunit 5 \\
\hline 38 & HGNC:11577 & $T A Z$ & Tafazzin \\
\hline 39 & HGNC:11610 & TCAP & Titin-cap \\
\hline 40 & HGNC:28472 & TMEM43 & Transmembrane protein 43 \\
\hline 41 & HGNC:11943 & TNNC1 & Troponin C1, slow skeletal and cardiac type \\
\hline 42 & HGNC:11947 & TNNI3 & Troponin 13, cardiac type \\
\hline 43 & HGNC:11949 & TNNT2 & Troponin T2, cardiac type \\
\hline
\end{tabular}




\begin{tabular}{cccc}
44 & HGNC:12010 & TPM1 & Tropomyosin 1 \\
45 & HGNC:12403 & $T T N$ & Titin \\
46 & HGNC:12405 & $T T R$ & Transthyretin \\
47 & HGNC:12665 & $V C L$ & Vinculin \\
\hline
\end{tabular}

Supplemental Table 2. Overview of all 47 clinical variables and their in- or exclusion at the several data processing steps

\begin{tabular}{|c|c|c|c|c|c|}
\hline Clinical Variable & $\begin{array}{c}\text { Variables } \\
\text { used for } \\
\text { data } \\
\text { imputation }\end{array}$ & $\begin{array}{c}\text { After } \\
\text { exclusion of } \\
\text { variables } \\
\text { with }>25 \% \\
\text { missing }\end{array}$ & $\begin{array}{c}\text { After } \\
\text { exclusion of } \\
\text { highly } \\
\text { correlated } \\
\text { variables } \\
(r>0.6)\end{array}$ & $\begin{array}{c}\text { Contribution } \\
\text { in defining } \\
\text { patient } \\
\text { clusters }\end{array}$ & $\begin{array}{c}\text { Decision } \\
\text { tree } \\
\text { modeling }\end{array}$ \\
\hline
\end{tabular}

\begin{tabular}{|c|c|c|c|c|c|c|}
\hline 1 & Age & $x$ & $x$ & $x$ & $x$ & \\
\hline 2 & Gender & $x$ & $x$ & $x$ & $x$ & \\
\hline 3 & Genetic mutation & $x$ & $x$ & & & \\
\hline 4 & $\begin{array}{l}\text { Genetic group (TTNtv, } \\
\text { LMNA, Other, Multiple) }\end{array}$ & $x$ & $x$ & $x$ & $x$ & \\
\hline 5 & Familial disease & $x$ & $x$ & $x$ & $x$ & \\
\hline 6 & Toxic trigger & $x$ & $x$ & $x$ & $x$ & \\
\hline 7 & Auto-immune disease & $x$ & $x$ & $x$ & $x$ & $x$ \\
\hline 8 & Cardiac inflammation & $x$ & $x$ & $x$ & $x$ & \\
\hline 9 & Significant viral load & $x$ & $x$ & $x$ & & \\
\hline 10 & Electrical trigger & $x$ & $x$ & & & \\
\hline 11 & NYHA functional class $\geq$ III & $x$ & $x$ & $x$ & $x$ & \\
\hline 12 & Hypertension & $x$ & $x$ & $x$ & $x$ & \\
\hline 13 & Body mass index & $x$ & $x$ & $x$ & $x$ & \\
\hline 14 & Diabetes mellitus & $x$ & $x$ & $x$ & $x$ & \\
\hline 15 & Heart rate & $x$ & $x$ & $x$ & $x$ & \\
\hline 16 & Systolic blood pressure & $x$ & $x$ & $\mathrm{X}$ & $x$ & \\
\hline 17 & Diastolic blood pressure & $x$ & $x$ & & & \\
\hline
\end{tabular}

$\begin{array}{ccc}18 & \text { LV Ejection fraction } & \mathrm{X} \\ 19 & \text { LV EDD index } & \mathrm{X} \\ 20 & \text { LV ESD } & \mathrm{X} \\ 21 & \text { LA Volume index } & \mathrm{X} \\ 22 & \text { LV Mass index } & \mathrm{X} \\ 23 & \text { E/A ratio } & \mathrm{X} \\ 24 & \text { E/e' ratio } & \mathrm{X} \\ 25 & \text { Posterior WT } & \mathrm{X} \\ 26 & \text { Intraventricular ST } & \mathrm{X}\end{array}$

\section{Echocardiography}

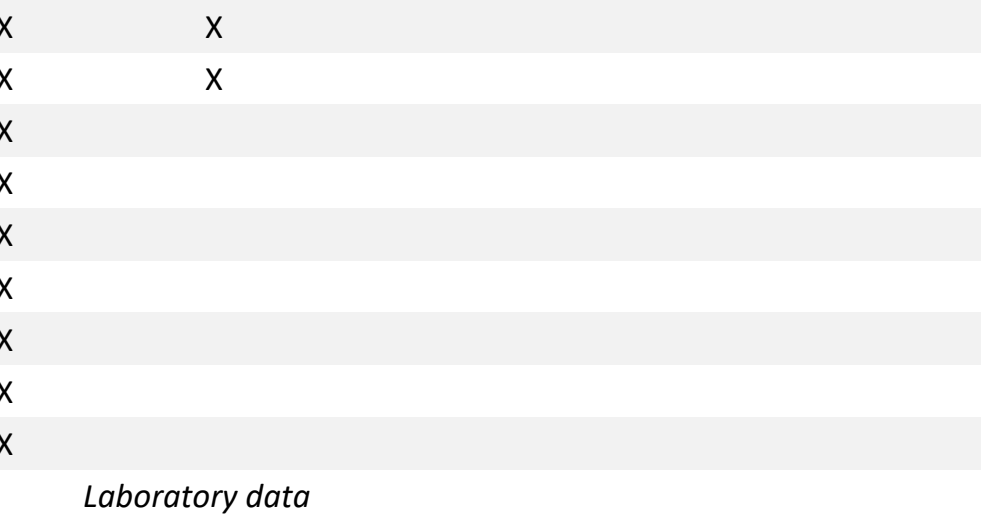

$\begin{array}{lcccccc}27 & \text { NT-pro BNP } & \text { X } & \text { X } & \text { X } & \text { X } \\ 28 & \text { Hs-Troponin T } & \text { X } & \text { X } & \text { X } & \text { X } & \text { X } \\ 29 & \text { Creatinine } & \text { X } & & & \\ 30 & \text { SIL-2R elevation } & \text { X } & & & \\ 31 & \text { Neopterin elevation } & \text { X } & & \end{array}$




\begin{tabular}{|c|c|c|c|c|c|c|}
\hline 33 & C-reactive protein & $x$ & $x$ & $x$ & $x$ & \\
\hline 34 & ASAT & $x$ & $x$ & $x$ & $x$ & \\
\hline 35 & ALAT & $x$ & $x$ & $x$ & $x$ & \\
\hline \multicolumn{7}{|c|}{ ECG/Holter } \\
\hline 36 & Atrial fibrillation & $x$ & $x$ & $x$ & $x$ & $x$ \\
\hline 37 & Non-sustained VT & $x$ & $x$ & $x$ & $x$ & \\
\hline 38 & Left bundle branch block & $x$ & $x$ & $x$ & $x$ & \\
\hline 39 & Atrioventricular block & $x$ & $x$ & $x$ & $x$ & \\
\hline 40 & $\begin{array}{c}\text { Out of hospital cardiac } \\
\text { arrest }\end{array}$ & $x$ & $x$ & $x$ & $x$ & \\
\hline \multicolumn{7}{|c|}{$M R I$} \\
\hline 41 & LV Mass index & $x$ & $x$ & $x$ & $x$ & \\
\hline 42 & LV EDV index & $x$ & $x$ & $x$ & $x$ & \\
\hline 43 & LV ESV index & $x$ & & & & \\
\hline 44 & LV Stroke volume index & $x$ & & & & \\
\hline 45 & LV Ejection fraction & $x$ & $x$ & $x$ & $x$ & $x$ \\
\hline 46 & $\begin{array}{l}\text { Late gadolinium } \\
\text { enhancement }\end{array}$ & $x$ & $x$ & $x$ & $\mathrm{x}$ & \\
\hline \multicolumn{7}{|c|}{ Endomyocardial Biopsy } \\
\hline 47 & Collagen volume fraction & $x$ & & & & \\
\hline
\end{tabular}

Supplemental Table 3. Performance metrics of the supervised decision tree model

\begin{tabular}{|c|c|c|c|c|c|}
\hline & $\begin{array}{l}\text { Group } 1 \\
(n=331)\end{array}$ & $\begin{array}{c}\text { Group } 2 \\
(n=83)\end{array}$ & $\begin{array}{l}\text { Group } 3 \\
(n=165)\end{array}$ & $\begin{array}{l}\text { Group } 4 \\
(n=216)\end{array}$ & $\begin{array}{c}\text { Sum } \\
(n=795)\end{array}$ \\
\hline \multicolumn{6}{|c|}{ Decision tree classification, $\%$} \\
\hline Group 1 & $80 \%$ & $1 \%$ & $11 \%$ & $8 \%$ & $100 \%$ \\
\hline Group 2 & $10 \%$ & $83 \%$ & $3 \%$ & $4 \%$ & $100 \%$ \\
\hline Group 3 & $24 \%$ & $9 \%$ & $51 \%$ & $16 \%$ & $100 \%$ \\
\hline Group 4 & $14 \%$ & $2 \%$ & $10 \%$ & $74 \%$ & $100 \%$ \\
\hline Performance metrics & & & & & Total \\
\hline Sensitivity & 0.74 & 0.69 & 0.65 & 0.72 & \\
\hline Specificity & 0.87 & 0.98 & 0.84 & 0.91 & \\
\hline Accuracy & 0.81 & 0.95 & 0.80 & 0.85 & 0.71 \\
\hline
\end{tabular}




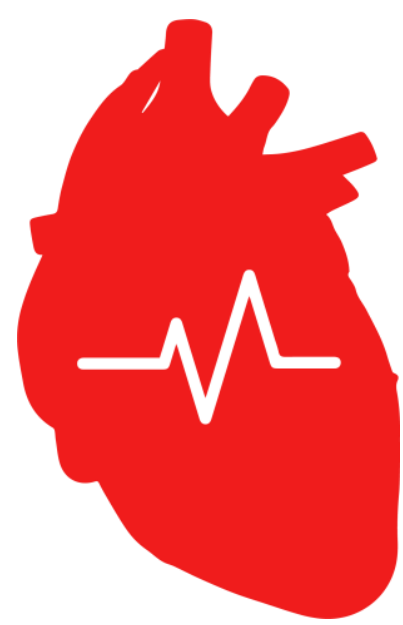

- 226 - 


\section{CHAPTER 9}

\section{Cardiac inflammation impedes response to cardiac resynchronization therapy in patiens with idiopathic dilated cardiomyopathy}

\author{
Job A.J. Verdonschot, $M D, M S c^{1,2}$ \\ Jort J. Merken, $M D^{l}$ \\ Antonius M.W. van Stipdonk, MD, $P h D^{2}$ \\ Philipp Pliger, $M D^{3}$ \\ Kasper W.J. Derks, $P h D^{2}$ \\ Ping Wang, $P h D^{2}$ \\ Michiel T.H.M. Henkens, $M D^{1}$ \\ Pieter van Paassen, $M D, P h D^{4}$ \\ Myrurgia A. Abdul Hamid, $M D^{5}$ \\ Vanessa P.M. van Empel, MD, PhD
}

\author{
Christian Knackstedt, $M D, P h D^{2}$ \\ Justin G.L.M. Luermans, MD, PhD1 \\ Harry J.G.M. Crijns, $M D$, $P h D^{l}$ \\ Hans-Peter Brunner-La Rocca, $M D^{1}$ \\ Han G. Brunner, $M D, P h D^{2,5,7}$ \\ Gerhard Poelzl, MD, PhD \\ Kevin Vernooy, $M D, P h D^{1,8}$ \\ Stephane R.B. Heymans, MD, $P h D^{19,10^{*}}$ \\ Mark R. Hazebroek, MD, PhD ${ }^{I^{*}}$
}

* Contributed equally

${ }^{1}$ Department of Cardiology, Cardiovascular Research Institute (CARIM), Maastricht University Medical Center, Maastricht, The Netherlands.

${ }^{2}$ Department of Clinical Genetics, Maastricht University Medical Center, Maastricht, The Netherlands. ${ }^{3}$ Innsbruck Medical University, Clinical Division of Cardiology and Angiology, Austria.

${ }^{4}$ Department of Immunology, Maastricht University Medical Center, Maastricht, The Netherlands.

${ }^{5}$ Department of Pathology, Maastricht University Medical Center, Maastricht, The Netherlands.

${ }^{6}$ Radboud University Medical Center, Department of Human Genetics, Donders Institute for Brain, Cognition and Behaviour, Nijmegen.

${ }^{7}$ GROW Institute for Developmental Biology and Cancer, Maastricht University Medical Centre, Maastricht, The Netherlands.

${ }^{8}$ Department of Cardiology, Radboud University Medical Center, Nijmegen, The Netherlands. ${ }^{9}$ Department of Cardiovascular Sciences, Centre for Molecular and Vascular Biology, KU Leuven, Belgium,

${ }^{10}$ The Netherlands Heart Institute, Nl-HI, Utrecht, The Netherlands. 


\begin{abstract}
Background: Cardiac resynchronization therapy (CRT) is an established therapy in patients with dilated cardiomyopathy (DCM) and conduction disorders. Still, one-third of the patients with DCM do not respond to CRT. This study aims to depict the underlying cardiac pathophysiological processes of non-response to CRT in DCM patients using endomyocardial biopsies (EMB).
\end{abstract}

Methods: Within the Maastricht and Innsbruck registries of DCM patients, 99 patients underwent EMB before CRT implantation, with histological quantification of fibrosis and inflammation, where inflammation was defined as $>14$ infiltrating cells $/ \mathrm{mm} 2$. Echocardiographic left ventricular end-systolic volume (LVESV) reduction $\geq 15 \%$ after 6 months was defined as response to CRT. RNA was isolated from cardiac biopsies of a representative subset of responders and non-responders.

Results: Sixty-seven patients responded (68\%), whereas 32 (32\%) did not respond to CRT. Cardiac inflammation prior to implantation was negatively associated with response to CRT ( $25 \%$ of responders, $47 \%$ of non-responders; odds ratio 0.3 [0.12-0.76]; $p=0.01$ ). EMB fibrosis did not relate to CRT response. Cardiac inflammation had added predictive value beyond wellknown clinical predictors of CRT response with c-statistics and likelihood ratio test $p<0.001$. Cardiac transcriptomic profiling of EMB reveals a strong pro-inflammatory and pro-fibrotic signature in the hearts of non-responders compared to responders. In particular, COL1A1, COL1A2, COL3A1, COL5A1, POSTN, CTGF, LOX, TGF61, PDGFRA, TNC, BGN and TSP2 were significantly higher expressed in the hearts of non-responders.

Conclusions: Cardiac inflammation along with a transcriptomic profile of high expression of combined pro-inflammatory and pro-fibrotic genes are associated with a poor response to CRT in DCM patients. 


\section{INTRODUCTION}

Cardiac resynchronisation therapy (CRT) is an established treatment for patients with heart failure (HF), impaired left ventricular (LV) function and ventricular conduction abnormalities ${ }^{1}$. In these patients, CRT improves exercise tolerance and quality of life, and reduces hospitalizations and mortality ${ }^{2}$. However, not all patients seem to benefit equally from this treatment ${ }^{3}$. Multiple patient- and therapy-related characteristics relate to CRT response, of which a native left bundle branch block (LBBB) pattern, female sex, sinus rhythm, and a wider QRS-duration are associated with a positive response to CRT ${ }^{145}$. Still, about one-third of the patients with DCM shows less than expected LV reverse remodeling as response to CRT ${ }^{67}$. The underlying pathophysiological processes related to non-response are incompletely understood. Endomyocardial biopsies (EMB) are a validated diagnostic tool to determine the underlying pathophysiology in DCM ${ }^{8}$. Since the lack of response to CRT is incompletely understood and there is a need for novel predictors ${ }^{5910}$, EMB provides a unique opportunity to determine the underlying myocardial molecular processes, such as inflammation and fibrosis. Although replacement fibrosis in ischemic cardiomyopathy (ICM), captured on cardiac magnetic resonance imaging (CMR), has been repeatedly shown to play an important role in response prediction and even tailoring CRT ${ }^{11}$, the role of histologic inflammation and fibrosis in CRT response have not been addressed. To further elucidate the underlying pathophysiological processes of non-response, and to delineate which biological pathways might be involved, we explored the clinical and cardiac molecular parameters that may help to unravel the CRT response in DCM.

\section{METHODS}

\section{Patient selection}

Data analysis was performed retrospectively with selection of DCM patients from the Maastricht Cardiomyopathy Registry (the Netherlands) enrolled between 2004 and 2018, and the Innsbruck Cardiomyopathy Registry (Austria) enrolled between 2005 and 2019, with inclusion and exclusion criteria as described previously ${ }^{12}{ }^{13}$. In short, consecutive DCM patients were included in the absence of a (i) myocardial infarction and/or significant coronary artery disease; (ii) primary valvular disease; (iii) hypertensive or congenital heart disease; (iv) acute myocarditis; (v) arrhythmogenic right ventricular dysplasia; (vi) hypertrophic, restrictive or peripartum cardiomyopathy, in accordance with the latest ESC proposal ${ }^{14}$.

From these registries, patients were selected based on two criteria: 1) Implanted with a CRT-device, indicated according to the current ESC guidelines ${ }^{15}$ and 2) EMBs prior to CRT implantation (Suppl. Figure 1). CRT criteria included: QRS-duration $\geq 130 \mathrm{~ms}$ with or without a complete left bundle branch block (LBBB), LVEF $\leq 35 \%$ who remain in NYHA-class $\geq$ Il despite optimal medical treatment. A complete LBBB was defined using the Strauss criteria as previously suggested: QRS duration $\geq 140 \mathrm{~ms}$ for men and $\geq 130 \mathrm{~ms}$ for women, along with midQRS notching or slurring in $\geq 2$ contiguous leads ${ }^{16}$. EMBs prior to CRT implantation were available as part of the standardized diagnostic pathway, where all patients undergo in-depth phenotyping - including EMB - in initially 'idiopathic' DCM cases ${ }^{17}$. The main indication for EMB was a LVEF $<45 \%$ after 3 months of optimal medical treatment in accordance with the latest ESC position statement ${ }^{18}$. EMB prior to CRT implantation occurred in $35 \%(77 / 217)$ of patients with an indication for CRT (Suppl. Figure 1). There was no significant difference in cardiac function or clinical presentation between CRT-DCM patients included in this study compared to CRT-DCM patients not included in this study (Suppl. Table 1). 
Genetic testing using a 47 DCM-associated gene panel was performed in DCM patients in Maastricht if consented ${ }^{12}$. Familial disease was defined as recommended by the ESC: (i) two or more individuals (first or second-degree relatives) have DCM fulfilling diagnostic criteria for 'definite' disease OR (ii) in the presence of an index patient fulfilling diagnostic criteria for DCM and a first-degree relative with autopsy-proven DCM and sudden death at $<50$ years of age ${ }^{14}$. The study was performed according to the declaration of Helsinki and was approved by the Medical Ethics Committee/Institutional Review Boards. All patients gave written informed consent. The data that support the findings of this study are available from the corresponding author on reasonable request.

\section{Baseline and Follow-up}

Patients from both registries were followed for at least 6 months after device implantation to assess therapy response, with a median of 6 months [interquartile range 6 to 7 months] between device implantation and follow-up echo. Echocardiography at baseline and 6 months was available for all patients. Additional cardiovascular magnetic resonance imaging at baseline was available in $75 \%$ of patients. Information about the occurrence of adverse events at follow-up was available from all patients included from the Maastricht Cardiomyopathy Registry, with a median follow-up time of 36 months [interquartile range 14 to 54 months] from device implantation. This follow-up information was retrieved from the hospital medical records, the Dutch Personal Records Database and/or telephone contact with the patient or their general practitioners. A combined clinical end-point was used to define clinical response to therapy, including at least one of the following events; 1 ) death due to cardiovascular disease, 2) heart transplantation, 3) heart failure that required a non-elective hospitalization, and 4) life-threatening arrhythmias (LTA) defined as non-fatal ventricular fibrillation (with or without ICD-shock), and/or sustained ventricular tachycardia with appropriate ICD shock. No patients were lost to follow-up. End of follow-up was defined as November 15 2019.

\section{Echocardiography and CRT response}

Every baseline and 6 month echocardiography was reviewed by two independent cardiologists. Imaging was performed in the standard left parasternal, apical, and subcostal views, using an IE33 from Philips Medical Systems (Best, The Netherlands). Left ventricular end-systolic volume (LVESV), LV end-diastolic volume (LVEDV), and LV ejection fraction (LVEF) were measured using the Simpson's biplane method. Primary endpoint was defined as CRT response with a LVESV decrease of $\geq 15 \%$.

\section{Endomyocardial biopsies}

EMBs were obtained in all patients prior to CRT implantation with a median of 2 (range 0-12) months. A minimum of 6 biopsies were taken in the right ventricle from the interventricular septum via the internal jugular vein using a transcatheter bioptome (Cordis, Miami, FL, USA). Three specimens were used for immunohistological analysis and three for the detection of viral genomes. Histopathological tests were done on $4 \mu \mathrm{m}$-thick tissue sections from formalin-fixed, paraffin-embedded EMBs, and stained with hematoxylin and eosin, Sirius red, $\mathrm{CD} 3+, \mathrm{CD} 45+$ and CD68+. Increased cardiac inflammation was defined as $\geq 14$ CD45, including up to 4 CD68-infiltrating cells $/ \mathrm{mm}^{2}$ according to the current ESC position statement ${ }^{18}$. Detection of viruses (Parvovirus B19 (B19V), Human Herpes Virus type-6 (HHV6)) were performed as previously described ${ }^{12}$. Polymerase chain reaction (PCR) and reverse transcriptase PCR analysis determined viral load. A significant viral load was defined as 
cardiotropic viruses higher than 500 copies per microgram DNA ${ }^{19}$. Collagen volume fraction (CVF) was quantified as percentage tissue positive for Sirius red of the total myocardial area. Perivascular and direct sub-endomyocardial collagen were not included in the quantification, as cutting artifacts may influence those.

\section{Cardiovascular magnetic resonance imaging}

Cine and late gadolinium enhancement (LGE) images were acquired on a $1.5 \mathrm{~T}$ MRI system (Intera, Philips Medical Systems, Best, The Netherlands). Late gadolinium imaging was performed $10 \mathrm{~min}$ after administration of an intravenous bolus of $0.2 \mathrm{mmol} / \mathrm{kg}$ body weight gadolinium-diethylenetriaminepentaacetic acid (Gadovist, Bayer, Berlin, Germany). Acquisition was performed using a two-dimensional (2D) T1-weighted gradient echo inversion recovery sequence and included images in standard long-axis planes and in a multi-slice shortaxis stack fully covering the LV. Inversion times were optimized to null the myocardium. Two blinded observers analyzed the cine and LGE images using dedicated software (CAAS MRV5.0, Pie Medical Imaging B.V., Maastricht, The Netherlands). LGE was considered present if seen in both long- and short-axis planes and extending beyond the localized ventricular insertion areas.

\section{RNA sequencing and bioinformatical analysis}

DCM patients included in the Maastricht Cardiomyopathy Registry, signed informed consent to store and analyze spare EMBs taken for clinical purposes. To evaluate disease-driving molecular mechanisms in CRT responders and non-responders, RNA was isolated from all available spare EMBs of DCM patients and checked for quality and integrity, as previously described ${ }^{12}$. The mRNA sequencing library was generated using TruSeq mRNA sample preparation kit (Illumina) and sequenced on the NextSeq 500 (Illumina). The bioinformatics tool GAGE [with Kyoto Encyclopaedia of Genes and Genomes (KEGG) pathway enrichment and Gene Ontology (GO) biological processes] was used for molecular pathway analysis.

We used knowledge-based network analysis with induced network approach by consensuspathDB (CPDB) online server (http://cpdb.molgen.mpg.de/) from Max Planck Institute for Molecular Genetics to identify the links among the top expressed cardiac transcripts, based on known knowledge of interaction networks (protein interactions and biochemical interactions) ${ }^{20}$. The network analysis also identifies additional proteins (intermediate nodes) based on knowledge-based interactions (with exclusion of lowconfidence interactions quantified by a $z$-score $\leq 20$ calculated for each intermediate node).

\section{Statistical analysis}

All quantitative measurements are presented as mean \pm standard deviation or median with IQR. Categorical data are expressed as absolute frequencies and percent values. MannWhitney $U$ test was used to test unpaired, not normally distributed data. Wilcoxon signedrank test was used in paired, not normally distributed data. Paired and unpaired T-test were used to analyze paired and unpaired normally distributed parameters, respectively.

Binary logistic regression analysis was performed to find associations between clinical variables and response to CRT. Receiver-operating-curves were generated for the different generated predictive models with corresponding area under the curve (AUC). 5-repeated 5fold cross validation (CV) was performed to assess the model's performance. The AUC in CV is expressed in median \pm median absolute deviation (MAD), and compared using MannWhitney $U$ test. Nested models of the clinical predictors, with the addition of cardiac 
inflammation were compared using a likelihood ratio-test. Kaplan-Meier survival curves were estimated and differences between groups were assessed by the log-rank test. Univariable Cox regression analysis was used to determine the hazard ratio (HR) and subsequent $95 \%$ confidence interval (CI). Statistical analysis was performed using SPSS 25.0 (IBM Corp., Armon, NY) software or R version 3.6.1 (R Foundation for Statistical Computing, Vienna, Austria). A pvalue $<0.05$ was considered statistically significant.

\section{RESULTS}

\section{Patient characteristics}

The present study included 99 consecutive DCM patients from two DCM registries, who underwent diagnostic EMB before CRT implantation. Those 99 patients represent a typical DCM-CRT population, with a median age of 59 years, $63 \%$ male, $73 \%$ at HF functional class II or III and a median baseline LVEF of $26 \%$ (Table 1). Sixty-seven percent had a native LBBB morphology, 77\% was in sinus rhythm and the mean QRS-width was 157 milliseconds.

Sixty-seven patients (68\%) had echocardiographic response to CRT, with a median LVESV decrease of $46 \%$ (IQR: $28-62 \%$ decrease), compared to a median decrease in LVESV of $3 \%$ (IQR: $14 \%$ decrease to $5 \%$ increase) in the non-responder group $(p<0.001)$. CRT responders had a higher event-free survival compared to non-responders (Log-Rank<0.001; Suppl. Figure 2; Suppl. Table 2).

\section{Increased cardiac inflammation in non-responders to CRT}

Forty-seven percent of the non-responders and $25 \%$ of the responders had increased cardiac inflammation in their EMB ( $p=0.009$; Table 1). Histological myocardial fibrosis was not related to CRT response (7.6\%, [IQR: 4.6 - 15.1\%] in non-responders and 7.5\%, [IQR: $4.5-13.1 \%$ ] in responders). There was also no significant difference in cardiac fibrosis using CMR-LGE, although there was a trend: $48 \%$ of the non-responders had LGE versus $29 \%$ of the responders. (Table 1).

Table 1. Patient characteristics of the study population.

\begin{tabular}{|c|c|c|c|c|}
\hline & $\begin{array}{l}\text { Total cohort } \\
\quad(n=99)\end{array}$ & $\begin{array}{c}\text { Non-responders } \\
(n=32)\end{array}$ & $\begin{array}{l}\text { Responders } \\
\quad(n=67)\end{array}$ & $p$-value \\
\hline Age of onset, years & 59 [51-67] & 60 [42-69] & $59[53-65]$ & NS \\
\hline Male, n (\%) & $63(64)$ & 22 (69) & $41(65)$ & NS \\
\hline Hypertension, n (\%) & $38(38)$ & $12(38)$ & 26 (39) & NS \\
\hline Diabetes Mellitus, n (\%) & $15(15)$ & $6(19)$ & $9(13)$ & NS \\
\hline Body Mass Index, $\mathrm{kg} / \mathrm{m}^{2}$ & $26[24-31]$ & $26[23-30]$ & $26[24-32]$ & NS \\
\hline Atrial Fibrillation, n (\%) & $67(68)$ & $13(41)$ & $10(15)$ & 0.005 \\
\hline NT-proBNP, pmol/L & $173[55-414]$ & 255 [77-495] & $120[43-335]$ & NS \\
\hline Creatinine, $\mu \mathrm{mol} / \mathrm{L}$ & 90 [76-116] & 90 [75-122] & 90 [79-115] & NS \\
\hline QRS duration pre-CRT, ms & $157 \pm 28$ & $154 \pm 33$ & $159 \pm 26$ & NS \\
\hline LBBB, n (\%) & $67(68)$ & $16(50)$ & $51(76)$ & 0.007 \\
\hline NYHA class II-III, n (\%) & $73(74)$ & $26(81)$ & $47(70)$ & NS \\
\hline Familial disease* & $10(13)$ & 2 (9) & $8(15)$ & NS \\
\hline Pathogenic $L M N A$ variant* & $4(5)$ & $2(9)$ & $2(4)$ & NS \\
\hline \multicolumn{5}{|l|}{ Echocardiography } \\
\hline LVEF, \% & 26 [19-34] & 31 [20-38] & 25 [18-32] & 0.04 \\
\hline LVESV, $\mathrm{ml}$ & 144 [103-198] & $123[82-171]$ & 149 [111-207] & 0.03 \\
\hline LVEDV, ml & 193 [158-234] & 173 [121-220] & $200[165-240]$ & 0.04 \\
\hline
\end{tabular}




\begin{tabular}{|c|c|c|c|c|}
\hline \multicolumn{5}{|l|}{ Endomyocardial biopsy } \\
\hline Biopsy CD3+, cells $/ \mathrm{mm}^{2}$ & $4[2-7]$ & $5[2-8]$ & $4[2-7]$ & NS \\
\hline Biopsy CD45+, cells $/ \mathrm{mm}^{2}$ & $7[4-12]$ & $9[4-12]$ & $7[4-11]$ & NS \\
\hline Biopsy CD68+, cells $/ \mathrm{mm}^{2}$ & $3[1-6]$ & $5[2-9]$ & $2[1-4]$ & NS \\
\hline $\begin{array}{c}\text { Increased cardiac } \\
\text { inflammation (ESC), } \mathrm{n}(\%)\end{array}$ & $32(32)$ & $15(47)$ & $17(25)$ & 0.009 \\
\hline $\begin{array}{l}\text { Myocardial fibrosis, } \\
\% / \text { myocardial area }\end{array}$ & $7.5[4.7-13.1]$ & $7.6[4.6-15.1]$ & $7.5[4.5-13.1]$ & NS \\
\hline Significant viral load, n (\%)* & $15(20)$ & $4(18)$ & $11(20)$ & NS \\
\hline \multicolumn{5}{|l|}{ CMR } \\
\hline CMR available, n (\%) & $74(75)$ & $21(66)$ & $53(79)$ & NS \\
\hline Indexed LVEDV $\left(\mathrm{ml} / \mathrm{m}^{2}\right)$ & $157 \pm 59$ & $142 \pm 55$ & $164 \pm 59$ & NS \\
\hline Indexed LVESV $\left(\mathrm{ml} / \mathrm{m}^{2}\right)$ & $119 \pm 53$ & $106 \pm 50$ & $125 \pm 54$ & NS \\
\hline CMR LVEF (\%) & $27 \pm 9$ & $28 \pm 9$ & $26 \pm 9$ & NS \\
\hline $\begin{array}{l}\text { Late gadolinium } \\
\text { enhancement, } \mathrm{n}(\%)\end{array}$ & $25(34)$ & $10(48)$ & $15(29)$ & NS \\
\hline \multicolumn{5}{|l|}{ Medication } \\
\hline Beta-blocker, n (\%) & $92(93)$ & $28(88)$ & $64(96)$ & NS \\
\hline ACEi/ARB, n (\%) & $90(91)$ & $29(91)$ & $61(91)$ & NS \\
\hline MRA, n (\%) & $49(50)$ & $16(50)$ & $33(49)$ & NS \\
\hline \multicolumn{5}{|c|}{ Abbreviations: CMR: cardiovascular magnetic resonance imaging; LV: left ventricle; LVEDV: left ventricular end-diastolic volume; LVESV. } \\
\hline \multirow{2}{*}{\multicolumn{5}{|c|}{$\begin{array}{l}\text { left ventricular end-systolic volume; SV: stroke volume; LVEF: left ventricular ejection fraction; CRT: cardiac resynchronization therapy; } \\
\text { ACEi: ACE-inhibitor; ARB: Angiotensin II-receptor blocker; MRA: mineralocorticoid receptor antagonist. }\end{array}$}} \\
\hline & & & & \\
\hline \multicolumn{5}{|c|}{ Values are depicted as mean \pm standard deviation, median [interquartile range] or number with percentage } \\
\hline
\end{tabular}

\section{Predictors of response}

Cardiac inflammation at EMB was an univariable negative predictor of response to CRT (OR $0.3[0.12-0.76], p=0.011$ ). Even after adjustment for well-known clinical predictors (AF, LBBB, QRS-width and $\operatorname{sex}^{4}$ ), cardiac inflammation remained a significant predictor (adjusted OR 0.22 [0.08-0.59], $p=0.003$ ).

To evaluate whether cardiac inflammation had predictive value beyond these clinical predictors, model performance of the clinical predictors with and without cardiac inflammation were tested. The in-sample AUC for the clinical predictors was 0.697, significantly increased to 0.784 after addition of cardiac inflammation (in cross validation: $0.628 \pm 0.11$ for the clinical model versus $0.714 \pm 0.1$ with cardiac inflammation added, $p<0.001$; Figure 1). Also the fit of the clinical model improved after adding cardiac inflammation to a range of models based on clinical predictors, showing its additional predictive value (likelihood ratio tests all $p<0.001$; Table 2).

Table 2. Cardiac inflammation adds predictive value beyond clinical parameters for the prediction of CRT response. A nested series of clinical predictors was evaluated with binary logistic regression and the addition of cardiac inflammation was assessed by a log likelihood ratio test.

\begin{tabular}{cc} 
Model & $\begin{array}{c}\text { p-value for addition of cardiac inflammation (likelihood } \\
\text { ratio test) }\end{array}$ \\
\hline sex, QRS-width & $1.58 \times 10^{-5}$ \\
sex, QRS-width, LBBB & $3.86 \times 10^{-5}$ \\
sex, QRS-width, LBBB, AF & $7.41 \times 10^{-5}$ \\
\hline Abbreviations: LBBB, left bundle branch block; AF, atrial fibrillation.
\end{tabular}


A

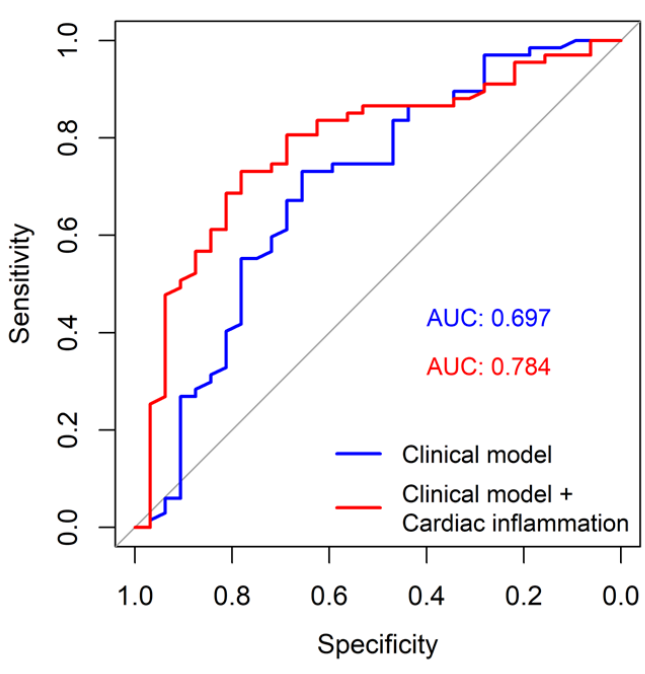

B

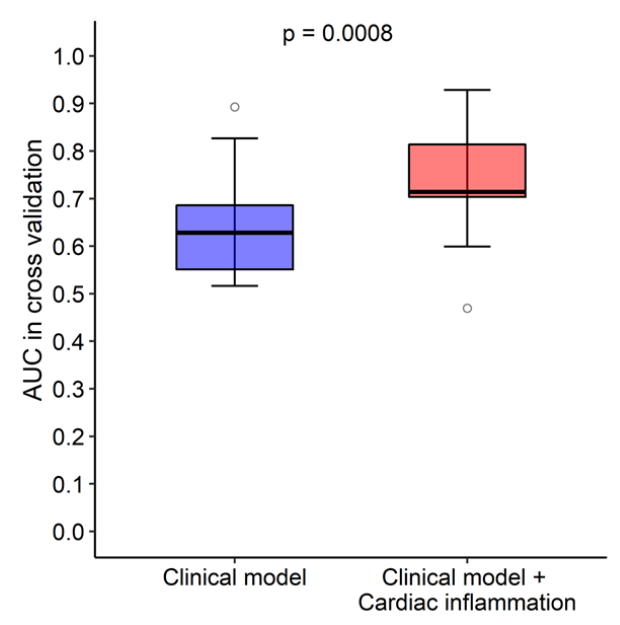

Figure 1. ROC curve for discrimination between responders and non-responders to CRT. Area under the curve (AUC) for the clinical model (left bundle branch block, atrial fibrillation, sex and QRS-width) and the clinical model with the addition of cardiac inflammation using the in-sample data (A). The robustness of the models were evaluated by 5 -repeated 5 -fold cross-validation. The AUC are plotted as median, interquartile range and $95 \%$ confidence interval, and compared using Mann-Whitney $\mathrm{U}$ test (B).

As $25 \%$ of the responders still had cardiac inflammation in their biopsy, we investigated if the duration of time between the taking of the biopsy and device implantation (EMB-device duration) had an effect on the treatment response (Suppl. Figure 3). The EMBdevice duration was no predictor for CRT response (OR 0.93 [0.85-1.01], $p=0.11$ ), but had a significant interaction with cardiac inflammation (interaction OR 0.73 [0.57-0.95], $p=0.017$ ). Within the group of DCM patients with cardiac inflammation, the non-responders had significantly more time between EMB and device implantation.

\section{Cardiac transcriptomics: upregulation of pro-inflammatory and pro-fibrotic pathways in non-responders}

To gain better insight into the molecular changes associated with non-response to CRT, RNAsequencing of EMB was performed in all patients with available spare EMBs, forming a representative subgroup ( $n=17 ; 9$ responders and 8 non-responders; Suppl. Table 3). Principal component analysis (PCA) revealed a clear separation based on transcriptomics between responders and non-responders. This indicates a distinct cardiac transcriptomic profile for non-responders (Figure 2). This distinct cardiac transcriptomic profile demonstrates significant alterations in extracellular matrix (ECM) and cardiac fibrosis pathways, using gene set enrichment analysis with predefined KEGG and GO-processes (Table 3). Genes involved in both modulation of inflammation and fibrosis (POSTN, CTGF, TGFB1, PDGFRA, TSP2) or fibrosis only (COL1A1, COL1A2, COL3A1, LOX) were the most enriched in these identified pathways and processes. These 9 genes were significantly higher expressed in the hearts of non-responders. 


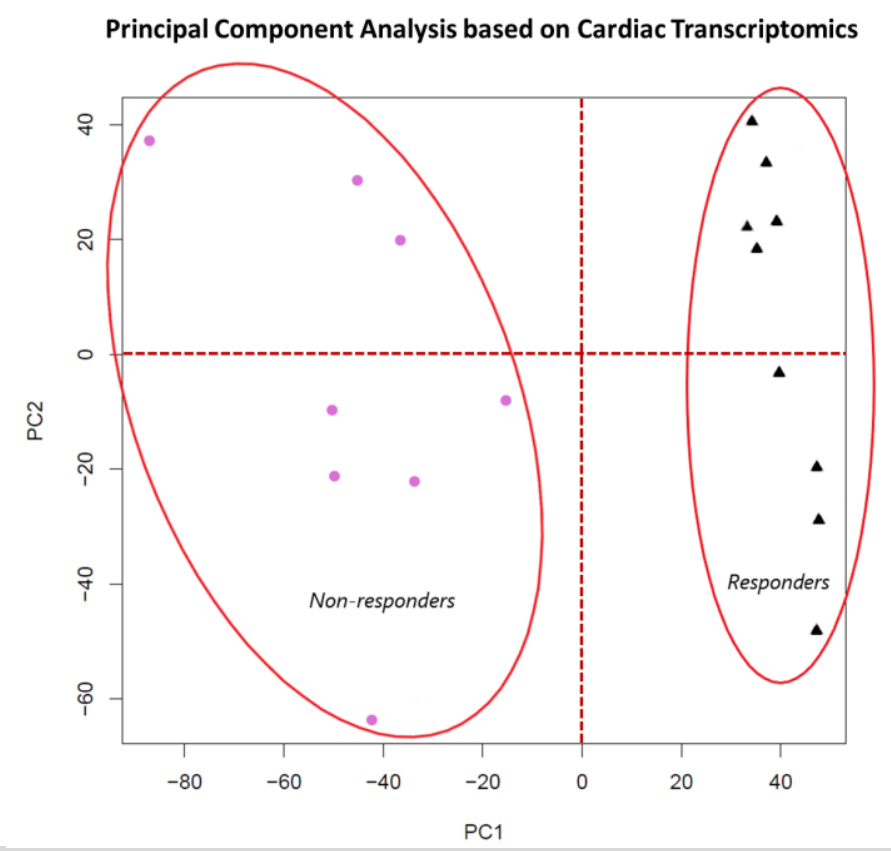

Figure 2. Principal component (PC) analysis based on cardiac RNA-sequencing data of responders versus nonresponders to cardiac resynchronization therapy. The transcriptomic profile of responders versus nonresponders is significantly different indicating strong differences in active molecular processes in the heart.

To evaluate the potential role of interactions with other proteins (and their respective genes) which are not characterized in the predefined KEGG and GO-processes, we used knowledge-based network analysis. This revealed additional protein-interactions (Suppl. Figure 4), where transcription levels of three of these additional knowledge-based intermediates were also significantly higher expressed in non-responders: COL5A1, TNC and $B G N$. The corresponding proteins of these genes play a role in the cardiac ECM modeling and the latter two are also involved in the regulation of inflammation (Figure 3).

\section{DISCUSSION}

The main finding of the present study is that a cardiac inflammatory response, both at histological and transcript levels, is associated with a non-response to CRT in DCM patients (Figure 4). Moreover, cardiac inflammation showed to have additional predictive value beyond a model of established clinical predictors of CRT response constituting of sex, QRSwidth, LBBB and AF. In total, $32 \%$ of our DCM patients did not respond to CRT, where nonresponse was related to adverse outcome, in line with previous studies ${ }^{21} 22$. Our findings may set the stage to understand the underlying pathophysiological pathways explaining the lack of response to CRT in DCM patients. Identifying molecular pathways that relate to the response to CRT in the heart, may help to identify those patients that will benefit most from CRT and distinguish them from those that need alternative therapies.

Table 3. Results of gene enrichment analysis using RNA-sequencing data and corresponding top associated genes

\begin{tabular}{cc|cc}
\hline \multicolumn{2}{c}{ Gene Enrichment Analysis } & \multicolumn{2}{c}{ Top Involved Genes in Enriched Pathways } \\
\hline KEGG pathways & $\boldsymbol{q}$-value & Gene & Protein \\
Focal adhesion & $7.43^{*} 10^{-3}$ & COL1A1 & Collagen type I alpha 1 \\
ECM-receptor interaction & $5.9 * 10^{-3}$ & COL1A2 & Collagen type I alpha 2
\end{tabular}




\begin{tabular}{|c|c|c|c|}
\hline \multirow[t]{3}{*}{ PI3K-Akt signaling pathway } & \multirow[t]{3}{*}{$5.3 * 10^{-3}$} & COL3A1 & Collagen type III alpha 1 \\
\hline & & POSTN & Periostin \\
\hline & & CTGF & Connective tissue growth factor \\
\hline GO-processes (GO:ID) & q-value & LOX & Lysyl oxidase \\
\hline Extracellular matrix organization (GO:0030198) & $1.7 * 10^{-6}$ & TGFB1 & Transforming growth factor $\beta 1$ \\
\hline $\begin{array}{l}\text { Extracellular structure organization } \\
\qquad(\text { GO:0043062) }\end{array}$ & $9.4 * 10^{-7}$ & PDGFRA & $\begin{array}{l}\text { Platelet-derived growth factor } \\
\text { receptor } \alpha\end{array}$ \\
\hline Cell adhesion (GO:0007155) & $1.5 * 10^{-5}$ & TSP2 & Thrombospondin 2 \\
\hline
\end{tabular}

Abbreviations: KEGG: Kyoto encyclopedia of genes and genomes; ECM: Extra-cellular matrix; GO: Gene ontology.

\section{The role of inflammation and fibrosis in CRT response: evidence from cardiac biopsies}

Our enrichment analysis reveals that gene transcripts of the pro-inflammatory and profibrotic matricellular proteins are significantly higher expressed in cardiac tissue of DCM who did not respond to CRT, namely POSTN, CTGF, TGFB1, PDGFRA, TSP2, COL1A1, COL1A2, COL3A1, COL5A1, LOX, TNC and BGN. This suggests that non-responders have ongoing inflammation with subsequent changes in the extracellular matrix (ECM), which could hamper the beneficial response to electrical therapy. Both cardiac inflammation and fibrosis are major processes in the failing heart which are closely linked to each other ${ }^{23}$. Non-structural proteins are important modulators of inflammation and the cardiac ECM ${ }^{24}$. Glycoproteins and proteoglycans orchestrate the remodeling of the myocardium during pressure overload, myocardial stress and injury (Figure 3). Importantly, most of the differentially expressed transcripts in non-responders are involved in the major signaling pathways of periostin (POSTN). Periostin is induced by transforming growth factor- $\beta$ (TGF- $\beta$ )- and phosphoinositide 3-kinase-protein kinase B (PI3K-Akt) signaling ${ }^{25}{ }^{26}$. Afterwards, POSTN together with matrixassociated lysyl oxidase (LOX) and tenascin-C (TNC) stimulate the transcription of collagen I (COL1A1; COL1A2) and connective tissue growth factor (CTGF), eventually promoting a cardiac pro-fibrotic phenotype ${ }^{26}$. Importantly, all these targets were higher expressed in the hearts of non-responders indicating a potential novel pathway for treatment. However, the higher expression of ECM pathways was not reflected in an increase in cardiac fibrosis on histologic or at imaging level using CMR-LGE. With respect to histology, this might be due to sampling bias as fibrosis has a predominantly focal aspect, in contrast to the more diffuse character of inflammation. As to imaging, it accurately localizes replacement fibrosis using LGE, but to a lesser extent with regard to quantification of interstitial fibrosis in the myocardium as a whole. Overall, it is possible that the histological cardiac inflammation is a precursor for cardiac fibrosis, which is already reflected in the cardiac transcriptome. The challenge lies in targeting this reversible cardiac inflammation before it proceeds to irreversible fibrotic tissue.

In this study, we used right ventricular (RV) EMBs, which could have led to falsenegative results regarding the finding of cardiac inflammation compared to LV EMBs and/or biventricular EMB ${ }^{27}$. Conversely, finding cardiac inflammation is more likely when there is RV dysfunction. We did not find such correlation between RV function and cardiac inflammation, in a sub-analysis of the Maastricht cohort with available RV measures (Suppl. Table 4). Also, RV function seems not predictive for CRT response (Suppl. Table 5).

\section{Signs of dyssynchrony}

LBBB, AF and cardiac inflammation were univariate predictors of CRT response, of which AF and LBBB are extensively been described ${ }^{428-30}$. Increased cardiac inflammation may lead to a nonspecific intraventricular conduction delay ${ }^{31} 32$, known to have more unfavorable CRT response rates 3033 . Consequently, although many non-responders meet current CRT 
indications, they may not have an actual (isolated) conduction disorder due to the ongoing cardiac inflammation ${ }^{33}$.

Whether increased myocardial inflammation with associated pro-inflammatory and fibrotic activity in non-responders is the cause or consequence of the conduction delay remains to be elucidated. Indeed, the electromechanical dysfunction in a failing heart is primarily related to the electromechanical delay in left and right ventricular activation, but also depends on the weakness in contraction strength of the myocardial fibers. Cardiac pacing is effective in removing the electrical component of the electromechanical delay, whereas the mechanical component is mainly dependent on the severity of the underlying myocardial disease. Ongoing myocardial inflammation may be the actual driving process hampering mechanical improvement in these non-responders. Therefore, we hypothesize that the observed conduction delay in DCM patients with increased cardiac inflammation could be a consequence of myocardial disease rather than a conduction disorder causing cardiac dysfunction.

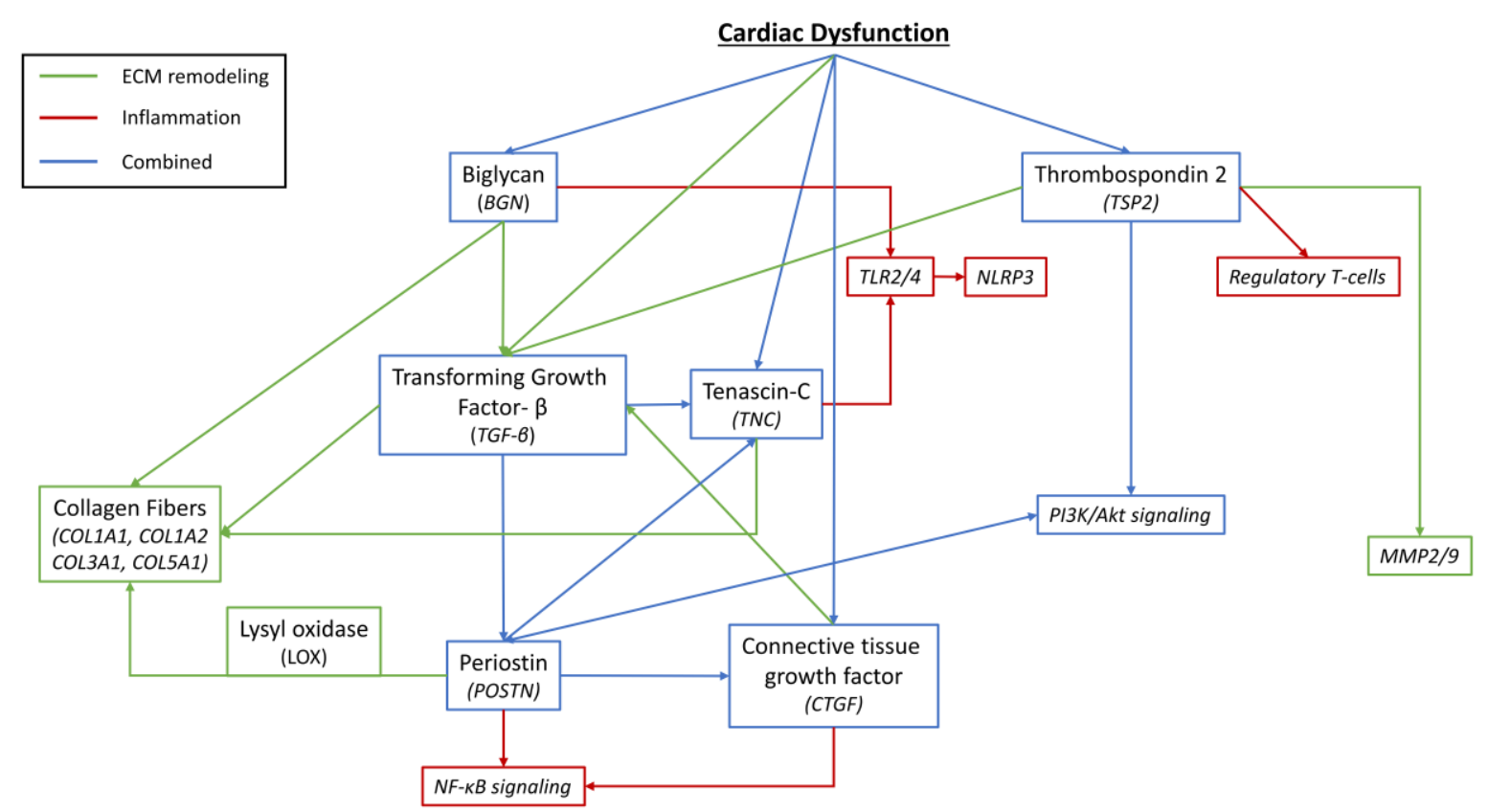

Figure 3. Biological interaction of matricellular proteins which are differentially expressed in the hearts of non-responders. Network of the biology behind all significant differentially expressed genes in CRT responders versus non-responders. Interactions are based on the induced knowledge-based network analysis and subsequent literature (Suppl. Figure 4). Arrows indicate downstream signaling within the pathway.

\section{Clinical implications and future directions}

Cardiac inflammation along with matrix alterations distinguished the non-responders from the responders in DCM. This suggests that evaluating the current ongoing processes that drive the DCM phenotype can provide a subset of patients with a less favorable response to CRT. Future studies should focus on less invasive diagnostic modalities that strongly correlate with histology, as we recognize EMBs prior to CRT implantation is not performed regularly in most centers. Therefore, we foresee a combination of advanced imaging - e.g. CMR complemented with circulating blood markers to accurately depict ongoing cardiac inflammation and fibrogenesis, making non-invasive individual evaluation of these specific patients possible. In addition, whether these findings of increased inflammation in the heart of non-responders would require additional immunosuppressive treatment is unclear and 
should be addressed in prospective trials ${ }^{13} 34$. Currently, none of the included patients was treated with immunosuppressive treatment, but this will eventually provide answers regarding the modifiability of these outcome determinants, and the possibility to turn a nonresponder into a responder. The observation that non-responders with cardiac inflammation had more time between EMB and device implantation compared to the responders with inflammation, warrants further investigation as to whether early intervention might be beneficial in those patients with inflammation in the EMB.

\section{Limitations}

The availability of EMBs before device implantation is quite rare, as DCM patients with an indication for CRT are often immediately considered for device implantation given the current guidelines. Therefore, the number of DCM patients we could include from both registries was relatively low and did not provide enough power to analyze all potential interaction terms. However, this study provides evidence that in-depth phenotyping before device implantation should be considered. The availability of spare EMBs is challenging given the invasive procedure, however we managed to include a representative subgroup for the tissue RNAsequencing with equal numbers in both groups. Finally, this is a retrospective analysis although inclusion of patients in the registry was done prospectively. Therefore, prospective testing of the hypothesis coming from the present findings is required.

\section{CONCLUSION}

Cardiac inflammation along with a transcriptomic profile of high expression of combined proinflammatory and pro-fibrotic genes are associated with a poor response to CRT in DCM patients.

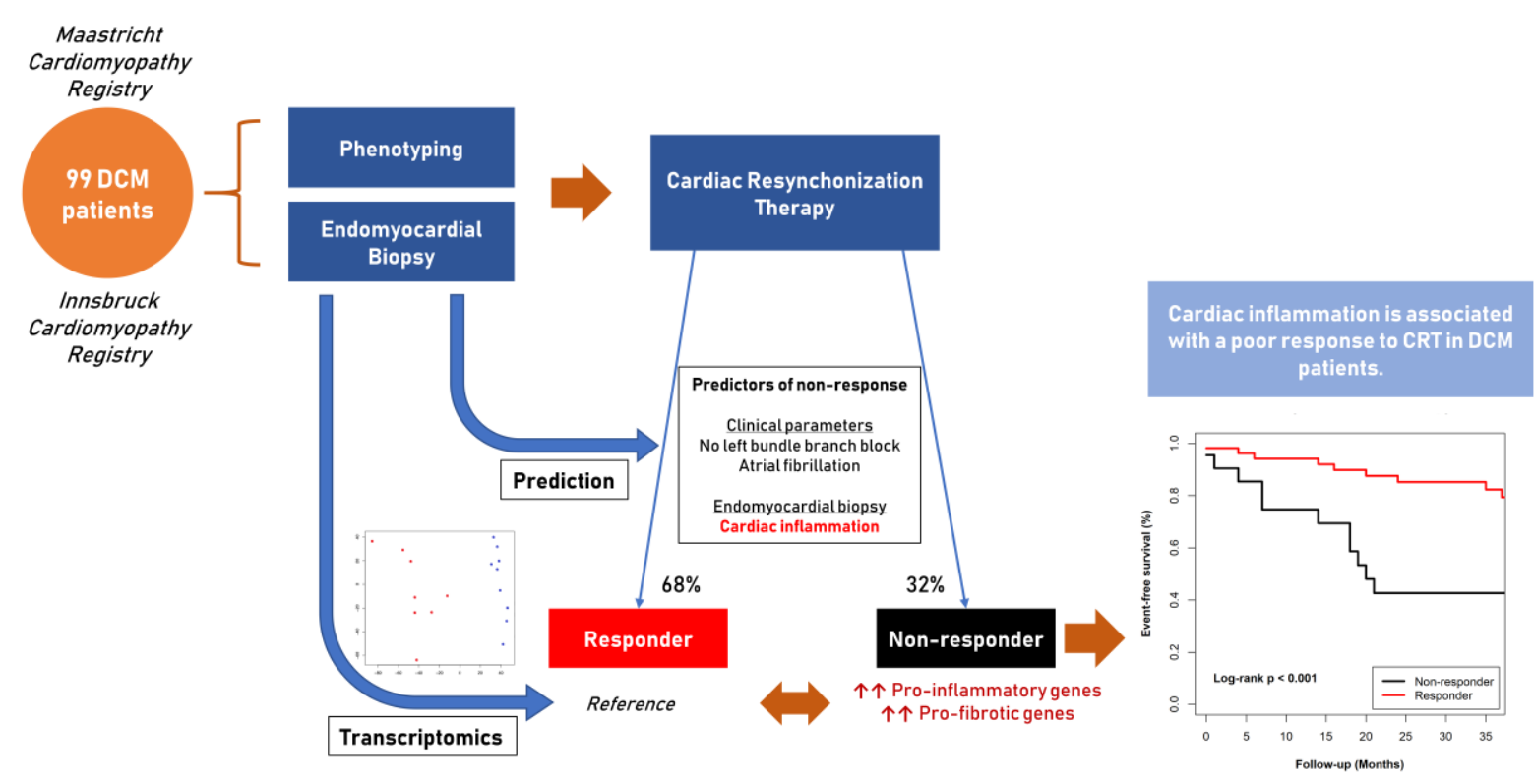

Figure 4. Prediction, outcome and molecular processes involved in response to cardiac resynchronization therapy. Cardiac inflammation determined in an endomyocardial biopsy is an independent predictor for CRT response, which was validated in an external cohort. RNA-sequencing of cardiac tissue revealed higher expression of genes involved in extracellular matrix remodeling in the non-responders. Response to CRT is an important predictor for long-term outcome of a DCM patient. 


\section{REFERENCES}

1. Brignole $M$, Auricchio A, Baron-Esquivias G, et al. 2013 ESC Guidelines on cardiac pacing and cardiac resynchronization therapy: the Task Force on cardiac pacing and resynchronization therapy of the European Society of Cardiology (ESC). Developed in collaboration with the European Heart Rhythm Association (EHRA). Eur Heart J 2013;34(29):2281-329.

2. Abraham WT, Fisher WG, Smith AL, et al. Cardiac resynchronization in chronic heart failure. $N$ Engl J Med 2002;346(24):1845-53.

3. Leyva F, Plummer CJ. National Institute for Health and Care Excellence 2014 guidance on cardiac implantable electronic devices: health economics reloaded. Europace 2015;17(3):33942.

4. Rickard J, Michtalik H, Sharma R, et al. Predictors of response to cardiac resynchronization therapy: A systematic review. Int J Cardiol 2016;225:345-52.

5. Feeny AK, Rickard J, Patel D, et al. Machine Learning Prediction of Response to Cardiac Resynchronization Therapy: Improvement Versus Current Guidelines. Circulation Arrhythmia and electrophysiology 2019;12(7):e007316.

6. Shanks M, Delgado V, Ng AC, et al. Clinical and echocardiographic predictors of nonresponse to cardiac resynchronization therapy. Am Heart $J$ 2011;161(3):552-7.

7. Daubert JC, Saxon L, Adamson PB, et al. 2012 EHRA/HRS expert consensus statement on cardiac resynchronization therapy in heart failure: implant and follow-up recommendations and management. Heart Rhythm 2012;9(9):1524-76.

8. Japp AG, Gulati A, Cook SA, et al. The Diagnosis and Evaluation of Dilated Cardiomyopathy. J Am Coll Cardiol 2016;67(25):2996-3010.

9. van Stipdonk AMW, Ter Horst I, Kloosterman M, et al. QRS Area Is a Strong Determinant of Outcome in Cardiac Resynchronization Therapy. Circulation Arrhythmia and electrophysiology 2018;11(12):e006497.

10. Huntjens PR, Ploux S, Strik M, et al. Electrical Substrates Driving Response to Cardiac Resynchronization Therapy: A Combined ClinicalComputational Evaluation. Circulation Arrhythmia and electrophysiology 2018;11(4):e005647.

11. Khan FZ, Virdee MS, Palmer CR, et al. Targeted left ventricular lead placement to guide cardiac resynchronization therapy: the TARGET study: a randomized, controlled trial. J Am Coll Cardiol 2012;59(17):1509-18.

12. Verdonschot JAJ, Hazebroek MR, Derks KWJ, et al. Titin cardiomyopathy leads to altered mitochondrial energetics, increased fibrosis and long-term life-threatening arrhythmias. Eur Heart J 2018

13. Merken J, Hazebroek M, Van Paassen $P$, et al. Immunosuppressive Therapy Improves Both Short- and Long-Term Prognosis in Patients With Virus-Negative Nonfulminant Inflammatory Cardiomyopathy. Circulation Heart failure 2018;11(2):e004228.

14. Pinto YM, Elliott PM, Arbustini E, et al. Proposal for a revised definition of dilated cardiomyopathy, hypokinetic non-dilated cardiomyopathy, and its implications for clinical practice: a position statement of the ESC working group on myocardial and pericardial diseases. Eur Heart J 2016;37(23):1850-8.

15. Ponikowski P, Voors AA, Anker SD, et al. 2016 ESC Guidelines for the diagnosis and treatment of acute and chronic heart failure: The Task Force for the diagnosis and treatment of acute and chronic heart failure of the European Society of Cardiology (ESC)Developed with the special contribution of the Heart Failure Association (HFA) of the ESC. Eur Heart J 2016;37(27):2129200.

16. Strauss DG, Selvester RH, Wagner GS. Defining left bundle branch block in the era of cardiac resynchronization therapy. Am J Cardiol 2011;107(6):927-34.

17. Verdonschot JAJ, Hazebroek MR, Ware JS, et al. Role of Targeted Therapy in Dilated Cardiomyopathy: The Challenging Road Toward a Personalized Approach. Journal of the American Heart Association 2019;8(11):e012514.

18. Caforio AL, Pankuweit S, Arbustini $\mathrm{E}$, et al. Current state of knowledge on aetiology, diagnosis, management, and therapy of myocarditis: a position statement of the European Society of Cardiology Working Group on Myocardial and Pericardial Diseases. Eur Heart J 2013;34(33):2636-48, 48a-48d.

19. Verdonschot J, Hazebroek M, Merken J, et al. Relevance of cardiac parvovirus B19 in myocarditis and dilated cardiomyopathy: review of the literature. Eur J Heart Fail 2016;18(12):1430-41.

20. Herwig R, Hardt C, Lienhard M, et al. Analyzing and interpreting genome data at the network level with ConsensusPathDB. Nat Protoc 2016;11(10):1889-907.

21. Goldenberg I, Moss AJ, Hall WJ, et al. Predictors of response to cardiac resynchronization therapy in the Multicenter Automatic Defibrillator 
Implantation Trial with Cardiac Resynchronization Therapy (MADIT-CRT). Circulation 2011;124(14):1527-36.

22. Rickard J, Cheng A, Spragg D, et al. Durability of the survival effect of cardiac resynchronization therapy by level of left ventricular functional improvement: fate of "nonresponders". Heart Rhythm 2014;11(3):412-6.

23. Suthahar N, Meijers WC, Sillje HHW, et al. From Inflammation to Fibrosis-Molecular and Cellular Mechanisms of Myocardial Tissue Remodelling and Perspectives on Differential Treatment Opportunities. Curr Heart Fail Rep 2017;14(4):235-50.

24. Rienks M, Papageorgiou AP, Frangogiannis NG, et al. Myocardial extracellular matrix: an everchanging and diverse entity. Circ Res 2014;114(5):872-88.

25. de Boer RA, De Keulenaer G, Bauersachs J, et al. Towards better definition, quantification and treatment of fibrosis in heart failure. A scientific roadmap by the Committee of Translational Research of the Heart Failure Association (HFA) of the European Society of Cardiology. Eur J Heart Fail 2019

26. Landry NM, Cohen S, Dixon IMC. Periostin in cardiovascular disease and development: a tale of two distinct roles. Basic Res Cardiol 2018;113(1):1.

27. Chimenti C, Frustaci A. Contribution and risks of left ventricular endomyocardial biopsy in patients with cardiomyopathies: a retrospective study over a 28-year period. Circulation 2013;128(14):1531-41.

28. Bilchick KC, Kamath S, DiMarco JP, et al. Bundlebranch block morphology and other predictors of outcome after cardiac resynchronization therapy in Medicare patients. Circulation 2010;122(20):2022-30.

29. Healey JS, Hohnloser SH, Exner DV, et al. Cardiac resynchronization therapy in patients with permanent atrial fibrillation: results from the Resynchronization for Ambulatory Heart Failure Trial (RAFT). Circulation Heart failure 2012;5(5):566-70.

30. Hsu JC, Solomon SD, Bourgoun $M$, et al. Predictors of super-response to cardiac resynchronization therapy and associated improvement in clinical outcome: the MADITCRT (multicenter automatic defibrillator implantation trial with cardiac resynchronization therapy) study. J Am Coll Cardiol 2012;59(25):2366-73.

31. Eschalier R, Ploux $S$, Ritter $P$, et al. Nonspecific intraventricular conduction delay: Definitions, prognosis, and implications for cardiac resynchronization therapy. Heart Rhythm 2015;12(5):1071-9.

32. Morimoto $S$, Kato $S$, Hiramitsu $S$, et al. Role of myocardial interstitial edema in conduction disturbances in acute myocarditis. Heart and vessels 2006;21(6):356-60.

33. van Stipdonk AM, Rad MM, Luermans JG, et al. Identifying delayed left ventricular lateral wall activation in patients with non-specific intraventricular conduction delay using coronary venous electroanatomical mapping. Neth Heart J 2016;24(1):58-65.

34. Frustaci A, Russo MA, Chimenti C. Randomized study on the efficacy of immunosuppressive therapy in patients with virus-negative inflammatory cardiomyopathy: the TIMIC study. Eur Heart J 2009;30(16):1995-2002. 


\section{SUPPLEMENTARY FIGURES}

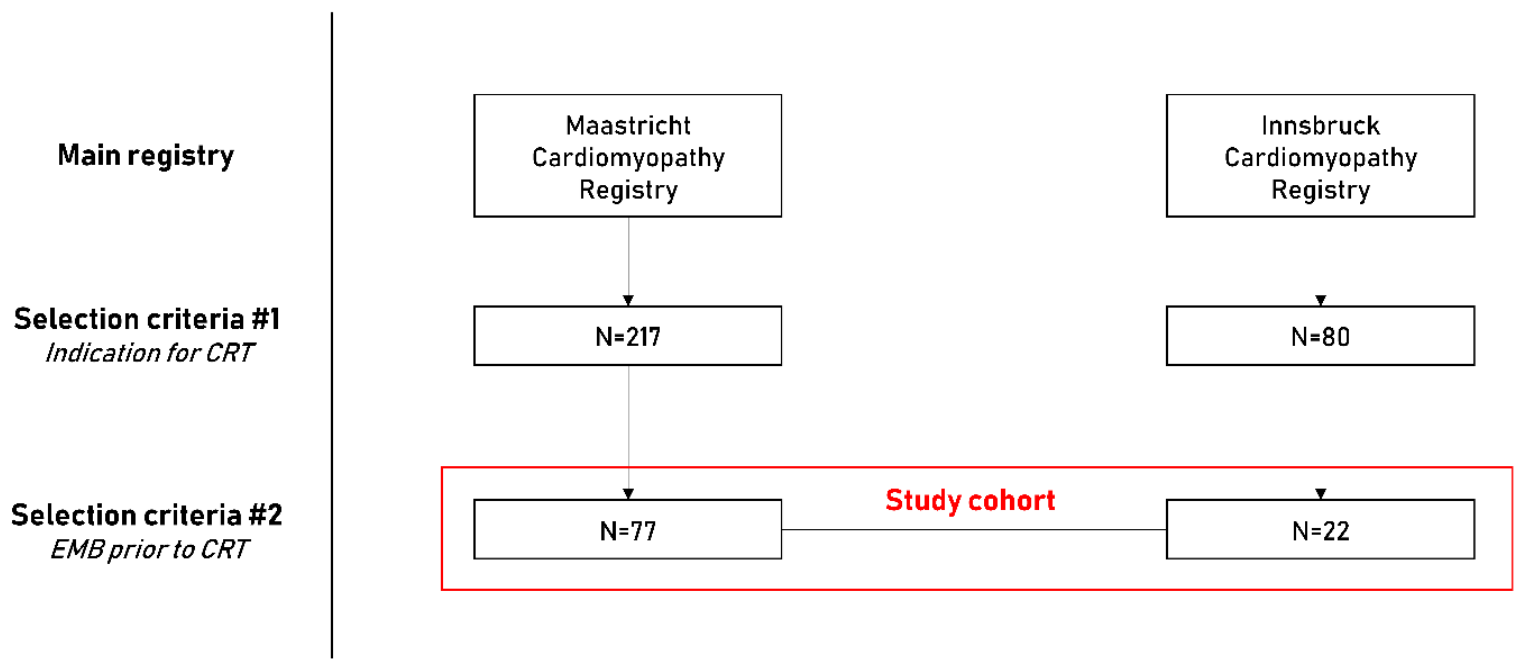

Supplemental Figure 1. Flowchart of patient selection from the Maastricht and Innsbruck Cardiomyopathy Registry. EMB: Endomyocardial Biopsy; CRT: Cardiac Resynchronization Therapy.

\section{Outcome stratified by Response to Cardiac Resynchronization Therapy}

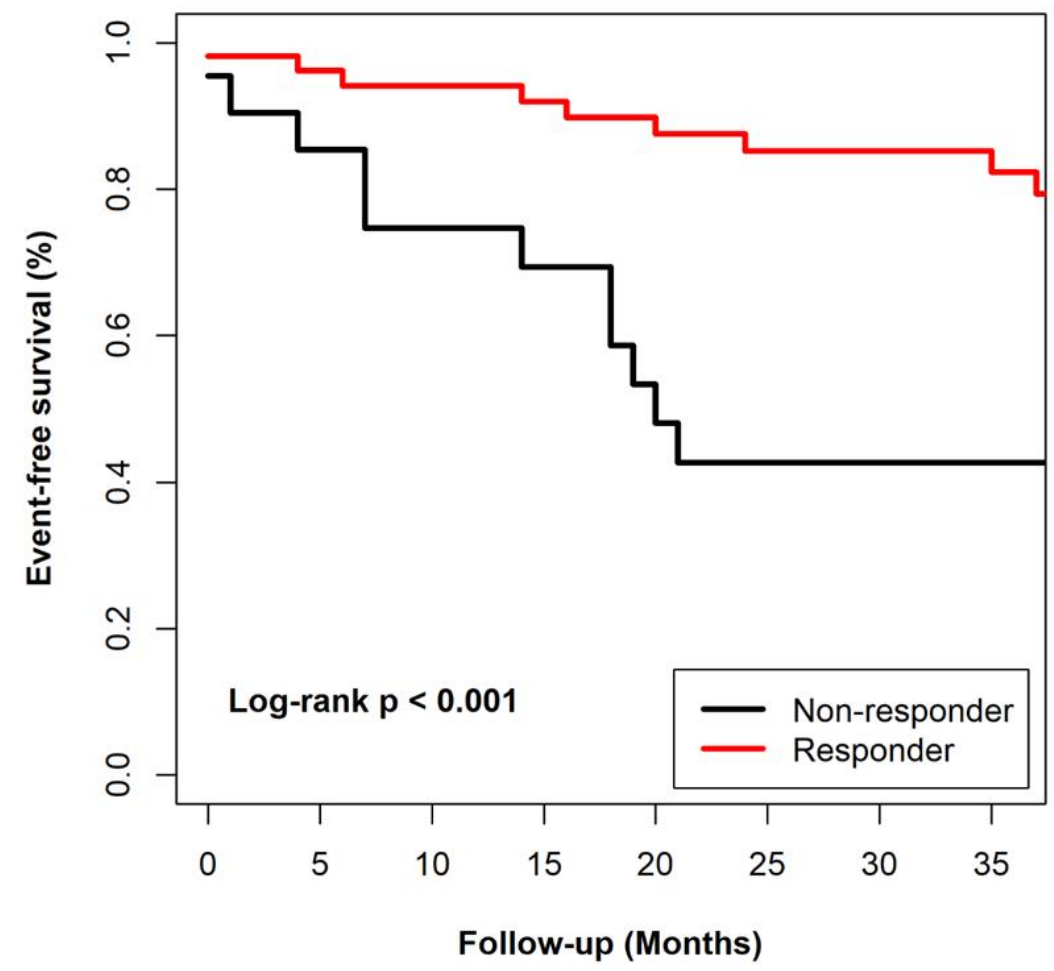

Supplemental Figure 2. Survival curves comparing freedom from the combined end-point in patient with and without response to CRT. CRT responders have a significant better survival compared to non-responders $(p<0.001)$.Curves are compared using the log-rank test. Follow-up time is shown as months since device implantation. 
Response to CRT based on cardiac inflammation and time between EMB and device implantation Significant cardiac inflammation

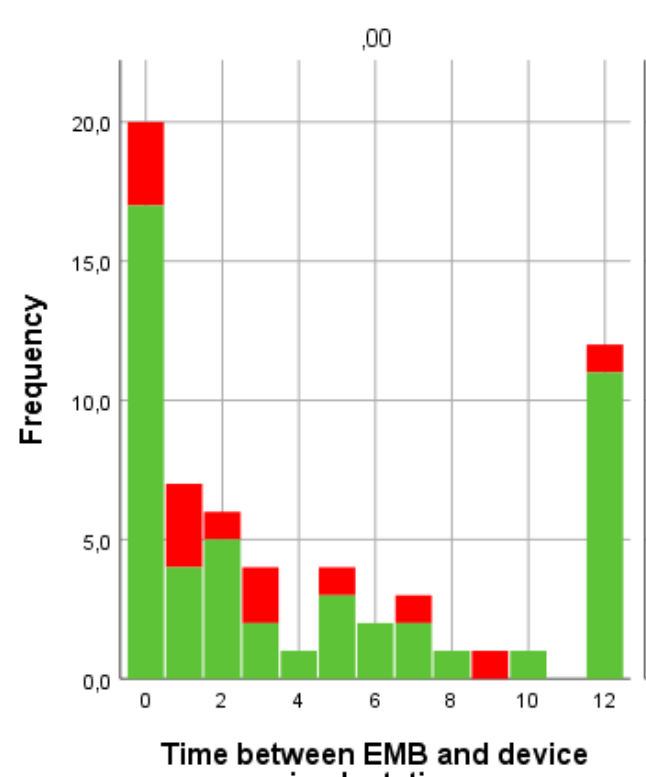
implantation

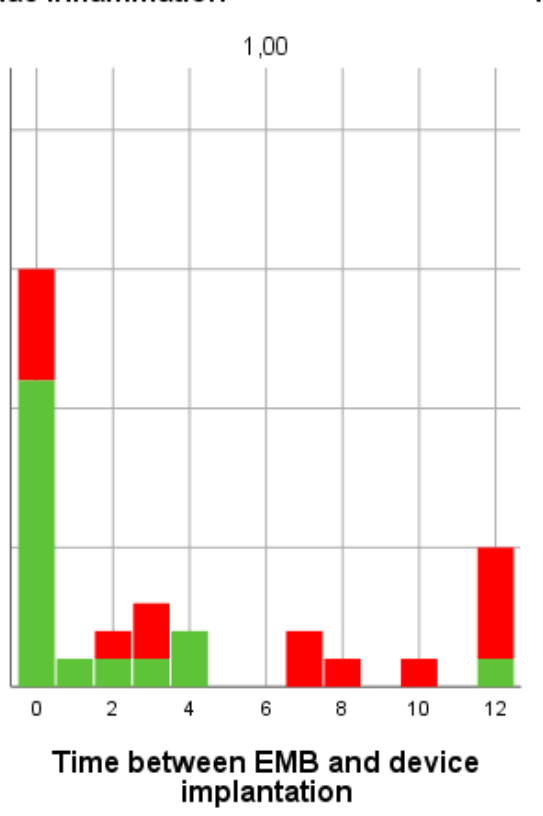

Responder

, 00

$\square, 00$

Supplemental Figure 3. Histogram depicting the duration of time between the taking of endomyocardial biopsies (EMB) and device implantation in months. The left panel indicates the patients without cardiac inflammation, the right panel indicates the patients with cardiac inflammation. Red indicates the nonresponders, green the responders to cardiac resynchronization therapy (CRT).

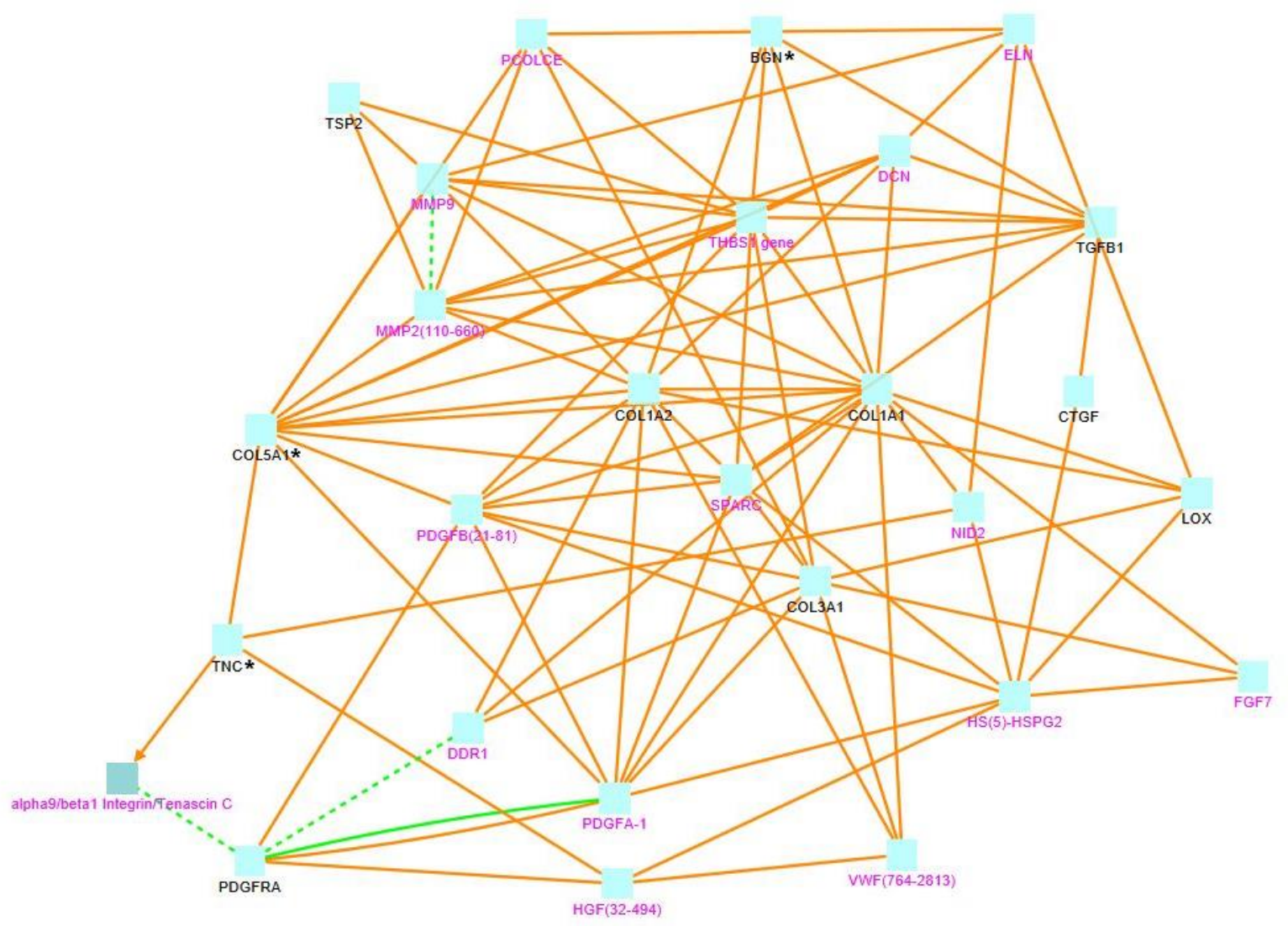


Supplemental Figure 4. Induced network analysis using the top genes selected from gene enrichment analysis on RNAseq data. This shows the interaction and connection between the top genes (black labels) with additional targets added to the network (pink). Black gene names indicate seed nodes (input); pink gene names indicate intermediate nodes (knowledge-based added to the network). Orange lines indicate protein-protein interactions and green lines indicate biochemical interactions. An asterisk depicts that the seed node was added after knowledge-based network analysis. All names used in the figure refer to their corresponding gene.

\section{SUPPLEMENTARY TABLES}

Supplemental Table 1. Comparison of the baseline variables between CRT patients included in the study and CRT patients not included in study from the Maastricht Cardiomyopathy Registry.

\begin{tabular}{|c|c|c|c|}
\hline & $\begin{array}{l}\text { CRT patients included in } \\
\text { study } \\
(\mathbf{n}=77)\end{array}$ & $\begin{array}{l}\text { CRT patients not included } \\
\text { in study } \\
(\mathbf{n}=140)\end{array}$ & $p$-value \\
\hline Age of onset, years & $60[43-67]$ & $58[52-66]$ & NS \\
\hline Male, n (\%) & $46(60)$ & 97 (69) & NS \\
\hline Hypertension, n (\%) & 30 (39) & $37(27)$ & NS \\
\hline Diabetes Mellitus, n (\%) & $10(13)$ & $16(11)$ & NS \\
\hline Body Mass Index, $\mathrm{kg} / \mathrm{m}^{2}$ & $26[24-32]$ & $27[24-30]$ & NS \\
\hline Atrial Fibrillation, $\mathrm{n}(\%)$ & $16(21)$ & $23(16)$ & NS \\
\hline NT-proBNP, pmol/L & 129 [43-374] & 92 [29-269] & 0.05 \\
\hline Creatinine, $\mu \mathrm{mol} / \mathrm{L}$ & $96[79-116]$ & 98 [81-113] & NS \\
\hline QRS duration pre-CRT, ms & $158 \pm 27$ & $152 \pm 31$ & NS \\
\hline LBBB, $\mathrm{n}(\%)$ & $50(65)$ & $86(61)$ & NS \\
\hline NYHA class II-III, n (\%) & $54(70)$ & $72(52)$ & NS \\
\hline \multicolumn{4}{|l|}{ Echocardiography } \\
\hline LVEF, \% & $27[20-35]$ & $26[21-35]$ & NS \\
\hline LVESV, ml & 143 [104-191] & $130[105-176]$ & NS \\
\hline LVEDV, ml & $193[158-234]$ & $192[145-235]$ & NS \\
\hline \multicolumn{4}{|l|}{ Medication } \\
\hline Beta-blocker, n (\%) & $72(94)$ & $127(91)$ & NS \\
\hline ACEi/ARB, n (\%) & $69(90)$ & $129(92)$ & NS \\
\hline MRA, n (\%) & $38(49)$ & $74(53)$ & NS \\
\hline
\end{tabular}

Abbreviations: LVEDV: left ventricular end-diastolic volume; LVESV: left ventricular end-systolic volume; SV: stroke volume; LVEF: left ventricular ejection fraction; CRT: cardiac resynchronization therapy; ACEi: ACE-inhibitor; ARB: Angiotensin II-receptor blocker; MRA: mineralocorticoid receptor antagonist.

Values are depicted as mean \pm standard deviation, median [interquartile range] or number with percentage

Supplemental Table 2. Long-term follow-up and adverse events in responders versus non-responders

\begin{tabular}{cccccc} 
& $\begin{array}{c}\text { Maastricht } \\
\text { cohort } \\
(\mathbf{n = 7 7 )}\end{array}$ & $\begin{array}{c}\text { Non-responders } \\
(\mathbf{n}=\mathbf{2 2})\end{array}$ & $\begin{array}{c}\text { Responders } \\
(\mathbf{n}=55)\end{array}$ & $\boldsymbol{p}$-value & $\begin{array}{c}\text { Hazard } \\
\text { Ratio [95\% Cl] }\end{array}$ \\
\hline Follow-up Duration & $36[14-54]$ & $22[6-41]$ & $44[20-56]$ & 0.02 & - \\
Death/HTx/LVAD & $14(18)$ & $9(41)$ & $5(9)$ & 0.002 & $0.18[0.06-0.53]$ \\
HF Hospitalization & $8(10)$ & $7(32)$ & $1(2)$ & 0.003 & $0.04[0.01-0.34]$ \\
Life-threatening Arrhythmia & $10(13)$ & $3(14)$ & $7(13)$ & 0.51 & - \\
Combined Event & $22(29)$ & $12(55)$ & $10(18)$ & 0.001 & $0.23[0.1-0.53]$ \\
\hline
\end{tabular}

Abbreviations: Cl: confidence interval; HTx: Heart transplantation; LVAD: Left ventricular assist device; HF: Heart failure

Data is depicted as median with interquartile range or as numbers with percentage 
Supplemental Table 3. Patient characteristics of DCM patients who had available EMB for RNA-sequencing.

\begin{tabular}{|c|c|c|c|}
\hline & $\begin{array}{c}\text { Non-responders } \\
(n=8)\end{array}$ & $\begin{array}{l}\text { Responders } \\
\qquad(n=9)\end{array}$ & $p$-value \\
\hline Age of onset, years & $67 \pm 10$ & $61 \pm 7$ & 0.16 \\
\hline Male, n (\%) & $3(38)$ & $4(44)$ & 0.99 \\
\hline Hypertension, n (\%) & $4(50)$ & $2(22)$ & 0.34 \\
\hline Diabetes Mellitus, n (\%) & $2(25)$ & $2(22)$ & 0.99 \\
\hline Atrial Fibrillation, $\mathrm{n}(\%)$ & $3(38)$ & $1(11)$ & 0.29 \\
\hline NT-proBNP, pmol/L & $108[68-1055]$ & $90[43-125]$ & 0.31 \\
\hline Creatinine, $\mu \mathrm{mol} / \mathrm{L}$ & $101[74-171]$ & 119 [86-142] & 0.42 \\
\hline QRS duration pre-CRT, ms & $158 \pm 27$ & $166 \pm 11$ & 0.45 \\
\hline LBBB, n (\%) & $4(50)$ & $8(89)$ & 0.13 \\
\hline NYHA II-III, n (\%) & $6(75)$ & $8(89)$ & 0.58 \\
\hline \multicolumn{4}{|l|}{ Echocardiography } \\
\hline LVEF, \% & $34 \pm 13$ & $27 \pm 6$ & 0.15 \\
\hline LVESV, ml & 105 [71-168] & 149 [107-192] & 0.25 \\
\hline LVEDV, $\mathrm{ml}$ & $171[123-211]$ & $186[149-260]$ & 0.25 \\
\hline \multicolumn{4}{|l|}{ Endomyocardial biopsy } \\
\hline Biopsy CD3+, cells $/ \mathrm{mm}^{2}$ & $6[3-13]$ & $2[1-3]$ & 0.03 \\
\hline Biopsy CD45+, cells $/ \mathrm{mm}^{2}$ & $12[10-16]$ & $4[2-6]$ & $<0.001$ \\
\hline Biopsy CD68+, cells $/ \mathrm{mm}^{2}$ & $5[2-13]$ & $2[1-6]$ & 0.38 \\
\hline $\begin{array}{c}\text { Increased cardiac } \\
\text { inflammation (ESC), n (\%) }\end{array}$ & $6(75)$ & $0(0)$ & 0.002 \\
\hline Myocardial fibrosis & $14.7[4.9-24.2]$ & $10.9[2.9-14.6]$ & 0.48 \\
\hline Significant viral load, n (\%) & $1(13)$ & $1(11)$ & 0.99 \\
\hline \multicolumn{4}{|l|}{ Medication } \\
\hline Beta-blocker, n (\%) & $8(100)$ & $9(100)$ & 0.99 \\
\hline ACEi/ARB, n (\%) & $6(75)$ & $8(89)$ & 0.58 \\
\hline MRA, n (\%) & $4(50)$ & $5(56)$ & 0.99 \\
\hline
\end{tabular}

Abbreviations: CMR: cardiovascular magnetic resonance imaging; LV: left ventricle; LVEDV: left ventricular end-diastolic volume; LVESV: left ventricular end-systolic volume; SV: stroke volume; LVEF: left ventricular ejection fraction; CRT: cardiac resynchronization therapy; ACEi: ACE-inhibitor; ARB: Angiotensin II-receptor blocker; MRA: mineralocorticoid receptor antagonist.

Values are depicted as mean \pm standard deviation, median [interquartile range] or number with percentage

Supplemental Table 4. Right ventricle function as measured with cardiac magnetic resonance imaging within the Maastricht subgroup, divided on response to cardiac resynchronization therapy.

\begin{tabular}{ccccc} 
& $\begin{array}{c}\text { Total cohort } \\
(\mathbf{n = 7 7})\end{array}$ & $\begin{array}{c}\text { Non-responders } \\
(\mathbf{n = 2 2})\end{array}$ & $\begin{array}{c}\text { Responders } \\
(\mathbf{n = 5 5 )}\end{array}$ & $\boldsymbol{p}$-value \\
\hline RV measure available on & $29(38)$ & $6(27)$ & $23(42)$ & NS \\
CMR, $\mathrm{n}(\%)$ & $81 \pm 28$ & $78 \pm 19$ & $81 \pm 30$ & NS \\
Indexed RVEDV (ml/m²) & $41 \pm 21$ & $34 \pm 9$ & $42 \pm 23$ & NS \\
Indexed RVESV (ml/m²) & $51 \pm 13$ & $56 \pm 7$ & $49 \pm 14$ & NS \\
\hline CMR RVEF (\%) & & &
\end{tabular}

Abbreviations: CMR: cardiovascular magnetic resonance imaging; NS: not significant; RV: right ventricle; RVEDV: right ventricular enddiastolic volume; RVESV: right ventricular end-systolic volume; RVEF: right ventricular ejection fraction.

Values are depicted as meantstandard deviation, or number with percentage 
Supplemental Table 5. Right ventricle function as measured with cardiac magnetic resonance imaging within the Maastricht subgroup, divided on the presence of cardiac inflammation in the endomyocardial biopsy.

\begin{tabular}{ccccc} 
& $\begin{array}{c}\text { Total cohort } \\
(\mathbf{n}=\mathbf{7 7})\end{array}$ & $\begin{array}{c}\text { No cardiac } \\
\text { inflammation } \\
(\mathbf{n = 5 2})\end{array}$ & $\begin{array}{c}\text { Cardiac inflammation } \\
(\mathbf{n = 2 5})\end{array}$ & $\boldsymbol{p}$-value \\
\hline RV measure available on & $29(38)$ & $17(33)$ & $12(48)$ & NS \\
CMR, $\mathrm{n}(\%)$ & $81 \pm 28$ & $83 \pm 31$ & $77 \pm 23$ & NS \\
Indexed RVEDV $\left(\mathrm{ml} / \mathrm{m}^{2}\right)$ & $41 \pm 21$ & $43 \pm 24$ & $38 \pm 16$ & NS \\
Indexed RVESV $\left(\mathrm{ml} / \mathrm{m}^{2}\right)$ & $51 \pm 13$ & $50 \pm 15$ & $52 \pm 11$ & NS \\
CMR RVEF $(\%)$ & & &
\end{tabular}

Abbreviations: CMR: cardiovascular magnetic resonance imaging; NS: not significant; RV: right ventricle; RVEDV: right ventricular enddiastolic volume; RVESV: right ventricular end-systolic volume; RVEF: right ventricular ejection fraction.

Values are depicted as mean \pm standard deviation, or number with percentage 
(ब) 


\title{
CHAPTER 10
}

\section{Metabolic profiling associates with disease severity in non-ischemic dilated cardiomyopathy}

\author{
Job A.J. Verdonschot, $M D, M S c^{1,2^{*}}$ \\ Ping Wang, $P h D^{2 *}$ \\ Marc van Bilsen, $P h D^{l}$ \\ Mark R. Hazebroek, MD, $P h D^{l}$ \\ Jort J. Merken, $M D^{\prime}$ \\ Els K. Vanhoutte, $M D, P h D^{2}$ \\ Michiel T.H.M. Henkens, $M D^{l}$ \\ Arthur van den Wijngaard, $P h D^{2}$ \\ Jan F.C. Glatz, $P h D^{l}$ \\ Ingrid P.C. Krapels, $M D, P h D^{1}$ \\ Han G. Brunner, $M D, P h D^{1,3,4}$ \\ Stephane R.B. Heymans, MD, PhD ${ }^{1,5,6}$ \\ Jörgen Bierau, $P h D^{2}$
}
* Contributed equally
${ }^{1}$ Department of Cardiology, Cardiovascular Research Institute (CARIM),
Maastricht University Medical Center, Maastricht, The Netherlands.
${ }^{2}$ Department of Clinical Genetics, Maastricht University Medical Center, Maastricht, The Netherlands.

${ }^{3}$ Radboud University Medical Center, Department of Human Genetics, Donders Institute for Brain, Cognition and Behaviour, Nijmegen

${ }^{4}$ GROW Institute for Developmental Biology and Cancer, Maastricht University Medical Centre,

Maastricht, The Netherlands

${ }^{5}$ Department of Cardiovascular Sciences, Centre for Molecular and Vascular Biology,

KU Leuven, Belgium.

${ }^{6}$ The Netherlands Heart Institute, Nl-HI, Utrecht, The Netherlands. 


\begin{abstract}
Aims: Metabolomic profiling may have diagnostic and prognostic value in heart failure. This study investigated whether targeted blood and urine metabolomics reflects disease severity in non-ischemic dilated cardiomyopathy (DCM) patients and compared its incremental value on top of NT-proBNP.
\end{abstract}

Methods and results: A total of 149 metabolites were measured in plasma and urine samples of 273 DCM patients with different stages of disease (DCM patients with LVRR (normal LVEF), $n=70$; asymptomatic $D C M, n=72$ and symptomatic $D C M, n=131$ ). Acylcarnitines, sialic acid, and glutamic acid are the most distinctive metabolites associated with disease severity, as repeatedly revealed by uni-biomarker linear regression, SPLSDA, Random Forest and conditional Random Forest analyses. However, the absolute difference of the metabolic profile among groups was marginal. A decision tree model based on the top metabolites did not surpass NT-proBNP in classifying stages. However, a combination of NT-proBNP and the top metabolites improved the decision tree to distinguish DCM patients with LVRR from symptomatic DCM (AUC $0.813 \pm 0.138$ versus $0.739 \pm 0.114 ; p=0.02$ ).

Conclusion: Functional cardiac recovery is reflected in metabolomics. These alterations reveal potential alternative treatment targets in advanced symptomatic DCM. The metabolic profile can complement NT-proBNP in determining disease severity in non-ischemic DCM. 


\section{INTRODUCTION}

Systolic dysfunction and dilation of the left and/or right ventricle characterize dilated cardiomyopathy (DCM) when myocardial ischemia and valvular disease is excluded ${ }^{1}$. DCM is a multifactorial and complex disease driven by multiple pathophysiological processes such as fibrotic, mechanical and metabolic alterations. Myocardial substrate and energy metabolism play an important role in the regulation of cardiac function, as a continuous energy generation is required for contraction ${ }^{2}$. Metabolic remodeling is a common feature of heart failure (HF), mainly because of impaired mitochondrial function and energy substrate adaptation in the challenged heart ${ }^{3}$. Alterations in cardiac metabolism are measurable in biological fluids of the patient ${ }^{2-4}$. Therefore, various studies explored the use of plasma metabolites as biomarkers for $\mathrm{HF}^{2}$. These studies show significant diagnostic and prognostic value of plasma metabolomic profiling in patients with ischemic HF, alone or in combination with B-type natriuretic peptide (BNP) ${ }^{5-9}$. However, data regarding metabolomics in non-ischemic DCM are scarce ${ }^{10}$, whereas the metabolic profile is likely to be different compared to ischemic HF 9 .

Modeling of large biological data sets represent a unique opportunity to better predict disease severity, as compared to single biomarkers. However, statistical classification is a critical component of utilizing metabolomics data for examining the phenotypical determinants ${ }^{11}$. Univariate associations or orthogonal partial least squares-discriminant analysis (OPLS-DA) are commonly used statistics 51012 . However, more advanced modeling, including sparse partial least squares-discriminant analysis (sPLSDA) and the machine learning method Random Forest analysis possess superior characteristics for analyzing large metabolomics datasets ${ }^{11}$.

This study aims to 1) associate disease severity with the metabolic profile in nonischemic DCM patients; and 2) compare the incremental value of measuring metabolites on top of NT-proBNP in determining disease severity.

\section{METHODS}

\section{Study population}

We included 273 consecutive DCM patients from February 2015 to August 2017, with inclusion and exclusion criteria as described previously ${ }^{13}$. In short, both DCM or hypokinetic non-dilated cardiomyopathy (HNDC; also called isolated LV dysfunction) according to the latest ESC proposal were included (DCM defined as LVEF $<50 \%$ with an indexed left ventricular end diastolic diameter (LVEDDi) $>33 \mathrm{~mm} / \mathrm{m}^{2}$ (men) or $>32 \mathrm{~mm} / \mathrm{m}^{2}$ (women) measured by echocardiography; and HNDC defined as LVEF $<50 \%$ with an LVEDDi $\leq 33 \mathrm{~mm} / \mathrm{m}^{2}$ (men) or $\leq 32$ $\mathrm{mm} / \mathrm{m}^{2}$ (women) measured by echocardiography) in the absence of a (i) history of myocardial infarction and/or significant coronary artery disease (stenosis $>50 \%$ ) determined by coronary angiography; (ii) primary valvular disease; (iii) hypertensive or congenital heart disease; (iv) acute myocarditis; (v) arrhythmogenic right ventricular dysplasia; (vi) hypertrophic, restrictive or peripartum cardiomyopathy ${ }^{14}$. The study was performed according to the declaration of Helsinki. All patients gave written informed consent.

Clinical examination and blood and urine sampling were performed at the outpatient clinic after the initial presentation. Therefore, patients could be classified into three distinct groups reflecting increasing disease severity: 1) DCM patients who achieved left ventricular reverse remodeling (LVRR; defined as a LVEF $>50 \%$ with a minimal increase of $5 \%$ from baseline) (group A; normal LVEF at the moment of sampling and examination), 2) 
asymptomatic DCM patients (group B; reduced LVEF and NYHA class I) and 3) symptomatic DCM patients (group C; reduced LVEF and NYHA class II or higher).

\section{Clinical measurements}

Clinical examination of all patients constituted of medical history-taking, physical examination, electrocardiogram (ECG), and echocardiography. Blood analysis was performed to screen for renal and liver function, lipid profile and cardiac stress. Echocardiographic measurements consisted of the standard parasternal, apical and subcostal views around the moment of blood and urine sampling for metabolomics analysis. Simpson's method was used to calculate LVEF in most patients. A minority of patients only had evaluation of LVEF by Teicholz method ( $n=42 ; 15.4 \%)$.

\section{Measurement of metabolites}

A targeted quantitative metabolomics approach was applied to measure metabolites in nonfasting plasma and spot urine samples, using an in-house metabolites screen system, to screen for inborn-errors of metabolism in an ISO 15189 accredited lab. The quantification of metabolites was based on internal isotopic standards. Supplemental Table 1 shows all measured metabolites, specific technical platforms of each metabolite category and corresponding references. Organic acids $(n=41)$, mucopolysaccharides $(n=1)$, sialic acids $(n=2)$, sugar alcohols $(n=10)$, globotriaosylsphingosine $(n=1)$, amino acids $(n=52)$ and acylcarnitines $(n=36)$ were measured in urine. In addition, amino acids $(n=48)$, acylcarnitines $(n=37)$, methylmalonic acids $(n=1)$ and very-long chain fatty acids $(n=5)$ were measured in plasma using various technical platforms specified in Supplemental Table 1. Raw anonymized metabolomics data were uploaded on figshare (data can be accessed via the DOI link: https://doi.org/10.6084/m9.figshare.8976719).

\section{Statistical analysis}

Clinical results are expressed as the mean \pm SD for continuous variables and as the number (percent) for categorical variables. Clinical data were compared by analysis of variance (ANOVA) and chi-square test, when appropriate. A $p$-value of $<0.05$ was considered significant. Metabolites are expressed as median \pm MAD (median absolute deviation).

\section{Missing data imputation}

Missing and zero values in the metabolomics data of measured samples (most likely due to left-censored missing not at random (MNAR, Suppl. Table 1) were imputed with half of the minimum (positive) reported value per analyte. Other missing values in both metabolic and clinical data (most likely due to missing at random (MAR, Suppl. Table 1) were imputed with the within-class median value per parameter stratified per group and gender. Processed data were $\log 2$ transformed to normalize the distribution, median-centered and scaled.

\section{Multivariate analysis methods}

A linear regression model (LM) tested the association between disease severity according to the predefined groups and each metabolite with adjustment for age and gender, using log transformed and standardized data. False discovery rate (FDR) q-values were calculated based on $p$-values for multiple testing correction. The variable importance is the absolute value of the t-statistic in the model. The variable importance of age and gender were derived from the LM without any metabolite. 
SPLSDA analysis of all metabolite data plus age and gender classified disease severity according to the three predefined groups. SPLSDA is a multivariate supervised method for the classification of high dimensional biological omics data, performing variable selection and dimension reduction simultaneously ${ }^{15}$. The analysis was run on log-transformed and standardized data including age and gender with splsda and related functions of mixOmics package in $\mathrm{R}$. The number of dimensions and variables to select on each dimension was tuned based on prediction distance in 5 times 10 -fold repeated cross-validation (CV). The generated model was further evaluated in CV for the variable frequency in selection. The variable importance was calculated as the product of the absolute value of the VIP of the useful component and the selection frequency.

Random forest analysis is a non-parametric machine-learning algorithm with an internal validation step using out-of-bag (OOB) subsampling, giving a fair estimation with robustness of biomarkers. We used two different forest analysis methods: standard random forest (RF) regression and conditional interference forest (CF) regression analysis. They classified groups according to the three predefined groups and ranked the variable importance of the individual variables. Unbiased CF with conditional inference trees is a more suitable method for predictor variables of different types of data with different degrees of complexity or data with high multicollinearity. ${ }^{16}$. It also measures conditional variable importance (VIP adjust) representing independent importance of a metabolite in the presence of other biomarkers. The analyses were run with resampling (ntree value) of 2000 times in the missing value implemented unprocessed metabolic data including age and gender. For both analyses, the mtry value was optimized using caret package in $\mathrm{R}$ to the value of 10 and 20, for RF and CF respectively. The variable importance was \%IncMSE in RF, VIP and VIP adjust in CF derived from the optimized models by randomForest package and party package in $R$. The variable importance of each method (LM, SPLSDA, RF, CF and CFadjust) was scaled to $0-100$ and the sum value as total variable importance. The top 25 hits in each method were selected and counted.

\section{Correlation network analysis}

The spearman correlations among top metabolites together with age, NT-proBNP and disease severity group, above the significant level of $\mid$ rho $\mid>0.2$ and $p$-value $<0.01$, were analyzed in Cytoscape (version 3.5) to construct correlation network with the EdgeBetweeness-weighted spring-embedded layout.

\section{Decision tree modeling}

Variables that were selected at least 4 times by the 5 different methods were used to build a decision tree model by conditional interference tree method, a recursive binary partitioning with less overfitting and variable selection problems. The mincriterion and maxdepth were tuned using caret and party package with CV in R. In addition, tree models of NT-proBNP with and without age were built for comparison.

To compare the performance of different tree models, 5 times 10-fold repeated stratified CV was performed on the subcohort of patients from group $A$ and $C(N=201)$. The ROC analysis obtained C-statistic, a measure of the area under the curve (AUC), to assess the model performance metrics in validation, expressed in median $\pm M A D{ }^{17}$. Mann-Whitney $U$ test was used to compare model performance metrics.

\section{RESULTS}


A total of 273 well-phenotyped non-ischemic DCM patients were divided into group $A(n=70)$, $B(n=72)$, and C $(n=131)$ according to disease severity as stated in the methods. Table 1 displays the baseline clinical characteristics and laboratory data. Most of the variables significantly differed among the groups. Symptomatic DCM patients were older, had a higher NT-proBNP and a lower renal function compared to DCM patients with LVRR. Gender did not differ among the groups.

\section{Classification of disease severity by metabolites using univariate analysis}

Out of 234 metabolite measurements on 149 unique compounds (Suppl. Table 1), 19.7\% showed association with HF severity after adjustment for gender and age with a p-value $<0.05$ in each linear regression model (LM). After correction for multiple testing ( $q$-value $<0.05$ ) only 18 metabolites, including 13 plasma acylcarnitines, 3-methylhistidine (3-MH), glutamic acid, cystathionine and urine sialic acid (total and free) associate to DCM patients in group $\mathrm{C}$, while urine carnosine, 3-hydroxyisovaleric acid and citric acid associate with DCM patients in group A (Figure 1).

\section{Classification of disease severity by metabolite profile using multivariate analyses}

sPLSDA tested whether metabolites could classify patients according to their corresponding group in a multivariate manner. Component 1 consisted of about half of the metabolites (variable $\mathrm{N}=108$ ) to get the most stable discrimination among groups (Suppl. Table 2), component 2 did not improve the model performance. However, the difference of the metabolic profile among groups was marginal (Figure 2).

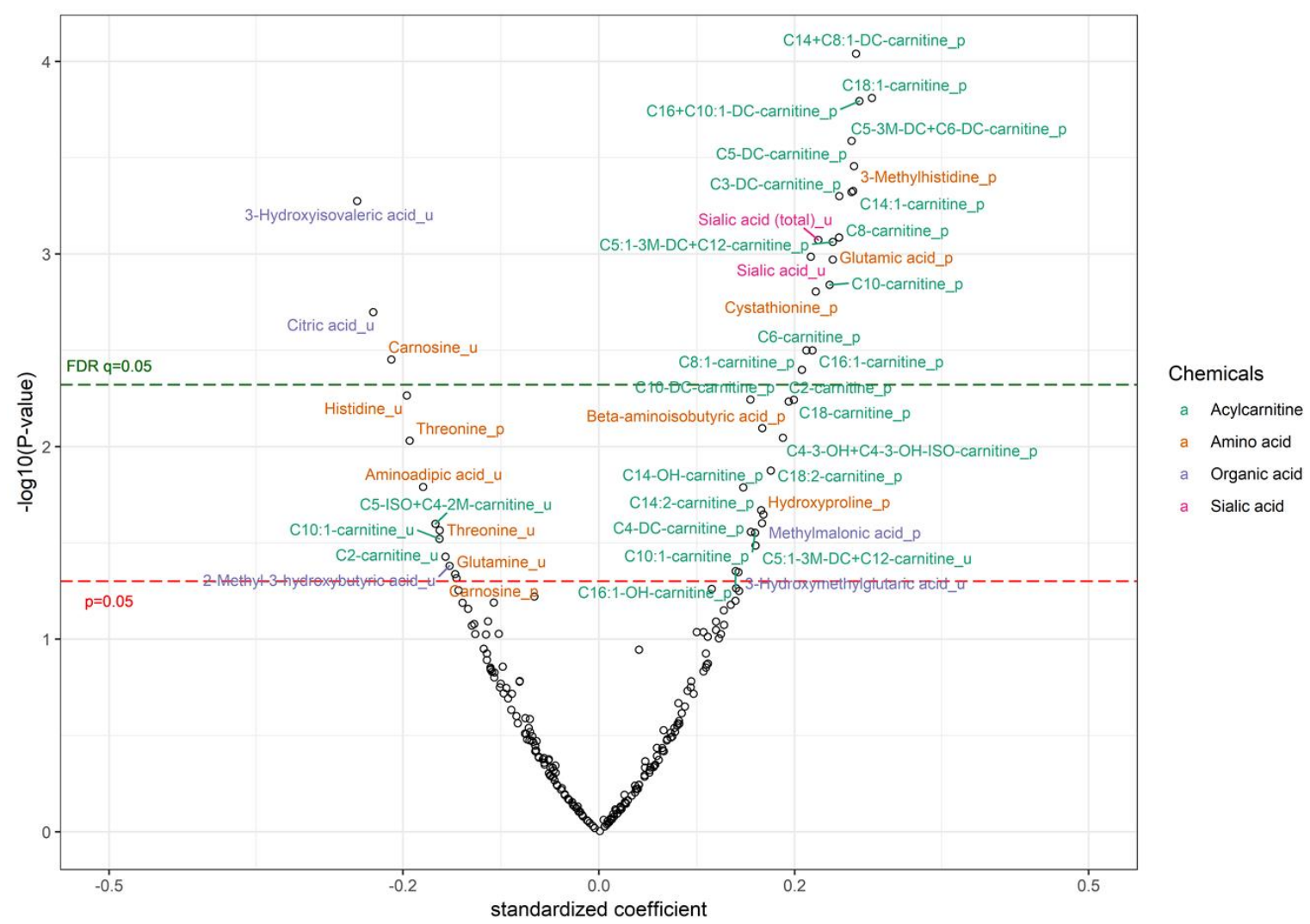

Figure 1. Univariate association of metabolites with disease severity. Volcano plot presentation of univariate association of metabolites with disease severity after age and gender adjustment analyzed in linear regression model. Metabolites with a $\mathrm{p}$-value $<0.05$ are labeled (above the red dotted line). P-value cutoff equivalent to FDR q-value 0.05 is also depicted (green dotted line). 
We used both standard RF and CF methods to robustly define the differences in metabolic profile in relation to disease severity. The optimized models provided a Root Mean Square Error (RMSE) of 0.792 and 0.789 , respectively (no difference between methods, $\mathrm{p}=0.73)$, indicating moderate discrimination among groups.

Table 1. Baseline characteristics of all DCM patients

\begin{tabular}{|c|c|c|c|c|}
\hline & $\begin{array}{c}\text { Group A } \\
\text { DCM with LVRR } \\
(n=70)\end{array}$ & $\begin{array}{c}\text { Group B } \\
\text { Asymptomatic } \\
\text { DCM patients } \\
(n=72)\end{array}$ & $\begin{array}{c}\text { Group C } \\
\text { Symptomatic } \\
\text { DCM patients } \\
(n=131)\end{array}$ & $\begin{array}{c}\text { p-value for } \\
\text { Trend }\end{array}$ \\
\hline Age, yrs & $51.7 \pm 13.3$ & $56.0 \pm 12.0$ & $59.1 \pm 10.0$ & $<0.001$ \\
\hline Male (\%) & $39(56)$ & $53(74)$ & $74(57)$ & 0.3 \\
\hline Body weight (kg) & $82 \pm 19$ & $84 \pm 19$ & $83 \pm 17$ & 0.77 \\
\hline Body mass index, $\mathrm{kg} / \mathrm{m} 2$ & $26.3 \pm 5.3$ & $26.5 \pm 5.0$ & $27.8 \pm 4.6$ & 0.09 \\
\hline NYHA I (\%) & $54(77)$ & $72(100)$ & $0(0)$ & $<0.001$ \\
\hline NYHA II (\%) & $16(23)$ & $0(0)$ & $96(73)$ & $<0.001$ \\
\hline NYHA III (\%) & $0(0)$ & $0(0)$ & $32(24)$ & $<0.001$ \\
\hline NYHA IV (\%) & $0(0)$ & $0(0)$ & $3(3)$ & 0.19 \\
\hline Heart rate, beats/min & $66 \pm 10$ & $67 \pm 13$ & $71 \pm 15$ & 0.008 \\
\hline Systolic blood pressure (mmHg) & $132 \pm 17$ & $136 \pm 18$ & $135 \pm 22$ & 0.95 \\
\hline Diastolic blood pressure (mmHg) & $79 \pm 16$ & $77 \pm 11$ & $79 \pm 13$ & 0.76 \\
\hline QRS complex, ms & $111 \pm 26$ & $115 \pm 30$ & $118 \pm 33$ & 0.36 \\
\hline Disease duration (months) & $33[13-76]$ & $13[5-47]$ & 10 [3-39] & $<0.001$ \\
\hline \multicolumn{5}{|l|}{ Echocardiography } \\
\hline LVEF, \% & $55 \pm 5$ & $39 \pm 9$ & $33 \pm 10$ & $<0.001$ \\
\hline LVEDD, mm & $52 \pm 6$ & $57 \pm 7$ & $59 \pm 8$ & $<0.001$ \\
\hline LVESD, mm & $37 \pm 6$ & $46 \pm 7$ & $49 \pm 9$ & $<0.001$ \\
\hline IVS, $\mathrm{mm}$ & $9 \pm 2$ & $9 \pm 1$ & $9 \pm 2$ & 0.19 \\
\hline PWT, mm & $8 \pm 1$ & $9 \pm 1$ & $9 \pm 2$ & 0.17 \\
\hline TR velocity $(\mathrm{m} / \mathrm{s})$ & $2.3 \pm 0.3$ & $2.2 \pm 0.2$ & $2.3 \pm 0.3$ & 0.22 \\
\hline Septal e' velocity $(\mathrm{cm} / \mathrm{s})$ & $8.1 \pm 3.3$ & $7.3 \pm 1.6$ & $5.8 \pm 1.7$ & 0.006 \\
\hline Lateral e' velocity $(\mathrm{cm} / \mathrm{s})$ & $11.8 \pm 4.7$ & $9.2 \pm 3.1$ & $8.3 \pm 3$ & 0.04 \\
\hline E/e' average & $6.8 \pm 1.9$ & $8.1 \pm 2.9$ & $11.11 \pm 6.6$ & 0.007 \\
\hline Indexed left atrial volume $\left(\mathrm{ml} / \mathrm{m}^{2}\right)$ & $36 \pm 12$ & $40 \pm 14$ & $47 \pm 22$ & 0.008 \\
\hline \multicolumn{5}{|l|}{ Comorbidity (\%) } \\
\hline Diabetes mellitus & $2(3)$ & $8(11)$ & $21(16)$ & 0.02 \\
\hline Hypercholesterolemia & $10(14)$ & $14(19)$ & $24(18)$ & 0.69 \\
\hline Moderate renal disease (eGFR 30-59) & $19(27)$ & $15(21)$ & $46(35)$ & 0.09 \\
\hline Severe renal disease (eGFR 10-29) & $1(1)$ & $0(0)$ & $5(4)$ & 0.18 \\
\hline Renal failure (eGFR <10) & $0(0)$ & $0(0)$ & $2(2)$ & 0.34 \\
\hline \multicolumn{5}{|l|}{ Laboratory data } \\
\hline NT-proBNP, pmol/L & $17[6-37]$ & $32[14-78]$ & $69[21-204]$ & $<0.001$ \\
\hline Total cholesterol, $\mathrm{mmol} / \mathrm{L}$ & $5.3 \pm 1.1$ & $4.8 \pm 1.2$ & $5.2 \pm 1.2$ & 0.08 \\
\hline Triglyceride, $\mathrm{mmol} / \mathrm{L}$ & $1.95 \pm 1.37$ & $1.62 \pm 1.15$ & $2.22 \pm 1.69$ & 0.33 \\
\hline LDL-C, mmol/L & $3.1 \pm 1.1$ & $2.7 \pm 1.2$ & $3.2 \pm 1.1$ & 0.3 \\
\hline $\mathrm{HDL}-\mathrm{C}, \mathrm{mmol} / \mathrm{L}$ & $1.3 \pm 0.3$ & $1.5 \pm 0.5$ & $1.3 \pm 0.3$ & 0.002 \\
\hline Serum sodium, mmol/L & $140 \pm 3$ & $140 \pm 2$ & $140 \pm 3$ & 0.97 \\
\hline
\end{tabular}




\begin{tabular}{|c|c|c|c|c|}
\hline Hemoglobin, mmol/L & $8.9 \pm 1.1$ & $9.0 \pm 0.8$ & $8.7 \pm 1.0$ & 0.04 \\
\hline ASAT, U/L & $26 \pm 12$ & $29 \pm 18$ & $27 \pm 10$ & 0.4 \\
\hline ALAT, U/L & $29 \pm 15$ & $33 \pm 19$ & $29 \pm 15$ & 0.31 \\
\hline Hemoglobin A1c (\%) & $5.72 \pm 0.6$ & $5.84 \pm 1.2$ & $6.06 \pm 1.2$ & 0.19 \\
\hline eGFR, $\mathrm{ml} / \mathrm{min} / 1.73 \mathrm{~m} 2$ & $68.2 \pm 12.4$ & $70.1 \pm 11.3$ & $62.6 \pm 18.2$ & $<0.001$ \\
\hline Creatinine, umol/L & $92 \pm 21$ & $93 \pm 22$ & $110 \pm 82$ & 0.05 \\
\hline Urea, $\mathrm{mmol} / \mathrm{L}$ & $6.0 \pm 2.0$ & $6.1 \pm 1.8$ & $7.8 \pm 5.7$ & 0.006 \\
\hline
\end{tabular}

\section{Metabolites associated with disease severity}

We compared the variable importance of all metabolites, as well as age and gender measured by the beforementioned 5 methods (LM, SPLSDA, RF, CF and CFadjust, Suppl. Table 2). In total, 40 different metabolites and age were selected as top 25 at least once by any of these methods (Figure 3). Eight acylcarnitines, sialic acid (total and free), glutamic acid, together with age were selected by all 5 methods. They appear to be the most distinctive metabolites associated with disease severity. In addition, 3 acylcarnitines, 3-methylhistidine and urine carnosine were selected at least 4 times. In contrast to age, gender was not an important variable in the classification.

The interaction among these 40 metabolites (together with age, NT-proBNP and disease severity) were explored by a correlation network (Figure 4). Plasma acylcarnitines are tightly grouped together. Glutamic acid is the only amino acid that closely correlated to the acylcarnitines. 3-Methylhistidine, sialic acid (total and free), and C5-3M-DC/C6-DC-carnitine were positively correlated to NT-proBNP, while urine histidine was inverse correlated.

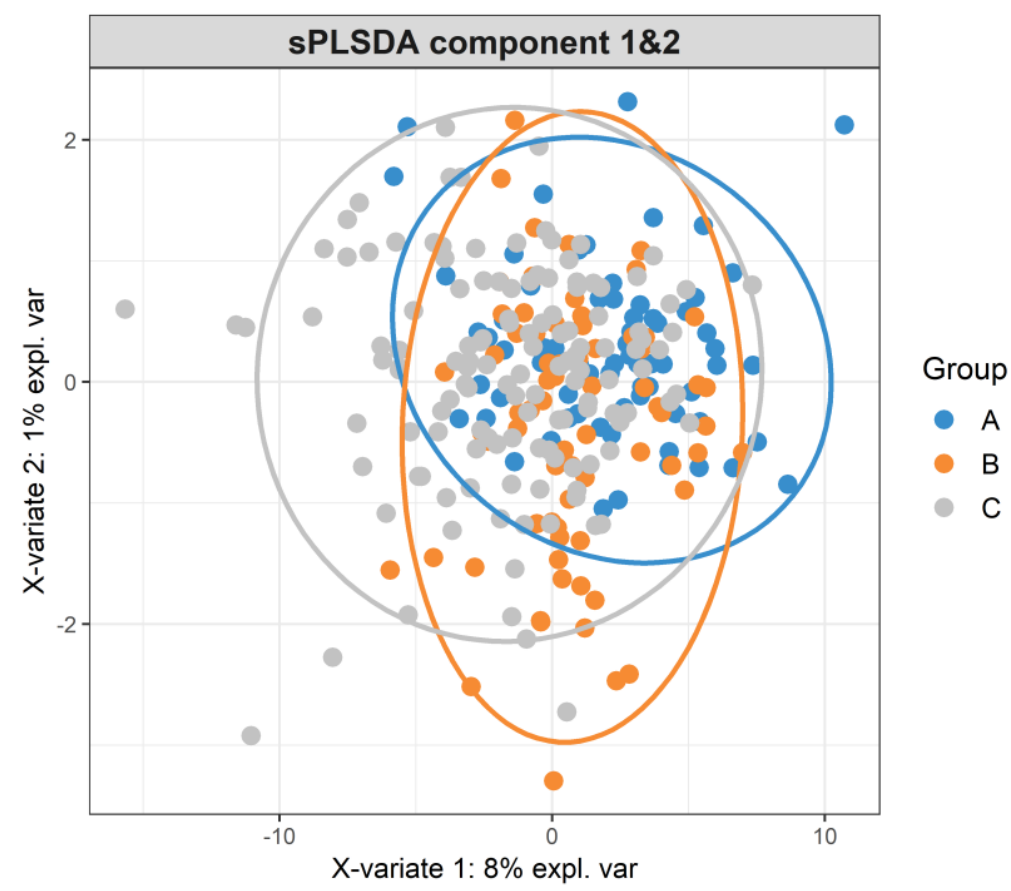

Figure 2. Classification of disease severity using metabolites with SPLSDA analysis. Sample score of patients projected into the space spanned by the two components of SPLSDA model with selected metabolites. 95\% confidence ellipses are plotted for each group to highlight the strength of the discrimination. 


\section{Comparison of metabolites with NT-proBNP in disease severity}

We used decision tree modeling as a tool for the clinical classification process to evaluate the value of metabolites in the clinical application. The 16 metabolites which were selected by at least 4 different methods were used as potential biomarkers. Plasma C16-acylcarnitine and urine sialic acid were selected by the model in addition to age for disease severity classification (Figure 5C). The metabolite model was comparable to NT-proBNP (Figure 5A), although less robust in CV (AUC $0.676 \pm 0.116$ versus $0.739 \pm 0.114, p=0.006$; Figure 5E). C16acylcarnitine and urine carnosine were selected by the model when combined with NTproBNP (Figure 5D). The combined model showed better classification and good robustness in CV (AUC 0.813 \pm 0.138 ; Figure 5E). This suggests incremental value of metabolites on top of NT-proBNP in defining disease severity. The addition of age (Figure 5B) lowered both classification capacity and robustness of NT-proBNP. Thus the value of age in the model was associated with metabolites, but not with NT-proBNP.

\section{DISCUSSION}

This study investigated the role of targeted metabolomics in relation to disease severity of non-ischemic DCM patients. The metabolic profile in symptomatic DCM patients was altered in comparison to DCM patients with LVRR, making the metabolic signature a marker for disease severity in addition to NT-proBNP in non-ischemic DCM. It also suggests that functional recovery of the heart is reflected in the metabolic signature. These metabolic alterations reveal potential treatment targets.

\section{Metabolic signature reflects disease severity in DCM patients}

Metabolomics studies in specific non-ischemic populations are relatively rare. Most reported studies using metabolic profiling include small populations $(n<50)$ of ischemic heart failure patients with reduced or preserved ejection fraction ${ }^{218}$. The large variety of metabolites reported as marker for HF in the various studies may be due to differences in disease severity between study populations, different methods, or statistical power ${ }^{2}$. Still, significant metabolic changes in plasma of DCM patients serve as specific non-ischemic DCM markers (such as $3-\mathrm{MH})$, as shown before in a small study population $(n=39)^{10}$. Also, strong metabolic changes related to increased NYHA class were noted in a recent study including 22 DCM patients ${ }^{19}$.

Based on our targeted metabolomics, the metabolic profile of DCM patients with different disease severity only showed marginal difference. Only symptomatic DCM patients (group C) showed a clear altered metabolic profile versus DCM patients with LVRR (group A). Asymptomatic DCM patients (group B) showed a metabolic profile in between, which made it difficult to distinguish from the other two groups. We used 5 methods, covering a broad range of methodology, to evaluate the importance of metabolites in relation to disease severity. This increases the robustness and reduces false positive findings of our data. A group of metabolites was repeatedly selected, underscoring their relation to disease severity in DCM patients. Furthermore, the metabolic signature could help to determine disease severity in DCM patients in addition to NT-proBNP alone.

We combined plasma and urine metabolites into one metabolic profile. However, there are differences between urine and plasma in biomarker presentation and variability in association with environmental factors ${ }^{2021}$. These factors make urine biomarkers less reliable compared to plasma biomarkers. Indeed, plasma C3-DC-carnitine was selected by all methods, although urine C3-DC-carnintine was only selected once. 


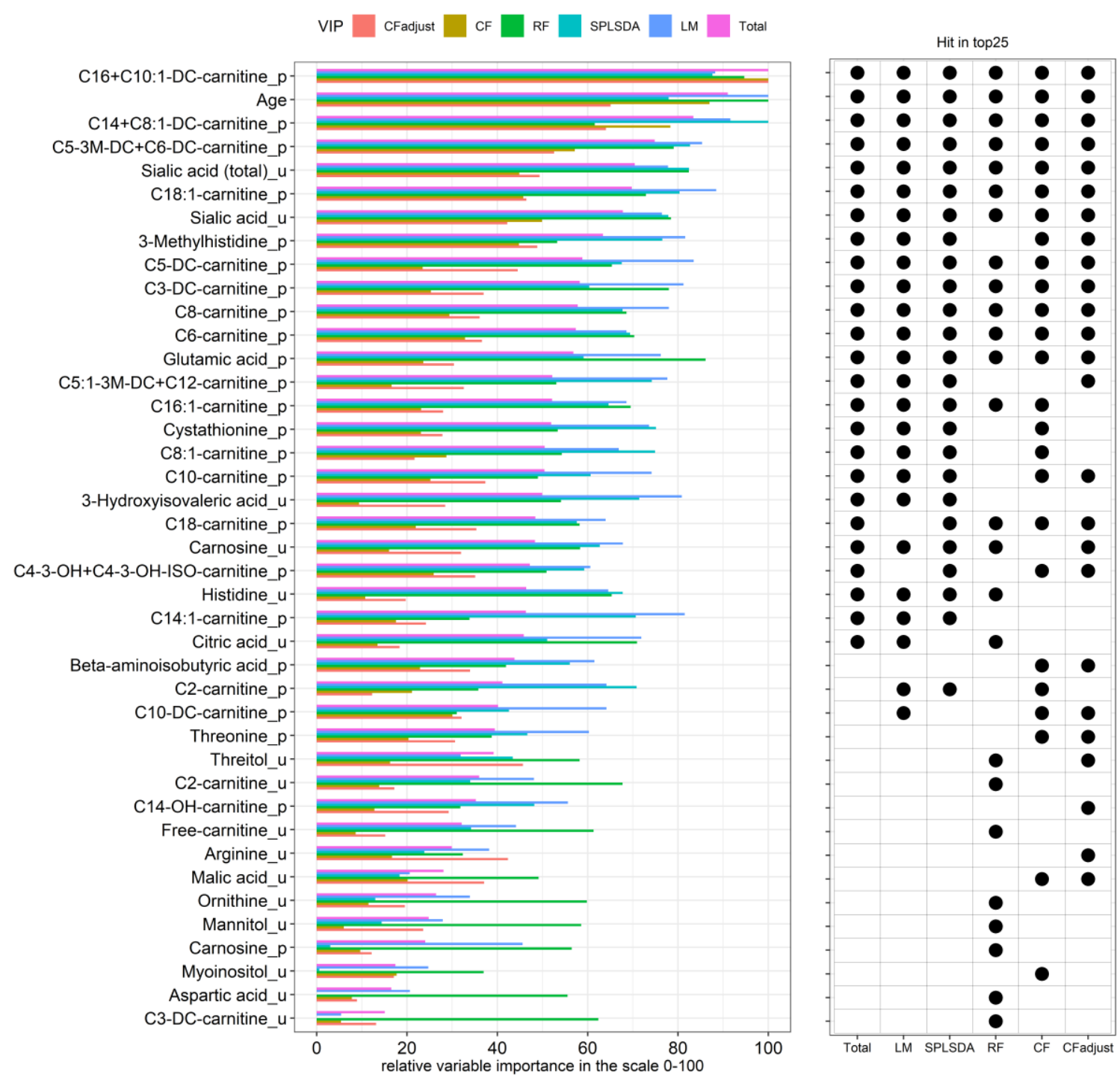

Figure 3. Top metabolites associated with disease severity as assessed by multiple methods. All individual metabolites and age that were selected in the top 25 by any of the methods are plotted with their relative variable importance in the scale of $0-100$.

$L M=$ linear regression model; SPLSDA = sPLSDA; RF = Random Forest; $C F=$ conditional interference forest; CFadjust = conditional variable importance in CF. The total variable importance is the sum of each relative value.

\section{Changes in metabolites reflect pathophysiological pathways driving disease progression in DCM}

Acylcarnitines, amino acids, sialic acids, sugar alcohols and organic acids are the top metabolites related to disease severity. Together, our results suggest a combined impairment of fatty acid and ketone body metabolism in symptomatic DCM, which is less pronounced when symptoms are absent. Myocardial metabolic disturbances often lead to accumulation or loss of specific metabolites from various metabolic pathways and these changes can be measured in the circulation ${ }^{2}$. The cardiac muscle has a high energy demand to sustain its contractile function, requiring an unimpeded supply of oxygen and substrates to maintain mitochondrial substrate oxidation and ATP production ${ }^{22}$. The healthy heart possesses the remarkable capacity to use a variety of substrates to be able to cope with differences in circulating substrate levels, including fatty acids, glucose, lactate and ketone bodies. However, during HF this metabolic flexibility gets compromised and the heart switches from 
long-chain fatty acids to glucose and ketone bodies as the substrates of preference ${ }^{2}$. A reduction in mitochondrial oxidative capacity associated with an impaired beta-oxidation of fatty acids lies at the base of the cardiac substrate switch in $\mathrm{HF}^{23}$. The accumulation of fatty acid intermediates, like acylcarnitines, in the affected myocardium is the consequence of this impaired beta-oxidation ${ }^{2}$. The increase in plasma acylcarnitine levels is also the main metabolic difference associated with disease severity in our DCM cohort. This lies in line with a subset of patients from the HF-ACTION (Exercise Training Program to Improve Clinical Outcomes in Individuals With Congestive Heart Failure) trial, where the elevation of plasma C16:0 and C18:1 acylcarnitines in patients with end-stage HF was the most important finding ${ }^{8}$. Furthermore, circulating acylcarnitines are increased in with $\mathrm{HF}$ and preserved EF (HFpEF) patients and further increased in HFrEF patients ${ }^{24}$. Increased circulating acylcarnitines was one of the main features in a cohort of principally ischemic HF patients compared to healthy subjects ${ }^{25}$. Like the latter study we noted an increase in circulating dicarboxylic (DC) acylcarnitines, suggesting that both mitochondrial beta-oxidation as well as peroxisomal omega-oxidation become implicated. However, short-chain (SC)-, medium-chain (MC)- and LC-acylcarnitine content was remarkably decreased in favor of an increase in ketone body intermediates in myocardial tissue of end-stage HF patients selected for heart transplantation 7. Myocardial fatty acid oxidation rates in the failing heart still raises controversy. It is thought that these discrepancies can partly be explained by the severity of disease ${ }^{223}$. Indeed, acylcarnitines had the most important role in determining disease severity differences using our statistical methods. Especially the LC-acylcarnitines (C14:0, C16:0 and C18:1) were among the top selected metabolites, showing the strong association of plasma acylcarnitines with advanced DCM.

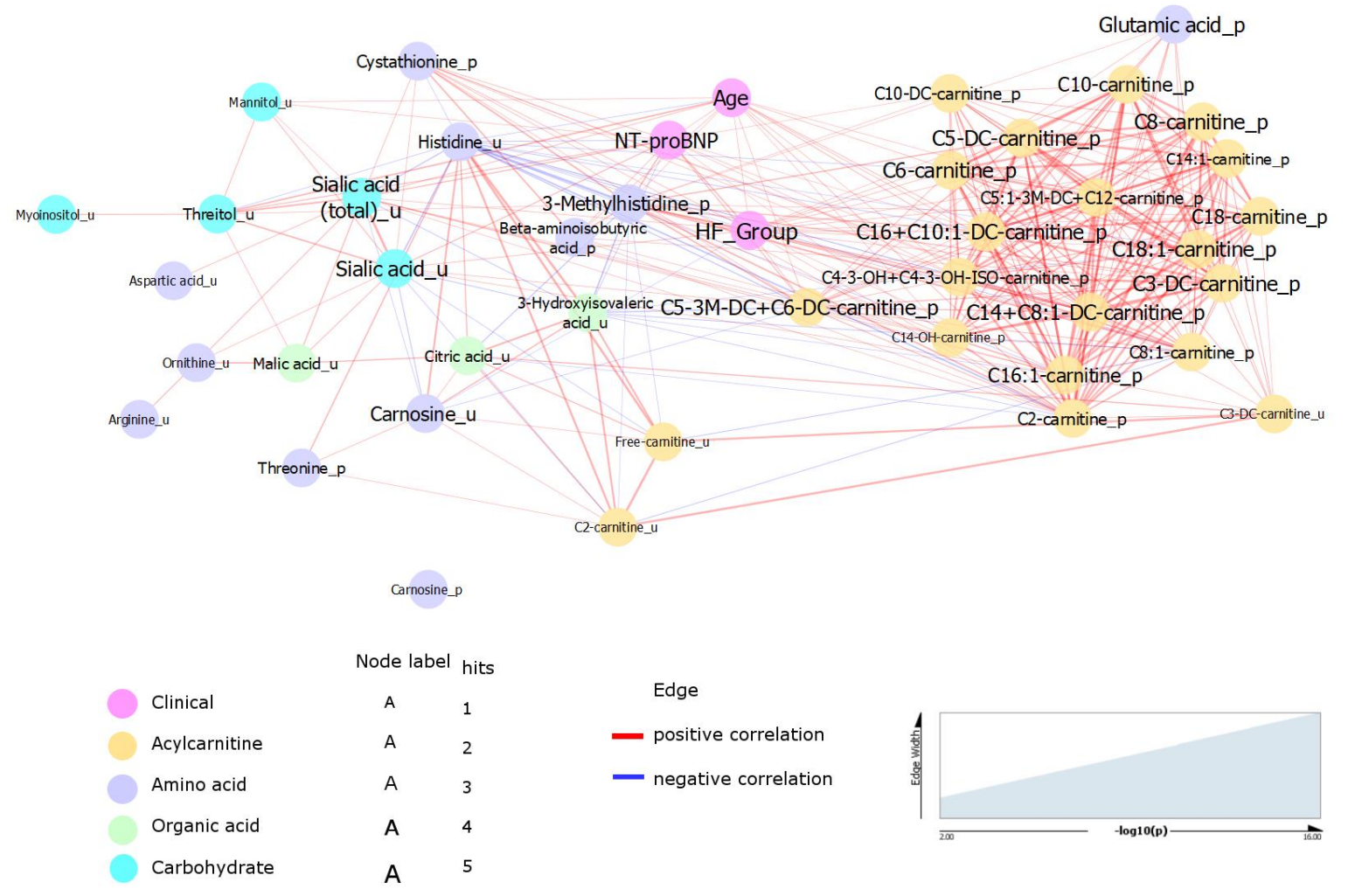

Figure 4. Correlation network of the top metabolites, age, NT-proBNP and disease severity. Spearman correlations above the significant level of $\mid$ rho $\mid>0.2$ and $p$-value $<0.01$ are analyzed and presented in the edge betweenness centrality-weighted spring-embedded layout, with distant nodes rescaled to enhance visualization. 
A

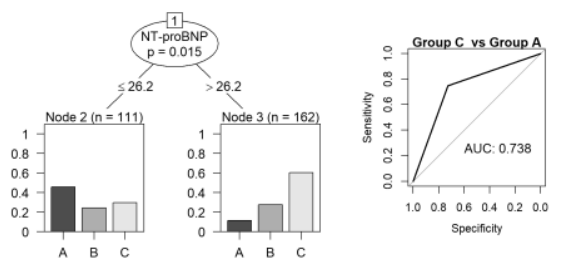

C

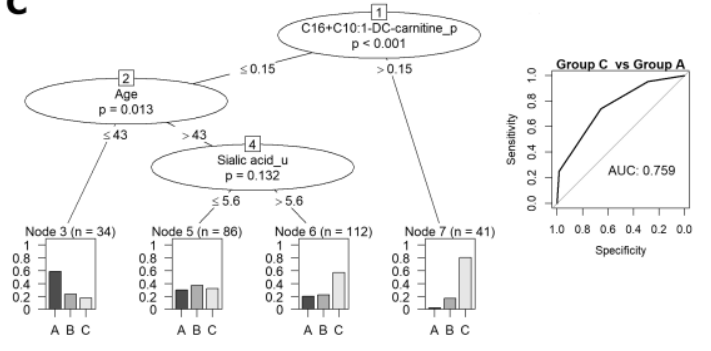

B
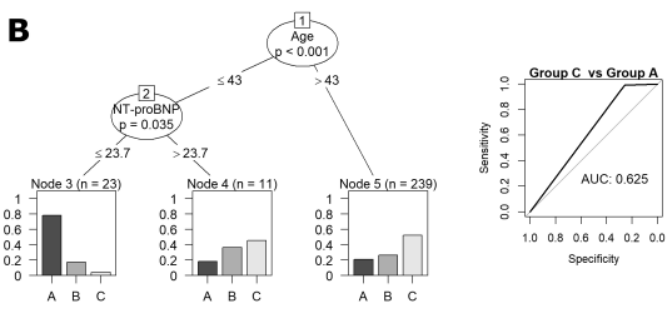

D

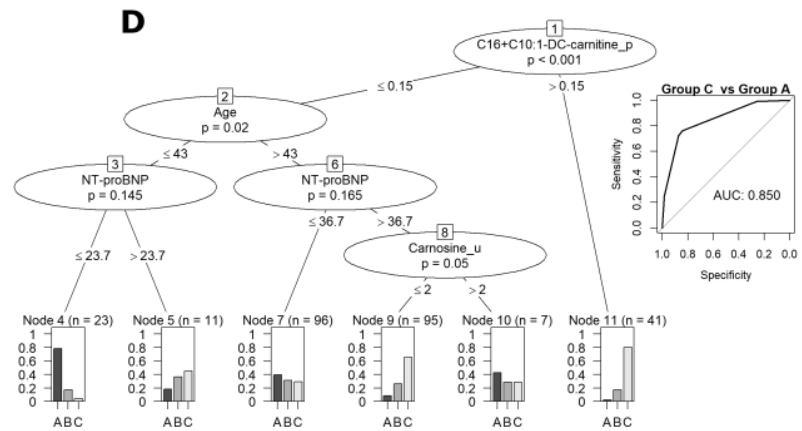

E

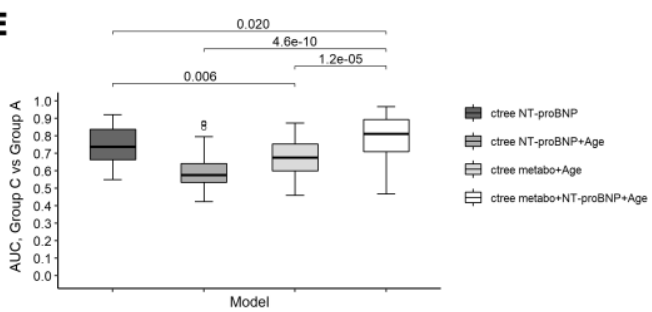

Figure 5. Decision tree models using NT-proBNP, age and metabolites. Conditional interference decision tree models using NT-proBNP (A), NT-proBNP and age (B), metabolites and age (C) and metabolites, NT-proBNP and age (D). The classification ability of each decision tree model to discriminate group $A$ from $C$ are shown by corresponding ROC curves with their associated AUC. The robustness of the models were evaluated by 5 times 10-fold CV. The AUC in CV are plotted with corresponding p-values using Mann-Whitney U test (E).

Units: NT-proBNP (pmol/L); age (years); C16+C10:1-DC-carnitine ( $\mu \mathrm{mol} / \mathrm{L})$; sialic acid $(\mathrm{mg} / \mathrm{mmol}$ creatinine); carnosine ( $\mu \mathrm{mol} / \mathrm{mmol}$ creatinine).

In contrast, 2-hydroxybutyrate, glycine, methylmalonate and myo-inositol were recently described as the main differentiating metabolites among HF severity using OPLS-DA ${ }^{12}$. However, it is unclear if acylcarnitines were measured in this study.

C5-3M-DC/C6-DC carnitine poorly correlates with other acylcarnitines, suggesting its involvement in pathways beyond fatty acid catabolism. Our LC-MS/MS method is not able to distinguish between 3-methylglutarylcarnitine and adipoylcarnitine. These two isomers appear in the same peak we detected. However, it has been shown in human plasma samples that this peak signal is mainly contributed by 3 -methylglutarylcarnitine ${ }^{26}$. Increased levels of 3-methylglutarylcarnitine serve as a biomarker for impaired leucine degradation with deficiency of 3-Hydroxy-3-Methylglutaryl-CoA Lyase (HMGCL) ${ }^{27}$, which plays a key role in ketogenesis in $\mathrm{DCM}{ }^{28}$. The heart switches from fatty acids to ketone bodies as an alternative energy source as HF gets more severe with increasing symptoms and decrease of LVEF ${ }^{3} 18$. The increase of 3-methylglutarylcarnitine in symptomatic DCM could indicate an impaired ketone body generation. However, this should be further investigated by measuring ketone bodies. 
Urine citric acid may reflect a blunted mitochondrial respiratory capacity, in line with the impaired fatty acid oxidation. 3-Hydroxyisovaleric acid is a byproduct of leucine degradation, and the urine concentration is associated to ketoacidosis ${ }^{29}$. The decreasing 3hydroxyisovaleric acid concentration associated with increase in disease severity may serve as a marker of decreased ketone body synthesis. Glutamic acid showed strong correlation with the plasma acylcarnitines, which may relate to its function in lipogenesis ${ }^{30}$. In addition, cystathionine and glutamic acid are both amino acids in the pathway of glutathione homeostasis in the myocardium, a pathway reflecting increased oxidative stress in more advanced DCM 3132 . Furthermore, cysteine is an amino acid which is synthesized from cystathionine. Cystathionine eventually removes homocysteine via the transsulfuration pathway ${ }^{53}$, a pathway which is up-regulated in advanced $\mathrm{HF}^{5}$. This suggests that the metabolic profile we see might indeed be correlated with a symptomatic and thus a more severe phenotype of DCM, and reflects the actual metabolic changes driving the disease progression.

3-Methylhistidine (3-MH) is a post-translationally modified amino acid derived from the contractile proteins actin and myosin ${ }^{34}$. Plasma 3-MH concentrations are higher in DCM patients compared to controls and possibly reflect enhanced myofibrillar degradation ${ }^{10}$. Carnosine is a dipeptide of histidine and beta-alanine. It acts as a protective agent for contracting muscles and cardiomyocytes and is therefore suggested as possible supplement in HF patients ${ }^{35}$.

Sialic acid is the common name for compounds derived from neuraminic acid and occurs mainly at terminal positions of glycoproteins (sialylation). Sialic acid is well-described in cardiovascular disease as a marker of systemic inflammation and is a predictor for mortality 3637 . Abnormal sialyation due to a genetic defect is reported as the cause of DCM in a mice model, possibly via altered $\mathrm{Ca}^{2+}$ handling ${ }^{38}$. Our results also support an association between sialyation and advanced DCM.

\section{Applicability of metabolomics in heart failure}

Better understanding of the metabolome in health and disease provides directions for new therapeutic targets ${ }^{3}$. Metabolomics is a promising tool for refining phenotypes and improving risk stratification ${ }^{39}$. Our work show that major metabolic disturbances occur in DCM, having a large overlap with all-cause HF. Increased circulating LC-acylcarnitines reflect impaired mitochondrial beta-oxidation in the failing heart, which could serve as a convincing diagnostic marker of disease and guide therapy ${ }^{2}$. Therapies to reverse this substrate switch are under development ${ }^{3}$. We showed that a decision tree model with metabolic markers in addition to NT-proBNP improved the classification and robustness in determining disease severity. However, other clinical parameters are also used to assess disease severity in clinical practice.

\section{Limitations}

A major limitation is the fact that these were non-fasting samples, and many of the measured metabolites can be influenced by diet. However, the fact that we found such distinctive differences irrespective of fasting state, ensures the strong metabolic profile related to cardiac function. Also, this reflects actual clinical practice as most sampling in the current clinics occurs in a chronic, non-fasting setting. We did not have access to samples of healthy controls, hence we were unable to compare our findings to a healthy control population. Our targeted metabolomics approach covers a broad spectrum of metabolites associated with inborn error of metabolism but does not include all potentially relevant metabolites such as 
ketone bodies. The discussion on heart metabolism in relation to whole body metabolic profile that we measured is at the assumption that the systemic metabolite profile in $\mathrm{HF}$ is heavily influenced by its cardiac origin, which we can't prove. Theoretically, an elevation in LC-acylcarnitines and other metabolites could also result from insulin resistance, renal dysfunction and medication use as possible confounders. Although we performed CV in our decision models, validation in an external cohort is necessary to determine the actual accuracy in clinical application. Finally, the sampling of plasma and urine was mostly performed in the chronic setting, usually after the occurrence of adverse events such as HF hospitalization. Therefore, we could not look for prognostic implications of the metabolic profile in our cohort.

\section{CONCLUSION}

Functional cardiac recovery is reflected in metabolomics. These alterations reveal potential alternative treatment targets in advanced symptomatic DCM. The metabolic profile can complement NT-proBNP in determining disease severity in non-ischemic DCM. 


\section{REFERENCES}

1. Japp AG, Gulati A, Cook SA, et al. The Diagnosis and Evaluation of Dilated Cardiomyopathy. J Am Coll Cardiol 2016;67(25):2996-3010.

2. Ussher JR, Elmariah S, Gerszten RE, et al. The Emerging Role of Metabolomics in the Diagnosis and Prognosis of Cardiovascular Disease. J Am Coll Cardiol 2016;68(25):2850-70.

3. Heggermont WA, Papageorgiou AP, Heymans $S$, et al. Metabolic support for the heart: complementary therapy for heart failure? Eur J Heart Fail 2016;18(12):1420-29.

4. Marcinkiewicz-Siemion $M$, Ciborowski $M$, Kretowski A, et al. Metabolomics - A wide-open door to personalized treatment in chronic heart failure? Int J Cardiol 2016;219:156-63.

5. Cheng ML, Wang $\mathrm{CH}$, Shiao MS, et al. Metabolic disturbances identified in plasma are associated with outcomes in patients with heart failure: diagnostic and prognostic value of metabolomics. J Am Coll Cardiol 2015;65(15):1509-20.

6. Sun H, Olson KC, Gao C, et al. Catabolic Defect of Branched-Chain Amino Acids Promotes Heart Failure. Circulation 2016;133(21):2038-49.

7. Bedi KC, Jr., Snyder NW, Brandimarto J, et al. Evidence for Intramyocardial Disruption of Lipid Metabolism and Increased Myocardial Ketone Utilization in Advanced Human Heart Failure. Circulation 2016;133(8):706-16.

8. Ahmad T, Kelly JP, McGarrah RW, et al. Prognostic Implications of Long-Chain Acylcarnitines in Heart Failure and Reversibility With Mechanical Circulatory Support. J Am Coll Cardiol 2016;67(3):291-9.

9. Lanfear DE, Gibbs JJ, Li J, et al. Targeted Metabolomic Profiling of Plasma and Survival in Heart Failure Patients. JACC Heart Fail 2017;5(11):823-32.

10. Alexander $D$, Lombardi R, Rodriguez $G$, et al. Metabolomic distinction and insights into the pathogenesis of human primary dilated cardiomyopathy. Eur J Clin Invest 2011;41(5):527-38.

11. Trainor PJ, DeFilippis AP, Rai SN. Evaluation of Classifier Performance for Multiclass Phenotype Discrimination in Untargeted Metabolomics. Metabolites 2017;7(2)

12. Deidda M, Piras C, Dessalvi CC, et al. Metabolomic approach to profile functional and metabolic changes in heart failure. J Transl Med 2015;13:297.

13. Verdonschot JAJ, Hazebroek MR, Derks KWJ, et al. Titin cardiomyopathy leads to altered mitochondrial energetics, increased fibrosis and long-term life-threatening arrhythmias. Eur Heart J 2018

14. Pinto YM, Elliott PM, Arbustini E, et al. Proposal for a revised definition of dilated cardiomyopathy, hypokinetic non-dilated cardiomyopathy, and its implications for clinical practice: a position statement of the ESC working group on myocardial and pericardial diseases. Eur Heart J 2016;37(23):1850-8.

15. Le Cao KA, Boitard S, Besse P. Sparse PLS discriminant analysis: biologically relevant feature selection and graphical displays for multiclass problems. BMC Bioinformatics 2011;12:253.

16. Strobl C, Boulesteix AL, Kneib T, et al. Conditional variable importance for random forests. $B M C$ Bioinformatics 2008;9:307.

17. Robin X, Turck N, Hainard A, et al. pROC: an opensource package for $\mathrm{R}$ and $\mathrm{S}+$ to analyze and compare ROC curves. BMC Bioinformatics 2011;12:77.

18. Hunter WG, Kelly JP, McGarrah RW, 3rd, et al. Metabolic Dysfunction in Heart Failure: Diagnostic, Prognostic, and Pathophysiologic Insights From Metabolomic Profiling. Curr Heart Fail Rep 2016;13(3):119-31.

19. Mueller-Hennessen M, Sigl J, Fuhrmann JC, et al. Metabolic profiles in heart failure due to nonischemic cardiomyopathy at rest and under exercise. ESC Heart Fail 2017;4(2):178-89.

20. Bouatra $S$, Aziat F, Mandal R, et al. The human urine metabolome. PLoS One 2013;8(9):e73076.

21. Kim K, Mall C, Taylor SL, et al. Mealtime, temporal, and daily variability of the human urinary and plasma metabolomes in a tightly controlled environment. PLoS One 2014;9(1):e86223.

22. Taegtmeyer $\mathrm{H}$, Young ME, Lopaschuk GD, et al. Assessing Cardiac Metabolism: A Scientific Statement From the American Heart Association. Circulation research 2016;118(10):1659-701.

23. Lopaschuk GD, Ussher JR, Folmes CD, et al. Myocardial fatty acid metabolism in health and disease. Physiol Rev 2010;90(1):207-58.

24. Hunter WG, Kelly JP, McGarrah RW, 3rd, et al. Metabolomic Profiling Identifies Novel Circulating Biomarkers of Mitochondrial Dysfunction Differentially Elevated in Heart Failure With Preserved Versus Reduced Ejection Fraction: Evidence for Shared Metabolic Impairments in Clinical Heart Failure. J Am Heart Assoc 2016;5(8)

25. Ruiz M, Labarthe F, Fortier A, et al. Circulating acylcarnitine profile in human heart failure: a surrogate of fatty acid metabolic dysregulation in mitochondria and beyond. Am J Physiol Heart Circ Physiol 2017;313(4):H768-H81. 
26. Kraus WE, Muoio DM, Stevens $R$, et al. Metabolomic Quantitative Trait Loci (mQTL) Mapping Implicates the Ubiquitin Proteasome System in Cardiovascular Disease Pathogenesis. PLoS genetics 2015;11(11):e1005553.

27. Roe CR, Millington DS, Maltby DA. Identification of 3-methylglutarylcarnitine. A new diagnostic metabolite of 3-hydroxy-3-methylglutarylcoenzyme A lyase deficiency. I Clin Invest 1986;77(4):1391-4.

28. Leung AA, Chan AK, Ezekowitz JA, et al. A Case of Dilated Cardiomyopathy Associated with 3Hydroxy-3-Methylglutaryl-Coenzyme A (HMG CoA) Lyase Deficiency. Case Rep Med 2009;2009:183125.

29. Landaas S. Increased urinary excretion of 3hydroxyisovaleric acid in patients with ketoacidosis. Clin Chim Acta 1974;54(1):39-46.

30. Belfiore F, lannello S. Fatty acid synthesis from glutamate in the adipose tissue of normal subjects and obese patients: an enzyme study. Biochem Mol Med 1995;54(1):19-25.

31. Ghosh S, Sulistyoningrum DC, Glier MB, et al. Altered glutathione homeostasis in heart augments cardiac lipotoxicity associated with diet-induced obesity in mice. I Biol Chem 2011;286(49):42483-93.

32. Irino $\mathrm{Y}$, Toh $\mathrm{R}$, Nagao $\mathrm{M}$, et al. 2-Aminobutyric acid modulates glutathione homeostasis in the myocardium. Sci Rep 2016;6:36749.

33. Huang $\mathrm{S}$, Li H, Ge J. A cardioprotective insight of the cystathionine gamma-lyase/hydrogen sulfide pathway. Int J Cardiol Heart Vasc 2015;7:51-57.

34. Young VR, Alexis SD, Baliga BS, et al. Metabolism of administered 3-methylhistidine. Lack of muscle transfer ribonucleic acid charging and quantitative excretion as 3-methylhistidine and its N-acetyl derivative. I Biol Chem 1972;247(11):3592-600.

35. Zhao J, Posa DK, Kumar V, et al. Carnosine protects cardiac myocytes against lipid peroxidation products. Amino Acids 2019;51(1):123-38. doi: 10.1007/s00726-0182676-6

36. Rajendiran KS, Ananthanarayanan RH, Satheesh $S$, et al. Elevated levels of serum sialic acid and high-sensitivity C-reactive protein: markers of systemic inflammation in patients with chronic heart failure. Br J Biomed Sci 2014;71(1):29-32.

37. Khalili P, Sundstrom J, Franklin SS, et al. Combined effects of brachial pulse pressure and sialic acid for risk of cardiovascular events during 40 years of follow-up in 37,843 individuals. J Hypertens 2012;30(9):1718-24.

38. Deng W, Ednie AR, Qi J, et al. Aberrant sialylation causes dilated cardiomyopathy and stress- induced heart failure. Basic Res Cardiol 2016;111(5):57.

39. Shah SH, Hunter WG. Realizing the Potential of Metabolomics in Heart Failure: Signposts on the Path to Clinical Utility. JACC Heart Fail 2017;5(11):833-36. 


\section{SUPPLEMENTARY FIGURES}
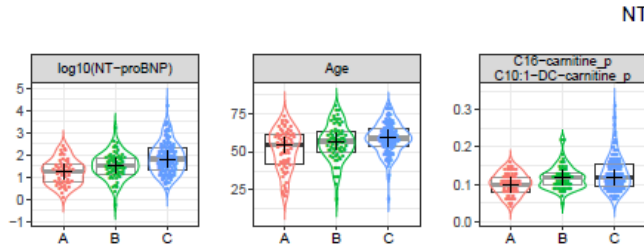

TT-proBNP, Age and Top metabolites boxplot + Violin plot + median
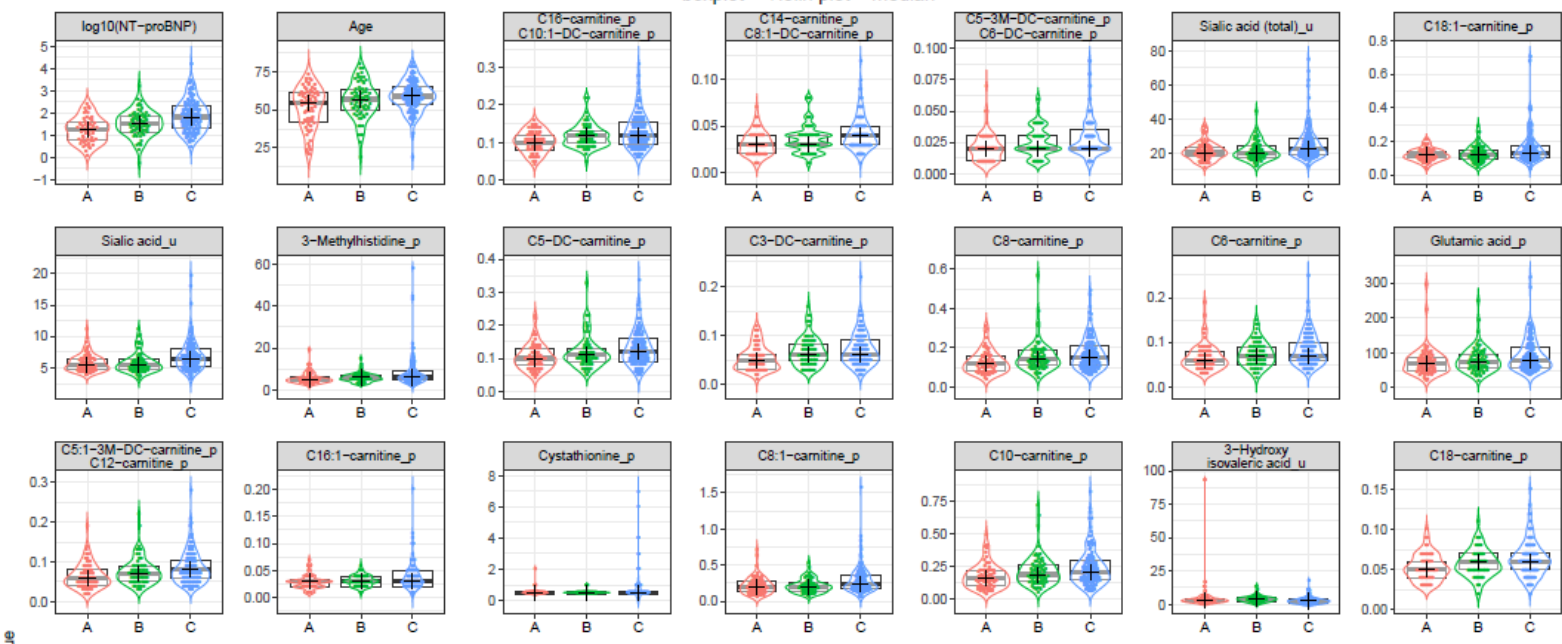

$\frac{\Phi}{3}$
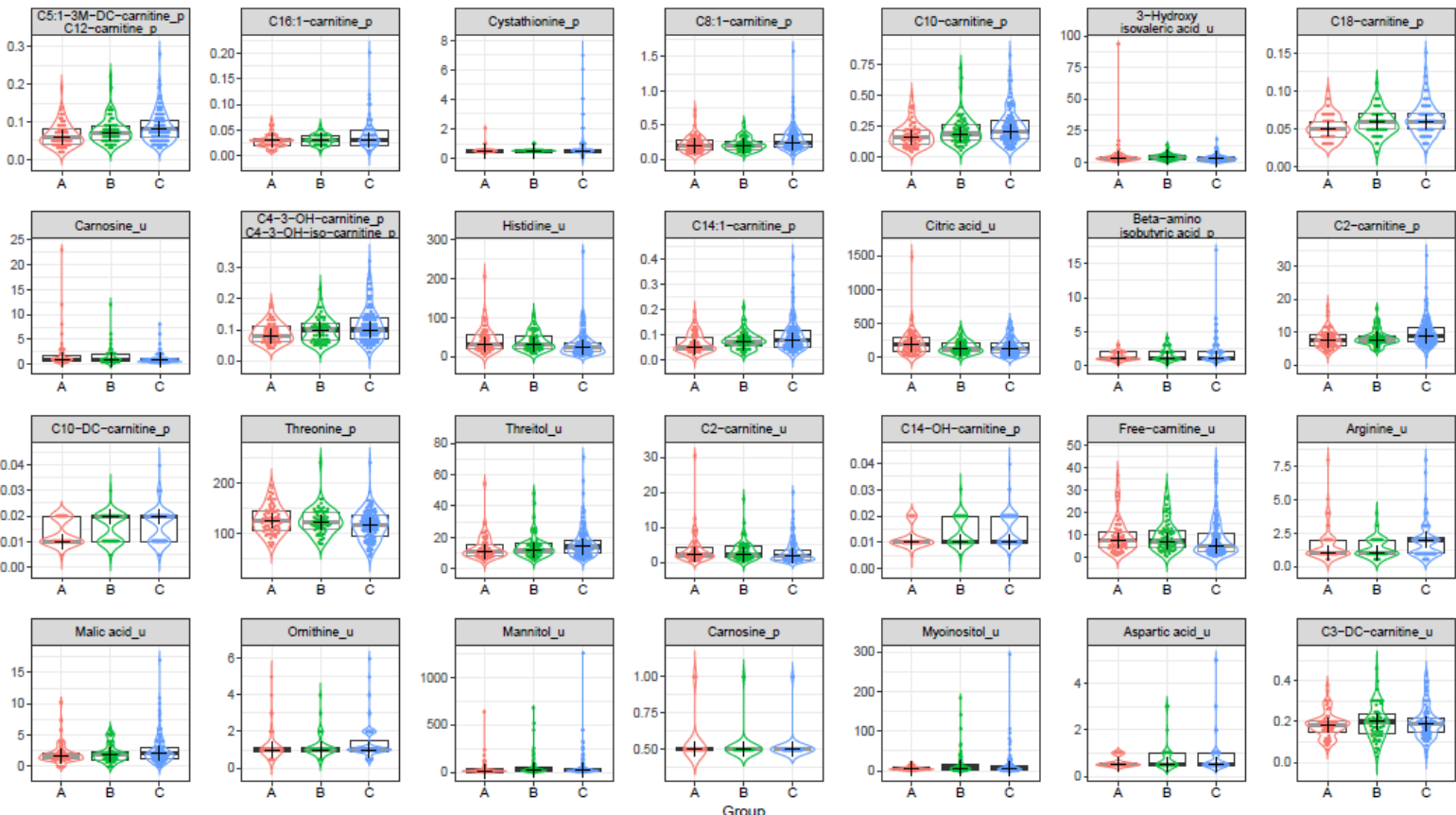

Supplemental Figure 1. Absolute values of NT-proBNP, age and top metabolites. Violin plot and boxplots with median highlighted for each parameter stratified on disease severity groups.

\section{SUPPLEMENTARY TABLES}

Supplemental Table 1. Summary statistics of all metabolites and their measurement methods.

This table can be accessed via

https://www.onlinejcf.com/cms/10.1016/i.cardfail.2019.09.004/attachment/8f2ea275-4559-41e4-b30a-

$6 c 92 f 87 d 4590 / m m c 2 . x / s x$

Supplemental Table 2. Complete list of variable importance for disease severity. All metabolites together with age and gender were assessed by 5 methods. LM = linear regression model; SPLSDA = SPLSDA; RF = Random Forest; $\mathrm{CF}=$ conditional interference forest; $\mathrm{CFa}=$ conditional variable importance in conditional interference forest. Their relative variable importance is in a scale of $0-100$. The total variable importance is the sum of each relative value.

\begin{tabular}{|c|c|c|c|c|c|c|c|c|c|c|c|c|c|c|}
\hline $\begin{array}{l}\text { 㞧 } \\
\text { 类 }\end{array}$ & 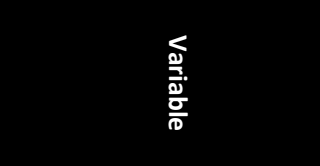 & $\begin{array}{l}\# \\
\text { 윰 } \\
\text { ㅁ }\end{array}$ & 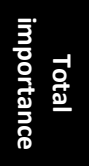 & 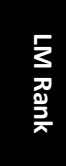 & $\begin{array}{l}5 \\
\mathbf{3} \\
\frac{5}{2}\end{array}$ & 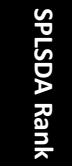 & $\begin{array}{l}\frac{y}{0} \\
\bar{n} \\
\frac{1}{5} \\
5\end{array}$ & 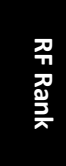 & 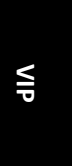 & 쥬 & 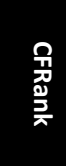 & 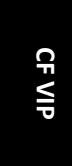 & 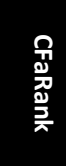 & $\begin{array}{l}\stackrel{0}{M} \\
\stackrel{M}{5}\end{array}$ \\
\hline 1 & C16+C10:1-DC-carnitine_p & 5 & $\begin{array}{l}100, \\
0\end{array}$ & 4 & 88,3 & 2 & 87,7 & 2 & 94,7 & & 1 & $\begin{array}{l}100, \\
0\end{array}$ & 1 & $\begin{array}{l}100, \\
0\end{array}$ \\
\hline 2 & Age & 5 & 91,0 & 1 & $\begin{array}{l}100 \\
0\end{array}$ & 6 & 77,9 & 1 & 100,0 & & 2 & 87,0 & 2 & 65,1 \\
\hline
\end{tabular}




\begin{tabular}{|c|c|c|c|c|c|c|c|c|c|c|c|c|c|}
\hline 3 & C14+C8:1-DC-carnitine_p & 5 & 83,4 & 2 & 91,6 & 1 & $\begin{array}{l}100, \\
0\end{array}$ & 17 & 61,6 & 3 & 78,3 & 3 & 64,0 \\
\hline 4 & $\begin{array}{l}\text { C5-3M-DC+C6-DC- } \\
\text { carnitine_p }\end{array}$ & 5 & 74,8 & 5 & 85,4 & 3 & 82,7 & 5 & 79,0 & 4 & 57,1 & 4 & 52,6 \\
\hline 5 & Sialic acid (total)_u & 5 & 70,4 & 12 & 77,8 & 4 & 82,4 & 4 & 82,4 & 7 & 44,8 & 5 & 49,4 \\
\hline 6 & C18:1-carnitine_p & 5 & 69,8 & 3 & 88,5 & 5 & 80,3 & 8 & 72,9 & 6 & 45,7 & 7 & 46,4 \\
\hline 7 & Sialic acid_u & 5 & 67,8 & 14 & 76,5 & 7 & 77,9 & 6 & 78,4 & 5 & 49,9 & 11 & 42,2 \\
\hline 8 & 3-Methylhistidine_p & 4 & 63,4 & 7 & 81,6 & 8 & 76,5 & 31 & 53,3 & 8 & 44,8 & 6 & 48,8 \\
\hline 9 & C5-DC-carnitine_p & 5 & 58,8 & 6 & 83,5 & 18 & 67,6 & 14 & 65,3 & 17 & 23,5 & 9 & 44,5 \\
\hline 10 & C3-DC-carnitine_p & 5 & 58,3 & 9 & 81,2 & 22 & 60,3 & 7 & 78,0 & 14 & 25,3 & 14 & 37,0 \\
\hline 11 & C8-carnitine_p & 5 & 57,8 & 11 & 78,0 & 17 & 67,7 & 12 & 68,6 & 11 & 29,4 & 16 & 36,1 \\
\hline 12 & C6-carnitine_p & 5 & 57,4 & 20 & 68,6 & 15 & 69,4 & 10 & 70,3 & 9 & 32,8 & 15 & 36,6 \\
\hline 13 & Glutamic acid_p & 5 & 56,9 & 15 & 76,2 & 24 & 59,1 & 3 & 86,1 & 16 & 23,6 & 24 & 30,4 \\
\hline 14 & $\begin{array}{l}\text { C5:1-3M-DC+C12- } \\
\text { carnitine p }\end{array}$ & 3 & 52,1 & 13 & 77,6 & 11 & 74,2 & 32 & 53,1 & 28 & 16,6 & 20 & 32,6 \\
\hline 15 & C16:1-carnitine_p & 4 & 52,1 & 19 & 68,6 & 19 & 64,6 & 11 & 69,5 & 18 & 23,1 & 27 & 28,0 \\
\hline 16 & Cystathionine_p & 3 & 51,9 & 17 & 73,6 & 9 & 75,1 & 30 & 53,4 & 19 & 23,1 & 28 & 27,8 \\
\hline 17 & C8:1-carnitine_p & 3 & 50,5 & 22 & 66,9 & 10 & 74,9 & 28 & 54,3 & 12 & 28,7 & 41 & 21,7 \\
\hline 18 & C10-carnitine_p & 4 & 50,4 & 16 & 74,2 & 21 & 60,7 & 45 & 49,0 & 15 & 25,2 & 12 & 37,3 \\
\hline 19 & 3-Hydroxyisovaleric acid_u & 2 & 50,0 & 10 & 80,8 & 12 & 71,4 & 29 & 54,1 & 54 & 9,4 & 26 & 28,5 \\
\hline 20 & C18-carnitine_p & 4 & 48,4 & 26 & 64,0 & 25 & 57,6 & 22 & 58,2 & 21 & 22,0 & 17 & 35,3 \\
\hline 21 & Carnosine_u & 4 & 48,3 & 21 & 67,8 & 20 & 62,7 & 21 & 58,3 & 31 & 16,0 & 22 & 32,0 \\
\hline 22 & $\begin{array}{l}\mathrm{C} 4-3-\mathrm{OH}+\mathrm{C} 4-3-\mathrm{OH}-\mathrm{ISO}- \\
\text { carnitine_p }\end{array}$ & 3 & 47,2 & 28 & 60,6 & 23 & 59,2 & 38 & 50,9 & 13 & 25,9 & 18 & 35,1 \\
\hline 23 & Histidine_u & 3 & 46,4 & 23 & 64,5 & 16 & 67,7 & 15 & 65,3 & 46 & 10,7 & 50 & 19,7 \\
\hline 24 & C14:1-carnitine_p & 2 & 46,3 & 8 & 81,5 & 14 & 70,7 & 100 & 33,8 & 26 & 17,6 & 33 & 24,2 \\
\hline 25 & Citric acid_u & 2 & 45,9 & 18 & 71,9 & 27 & 51,0 & 9 & 70,9 & 35 & 13,5 & 61 & 18,4 \\
\hline 26 & $\begin{array}{l}\text { Beta-aminoisobutyric } \\
\text { acid_p }\end{array}$ & 2 & 43,8 & 27 & 61,5 & 26 & 56,1 & 59 & 41,9 & 20 & 22,9 & 19 & 33,9 \\
\hline 27 & C2-carnitine_p & 3 & 41,1 & 25 & 64,2 & 13 & 70,8 & 91 & 35,8 & 22 & 21,1 & 165 & 12,3 \\
\hline 28 & C10-DC-carnitine_p & 3 & 40,2 & 24 & 64,2 & 35 & 42,6 & 124 & 31,0 & 10 & 30,1 & 21 & 32,1 \\
\hline 29 & Threonine_p & 2 & 39,5 & 29 & 60,3 & 29 & 46,7 & 78 & 38,8 & 23 & 20,3 & 23 & 30,6 \\
\hline 30 & Threitol_u & 2 & 39,2 & 90 & 31,9 & 33 & 43,4 & 23 & 58,2 & 29 & 16,2 & 8 & 45,7 \\
\hline 31 & C18:2-carnitine_p & 0 & 37,2 & 30 & 57,3 & 32 & 45,6 & 51 & 44,8 & 38 & 12,2 & 29 & 26,4 \\
\hline 32 & C2-carnitine_u & 1 & 36,0 & 42 & 48,1 & 50 & 34,0 & 13 & 67,7 & 34 & 13,9 & 71 & 17,2 \\
\hline 33 & C14-OH-carnitine_p & 1 & 35,2 & 32 & 55,6 & 28 & 48,2 & 117 & 31,8 & 37 & 12,8 & 25 & 29,2 \\
\hline 34 & C10:1-carnitine_u & 0 & 34,9 & 40 & 50,1 & 46 & 37,4 & 41 & 49,4 & 33 & 14,8 & 32 & 24,3 \\
\hline 35 & Aminoadipic acid_u & 0 & 33,2 & 31 & 55,7 & 47 & 36,7 & 42 & 49,3 & 80 & 7,4 & 56 & 19,3 \\
\hline 36 & Threonine_u & 0 & 32,5 & 37 & 51,1 & 30 & 46,4 & 36 & 51,9 & 84 & 7,3 & 220 & 8,7 \\
\hline 37 & Free-carnitine_u & 1 & 32,1 & 50 & 44,2 & 49 & 34,2 & 18 & 61,3 & 64 & 8,6 & 92 & 15,2 \\
\hline 38 & $\begin{array}{l}\text { 2-Methyl-3-hydroxybutyric } \\
\text { acid_u }\end{array}$ & 0 & 31,6 & 43 & 47,1 & 42 & 39,8 & 60 & 41,8 & 55 & 9,3 & 38 & 23,2 \\
\hline 39 & C5-ISO+C4-2M-carnitine_u & 0 & 31,5 & 36 & 51,8 & 48 & 34,6 & 37 & 51,7 & 61 & 9,1 & 138 & 13,3 \\
\hline 40 & C14:2-carnitine_p & 0 & 30,5 & 33 & 53,3 & 31 & 46,1 & 83 & 38,0 & 146 & 5,8 & 153 & 12,9 \\
\hline 41 & Methylmalonic acid_p & 0 & 30,4 & 35 & 51,9 & 43 & 39,1 & 61 & 41,5 & 48 & 10,4 & 158 & 12,7 \\
\hline 42 & Arginine_u & 1 & 29,9 & 71 & 38,2 & 58 & 23,8 & 111 & 32,3 & 27 & 16,7 & 10 & 42,4 \\
\hline 43 & C16:1-OH-carnitine_p & 0 & 29,0 & 44 & 46,5 & 36 & 42,4 & 46 & 46,0 & 176 & 5,0 & 214 & 9,4 \\
\hline 44 & C10:1-carnitine_p & 0 & 28,4 & 39 & 50,8 & 39 & 41,0 & 92 & 35,5 & 50 & 9,9 & 209 & 9,6 \\
\hline 45 & C8-DC-carnitine_u & 0 & 28,4 & 53 & 42,9 & 54 & 25,7 & 26 & 55,5 & 41 & 11,7 & 191 & 10,7 \\
\hline 46 & Glutamine_u & 0 & 28,2 & 46 & 46,1 & 41 & 39,9 & 48 & 45,6 & 226 & 3,1 & 181 & 11,3 \\
\hline
\end{tabular}




\begin{tabular}{|c|c|c|c|c|c|c|c|c|c|c|c|c|c|}
\hline 47 & Malic acid_u & 2 & 28,1 & 133 & 20,6 & 62 & 18,3 & 43 & 49,1 & 24 & 20,1 & 13 & 37,1 \\
\hline 48 & Homocitrulline_p & 0 & 28,1 & 49 & 44,3 & 34 & 43,3 & 134 & 29,3 & 30 & 16,1 & 169 & 12,1 \\
\hline 49 & Erythritol_u & 0 & 27,6 & 76 & 35,9 & 40 & 40,4 & 72 & 39,8 & 49 & 10,2 & 83 & 16,5 \\
\hline 50 & Total-carnitine_p & 0 & 26,7 & 58 & 41,7 & 37 & 41,4 & 93 & 35,5 & 159 & 5,5 & 94 & 15,1 \\
\hline 51 & Ornithine_u & 1 & 26,4 & 81 & 33,9 & 74 & 13,0 & 19 & 59,8 & 42 & 11,5 & 53 & 19,5 \\
\hline 52 & C4+C4-ISO-carnitine_p & 0 & 26,2 & 56 & 42,4 & 44 & 38,1 & 74 & 39,7 & 58 & 9,1 & 228 & 7,3 \\
\hline 53 & Mannitol_u & 1 & 24,8 & 102 & 27,9 & 71 & 14,4 & 20 & 58,6 & 130 & 6,0 & 37 & 23,6 \\
\hline 54 & Hydroxyproline_p & 0 & 24,7 & 34 & 52,8 & 75 & 12,8 & 84 & 37,1 & 67 & 8,5 & 59 & 18,8 \\
\hline 55 & Ornithine_p & 0 & 24,6 & 79 & 34,3 & 52 & 29,7 & 77 & 39,2 & 83 & 7,3 & 58 & 19,1 \\
\hline 56 & Proline_u & 0 & 24,5 & 62 & 39,8 & 55 & 25,5 & 70 & 40,1 & 87 & 7,0 & 84 & 16,5 \\
\hline 57 & Vanillactic acid_u & 0 & 24,2 & 68 & 38,6 & 57 & 24,4 & 34 & 52,6 & 95 & 6,6 & 232 & 5,5 \\
\hline 58 & Carnosine_p & 1 & 24,0 & 47 & 45,6 & 92 & 3,0 & 24 & 56,5 & 52 & 9,6 & 167 & 12,2 \\
\hline 59 & Anserine_u & 0 & 23,7 & 70 & 38,5 & 60 & 20,0 & 122 & 31,5 & 45 & 11,2 & 31 & 24,3 \\
\hline 60 & $\begin{array}{l}\text { 3-Hydroxymethylglutaric } \\
\text { acid_u }\end{array}$ & 0 & 23,2 & 45 & 46,3 & 56 & 24,7 & 113 & 32,0 & 71 & 8,1 & 166 & 12,2 \\
\hline 61 & Arabitol_u & 0 & 23,1 & 138 & 19,1 & 63 & 17,1 & 27 & 54,7 & 32 & 15,5 & 85 & 16,1 \\
\hline 62 & Aminoadipic acid_p & 0 & 22,4 & 65 & 38,9 & 81 & 11,2 & 95 & 35,1 & 56 & 9,2 & 30 & 25,1 \\
\hline 63 & C4-DC-carnitine_p & 0 & 22,4 & 38 & 50,9 & 45 & 37,8 & 204 & 17,4 & 169 & 5,2 & 225 & 8,1 \\
\hline 64 & C10:2-carnitine_p & 0 & 22,3 & 48 & 44,4 & 38 & 41,1 & 192 & 19,5 & 192 & 4,6 & 206 & 9,7 \\
\hline 65 & C4+C4-ISO-carnitine_u & 0 & 22,3 & 55 & 42,6 & 84 & 8,6 & 35 & 52,3 & 63 & 8,8 & 230 & 6,7 \\
\hline 66 & Homocystine_u & 0 & 21,7 & 73 & 36,6 & 70 & 14,7 & 53 & 44,4 & $\begin{array}{l}110 \\
5\end{array}$ & 6,4 & 113 & 14,3 \\
\hline 67 & C10-carnitine_u & 0 & 21,5 & 61 & 39,9 & 61 & 20,0 & 170 & 23,3 & 36 & 13,0 & 54 & 19,5 \\
\hline 68 & $\begin{array}{l}\text { Piperideine-6-carboxylic } \\
\text { acid_u }\end{array}$ & 0 & 21,0 & 51 & 44,1 & 91 & 3,5 & 50 & 45,0 & $\begin{array}{l}110 \\
5\end{array}$ & 6,4 & 113 & 14,3 \\
\hline 69 & Ethanolamine_u & 0 & 20,7 & 57 & 41,9 & 53 & 25,7 & 145 & 28,0 & 170 & 5,2 & 186 & 11,0 \\
\hline 70 & $\begin{array}{l}\text { C5:1-3M-DC+C12- } \\
\text { carnitine_u }\end{array}$ & 0 & 20,6 & 41 & 49,4 & 59 & 21,7 & 157 & 25,7 & 205 & 4,0 & 192 & 10,7 \\
\hline 71 & Isovalerylglycine_u & 0 & 20,5 & 95 & 30,9 & & 0,0 & 49 & 45,1 & 44 & 11,2 & 36 & 23,6 \\
\hline 72 & Cysteine-S-sulfate_u & 0 & 19,8 & 54 & 42,6 & 66 & 16,6 & 138 & 28,6 & 228 & 3,0 & 78 & 16,8 \\
\hline 73 & C18:1-OH-carnitine_u & 0 & 19,3 & 91 & 31,8 & & 0,0 & 66 & 40,6 & 53 & 9,4 & 35 & 23,7 \\
\hline 74 & C8:1-carnitine_u & 0 & 19,2 & 103 & 27,4 & 107 & 0,4 & 40 & 50,1 & 57 & 9,2 & 64 & 18,0 \\
\hline 75 & Serine_u & 0 & 19,2 & 77 & 35,0 & 67 & 16,4 & 89 & 36,5 & 139 & 5,9 & 179 & 11,3 \\
\hline 76 & Lactic acid_u & 0 & 19,2 & 87 & 33,2 & & 0,0 & 39 & 50,5 & 186 & 4,7 & 80 & 16,6 \\
\hline 77 & C8-DC-carnitine_p & 0 & 19,1 & 66 & 38,8 & 51 & 31,6 & 213 & 15,2 & 221 & 3,4 & 89 & 15,5 \\
\hline 78 & C26:0_p & 0 & 18,6 & 125 & 22,2 & & 0,0 & 33 & 53,1 & 40 & 12,1 & 93 & 15,1 \\
\hline 79 & $\begin{array}{l}\text { C4:1-3M+C4:1-2M+C5:1- } \\
\text { carnitine_p }\end{array}$ & 0 & 18,2 & 85 & 33,4 & 76 & 12,5 & 79 & 38,8 & 137 & 5,9 & 203 & 9,9 \\
\hline 80 & Homocystine_p & 0 & 18,0 & 60 & 40,2 & 64 & 17,1 & 214 & 14,7 & 102 & 6,4 & 42 & 21,3 \\
\hline 81 & Oxoglutaric acid_u & 0 & 17,7 & 99 & 30,0 & 102 & 0,6 & 94 & 35,5 & 70 & 8,2 & 34 & 23,9 \\
\hline 82 & Lysogb3_u & 0 & 17,6 & 75 & 35,9 & & 0,0 & 119 & 31,7 & 66 & 8,5 & 40 & 21,7 \\
\hline 83 & Beta-Alanine_u & 0 & 17,6 & 59 & 40,2 & 78 & 12,1 & 177 & 22,7 & 62 & 9,0 & 129 & 13,6 \\
\hline 84 & Myoinositol_u & 1 & 17,4 & 112 & 24,7 & 103 & 0,6 & 85 & 37,0 & 25 & 17,7 & 75 & 17,1 \\
\hline 85 & Arginine_p & 0 & 17,4 & 82 & 33,9 & 104 & 0,5 & 105 & 33,2 & 60 & 9,1 & 46 & 20,2 \\
\hline 86 & $\begin{array}{l}\text { 3-Hydroxyisobutyric } \\
\text { acid_u }\end{array}$ & 0 & 17,2 & 100 & 29,2 & 72 & 14,0 & 148 & 27,6 & 68 & 8,2 & 76 & 17,1 \\
\hline 87 & Free-carnitine_p & 0 & 17,0 & 124 & 22,3 & 73 & 13,6 & 97 & 34,6 & 211 & 3,8 & 45 & 20,9 \\
\hline 88 & C3-carnitine_u & 0 & 16,8 & 86 & 33,3 & 65 & 16,7 & 173 & 23,0 & 120 & 6,2 & 96 & 15,0 \\
\hline 89 & Glycine_u & 0 & 16,7 & 63 & 39,7 & 68 & 16,2 & 183 & 21,6 & 220 & 3,4 & 160 & 12,6 \\
\hline 90 & Isobutyrylglycine_u & 0 & 16,6 & 141 & 18,7 & & 0,0 & 47 & 45,6 & 51 & 9,7 & 55 & 19,4 \\
\hline
\end{tabular}




\begin{tabular}{|c|c|c|c|c|c|c|c|c|c|c|c|c|c|}
\hline 91 & Aspartylglycosamine_u & 0 & 16,6 & 72 & 38,0 & 85 & 7,8 & 149 & 27,6 & 89 & 7,0 & 155 & 12,8 \\
\hline 92 & Aspartic acid_u & 1 & 16,5 & 132 & 20,7 & & 0,0 & 25 & 55,6 & 75 & 7,8 & 218 & 8,9 \\
\hline 93 & $\begin{array}{l}\text { Cys-homo-Cys-mixed } \\
\text { disulfide_u }\end{array}$ & 0 & 16,4 & 83 & 33,7 & & 0,0 & 82 & 38,1 & $\begin{array}{l}110, \\
5\end{array}$ & 6,4 & 113 & 14,3 \\
\hline 94 & Methylglutaric acid_u & 0 & 16,4 & 121 & 22,8 & & 0,0 & 58 & 41,9 & 150 & 5,7 & 39 & 22,1 \\
\hline 95 & Methylsuccinic acid_u & 0 & 16,3 & 200 & 7,4 & 82 & 10,3 & 44 & 49,1 & 43 & 11,5 & 128 & 13,6 \\
\hline 96 & Suberic acid_u & 0 & 16,0 & 89 & 31,9 & & 0,0 & 76 & 39,3 & 122 & 6,1 & 137 & 13,4 \\
\hline 97 & Homocitrulline_u & 0 & 15,8 & 67 & 38,6 & & 0,0 & 104 & 33,3 & 214 & 3,6 & 122 & 13,9 \\
\hline 98 & Citrulline_u & 0 & 15,4 & 64 & 39,1 & 80 & 11,5 & 197 & 18,7 & 140 & 5,9 & 152 & 12,9 \\
\hline 99 & C5-ISO+C4-2M-carnitine_p & 0 & 15,2 & 118 & 23,4 & & 0,0 & 67 & 40,6 & 69 & 8,2 & 101 & 14,7 \\
\hline 100 & Asparagine_u & 0 & 15,2 & 97 & 30,0 & 90 & 4,8 & 112 & 32,3 & 131 & 6,0 & 126 & 13,6 \\
\hline 101 & C3-DC-carnitine_u & 1 & 15,1 & 212 & 5,4 & & 0,0 & 16 & 62,4 & 165 & 5,4 & 140 & 13,2 \\
\hline 102 & Alpha-aminobutyric acid_u & 0 & 14,6 & 106 & 26,0 & 77 & 12,4 & 132 & 29,6 & 224 & 3,3 & 145 & 13,1 \\
\hline 103 & Sebacic acid_u & 0 & 14,5 & 80 & 34,0 & & 0,0 & 125 & 30,6 & 100 & 6,5 & 156 & 12,7 \\
\hline 104 & Glycine_p & 0 & 14,5 & 123 & 22,6 & & 0,0 & 69 & 40,3 & 81 & 7,4 & 134 & 13,4 \\
\hline 105 & Alanine_u & 0 & 14,3 & 109 & 25,1 & & 0,0 & 90 & 36,4 & 175 & 5,0 & 82 & 16,5 \\
\hline 106 & $\begin{array}{l}\text { Gamma-aminobutyric } \\
\text { acid_p }\end{array}$ & 0 & 14,3 & 84 & 33,6 & & 0,0 & $\begin{array}{l}140 \\
5\end{array}$ & 28,4 & $\begin{array}{l}110, \\
5\end{array}$ & 6,4 & 113 & 14,3 \\
\hline 107 & 1-Methylhistidine_u & 0 & 14,2 & 93 & 31,0 & 86 & 7,5 & 150 & 27,4 & 143 & 5,8 & 194 & 10,5 \\
\hline 108 & Oxoadipic acid_u & 0 & 14,1 & 52 & 43,4 & & 0,0 & 178 & 22,7 & 125 & 6,0 & 205 & 9,8 \\
\hline 109 & Ethanolamine_p & 0 & 14,0 & 78 & 34,5 & 79 & 11,6 & 223 & 10,7 & 82 & 7,3 & 73 & 17,2 \\
\hline 110 & Hydroxyproline_u & 0 & 13,7 & 162 & 15,0 & & 0,0 & 65 & 40,7 & 78 & 7,6 & 77 & 16,8 \\
\hline 111 & Pipecolic acid_u & 0 & 13,6 & 126 & 22,0 & & 0,0 & 81 & 38,6 & 160 & 5,5 & 130 & 13,5 \\
\hline 112 & $\begin{array}{l}\text { C5-3M-3OH-DC- } \\
\text { carnitine_p }\end{array}$ & 0 & 13,5 & 115 & 23,9 & 69 & 16,1 & 166 & 23,9 & 232 & 2,2 & 150 & 13,0 \\
\hline 113 & Alloisoleucine_p & 0 & 13,4 & 134 & 20,1 & & 0,0 & 120 & 31,6 & 73 & 7,9 & 57 & 19,2 \\
\hline 114 & Ethylmalonic acid_u & 0 & 13,3 & 69 & 38,6 & & 0,0 & 176 & 22,7 & 229 & 2,6 & 104 & 14,5 \\
\hline 115 & Mucopolysaccharides_u & 0 & 13,2 & 140 & 18,7 & & 0,0 & 71 & 40,1 & 76 & 7,8 & 178 & 11,4 \\
\hline 116 & 3-Methylcrotonylglycine_u & 0 & 13,1 & 158 & 16,1 & & 0,0 & 126 & 30,6 & 47 & 10,5 & 47 & 20,2 \\
\hline 117 & 3-Methyladipic acid_u & 0 & 12,9 & 144 & 18,2 & & 0,0 & 56 & 42,3 & 99 & 6,5 & 213 & 9,4 \\
\hline 118 & Glycylproline_u & 0 & 12,8 & 131 & 21,1 & 98 & 1,0 & 107 & 32,9 & 92 & 6,7 & 105 & 14,5 \\
\hline 119 & Methionine_u & 0 & 12,8 & 107 & 25,9 & & 0,0 & 116 & 31,9 & 90 & 7,0 & 180 & 11,3 \\
\hline 120 & Methylmalonic acid_u & 0 & 12,7 & 129 & 21,9 & & 0,0 & 114 & 32,0 & 142 & 5,8 & 87 & 15,9 \\
\hline 121 & 5-Hydroxylysine_u & 0 & 12,6 & 127 & 22,0 & & 0,0 & 110 & 32,6 & 138 & 5,9 & 103 & 14,5 \\
\hline 122 & $\begin{array}{l}\text { C5-3M-DC+C6-DC- } \\
\text { carnitine_u }\end{array}$ & 0 & 12,6 & 191 & 8,6 & 94 & 2,0 & 52 & 44,7 & 133 & 6,0 & 125 & 13,8 \\
\hline 123 & Glutaric acid_u & 0 & 12,5 & 168 & 14,6 & & 0,0 & 101 & 33,4 & 134 & 5,9 & 44 & 20,9 \\
\hline 124 & C5-DC-carnitine_u & 0 & 12,5 & 186 & 9,4 & & 0,0 & 57 & 42,0 & 190 & 4,6 & 60 & 18,5 \\
\hline 125 & Saccharopine_u & 0 & 12,4 & 110 & 25,1 & & 0,0 & 130 & 29,6 & 118 & 6,3 & 146 & 13,1 \\
\hline 126 & Sarcosine_p & 0 & 12,3 & 101 & 28,5 & & 0,0 & 154 & 26,3 & 154 & 5,6 & 133 & 13,5 \\
\hline 127 & C18:2-carnitine_u & 0 & 12,3 & 155 & 16,7 & & 0,0 & 68 & 40,5 & 163 & 5,4 & 184 & 11,1 \\
\hline 128 & 5-Aminolevulinic acid_u & 0 & 12,3 & 145 & 18,2 & & 0,0 & 64 & 40,7 & 182 & 4,7 & 201 & 10,1 \\
\hline 129 & Argininosuccinic acid_p & 0 & 12,3 & 116 & 23,6 & 99 & 0,9 & $\begin{array}{l}140 \\
5\end{array}$ & 28,4 & $\begin{array}{l}110, \\
5\end{array}$ & 6,4 & 113 & 14,3 \\
\hline 130 & C18:1-OH-carnitine_p & 0 & 12,3 & 113 & 24,3 & 100 & 0,8 & 108 & 32,9 & 97 & 6,6 & 217 & 9,0 \\
\hline 131 & Cystathionine_u & 0 & 12,2 & 172 & 12,9 & 101 & 0,6 & 54 & 43,2 & 93 & 6,7 & 204 & 9,8 \\
\hline 132 & Pipecolic acid_p & 0 & 12,0 & 108 & 25,5 & & 0,0 & 159 & 25,4 & 198 & 4,2 & 72 & 17,2 \\
\hline 133 & Aspartic acid_p & 0 & 11,8 & 111 & 25,1 & & 0,0 & 172 & 23,2 & 72 & 8,1 & 90 & 15,4 \\
\hline 134 & Hexanoylglycine_u & 0 & 11,8 & 130 & 21,7 & & 0,0 & 102 & 33,4 & 124 & 6,1 & 198 & 10,1 \\
\hline 135 & Citrulline_p & 0 & 11,7 & 190 & 9,1 & 97 & 1,2 & 62 & 41,4 & 103 & 6,4 & 157 & 12,7 \\
\hline
\end{tabular}




\begin{tabular}{|c|c|c|c|c|c|c|c|c|c|c|c|c|c|}
\hline 136 & Homocarnosine_u & 0 & 11,7 & 105 & 26,3 & 93 & 2,8 & 187 & 21,1 & 98 & 6,6 & 120 & 14,2 \\
\hline 137 & Sarcosine_u & 0 & 11,7 & 135 & 20,1 & & 0,0 & 131 & 29,6 & 104 & 6,4 & 98 & 14,8 \\
\hline 138 & Propionylglycine_u & 0 & 11,6 & 74 & 36,5 & & 0,0 & 175 & 22,9 & 222 & 3,4 & 226 & 8,0 \\
\hline 139 & Beta-Alanine_p & 0 & 11,5 & 96 & 30,4 & & 0,0 & 163 & 24,7 & 149 & 5,7 & 211 & 9,5 \\
\hline 140 & C16-OH-carnitine_p & 0 & 11,4 & 128 & 22,0 & 87 & 5,4 & 167 & 23,6 & 152 & 5,6 & 147 & 13,0 \\
\hline 141 & $\begin{array}{l}\mathrm{C} 4-3-\mathrm{OH}+\mathrm{C} 4-3-\mathrm{OH}-\mathrm{ISO}- \\
\text { carnitine_u }\end{array}$ & 0 & 11,3 & 197 & 7,8 & & 0,0 & 55 & 43,1 & 177 & 4,9 & 132 & 13,5 \\
\hline 142 & Taurine_u & 0 & 11,2 & 92 & 31,5 & 83 & 9,1 & 200 & 18,1 & 223 & 3,3 & 229 & 7,0 \\
\hline 143 & Butyrylglycine_u & 0 & 11,1 & 208 & 6,3 & & 0,0 & 63 & 41,2 & 178 & 4,8 & 86 & 16,0 \\
\hline 144 & C5-OH-carnitine_p & 0 & 11,1 & 149 & 17,3 & & 0,0 & 98 & 34,4 & 127 & 6,0 & 195 & 10,5 \\
\hline 145 & Anserine_p & 0 & 10,9 & 163 & 15,0 & & 0,0 & 121 & 31,6 & $\begin{array}{l}110 \\
5\end{array}$ & 6,4 & 113 & 14,3 \\
\hline 146 & Proline_p & 0 & 10,7 & 120 & 23,2 & & 0,0 & 194 & 19,3 & 59 & 9,1 & 97 & 15,0 \\
\hline 147 & Formiminoglutamic acid_p & 0 & 10,7 & 148 & 17,3 & & 0,0 & $\begin{array}{l}140 \\
5\end{array}$ & 28,4 & $\begin{array}{l}110 \\
5\end{array}$ & 6,4 & 113 & 14,3 \\
\hline 148 & C24:0_p & 0 & 10,6 & 164 & 15,0 & & 0,0 & 128 & 30,1 & 96 & 6,6 & 102 & 14,6 \\
\hline 149 & $\begin{array}{l}\text { Gamma-aminobutyric } \\
\text { acid_u }\end{array}$ & 0 & 10,6 & 152 & 16,8 & & 0,0 & $\begin{array}{l}140 \\
5\end{array}$ & 28,4 & $\begin{array}{l}110 \\
5\end{array}$ & 6,4 & 113 & 14,3 \\
\hline 150 & Succinic acid_u & 0 & 10,5 & 147 & 17,7 & & 0,0 & 118 & 31,7 & 126 & 6,0 & 200 & 10,1 \\
\hline 151 & C14:1-carnitine_u & 0 & 10,5 & 225 & 3,2 & & 0,0 & 75 & 39,6 & 193 & 4,5 & 62 & 18,2 \\
\hline 152 & Lysine_u & 0 & 10,4 & 88 & 32,4 & 95 & 1,5 & 186 & 21,2 & 179 & 4,8 & 233 & 5,2 \\
\hline 153 & 5-Hydroxylysine_p & 0 & 10,3 & 104 & 26,8 & & 0,0 & 203 & 17,5 & 156 & 5,6 & 95 & 15,0 \\
\hline 154 & Phytanic acid_p & 0 & 10,2 & 209 & 6,0 & & 0,0 & 129 & 29,7 & 65 & 8,5 & 48 & 20,1 \\
\hline 155 & Pristanic acid_p & 0 & 10,1 & 183 & 10,4 & & 0,0 & 96 & 34,7 & 136 & 5,9 & 149 & 13,0 \\
\hline 156 & Isoleucine_u & 0 & 9,9 & 122 & 22,8 & & 0,0 & 207 & 16,8 & 128 & 6,0 & 68 & 17,6 \\
\hline 157 & C8-carnitine_u & 0 & 9,9 & 234 & 1,5 & & 0,0 & 87 & 36,8 & 91 & 6,9 & 66 & 17,7 \\
\hline 158 & Cystine_p & 0 & 9,9 & 184 & 10,3 & 89 & 5,0 & 135 & 29,1 & 172 & 5,0 & 135 & 13,4 \\
\hline 159 & Tyrosine_u & 0 & 9,8 & 169 & 13,9 & & 0,0 & 144 & 28,0 & 79 & 7,5 & 148 & 13,0 \\
\hline 160 & Methionine_p & 0 & 9,8 & 98 & 30,0 & & 0,0 & 202 & 17,6 & 117 & 6,3 & 223 & 8,4 \\
\hline 161 & 4-Hydroxybutyric acid_u & 0 & 9,8 & 161 & 15,4 & & 0,0 & 156 & 26,2 & $\begin{array}{l}110 \\
5\end{array}$ & 6,4 & 113 & 14,3 \\
\hline 162 & C16:1-carnitine_u & 0 & 9,7 & 94 & 31,0 & 88 & 5,0 & 217 & 13,5 & 217 & 3,5 & 215 & 9,3 \\
\hline 163 & Valine_p & 0 & 9,7 & 204 & 6,9 & & 0,0 & 73 & 39,8 & 187 & 4,7 & 188 & 10,9 \\
\hline 164 & 3-Hydroxysebacic acid_u & 0 & 9,6 & 218 & 4,0 & & 0,0 & 80 & 38,7 & 153 & 5,6 & 136 & 13,4 \\
\hline 165 & Adipic acid_u & 0 & 9,6 & 119 & 23,3 & & 0,0 & 171 & 23,3 & 202 & 4,1 & 187 & 10,9 \\
\hline 166 & Glutamine_p & 0 & 9,6 & 170 & 12,9 & & 0,0 & 88 & 36,8 & 231 & 2,3 & 210 & 9,5 \\
\hline 167 & C18-OH-carnitine_u & 0 & 9,2 & 182 & 10,4 & 96 & 1,5 & 115 & 32,0 & 164 & 5,4 & 196 & 10,4 \\
\hline 168 & 1-Methylhistidine_p & 0 & 9,1 & 196 & 8,2 & & 0,0 & 133 & 29,5 & 218 & 3,5 & 63 & 18,2 \\
\hline 169 & Fumaric acid_u & 0 & 9,0 & 142 & 18,4 & & 0,0 & 158 & 25,7 & 188 & 4,6 & 199 & 10,1 \\
\hline 170 & Taurine_p & 0 & 9,0 & 137 & 20,0 & & 0,0 & 211 & 15,5 & 162 & 5,4 & 65 & 17,8 \\
\hline 171 & 3-Hydroxybutyric acid_u & 0 & 8,9 & 160 & 15,6 & & 0,0 & 143 & 28,2 & 184 & 4,7 & 202 & 9,9 \\
\hline 172 & $\begin{array}{l}\text { Beta-aminoisobutyric } \\
\text { acid_u }\end{array}$ & 0 & 8,7 & 151 & 17,1 & & 0,0 & 220 & 12,4 & 39 & 12,1 & 88 & 15,8 \\
\hline 173 & Formiminoglutamic acid_u & 0 & 8,6 & 159 & 15,6 & & 0,0 & 169 & 23,3 & 197 & 4,2 & 124 & 13,8 \\
\hline 174 & Tiglylglycine_u & 0 & 8,5 & 177 & 11,9 & & 0,0 & 136 & 29,1 & 88 & 7,0 & 219 & 8,8 \\
\hline 175 & Valine_u & 0 & 8,5 & 167 & 14,7 & & 0,0 & 196 & 18,8 & 105 & 6,4 & 79 & 16,7 \\
\hline 176 & Glutamic acid_u & 0 & 8,4 & 146 & 18,0 & & 0,0 & 181 & 22,0 & 207 & 3,9 & 162 & 12,3 \\
\hline 177 & 2-Methylcitric acid_u & 0 & 8,4 & 227 & 2,9 & & 0,0 & 86 & 36,9 & 167 & 5,3 & 189 & 10,8 \\
\hline 178 & Xylitol_u & 0 & 8,3 & 233 & 1,6 & & 0,0 & 109 & 32,8 & 129 & 6,0 & 91 & 15,4 \\
\hline 179 & C18-OH-carnitine_p & 0 & 8,1 & 156 & 16,7 & & 0,0 & 165 & 24,4 & 151 & 5,7 & 224 & 8,1 \\
\hline
\end{tabular}




\begin{tabular}{|c|c|c|c|c|c|c|c|c|c|c|c|c|c|}
\hline 180 & $\begin{array}{l}\text { C5-3M-3OH-DC- } \\
\text { carnitine_u }\end{array}$ & 0 & 8,1 & 202 & 7,1 & & 0,0 & 106 & 33,2 & 147 & 5,8 & 221 & 8,7 \\
\hline 181 & Alpha-aminobutyric acid_p & 0 & 8,1 & 189 & 9,1 & & 0,0 & 155 & 26,2 & 77 & 7,7 & 175 & 11,5 \\
\hline 182 & Phosphoethanolamine_u & 0 & 8,0 & 223 & 3,5 & & 0,0 & 99 & 34,0 & 191 & 4,6 & 163 & 12,3 \\
\hline 183 & C6-carnitine_u & 0 & 7,7 & 165 & 14,8 & & 0,0 & 219 & 12,7 & 195 & 4,4 & 43 & 21,1 \\
\hline 184 & Leucine_p & 0 & 7,7 & 210 & 5,9 & & 0,0 & 153 & 26,7 & 135 & 5,9 & 119 & 14,2 \\
\hline 185 & $\begin{array}{l}\text { Piperideine-6-carboxylic } \\
\text { acid_p }\end{array}$ & 0 & 7,6 & 188 & 9,3 & & 0,0 & 180 & 22,4 & 119 & 6,2 & 100 & 14,7 \\
\hline 186 & C10-DC-carnitine_u & 0 & 7,3 & 207 & 6,5 & & 0,0 & 185 & 21,5 & 121 & 6,1 & 74 & 17,1 \\
\hline 187 & Glycolic acid_u & 0 & 7,3 & 117 & 23,6 & & 0,0 & 206 & 16,8 & 235 & 1,0 & 207 & 9,7 \\
\hline 188 & C4-DC-carnitine_u & 0 & 7,3 & 203 & 7,0 & & 0,0 & 189 & 20,6 & 141 & 5,9 & 67 & 17,6 \\
\hline 189 & C16-OH-carnitine_u & 0 & 7,3 & 176 & 12,0 & & 0,0 & 174 & 22,9 & 181 & 4,7 & 177 & 11,5 \\
\hline 190 & Ribitol_u & 0 & 7,2 & 143 & 18,4 & & 0,0 & 232 & 7,4 & 168 & 5,3 & 49 & 19,7 \\
\hline 191 & Acetyl-aspartic acid_u & 0 & 7,2 & 228 & 2,6 & & 0,0 & 151 & 27,4 & 144 & 5,8 & 99 & 14,8 \\
\hline 192 & Histidine_p & 0 & 7,2 & 187 & 9,3 & & 0,0 & 209 & 16,0 & 155 & 5,6 & 52 & 19,6 \\
\hline 193 & C22:0_p & 0 & 7,2 & 235 & 1,0 & & 0,0 & 123 & 31,1 & 194 & 4,4 & 121 & 14,0 \\
\hline 194 & Suberylglycine_u & 0 & 7,1 & 206 & 6,7 & & 0,0 & 160 & 25,3 & 171 & 5,2 & 139 & 13,3 \\
\hline 195 & Asparagine_p & 0 & 7,0 & 217 & 4,6 & & 0,0 & 152 & 27,1 & 174 & 5,0 & 144 & 13,1 \\
\hline 196 & Argininosuccinic acid_u & 0 & 6,9 & 114 & 24,3 & & 0,0 & 221 & 11,3 & 213 & 3,6 & 197 & 10,3 \\
\hline 197 & Cystine_u & 0 & 6,9 & 221 & 3,7 & & 0,0 & 137 & 29,1 & 173 & 5,0 & 174 & 11,6 \\
\hline 198 & Phosphoethanolamine_p & 0 & 6,9 & 153 & 16,7 & & 0,0 & 208 & 16,1 & 216 & 3,5 & 154 & 12,8 \\
\hline 199 & C10-OH-carnitine_p & 0 & 6,7 & 213 & 5,3 & & 0,0 & 179 & 22,4 & 199 & 4,1 & 81 & 16,6 \\
\hline 200 & $\begin{array}{l}C 4: 1-3 M+C 4: 1-2 M+C 5: 1- \\
\text { carnitine u }\end{array}$ & 0 & 6,7 & 139 & 18,9 & & 0,0 & 191 & 20,0 & 185 & 4,7 & 234 & 4,8 \\
\hline 201 & Tryptophan_p & 0 & 6,6 & 185 & 9,5 & & 0,0 & 188 & 21,0 & 180 & 4,7 & 151 & 12,9 \\
\hline 202 & C14+C8:1-DC-carnitine_u & 0 & 6,6 & 166 & 14,7 & & 0,0 & 184 & 21,5 & 206 & 4,0 & 227 & 7,7 \\
\hline 203 & 2-Methylbutyrylglycine_u & 0 & 6,5 & 175 & 12,1 & & 0,0 & 198 & 18,4 & 201 & 4,1 & 142 & 13,1 \\
\hline 204 & Gender & 0 & 6,5 & 219 & 4,0 & & 0,0 & 147 & 27,7 & 234 & 2,1 & 123 & 13,9 \\
\hline 205 & Leucine_u & 0 & 6,4 & 230 & 2,5 & & 0,0 & 164 & 24,5 & 145 & 5,8 & 106 & 14,4 \\
\hline 206 & 3-Hydroxyglutaric acid_u & 0 & 6,4 & 195 & 8,2 & & 0,0 & 182 & 21,6 & 166 & 5,4 & 170 & 12,0 \\
\hline 207 & Cysteine-S-sulfate_p & 0 & 6,4 & 180 & 11,1 & & 0,0 & 230 & 8,3 & 74 & 7,9 & 51 & 19,7 \\
\hline 208 & Serine_p & 0 & 6,3 & 220 & 3,8 & & 0,0 & 146 & 27,9 & 208 & 3,9 & 183 & 11,1 \\
\hline 209 & C16+C10:1-DC-carnitine_u & 0 & 6,2 & 157 & 16,2 & 106 & 0,5 & 212 & 15,4 & 219 & 3,5 & 190 & 10,8 \\
\hline 210 & C5-OH-carnitine_u & 0 & 6,2 & 174 & 12,7 & & 0,0 & 201 & 18,0 & 225 & 3,2 & 161 & 12,4 \\
\hline 211 & C14:2-carnitine_u & 0 & 6,2 & 171 & 12,9 & & 0,0 & 205 & 17,1 & 101 & 6,5 & 208 & 9,6 \\
\hline 212 & Tyrosine_p & 0 & 6,1 & 229 & 2,6 & & 0,0 & 161 & 25,0 & 123 & 6,1 & 168 & 12,1 \\
\hline 213 & C18:1-carnitine_u & 0 & 6,1 & 150 & 17,1 & & 0,0 & 216 & 13,7 & 212 & 3,6 & 182 & 11,2 \\
\hline 214 & Lysine_p & 0 & 6,1 & 205 & 6,8 & & 0,0 & 162 & 24,7 & 183 & 4,7 & 212 & 9,4 \\
\hline 215 & Galactose_u & 0 & 6,0 & 211 & 5,6 & & 0,0 & 195 & 19,1 & 86 & 7,2 & 131 & 13,5 \\
\hline 216 & C10-OH-carnitine_u & 0 & 5,9 & 173 & 12,7 & & 0,0 & 222 & 11,2 & 215 & 3,6 & 69 & 17,5 \\
\hline 217 & Phenylpropionylglycine_u & 0 & 5,8 & 222 & 3,6 & & 0,0 & 103 & 33,3 & 85 & 7,3 & 236 & 0,0 \\
\hline 218 & 3-Methylglutaconic acid_u & 0 & 5,7 & 214 & 5,2 & & 0,0 & 127 & 30,1 & 236 & 0,0 & 222 & 8,7 \\
\hline 219 & Sorbitol_u & 0 & 5,6 & 232 & 1,6 & & 0,0 & 168 & 23,6 & 157 & 5,5 & 159 & 12,7 \\
\hline 220 & Isoleucine_p & 0 & 5,0 & 192 & 8,6 & & 0,0 & 215 & 14,5 & 132 & 6,0 & 172 & 11,9 \\
\hline 221 & Alanine_p & 0 & 4,9 & 179 & 11,7 & & 0,0 & 218 & 12,9 & 209 & 3,9 & 173 & 11,8 \\
\hline 222 & C18-carnitine_u & 0 & 4,9 & 199 & 7,4 & & 0,0 & 199 & 18,3 & 161 & 5,5 & 216 & 9,1 \\
\hline 223 & C3-carnitine_p & 0 & 4,9 & 154 & 16,7 & 105 & 0,5 & 231 & 8,2 & 230 & 2,5 & 164 & 12,3 \\
\hline 224 & Pyroglutamic acid_u & 0 & 4,8 & 226 & 3,1 & & 0,0 & 210 & 15,5 & 204 & 4,0 & 70 & 17,2 \\
\hline
\end{tabular}




\begin{tabular}{|c|c|c|c|c|c|c|c|c|c|c|c|c|c|}
\hline 225 & Galactitol_u & 0 & 4,8 & 181 & 10,7 & & 0,0 & 193 & 19,4 & 203 & 4,0 & 231 & 5,6 \\
\hline 226 & Phenylalanine_p & 0 & 4,6 & 178 & 11,9 & 108 & 0,0 & 226 & 10,1 & 210 & 3,8 & 143 & 13,1 \\
\hline 227 & 3-Methylhistidine_u & 0 & 4,5 & 136 & 20,0 & & 0,0 & 235 & 3,4 & 189 & 4,6 & 193 & 10,6 \\
\hline 228 & $\begin{array}{l}\text { Cys-homo-Cys-mixed } \\
\text { disulfide } p\end{array}$ & 0 & 4,0 & 193 & 8,4 & & 0,0 & 228 & 8,8 & 148 & 5,7 & 141 & 13,2 \\
\hline 229 & 5-Aminolevulinic acid_p & 0 & 3,5 & 216 & 4,6 & & 0,0 & 227 & 10,1 & 158 & 5,5 & 127 & 13,6 \\
\hline 230 & Acetoacetic acid_u & 0 & 3,4 & 194 & 8,3 & & 0,0 & 234 & 4,8 & 116 & 6,3 & 113 & 14,3 \\
\hline 231 & C14-OH-carnitine_u & 0 & 3,3 & 198 & 7,5 & & 0,0 & 224 & 10,2 & 196 & 4,3 & 185 & 11,1 \\
\hline 232 & Alloisoleucine_u & 0 & 3,3 & 231 & 2,2 & & 0,0 & 225 & 10,2 & $\begin{array}{l}110 \\
5\end{array}$ & 6,4 & 113 & 14,3 \\
\hline 233 & C10:2-carnitine_u & 0 & 3,0 & 224 & 3,5 & & 0,0 & 190 & 20,1 & 200 & 4,1 & 235 & 4,0 \\
\hline 234 & C16:1-OH-carnitine_u & 0 & 2,8 & 201 & 7,3 & & 0,0 & 229 & 8,6 & 227 & 3,0 & 171 & 11,9 \\
\hline 235 & Phenylalanine_u & 0 & 2,0 & 215 & 5,0 & & 0,0 & 233 & 5,6 & 233 & 2,1 & 107 & 14,3 \\
\hline 236 & Tryptophan_u & 0 & 0,0 & 236 & 0,0 & & 0,0 & 236 & 0,0 & 94 & 6,6 & 176 & 11,5 \\
\hline
\end{tabular}




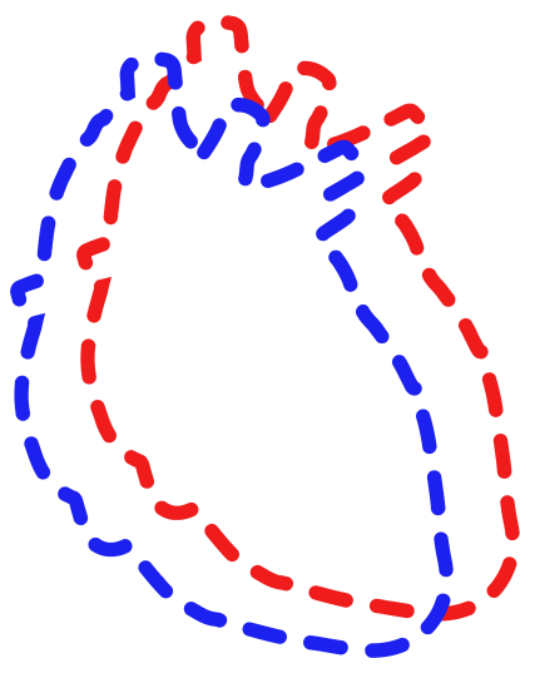

- 270 - 


\title{
CHAPTER 11
}

\section{The role of targeted therapy in dilated cardiomyopathy; the challenging road towards a personalized approach}

\author{
Job A.J. Verdonschot, $M D, M S c^{1,2}$ \\ Mark R. Hazebroek, $M D^{1}$ \\ James $S$. Ware, MRCP, $P h D^{3,4,5}$ \\ Sanjay K. Prasad, $M D^{3,4}$ \\ Stephane R.B. Heymans, MD, PhD ${ }^{1,6,7}$
}

${ }^{1}$ Department of Cardiology, Cardiovascular Research Institute (CARIM), Maastricht University Medical Center, Maastricht, The Netherlands.

${ }^{2}$ Department of Clinical Genetics, Maastricht University Medical Center, Maastricht, The Netherlands.

${ }^{3}$ Cardiovascular Research Centre, Royal Brompton \& Harefield Hospitals NHS Trust, London, United Kingdom.

${ }^{4}$ National Heart and Lung Institute, Imperial College London, London, United Kingdom.

${ }^{5}$ London Institute of Medical Sciences, Imperial College London, London, United Kingdom.

${ }^{6}$ Department of Cardiovascular Sciences, Centre for Molecular and Vascular Biology,

KU Leuven, Belgium.

${ }^{7}$ The Netherlands Heart Institute, Nl-HI, Utrecht, The Netherlands. 


\section{INTRODUCTION}

Dilated cardiomyopathy (DCM) is characterized by left ventricular (LV) or biventricular systolic dysfunction and dilation, not explained by abnormal loading conditions or coronary artery disease (CAD) ${ }^{1}$. DCM has a prevalence between 1 in 250 to 1 in 500. End-stage DCM is the leading indication for heart transplantation. DCM is a condition of heterogeneous etiology, underlining the importance of in-depth geno- and phenotyping in this multifactorial disease. Using contemporary diagnostic workup, an underlying etiology can be identified in between 50 to $75 \%$ of patients with multiple etiologies seen in approximately $25 \%{ }^{2}$.

Current therapy of DCM is mainly based on neurohumoral blockade as for other forms of heart failure (HF) with reduced ejection fraction (HFrEF) ${ }^{3}$. In spite of optimal medical therapy, the premature morbidity and mortality rate remains unacceptably high driving the need for novel and more individualized therapeutic options. However targeted therapy is largely lacking in clinical practice, but may be of significant added value as seen in the field of oncology ${ }^{4}$. Herein, the development of targeted therapies has greatly improved outcome in many cancer patients. The recently proposed MOGE(S) (Morphology, Organ Involvement, Genetic, Etiology, Stage of disease) nomenclature aims to better classify the multifactorial pathogenesis of DCM including upstream etiologies such as genetic variants and acquired diseases - toxic agents, viruses, immune disorders, hormonal changes and arrhythmias - that interact and lead to overlapping disease-driving molecular mechanisms that finally impair myocardial function ${ }^{12}$. These downstream disease-driving mechanisms can contribute 'variably' to the disease in the same patient. As such, parallels to oncology are challenging as biologic processes are not point mutations in a specific oncogene, but rather broad biologic pathways. Nevertheless, the MOGE(S) classification may provide the first step towards improved classification and more personalized approached to care. Using imaging complemented by genetics, blood biomarkers and occasionally cardiac sampling, a refined diagnosis of the underlying causes and downstream key processes can provide targeted treatment strategies. Furthermore, well-characterized geno- and phenotyping provides further understanding of the individual prognosis and disease penetrance within a family ${ }^{2}$. The extensive phenotyping of a patient should eventually lead to a diagnosis at the individual level: determining etiology and downstream molecular process activity in the patient. Such 'fingerprinting' of a patient could be the determinant for the treatment that will be most beneficial for the individual.

Many trials involving new therapies targeting key disease-driving mechanisms were published in the past decade. Although results are often promising, almost none of them have yet emerged in the mainstream in guideline-driven management of DCM patients. Still, many are promising, and the current overview aims to translate the information from these studies into opportunities for future novel or validation research.

\section{PATHOPHYSIOLOGY}

DCM is best regarded not a single disease entity, but rather as a nonspecific phenotype, with a final common response of the myocardium to a number of genetic and environmentallyacquired insults, which were extensively discussed in a previous review ${ }^{1}$. This in turn leads to variable presentation and often patients do not have a clinically recognized acute onset of the disease etiology. Therefore, knowledge regarding the exact stages and molecular changes for most etiologies is quite limited. Importantly, overall they share similar downstream pathophysiological mechanisms, which may have contributed 'variably' in the same patient,ultimately leading to the end-stage phenotype of cardiac dilation and systolic dysfunction 
(Figure 1). Thus, the finesse lies in understanding and recognizing the upstream etiology or trigger to create a fingerprint of the key disease-driving molecular mechanisms, so timely initiation of targeted therapy can be initiated to prevent irreversible progression of the disease. Several key molecular processes are at play during the development of DCM including altered cardiac metabolism and fibrosis, which provide novel opportunities for treatment.

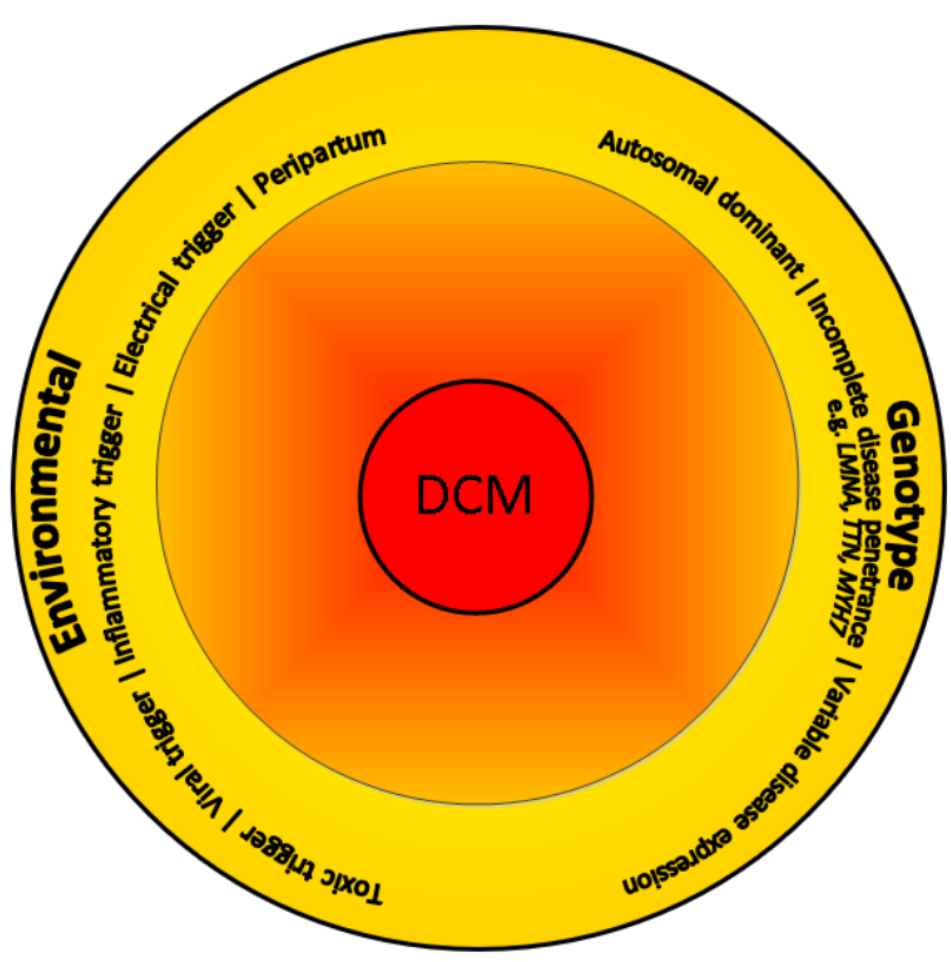

Figure 1. Different upstream environmental triggers on an individual genetic background can lead to dilated cardiomyopathy. DCM = dilated cardiomyopathy characterized by dilatation of the left and/or right ventricle and reduced cardiac function in the absence of ischemia and/or hemodynamic stress; $\mathrm{CM}=$ cardiomyocyte.

\section{CLINICAL PRESENTATION}

The majority of DCM patients will present insidiously when overt disease already has developed. Often the initial trigger or exact start of symptoms or 'tipping point' is not known. Only a minority of patients will have symptoms at onset, largely depending on the etiology, with peripartum or (viral) myocarditis being classic examples ${ }^{56}$. In the latter, the acute onset can range from days to weeks, infection by a virus triggers inflammation and cardiac injury, and subsequently a shift to an adaptive immune response. Of note, in many subjects the damage is minimal and no DCM develops ${ }^{7}$. Following acute myocarditis a subset of around $25-30 \%$ may deteriorate with worsening heart-failure culminating in end-stage DCM. They remain at increased arrhythmic risk both in the short and long-term ${ }^{7}$. Whether this is also the case in other upstream etiologies of DCM is not known, as their acute pathological phases, if any, are difficult to recognize due to the slow onset of symptoms.

As most DCM patients will present themselves when significant adverse remodeling is already manifest, focusing on the downstream molecular pathways at play throughout this phase is clinically most relevant. Importantly, several key processes may overlap during the disease course which makes estimating disease duration challenging. Therefore, targeting key 
disease-driving processes at the time of evaluation could be opportunities for treatment strategies, irrespective of the disease duration. Moreover, approximately one-third of DCM patients with recovered LVEF may relapse during the chronic disease 89 . Indeed, abrupt worsening of cardiac function or an increased ventricular arrhythmic burden can be driven not only by DCM progression but also by the development of new co-morbidities, such as common cardiovascular risk factors.

\section{PRECISION PHENOTYPING}

Phenotyping is essential for a heterogeneous disease such as DCM. A previous study in HF with preserved ejection fraction (HFpEF) patients, elegantly demonstrated the benefits of deep phenotyping in combination with unbiased clustering analysis (so-called phenomapping) ${ }^{10}$. Phenomapping in the heterogeneous HFpEF population defined more homogeneous patient subclasses who benefit from specific therapies, showing the importance of structural phenotyping. Phenotyping in DCM is essential to provide optimal treatment, as the key targets for treatment per patient are dependent on the upstream etiology and downstream active processes, reflecting patient subgroups (Table 1). Many tools can help us to determine the upstream trigger(s), which are extensively reviewed elsewhere (Figure 2) ${ }^{1}$. It is key to acquire a detailed history combined with clinical examination and echo or CMR assessment. Genetic testing and cardiac tissue sampling are useful in establishing underlying etiologies or susceptibilities. Detailed characterization of remodeling, particularly with CMR, provide further insight into etiology and prognosis, and may guide treatment strategy. Specifically, it is useful to accurately quantify the degree of remodeling, to look for inflammation, exclude underlying infarction and to assess the interstitial and replacement patterns of fibrosis ${ }^{11-13}$. Moreover, genetic testing has evolved tremendously in the past few years from single-gene to whole genome sequencing. The translation of this large generation of data towards clinical utility remains challenging as we often do not have enough knowledge to classify all found variants. Therefore, most centers performing genetic evaluations use a limited panel constituting of approximately 50 genes which are known to be DCM-associated. Titin (TTN), lamin A/C (LMNA) and myosin heavy chain 7 (MYH7) are the most well-known genes to be causative of DCM. Genetic analysis shows its importance as gene-environment interactions show prognostic value and determine the severity of disease presentation 214 . For example, alcohol cardiomyopathy has a more severe disease presentation when there is an underlying $T T N$ mutation ${ }^{14}$. Truncating TTN variants (TTNtv) are also seen in the general population, suggesting it does not always lead to a clear phenotype ${ }^{15}$. Also, rodent studies show a very mild effect of TTNtv on cardiac function in the absence of an additional trigger ${ }^{15}$ ${ }^{16}$. However, TTNtv do often give a subclinical phenotype in the general population, probably predisposing to heart failure under stress conditions ${ }^{15}$. The understanding of modifying environmental and/or genetic factors unveiling the cardiac phenotype is an important area of research. This highlights the importance of a complete work-up in a patient, as one etiology does not exclude others and often the underlying etiology includes a second 'insult' to unmask the phenotype. The goal lies in identification of those patients at-risk of developing DCM and use a combined diagnostic approach for earlier disease detection, with the opportunity to delay or possibly arrest disease progression. 
Table 1. Diagnostic options and their potential treatment targets of specific DCM etiologies

Causes and processes cardiotoxic chemotherapy)

Inflammation

EMB

12-lead ECG,

Electrical ambulatory ECG monitoring

Peripartum

(history of) Pregnancy

Cardiac metabolism

Blood, tissue and/or

Detailed family history, genetic testing using gene-panels (in adult disease) and WES (in pediatric disease), identifying specific clinical features (e.g. early LMNA)

Detailed history of toxin exposure; possibility for urine toxicology screen

MB, blood sampling (e.g. sIL2-R, CRP, galectin-3), CMR

$E M B$, blood sampling, imaging, autoantibody screen, presence of extracardiac features

$\begin{array}{cc}\text { Viral } & \text { EMB } \\ \text { Electrical } & \begin{array}{c}\text { 12-lead ECG, } \\ \text { ambulatory ECG } \\ \text { monitoring }\end{array} \\ \text { Peripartum } & \text { (history of) Pregnancy }\end{array}$
urine metabolomics

Cardiac fibrosis $\quad$ EMB, CMR, blood

Finding of a variant which is classified as a pathogenic mutation with or without familial DCM
Disease onset during/after toxin exposure; regression or resolution after withholding Immune cell infiltration in EMB, increased T2-signal, raised CRP/ESR

\section{Presence of} inflammation and positive autoantibody titers

\section{Cardiotropic virus} presence with a viral load > 500

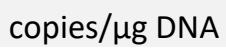
$>10.000-25.000$ PVCs/day; (supra)ventricular tachycardia Disease onset during pregnancy up to 6 months postpartum

\section{Elevated} acylcarnitines, increase in ketone bodies

Increased CFV; midmyocardial LE; increased fibrosis blood markers
Early ICD therapy in LMNA DCM; p38 signaling in LMNA DCM; gene correction in patients with truncating variants

No specific targets: withholding or reducing exposure; use of cardioprotective agents in anthracycline toxicity (dexrazoxane)

Pro-inflammatory pathways (e.g. IL-1ß)

Auto-antibodies (e.g. $\beta(1)$ AABs)

Virus and subsequent cardiac inflammation

\section{Cleaved 16kDa N-terminal fragment of prolactin}

Multiple strategies possible interfering with the metabolic substrate switch (mainly involving mitochondrial pathways) RAAS-pathway; Angiotensin II-Galectin-3interleukin-6 axis; matricellular proteins; syndecan-4-osteopontinlysyl oxidase-like axis

WES = Whole Exome Sequencing; DCM = Dilated Cardiomyopathy; ICD = Implantable Cardiac Defibrillator; EMB = Endomyocardial Biopsy; $C M R=$ Cardiac Magnetic Resonance; $C R P=C$-reactive Protein; $E S R=$ Erythrocyte Sedimentation Rate; PVC = Premature Ventricular Complex; $E C G=$ Electrocardiogram; $A A B=$ Autoantibodies; $C F V=$ Collagen Fraction Volume; $L E=$ Late Enhancement 
Diagnostic work-up

Characterization

\begin{tabular}{|c|c|c|}
\hline Medical History & Toxic exposure, obstetric history \\
\hline Coronary Angiography (CAG) & Exclude ischemia \\
\hline $\begin{array}{c}\text { Echocardiography } \\
\text { Cardiac Magnetic Resonance } \\
\text { Imaging (CMR) }\end{array}$ & Tissue characterization \\
\hline $\begin{array}{c}\text { Endomyocardial Biopsy } \\
\text { (EMB) }\end{array}$ & $\begin{array}{c}\text { Inflammation, auto-immunity, } \\
\text { Infectious triggers }\end{array}$ \\
\hline $\begin{array}{c}\text { Blood biomarkers } \\
\text { (CRP, sIL-2R, cardiac auto- } \\
\text { antibodies) }\end{array}$ & $\begin{array}{c}\text { Arrhythmias and conduction } \\
\text { disease }\end{array}$ \\
\hline Electrocardiography (ECG) \\
\hline Holter monitoring
\end{tabular}

Figure 2. Complete diagnostic work-up of a dilated cardiomyopathy (DCM) patient to characterize the cardiac function and underlying etiology.

\section{CURRENT STATE OF GUIDELINE-DRIVEN THERAPY}

The current treatment of DCM patients does not vary from general HF management, with the pharmacological cornerstone consisting of beta-blockers, RAS-inhibitors, aldosterone antagonists and diuretics ${ }^{17}$. One of the most recent guideline-changing breakthroughs in general HF treatment was the introduction of the angiotensin receptor-neprilysin inhibitor (ARNi) sacubitril-valsartan ${ }^{18}$. The major PARADIGM-trial showed a risk reduction of death and HF hospitalization in chronic HF patients treated with sacubitril-valsartan instead of enalapril. About $60 \%$ of the included patients had an ischemic etiology, making the drug still relatively new in the field of DCM. The results of the PARADIGM-trial have been analyzed post-hoc in several subgroups ${ }^{19}$. It remains an opportunity to perform an additional post-hoc analysis in only non-ischemic HF patients to evaluate its effect in this subgroup.

\section{TARGETING THE UNDERLYING ETIOLOGY OF DCM - IDENTIFYING UPSTREAM CAUSES}

In DCM it would be the goal to initiate therapies targeting key molecular processes driven by upstream etiologies. Knowledge regarding etiologies of DCM has expanded significantly over the last years. The increasing number of patients in registries and combining international 
databases provides us with valuable information regarding clinical presentation and prognosis of specific etiologies. Current guidelines do not include treatment strategies directed at key molecular processes driven by upstream etiologies, as evidence is mostly coming from single-center pilot and retrospective cohort studies (Table 2). Now is the time to make the next step towards multicenter, randomized trials (Table $\mathbf{3}$ ).

\section{Genetic causes}

Inheritance of a pathogenic gene variant is currently considered an irreversible cause and clinical management is based on the increasing genotype-phenotype knowledge. To date the monogenetic dogma is applied in clinical practice: whereas genetic DCM is mainly considered as an autosomal dominant disease in which one gene variant contributes to the DCM phenotype. However, increasing insights suggests that DCM is besides a monogenic disease potentially a polygenic, , complex disease involving the interaction among multiple gene variants. Whether this complex disease model will result in therapy guidance is yet to be discovered. Currently, guided therapy in the field of (mono)genetics can roughly be divided into three different approaches. The first approach is based on amelioration of the clinical consequences related to the specific gene mutation, based on the current genotypephenotype knowledge. The best example is a lower threshold for ICD-implantation in patients with a pathogenic lamin $\mathrm{A} / \mathrm{C}(L M N A)$ mutation, which is related with a higher risk of rhythm disturbances and sudden cardiac death ${ }^{20}$. For most other mutations, there is still a dearth of clinical and prognostic data to understand clinical consequences. Also, they often have a lower disease penetrance and more clinical variability among gene mutation carriers compared to $L M N A$ mutations ${ }^{21}$. This is likely due to the effect of additional environmental factors needed to trigger the phenotype on top of the genotype, creating the concept of susceptibility genes 2614 . This is especially relevant for truncating TTN mutations, which are the most prevalent among genetic causes for DCM.

A second approach is to target the molecular consequences of a specific gene mutation. Most cardiomyopathy-related genes and their subsequent proteins have specific functions in the cardiomyocyte. Loss (or gain) of function of some of these proteins will lead to intracellular changes of the signal transduction for which the cardiomyocyte tries to adapt. These molecular changes are well investigated in LMNA-mutated mice ${ }^{22}$. The most distinct change is the increased cardiac activity of the ERK1/2, JNK and p38 MAP kinases. Interestingly, when the mice were treated with the 38 inhibitor ARRY-371797, it prevented left ventricular dilation and deterioration of $\mathrm{EF}^{22}$. In addition, p38 inhibition was able to rescue $L M N A$-related severe biomechanical defects in neonatal rat ventricular myocytes ${ }^{23}$. The increasing evidence of beneficial p38 inhibition led to an international phase 3 clinical trial which is currently running (NCT03439514). This double-blinded, randomized, placebo-controlled study investigates the benefit of ARRY-371797 on change in 6-minute walk distance at 12 weeks in 160 patients with symptomatic DCM due to a LMNA gene mutation, which will be the first study towards genotype-specific therapy. With the increasing possibilities of transcriptomics and proteomics, it is likely that the altered signal transduction of other gene mutations will be unraveled. Truncating TTN mutations are a good example, as evidence is accumulating regarding changes in the cardiac metabolism and energy homeostasis ${ }^{24}$. The increase in the energy metabolism could be a mechanism of metabolic compensation for the sarcomere insufficiency ${ }^{25}$. This change in metabolism related to truncating TTN mutations can be a valid downstream target for intervention to prevent disease onset towards DCM. 
Table 2. Overview of all studies investigating etiology-directed treatments in dilated cardiomyopathy patients

$$
\begin{array}{lllll}
\text { Therapy } & \text { Year } & \text { Brief study design } & \mathrm{Nr} & \text { Follow-up }
\end{array}
$$

\begin{tabular}{|c|c|c|c|c|c|c|c|}
\hline \multicolumn{8}{|c|}{ Genetics } \\
\hline$\frac{\text { Signal transduction }}{\text { alteration }}$ & ARRY-371797 & 2018 & RCT & $\begin{array}{l}\text { Estimation } \\
\quad 160\end{array}$ & 6 months & $\begin{array}{l}\text { Study is still ongoing, estimated } \\
\text { study completion } 2020\end{array}$ & NCT03439514 \\
\hline \multicolumn{8}{|c|}{ Acquired triggers } \\
\hline \multirow[t]{5}{*}{ Inflammation } & Prednisone + Azathioprine & 2001 & RCT & 84 & 24 months & $\mathrm{C}$ & 35 \\
\hline & Prednisone + Azathioprine & 2009 & $\mathrm{RCT}$ & 85 & 6 months & $\mathrm{C}$ & 33 \\
\hline & Prednisone + Azathioprine & 2018 & $\begin{array}{l}\text { Retrospective, case- } \\
\text { control matched study }\end{array}$ & 180 & 31 months & $C, L$ & 38 \\
\hline & Anakinra & 2016 & $\mathrm{RCT}$ & 30 & 14 days & $\begin{array}{l}\text { Decreased systemic } \\
\text { inflammation }\end{array}$ & 42 \\
\hline & Anakinra & 2017 & $\mathrm{RCT}$ & 60 & 24 weeks & $S$ & 43 \\
\hline \multirow[t]{3}{*}{ Viral } & Interferon- $\beta$ & 2003 & Open & 22 & 24 weeks & $\mathrm{C}$ & 49 \\
\hline & $\begin{array}{l}\text { Intravenous } \\
\text { Immunoglobulins }\end{array}$ & 2010 & Open & 17 & 6 months & C & 50 \\
\hline & $\begin{array}{c}\text { Intravenous } \\
\text { Immunoglobulins }\end{array}$ & 2018 & $\mathrm{RCT}$ & 50 & 6 months & $\begin{array}{l}\text { Study recently completed, } \\
\text { results are pending }\end{array}$ & NCT00892112 \\
\hline \multirow[t]{2}{*}{ Auto-immunity } & $\begin{array}{l}\text { Immunoadsorption and Ig } \\
\text { replacement }\end{array}$ & 1996 & Open & 8 & Variable & $\mathrm{C}, \mathrm{S}$ & 108 \\
\hline & Immunoadsorption & 2000 & $\begin{array}{l}\text { Case-control study, } \\
\text { adsorption on top of OMT }\end{array}$ & 34 & 12 months & $\mathrm{C}, \mathrm{S}$ & 46 \\
\hline$\underline{\text { Toxic }}$ & Mesenchymal stem cells & 2018 & $\mathrm{RCT}$ & $\begin{array}{l}\text { Estimation } \\
\quad 36\end{array}$ & 12 months & $\begin{array}{l}\text { Study is still ongoing, estimated } \\
\text { study completion } 2019\end{array}$ & NCT02509156 \\
\hline \multirow[t]{2}{*}{ Peripartum } & Bromocriptine & 2010 & $\begin{array}{l}\text { Open, randomized pilot } \\
\text { study, } \mathrm{Br} \text { on top of OMT }\end{array}$ & 20 & 6 months & $C, L$ & 70 \\
\hline & Bromocriptine & 2017 & $\mathrm{RCT}$ & 63 & 6 months & $\begin{array}{l}\text { Short- and longterm } \mathrm{Br} \text { usage on } \\
\text { top of OMT are equal beneficial }\end{array}$ & 71 \\
\hline \multirow[t]{3}{*}{ Electrical } & Cathether ablation of AF & 2004 & $\begin{array}{c}\text { Prospective, case-control } \\
\text { matched study }\end{array}$ & 58 & 12 months & $C, S$ & 67 \\
\hline & Cathether ablation of PVC & 2011 & Retrospective cohort study & 69 & 11 months & C & 62 \\
\hline & $\begin{array}{l}\text { Catheter ablation vs } \\
\text { Antiarrhytmic drugs }\end{array}$ & 2014 & $\begin{array}{l}\text { Retrospective case-control } \\
\text { matched study }\end{array}$ & 510 & 12 months & $\mathrm{C}, \mathrm{S}$ & 66 \\
\hline
\end{tabular}




\begin{tabular}{|c|c|c|c|c|c|c|c|}
\hline & Rhythm vs Rate control & 2009 & $\mathrm{RCT}$ & 61 & 12 months & $C, S$ & 65 \\
\hline & Rhythm vs Rate control & 2010 & RCT & 614 & 36 months & $\begin{array}{l}\text { Lenient rate control is as } \\
\text { effective as strict rate control } \\
\text { and is easier to achieve }\end{array}$ & 64 \\
\hline & $\begin{array}{l}\text { Catheter ablation vs } \\
\text { Amiodaron }\end{array}$ & 2016 & RCT & 203 & 24 months & $\begin{array}{l}\text { Superior in achieving freedom of } \\
\text { AF, better long-term outcome }\end{array}$ & 68 \\
\hline \multicolumn{8}{|c|}{ Pathophysiological processes } \\
\hline \multirow[t]{3}{*}{ Myocardial damage } & $\begin{array}{l}\text { Bone marrow-derived } \\
\text { stem cells }\end{array}$ & 2015 & $\mathrm{RCT}$ & 60 & 12 months & C & 84 \\
\hline & Hematopoietic stem cells & 2013 & Open, randomized & 110 & 60 months & $C, S, L$ & 82 \\
\hline & Mesenchymal stem cells & 2017 & RCT & 37 & 12 months & $C, S$ & 109 \\
\hline$\frac{\text { Biomechanical }}{\text { defects }}$ & MYK-491 & 2018 & RCT & $\begin{array}{l}\text { Estimation } \\
\quad 56\end{array}$ & 49 days & $\begin{array}{l}\text { Study is still ongoing, estimated } \\
\text { study completion } 2019\end{array}$ & NCT03447990 \\
\hline \multirow[t]{4}{*}{$\frac{\text { Altered cardiac }}{\underline{\text { metabolism }}}$} & Etomoxir & 2000 & $\begin{array}{c}\text { First trial with etomoxir in } \\
\text { DCM individuals }\end{array}$ & 9 & 3 months & $\mathrm{C}$ & 90 \\
\hline & Perhexiline & 2015 & $\mathrm{RCT}$ & 50 & 2 months & $\mathrm{S}$ & 93 \\
\hline & Trimetazidine & 2008 & RCT & 19 & 3 months & $\mathrm{C}$ & 86 \\
\hline & Trimetazidine & 2013 & $\mathrm{RCT}$ & 80 & 6 months & $C, S$ & 87 \\
\hline Fibrosis & Spironolacton & 2005 & Open & 25 & 12 months & C & 96 \\
\hline
\end{tabular}


The ultimate therapy is to address the genetic perturbation in the individual patient. Several methods are under investigation to achieve this therapeutic goal such as 1) CRISPR/Cas9 which can target specific single mutations, 2) exon skipping, targeting all mutations present in one or two exons and their associated introns, 3) gene replacement targeting all mutations at once by gene transfer of the full-length cDNA ${ }^{26}$. Since its discovery in 2013, CRISPR/Cas9 is now becoming a valuable tool to investigate pathogenicity of gene mutations, create experimental models and to develop a genome editing therapy for specific gene mutations such as $D M D 2728$. In vivo genome editing with CRISPR/Cas9 restored dystrophic cardiomyopathy structurally and functionally in an animal model ${ }^{27}$. Exon skipping is especially useful in truncating mutations, as the exon containing the new stop-codon can be removed, preventing an incomplete transcript. Truncating mutations in TTN are the most well-known and exon skipping in patient-specific cardiomyocytes derived from induced pluripotent stem cells could be rescued preventing defective myofibril assembly and stability 29. Also, exon skipping prevented the development of DCM and improved contractile performance in TTN-mutated mice ${ }^{29}$. Gene replacement is a promising therapy for specific severe forms of genetic DCM when a mutation results in a low level or absence of the corresponding protein, which is mostly seen in pediatric cases of DCM. Evidence for the feasibility of gene replacement in a mouse model and human induced pluripotent stem cellderived cardiomyocytes carrying gene mutations in MYBPC3 has been shown ${ }^{26}$. Gene replacement has been realized by transducing the cells with adeno-associated virus (AAV) ${ }^{30}$. This successfully increased the expression of MYBPC3 transcripts and suppressed the disease phenotype. Although most of the genome editing has shown to be feasible and promising in cells and mice, there are still many questions which need to be answered before progressing to human trials with the mentioned three techniques. These include the safety of viral delivery and delivering the vector to the right place in the right dose, which will be challenging. Gene replacement therapy depends on AAV with a high cardiac tropism and a cardiac-specific promotor, which is AAV9 in mouse models. However, the best AAV serotype for the human heart still needs to be determined. Non-specific cardiac tropism can lead to off-target effects, strong host immunogenicity against the virus, insufficient AAV potency and efficacy and incomplete incorporation ${ }^{31}$. Interestingly, intracoronary infusion of AAV1SERCA2a in $123 \mathrm{HFrEF}$ patients was not associated with improvement in clinical outcome ${ }^{32}$. Despite the failure of this study, these findings should stimulate further research into the use of gene therapy to treat HF patients.

Table 3. Current situation and future directions for targeted therapies in dilated cardiomyopathy

\begin{tabular}{|c|c|c|c|}
\hline & Current state & Ongoing projects & Future directions \\
\hline \multicolumn{4}{|c|}{ Upstream triggers } \\
\hline Genetic & $\begin{array}{l}\text { Prevention with device } \\
\text { therapy in gene mutations } \\
\text { susceptible for malignant } \\
\text { arrhythmias (ie. LMNA) }\end{array}$ & $\begin{array}{l}\text { Phase } 3 \text { clinical trial } \\
\text { using p38 inhibition } \\
\text { in } L M N A D C M \\
\text { patients }\end{array}$ & $\begin{array}{l}\text { - Unravel molecular consequences } \\
\text { of specific gene mutations } \\
\text { - Gene correction therapies } \\
\text { towards a clinical application }\end{array}$ \\
\hline Inflammation & $\begin{array}{l}\text { No guideline-directed } \\
\text { therapy; although there is } \\
\text { evidence from retrospective } \\
\text { studies showing benefit } \\
\text { from immunosuppression }\end{array}$ & $\begin{array}{l}\text { Phase } 2 \mathrm{~B} \text { RCT in } \\
\text { acute myocarditis } \\
\text { using anakinra versus } \\
\text { standard care }\end{array}$ & $\begin{array}{c}\text { - Multicenter RCT using } \\
\text { immunosuppressive therapy in } \\
\text { inflammatory DCM }\end{array}$ \\
\hline $\begin{array}{l}\text { Auto- } \\
\text { immunity }\end{array}$ & $\begin{array}{l}\text { No guideline-directed } \\
\text { therapy; although there is }\end{array}$ & - & $\begin{array}{l}\text { - Multicenter RCT using } \\
\text { immunoadsorption in DCM with } \\
\text { cardiotoxic autoantibodies }\end{array}$ \\
\hline
\end{tabular}


evidence showing benefit from immunoadsorption

No guideline-directed therapy; although there are retrospective studies and

Viral case reports showing benefit from intravenous immunoglobulins

No guideline-directed therapy; withholding or reducing exposure has shown to be the most effective; in some cases, cardioprotective compounds for anthracycline toxicity are advised.

\section{Phase 3 RCT using}

IVIg for chronic

PVB19-related DCM

rapies (val/-ganciclovir)

- Multicenter RCT for IVIg if phase 3 trial is positive

- Unravel molecular changes in cocaine-induced DCM

- Phase 2 RCT using stem cell therapy in cardiotoxic

Phase 1 RCT using MPCs in anthracyclineinduced DCM chemotherapy-induced DCM

- Define the timing, dose and duration of prophylactic therapy to prevent HF onset in those patients receiving cardiotoxic chemotherapy at risk - Better insight in the

Early treatment of electrical disturbance $(\mathrm{AF}$, ablation;

Electrical left bundle branch block, CRTD)

No guideline-directed therapy; although one Peripartum prospective study showed benefit from bromocriptine

pathomechanisms of the interplay between between heart failure and electrical disturbances to detect their causal relationships and better stratify patients who will benefit from (device) therapy - A placebo-controlled study with bromocriptine to assess safety and efficacy

- More data regarding long-term outcome of PPCM after bromocriptine usage

\section{Downstream processes}

\begin{tabular}{|c|c|}
\hline $\begin{array}{c}\text { Biomechanical } \\
\text { defects }\end{array}$ & $\begin{array}{c}\text { No guideline-directed } \\
\text { therapy }\end{array}$ \\
\hline $\begin{array}{c}\text { Myocardial } \\
\text { damage }\end{array}$ & $\begin{array}{l}\text { No guideline-directed } \\
\text { therapy; although multiple } \\
\text { studies investigated the } \\
\text { benefit from stem cell } \\
\text { therapy }\end{array}$ \\
\hline $\begin{array}{c}\text { Cardiac } \\
\text { metabolism }\end{array}$ & $\begin{array}{l}\text { No guideline-directed } \\
\text { therapy; although multiple } \\
\text { compounds have been } \\
\text { investigated showing benefit }\end{array}$ \\
\hline $\begin{array}{l}\text { Cardiac } \\
\text { fibrosis }\end{array}$ & $\begin{array}{c}\text { No guideline-directed } \\
\text { therapy }\end{array}$ \\
\hline
\end{tabular}

Phase 1 and phase 2

RCT using MYK-491 in DCM
- Follow-up research based on results of current phase 1 and 2 RCT

- Investigate the role of omecamtiv in (genetic) DCM.

- Phase 3 RCT combining G-CSF and $B M C$ in DCM as follow-up on the REGENERATE-DCM

- More data regarding long-term outcome of DCM after trimetazidine

- Investigating extra-cardiac effects of perhexiline usage - Specific anti-fibrotic medication tailored to arrhythmic burden or reversibility of fibrosis implantation in DCM patients with LGE on CMR
- Exploring the role of novel antifibrotic therapies with regards to cardiac fibrosis (i.e. vanticumab,

Abbreviations: AF indicates atrial fibrillation; BMC, bone marrow-derived stem cells; CMR, cardiovascular magnetic resonance; CRTD, cardiac resynchronization therapy device; DCM, dilated cardiomyopathy; G-CSF, granulocyte colony-stimulating factor; HF, heart failure; ICD, implantable cardioverter-defibrillator; ILR, implantable loop recorder; IVIg, intravenous immunoglobulin; LGE, late gadolinium 


\section{Inflammatory triggers}

So far 3 randomized trials have examined the effect of immunosuppressive therapies in DCM 33-35. The 2 largest trials of these assessed the effect of prednisone and azathioprine. However, they were conducted before the era of quantitative immunohistochemistry for the assessment of infiltrates, using solely hematoxylin and eosin staining and the Dallas criteria 34-36. In addition, molecular analysis for viral presence in myocardial biopsies was not available. Both trials showed no beneficial effect on mortality, wherethe IMAC trial also failed to show beneficial effects on cardiac function. Interestingly, Frustaci et al. demonstrated improved and sustained cardiac function in DCM patients treated with immunosuppressive therapy on top of HF therapy as compared to those that received standard HF treatment alone. The importance of discrimination between virus-positive and -negative patients was exemplified by a post hoc stratification of patients treated with prednisone and azathioprine, showing beneficial effects predominantly in the virus-negative group ${ }^{37}$. Subsequently, a prospective study using immunosuppressive therapy in 85 patients with aggressive cardiac inflammation, but virus absence revealed a significantly improved cardiac function at 6 months in the immunosuppression group as compared to standard HF therapy only. Besides the potential beneficial effects on cardiac function, long-term transplantation-free survival can also be improved using this immunosuppressive regimen ${ }^{38}$. Based on these results, the most recent ESC recommendations state that immunosuppression may be considered in infection-negative myocarditis refractory to standard therapy in patients with no contraindications to immunosuppression ${ }^{39}{ }^{40}$. In contrast, these recommendations are not advocated by the AHA guidelines ${ }^{41}$. To definitively prove potential benefits of immunosuppression in this subset of patients, a multicenter trial was initiated but terminated due to low inclusion rate (NCT01877746). Therefore, confirmation of a randomized, prospective, placebo-controlled multicenter trial will be necessary before a consensus is reached in guidelines.

Recently, the role of IL-1 $\beta$ blockade, namely anakinra, has raised interest as clinical pilot studies in inflammatory DCM showed beneficial effects on hemodynamics, inflammation and clinical performance ${ }^{42} 43$. Anakinra is a nonglycosylated protein that differs from the sequence of the native IL-1 receptor antagonist by one methionine added to its $\mathrm{N}$-terminus. The IL-1 family of ligands and receptors is the main cytokine family associated with acute and chronic inflammation. Recently, 30 patients with acute decompensated HF and elevated CRP levels were randomized to either receive anakinra or matching placebo ${ }^{42}$. Anakinra reduced CRP by $61 \%$ versus baseline, compared with a $6 \%$ reduction among patients receiving placebo. Moreover, anakinra was associated with a greater recovery in LVEF compared with placebo. No significant differences between treatment groups in the initial length of stay or total hospital days during the 14 days was observed. Based on these favorable results, safety and efficacy of anakinra was evaluated in the Recently Decompensated Heart Failure Anakinra Response Trial (REDHART), involving 60 patients with recently decompensated heart failure and systolic dysfunction. Anakinra treatment reduces serum CRP levels in a sustained manner and improves peak Vo2. However, IL-1B blockade does not improve cardiac function or improve prognosis in terms of death or HF hospitalization at 24 weeks as compared to placebo 43. The study was however not powered to detect differences in outcome and should be used as an estimate of effect size to design appropriately powered studies. Moreover, the ACTION Study Group in France initiated a Phase 2B randomized controlled trial in acute myocarditis 
patients using anakinra versus standard care (NCT03018834). The study is estimated to be completed in 2021. Blockade of IL-1B seems to be a promising therapy target in HF, as a recent subgroup analysis using placebo-controlled data of the CANTOS trial in which canakinumab (a monoclonal antibody against IL-1B) showed a dose-dependent reduction in heart failure hospitalization and heart failure related mortality in 2173 patients with heart failure at baseline ${ }^{44}$. Hence, one of the inclusion-criteria for the CANTOS trial was a baseline CRP $\geq 2$ $\mathrm{mg} / \mathrm{L}$, thus creating a selected population with signs of elevated systemic inflammation who benefitted from canakinumab treatment.

\section{Auto-immunity}

The concept of immunoadsorption involves the removal of cardiotoxic autoantibodies, together with cytokines from the circulating system, as they might cause damage to the myocardium ${ }^{45}$. To avoid infection, the adsorbed immunoglobulins (namely IgG, little IgA and IgM) are replaced using administration of $0.5 \mathrm{~g} / \mathrm{kg}$ body weight polyclonal IgG after treatment. Although studies are relatively small, an improvement of LV function is reported in approximately $60 \%$ of patients treated ${ }^{46}$. Using gene expression analysis from the myocardial tissue, pre-treatment responders and non-responders can be potentially distinguished ${ }^{47}$. Although these results seem attractive, confirmation by a randomized treatment trial is needed.

\section{Viral triggers}

These include antiviral therapies targeting cardiotropic viruses such as Herpes simplex virus (HSV) 1 and 2, cytomegalovirus (CMV), Epstein-Barr virus (EBV), varicella virus, human herpes virus (HHV) 6 and 7, Parvovirus B19 (B19V), respiratory syncytial virus, hepatitis C virus, and HIV. Specific antiviral therapies comprise Val-/Ganciclovir, acyclovir, Foscanet, high-dose intravenous immunoglobulins (IVIG) and antiretroviral therapy administration in human immunodeficiency virus infection ${ }^{48}$. Of note, only a few are tested in a trial setting for DCM, with limited randomized control data.

In enterovirus-positive DCM patients, spontaneous enterovirus elimination and elimination using interferon- $\beta$ are both associated with both clinical and hemodynamic improvement ${ }^{49}$. Of note, half of these patients spontaneously eliminated their enterovirus without specific treatment, suggesting that not merely the presence of viral genomes but also the replication status and viral load are important criteriafor antiviral therapy ${ }^{5}$.

Retrospective studies demonstrated that DCM patients with a high viral load may benefit from IVIG ${ }^{50}$. Although a randomized trial in 62 patients with recent-onset DCM $(<6$ months) did not show beneficial effects on mortality and LVEF after 6 and 12 months, it lacked evaluation of viral infection and only $12 \%$ exhibited cardiac inflammation ${ }^{34}$. Currently, a single center randomized controlled trial using high-dose IVIG ( $2.0 \mathrm{~g} / \mathrm{kg}$ body weight) for chronic PVB19-related DCM to reduce PVB19 viral load was completed in August 2018, enrolling a total of 50 patients. (NCT00892112).

\section{Toxic triggers}

Toxic triggers are most known for structural and functional changes in the myocardium due to increased myocyte loss, which is largely irreversible ${ }^{51-53}$. The main advice remains to reduce or withdraw the introduced toxic trigger such as excess alcohol, cocaine or cancer therapy. 
Anthracyclines are best known for their cardiotoxic effect. They manifest their cardiotoxicity via multiple mechanisms: increase in oxidative stress, modulation in topoisomerase activity, alteration in the multidrug-resistant (MDR) efflux proteins and a decrease in mesenchymal progenitor cells (MPC) ${ }^{53}$. The decrease in MPCs reduces the cardioreparative capacity of the heart when its exposed to stress ${ }^{54}$. A phase I randomized, placebo-controlled trial currently evaluates the safety and feasibility of administering MPCs to patients with anthracycline-induced DCM (NCT02509156). This trial will lay the foundation for a specific treatment regarding chemotherapy-induced DCM in addition to prompt treatment with general HF therapy ${ }^{55}$. Multiple trials investigated the potential of prophylactic cardioprotective therapy using general HF medication or specific compounds such as dexrazoxane, an iron chelator decreasing the formation of superoxide radicals ${ }^{56}$. However, current data does not support the routine prophylactic use of HF treatment in patients receiving cardiotoxic chemotherapies. In contrast, the use of dexrazoxane is licensed for clinical use in cancer patients undergoing anthracycline dosing in excess of $300 \mathrm{mg} / \mathrm{m}^{2}$ or epirubicin greater than $550 \mathrm{mg} / \mathrm{m}^{2}$. Therefore, it may be considered in patients receiving these high doses of cardiotoxic chemotherapy ${ }^{57}$. Ongoing studies will help to better define the timing, dose and duration of prophylactic therapy to prevent HF onset in those patients at risk. Also, high-throughput screenings to identify novel cardioprotective compounds are ongoing, although none of them reached clinical trials yet ${ }^{58}$.

A cocaine use disorder can lead to a broad spectrum of cardiovascular complications 52. Careful diagnostics is therefore of importance when seeing a patient with suspicion of cocaine-related DCM. Cocaine exerts its toxic effect via multiple pathways: myocardial scarring, impaired intracellular calcium handling, apoptosis, increase of oxidative stress and acute effects of the catecholamines. However, current treatment advice is to treat according to the general $\mathrm{HF}$ guidelines and trials investigating specific treatment regimens are lacking.

Alcohol in low concentrations is beneficial for the heart, in contrast to the other toxic triggers ${ }^{51}$. Long-term heavy alcohol consumption may lead to alcoholic DCM, most likely via structural damage to the cardiomyocyte leading to apoptosis ${ }^{51}$. Also, ethanol and its metabolites are thought to be toxic for the sarcoplasm and mitochondria, altering the calcium sensitivity at the myofilament level. Specific guideline for the treatment of alcoholic DCM are lacking. Most studies focus on the clinical effect of alcohol decrease or complete abstention 51. Patients who reduced their alcohol intake to moderate levels had a similar survival and cardiac function recovery compared to complete alcohol abstainers ${ }^{59}$. Overall, the alcoholic DCM patients had a better survival compared with the general group of DCM patients. Therefore, alcohol reduction on top of general HF treatment is the keystone of therapy in alcoholic DCM.

\section{Electrical triggers}

Arrhythmia-induced DCM is a well-known potentially reversible condition in which DCM is induced or mediated by atrial or ventricular arrhythmias and has been extensively reviewed previously ${ }^{60}$. It may follow many types of cardiac arrhythmia: supraventricular tachyarrhythmias, ventricular tachycardia, or frequent ventricular ectopy. However, the diagnosis of arrhythmia-induced cardiomyopathy is often difficult, as both are frequently diagnosed simultaneously. DCM in response to an arrhythmia may take months to years to develop, although rapid declines in ventricular function with development of heart failure symptoms is also observed in recurrent (tachy)arrhythmias. If this beneficial response remains absent, one must consider other factors influencing the arrhythmic phenotype such 
as genetic mutations, i.e. LMNA or SCN5A mutations ${ }^{20}$. To date, the exact mechanisms of most genetic and non-genetic arrhythmias in DCM are not completely understood, but involve the loss of normal extracellular matrix and contractile dysfunction, alterations in cellular growth and viability, defects in $\mathrm{Ca} 2+$ handling, and neurohormonal activation results in DCM ${ }^{60}$.

Managing patients with suspected arrhythmic-induced DCM is to attempt careful and aggressive control of rate and rhythm, with the focus on arrhythmia elimination by catheter ablation whenever possible. Identifying the underlying condition is important to predict treatment response. In a study of 27 patients with frequent premature ventricular complexes (PVCs) and DCM, 22 had improvement in LVEF following PVC suppression; 5 did not. Four of the 5 patients with LVEF that did not improve had evidence of irreversible myocardial fibrosis detected by LGE CMR ${ }^{61}$. A high PVC burden of $>10,000$ PVCs/day or $>10 \%$ to $24 \%$ of total heartbeats/day can cause DCM. Reducing the PVC burden to $<5,000 /$ day can improve LVEF ${ }^{62}$. Moreover, elimination of PVCs with ablation has been shown to improve LVEF, ventricular dimensions, mitral regurgitation, and functional status. In an observational series, ablation was superior to antiarrhythmic therapy in reducing PVCs and improving LVEF ${ }^{63}$.

In atrial fibrillation (AF), several trials evaluating rhythm control to rate control strategies have been conducted. Although they did not focus on arrhythmic-induced DCM, several lessons can be learned. In general, rhythm control does not provide a benefit in allcause mortality or worsening heart failure as compared to rate control, with similar findings in strict versus lenient rate control ${ }^{64}$. In AF-mediated DCM, rhythm control is superior to rate control in improving LVEF, pro-BNP levels, and quality of life ${ }^{65}$. Moreover, in AF-mediated $D C M$, restoring and maintaining sinus rhythm can accelerate clinical recovery and reverse DCM over several in months ${ }^{66}$. Indeed, restoration and maintenance of sinus rhythm by catheter ablation in patients with congestive heart failure and AF significantly improves cardiac function, symptoms, exercise capacity, and quality of life ${ }^{67}$. In atrial flutter, catheter ablation is recommended in arrhythmia-induced DCM, as rate control is more difficult than in $\mathrm{AF}^{68}$.

\section{Peripartum cardiomyopathy}

Peripartum cardiomyopathy (PPCM) is a form of DCM with deterioration of cardiac function typically between the last trimester of pregnancy and up to six months postpartum. It is recognized as a major cause of pregnancy related heart failure ${ }^{69}$. To date, no evidence-based specific therapy is recommended in the current guidelines. The exact disease pathophysiology is not known, however, high levels of cleaved 16kDa N-terminal fragment of prolactin have been shown to be an important mediator. Bromocriptine is a dopamine-D2-receptor agonist and inhibits prolactin release. A pilot study in acute severe PPCM showed improvement in LVEF and prognosis when bromocriptine was added to standard HF therapy ${ }^{70}$. A later randomized multicenter clinical trial confirmed these findings and showed no significant difference between short- and long-term treatment with bromocriptine ${ }^{71}$. Importantly, the trial did not include a non-use control group, leaving many unanswered questions. Ongoing observatory registries will provide further information regarding benefit and follow-up of bromocriptine usage ${ }^{72}$. Despite European experience and guidelines, a placebo-controlled trial of bromocriptine for peripartum CMP is very much needed. 


\section{TARGETING DOWNSTREAM MOLECULAR PROCESSES DRIVING DISEASE PROGRESSION}

Despite many downstream processes driving the progression to DCM overlap among different upstream etiologies, the timing and severity of these processes may vary. Clinical outcome is eventually determined by the interplay between trigger, host response, and therapeutic intervention. The alterations in cardiac metabolism and fibrosis are popular targets for intervention. In contrast, cardiomyocyte apoptosis, stretch and damage are less suitable for intervention as they are often irreversible. However, cell therapy does provide potential as a future therapy to remedy cardiomyocyte damage and loss.

\section{Cardiomyocyte dysfunction}

DCM is characterized by biomechanical defects leading to inadequate cardiac contraction. Positive inotropic agents, such as dobutamine and milrinone, are indicated as a therapy in patients with end-stage HF and cardiogenic shock ${ }^{73}$. Milrinone is a bipyridine and inhibits phosphodiesterase-3 (PDE3) eventually leading to an increased cellular calcium influx with subsequent stimulation of myocardial contractility. The OPTIME-CHF study was a large randomized trial to investigate the incremental value of milrinone on top of standard $\mathrm{HF}$ therapy in patients with acute $\mathrm{HF}^{74}$. This study showed significant side-effects of milrinone, such as sustained hypotension and arrhythmias. A post hoc analysis showed that milrinone was associated with higher mortality and rehospitalization in ischemic HF but was neutral to beneficial in non-ischemic HF patients ${ }^{75}$. However, we should be careful in the interpretation of this post hoc analysis as the study is not initially designed for this purpose. For example, a subset analysis of the PRAISE I study showed a significant reduction in mortality in the nonischemic subgroup treated with amlodipine ${ }^{76}$. The well-designed PRAISE 2 study with the purpose to investigate the potential of amlodipine to reduce mortality in patients with nonischemic HF, failed to show this effect with trends in favor of placebo ${ }^{77}$. There are currently no therapies that address the underlying biomechanical causes in DCM, one of the newest therapies aims at increasing the contractile function of the heart with minimal adverse effects on myocardial relaxation. The compound, named MYK-491, is currently being investigated in a randomized, double-blind, placebo-controlled, cross-over single-ascending dose phase $1 \mathrm{~b}$ trial including DCM patients (NCT03447990). In contrast to milrinone, MYK-491 is an allosteric activator of myosin, thereby not increasing calcium levels in the cardiomyocytes and limiting potential side-effects. If the safety and tolerability and echocardiographic measures of cardiac contractility will be positive, MYK-491 will be pursued in future research. A different inotropic compound is omecamtiv mecarbil, which showed favorable hemodynamic results in patients with chronic HF in a phase II clinical trial ${ }^{78}$. In patients with acute HF, omecamtiv mecarbil did not meet the primary endpoint of dyspnea improvement ${ }^{79}$. Both phase II trials (ATOMIC-AHF and COSMIC-HF) showed small increases in plasma troponin concentrations in treated patients. Overall, there was no relationship with drug omecamtiv concentrations and occurrence of adverse events. The origin of these raised troponin concentrations is unknown and needs to be addressed in larger outcome trials. A phase III morbidity/mortality trial in patients with chronic HF (NCT02929329) is currently recruiting patients. Interestingly, omecamtiv mecarbil showed to augment cardiac contractility in DCM using a DCM mouse model containing a tropomyosin mutation ${ }^{80}$. This makes omecamtiv mecarbil a promising compound to treat DCM patients with sarcomeric gene mutations. 


\section{Cardiomyocyte damage and loss}

Stem cell therapy to compensate for damaged/lost myocardial tissue aims to improve cardiac function in HF. Although the main focus of this approach was on ischemic HF, multiple clinical trials investigated the value of cell therapy in DCM, which is extensively reviewed elsewhere 81. The most widely used method for delivery is intracoronary. Although showed to be safe and simple, these stem cells cannot reach the inadequate perfused myocardium. Also, homing and retention of the stem cells at the right location is still difficult. A variety of stem cell types is investigated in DCM: bone marrow-derived (BMC), hematopoietic (HSC) and mesenchymal (MSC) stem cells. Initially, cytokines and SCs were investigated separately and both had shown beneficial effects on intermediate outcome such as cardiac function ${ }^{82} 83$. REGENERATE-DCM was the first phase II randomized, placebo-controlled trial showing a significant improvement in cardiac function, symptoms and biochemical parameters after a combined treatment of granulocyte colony-stimulating factor (G-CSF) and BMC therapy ${ }^{84}$. The results of the REGENERATE-DCM cannot be translated to long-term outcome; therefore a phase III trial is necessary to investigate these promising beneficial effects of combining $G$ CSF and BMC in DCM.

\section{Altered cardiac metabolism}

One of the key hallmarks in HF is the decreased oxidative metabolism of the cardiomyocyte 85. This in turn leads to a switch from fatty acid oxidation towards increased glucose metabolism to fulfill the energy demand of the contracting cardiac muscle. A large part of the glucose utilization is used for anaerobic glycolysis, which is an energy inefficient process. Eventually, the accumulating metabolic changes in HF will lead to an energy deficit which maintains the progression of the disease ${ }^{85}$. Multiple compounds have been tested to intervene different stages of metabolic changes, as extensively described in a previous review 85 .

One possible intervention mechanism is to stimulate the heart to use glucose as primary substrate by inhibiting the fatty acid oxidation. In this case, the heart will use its available oxygen in a more efficient way. Trimetazidine is a thiolase I inhibitor. Thiolase I catalyses the last step of the beta-oxidation in the mitochondria and its inhibition will shift the substrate utilization towards glucose. Use of trimetazidine on top of optimal medical treatment (OMT) showed a significant increase in LVEF in DCM ${ }^{86}$. These findings were replicated in a later double-blinded study including DCM patients with diabetes ${ }^{87}$. In addition, they also showed that theinflammatory response was decreased, and the physical performance of the patients was better. A recent meta-analysis of trimetazidine use in DCM showed its effectiveness regarding LVEF improvement and reduction in cardiac dimensions 88. Although there is no long-term follow-up in DCM patients, a meta-analysis showed a decreased risk for hospitalization but no effect on all-cause mortality in general HF ${ }^{89}$. Trimetazidine is the compound for metabolic support which is the most ready for larger-scale trials in DCM.

Carnitine palmitoyl transferase (CPT) is part of the acyl-carnitine shuttle of the mitochondria, which plays a crucial role in the fatty acid transport. Etomoxir is a compound which inhibits CPT and thereby inhibits the beta-oxidation, stimulating glucose as the primary substrate ${ }^{85}$. The first trial with etomoxir included 90\% non-ischemic DCM patients and showed improvements in LVEF and clinical status ${ }^{90}$. However, a larger follow-up study with a general HF population was terminated prematurely due to adverse side effects ${ }^{91}$. Perhexiline is an alternative CPT-inhibitor which showed its value in hypertrophic cardiomyopathy and 
ischemic HF ${ }^{92}$. Recently, a phase 2 randomized, double-blinded, placebo-controlled study was published, only including non-ischemic DCM patients ${ }^{93}$. Although they noted no benefit in cardiac function, the symptoms improved in patients receiving perhexiline. More importantly, treatment with perhexiline increased the energy status of the heart without detectable changes of substrate utilization. This suggested that the treatment duration in this study was too short or that the effects of perhexiline are much broader than only CPT inhibition. However, perhexiline was taken off the market in many countries due to its toxicity in patients with CYP2D6 polymorphisms, which constitutes a significant proportion in the Caucasian population.

Although metabolic changes are a major effect of HF in the heart, therapy targeting these changes have not seen clinical implementation yet. Partly due to our incomplete knowledge regarding the specific metabolic changes in the heart. New methods such as metabolomics will shed more light on the molecular changes which will help us to better identify the best patients and stage of the disease when metabolic support can be the most effective. The current available larger studies used heterogeneous HF populations, probably leading to the mixed results. Pre-clinical and clinical data in the general HF population with newer molecules were promising ${ }^{85}$, and investigating these newer compounds in DCM would be valuable.

\section{Cardiac fibrosis}

Fibrosis is an essential component of tissue repair that follows tissue injury and is usually associated with inflammation. Progressive fibrosis reflects a pathologic state and results in scarring and impairment of organ function. Myocardial fibrosis is a hallmark in end-stage DCM, starting with potentially reversible diffuse reactive fibrosis finally transitioning to irreversible focal scarring fibrosis ${ }^{94}$. The main pathways and biological entities involved in the development of myocardial fibrosis are the mineralocorticoid and transforming growth factor- $\beta$ (TGF- $\beta$ ) pathways, and non-structural matrix proteins and miRNAs ${ }^{95}$. To date, the impact of gene mutations and additional environmental-acquired factors on the extent of myocardial fibrosis is largely unknown. Some insights have been gained by examining subsets of patients with DCM such as those with LMNA mutations where premature scarring fibrosis is associated with increased risk of arrhythmias ${ }^{20}$. In this regard, targeting fibrosis can be roughly divided into two categories.

The first is delaying or preventing the onset of myocardial fibrosis, with early detection of potentially treatable causes to prevent further progression towards irreversible fibrosis in DCM patients or those at-risk of developing DCM. The second is regression or stabilization of established myocardial fibrosis through targeting of key molecular processes. Although these key molecular processes can be targeted in several ways, most strategies do not specifically target fibrosis.

Mineralocorticoid receptor antagonists (MRAs), already part of standard HF therapy, may be interesting anti-fibrotic drugs as they act on extracellular matrix (ECM) remodeling, decrease collagen biosynthesis biomarkers and improve outcomes in patients with heart failure and DCM ${ }^{9697}$. In DCM patients, myocardial fibrosis on histologic level can be reduced using MRAs accompanied by a decrease in collagen biosynthesis biomarkers ${ }^{96}$. Moreover, myocardial galectin-3 (gal-3) plasma concentrations are increased by aldosterone and angiotensin II early in the fibrotic cascade and can be reduced by MRAs, consistent with an effect of these agents on tissue pathology ${ }^{97}$. Oher potential therapeutic targets include transforming growth factor- $\beta$ (TGF- $\beta$ ), interleukins (ILs) and wnt signaling, as they can 
transform quiescent fibroblasts into active collagen-producing myofibroblasts ${ }^{98}$. Although the molecular TGF- $\beta$ pathway is important in virtually all types of fibrosis, its pleiotropic effects makes it an unattractive target.Cardiotrophin-1, a member of the IL-6 superfamily, is a pro-fibrotic factor and is increased in the myocardium of patient with HF of different etiologies, including DCM ${ }^{99}$. Finally, inhibition of wnt signaling reduced fibrosis and cardiac recovery in mice ${ }^{100}$. So far, pharmacological studies are performed only in gal-3 and wnt signaling. Gal-3 inhibitors include carbohydrates such as modified citrus pectin (MCP), which is used in an ongoing trial including patients with high blood pressure to reduce cardiac fibrosis (NCT01960946). Interestingly, a phase 1 trial using a monoclonal antibody called vantictumab, which inhibits downstream signaling of wnt, was completed in December 2017 of which results are awaiting (NCT01973309). Thus, targeting the Angiotensin II-Galectin-3interleukin(IL)- 6 axis using MRAs or Gal-3 inhibitors or inhibiting wnt signaling pathway by vantictumab could specifically reduce cardiac fibrosis.

Matricellular proteins have a potential in anti-fibrotic therapies, as they can degrade collagen and modulate the function of cardiac fibroblasts, inflammatory cells, and cardiomyocytes. Recently, the first proof of principle for a therapeutic potential has evolved for osteoglycin. Absence of osteoglycin in mice reduced collagen cross-linking in the infarct scar after myocardial infarction, thereby increasing cardiac rupture and dilatation. Osteoglycin administration through gene therapy enhanced collagen maturation and thereby prevented LV dilatation and dysfunction after myocardial infarction ${ }^{101}$. To date, no human trials exist.

Various small non-coding RNAs (miRs), post-transcriptional regulators of gene expression, are involved in cardiac fibrosis. Myocardial fibrosis in patients with diverse cardiac disease is accompanied by increased expression of profibrotic miRs including miR-21, miR208a, and miR-499-5p, and decreased expression of antifibrotic miRs suchs as miR-29, miR 19b, miR-1, miR-133a, and miR-122 ${ }^{95}$. More importantly, miRNAs can be easily manipulated and provide a novel class of anti-fibrotic agents. Although no human trials including heart failure patients receiving miRs are conducted yet, the anti-fibrotic miR-122 reached phase II trial for treating hepatitis ${ }^{102}$. Thus, the advancement of miR-based therapeutics into the clinical testing of heart failure is on the horizon.

Besides quantity is the quality of myocardial fibrosis, in terms of collagen cross-linking, is an equally important feature ${ }^{94}$. Excessively cross-linked collagen is difficult to degrade and critically affects extra-cellular matrix turnover. Syndecan-4-osteopontin-Lysyl Oxidase-Like (LOXL) axis is important in the formation of insoluble cross-linked collagen, and therapeutic strategies that target such pathways could also ameliorate the effects of myocardial fibrosis 103. A monoclonal antibody against LOXL2, called simtuzumab, is currently being tested in a phase Ilb trial in subjects with compensated cirrhosis secondary to non-alcoholic steatohepatitis (NCT01672879). So far, no clinical trials in cardiac diseases have been initiated.

Interestingly, in the field of idiopathic pulmonary fibrosis (IPF) two anti-fibrotic medications, nintedanib and pirfenidone, have been shown to be safe and effective in the treatment of IPF and are currently recommended for use in these patients ${ }^{104}$. Nintanib is a tyrosine kinase inhibitor that targets growth factor pathways and pirfenidone has a number of anti-inflammatory and anti-fibrotic effects, including inhibition of collagen synthesis, down-regulation of TGF- $\beta$ and tumor necrosis factor alpha, and a reduction in fibroblast proliferation ${ }^{105}$. Currently, a phase II trial is conducted to evaluate the efficacy and safety of pirfenidone in patients with heart failure and preserved ejection fraction (NCT02932566). 


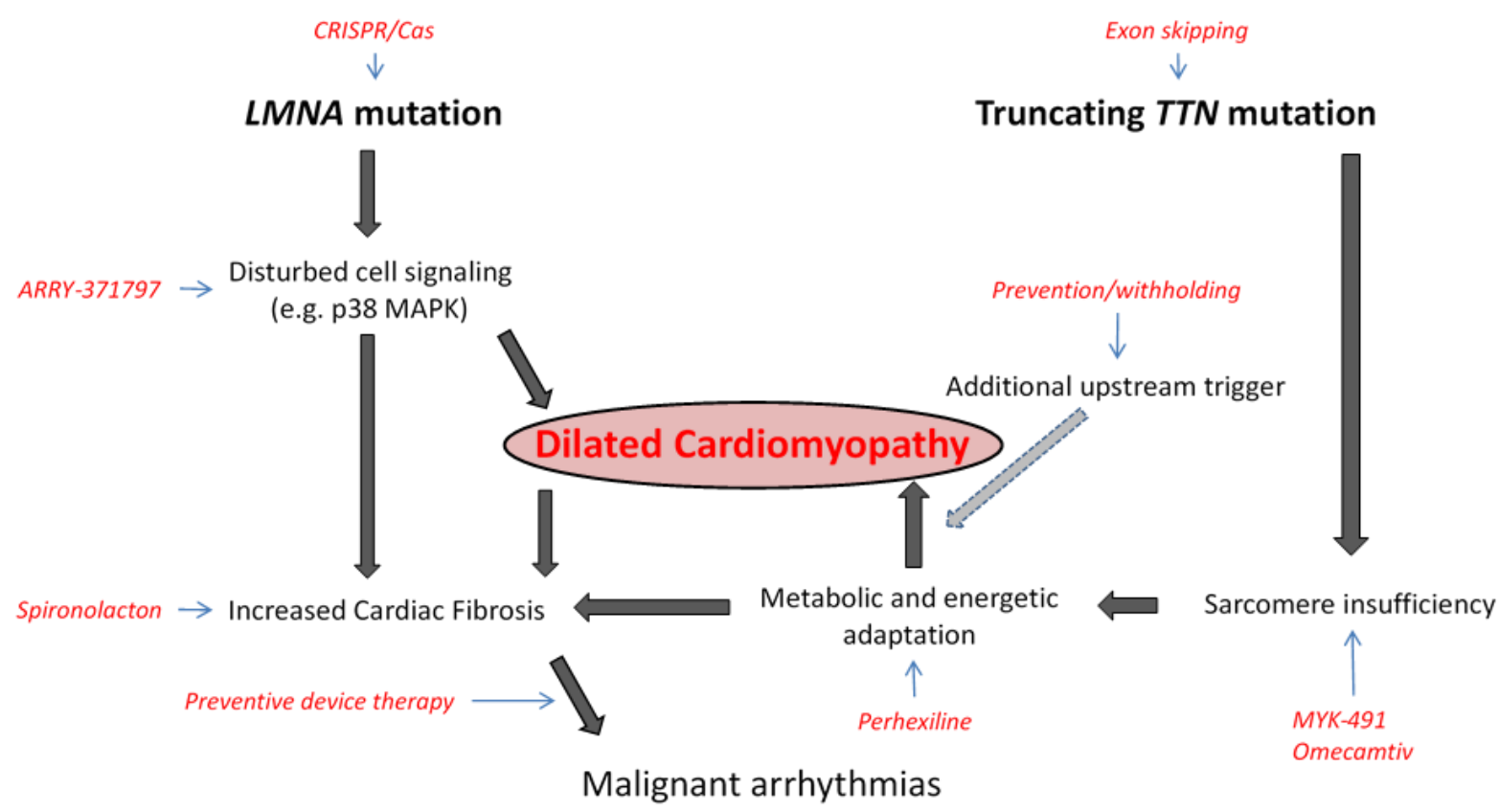

Figure 3. Two examples of genetic mutations leading to dilated cardiomyopathy and their potential treatment targets interfering with disease progression at different levels.

\section{CLINICAL IMPLICATIONS AND FUTURE PERSPECTIVES}

The greatest pitfall of evidence-based etiology-directed therapy is the small number of patients included in studies $(<100)$ (Table 2 ). This is mainly due to the heterogeneous nature of DCM and incomplete characterization of etiology, which limits the numbers of specific subgroups of patients. Often echocardiographic analysis is available without CMR, and EMB is not standard in all countries/practices. Although the quality of most studies is good, the majority remains single-center experience. Therefore, clinical implications are limited. The only guideline-changing therapies over the past few years were tested in general HF, of which DCM is often a small subset. This retains the current dogma of non-personalized treatment.

Understanding the molecular consequences of genetic DCM will provide novel and specific treatment targets. LMNA DCM is a good example, as the altered intracellular signaling is well studied. Therefore, multiple therapies can interfere with the disease course, upstream and downstream (Figure 3). With the high prevalence of TTN-associated DCM, its molecular and phenotypical signature is becoming more clear. This will also provide therapeutic options for these specific patients. Although we have a broad selection of therapies, we now have to fine-tune the therapy based on upstream triggers and their downstream active processes (Figure 3).

Genetic diagnostics adds a different dimension to the spectrum. The increase in genotype-phenotype knowledge will greatly benefit prevention and a personalized approach. Early detection and preventive medicine will be an important focus of future research. Clinical and genetic screening of DCM relatives is a perfect example for identifying carriers of a pathogenic mutation who are at risk for developing the phenotype. Herein, echocardiographic strain may provide a useful tool to detect subclinical disease in 'healthy' DCM relatives and moreover carries prognostic relevance. However, there is great clinical variability and incomplete penetrance within families due to factors we do not understand yet. Moreover, whether prophylactic treatment is beneficial and able to prevent disease 
onset is unknown. This is an important area of research as it will greatly benefit patient care and reassurance among genetic DCM families.

Another important clinical issue is the safety of medication withdrawal in currently asymptomatic DCM patients with complete cardiac recovery. Notably, DCM patients are relatively young and reluctant to take lifelong medication without continued beneficial effects. A recent open-label, randomized, pilot trial suggested that around $40 \%$ patients with recovered DCM will have a relapse within 6 months after phased medication discontinuation

${ }^{106}$. However, this also showed that $60 \%$ of DCM patients did not have a relapse over that time period. Future research should focus on identifying subgroups of DCM patients in whom pharmacological treatment for HF can be safely withdrawn. One such possible marker for identifying subgroups is global longitudinal strain (GLS) analysis on echocardiography. A recent study showed that $79 \%$ of recovered HF patients still had an abnormal GLS, despite normalization of geometry and/or LVEF ${ }^{107}$. Furthermore, the abnormal GLS is a predictor for worse clinical outcome in general recovered HF patients.

The ultimate goal is to link the key disease-driving mechanisms in DCM patients with specific therapies. These targeted therapies will be most beneficial in those patients that do not improve sufficiently using the established guideline-directed medical therapies ${ }^{17}$. This should set the stage for further in depth pheno- and genotyping using the proposed diagnostic DCM work-up (Figure 2). As such, standardized classification of DCM subclasses according to the upstream etiology and downstream molecular processes at the time of clinical evaluation is crucial. Herein, the MOGE(S) classification can provide the first steps towards standardized classification of these subclasses whom might benefit most from targeted therapy (e.g. immunosuppressive, anti-fibrotic therapies). Once global standardized classification and subsequent stratification validates the proposed up- and downstream subclasses, multicenter trials using targeted therapies adjunctive to established guideline-directed medical therapy can be initiated to prove their incremental value. In the future, recruitment should start in global expert centers with the availability of advanced imaging technologies (e.g. CMR), genetic evaluation and preferably myocardial biopsy. Treatment efficacy should be monitored using established (natriuretic peptides, troponin) and relevant circulating biomarkers for the specific therapy (e.g. inflammatory markers for immunosuppression, fibrosis markers for antifibrotic therapy). In addition, assessment of short-term (6 - 12 months) functional improvement such as HF questionnaires, exercise tolerance, and cardiac function together with 'hard' endpoints including HF hospitalization, life-threatening arrhythmias, implantation of ventricular assist device, heart-transplantation, and death are crucial to evaluate long-term beneficial effects of the targeted therapy.

\section{CONCLUSION}

DCM constitutes a broad clinical phenotype that arises from a final common response to a number of genetic and environmental-acquired insults. Detecting the underlying dominant etiology and the ongoing downstream pathophysiological processes is the key to initiate targeted treatment strategies. Several small targeted trials have been conducted, but as of now the era has arrived to conduct large, multicenter trials that result in breakthroughs in targeted treatment that improves prognosis in these patients. 


\section{REFERENCES}

1. Japp AG, Gulati A, Cook SA, et al. The Diagnosis and Evaluation of Dilated Cardiomyopathy. J Am Coll Cardiol 2016;67(25):2996-3010.

2. Hazebroek MR, Moors S, Dennert $R$, et al. Prognostic Relevance of Gene-Environment Interactions in Patients With Dilated Cardiomyopathy: Applying the MOGE(S) Classification. J Am Coll Cardiol 2015;66(12):1313-23.

3. Writing Committee $\mathrm{M}$, Yancy CW, Jessup $\mathrm{M}$, et al. 2013 ACCF/AHA guideline for the management of heart failure: a report of the American College of Cardiology Foundation/American Heart Association Task Force on practice guidelines. Circulation 2013;128(16):e240-327.

4. Pao W, Fodor I. Catalyzing the field of precision oncology, one basket at a time. Nat Med 2018;24(4):387-88.

5. Verdonschot J, Hazebroek M, Merken J, et al. Relevance of cardiac parvovirus B19 in myocarditis and dilated cardiomyopathy: review of the literature. Eur J Heart Fail 2016;18(12):1430-41.

6. Ware JS, Li J, Mazaika E, et al. Shared Genetic Predisposition in Peripartum and Dilated Cardiomyopathies. N Engl J Med 2016;374(3):233-41.

7. Anzini M, Merlo M, Sabbadini G, et al. Long-term evolution and prognostic stratification of biopsyproven active myocarditis. Circulation 2013;128(22):2384-94.

8. Merlo M, Cannata A, Gobbo M, et al. Evolving concepts in dilated cardiomyopathy. Eur J Heart Fail 2018;20(2):228-39.

9. Gupta A, Goyal P, Bahl A. Frequency of recovery and relapse in patients with nonischemic dilated cardiomyopathy on guideline-directed medical therapy. Am J Cardiol 2014;114(6):883-9.

10. Shah SJ, Katz DH, Selvaraj S, et al. Phenomapping for novel classification of heart failure with preserved ejection fraction. Circulation 2015;131(3):269-79.

11. Puntmann VO, Carr-White G, Jabbour A, et al. T1Mapping and Outcome in Nonischemic Cardiomyopathy: All-Cause Mortality and Heart Failure. JACC Cardiovasc Imaging 2016;9(1):4050.

12. Halliday BP, Baksi AJ, Gulati A, et al. Outcome in Dilated Cardiomyopathy Related to the Extent, Location, and Pattern of Late Gadolinium Enhancement. JACC Cardiovasc Imaging 2018

13. Gulati A, Jabbour A, Ismail TF, et al. Association of fibrosis with mortality and sudden cardiac death in patients with nonischemic dilated cardiomyopathy. JAMA 2013;309(9):896-908.

14. Ware JS, Amor-Salamanca A, Tayal U, et al. Genetic Etiology for Alcohol-Induced Cardiac Toxicity. J Am Coll Cardiol 2018;71(20):2293-302.

15. Schafer S, de Marvao A, Adami E, et al. Titintruncating variants affect heart function in disease cohorts and the general population. Nature genetics 2017;49(1):46-53.

16. Radke $\mathrm{MH}$, Peng J, Wu Y, et al. Targeted deletion of titin N2B region leads to diastolic dysfunction and cardiac atrophy. Proc Natl Acad Sci U S A 2007;104(9):3444-9.

17. Yancy CW, Jessup M, Bozkurt B, et al. 2017 ACC/AHA/HFSA Focused Update of the 2013 ACCF/AHA Guideline for the Management of Heart Failure: A Report of the American College of Cardiology/American Heart Association Task Force on Clinical Practice Guidelines and the Heart Failure Society of America. Circulation 2017;136(6):e137-e61.

18. McMurray JJ, Packer M, Desai AS, et al. Angiotensin-neprilysin inhibition versus enalapril in heart failure. N Engl J Med 2014;371(11):9931004.

19. Okumura N, Jhund PS, Gong J, et al. Importance of Clinical Worsening of Heart Failure Treated in the Outpatient Setting: Evidence From the Prospective Comparison of ARNI With ACEI to Determine Impact on Global Mortality and Morbidity in Heart Failure Trial (PARADIGM-HF). Circulation 2016;133(23):2254-62.

20. Halliday BP, Cleland JGF, Goldberger JJ, et al. Personalizing Risk Stratification for Sudden Death in Dilated Cardiomyopathy: The Past, Present, and Future. Circulation 2017;136(2):215-31.

21. Kayvanpour E, Sedaghat-Hamedani F, Amr A, et al. Genotype-phenotype associations in dilated cardiomyopathy: meta-analysis on more than 8000 individuals. Clin Res Cardiol 2016

22. Muchir A, Wu W, Choi JC, et al. Abnormal p38alpha mitogen-activated protein kinase signaling in dilated cardiomyopathy caused by lamin A/C gene mutation. Human molecular genetics 2012;21(19):4325-33.

23. Laurini $E$, Martinelli V, Lanzicher $T$, et al. Biomechanical defects and rescue of cardiomyocytes expressing pathologic nuclear lamins. Cardiovasc Res 2018;114(6):846-57.

24. Verdonschot JAJ, Hazebroek MR, Derks KWJ, et al. Titin cardiomyopathy leads to altered mitochondrial energetics, increased fibrosis and long-term life-threatening arrhythmias. Eur Heart J 2018

25. Ware JS, Cook SA. Role of titin in cardiomyopathy: from DNA variants to patient 
stratification. Nature reviews Cardiology 2018;15(4):241-52.

26. Carrier L, Mearini G, Stathopoulou K, et al. Cardiac myosin-binding protein C (MYBPC3) in cardiac pathophysiology. Gene 2015;573(2):18897.

27. El Refaey M, Xu L, Gao Y, et al. In Vivo Genome Editing Restores Dystrophin Expression and Cardiac Function in Dystrophic Mice. Circulation research 2017;121(8):923-29.

28. Ma N, Zhang J, Itzhaki I, et al. Determining the Pathogenicity of a Genomic Variant of Uncertain Significance Using CRISPR/Cas9 and HumanInduced Pluripotent Stem Cells. Circulation 2018

29. Gramlich M, Pane LS, Zhou Q, et al. Antisensemediated exon skipping: a therapeutic strategy for titin-based dilated cardiomyopathy. EMBO Mol Med 2015;7(5):562-76.

30. Prondzynski M, Kramer E, Laufer SD, et al. Evaluation of MYBPC3 trans-Splicing and Gene Replacement as Therapeutic Options in Human iPSC-Derived Cardiomyocytes. Mol Ther Nucleic Acids 2017;7:475-86.

31. Colella P, Ronzitti G, Mingozzi F. Emerging Issues in AAV-Mediated In Vivo Gene Therapy. Mol Ther Methods Clin Dev 2018;8:87-104.

32. Greenberg B, Butler J, Felker GM, et al. Calcium upregulation by percutaneous administration of gene therapy in patients with cardiac disease (CUPID 2): a randomised, multinational, doubleblind, placebo-controlled, phase $2 \mathrm{~b}$ trial. Lancet (London, England) 2016;387(10024):1178-86.

33. Frustaci A, Russo MA, Chimenti C. Randomized study on the efficacy of immunosuppressive therapy in patients with virus-negative inflammatory cardiomyopathy: the TIMIC study. Eur Heart J 2009;30(16):1995-2002.

34. McNamara DM, Holubkov R, Starling RC, et al. Controlled trial of intravenous immune globulin in recent-onset dilated cardiomyopathy. Circulation 2001;103(18):2254-9.

35. Wojnicz R, Nowalany-Kozielska E, Wojciechowska C, et al. Randomized, placebocontrolled study for immunosuppressive treatment of inflammatory dilated cardiomyopathy: two-year follow-up results. Circulation 2001;104(1):39-45.

36. Aretz HT, Billingham ME, Edwards WD, et al. Myocarditis. A histopathologic definition and classification. Am J Cardiovasc Pathol 1987;1(1):3-14.

37. Frustaci A, Chimenti C, Calabrese F, et al. Immunosuppressive therapy for active lymphocytic myocarditis: virological and immunologic profile of responders versus nonresponders. Circulation 2003;107(6):857-63.
38. Merken J, Hazebroek M, Van Paassen P, et al. Immunosuppressive Therapy Improves Both Short- and Long-Term Prognosis in Patients With Virus-Negative Nonfulminant Inflammatory Cardiomyopathy. Circulation Heart failure 2018;11(2):e004228.

39. Caforio AL, Pankuweit S, Arbustini $E$, et al. Current state of knowledge on aetiology, diagnosis, management, and therapy of myocarditis: a position statement of the European Society of Cardiology Working Group on Myocardial and Pericardial Diseases. Eur Heart J 2013;34(33):2636-48, 48a-48d.

40. Pinto YM, Elliott PM, Arbustini E, et al. Proposal for a revised definition of dilated cardiomyopathy, hypokinetic non-dilated cardiomyopathy, and its implications for clinical practice: a position statement of the ESC working group on myocardial and pericardial diseases. Eur Heart J 2016;37(23):1850-8.

41. Bozkurt B, Colvin M, Cook J, et al. Current Diagnostic and Treatment Strategies for Specific Dilated Cardiomyopathies: A Scientific Statement From the American Heart Association. Circulation 2016;134(23):e579-e646.

42. Van Tassell BW, Abouzaki NA, Oddi Erdle C, et al. Interleukin-1 Blockade in Acute Decompensated Heart Failure: A Randomized, Double-Blinded, Placebo-Controlled Pilot Study. J Cardiovasc Pharmacol 2016;67(6):544-51.

43. Van Tassell BW, Canada J, Carbone S, et al. Interleukin-1 Blockade in Recently Decompensated Systolic Heart Failure: Results From REDHART (Recently Decompensated Heart Failure Anakinra Response Trial). Circulation Heart failure 2017;10(11)

44. Everett BM, Cornel J, Lainscak M, et al. AntiInflammatory Therapy with Canakinumab for the Prevention of Hospitalization for Heart Failure. Circulation 2018

45. Wallukat G, Muller J, Hetzer R. Specific removal of beta1-adrenergic autoantibodies from patients with idiopathic dilated cardiomyopathy. N Engl J Med 2002;347(22):1806.

46. Muller J, Wallukat G, Dandel $M$, et al. Immunoglobulin adsorption in patients with idiopathic dilated cardiomyopathy. Circulation 2000;101(4):385-91.

47. Ameling S, Herda LR, Hammer E, et al. Myocardial gene expression profiles and cardiodepressant autoantibodies predict response of patients with dilated cardiomyopathy to immunoadsorption therapy. Eur Heart J 2013;34(9):666-75.

48. Heymans S, Eriksson U, Lehtonen J, et al. The Quest for New Approaches in Myocarditis and Inflammatory Cardiomyopathy. J Am Coll Cardiol 2016;68(21):2348-64. 
49. Kuhl U, Pauschinger $M$, Schwimmbeck PL, et al. Interferon-beta treatment eliminates cardiotropic viruses and improves left ventricular function in patients with myocardial persistence of viral genomes and left ventricular dysfunction. Circulation 2003;107(22):2793-8.

50. Dennert R, Velthuis S, Schalla S, et al. Intravenous immunoglobulin therapy for patients with idiopathic cardiomyopathy and endomyocardial biopsy-proven high PVB19 viral load. Antivir Ther 2010;15(2):193-201.

51. Guzzo-Merello G, Cobo-Marcos M, GallegoDelgado $\mathrm{M}$, et al. Alcoholic cardiomyopathy. World J Cardiol 2014;6(8):771-81.

52. Havakuk O, Rezkalla SH, Kloner RA. The Cardiovascular Effects of Cocaine. J Am Coll Cardiol 2017;70(1):101-13.

53. Lenneman CG, Sawyer DB. Cardio-Oncology: An Update on Cardiotoxicity of Cancer-Related Treatment. Circulation research 2016;118(6):1008-20.

54. De Angelis A, Piegari E, Cappetta D, et al. Anthracycline cardiomyopathy is mediated by depletion of the cardiac stem cell pool and is rescued by restoration of progenitor cell function. Circulation 2010;121(2):276-92.

55. Cardinale D, Colombo A, Sandri MT, et al. Prevention of high-dose chemotherapy-induced cardiotoxicity in high-risk patients by angiotensin-converting enzyme inhibition. Circulation 2006;114(23):2474-81.

56. Levis BE, Binkley PF, Shapiro CL. Cardiotoxic effects of anthracycline-based therapy: what is the evidence and what are the potential harms? Lancet Oncol 2017;18(8):e445-e56.

57. Hensley ML, Hagerty KL, Kewalramani $\mathrm{T}$, et al. American Society of Clinical Oncology 2008 clinical practice guideline update: use of chemotherapy and radiation therapy protectants. J Clin Oncol 2009;27(1):127-45.

58. Gergely S, Hegedus C, Lakatos $P$, et al. High Throughput Screening Identifies a Novel Compound Protecting Cardiomyocytes from Doxorubicin-Induced Damage. Oxid Med Cell Longev 2015;2015:178513.

59. Guzzo-Merello G, Segovia J, Dominguez F, et al. Natural history and prognostic factors in alcoholic cardiomyopathy. JACC Heart Fail 2015;3(1):78-86.

60. Gopinathannair R, Etheridge SP, Marchlinski FE, et al. Arrhythmia-Induced Cardiomyopathies: Mechanisms, Recognition, and Management. J Am Coll Cardiol 2015;66(15):1714-28.

61. Hasdemir C, Yuksel A, Camli D, et al. Late gadolinium enhancement CMR in patients with tachycardia-induced cardiomyopathy caused by idiopathic ventricular arrhythmias. Pacing Clin Electrophysiol 2012;35(4):465-70.

62. Mountantonakis SE, Frankel DS, Gerstenfeld EP, et al. Reversal of outflow tract ventricular premature depolarization-induced cardiomyopathy with ablation: effect of residual arrhythmia burden and preexisting cardiomyopathy on outcome. Heart Rhythm 2011;8(10):1608-14.

63. Zhong L, Lee $\mathrm{YH}$, Huang XM, et al. Relative efficacy of catheter ablation vs antiarrhythmic drugs in treating premature ventricular contractions: a single-center retrospective study. Heart Rhythm 2014;11(2):187-93.

64. Van Gelder IC, Groenveld HF, Crijns HJ, et al. Lenient versus strict rate control in patients with atrial fibrillation. $N$ Engl J Med 2010;362(15):1363-73.

65. Shelton RJ, Clark AL, Goode K, et al. A randomised, controlled study of rate versus rhythm control in patients with chronic atrial fibrillation and heart failure: (CAFE-II Study). Heart (British Cardiac Society) 2009;95(11):92430.

66. Nedios S, Sommer P, Dagres $\mathrm{N}$, et al. Long-term follow-up after atrial fibrillation ablation in patients with impaired left ventricular systolic function: the importance of rhythm and rate control. Heart Rhythm 2014;11(3):344-51.

67. Hsu LF, Jais $P$, Sanders $P$, et al. Catheter ablation for atrial fibrillation in congestive heart failure. $N$ Engl J Med 2004;351(23):2373-83.

68. Di Biase L, Mohanty P, Mohanty S, et al. Ablation Versus Amiodarone for Treatment of Persistent Atrial Fibrillation in Patients With Congestive Heart Failure and an Implanted Device: Results From the AATAC Multicenter Randomized Trial. Circulation 2016;133(17):1637-44.

69. Sliwa K, Fett J, Elkayam U. Peripartum cardiomyopathy. Lancet (London, England) 2006;368(9536):687-93.

70. Sliwa K, Blauwet L, Tibazarwa K, et al. Evaluation of bromocriptine in the treatment of acute severe peripartum cardiomyopathy: a proof-ofconcept pilot study. Circulation 2010;121(13):1465-73.

71. Hilfiker-Kleiner D, Haghikia A, Berliner D, et al. Bromocriptine for the treatment of peripartum cardiomyopathy: a multicentre randomized study. Eur Heart J 2017;38(35):2671-79.

72. Sliwa K, Mebazaa A, Hilfiker-Kleiner D, et al. Clinical characteristics of patients from the worldwide registry on peripartum cardiomyopathy (PPCM): EURObservational Research Programme in conjunction with the Heart Failure Association of the European 
Society of Cardiology Study Group on PPCM. Eur J Heart Fail 2017;19(9):1131-41.

73. Tariq S, Aronow WS. Use of Inotropic Agents in Treatment of Systolic Heart Failure. Int J Mol Sci 2015;16(12):29060-8.

74. Cuffe MS, Califf RM, Adams KF, Jr., et al. Shortterm intravenous milrinone for acute exacerbation of chronic heart failure: a randomized controlled trial. JAMA 2002;287(12):1541-7.

75. Felker GM, Benza RL, Chandler AB, et al. Heart failure etiology and response to milrinone in decompensated heart failure: results from the OPTIME-CHF study. J Am Coll Cardiol 2003;41(6):997-1003.

76. Packer M, O'Connor CM, Ghali JK, et al. Effect of amlodipine on morbidity and mortality in severe chronic heart failure. Prospective Randomized Amlodipine Survival Evaluation Study Group. N Engl J Med 1996;335(15):1107-14.

77. Packer $\mathrm{M}$, Carson $\mathrm{P}$, Elkayam $\mathrm{U}$, et al. Effect of amlodipine on the survival of patients with severe chronic heart failure due to a nonischemic cardiomyopathy: results of the PRAISE-2 study (prospective randomized amlodipine survival evaluation 2). JACC Heart Fail 2013;1(4):308-14.

78. Teerlink JR, Felker GM, McMurray JJ, et al. Chronic Oral Study of Myosin Activation to Increase Contractility in Heart Failure (COSMIC$\mathrm{HF}$ ): a phase 2, pharmacokinetic, randomised, placebo-controlled trial. Lancet (London, England) 2016;388(10062):2895-903.

79. Teerlink JR, Felker GM, McMurray JJV, et al. Acute Treatment With Omecamtiv Mecarbil to Increase Contractility in Acute Heart Failure: The ATOMIC-AHF Study. J Am Coll Cardiol 2016;67(12):1444-55.

80. Utter MS, Ryba DM, Li BH, et al. Omecamtiv Mecarbil, a Cardiac Myosin Activator, Increases Ca2+ Sensitivity in Myofilaments With a Dilated Cardiomyopathy Mutant Tropomyosin E54K. J Cardiovasc Pharmacol 2015;66(4):347-53.

81. Vrtovec B. Cell Therapy for Nonischemic Cardiomyopathy: Current Status and Future Perspectives. Circulation research 2018;122(1):28-30.

82. Vrtovec B, Poglajen G, Lezaic L, et al. Effects of intracoronary CD34+ stem cell transplantation in nonischemic dilated cardiomyopathy patients: 5year follow-up. Circulation research 2013;112(1):165-73.

83. Joseph J, Rimawi A, Mehta P, et al. Safety and effectiveness of granulocyte-colony stimulating factor in mobilizing stem cells and improving cytokine profile in advanced chronic heart failure. Am J Cardiol 2006;97(5):681-4.
84. Hamshere $S$, Arnous $S$, Choudhury $T$, et al. Randomized trial of combination cytokine and adult autologous bone marrow progenitor cell administration in patients with non-ischaemic dilated cardiomyopathy: the REGENERATE-DCM clinical trial. Eur Heart J 2015;36(44):3061-9.

85. Heggermont WA, Papageorgiou AP, Heymans S, et al. Metabolic support for the heart: complementary therapy for heart failure? Eur J Heart Fail 2016;18(12):1420-29.

86. Tuunanen $H$, Engblom $E$, Naum $A$, et al. Trimetazidine, a metabolic modulator, has cardiac and extracardiac benefits in idiopathic dilated cardiomyopathy. Circulation 2008;118(12):1250-8.

87. Zhao $P$, Zhang J, Yin XG, et al. The effect of trimetazidine on cardiac function in diabetic patients with idiopathic dilated cardiomyopathy. Life Sci 2013;92(11):633-8.

88. Fan Q, Niu Z, Ma L. Meta-analysis of trimetazidine treatment for cardiomyopathy. Biosci Rep 2018;38(3)

89. Zhang $L, L u Y$, Jiang $H$, et al. Additional use of trimetazidine in patients with chronic heart failure: a meta-analysis. J Am Coll Cardiol 2012;59(10):913-22.

90. Schmidt-Schweda S, Holubarsch C. First clinical trial with etomoxir in patients with chronic congestive heart failure. Clin Sci (Lond) 2000;99(1):27-35.

91. Holubarsch CJ, Rohrbach M, Karrasch M, et al. A double-blind randomized multicentre clinical trial to evaluate the efficacy and safety of two doses of etomoxir in comparison with placebo in patients with moderate congestive heart failure: the ERGO (etomoxir for the recovery of glucose oxidation) study. Clin Sci (Lond) 2007;113(4):20512.

92. Lee L, Campbell R, Scheuermann-Freestone $M$, et al. Metabolic modulation with perhexiline in chronic heart failure: a randomized, controlled trial of short-term use of a novel treatment. Circulation 2005;112(21):3280-8.

93. Beadle RM, Williams LK, Kuehl $M$, et al. Improvement in cardiac energetics by perhexiline in heart failure due to dilated cardiomyopathy. JACC Heart Fail 2015;3(3):20211.

94. Lopez B, Gonzalez A, Ravassa S, et al. Circulating Biomarkers of Myocardial Fibrosis: The Need for a Reappraisal. J Am Coll Cardiol 2015;65(22):2449-56.

95. Heymans S, Gonzalez A, Pizard A, et al. Searching for new mechanisms of myocardial fibrosis with diagnostic and/or therapeutic potential. Eur J Heart Fail 2015;17(8):764-71. 
96. Izawa H, Murohara T, Nagata K, et al. Mineralocorticoid receptor antagonism ameliorates left ventricular diastolic dysfunction and myocardial fibrosis in mildly symptomatic patients with idiopathic dilated cardiomyopathy: a pilot study. Circulation 2005;112(19):2940-5.

97. Sharma UC, Pokharel S, van Brakel TJ, et al. Galectin-3 marks activated macrophages in failure-prone hypertrophied hearts and contributes to cardiac dysfunction. Circulation 2004;110(19):3121-8.

98. Pellman J, Zhang J, Sheikh F. Myocyte-fibroblast communication in cardiac fibrosis and arrhythmias: Mechanisms and model systems. J Mol Cell Cardiol 2016;94:22-31.

99. Zolk O, Ng LL, O'Brien RJ, et al. Augmented expression of cardiotrophin-1 in failing human hearts is accompanied by diminished glycoprotein 130 receptor protein abundance. Circulation 2002;106(12):1442-6.

100. Laeremans $H$, Hackeng TM, van Zandvoort MA, et al. Blocking of frizzled signaling with a homologous peptide fragment of wnt3a/wnt5a reduces infarct expansion and prevents the development of heart failure after myocardial infarction. Circulation 2011;124(15):1626-35.

101. Van Aelst LN, Voss S, Carai P, et al. Osteoglycin prevents cardiac dilatation and dysfunction after myocardial infarction through infarct collagen strengthening. Circulation research 2015;116(3):425-36.

102. Janssen HL, Reesink HW, Lawitz EJ, et al. Treatment of HCV infection by targeting microRNA. N Engl J Med 2013;368(18):1685-94.

103. Lopez B, Gonzalez A, Lindner D, et al. Osteopontin-mediated myocardial fibrosis in heart failure: a role for lysyl oxidase? Cardiovasc Res 2013;99(1):111-20.

104. Raghu G, Rochwerg B, Zhang Y, et al. An Official ATS/ERS/JRS/ALAT Clinical Practice Guideline: Treatment of Idiopathic Pulmonary Fibrosis. An Update of the 2011 Clinical Practice Guideline. Am J Respir Crit Care Med 2015;192(2):e3-19.

105. Kolb M, Bonella F, Wollin L. Therapeutic targets in idiopathic pulmonary fibrosis. Respir Med 2017;131:49-57.

106. Halliday BP, Wassall R, Lota AS, et al. Withdrawal of pharmacological treatment for heart failure in patients with recovered dilated cardiomyopathy (TRED-HF): an open-label, pilot, randomised trial. Lancet (London, England) 2018

107. Merken J, Brunner-La Rocca HP, Weerts J, et al. Heart Failure With Recovered Ejection Fraction. $J$ Am Coll Cardiol 2018;72(13):1557-58.

108. Wallukat G, Reinke P, Dorffel WV, et al. Removal of autoantibodies in dilated cardiomyopathy by immunoadsorption. Int J Cardiol 1996;54(2):1915.

109. Hare JM, DiFede DL, Rieger AC, et al. Randomized Comparison of Allogeneic Versus Autologous Mesenchymal Stem Cells for Nonischemic Dilated Cardiomyopathy: POSEIDON-DCM Trial. J Am Coll Cardiol 2017;69(5):526-37. 
- 298 - 


\section{Part III}

Screening of asymptomatic family members

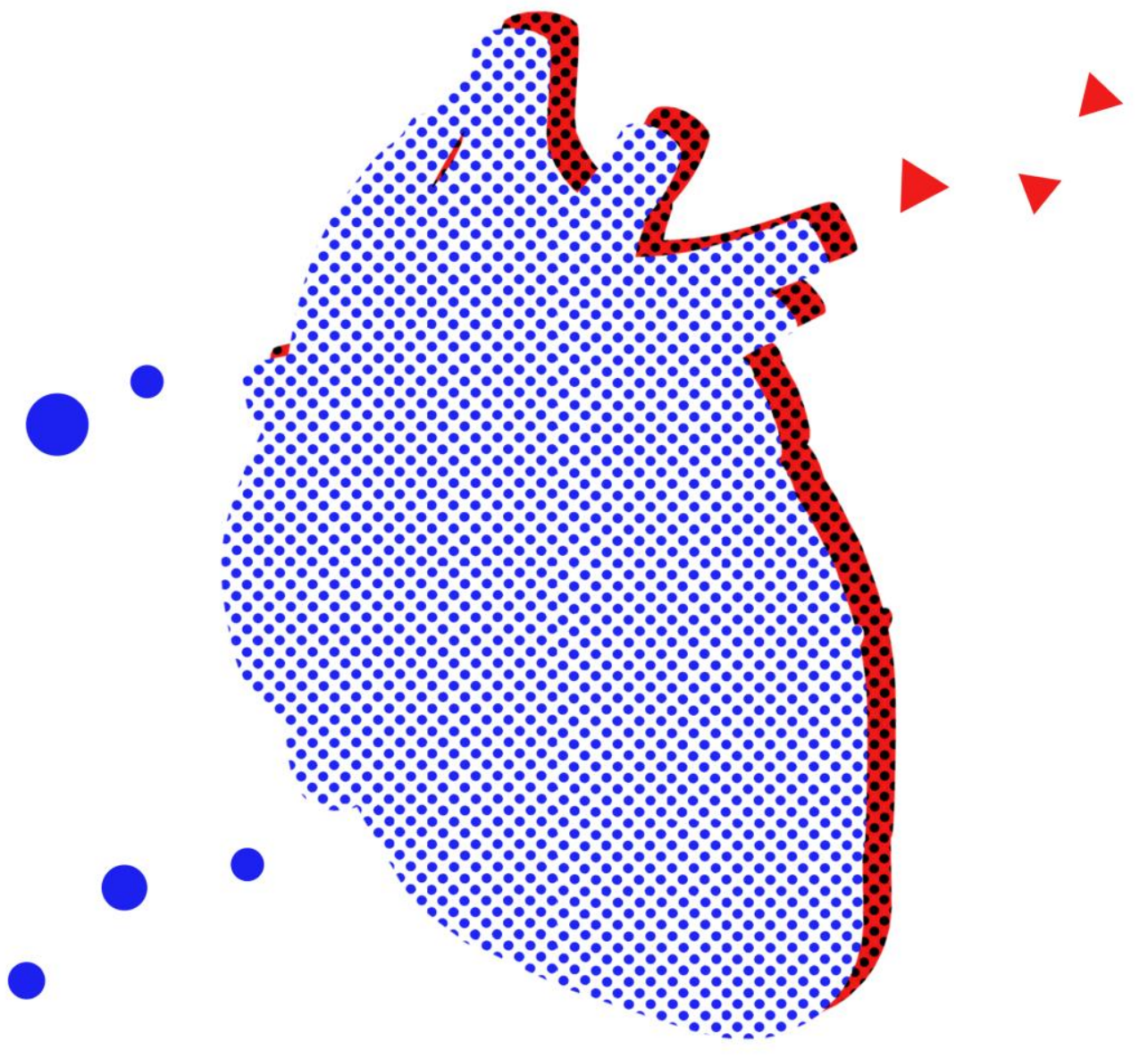




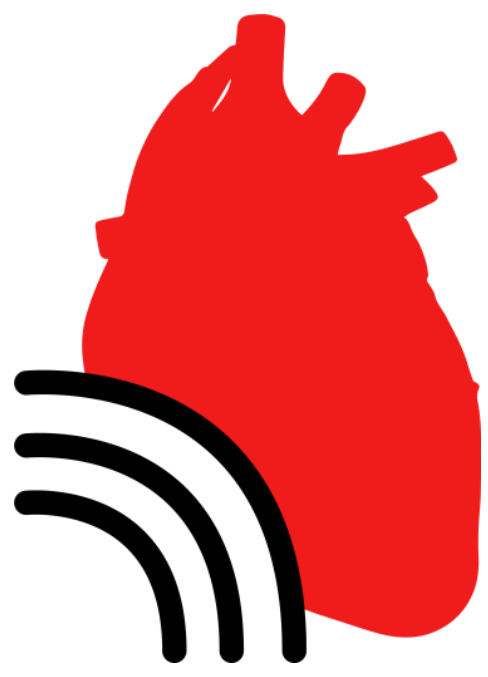

- 300 - 


\title{
CHAPTER 12
}

\section{Value of Speckle Tracking-Based Deformation Analysis in the Screening of Asymptomatic Dilated Cardiomyopathy Relatives}

\author{
Job A.J. Verdonschot, $M D, M S c^{1,2 *}$ \\ Jort J. Merken, $M D^{I^{*}}$ \\ Hans-Peter Brunner-La Rocca, $M D^{1}$ \\ Mark R. Hazebroek, $M D, P h D^{l}$ \\ Casper G.M.J. Eurlings, $M D^{l}$ \\ Eline Thijssen, $B S c^{l}$ \\ Ping Wang, $P h D^{2}$ \\ Jerremy Weerts, $B S c^{2}$ \\ Vanessa van Empel, $M D, P h D^{l}$ \\ Georg Schummers ${ }^{3}$ \\ Marcus Schreckenberg ${ }^{3}$ \\ Arthur van den Wijngaard, $P h D^{2}$ \\ Joost Lumens, $P h D^{4}$ \\ Han G. Brunner, $M D, P h D^{2,5,6}$ \\ Stephane R.B. Heymans, $M D, P h D^{1,7,8}$ \\ Ingrid P.C. Krapels, $M D, P h D^{2+}$ \\ Christian Knackstedt, $M D, P h D^{2,+}$
}

$*,+=$ Contributed equally

${ }^{1}$ Department of Cardiology, Cardiovascular Research Institute (CARIM),

Maastricht University Medical Center, Maastricht, The Netherlands.

${ }^{2}$ Department of Clinical Genetics, Maastricht University Medical Center, Maastricht, The Netherlands.

${ }^{3}$ TOMTEC Imaging Systems GmbG, Unterschleissheim, Germany.

${ }^{4}$ Department of Biomedical Engineering, Cardiovascular ResearchInstitute (CARIM),

Maastricht University Medical Center, Maastricht, The Netherlands.

${ }^{5}$ Radboud University Medical Center, Department of Human Genetics, Donders Institute for Brain, Cognition and Behaviour, Nijmegen

${ }^{6}$ GROW Institute for Developmental Biology and Cancer, Maastricht University Medical Centre, Maastricht, The Netherlands

${ }^{7}$ Department of Cardiovascular Sciences, Centre for Molecular and Vascular Biology,

KU Leuven, Belgium.

${ }^{8}$ The Netherlands Heart Institute, Nl-HI, Utrecht, The Netherlands. 


\begin{abstract}
Objectives: To investigate the prevalence of systolic dysfunction using global longitudinal strain (GLS) and its prognostic value in relatives of dilated cardiomyopathy (DCM) patients with normal left-ventricular ejection fraction (LVEF).
\end{abstract}

Background: DCM relatives are advised to undergo cardiac assessment including echocardiography, irrespective of the genetic status of the index patient. Even though LVEF is normal, the question remains whether this indicates absence of disease, or simply normal cardiac volumes. GLS may provide additional information regarding (sub)clinical cardiac abnormalities and thus allow earlier disease detection.

Methods: 251 DCM relatives and 251 control subjects with a normal LVEF ( $\geq 55 \%)$ were screened. Automated software measured the GLS on echocardiographic two-, three-, and four chamber views. The cut-off value for abnormal strain was $>-21.5$. Median follow-up was 40 months [5-80]. Primary outcome was the combination of death and cardiac hospitalization.

Results: 120 relatives and 83 controls showed abnormal GLS (48\% vs 33\%, respectively, $\mathrm{p}<0.001)$. Abnormal GLS was independently associated with DCM relatives and cardiovascular risk factors, rather than genetic mutations. Subjects with abnormal GLS had more frequent cardiac hospitalizations and a higher mortality as compared to subjects with normal GLS (HR 3.29 [1.58-6.87]; $p=0.001$ ). Additionally, follow-up LVEF was measured in a subset of relatives, which decreased significantly in those with abnormal as compared to normal GLS $(p=0.006)$.

Conclusions: Relatives from DCM patients had a significantly higher prevalence of systolic dysfunction detected by GLS despite normal LVEF compared to control subjects, independent of age, gender, comorbidities and genotype. Abnormal GLS was associated with LVEF deterioration, cardiac hospitalization and death. 


\section{INTRODUCTION}

'Idiopathic' dilated cardiomyopathy (DCM) is a common cause of heart failure (HF) in young adult patients and is frequently seen at the outpatient clinic ${ }^{1}$. Adequate diagnosis is not only important for the patients but also for their family as all relatives of DCM patients are at risk of developing HF. Importantly, this is irrespective of the particular cause or a proven genetic mutation in the index patient due to familial susceptibility ${ }^{2}$. Although often no causal genetic mutations are found in DCM families, frequent cardiac screening is advised for all first-degree relatives by the latest position papers of the American Heart Association (AHA) and European Society of Cardiology (ESC) in order to detect cardiac abnormalities such as left ventricular (LV) enlargement or reduced LV ejection fraction (LVEF) ${ }^{3-6}$. This constitutes a substantial proportion of patients seen by the general cardiologist, given the estimated prevalence of DCM of 1:250-1:500 and an average of 4-5 first-degree relatives in Western countries ${ }^{7}$. Also, relatives are of different age categories and cardiovascular risk factors accumulate with increasing age, potentially influencing cardiac function irrespective of family history.

Of course, readily identification and treatment of disease is warranted in this group of individuals to prevent disease progression and occurrence of adverse cardiac events e.g. sudden cardiac death. However, subtle functional or structural changes of the myocardium are likely to be missed when using classical echocardiographic parameters such as LVEF, as ventricular volumes may be preserved or subtle changes of LVEF remain concealed due to measurement noise in early stages of cardiac disease ${ }^{8}$. Global longitudinal strain (GLS) seems to be able to detect subtle changes preceding deterioration of LVEF in different $\mathrm{HF}$ cohorts ${ }^{8}$. Therefore, this study evaluates the role of GLS in asymptomatic DCM relatives with normal LVEF to identify subtle cardiac abnormalities and associate those findings to distinctive clinical parameters, genotype and prognosis. The findings are compared to control subjects with normal LVEF and similar age, gender and comorbidity distribution, to be able to investigate the true family effect in DCM relatives.

\section{METHODS}

\section{Study subjects}

DCM relatives - This retrospective study was carried out in a population of relatives of genotyped DCM patients who were seen for genetic analysis at the Maastricht University Medical Centre (MUMC+) between 2009 and 2017. Initially, pedigree analysis and sequencing of a 47 cardiomyopathy-associated gene panel was performed in all index patients to determine genetic status (Suppl. Table 1). Genetic counseling, testing and analysis was performed previously as described ${ }^{9}$. Genetic DCM was defined as a confirmed class 4 or 5 pathogenic mutation; a list of all pathogenic mutations can be found in Supplemental Table 2.

All index patients were counseled to inform their first-degree relatives about their cardiac disease and the necessity for cardiac screening regardless of genetic status. Pedigrees of 607 unrelated DCM index patients were retrospectively analyzed. In total, 333 relatives from 158 different families were seen in our hospital for cardiac screening, which consisted of medical history, physical examination, ECG and echocardiography. Only first-degree relatives with a completely normal $\operatorname{LVEF}(\geq 55 \%)$ were included for analysis ${ }^{10}$ (Figure 1). The study was performed according to the declaration of Helsinki and was approved by the Medical Ethics Committee of Maastricht University Medical Centre (METC 16-4-222). 
Control subjects - Control subjects were selected from the Outpatient Cardiology Clinic Registry (CVC database) from MUMC including patients referred due to chest pain, dyspnea or palpitations between April 2006 and February 2008. Cardiac screening included physical examination, ECG, and echocardiography. A total of 1610 out of 2110 patients demonstrated normal LVEF ( $\geq 55 \%)$ on echocardiography. Control patients were randomly selected from these 1610 patients. A cohort of 251 control subjects was selected from the outpatient database with similar age, gender and comorbidity distribution (hypertension, diabetes, atrial fibrillation, coronary artery disease).

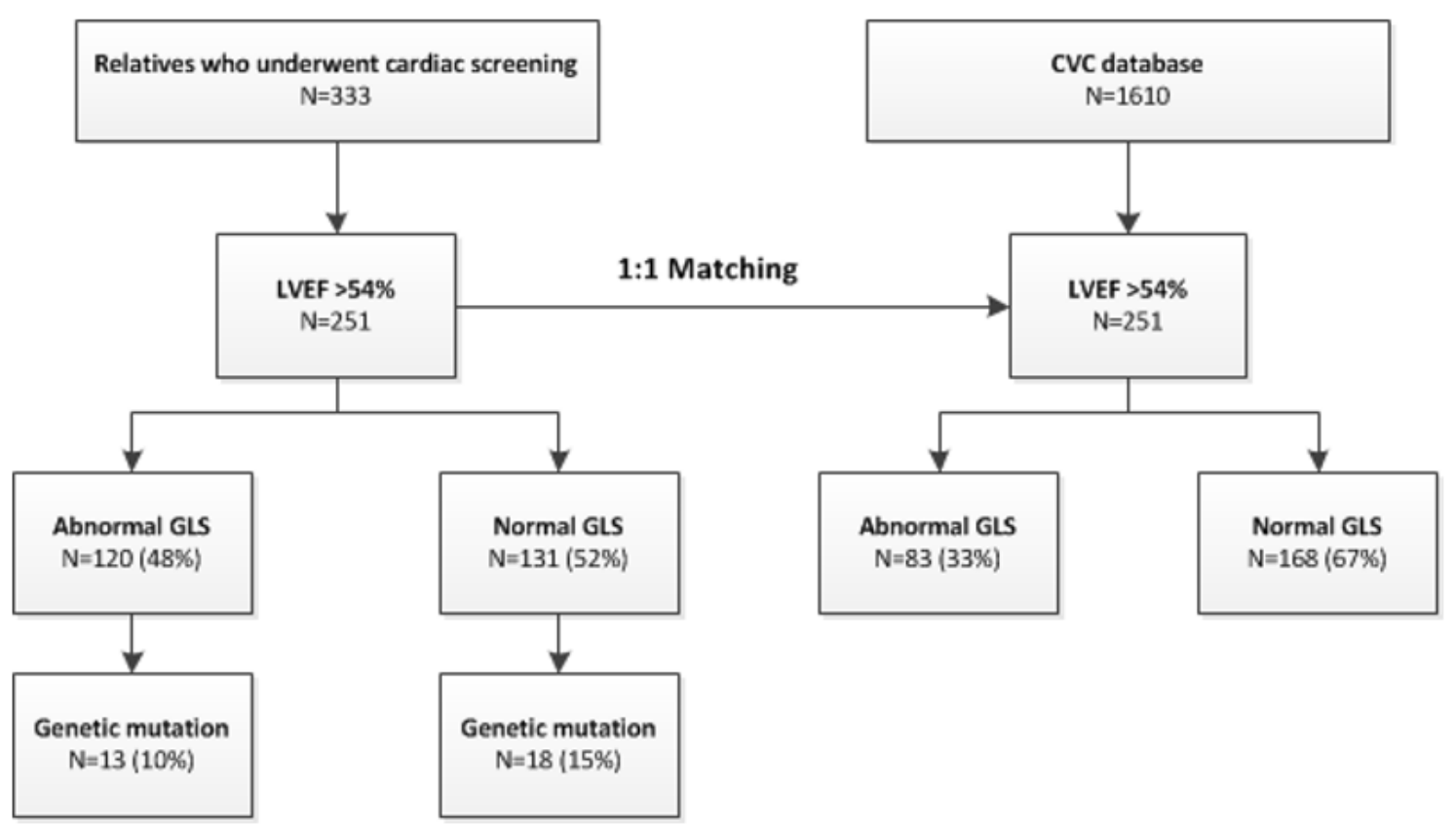

Figure 1. Flowchart of patient selection. Echocardiograms of 333 relatives were available at our center. Eventually, 251 patients had a normal $\operatorname{LVEF}(\geq 55 \%)$ and an echocardiography, which was suitable for analysis of GLS. These 251 relatives had no significant differences in baseline characteristics compared with a control cohort of 251 control subjects.

\section{Echocardiographic protocol}

All echocardiographic images were made during clinical routine using a Philips IE33 ultrasound system obtaining standard parasternal, apical, and subcostal views according to current recommendations ${ }^{10}$. Standard measurements were retrieved from the clinical echocardiographic database including cardiac dimensions, LVEF using Simpson's, and presence of any relevant valve disease ${ }^{11}$. Still, a minority of patients only had evaluation of LVEF by Teichholz method ( $n=67,13.3 \%)$.

In addition to the existing measurements, analysis of LV function with speckle tracking based GLS was performed, applying a dedicated software package (AutoSTRAIN, TOMTECARENA* 1.2, TOMTEC Imaging Systems GmbH, Unterschleissheim, Germany) with a recently published algorithm ${ }^{12}$. Briefly, apical 2-, 3- and 4- chamber views of all patients are uploaded onto a computer. Three experienced physicians reviewed all echocardiograms for image quality and completeness (CK, JM, JV). Incomplete echocardiographs or studies of insufficient quality were not taken into consideration for analysis. The user is requested to indicate the correct loops to the software. Regional and global longitudinal peak systolic strain is calculated applying a contour detection algorithm. Furthermore, the contours suggested by 
the automated software were revised and corrected if felt necessary by four independent investigators (CK, JM, JV, JW). For this analysis, we used a vendor specific cut-off value of 21.5 to define abnormal GLS as previously described ${ }^{13}$.

\section{Follow-up}

Minimum follow-up duration after the first outpatient visit was 1 month, up to 8 years. Total median follow-up of the whole cohort was 40 months (Interquartile range [5-80]). Follow-up data on death and cardiac hospitalization were collected using medical records. The primary outcome endpoint was the combination of death and cardiac hospitalization.

In addition to baseline measurement, 69 DCM relatives (27.5\%) with a normal LVEF at baseline underwent echocardiography during follow-up. LVEF deterioration was defined as a LVEF $<55 \%$ with a minimal decrease of $5 \%$ at follow-up.

\section{Statistical analysis}

Data are presented as frequencies, mean \pm SD or median (interquartile range). Comparisons between groups were performed using chi-square tests for categorical data and t-test or Mann-Whitney test for continuous data, as appropriate. Kruskall-Wallis test was used to analyze to compare continuous data among three groups.

Uni- and multivariable regression analysis with generalized estimating equations (GEE) approach in a binomial model was performed to test the association between (clinical) parameters and abnormal GLS in the total cohort $(n=502)$. A GEE approach was performed with an exchangeable correlation structure to adjust for correlated observations within families, as there are large families with multiple first-degree relatives included in the DCM relatives cohort. For multivariable analysis, we first included all parameters with a cut-off for entry of $p<0.1$ in the univariable analysis. The final multivariable model was created by backward elimination with a cut-off of $p>0.05$.

To assess which clinical variables influence the progression of LVEF in a longitudinal fashion, univariate analysis was performed using generalized estimating equations (GEE) in a subset of study cohort with multiple echoes $(n=69)$. Univariable Cox proportional hazards regression analysis was performed to assess clinical and demographic covariates associated event-free survival. The level of significance was $p<0.05$ and tests were 2-sided. Calculations were done using SPSS version 23.0, and R environment version 3.5.

\section{RESULTS}

Baseline characteristics of the study and control population did not show significant differences (Table 1 and 2). One hundred and twenty DCM relatives (48\%) compared to 83 healthy controls (33\%) had an abnormal GLS despite having a normal LVEF and volumetric parameters ( $p<0.001$; Figure 1; Suppl. Table 2).

\section{Genetic status of DCM relatives}

Forty-one (26\%) of the 158 index DCM patients, used for the selection of DCM relatives, had a genetic mutation with predominantly TTN and LMNA mutations (Suppl. Table 3). In total, $92(28 \%)$ of the 333 relatives who underwent cardiac screening were related to these 41 DCM index patients with a genetic mutation (Suppl. Figure 1). Thus, the majority of the DCM relatives who underwent cardiac screening are related to DCM index patients without a proven genetic mutation, reflecting typical daily practice. DCM relatives with LVEF $<55 \%$ at baseline were excluded, showing no significant difference in patient selection between DCM 
relatives related to mutation-positive as compared to mutation-negative DCM index patients (30\% vs $21 \%$; $p=0.07$, Suppl. Figure 1 ). In total, 44 DCM relatives of mutation positive DCM index patients underwent genetic testing: 32 carriers and 12 without the familial mutation (Suppl. Table 3).

\section{GLS difference between DCM relatives and control subjects}

The absolute GLS value in DCM relatives was worse compared to the control population (21.6 [19-24] vs -23.1 [21-26], respectively; $p<0.001$; Figure 2A, Table 2). Importantly, DCM relatives with a genetic mutation had a significant worse absolute GLS value as compared to relatives without a genetic mutation and control subjects $(-20.1$ [18-23] vs -21.7 [20-24] vs 23.1 [21-26], respectively; Kruskall-Wallis $\mathrm{p}<0.001$; Figure 2B).

\section{Clinical parameters influencing GLS}

All clinical factors associated with abnormal GLS in univariable analysis are depicted in Table 3. Importantly, in the multivariable regression analysis with GEE approach, male gender, increased age, chemotherapy, CAD, chronic obstructive pulmonary disease (COPD) and status as DCM relative remained independent factors associated with abnormal GLS (Table 3). Interestingly, a DCM relative had a two-fold increased risk of an abnormal GLS compared to the non-related control subjects after correcting for gender, age and comorbidities (OR 2.25 (1.51-3.35); $p<0.001 ;$ Central Illustration).

Table 1. Baseline characteristics of the DCM relatives and controls

\begin{tabular}{|c|c|c|c|}
\hline Baseline variables & DCM relatives $(n=251)$ & Control $(n=251)$ & p-value \\
\hline Male gender, n (\%) & $113(45)$ & $129(51)$ & 0.15 \\
\hline Age (years) & $46 \pm 17$ & $46 \pm 13$ & 0.65 \\
\hline Body Mass Index (BMI) & $26 \pm 4$ & $26 \pm 5$ & 0.7 \\
\hline NYHA III-IV, n (\%) & $8(3)$ & $16(6)$ & 0.09 \\
\hline cLBBB & $6(2)$ & $15(6)$ & 0.07 \\
\hline Chemotherapy & $7(3)$ & $16(6)$ & 0.13 \\
\hline \multicolumn{4}{|l|}{ Genetic Status, n (\%) } \\
\hline Familial DCM & $126(50)$ & $0(0)$ & $<0.001$ \\
\hline Relative of index with mutation & $61(24)$ & $0(0)$ & $<0.001$ \\
\hline Familial gene mutation present & $31(12)$ & $0(0)$ & $<0.001$ \\
\hline \multicolumn{4}{|l|}{ Cardiovascular History, n (\%) } \\
\hline Coronary artery disease & $14(6)$ & $7(3)$ & 0.11 \\
\hline Stroke & $6(2)$ & $2(1)$ & 0.17 \\
\hline CABG & $3(1)$ & $0(0)$ & 0.12 \\
\hline $\mathrm{PCl}$ & $6(2)$ & $5(2)$ & 0.74 \\
\hline Valvular disease & $42(17)$ & $49(20)$ & 0.42 \\
\hline \multicolumn{4}{|l|}{ Comorbidities, n (\%) } \\
\hline Atrial fibrillation & $2(1)$ & $4(2)$ & 0.69 \\
\hline Hypertension & $65(26)$ & $57(23)$ & 0.24 \\
\hline COPD & $10(4)$ & $13(5)$ & 0.56 \\
\hline Hypercholesterolemia & $30(12)$ & $44(18)$ & 0.13 \\
\hline Diabetes mellitus & $21(8)$ & $15(6)$ & 0.25 \\
\hline \multicolumn{4}{|l|}{ Medication, n (\%) } \\
\hline Beta-blocker & $29(12)$ & $45(18)$ & 0.33 \\
\hline ACE-i & $17(7)$ & $19(8)$ & 0.73 \\
\hline ARB & $18(7)$ & $24(10)$ & 0.84 \\
\hline MRA & $2(1)$ & $1(0)$ & 0.59 \\
\hline Diuretics & $17(7)$ & $13(5)$ & 0.16 \\
\hline Calcium antagonists & $11(4)$ & 22 (9) & 0.19 \\
\hline
\end{tabular}


Abbreviations: $A C E-I$, angiotensin converting enzyme-inhibitor; $A R B$, angiotensin II receptor blocker; CABG, coronary artery bypass graft; CLBBB, complete left bundle branch block; COPD, chronic obstructive pulmonary disease; DCM, dilated cardiomyopathy; MRA. Mineralocorticoid receptor antagonist; $\mathrm{PCl}$, percutaneous coronary intervention.

\section{Abnormal GLS is associated with LVEF deterioration in DCM relatives}

Follow-up echocardiograms were available in 69 DCM relatives (26 normal GLS; 43 abnormal GLS; Suppl. Table 4). Relatives with a follow-up echo were more often related to an index patient with a genetic mutation or were carrier of a genetic mutation themselves. Also, coronary artery and valvular disease were more prevalent in these relatives with a follow-up echo (Suppl. Table 5 and 6).

There was a median time span of 36 [24-63] months between baseline and follow-up echocardiograms; which did not differ between the two groups (DCM relatives with normal versus abnormal GLS) ( $p=0.99)$. Patients with an abnormal GLS showed a significant decrease in LVEF over time $(60 \pm 0.6$ to $56 \pm 1.1 ; p=0.006)$ in contrast to patients with a normal GLS at baseline ( $61 \pm 0.7$ to $60 \pm 0.9 ; p=0.22$; Figure 3A). Moreover, relatives with an abnormal GLS had more frequently an abnormal LVEF $(<55 \%)$ at follow-up compared to relatives with a normal GLS (17 (40\%) versus 3 (12\%); $p=0.013$, respectively; Figure 3B). An abnormal GLS at baseline was associated with a LVEF decrease over time using GEE analysis (-2.71\%, 95\% Cl: -4.4 - 1.03; $p=0.002$; Table 4).

A Absolute Global Longitudinal Strain

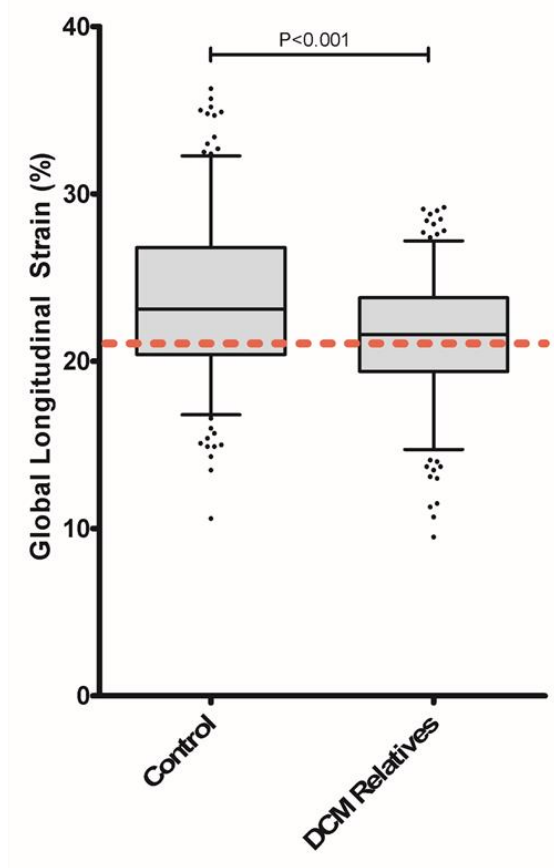

B

Absolute Global Longitudinal Strain values and genetic status

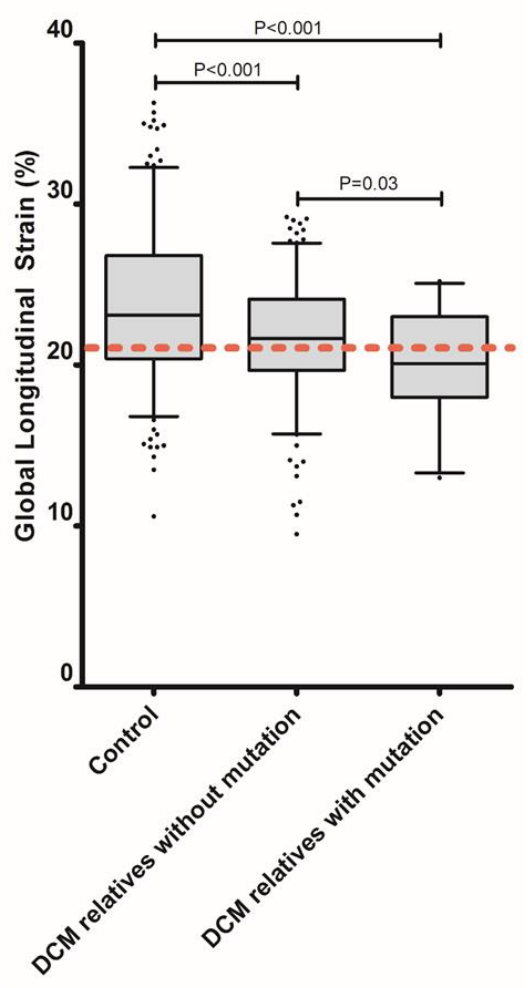

Figure 2. Absolute GLS values in controls and DCM relatives. Control subjects have a lower absolute GLS compared to DCM relatives (A) with or without a genetic mutation (B). Boxes represent the median and interquartile range, whiskers are 5-95\% range, and dots represent the outliers. The dotted red line is the used GLS cut-off value of -21.5. P-values indicate results of Mann-Whitney tests, Kruskall-Wallis test for comparing GLS among all three groups: $p<0.001$. 


\section{Detrimental long-term prognosis in DCM relatives with an abnormal GLS at baseline}

An abnormal GLS at baseline resulted in a worse event-free survival as compared to subjects with a normal GLS in DCM relatives (HR 3.37 [1.11-10.2]; $p=0.03$; Table 5). In addition to an abnormal GLS, age, hypercholesterolemia, CAD, hypertension, NYHA III or IV and chemotherapy in the previous history were all associated with a worse event-free survival in DCM relatives.

Abnormal GLS was also associated with worse outcome in the whole study group $(n=502)$ including DCM relatives and controls (HR 3.29 [1.58-6.87]; log rank $p=0.001 ;$ Table 5, Figure 4), even after correcting for age (HR 2.51 [1.18-5.33]; $p=0.017)$. In total, 17 (8\%) subjects with an abnormal baseline GLS were hospitalized for cardiac reasons, which was significantly more frequent compared to $8(3 \%)$ cardiac hospitalizations among subjects with a normal GLS at baseline (HR 2.84 [1.2-6.6]; $p=0.015$; Suppl. Table 7). During follow-up, a total of 11 cardiovascular deaths occurred, of which 9 in the subjects with an abnormal GLS (HR 5.6 [1.2-26.2]; $p=0.027$ ). There were no significant differences in events between the control subjects and DCM relatives (Suppl. Table 8 ).

\section{DISCUSSION}

This is the largest study investigating the use of GLS to detect early disease stage in relatives of DCM patients with a normal LVEF in comparison to individuals without a familial background of DCM. Our most important findings are that (1) abnormal GLS is more common in DCM relatives as compared to controls, (2) abnormal GLS is mainly influenced by classical cardiovascular risk factors and family history of DCM instead of a proven genetic mutation, and (3) abnormal GLS is a predictor of LVEF deterioration, cardiac hospitalization and death. Overall, abnormal GLS seems to reflect systolic dysfunction despite normal LVEF and could be of additional value for the physician in advising DCM relatives regarding cardiac screening frequency.

Table 2. Echocardiographic baseline parameters of the DCM relatives and control subjects

\begin{tabular}{cccc}
\hline Baseline variables & $\begin{array}{c}\text { DCM relatives } \\
(\mathbf{n = 2 5 1 )}\end{array}$ & Control (n=251) & p-value \\
\hline Systolic function & & & \\
LVEF (\%) & $61 \pm 3.8$ & $61 \pm 3.9$ & 0.23 \\
LVEDD (mm) & $49 \pm 4.9$ & $49 \pm 4.7$ & 0.15 \\
LVESD (mm) & $33 \pm 3.6$ & $33 \pm 3.7$ & 0.06 \\
IVS (mm) & $8 \pm 1.8$ & $8 \pm 1.2$ & 0.36 \\
PWT (mm) & $8 \pm 1.3$ & $8 \pm 1.1$ & 0.1 \\
LA volume index (ml/m $\left.{ }^{2}\right)$ & $33 \pm 10.6$ & $31 \pm 8.5$ & 0.49 \\
GLS (\%) & $-21.6[-19--24]$ & $-23.1[-21--26]$ & $<0.001$ \\
Abnormal GLS, $\mathrm{n}(\%)$ & $120(48)$ & $83(33)$ & 0.001 \\
Diastolic function & & & \\
E/A ratio & $1.2 \pm 0.4$ & $1.3 \pm 0.4$ & 0.07 \\
TI velocity (cm/sec) & $2.2 \pm 0.3$ & $2.3 \pm 0.4$ & 0.1 \\
\hline
\end{tabular}

Abbreviations: GLS, global longitudinal strain; IVS, inter ventricular septum; LA, left atrium; LVEDD, left ventricular end-diastolic diameter; $L V E F$, left ventricular ejection fraction; LVESD, left ventricular end-systolic diameter; PWT, posterior wall thickness. 
Table 3. Significant abnormal GLS associated baseline variables at univariable and multivariable regression analysis with generalized estimating equations (GEE) approach in the whole cohort $(n=502)$

\begin{tabular}{ccccccc} 
& \multicolumn{3}{c}{ Univariable analysis } & \multicolumn{3}{c}{ Multivariable analysis } \\
Baseline variables & OR & $\mathbf{9 5 \%} \mathbf{~ C l}$ & P Value & OR & 95\% Cl & P-value \\
\hline DCM relative & 1.86 & $1.31-2.67$ & $<0.001$ & 2.25 & $1.51-3.35$ & $<0.001$ \\
Female gender & 0.44 & $0.30-0.63$ & $<0.001$ & 0.39 & $0.26-0.58$ & $<0.001$ \\
Age (years) & 1.04 & $1.02-1.05$ & $<0.001$ & 1.03 & $1.02-1.05$ & $<0.001$ \\
Body mass index $\left(\mathrm{kg} / \mathrm{m}^{2}\right)$ & 1.07 & $1.03-1.12$ & $<0.001$ & --- & --- & --- \\
Hypertension & 2.89 & $1.91-4.37$ & $<0.001$ & --- & --- & --- \\
Coronary artery disease & 6.72 & $2.40-18.78$ & $<0.001$ & 3.47 & $1.33-9.05$ & 0.011 \\
COPD & 2.92 & $1.21-7.02$ & 0.02 & 3.40 & $1.38-8.38$ & 0.008 \\
Hypercholesterolemia & 1.95 & $1.20-3.17$ & 0.007 & --- & --- & --- \\
Diabetes mellitus & 3.66 & $1.81-7.37$ & $<0.001$ & --- & --- & --- \\
Chemotherapy & 2.94 & $1.26-6.87$ & 0.013 & 2.88 & $1.10-7.55$ & 0.032 \\
\hline
\end{tabular}

Abbreviations: $O R=$ Odds Ratio; $C l$ = Confidence Interval; $D C M$ = Dilated Cardiomyopathy; $C O P D=$ Chronic Obstructive Pulmonary Disease

\section{Clinical and genetic factors influencing GLS in DCM relatives}

Common cardiovascular risk factors such as CAD and influence the myocardial tissue, subsequently reflected by an abnormal GLS ${ }^{14}{ }^{15}$. This observation is in line with our data, showing an independent association of gender, age and CAD with abnormal GLS. The Norwegian HUNT study showed a similar influence of gender and age on the GLS values in a healthy population of 1266 individuals ${ }^{14}$. In general, abnormal GLS is more common in men with increasing age. A novel addition of the present study is the comparison between DCM relatives and controls showing the influence of being related to a DCM patient in addition to clinical factors. This indicates a certain genetic susceptibility in DCM relatives, which is likely to be more complex than the current monogenetic dogma.

Table 4. Association between baseline parameters and LVEF in univariate models for the subset of DCM relatives with follow-up echoes in GEE models ( $n=69)$.

\begin{tabular}{cccc} 
& \multicolumn{3}{c}{ Univariable analysis } \\
Baseline variables & $\boldsymbol{\beta}$ & $\mathbf{9 5 \%} \mathbf{~ C l}$ & P Value \\
\hline Abnormal GLS & -2.71 & $-4.4--1.03$ & 0.002 \\
Coronary artery disease & -4.54 & $-7.12--1.95$ & $<0.001$ \\
Diabetes mellitus & -5.17 & $-7.81--2.53$ & $<0.001$ \\
\hline
\end{tabular}

Abbreviations: $\mathrm{Cl}=$ Confidence Interval; $\mathrm{GLS}=$ Global Longitudinal Strain.

The $B$ reflects the change in \% LVEF.

We found 31 DCM relatives with a genetic mutation, but no strong correlation with GLS after multivariable correction, although DCM relatives with a genetic mutation had a significant worse absolute GLS value. A decrease in absolute GLS was previously shown in a cohort of relatives with sarcomeric mutations (MYH7, TPM1 and TNNT2), indicating early subtle abnormalities in myocardial function in gene mutation carriers ${ }^{16}$. Similar results are recently shown in mutation-positive DCM relatives ${ }^{17}$. However, those previous studies mainly focused on DCM relatives with a proven genetic mutation. As only $\sim 20-25 \%$ of DCM patients have a proven genetic mutation after extensive genetic screening, these relatives constitute only a minority of the subjects referred for cardiac screening ${ }^{18}$. Therefore, our study mirrors clinical practice and adds significant insight into the potential clinical relevance of abnormal GLS in subjects referred for cardiac screening, i.e. DCM relatives with normal LVEF, irrespective of proven genetic mutations, highlighting the need for cardiac screening in all first-degree DCM relatives. This is further exemplified by another small study showing 
reduced absolute GLS values in a cohort of first-degree DCM relatives when comparing to controls ${ }^{19}$.
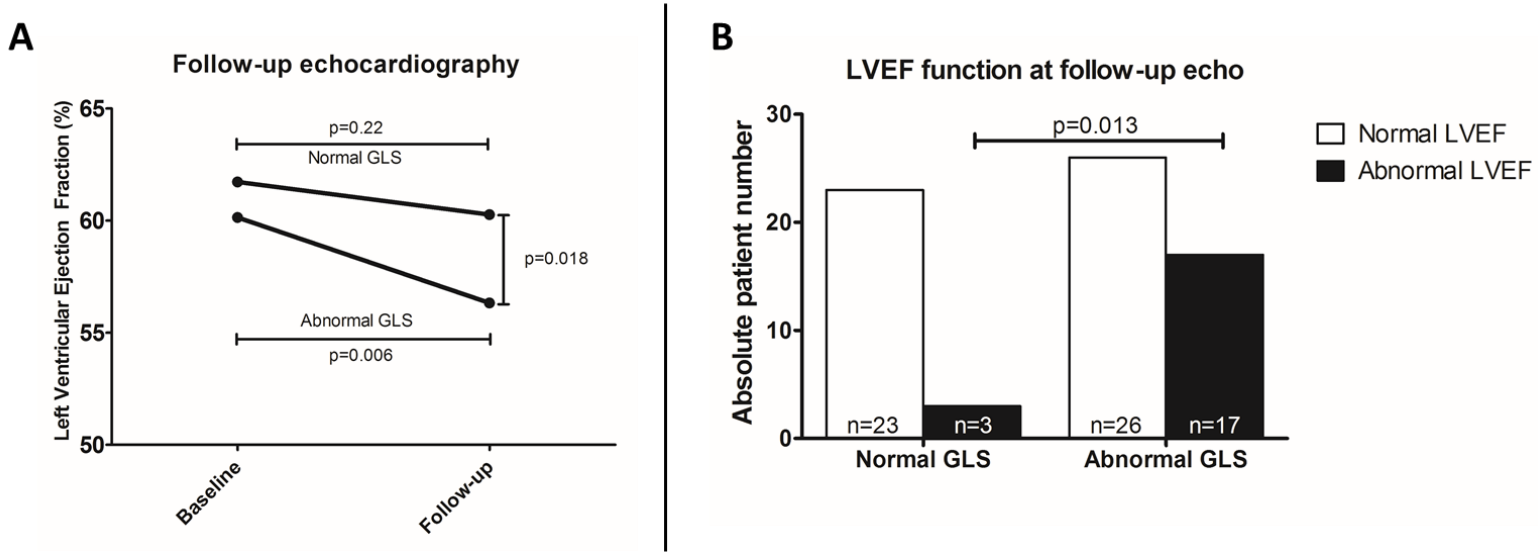

Figure 3. LVEF at follow-up echocardiography in relation to baseline GLS in DCM relatives ( $n=69)$. Relatives with an abnormal GLS at baseline have a significantly lower LVEF at follow-up compared to relatives with a normal GLS at baseline (A). Also, more patients have an abnormal LVEF $(<55 \%)$ at follow-up in the group of abnormal GLS at baseline (B).

\section{Prognostic value of GLS}

Our study indicates that abnormal GLS seems to be a predictor of LVEF deterioration, cardiac hospitalization and death in DCM relatives. GLS as a predictor of LVEF deterioration in DCM relatives builds upon knowledge of other forms of HF. An abnormal GLS is the most important marker for LVEF deterioration in HF patients with a recovered LVEF whereas a normal GLS predicted stable LVEF during follow-up ${ }^{20}$. Also, an abnormal GLS is a strong predictor for LVEF deterioration in patients receiving cardiotoxic chemotherapy ${ }^{21}$. Our study adds another important population in which GLS may be an appropriate tool for early disease detection ${ }^{22}$.

To date, studies using the prognostic value of GLS in DCM relatives are lacking. We found that abnormal GLS is associated with a higher risk for cardiac hospitalization and death at follow-up both in this group and in control subjects with normal LVEF. The latter is in line with previous findings. Thus, a Norwegian population-based study with healthy subjects without cardiovascular disease or diabetes showed independent prognostic value of GLS on long-term risk of cardiovascular morbidity and mortality with a median follow-up of 11 years 23. Furthermore, there is strong evidence of the prognostic value of GLS, which appears to have superior prognostic value to LVEF in predicting death and HF hospitalization in patients with different forms of HF ${ }^{24-26}$. Prognostic data of GLS specifically in DCM patients is mainly available from cardiac magnetic resonance (CMR)-derived feature tracking GLS, which shows to be an independent predictor of mortality ${ }^{2728}$. Data of echo-derived GLS prognostic value in DCM is scarce, with only one study showing GLS to be a predictor for ventricular arrhythmias ${ }^{29}$. Our study shows the prognostic value of abnormal GLS also in otherwise healthy relatives of DCM patients with normal EF.

\section{Potential clinical implications}

Identifying and treating DCM relatives, who are at-risk of developing DCM, at an early stage might minimize the risk of cardiac deterioration, hospitalization and death ${ }^{30}$. Current guidelines stratify patients in definite, probable, or possible disease based on the genetic and clinical status ${ }^{18}$. However, the genetic status is not always known, and echocardiographic screening is often normal using the classical parameters. When genetic information is not 
available, current ESC position statement advise systematic cardiac screening every 2-5 years until the age of 60-65 years in first-degree relatives of DCM patients ${ }^{4}$. The AHA guidelines are less stringent, where the treating physician can decide to perform periodic echocardiographic screening ${ }^{6}$. When an asymptomatic relative carries a pathogenic mutation, repeated cardiac evaluation should be performed every 1-3 years according to the ESC guidelines and every 35 years according to the AHA guidelines ${ }^{6}$. As reflected by this lack of consensus between the European and American guidelines, the level of evidence regarding echocardiographic screening of DCM relatives and determining their subsequent follow-up periods is low. Based on our findings, classical echocardiographic measurements could be enriched by determination of GLS in all DCM relatives. Furthermore, cardiac follow-up could be extended with a longer interval when GLS is normal. Although there is accumulating evidence for GLS as a marker of subtle early disease, clinical decision-making based on GLS should be further determined in a prospective manner to evaluate its usefulness in predicting cardiac deterioration or onset of disease. When investigating the clinical value of GLS, it also needs to be considered if starting medical treatment early (i.e. ACE-inhibitors) in these relatives with abnormal GLS may improve outcome.

Table 5. Significant baseline parameters associated with long-term outcome at follow-up using univariable cox regression analysis in DCM relatives and control subjects.

\begin{tabular}{|c|c|c|c|c|c|c|}
\hline \multirow[b]{2}{*}{ Baseline variables } & \multicolumn{3}{|c|}{$\frac{\text { Long-term outcome }}{D C M \text { relatives }(n=251)}$} & \multicolumn{3}{|c|}{$\frac{\text { Long-term outcome }}{\text { Total cohort }(n=502)}$} \\
\hline & HR & $95 \% \mathrm{Cl}$ & P Value & HR & $95 \% \mathrm{Cl}$ & P Value \\
\hline Abnormal GLS & 3.37 & $1.11-10.2$ & 0.03 & 3.29 & $1.58-6.87$ & 0.001 \\
\hline Age (years) & 1.07 & 1.03-1.11 & $<0.001$ & 1.05 & $1.02-1.07$ & $<0.001$ \\
\hline Hypercholesterolemia & 3.36 & $1.26-8.99$ & 0.02 & --- & --- & --- \\
\hline Coronary artery disease & 10.54 & $4.06-27.3$ & $<0.001$ & 5.83 & $2.64-12.8$ & $<0.001$ \\
\hline Diabetes mellitus & --- & --- & --- & 2.62 & $1.14-6.01$ & 0.02 \\
\hline Hypertension & 5.08 & $1.91-13.5$ & 0.001 & 2.62 & $1.35-5.09$ & 0.004 \\
\hline NYHA III-IV & 6.05 & $1.68-21.79$ & 0.006 & 2.95 & $1.14-7.61$ & 0.03 \\
\hline Chemotherapy & 5.75 & $1.59-20.8$ & 0.008 & 3.48 & $1.43-8.46$ & 0.006 \\
\hline
\end{tabular}

Abbreviations: $H R=$ Hazard Ratio; $\mathrm{Cl}=$ Confidence Interval; $G L S=$ Global Longitudinal Strain; NYHA = New York Heart Association classification.

\section{Limitations}

This study represents a single-center, retrospective data analysis. A prospective multi-center study will be needed to investigate if clinical decision-making based on GLS is safe and more cost-effective compared to the current recommendations. We were unable to include all relatives from all families due to the voluntary character of the screening. Also, indications for follow-up echocardiography was depending on clinical background, current guidelines and treating cardiologist; therefore, a certain selection bias cannot be excluded. This indicates that many relatives of DCM patients do not undergo cardiac screening, for many reasons. Due to the relatively low event rate and available follow-up echocardiograms, the current study had insufficient power to perform multivariable modeling to test for independent predictors of LVEF deterioration and long-term outcome. Therefore, these results should be interpreted with caution. The number of asymptomatic unique mutation carriers was relatively low in our population, leading to a genetically heterogeneous group. Also, a 47-gene panel was used to screen for genetic mutation in the DCM index patients. However, we cannot exclude the contribution of other genes to the phenotype in the index patients and the carrier status in their relatives. 


\section{CONCLUSION}

Relatives from DCM patients have a higher chance of systolic dysfunction reflected by an abnormal GLS, independent of age, gender, comorbidities and genotype. An abnormal GLS is associated with LVEF deterioration, cardiac hospitalization and death in asymptomatic DCM relatives with normal LVEF. GLS seems to be a promising tool for cardiac screening of relatives and could be used to identify patients at risk for adverse events who will benefit most from regular follow-up and treatment.

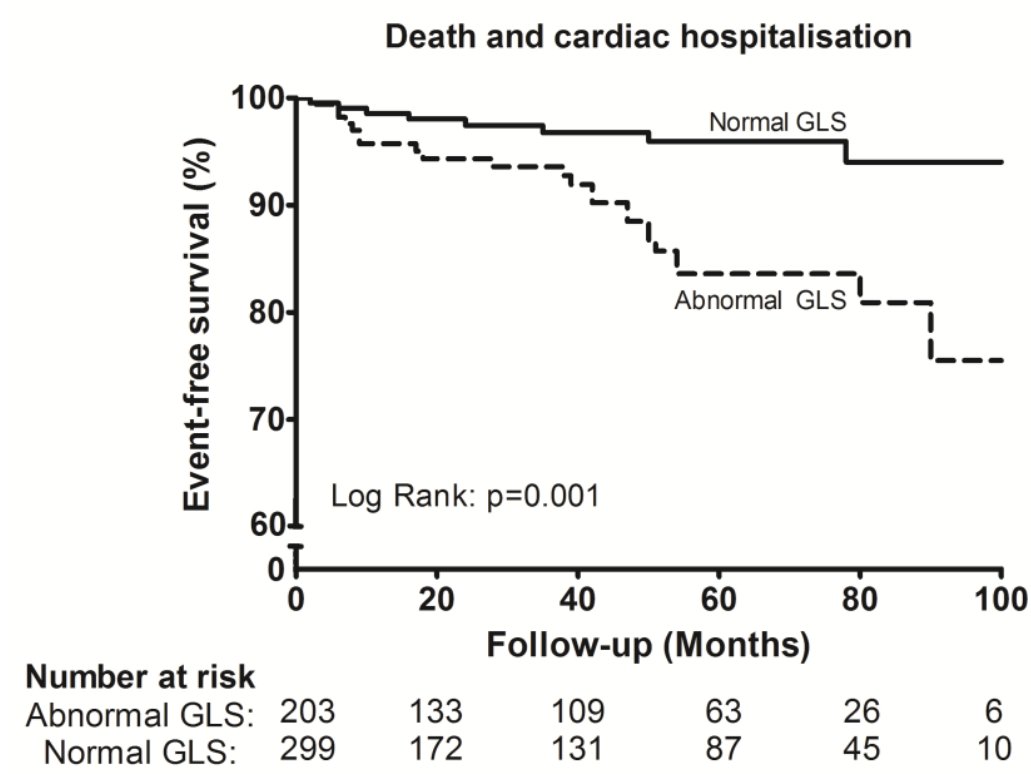

Figure 4. Baseline GLS value and long-term outcome in the whole cohort $(n=502)$. Subjects with an abnormal GLS at baseline have an increased risk for cardiac hospitalization and death. 


\section{Asymptomatic \\ Asymptomatic DCM Relatives Control subjects}

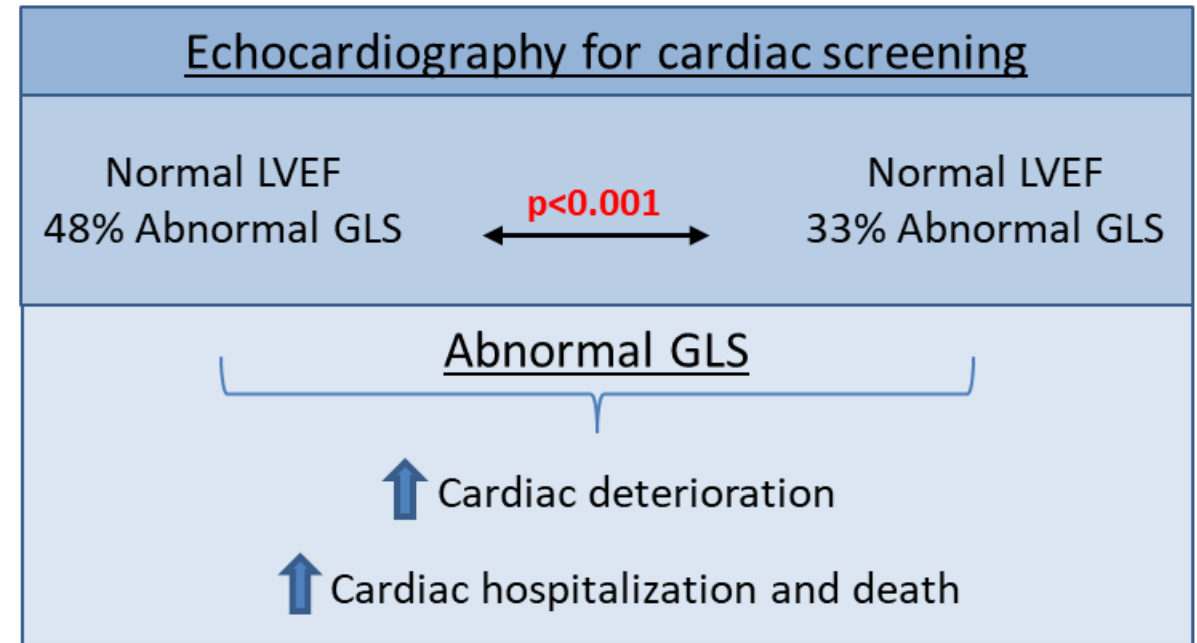

Central Illustration. Global Longitudinal Strain (GLS) analysis in the screening of asymptomatic DCM relatives. First-degree relatives of DCM patients are frequently seen at the outpatient clinic for cardiac screening. The left ventricular ejection fraction (LVEF) is often normal, however, DCM relatives have a higher chance of an abnormal GLS. An abnormal GLS is associated with an increased risk of LVEF deterioration, cardiac hospitalization and death 


\section{REFERENCES}

1. Japp AG, Gulati A, Cook SA, et al. The Diagnosis and Evaluation of Dilated Cardiomyopathy. J Am Coll Cardiol 2016;67(25):2996-3010.

2. Lee DS, Pencina MJ, Benjamin EJ, et al. Association of parental heart failure with risk of heart failure in offspring. $N$ Engl J Med 2006;355(2):138-47.

3. Mahon NG, Murphy RT, MacRae CA, et al. Echocardiographic evaluation in asymptomatic relatives of patients with dilated cardiomyopathy reveals preclinical disease. Annals of internal medicine 2005;143(2):108-15.

4. Charron P, Arad M, Arbustini E, et al. Genetic counselling and testing in cardiomyopathies: a position statement of the European Society of Cardiology Working Group on Myocardial and Pericardial Diseases. Eur Heart $J$ 2010;31(22):2715-26.

5. Fatkin D, Yeoh T, Hayward CS, et al. Evaluation of left ventricular enlargement as a marker of early disease in familial dilated cardiomyopathy. Circulation Cardiovascular genetics 2011;4(4):342-8.

6. Bozkurt B, Colvin M, Cook J, et al. Current Diagnostic and Treatment Strategies for Specific Dilated Cardiomyopathies: A Scientific Statement From the American Heart Association. Circulation 2016;134(23):e579-e646.

7. Hershberger RE, Hedges DJ, Morales A. Dilated cardiomyopathy: the complexity of a diverse genetic architecture. Nature reviews Cardiology 2013;10(9):531-47.

8. Potter E, Marwick TH. Assessment of Left Ventricular Function by Echocardiography: The Case for Routinely Adding Global Longitudinal Strain to Ejection Fraction. JACC CardiovasC Imaging 2018;11(2 Pt 1):260-74.

9. Verdonschot JAJ, Hazebroek MR, Derks KWJ, et al. Titin cardiomyopathy leads to altered mitochondrial energetics, increased fibrosis and long-term life-threatening arrhythmias. Eur Heart J 2018

10. Lang RM, Badano LP, Mor-Avi V, et al. Recommendations for cardiac chamber quantification by echocardiography in adults: an update from the American Society of Echocardiography and the European Association of Cardiovascular Imaging. J Am Soc Echocardiogr 2015;28(1):1-39 e14.

11. Nagueh SF, Smiseth $\mathrm{OA}$, Appleton $\mathrm{CP}$, et al. Recommendations for the Evaluation of Left Ventricular Diastolic Function by Echocardiography: An Update from the American Society of Echocardiography and the
European Association of Cardiovascular Imaging.

J Am Soc Echocardiogr 2016;29(4):277-314.

12. Knackstedt C, Bekkers SC, Schummers G, et al. Fully Automated Versus Standard Tracking of Left Ventricular Ejection Fraction and Longitudinal Strain: The FAST-EFs Multicenter Study. J Am Coll Cardiol 2015;66(13):1456-66.

13. Merken J, Brunner-La Rocca HP, Weerts J, et al. Heart Failure With Recovered Ejection Fraction. J Am Coll Cardiol 2018;72(13):1557-58.

14. Dalen $H$, Thorstensen A, Aase SA, et al. Segmental and global longitudinal strain and strain rate based on echocardiography of 1266 healthy individuals: the HUNT study in Norway. Eur J Echocardiogr 2010;11(2):176-83.

15. Ng AC, Delgado V, Bertini M, et al. Myocardial steatosis and biventricular strain and strain rate imaging in patients with type 2 diabetes mellitus. Circulation 2010;122(24):2538-44.

16. Lakdawala NK, Thune JJ, Colan SD, et al. Subtle abnormalities in contractile function are an early manifestation of sarcomere mutations in dilated cardiomyopathy. Circulation Cardiovascular genetics 2012;5(5):503-10.

17. van der Bijl P, Bootsma M, Hiemstra YL, et al. Left ventricular 2D speckle tracking echocardiography for detection of systolic dysfunction in genetic, dilated cardiomyopathies. European heart journal cardiovascular Imaging 2018

18. Pinto YM, Elliott PM, Arbustini E, et al. Proposal for a revised definition of dilated cardiomyopathy, hypokinetic non-dilated cardiomyopathy, and its implications for clinical practice: a position statement of the ESC working group on myocardial and pericardial diseases. Eur Heart J 2016;37(23):1850-8.

19. Sefa Okten M, Tuluce K, Yakar Tuluce $S$, et al. Screening first-degree relatives of patients with idiopathic dilated cardiomyopathy. Herz 2017;42(7):669-76.

20. Adamo L, Perry A, Novak E, et al. Abnormal Global Longitudinal Strain Predicts Future Deterioration of Left Ventricular Function in Heart Failure Patients With a Recovered Left Ventricular Ejection Fraction. Circulation Heart failure 2017;10(6)

21. Charbonnel C, Convers-Domart R, Rigaudeau S, et al. Assessment of global longitudinal strain at low-dose anthracycline-based chemotherapy, for the prediction of subsequent cardiotoxicity. European heart journal cardiovascular Imaging 2017;18(4):392-401.

22. Smiseth OA, Torp H, Opdahl A, et al. Myocardial strain imaging: how useful is it in clinical decision making? Eur Heart J 2016;37(15):1196-207. 
23. Biering-Sorensen T, Biering-Sorensen SR, Olsen FJ, et al. Global Longitudinal Strain by Echocardiography Predicts Long-Term Risk of Cardiovascular Morbidity and Mortality in a LowRisk General Population: The Copenhagen City Heart Study. Circ Cardiovasc Imaging 2017;10(3)

24. Kalam K, Otahal P, Marwick TH. Prognostic implications of global LV dysfunction: a systematic review and meta-analysis of global longitudinal strain and ejection fraction. Heart (British Cardiac Society) 2014;100(21):1673-80.

25. Sengelov M, Jorgensen PG, Jensen JS, et al. Global Longitudinal Strain Is a Superior Predictor of All-Cause Mortality in Heart Failure With Reduced Ejection Fraction. JACC Cardiovasc Imaging 2015;8(12):1351-59.

26. Mignot A, Donal E, Zaroui A, et al. Global longitudinal strain as a major predictor of cardiac events in patients with depressed left ventricular function: a multicenter study. J Am Soc Echocardiogr 2010;23(10):1019-24.

27. Romano S, Judd RM, Kim RJ, et al. FeatureTracking Global Longitudinal Strain Predicts Death in a Multicenter Population of Patients with Ischemic and Nonischemic Dilated Cardiomyopathy Incremental to Ejection Fraction and Late Gadolinium Enhancement. JACC Cardiovasc Imaging 2018

28. Buss SJ, Breuninger K, Lehrke S, et al. Assessment of myocardial deformation with cardiac magnetic resonance strain imaging improves risk stratification in patients with dilated cardiomyopathy. European heart journal cardiovascular Imaging 2015;16(3):307-15.

29. Haugaa KH, Goebel B, Dahlslett T, et al. Risk assessment of ventricular arrhythmias in patients with nonischemic dilated cardiomyopathy by strain echocardiography. J Am Soc Echocardiogr 2012;25(6):667-73.

30. Pankuweit S. Lamin $\mathrm{A} / \mathrm{C}$ mutations in patients with dilated cardiomyopathy. Eur Heart J 2017 


\section{SUPPLEMENTARY FIGURES}

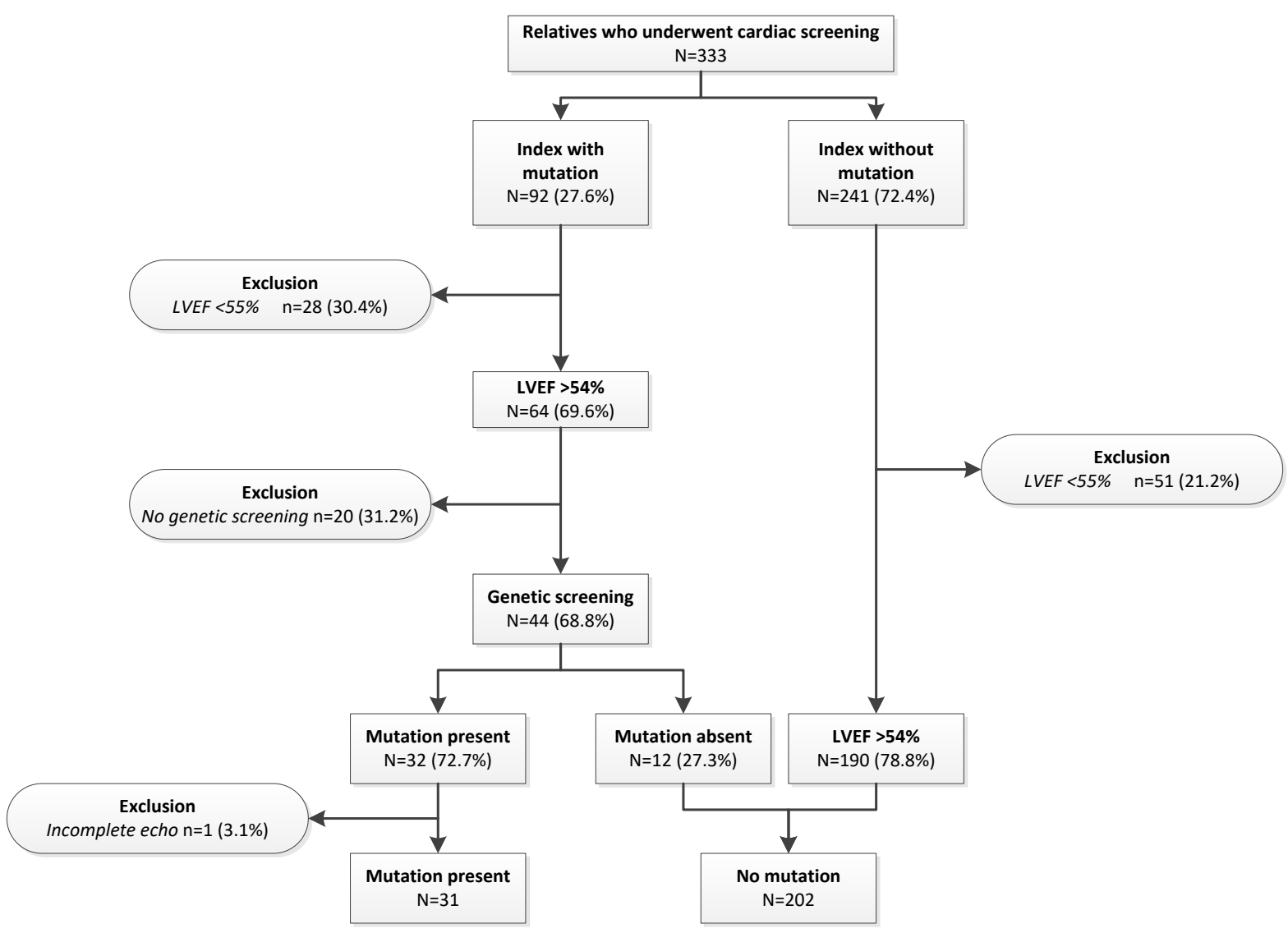

Supplemental Figure 1. Flowchart of subject selection of DCM relatives for the analysis based on genotype

\section{SUPPLEMENTARY TABLES}

Supplemental Table 1. Overview of all 47 genes used in the Maastricht Cardiomyopathy gene-panel.

\begin{tabular}{cccc} 
& HGNC.ID & HGNC.symbol & HGNC.Name \\
\hline 1 & HGNC:143 & ACTC1 & Actin, alpha, cardiac muscle 1 \\
2 & HGNC:164 & ACTN2 & Actinin alpha 2 \\
3 & HGNC:15819 & ANKRD1 & Ankyrin repeat domain 1 \\
4 & HGNC:939 & BAG3 & BCL2 associated athanogene 3 \\
5 & HGNC:20407 & CALR3 & Calreticulin 3 \\
6 & HGNC:1529 & CAV3 & Caveolin 3 \\
7 & HGNC:2389 & CRYAB & Crystallin alpha B \\
8 & HGNC:2472 & CSRP3 & Cysteine and glycine rich protein 3 \\
9 & HGNC:2511 & CTNNA3 & Catenin alpha 3 \\
10 & HGNC:2770 & DES & Desmin \\
11 & HGNC:3036 & DSC2 & Desmocollin 2 \\
12 & HGNC:3049 & DSG2 & Desmoglein 2 \\
13 & HGNC:3052 & DSP & Desmoplakin \\
14 & HGNC:3331 & EMD & Emerin \\
15 & HGNC:3702 & FHL1 & GLA \\
16 & HGNC:4296 & & Four and a half LIM domains 1 \\
\hline
\end{tabular}




\begin{tabular}{|c|c|c|c|}
\hline 17 & HGNC:14202 & JPH2 & Junctophilin 2 \\
\hline 18 & HGNC:6207 & JUP & Junction plakoglobin \\
\hline 19 & HGNC:6484 & LAMA4 & Laminin subunit alpha 4 \\
\hline 20 & HGNC:6501 & LAMP2 & Lysosomal associated membrane protein 2 \\
\hline 21 & HGNC:15710 & $\angle D B 3$ & LIM domain binding 3 \\
\hline 22 & HGNC:6636 & LMNA & Lamin A/C \\
\hline 23 & HGNC:21086 & MIB1 & Mindbomb E3 ubiquitin protein ligase 1 \\
\hline 24 & HGNC:7551 & МYBPC3 & Myosin binding protein C, cardiac \\
\hline 25 & HGNC:7576 & MYH6 & Myosin heavy chain 6 \\
\hline 26 & HGNC:7577 & MYH7 & Myosin heavy chain 7 \\
\hline 27 & HGNC:7583 & MYL2 & Myosin light chain 2 \\
\hline 28 & HGNC:7584 & MYL3 & Myosin light chain 3 \\
\hline 29 & HGNC:1330 & MYOZ2 & Myozenin 2 \\
\hline 30 & HGNC:23246 & MYPN & Myopalladin \\
\hline 31 & HGNC:29557 & NEXN & Nexilin F-actin binding protein \\
\hline 32 & HGNC:9024 & PKP2 & Plakophilin 2 \\
\hline 33 & HGNC:9080 & PLN & Phospholamban \\
\hline 34 & HGNC:14000 & PRDM16 & $\mathrm{PR} / \mathrm{SET}$ domain 16 \\
\hline 35 & HGNC:9386 & PRKAG2 & Protein kinase AMP-activated non-catalytic subunit gamma 2 \\
\hline 36 & HGNC:27424 & $R B M 20$ & RNA binding motif protein 20 \\
\hline 37 & HGNC:10593 & SCN5A & Sodium voltage-gated channel alpha subunit 5 \\
\hline 38 & HGNC:11577 & $T A Z$ & Tafazzin \\
\hline 39 & HGNC:11610 & TCAP & Titin-cap \\
\hline 40 & HGNC:28472 & TMEM43 & Transmembrane protein 43 \\
\hline 41 & HGNC:11943 & TNNC1 & Troponin C1, slow skeletal and cardiac type \\
\hline 42 & HGNC:11947 & TNNI3 & Troponin 13 , cardiac type \\
\hline 43 & HGNC:11949 & TNNT2 & Troponin T2, cardiac type \\
\hline 44 & HGNC:12010 & TPM1 & Tropomyosin 1 \\
\hline 45 & HGNC:12403 & TTN & Titin \\
\hline 46 & HGNC:12405 & TTR & Transthyretin \\
\hline 47 & HGNC:12665 & $V C L$ & Vinculin \\
\hline
\end{tabular}

Supplemental table 2. Baseline characteristics of the subjects of the whole cohort according to GLS-status

\begin{tabular}{cccc}
\hline Baseline variables & $\begin{array}{c}\text { Normal GLS } \\
(\mathbf{n = 2 9 9 )}\end{array}$ & $\begin{array}{c}\text { Abnormal GLS } \\
(\mathbf{n = 2 0 3 )}\end{array}$ & p-value \\
\hline Male gender, $\mathrm{n}(\%)$ & $119(40)$ & $123(61)$ & $<0.001$ \\
Age (years) & $43 \pm 14$ & $51 \pm 15$ & 0.001 \\
Body Mass Index (BMI) & $25 \pm 4$ & $27 \pm 5$ & 0.16 \\
NYHA III-IV, $\mathrm{n}(\%)$ & $11(4)$ & $13(6)$ & 0.83 \\
CLBBB & $13(4)$ & $8(4)$ & $<0.001$ \\
Genetic Status, $\mathrm{n}(\%)$ & & & 0.001 \\
DCM relative & $131(44)$ & $120(59)$ & 0.23 \\
Familial DCM & $61(20)$ & $65(32)$ & 0.41 \\
Relative of index with mutation & $29(10)$ & $32(16)$ & 0.34 \\
Familial gene mutation present & $13(4)$ & $18(9)$ & $<0.001$ \\
Cardiovascular History, $\mathrm{n}(\%)$ & & & 0.56 \\
Coronary artery disease & $4(1)$ & $17(8)$ & 0.57
\end{tabular}




\begin{tabular}{cccc} 
PCI & $3(1)$ & $8(4)$ & 0.03 \\
Valvular disease & $48(16)$ & $43(21)$ & 0.14 \\
Comorbidities, $\mathrm{n}(\%)$ & & & 0.23 \\
\hline Atrial fibrillation & $2(1)$ & $4(2)$ & $<0.001$ \\
Hypertension & $49(16)$ & $73(36)$ & 0.012 \\
COPD & $8(3)$ & $15(7)$ & 0.007 \\
Hypercholesterolemia & $34(11)$ & $40(20)$ & $<0.001$ \\
Diabetes mellitus & $11(4)$ & $25(12)$ & 0.013 \\
Chemotherapy & $8(3)$ & $15(7)$ & $<0.001$ \\
Medication, $\mathrm{n}(\%)$ & & & 0.025 \\
Beta-blocker & $25(8)$ & $49(24)$ & $<0.001$ \\
ACE-i & $15(5)$ & $21(10)$ & 0.57 \\
ARB & $14(5)$ & $28(14)$ & 0.009 \\
MRA & $1(0)$ & $2(1)$ & 0.014 \\
Diuretics & $11(4)$ & $19(9)$ & $<0.001$ \\
Calcium antagonist & $13(4)$ & $20(10)$ & $48(24)$ \\
Statins & $33(11)$ & & \\
\hline
\end{tabular}

Abbreviations, $A C E-I$, angiotensin converting enzyme-inhibitor;ARB, angiotensin II receptor blocker; CABG, coronary artery bypass graft; CLBBB, complete left bundle branch block; COPD, chronic obstructive pulmonary disease; DCM, dilated cardiomyopathy; MRA. Mineralocorticoid receptor antagonist; $P C I$, percutaneous coronary intervention.

Supplemental Table 3. Genetic mutations in index DCM patients and their asymptomatic relatives

\begin{tabular}{|c|c|c|c|c|c|}
\hline Gene & cDNA variant & Amino acid change & $\begin{array}{c}\text { No. of } \\
\text { index } \\
\text { patients }\end{array}$ & $\begin{array}{l}\text { No. of } \\
\text { asymptomatic } \\
\text { relatives }\end{array}$ & $\begin{array}{c}\text { No. of } \\
\text { relatives with } \\
\text { proven } \\
\text { mutation }\end{array}$ \\
\hline TTN & c.47137_47138de & p.(Ser15713Leufs*2) & 1 & 1 & 0 \\
\hline$T T N$ & c. $50296 C>T$ & p. $\left(\operatorname{Arg} 16766^{*}\right)$ & 1 & 3 & 1 \\
\hline TTN & c.58327del & p.(Ala19443Leufs*2) & 1 & 1 & 1 \\
\hline TTN & c. $60931 \mathrm{C}>\mathrm{T}$ & p. $\left(\operatorname{Arg} 20311^{*}\right)$ & 1 & 1 & 0 \\
\hline TTN & c. $63794-1 G>A$ & p.(?) & 1 & 5 & 1 \\
\hline TTN & c. $67863 \mathrm{C}>\mathrm{G}$ & p.(Tyr22621*) & 1 & 5 & 5 \\
\hline TTN & c. $73332 C>A$ & p. $\left(\right.$ Cys $\left.24444^{*}\right)$ & 1 & 1 & 0 \\
\hline TTN & c. $87782 \mathrm{del}$ & p.(Pro29261GInfs*10) & 1 & 2 & 2 \\
\hline$T T N$ & c.97427dup & p. (Thr32477Asnfs*13) & 1 & 1 & 1 \\
\hline LMNA & c. $481 G>A$ & p.(Glu161Lys) & 2 & 2 & 2 \\
\hline LMNA & c. $357-2 A>G$ & p.(?) & 1 & 1 & 1 \\
\hline LMNA & c. $208 \mathrm{G}>\mathrm{A}$ & p.(Val70lle) & 1 & 3 & 0 \\
\hline LMNA & c. $1300 \mathrm{G}>\mathrm{A}$ & p.(Ala434Thr) & 1 & 1 & 1 \\
\hline$D S P$ & c.7773_7776del & p.(Ser2591Argfs*11) & 1 & 1 & 0 \\
\hline$D S P$ & c.6393del & p.(Gly2133Valfs*2) & 1 & 1 & 0 \\
\hline$D S P$ & c.3383_3384del & p.(Val1128Glyfs*5) & 1 & 1 & 1 \\
\hline TPM1 & c. $829 \mathrm{G}>\mathrm{A}$ & p.(Ala277Thr) & 1 & 3 & 3 \\
\hline TPM1 & c. $284 \mathrm{~T}>\mathrm{C}$ & p.(Val95Ala) & 1 & 3 & 2 \\
\hline TNNC1 & c. $317+1 G>A$ & p.(Gly68Glufs*12) & 1 & 2 & 2 \\
\hline MYL3 & c. $476 \mathrm{C}>\mathrm{T}$ & p.(Thr159Met) & 1 & 3 & 0 \\
\hline MYH7 & c. $2167 C>T$ & p.(Arg723Cys) & 1 & 2 & 2 \\
\hline МYBPCЗ & c. $2905 C>T$ & p. $(G \ln 969 *)$ & 1 & 2 & 1 \\
\hline МYBPCЗ & c. $3065 \mathrm{G}>\mathrm{C}$ & p.(Arg1022Pro) & 1 & 1 & 1 \\
\hline NEXN & c.1909_1912del & p.(Tyr637Alafs*48) & 1 & 1 & 0 \\
\hline MYOT & c. $179 \mathrm{C}>\mathrm{T}$ & p.(Ser60Phe) & 1 & 1 & 0 \\
\hline SCN5A & c. $311 G>A$ & p.(Arg104GIn) & 1 & 12 & 4 \\
\hline SCN5A & c. $4057 \mathrm{G}>\mathrm{A}$ & p.(Val1353Met) & 1 & 2 & 0 \\
\hline$R B M 20$ & c. $1764 \mathrm{~T}>\mathrm{G}$ & p.(Ile588Met) & 1 & 1 & 1 \\
\hline MIB1 & c. $912 \mathrm{G}>\mathrm{A}$ & p. $\left(\operatorname{Trp} 304^{*}\right)$ & 1 & 1 & 1 \\
\hline
\end{tabular}

Genome Reference Consortium Human Build 37 (GRCh37 (h19)) 
Supplemental Table 4. Values of the follow-up echoes of the DCM relatives $(n=69)$ with and without abnormal GLS

\begin{tabular}{|c|c|c|c|}
\hline Baseline variables & $\begin{array}{c}\text { Normal GLS } \\
\quad(n=26)\end{array}$ & $\begin{array}{c}\text { Abnormal GLS } \\
(n=43)\end{array}$ & p-value \\
\hline Follow-up duration (months) & $35[23-63]$ & 36 [24-65] & 0.99 \\
\hline \multicolumn{4}{|l|}{ Systolic function } \\
\hline LVEF (\%) & $60 \pm 5$ & $56 \pm 7$ & 0.018 \\
\hline LVEDD (mm) & $50 \pm 6$ & $51 \pm 7$ & 0.82 \\
\hline LVESD (mm) & $34 \pm 4$ & $36 \pm 5$ & 0.12 \\
\hline $\mathrm{LA}(\mathrm{mm})$ & $38 \pm 5$ & $40 \pm 7$ & 0.13 \\
\hline $\mathrm{RV}(\mathrm{mm})$ & $41 \pm 20$ & $38 \pm 12$ & 0.54 \\
\hline IVS (mm) & $8 \pm 2$ & $9 \pm 2$ & 0.034 \\
\hline PWT (mm) & $8 \pm 2$ & $9 \pm 2$ & 0.22 \\
\hline LVEF <55\%, n (\%) & $3(12)$ & $17(40)$ & 0.013 \\
\hline \multicolumn{4}{|l|}{ Diastolic function } \\
\hline E-wave & $71 \pm 17$ & $70 \pm 26$ & 0.78 \\
\hline A-wave & $58 \pm 17$ & $65 \pm 20$ & 0.16 \\
\hline E/A ratio & $1.4[1.0-1.7]$ & $1.0[0.7-1.3]$ & 0.028 \\
\hline $\mathrm{e}^{\prime}$ velocity $(\mathrm{cm} / \mathrm{sec})$ & $10.5 \pm 3$ & $8.0 \pm 3$ & 0.003 \\
\hline$E / e^{\prime}$ & $7.4 \pm 3$ & $9.8 \pm 6$ & 0.084 \\
\hline TI velocity $(\mathrm{cm} / \mathrm{sec})$ & $2.2 \pm 0.2$ & $2.3 \pm 0.4$ & 0.35 \\
\hline
\end{tabular}

Abbreviations, GLS, global longitudinal strain; IVS, inter ventricular septum; $L A$, left atrium; $L V E D D$, left ventricular end-diastolic diameter; $L V E F$, left ventricular ejection fraction; LVESD, left ventricular end-systolic diameter; PWT, posterior wall thickness; RV, right ventricle.

Supplemental Table 5. Baseline characteristics of the DCM relatives with and without follow-up echo

\begin{tabular}{|c|c|c|c|}
\hline Baseline variables & $\begin{array}{l}\text { Relatives with FU } \\
\text { echo }(n=69)\end{array}$ & $\begin{array}{l}\text { Relatives without FU echo } \\
\qquad(\mathrm{n}=182)\end{array}$ & p-value \\
\hline Male gender, n (\%) & $39(57)$ & $74(41)$ & 0.024 \\
\hline Age (years) & $45 \pm 18$ & $46 \pm 17$ & 0.65 \\
\hline Body Mass Index (BMI) & $27 \pm 5$ & $25 \pm 4$ & 0.013 \\
\hline NYHA III-IV, n (\%) & $3(4)$ & $5(3)$ & 0.69 \\
\hline CLBBB & $3(4)$ & $3(2)$ & 0.35 \\
\hline Chemotherapy & $1(1)$ & $6(3)$ & 0.68 \\
\hline \multicolumn{4}{|l|}{ Genetic Status, n (\%) } \\
\hline Familial DCM & $42(61)$ & $84(46)$ & 0.06 \\
\hline Relative of index with mutation & $26(38)$ & 35 (19) & 0.003 \\
\hline Familial gene mutation present & $13(19)$ & $18(10)$ & 0.038 \\
\hline \multicolumn{4}{|l|}{ Cardiovascular History, n (\%) } \\
\hline Coronary artery disease & $8(12)$ & $6(3)$ & 0.027 \\
\hline Stroke & $4(6)$ & $2(1)$ & 0.68 \\
\hline CABG & $1(1)$ & $2(1)$ & 0.99 \\
\hline $\mathrm{PCl}$ & $4(6)$ & $2(1)$ & 0.06 \\
\hline Valvular disease & $19(28)$ & $23(13)$ & 0.005 \\
\hline \multicolumn{4}{|l|}{ Comorbidities, n (\%) } \\
\hline Atrial fibrillation & $1(1)$ & $1(1)$ & 0.48 \\
\hline Hypertension & $27(39)$ & $38(21)$ & 0.004 \\
\hline COPD & $4(6)$ & $6(3)$ & 0.47 \\
\hline Hypercholesterolemia & $10(14)$ & $20(11)$ & 0.52 \\
\hline Diabetes mellitus & $5(7)$ & $16(9)$ & 0.66 \\
\hline \multicolumn{4}{|l|}{ Medication, $\mathrm{n}(\%)$} \\
\hline Beta-blocker & $16(23)$ & $13(7)$ & 0.001 \\
\hline ACE-i & $8(12)$ & $9(5)$ & 0.09 \\
\hline ARB & $9(13)$ & $9(5)$ & 0.04 \\
\hline MRA & $2(3)$ & $0(0)$ & 0.08 \\
\hline Diuretics & $8(12)$ & $9(5)$ & 0.1 \\
\hline Calcium antagonists & $5(7)$ & $6(3)$ & 0.3 \\
\hline
\end{tabular}


Abbreviations, ACE-I, angiotensin converting enzyme-inhibitor;ARB, angiotensin II receptor blocker; CABG, coronary artery bypass graft; CLBBB, complete left bundle branch block; COPD, chronic obstructive pulmonary disease; DCM, dilated cardiomyopathy; MRA. Mineralocorticoid receptor antagonist; $\mathrm{PCl}$, percutaneous coronary intervention.

Supplemental Table 6. Echocardiographic baseline parameters of the DCM relatives with and without followup echo

\begin{tabular}{|c|c|c|c|}
\hline Baseline variables & $\begin{array}{c}\text { Relatives with FU } \\
\text { echo }(n=69)\end{array}$ & $\begin{array}{c}\text { Relatives without FU } \\
\text { echo ( } n=182)\end{array}$ & p-value \\
\hline \multicolumn{4}{|l|}{ Systolic function } \\
\hline LVEF (\%) & $61 \pm 3.7$ & $61 \pm 3.8$ & 0.77 \\
\hline $\operatorname{LVEDD}(\mathrm{mm})$ & $50 \pm 6$ & $49 \pm 5$ & 0.07 \\
\hline $\operatorname{LVESD}(\mathrm{mm})$ & $34 \pm 4$ & $33 \pm 3$ & 0.16 \\
\hline IVS (mm) & $9 \pm 1.5$ & $8 \pm 1.9$ & 0.018 \\
\hline PWT (mm) & $9 \pm 1.5$ & $8 \pm 1.1$ & 0.007 \\
\hline LA volume index $\left(\mathrm{ml} / \mathrm{m}^{2}\right)$ & $31 \pm 8$ & $33 \pm 11$ & 0.29 \\
\hline GLS (\%) & $-20.6[-18--23]$ & $-22.1[-20--24]$ & 0.001 \\
\hline Abnormal GLS, n (\%) & $43(62)$ & $26(14)$ & 0.005 \\
\hline \multicolumn{4}{|l|}{ Diastolic function } \\
\hline $\mathrm{E} / \mathrm{A}$ ratio & $1.3 \pm 0.5$ & $1.2 \pm 0.3$ & 0.34 \\
\hline TI velocity $(\mathrm{cm} / \mathrm{sec})$ & $2.3 \pm 0.3$ & $2.2 \pm 0.3$ & 0.09 \\
\hline
\end{tabular}

Abbreviations, GLS, global longitudinal strain; IVS, inter ventricular septum; LA, left atrium; LVEDD, left ventricular end-diastolic diameter; $L V E F$, left ventricular ejection fraction; LVESD, left ventricular end-systolic diameter; PWT, posterior wall thickness.

Supplemental Table 7. Survival end-points and hazard ratios between normal and abnormal GLS subjects in the whole cohort $(n=502)$

\begin{tabular}{|c|c|c|c|c|}
\hline & $\begin{array}{c}\text { Normal GLS } \\
\quad(n=299)\end{array}$ & $\begin{array}{c}\text { Abnormal GLS } \\
(n=203)\end{array}$ & P-value & $\begin{array}{c}\text { Hazard Ratio } \\
\text { [95\%Cl] }\end{array}$ \\
\hline Follow-up duration (months) & $42[3-86]$ & 38 [3-77] & 0.95 & - \\
\hline CV Death & $2(1)$ & $9(4)$ & 0.027 & $5.63[1.21-26.16]$ \\
\hline Cardiac hospitalisation & $8(3)$ & $17(8)$ & 0.015 & $2.84[1.23-6.59]$ \\
\hline Combined end-point & $10(3)$ & $25(12)$ & 0.001 & $3.29[1.58-6.87]$ \\
\hline
\end{tabular}

Abbreviations: GLS = Global Longitudinal Strain; CV = Cardiovascular.

Supplemental Table 8. Detailed information regarding all events in DCM relatives (R) control subjects (C)

\begin{tabular}{cc|ccc|}
$\begin{array}{c}\text { Patient study } \\
\text { number }\end{array}$ & Group & Age & Event & Detail \\
\hline R627 & Abnormal GLS & 71 & Death & VT storm \\
R841 & Abnormal GLS & 72 & Death & Heart Failure \\
R805 & Abnormal GLS & 56 & Death & Heart Failure \\
R1031 & Abnormal GLS & 72 & Death & Heart Failure \\
R845 & Abnormal GLS & 81 & Death & Heart Failure \\
R646 & Normal GLS & 44 & Death & Sudden Death \\
R1108 & Normal GLS & 78 & Death & Heart Failure \\
C95 & Abnormal GLS & 74 & Death & Infection during cardiac hospitalisation \\
C143 & Abnormal GLS & 83 & Death & Heart Failure \\
C13 & Abnormal GLS & 47 & Death & Respiratory Failure after cardiac \\
C106 & Abnormal GLS & 41 & Death & surgery \\
R627 & Abnormal GLS & 66 & Hospitalisation & Appropriate ICD shock after sustained \\
& & & monomorphic VT
\end{tabular}




\begin{tabular}{|c|c|c|c|c|}
\hline R630 & Abnormal GLS & 72 & Hospitalisation & Unstable AP with $\mathrm{PCl}$ \\
\hline R702 & Abnormal GLS & 60 & Hospitalisation & Appropriate ICD shock after VF \\
\hline R1071 & Abnormal GLS & 60 & Hospitalisation & Cardiac arrest with total AV-block \\
\hline $\mathrm{R} 713$ & Abnormal GLS & 50 & Hospitalisation & Unstable AP with $\mathrm{PCl}$ \\
\hline R944 & Abnormal GLS & 64 & Hospitalisation & $\begin{array}{l}\text { Three vessel disease and emergency } \\
\text { CABG }\end{array}$ \\
\hline R977 & Abnormal GLS & 65 & Hospitalisation & Collaps with total AV block \\
\hline R612 & Abnormal GLS & 77 & Hospitalisation & Unstable AP with PCI \\
\hline R1208 & Abnormal GLS & 71 & Hospitalisation & Heart Failure \\
\hline R1105 & Abnormal GLS & 53 & Hospitalisation & Unstable AP with CABG \\
\hline R1039 & Normal GLS & 71 & Hospitalisation & Heart Failure \\
\hline R697 & Normal GLS & 18 & Hospitalisation & Collaps with new LBBB \\
\hline C11 & Normal GLS & 19 & Hospitalisation & Collaps \\
\hline C61 & Normal GLS & 45 & Hospitalisation & Rhythm disorder \\
\hline C204 & Normal GLS & 55 & Hospitalisation & Rhythm disorder \\
\hline C100 & Normal GLS & 47 & Hospitalisation & Unstable AP with $\mathrm{PCl}$ \\
\hline C52 & Normal GLS & 35 & Hospitalisation & Collaps \\
\hline C128 & Normal GLS & 49 & Hospitalisation & Dyspnea \\
\hline C197 & Abnormal GLS & 48 & Hospitalisation & Dyspnea \\
\hline $\mathrm{C} 212$ & Abnormal GLS & 60 & Hospitalisation & Unstable AP with $\mathrm{PCl}$ \\
\hline C168 & Abnormal GLS & 38 & Hospitalisation & Dyspnea \\
\hline C217 & Abnormal GLS & 59 & Hospitalisation & Unstable AP with $\mathrm{PCl}$ \\
\hline C200 & Abnormal GLS & 49 & Hospitalisation & Rhythm disorder \\
\hline C166 & Abnormal GLS & 33 & Hospitalisation & Dyspnea \\
\hline C33 & Abnormal GLS & 32 & Hospitalisation & Dyspnea \\
\hline
\end{tabular}

Abbreviations: $R=$ DCM relatives; $C=$ Control subject.

$G L S=$ Global Longitudinal Strain, VT = Ventricular Tachycardia; $I C D=I m p l a n t a b l e$ Cardiac Defibrillator; $A P=A n g i n a P e c t o r i s ; P C I=$

Percutaneous Coronary Intervention; $V F=$ Ventricular Fibrillation; $C A B G=$ Coronary Artery Bypass Graft; $A V=A t r i o v e n t r i c u l a r ; ~ L B B B=L e f t$ Bundle Branch Block. 


\title{
EDITORIAL COMMENT
}

The value of strain in familial dilated cardiomyopathy screening

\author{
Sanjay K. Prasad, $M D^{1,2}$ \\ Upasana Tayal, MD, PhD ${ }^{1,2}$ \\ ${ }^{1}$ National Heart Lung Institute, Imperial College London, UK. \\ ${ }^{2}$ Cardiovascular Research Centre, Royal Brompton Hospital, London, UK.
}

JOURNAL OF THE AMERICAN COLLEGE OF CARDIOLOGY: CARDIOVASCULAR IMAGING

2020. FEB; 13 (2 Pt 2): 559-561. 
Dilated cardiomyopathy (DCM) has a familial basis in $20 \%$ to $30 \%$ of cases, though up to $60 \%$ has been suggested ${ }^{1}$. However, in familial DCM only up to $40 \%$ of cases may have an identifiable genetic basis ${ }^{2}$. Gene based cascade screening is therefore only suitable for a limited proportion of patients with DCM. In addition, as of yet, there are no reproducible genotype phenotype correlations in DCM that sufficiently discriminate a genetic etiology. Therefore, cascade screening, the search for asymptomatic disease in relatives, is largely undertaken through clinical assessment and imaging. However, the screening intervals are not universally defined across European and American guidelines, reflecting a weak evidence base. Current American Heart Association recommendations suggest periodic echocardiographic screening of first-degree relatives ${ }^{3}$, whereas European Society of Cardiology recommendations are more prescriptive, recommending clinical screening including imaging of first-degree relatives at 2- to 5-year intervals until 60 to 65 years of age ${ }^{4}$. If an asymptomatic relative is found to carry a pathogenic genetic variant then repeated cardiac evaluation is recommended every 1 to 3 years (European Society of Cardiology guidelines) or every 3 to 5 years (American Heart Association guidelines). Separate to the issue of screening timelines, whether left ventricular ejection fraction (LVEF) is a sufficiently sensitive discriminator of early disease in this cohort is a matter of debate. DCM is a now thought to be a spectrum of disease, from hypokinetic nondilated phenotypes to the classical dilated, impaired LV ${ }^{5}$. Therefore, the quest is on to detect phenotypes even earlier in the DCM cascade. This has important utility in guiding patient management. Myocardial strain is an attractive imaging biomarker in this context. Strain is a unitless measure of tissue deformation. As the LV contracts, the myocardium shortens in a longitudinal and circumferential dimension (negative strain) and thickens or lengthens in the radial direction (positive strain). Longitudinal strain measures different aspects of myocardial deformation compared with LVEF, which largely reflects radial contraction. Longitudinal strain is a measure of the active shortening of the LV in the longitudinal direction and represents the function of subendocardial longitudinal myocardial fibers. These fibers are more sensitive to reduced coronary perfusion and increased wall stress; therefore, longitudinal dysfunction may be an early marker of LV dysfunction ${ }^{6}$. The prognostic value of myocardial strain is well established in a range of conditions including dilated cardiomyopathy ${ }^{7}$, ischemic heart disease ${ }^{8}$, and allcause heart failure ${ }^{9}$. Myocardial strain has also been recently shown to predict LV recovery in nonischemic cardiomyopathy patients ${ }^{10}$. Therefore, it is highly plausible that myocardial strain could detect subclinical disease in asymptomatic relatives of patients with DCM.

In this issue of iJACC, Verdonschot et al. ${ }^{11}$ evaluate the use of echocardiography speckle tracking strain in relatives of patients with DCM. They make an important observation that more DCM relatives with normal LVEF had impaired global longitudinal strain (GLS) compared with a cohort of individuals with normal LVEF referred for investigation of cardiac symptoms (48\% vs. 33\%) and that GLS was lower in the DCM relatives compared with the "control" cohort. In subset analyses (69 individuals from 251), they find that abnormal GLS is associated with a slightly reduced LVEF on follow-Up (LVEF 60\% to 56\%) and across the whole cohort replicate previous studies that show that an abnormal GLS is an adverse prognostic indicator. The authors propose that these findings support the use of GLS in the screening of asymptomatic DCM relatives. It is important to stress that the authors neither show nor suggest that abnormal strain is unique to cardiomyopathy relatives and that abnormal strain in this setting should change therapeutic management; however, it does imply that such patients should remain under some form of surveillance. The control cohort was notable for 
a number of individuals with comorbid cardiovascular conditions including hypertension, and all had presented with cardiovascular symptoms. Indeed, the number of cardiovascular events in follow-up suggest that this was not a truly healthy cohort.

There are a number of steps required in taking these findings forward in a prospective setting. One is for the cardiovascular community to standardize strain measurements and normal ranges, independent of vendor specific software and to be able to compare strain data across imaging modalities. For echocardiography at least, the American Society of Echocardiography, the European Association of Cardiovascular Imaging, and the ultrasound imaging industry undertook a joint standardization initiative for GLS, which has led to improved intra- and interobserver variability ${ }^{12}$. Other strain measurements remain to be standardized. There is no universally accepted method of evaluating strain. Reproducibility of the strain measures will, to some extent, determine the diagnostic utility of myocardial strain in subtle phenotypes, particularly given the relatively small changes in strain between DCM relatives and the "control" cohort. Harnessing this information at an individual patient level rather than at the cohort level remains a challenge. Additionally, it is too early to adopt these measures in routine clinical practice as yet because having identified early disease, what does the practicing clinician do? There is no evidence to support a change in practice, and the evidence base is limited so current options consist of more regular time- and cost-intensive screening or the intuitive but evidence-free institution of heart failure therapies. One school of thought is that screening should identify early disease and by the time LVEF is impaired, the valuable opportunity for introduction of early therapy is lost. Critics argue that there is no evidence base to support treatment to improve outcomes at this early stage and that limited health resources should be directed to existing patients with disease and medications known to improve prognosis. Importantly, we do not want to be labeling otherwise well individuals with a pathology that we are not yet placed to treat or modify the associated risk. However, while the outcome in DCM is improving ${ }^{13}{ }^{14}$, many patients remain affected by the substantial morbidity and mortality burden. Striving for earlier disease detection is a laudable aim and as supported by the findings of this study. To complement these efforts, future research should be directed at testing the hypothesis that early detection of disease and subsequent introduction of disease modifying therapy improves outcomes. 


\section{REFERENCES}

1. Petretta M, Pirozzi F, Sasso L, et al. Review and metaanalysis of the frequency of familial dilated cardiomyopathy. Am J Cardiol 2011;108(8):11716.

2. Hershberger RE, Hedges DJ, Morales A. Dilated cardiomyopathy: the complexity of a diverse genetic architecture. Nature reviews Cardiology 2013;10(9):531-47.

3. Bozkurt B, Colvin M, Cook J, et al. Current Diagnostic and Treatment Strategies for Specific Dilated Cardiomyopathies: A Scientific Statement From the American Heart Association. Circulation 2016;134(23):e579-e646.

4. Charron P, Arad M, Arbustini E, et al. Genetic counselling and testing in cardiomyopathies: a position statement of the European Society of Cardiology Working Group on Myocardial and Pericardial Diseases. Eur Heart $J$ 2010;31(22):2715-26.

5. Pinto YM, Elliott PM, Arbustini E, et al. Proposal for a revised definition of dilated cardiomyopathy, hypokinetic non-dilated cardiomyopathy, and its implications for clinical practice: a position statement of the ESC working group on myocardial and pericardial diseases. Eur Heart J 2016;37(23):1850-8.

6. Buckberg G, Hoffman JI, Mahajan A, et al. Cardiac mechanics revisited: the relationship of cardiac architecture to ventricular function. Circulation 2008;118(24):2571-87.

7. Chimura M, Onishi T, Tsukishiro $Y$, et al. Longitudinal strain combined with delayedenhancement magnetic resonance improves risk stratification in patients with dilated cardiomyopathy. Heart (British Cardiac Society) 2017;103(9):679-86.

8. Ersboll $M$, Valeur $N$, Mogensen UM, et al. Prediction of all-cause mortality and heart failure admissions from global left ventricular longitudinal strain in patients with acute myocardial infarction and preserved left ventricular ejection fraction. J Am Coll Cardiol 2013;61(23):2365-73.

9. Mignot A, Donal E, Zaroui A, et al. Global longitudinal strain as a major predictor of cardiac events in patients with depressed left ventricular function: a multicenter study. J Am Soc Echocardiogr 2010;23(10):1019-24.

10. Swat SA, Cohen D, Shah SJ, et al. Baseline Longitudinal Strain Predicts Recovery of Left Ventricular Ejection Fraction in Hospitalized Patients With Nonischemic Cardiomyopathy. Journal of the American Heart Association 2018;7(20):e09841.
11. Verdonschot JAJ, Merken JJ, Brunner-La Rocca $H P$, et al. Value of Speckle Tracking-Based Deformation Analysis in Screening Relatives of Patients With Asymptomatic Dilated Cardiomyopathy. JACC Cardiovasc Imaging 2020;13(2 Pt 2):549-58.

12. Voigt JU, Pedrizzetti G, Lysyansky $P$, et al. Definitions for a common standard for 2D speckle tracking echocardiography: consensus document of the EACVI/ASE/Industry Task Force to standardize deformation imaging. European heart journal cardiovascular Imaging 2015;16(1):1-11.

13. Kober L, Thune JJ, Nielsen JC, et al. Defibrillator Implantation in Patients with Nonischemic Systolic Heart Failure. $N$ Engl J Med 2016;375(13):1221-30.

14. Tayal U, Newsome S, Buchan R, et al. Phenotype and Clinical Outcomes of Titin Cardiomyopathy. $J$ Am Coll Cardiol 2017;70(18):2264-74. 


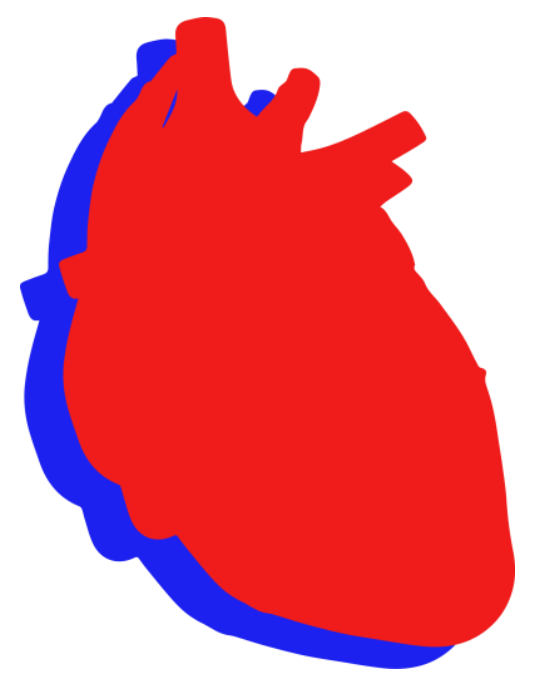

- 326 - 


\section{CHAPTER 13}

General Discussion 
This thesis explored the genetic landscape of dilated cardiomyopathy (DCM) in relation to a broad spectrum of etiologies and phenotypic manifestations. Specific clinical characteristics have been identified which can improve risk stratification of genetic and non-genetic DCM. This chapter will elaborate on the topics of this thesis and discuss the future of genetics in DCM.

\section{Re-evaluating the size of gene panels in clinical care}

Part I describes the clinical consequences of (likely) pathogenic variants in DCM patients. The genetic understanding of DCM developed rapidly since the discovery of the first pathogenic gene variants. Sixty-eight genes are associated with DCM in the Human Genome Mutation Database (HGMD) ${ }^{1}$. Panels covering these genes are currently preferred over targeted gene sequencing, as this approach is more cost and time-efficient in the clinic ${ }^{2}$. A large amount of genetic data is generated with gene panels that needs to be correctly interpreted in a clinical context. Previous studies reporting on genes associated with DCM did not correct for background population variation. The Exome Aggregation Consortium (ExAC) database was released in 2015, containing rare variants found in over 60.000 individuals of diverse ancestries ${ }^{34}$. This provided the opportunity to reevaluate the proposed disease association of gene variants. In some instances, this comparison showed no enrichment in patients relative to controls, which put the pathogenicity of the specific variant in question ${ }^{5}$. The American College of Medical Genetics and Genomics (ACMG) provided criteria for classifying variants which incorporated the prevalence in the ExAC database ${ }^{6}$. The ACMG criteria offer an international, standardized way to interpret variants.

In chapter 2, we systematically applied the ACMG criteria to variants that were found in 689 DCM patients undergoing genetic testing using a panel of 48 genes. One-hundred and thirty-two variants were classified as (likely) pathogenic distributed over 129 individual patients (18.7\%). Figure 1 shows the cumulative detection rate of (likely) pathogenic variants versus variants of unknown significance (VUS) when adding genes to a screening panel. There is no increase in (likely) pathogenic variants after 17 genes, but instead a steep increase in the number of VUS. Restricting the number of genes in clinically used gene panels, will reduce the number of false-positive results and VUS. Both of which can negatively affect patients and their family.

The true percentage of genetic DCM is difficult to estimate from the current literature for a number of reasons. Studies performed before the ACMG criteria reported (likely) pathogenic variants in up to $57 \%$ of DCM patients ${ }^{7-9}$. In order to compare results between studies, all genetic variants should be re-evaluated in a uniform way. A necessary step is to first determine which genes have robust evidence of disease association. The specific DCMframework for the ACMG criteria can then be applied to individual variants. Significant enrichment of rare variation in DCM patients constitutes strong evidence in favor of pathogenicity of specific genes. In line with the findings of this thesis, only 12 genes show significant enrichment of rare variation in DCM patients, when compared to the ExAC database ${ }^{1}$. This does not exclude the pathogenicity of other genes, but emphasizes the difficulty in interpreting variants in other genes in a diagnostic context without strong functional or genetic data. Uncertainty associated with clinical genetic testing in DCM can be significantly reduced by restricting the genes on screening panels to well-validated genes. Previous work in hypertrophic cardiomyopathy (HCM) showed improved diagnostic and prognostic utility when only robustly validated genes were used in gene panels ${ }^{10-12}$. Emerging literature now underline the same possible trend for DCM ${ }^{15}$. Together with the work 
described in this thesis (chapter 2), it supports the necessity for a thorough re-evaluation of DCM gene panels as used in current clinical practice.

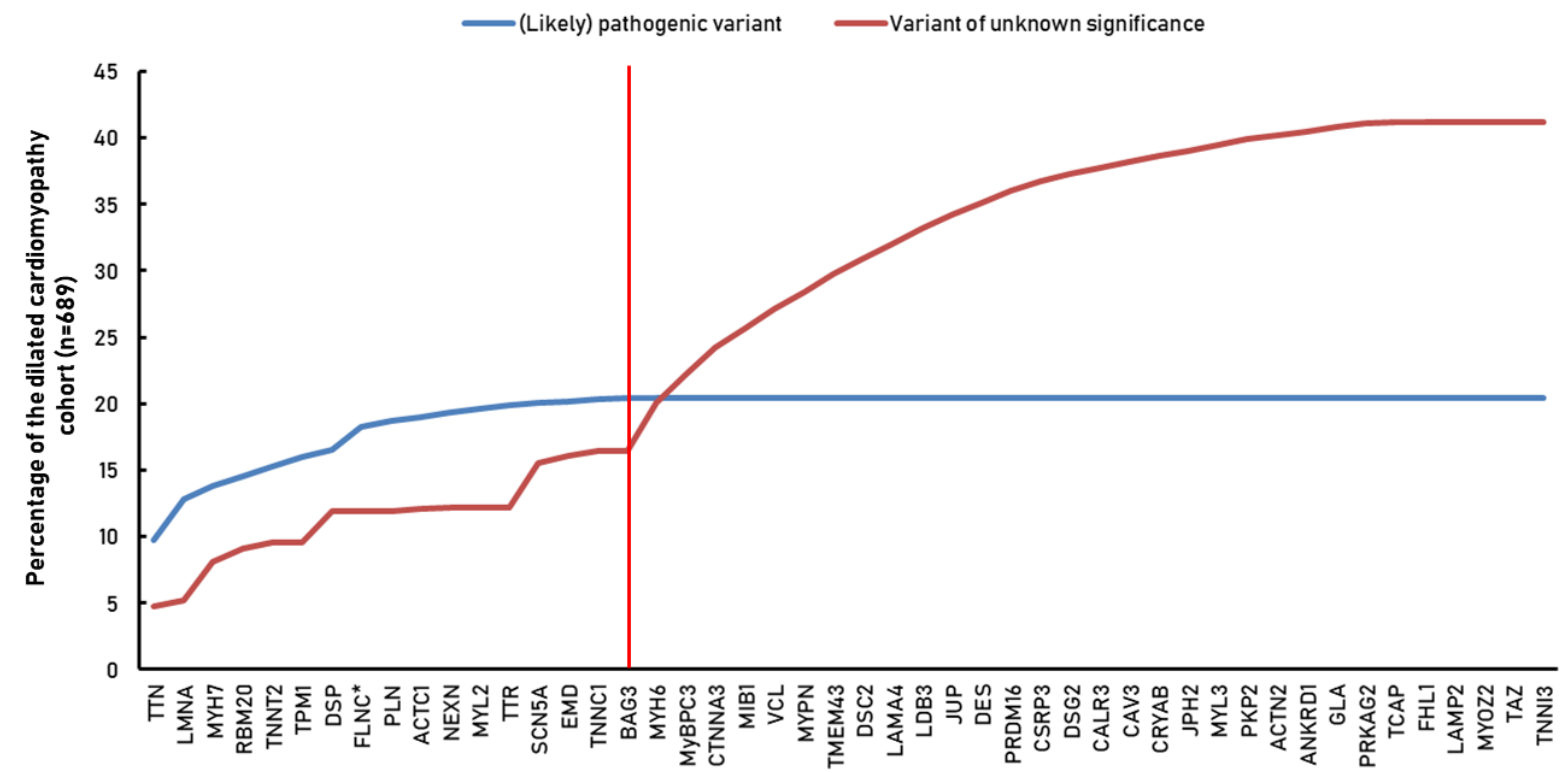

Figure 1. Increase in detection rate of pathogenic gene variants and VUSses with expanding the number of genes tested. The patient cohort and genetic data are described in chapter 2 . An asterisk indicates that the gene is only been tested in a selection of the total cohort (the 48-gene panel used since June 2018).

\section{Contribution of genetics to the clinical care of a dilated cardiomyopathy patient}

Genetic results are the cornerstone of every cardiomyopathy classification system ${ }^{13-15}$. Current practice tends to focus genetic testing largely on familial forms, in the absence of acquired diseases. In chapter $\mathbf{2}$, we performed genetic testing combined with in-depth phenotyping in 689 consecutive, unselected DCM patients. The overall genetic yield was 19\%, varying from $13 \%$ in non-familial to $36 \%$ in familial forms, and $18 \%$ in patients with an acquired cause of DCM. Clearly, neither the absence of a family history, nor the presence of an acquired cause excludes the chance of finding a (likely) pathogenic variant. Family segregation data in 36 non-familial DCM patients showed a relative with clinically asymptomatic DCM in 16 families (46\%). Limiting genetic testing to familial DCM patients would have missed these asymptomatic DCM patients in an early stage. Previous studies documented that a family history provided by patients is frequently incomplete and may miss familial DCM ${ }^{16}{ }^{17}$. Based on these findings, a negative familial history or the presence of an acquired cause should not exclude a DCM patient from genetic testing.

A positive familial history remains the strongest predictor of a pathogenic variant. In addition, electrical disturbances like atrial fibrillation (AF), non-sustained ventricular tachycardia (NSVT) and an atrioventricular block (AVB) are common findings in a genetic DCM patient. In chapter 2, we showed that the strong association between genetic variants and the electrical disturbances predict adverse outcome, especially for life-threatening arrythmias (LTA). The arrhythmogenic phenotype of many genes is becoming more evident ${ }^{18-21}$, which calls for reconsideration of recommendations in current guidelines. Early preventive implantable cardioverter-defibrillator (ICD) implantation is currently advised only for patients carrying a $L M N A$ variant ${ }^{22}$. Similar preventive measures may be warranted in carriers of other highly arrhythmogenic gene variants such as FLNC, RBM20, and TTN. The (arrhythmogenic) clinical phenotype of TTN was described in more detail in chapter 3. Holter monitoring at baseline showed a high prevalence of NSVT in DCM patients with a truncating TTN variant 
(TTNtv), in line with literature ${ }^{23-25}$. In a secondary analysis, DCM patients with a TTNtv appeared to have more LTA compared to DCM patients without TTNtv after a median of 45 months of follow-up. The long-term arrhythmic phenotype of TTNtv remains under debate, as there are studies confirming ${ }^{26}$, but also opposing this finding ${ }^{19}{ }^{27}$. The strong association with baseline NSVT and increased endomyocardial fibrosis can mechanistically explain the arrhythmic events in our cohort. To work towards guideline recommendations, future studies should focus on risk stratification for arrhythmic events: TTNtv can be an important arrhythmic risk factor within a subgroup of DCM patients ${ }^{26}$. A collective effort is needed to evaluate such multi-parametric risk stratification model in $D C M{ }^{28}$. A systematic review or meta-analysis could be the first step in evaluating the arrhythmic potential of TTNtv in various DCM subgroups. In chapter 4, we evaluate truncating variants in FLNC (FLNCtv), which were discovered and recognized as DCM-causing in $2016^{29}$. FLNCtv are found in DCM with high prevalence and penetrance. The clinical course is often malignant as confirmed by multiple studies ${ }^{29-32}$. FLNC-associated DCM is characterized by ventricular arrhythmias, myocardial fibrosis, and a high risk of sudden cardiac death, comparable to the clinical course of LMNA ${ }^{33}$ 34. The particular comparison with LMNA could indicate the potential benefit of early preventive ICD implantation in DCM patients carrying a FLNCtv. Currently, all evidence is at level B: small studies, retrospective studies and registry data. This thesis confirms the arrhythmogenic phenotype of FLNCtv-associated DCM by analyzing the phenotypes of all published FLNCtv in DCM. A large comparative study identifying the risk factors for malignant ventricular arrhythmias would be a next step forward ${ }^{34}$.

Preventing and reversing disease progression is the therapeutic goal in the short term, which is referred to as left ventricular reverse remodeling (LVRR) ${ }^{35}$. The impact of pathogenic gene variants on the potential to achieve LVRR was analyzed in chapter 6. Overall, the presence of a pathogenic variant is inversely correlated to the rate of LVRR, after adjusting for clinical phenotype, with the exception of TTNtv. In fact, TTNtv was positively associated with LVRR, in line with previous studies 23243637 . Despite the adequate response to current heart failure medication, some TTNtv DCM patients can have severe arrhythmic outcomes. Therefore, we need to determine the specific risk factors predicting LTA in TTNtv carriers, as previously done in $L M N A$ carriers to tailor recommendations regarding preventive measures in each specific patient.

Current observations and subsequent recommendations aim to treat the clinical consequences of genetic abnormalities, such as the high arrhythmic burden. In chapter 11 we reflect on ongoing research addressing genetics in DCM at different levels: targeting the molecular consequences or even treating the genetic perturbation itself. There is increased activity of the ERK1/2, JNK and p38 MAP kinases in $L M N A$-associated DCM ${ }^{38}$. Treatment with the specific $\mathrm{p} 38$ inhibitor ARRY-371797 prevented LVEF deterioration in LMNA-mutated mice. A phase 3 clinical trial is currently investigating the benefit of ARRY-371797 in LMNAassociated DCM patients (NCT03439514). In vivo genome editing with CRISPR/Cas9 restored dystrophic cardiomyopathy in a murine ${ }^{39}$ and pig model ${ }^{40}$. Although these results are promising and will provide therapeutic options guided by genetics, there are still many questions unanswered before clinical implementation. These include the safety, specificity and efficacy of the specific therapies.

\section{Dilated cardiomyopathy is not just a monogenic disease}

The DCM Precision Medicine Study was initiated in 2017 to investigate the hypothesis that most DCM, whether familial or non-familial, has a genetic basis ${ }^{41}$. An impressive number of 
DCM (candidate) genes were described over the past 20 years. Now we are re-appraising the contribution of each of these genes to monogenic DCM ${ }^{1}$. The proportion of patients with monogenic dominant forms is about $20 \%$ of the DCM patient population, as described in chapter 2. This does not exclude a genetic contribution to disease susceptibility in the other DCM patients, but puts it in a more interesting and challenging perspective.

The disease penetrance of established DCM-associated genes is apparently high enough to lead to DCM. Variants in genes with a lesser etiological contribution may require additional stressors to contribute to DCM development. These stressors could be genetic, environmental and/or metabolic. Collectively, these less penetrant variants could also alter disease expression in monogenic DCM in an oligogenic fashion. For example, TTNtv have a relative high penetrance in DCM relatives, with lower penetrance in the general population 42. Our understanding of factors that determine penetrance, whether genetic or non-genetic, is limited but evolving. In chapter 5 we applied an innovative bio-informatic tool to find novel genes associated with DCM. In a cohort of 142 DCM patients studied by whole exome sequencing, we did not find any variant which could explain the observed phenotype, confirming that most genes causing highly penetrant DCM have been identified already. We did find a variant in PDLIM5 which seemed to modulate disease expression in a family with DCM due to TTNtv. This could be an example how lower penetrant variants can contribute to the phenotype.

Unraveling the exact etiological contribution per gene and (environmental) stressor can be approached from several angles. One possibility is to focus on a specific subgroup of DCM patients with a single known etiological factor. For example, efforts have been made to unravel the genetic susceptibility to myocarditis leading to DCM ${ }^{43}{ }^{44}$. Another option would be to explore specific genetic models. Recently, non-inherited somatic mutation in circulating (immune) cells have been associated with increased inflammation and subsequent cardiovascular risk ${ }^{45-47}$. The role of such somatic mutations in the development of (inflammatory) DCM is yet to be explored.

A genome-wide association study (GWAS) is an unbiased case-control approach to find common genetic variants that each contribute only a small fraction to the likelihood that an individual develops a specific disease ${ }^{48-50}$. So far, DCM GWAS studies have been underpowered, and identified susceptibility variants in both cardiac and non-cardiac genes. Because these variants collectively explain only a very small fraction of the total genetic burden (heritability) they cannot be applied in a clinical setting. In the field of coronary artery disease (CAD), genetic variants identified with much larger GWAS sample sizes, are sufficiently powered to allow translation to polygenic risk scores (PRS): a score reflecting the risk of an individual for CAD based on their genetic profile ${ }^{5152}$. PRS can be calculated from thousands to millions of genetic variants, weighted by variant-level strength of association with disease. The first results and applications are promising, providing an outlook for polygenic applications in DCM, once large enough cohorts have been amassed.

The gene-environment concept presumes that an additional non-genetic stressor is needed to unfold the disease in carriers of a pathogenic variant ${ }^{53}$. However, the definition of a non-genetic stressor is very broad and yet undefined. This makes it difficult to recognize and quantify these stressors in a patient. For instance, cardiotoxic chemotherapy and coronary artery disease are clearly defined, in contrast to obesity and exercise which are not regularly scored as a potential contributor 545556 . Clear definitions, cut-off values and recommendations on which non-genetic stressors to investigate are lacking but crucial, 
rendering it difficult to achieve a complete overview of gene-environment interactions in DCM.

\section{Patient clustering}

We are making advances in comprehending the clinical characteristics and subsequent therapeutic implications of the various forms of monogenic DCM, as discussed in Part I. In Part II of this thesis, we aimed to add the genotype-phenotype knowledge to the classical definition of DCM, thereby integrating (genetic or acquired) etiology, cardiac function, disease stage and comorbidities in order to classify and stratify subgroups on a more patient-specific level.

DCM is a highly heterogeneous disease, both in terms of etiology and phenotypic manifestations. The phenotype develops as a final response to genetic and acquired disease triggers as described in chapter $11^{535758}$. Many trials investigating etiology-directed therapies were published in the past decades, but almost none is implemented in treatment guidelines of DCM patients. The lack of implementation is mainly due to the absence of validation in multicenter trials, but also due to under-recognition of the complete complexity of DCM. In the PercutaneOus StEm Cell Injection Delivery Effects On Neomyogenesis in Dilated CardioMyopathy (POSEIDON-DCM) study, patients were included without further phenotyping ${ }^{5960}$. A post-hoc analysis of the POSEIDON-DCM study showed that the presence of a pathogenic gene variant played an important role in the responsiveness to the investigated therapy ${ }^{61}$. The finding emphasizes the inadequacy of grouping patients on only etiological or functional parameters, and encourages the integration of clinical parameters to comprehend the disease and corresponding management.

In $18 \%$ of the patients with an acquired trigger, we also found a (likely) pathogenic variant (chapter 2), in line with the literature showing increasing overlap between different DCM etiologies 43566263 . An underlying genetic predisposition can negatively influence the response to etiology-directed therapies, emphasizing the interaction between genes and acquired diseases ${ }^{61}$. However, the exact contribution per gene and/or acquired disease to the final phenotype is not embraced by the current concept of gene-environment interactions, i.e. the variable penetrance per gene is not taken into account. Besides the etiology, the phenotypical presentation (e.g. presence or absence of electrical phenotypes, degree of chamber enlargement) and clinical course (e.g. response to conventional medical therapy) differ greatly among patients, as depicted in chapter 2 and chapter $\mathbf{6}$. We attempted to catch the heterogeneity by using machine learning methods on phenotypical data of DCM patients in chapter 8 . Four subgroups of DCM patients were proposed. Two of them represent opposite poles of severity, and two groups appear to have distinct clinical features: [1] a high rate of auto-immune diseases, and [2] a high prevalence of pathogenic gene variants (57\%) and associated cardiac arrhythmias. Classifying a patient in a specific subgroup had prognostic implications: both distinct subgroups had a significantly higher event rate compared to the DCM patients with mild systolic dysfunction. In chapter 2, we highlighted the adverse prognosis of DCM patients who carry a pathogenic gene variant. The benefit of applying advanced cluster methods is that it classifies genetic DCM in separate groups, with associated prognostic differences, thus integrating etiological and phenotypical factors. Future research should focus on the potential biological differences among identified subgroups, as this may inform personalized treatment strategies. 


\section{From genetic testing to RNA sequencing}

RNA plays a role in numerous biological processes. Next-generation sequencing (NGS) technologies of RNA can improve the diagnosis and treatment of human disease ${ }^{64-66}$. Although RNA-sequencing holds great promise, considerable hurdles need to be overcome before it can be implemented in a clinical laboratory setting ${ }^{64}$. In this thesis, we explored the potential of messenger RNA (mRNA) sequencing to provide underlying biological information in DCM subgroups.

The clinical implications of a truncating titin variant (TTNtv) were described in chapter 3. Insights from myocardial mRNA sequencing demonstrated up-regulation of the mitochondrial electron transport chain (ETC) in TTNtv hearts, without affecting other building blocks of cardiac energy metabolism. These findings could reflect an increased efficiency of mitochondrial ATP production trying to overcome a TTNtv-related sarcomeric defect ${ }^{67}$. The same molecular signature of increased ETC expression was found in TTNtv mutated rat hearts, although here it was associated with a cardiac metabolic shift towards anaerobic glycolysis ${ }^{68}$. Mitochondrial abnormalities, including decreased mitochondrial respiration, increased reactive oxygen species and increased levels of mitochondrial protein ubiquitination were observed in these rat hearts ${ }^{69}$. The observed metabolic changes are thought to represent adaptive mechanisms to the sarcomere deficit, keeping the heart in a compensated state inflexible to further stress ${ }^{68} 69$. Introduction of additional stress factors, such as cardiotoxic chemotherapy or pressure overload, then uncover the TTNtv phenotype 425662 . The observed metabolic phenotype and accompanying changes in signaling pathways provide a target for therapy in TTNtv patients. As a first step, the effect of cardiac stress on mitochondrial function TTNtv hearts needs further investigation. It has been shown that most individuals who carry a TTNtv do not develop DCM under conditions of increased hemodynamical stress, such as during pregnancy. The variable penetrance and expressivity suggest that there are other (unidentified) genetic and environmental factors accounting for the interindividual variation in susceptibility. Another explanation is that there could be two distinct populations of TTNtv, one more disease-causing compared to the other, which was further explored in chapter 7.

We related the transcriptomic profile of genetic DCM patients to the phenotype in chapter 7. There were two major findings of this study. The first observation was the distinction in subgroups of TTNtv DCM patients based on their transcriptomic profile. This translated to significant differences in clinical presentation: one subgroup had a milder disease course starting at a younger age, and the other subgroup had a more malignant course with prominent electrical disturbances, comparable to $L M N A$-associated DCM. It is possible, that these clinical presentations could reflect different stages of disease. The transcriptomic profiles of other genetic subgroups clustered separately from both TTNtv subgroups, despite having similarities in phenotype. The second observation was the distinct transcriptomic profiles of TTNtv and LMNA DCM patients, despite clinical similarities to other pathogenic variant carriers such as RBM2O and MYH7. The gene-specific transcriptomic profiles can be a first lead towards altered signaling pathways which can be modulated to improve cardiac function.

In chapter 9 we showed that a primary tissue diagnosis improved the prediction of an individual patient's disease trajectory: immunohistochemical cardiac inflammation was a negative predictor of therapy response in DCM patients receiving cardiac resynchronization therapy (CRT). We used transcriptomics to validate the tissue activity beyond the observed histology. DCM patients who did not respond sufficiently to CRT had higher expression of proinflammatory genes in their hearts compared to the responders. Thus multi-level (histological 
and transcriptomic) signs of inflammation correspond to a non-response to CRT in DCM patients. Current guidelines recommend CRT based on 3 clinical parameters (heart rhythm, left bundle branch block and NYHA-class) ${ }^{70}$. Machine learning tools including up to 9 clinical parameters did not improve response prediction beyond guideline recommendations ${ }^{71}$. Our proposed tissue diagnosis could offer additional insight in non-response to CRT. Future studies should focus on the modifiability of the tissue pathology, and the possibility to turn a non-responder into a responder.

One big opportunity of RNA-sequencing, with immediate clinical utility, is the detection and interpretation of genetic variants in undiagnosed, suspected genetic conditions. RNA-sequencing can validate candidate splice-disrupting variants, and identify splice-altering variants in both exonic and deep-intronic regions ${ }^{72-74}$. In chapter 2 , we show that analysis of $(\mathrm{m}) \mathrm{RNA}$ can validate the implications of novel splice-disrupting variants in well-known DCM associated genes, aiding the classification of three splice variants (LMNA c.357-2A>G and c.810G $>$ A; TNNC1 c.317+1G>A). However, RNA-sequencing can also be of great potential in finding a genetic cause in 'unsolved' familial cardiomyopathies ${ }^{75} 76$. Whole genome sequencing (WGS) together with RNA-sequencing identified additional genetic causes in $42 \%$ of unsolved hypertrophic cardiomyopathy (HCM) families ${ }^{77}$. This approach is yet to be explored for DCM.

The combination of genomics with transcriptomics creates a valuable synergy in DCM research. Metabolomics is emerging as an important tool that can aid clinicians in better understanding the pathogenesis of $\mathrm{DCM}^{78}$, and can provide additional information beyond DNA and RNA. Metabolomic analysis has been used to characterize metabolism in a LMNA mouse model of DCM ${ }^{79}$, and to functionally test the altered transcriptomic profile in TTNtv rat hearts ${ }^{68}$ or pathogenic variants in genes implicated in metabolic pathways ${ }^{80}$. We explored the potential of metabolomics to refine DCM diagnostics in chapter 10. Metabolomics does accurately reflect the severity of cardiac failure in DCM patients. Our results suggest a combined impairment of fatty acid and ketone body metabolism in symptomatic DCM, which is less pronounced when symptoms are absent. Identifying metabolic impairments can refine heart failure phenotypes, enhance risk stratification, and eventually identify therapeutic targets 8182 . However, while these results confirm the potential of metabolomics, clinical application is still many steps away ${ }^{82}$.

Trying to integrate the complete -omics-spectrum (from genomics to metabolomics) is commendable, and could provide a more complete view of the disease. However, multiomics approaches are extremely complex ${ }^{83}$ and would require large consortia such as the Heart Omics in AGEing (HOMAGE) ${ }^{84}$.

\section{Screening of asymptomatic family members}

The focus of Part III was on the (asymptomatic) relatives of DCM patients. The finding of a genetic etiology in DCM patients can have profound impact for their relatives. In chapter 2 we showed that family segregation can detect DCM in individuals who have no symptoms. We also detected relatives carrying the familial variant without having a phenotype (yet). In the absence of reliable disease penetrance per gene and per variant, all (first-degree) relatives of DCM patients should undergo regular cardiac evaluation, irrespective of a genetic etiology 168586 . The frequency of serial follow-up remains unclear, especially when there are no cardiac abnormalities during screening. In addition, the sensitivity of left ventricular ejection fraction (LVEF) to detect early disease in the DCM spectrum is questionable. Longitudinal strain is a measure of the active shortening of the LV in the longitudinal direction, representing the 
function of subendocardial myocardial fibers. These fibers are sensitive to reduced coronary perfusion and increased wall stress ${ }^{8788}$. Therefore longitudinal dysfunction may be a better marker for early disease compared to LVEF, which mainly reflects the radial contraction.

The value of global longitudinal strain (GLS) analysis in DCM relatives with a normal left ventricular ejection fraction (LVEF) was evaluated in chapter 12. Relatives of patients with DCM had more often impaired GLS compared to a matched cohort with normal LVEF ( $48 \%$ vs. 33\%). Impaired GLS at baseline was associated with a decrease in LVEF during follow-up, potentially indicating that impaired GLS precedes a decrease in LVEF. These findings support a role for GLS in the screening of asymptomatic relatives: it could imply that relatives with abnormal GLS would need to undergo more regular screening compared to relatives with normal GLS. Although GLS shows promise in different clinical scenarios ${ }^{89-92}$, several issues remain. Strain is an unitless measure and the values and normal ranges are still highly dependent on the used software ${ }^{93}$. Standardization of strain measurement and clear cut-off values independent of used software will be required before clinical implementation ${ }^{94} 95$. In addition, the field needs to reach consensus about the therapeutic implications of an abnormal GLS. There are two possible scenarios: [1] shorter time intervals for cardiac screening or [2] start of heart failure therapy. Waiting until the LVEF declines to start with medication, could miss a valuable opportunity for treatment in the 'early' disease stage. As we showed in chapter 12 that early detection of disease is possible, future research should aim to test whether we can prevent LVEF deterioration and improve outcome by starting treatment early. 


\section{REFERENCES}

1. Mazzarotto F, Tayal U, Buchan RJ, et al. Reevaluating the Genetic Contribution of Monogenic Dilated Cardiomyopathy. Circulation 2020;141(5):387-98.

2. McNally EM, Mestroni L. Dilated Cardiomyopathy: Genetic Determinants and Mechanisms. Circulation research 2017;121(7):731-48.

3. Lek M, Karczewski KJ, Minikel EV, et al. Analysis of protein-coding genetic variation in 60,706 humans. Nature 2016;536(7616):285-91.

4. Karczewski KJ, Weisburd B, Thomas B, et al. The ExAC browser: displaying reference data information from over 60000 exomes. Nucleic Acids Res 2017;45(D1):D840-d45.

5. Walsh R, Thomson KL, Ware JS, et al. Reassessment of Mendelian gene pathogenicity using 7,855 cardiomyopathy cases and 60,706 reference samples. Genetics in medicine : official journal of the American College of Medical Genetics 2017;19(2):192-203.

6. Richards S, Aziz N, Bale S, et al. Standards and guidelines for the interpretation of sequence variants: a joint consensus recommendation of the American College of Medical Genetics and Genomics and the Association for Molecular Pathology. Genetics in medicine : official journal of the American College of Medical Genetics 2015;17(5):405-24.

7. Akinrinade $\mathrm{O}$, Ollila L, Vattulainen S, et al. Genetics and genotype-phenotype correlations in Finnish patients with dilated cardiomyopathy. Eur Heart $J$ 2015;36(34):2327-37.

8. Dal Ferro $M$, Stolfo $D$, Altinier A, et al. Association between mutation status and left ventricular reverse remodelling in dilated cardiomyopathy. Heart (British Cardiac Society) 2017

9. Haas J, Frese KS, Peil B, et al. Atlas of the clinical genetics of human dilated cardiomyopathy. Eur Heart J 2015;36(18):1123-35a.

10. Ho CY, Day SM, Ashley EA, et al. Genotype and Lifetime Burden of Disease in Hypertrophic Cardiomyopathy: Insights from the Sarcomeric Human Cardiomyopathy Registry (SHaRe). Circulation 2018;138(14):1387-98.

11. Ingles J, Goldstein J, Thaxton C, et al. Evaluating the Clinical Validity of Hypertrophic Cardiomyopathy Genes. Circulation Genomic and precision medicine 2019;12(2):e002460.

12. Walsh R, Buchan R, Wilk A, et al. Defining the genetic architecture of hypertrophic cardiomyopathy: re-evaluating the role of nonsarcomeric genes. Eur Heart J 2017;38(46):346168.
13. Maron BJ, Towbin JA, Thiene G, et al. Contemporary definitions and classification of the cardiomyopathies: an American Heart Association Scientific Statement from the Council on Clinical Cardiology, Heart Failure and Transplantation Committee; Quality of Care and Outcomes Research and Functional Genomics and Translational Biology Interdisciplinary Working Groups; and Council on Epidemiology and Prevention. Circulation 2006;113(14):1807-16.

14. Elliott $P$, Andersson B, Arbustini $E$, et al. Classification of the cardiomyopathies: a position statement from the European Society of Cardiology Working Group on Myocardial and Pericardial Diseases. Eur Heart J 2008;29(2):270-6.

15. Arbustini E, Narula N, Tavazzi L, et al. The MOGE(S) classification of cardiomyopathy for clinicians. J Am Coll Cardiol 2014;64(3):304-18.

16. Hershberger RE, Givertz MM, Ho CY, et al. Genetic Evaluation of Cardiomyopathy-A Heart Failure Society of America Practice Guideline. J Card Fail 2018;24(5):281-302.

17. Waddell-Smith KE, Donoghue T, Oates $S$, et al. Inpatient detection of cardiac-inherited disease: the impact of improving family history taking. Open Heart 2016;3(1):e000329.

18. Cannatà A, De Angelis G, Boscutti A, et al. Arrhythmic risk stratification in non-ischaemic dilated cardiomyopathy beyond ejection fraction. Heart (British Cardiac Society) 2020;106(9):656-64.

19. Gigli M, Merlo M, Graw SL, et al. Genetic Risk of Arrhythmic Phenotypes in Patients With Dilated Cardiomyopathy. J Am Coll Cardiol 2019;74(11):1480-90.

20. McNally EM, Amaral AP. Predicting Arrhythmia Risk in Dilated Cardiomyopathy Using Genetic Mutation Status. J Am Coll Cardiol 2019;74(11):1491-93.

21. Peters S, Kumar S, Elliott $P$, et al. Arrhythmic Genotypes in Familial Dilated Cardiomyopathy: Implications for Genetic Testing and Clinical Management. Heart, lung \& circulation 2019;28(1):31-38.

22. Priori SG, Blomstrom-Lundqvist C, Mazzanti A, et al. 2015 ESC Guidelines for the management of patients with ventricular arrhythmias and the prevention of sudden cardiac death: The Task Force for the Management of Patients with Ventricular Arrhythmias and the Prevention of Sudden Cardiac Death of the European Society of Cardiology (ESC). Endorsed by: Association for European Paediatric and Congenital Cardiology (AEPC). Eur Heart J 2015;36(41):2793-867.

23. Jansweijer JA, Nieuwhof K, Russo F, et al. Truncating titin mutations are associated with a mild and treatable form of dilated 
cardiomyopathy. Eur J Heart Fail 2016;19(4):51221.

24. Roberts AM, Ware JS, Herman DS, et al. Integrated allelic, transcriptional, and phenomic dissection of the cardiac effects of titin truncations in health and disease. Science translational medicine 2015;7(270):270ra6.

25. Tayal U, Newsome S, Buchan R, et al. Truncating Variants in Titin Independently Predict Early Arrhythmias in Patients With Dilated Cardiomyopathy. J Am Coll Cardiol 2017;69(19):2466-68.

26. Corden B, Jarman J, Whiffin N, et al. Association of Titin-Truncating Genetic Variants With Lifethreatening Cardiac Arrhythmias in Patients With Dilated Cardiomyopathy and Implanted Defibrillators. JAMA Netw Open 2019;2(6):e196520.

27. Tayal U, Newsome S, Buchan R, et al. Phenotype and Clinical Outcomes of Titin Cardiomyopathy. $J$ Am Coll Cardiol 2017;70(18):2264-74.

28. Tayal U, Prasad SK. Titin cardiomyopathy: why we need to go big to understand the giant. Eur Heart $J$ 2018;39(10):874-75.

29. Ortiz-Genga MF, Cuenca S, Dal Ferro $M$, et al. Truncating FLNC Mutations Are Associated With High-Risk Dilated and Arrhythmogenic Cardiomyopathies. J Am Coll Cardiol 2016;68(22):2440-51.

30. Begay RL, Graw SL, Sinagra G, et al. Filamin C Truncation Mutations Are Associated With Arrhythmogenic Dilated Cardiomyopathy and Changes in the Cell-Cell Adhesion Structures. JACC Clinical electrophysiology 2018;4(4):504-14.

31. Sammani A, Kayvanpour E, Bosman LP, et al. Predicting sustained ventricular arrhythmias in dilated cardiomyopathy: a meta-analysis and systematic review. ESC heart failure 2020

32. Augusto JB, Eiros R, Nakou E, et al. Dilated cardiomyopathy and arrhythmogenic left ventricular cardiomyopathy: a comprehensive genotype-imaging phenotype study. European heart journal cardiovascular Imaging 2020;21(3):326-36.

33. Hasselberg NE, Haland TF, Saberniak J, et al. Lamin A/C cardiomyopathy: young onset, high penetrance, and frequent need for heart transplantation. Eur Heart J 2017

34. van Rijsingen IA, Arbustini E, Elliott PM, et al. Risk factors for malignant ventricular arrhythmias in lamin a/c mutation carriers a European cohort study. J Am Coll Cardiol 2012;59(5):493-500.

35. Merlo $M$, Pyxaras SA, Pinamonti $B$, et al. Prevalence and prognostic significance of left ventricular reverse remodeling in dilated cardiomyopathy receiving tailored medical treatment. J Am Coll Cardiol 2011;57(13):1468-76.
36. Luk K, Bakhsh A, Giannetti N, et al. Recovery in Patients With Dilated Cardiomyopathy With Lossof-Function Mutations in the Titin Gene. JAMA Cardiol 2017;2(6):700-02.

37. Felkin LE, Walsh R, Ware JS, et al. Recovery of Cardiac Function in Cardiomyopathy Caused by Titin Truncation. JAMA Cardiol 2016;1(2):234-5.

38. Muchir A, Wu W, Choi JC, et al. Abnormal p38alpha mitogen-activated protein kinase signaling in dilated cardiomyopathy caused by lamin A/C gene mutation. Human molecular genetics 2012;21(19):4325-33.

39. El Refaey M, Xu L, Gao Y, et al. In Vivo Genome Editing Restores Dystrophin Expression and Cardiac Function in Dystrophic Mice. Circulation research 2017;121(8):923-29.

40. Moretti A, Fonteyne L, Giesert F, et al. Somatic gene editing ameliorates skeletal and cardiac muscle failure in pig and human models of Duchenne muscular dystrophy. Nat Med 2020;26(2):207-14.

41. Kinnamon DD, Morales A, Bowen DJ, et al. Toward Genetics-Driven Early Intervention in Dilated Cardiomyopathy: Design and Implementation of the DCM Precision Medicine Study. Circulation Cardiovascular genetics 2017;10(6)

42. Ware JS, Cook SA. Role of titin in cardiomyopathy: from DNA variants to patient stratification. Nature reviews Cardiology 2018;15(4):241-52.

43. Belkaya S, Kontorovich AR, Byun $M$, et al. Autosomal Recessive Cardiomyopathy Presenting as Acute Myocarditis. I Am Coll Cardiol 2017;69(13):1653-65.

44. Dou $Q$, Peng $Y$, Zhou $B$, et al. Association of innate immune IFIH1 gene polymorphisms with dilated cardiomyopathy in a Chinese population. Immunological investigations 2014;43(7):627-37.

45. Jaiswal S, Libby P. Clonal haematopoiesis: connecting ageing and inflammation in cardiovascular disease. Nature reviews Cardiology 2020;17(3):137-44.

46. Jaiswal S, Natarajan P, Silver AJ, et al. Clonal Hematopoiesis and Risk of Atherosclerotic Cardiovascular Disease. $N$ Engl J Med 2017;377(2):111-21.

47. Fuster JJ, Walsh K. Somatic Mutations and Clonal Hematopoiesis: Unexpected Potential New Drivers of Age-Related Cardiovascular Disease. Circulation research 2018;122(3):523-32.

48. Meder B, Ruhle F, Weis $T$, et al. A genome-wide association study identifies $6 \mathrm{p} 21$ as novel risk locus for dilated cardiomyopathy. Eur Heart J 2014;35(16):1069-77.

49. Villard E, Perret C, Gary F, et al. A genome-wide association study identifies two loci associated with heart failure due to dilated cardiomyopathy. Eur Heart J 2011;32(9):1065-76. 
50. Stark K, Esslinger UB, Reinhard W, et al. Genetic association study identifies HSPB7 as a risk gene for idiopathic dilated cardiomyopathy. PLOS genetics 2010;6(10):e1001167.

51. Elliott J, Bodinier B, Bond TA, et al. Predictive Accuracy of a Polygenic Risk Score-Enhanced Prediction Model vs a Clinical Risk Score for Coronary Artery Disease. Jama 2020;323(7):63645.

52. Inouye M, Abraham G, Nelson CP, et al. Genomic Risk Prediction of Coronary Artery Disease in 480,000 Adults: Implications for Primary Prevention. J Am Coll Cardiol 2018;72(16):1883-93.

53. Piran S, Liu P, Morales A, et al. Where genome meets phenome: rationale for integrating genetic and protein biomarkers in the diagnosis and management of dilated cardiomyopathy and heart failure. J Am Coll Cardiol 2012;60(4):283-9.

54. Fumagalli C, Maurizi N, Day SM, et al. Association of Obesity With Adverse Long-term Outcomes in Hypertrophic Cardiomyopathy. JAMA Cardiol 2019;5(1):1-8.

55. Skjølsvik ET, Hasselberg NE, Dejgaard LA, et al. Exercise is Associated With Impaired Left Ventricular Systolic Function in Patients With Lamin A/C Genotype. Journal of the American Heart Association 2020;9(2):e012937.

56. Garcia-Pavia P, Kim Y, Restrepo-Cordoba MA, et al. Genetic Variants Associated With Cancer TherapyInduced Cardiomyopathy. Circulation 2019;140(1):31-41.

57. Verdonschot JAJ, Hazebroek MR, Ware JS, et al. Role of Targeted Therapy in Dilated Cardiomyopathy: The Challenging Road Toward a Personalized Approach. Journal of the American Heart Association 2019;8(11):e012514.

58. Hazebroek MR, Moors S, Dennert R, et al. Prognostic Relevance of Gene-Environment Interactions in Patients With Dilated Cardiomyopathy: Applying the MOGE(S) Classification. J Am Coll Cardiol 2015;66(12):131323.

59. Hare JM, Fishman JE, Gerstenblith G, et al. Comparison of allogeneic vs autologous bone marrow-derived mesenchymal stem cells delivered by transendocardial injection in patients with ischemic cardiomyopathy: the POSEIDON randomized trial. Jama 2012;308(22):2369-79.

60. Hare JM, DiFede DL, Rieger AC, et al. Randomized Comparison of Allogeneic Versus Autologous Mesenchymal Stem Cells for Nonischemic Dilated Cardiomyopathy: POSEIDON-DCM Trial. J Am Coll Cardiol 2017;69(5):526-37.

61. Rieger AC, Myerburg RJ, Florea V, et al. Genetic determinants of responsiveness to mesenchymal stem cell injections in non-ischemic dilated cardiomyopathy. EBioMedicine 2019;48:377-85.
62. Ware JS, Amor-Salamanca A, Tayal U, et al. Genetic Etiology for Alcohol-Induced Cardiac Toxicity. J Am Coll Cardiol 2018;71(20):2293-302.

63. Ware JS, Li J, Mazaika E, et al. Shared Genetic Predisposition in Peripartum and Dilated Cardiomyopathies. N Engl J Med 2016;374(3):23341.

64. Byron SA, Van Keuren-Jensen KR, Engelthaler DM, et al. Translating RNA sequencing into clinical diagnostics: opportunities and challenges. Nature reviews Genetics 2016;17(5):257-71.

65. Buzdin A, Sorokin M, Garazha A, et al. RNA sequencing for research and diagnostics in clinical oncology. Seminars in cancer biology 2020;60:31123.

66. Lu D, Thum T. RNA-based diagnostic and therapeutic strategies for cardiovascular disease. Nature reviews Cardiology 2019;16(11):661-74.

67. Hinson JT, Chopra A, Nafissi N, et al. HEART DISEASE. Titin mutations in iPS cells define sarcomere insufficiency as a cause of dilated cardiomyopathy. Science (New York, NY) 2015;349(6251):982-6.

68. Schafer S, de Marvao A, Adami E, et al. Titintruncating variants affect heart function in disease cohorts and the general population. Nature genetics 2017;49(1):46-53.

69. Zhou J, Ng B, Ko NSJ, et al. Titin truncations lead to impaired cardiomyocyte autophagy and mitochondrial function in vivo. Human molecular genetics 2019;28(12):1971-81.

70. Cowie MR, Sarkar S, Koehler J, et al. Development and validation of an integrated diagnostic algorithm derived from parameters monitored in implantable devices for identifying patients at risk for heart failure hospitalization in an ambulatory setting. Eur Heart J 2013;34(31):2472-80.

71. Feeny AK, Rickard J, Patel D, et al. Machine Learning Prediction of Response to Cardiac Resynchronization Therapy: Improvement Versus Current Guidelines. Circulation Arrhythmia and electrophysiology 2019;12(7):e007316.

72. Cummings BB, Marshall JL, Tukiainen T, et al. Improving genetic diagnosis in Mendelian disease with transcriptome sequencing. Science translational medicine 2017;9(386)

73. Lee $H$, Huang $A Y$, Wang LK, et al. Diagnostic utility of transcriptome sequencing for rare Mendelian diseases. Genetics in medicine : official journal of the American College of Medical Genetics 2020;22(3):490-99.

74. Gonorazky HD, Naumenko S, Ramani AK, et al. Expanding the Boundaries of RNA Sequencing as a Diagnostic Tool for Rare Mendelian Disease. American journal of human genetics 2019;104(3):466-83. 
75. Ribeiro $M$, Furtado $M$, Martins S, et al. RNA Splicing Defects in Hypertrophic Cardiomyopathy: Implications for Diagnosis and Therapy. Int J Mol Sci 2020;21(4)

76. Mendes de Almeida R, Tavares J, Martins S, et al. Whole gene sequencing identifies deep-intronic variants with potential functional impact in patients with hypertrophic cardiomyopathy. PLoS One 2017;12(8):e0182946.

77. Bagnall RD, Ingles J, Dinger ME, et al. Whole Genome Sequencing Improves Outcomes of Genetic Testing in Patients With Hypertrophic Cardiomyopathy. J Am Coll Cardiol 2018;72(4):41929.

78. Ussher JR, Elmariah S, Gerszten RE, et al. The Emerging Role of Metabolomics in the Diagnosis and Prognosis of Cardiovascular Disease. J Am Coll Cardiol 2016;68(25):2850-70.

79. West JA, Beqqali A, Ament Z, et al. A targeted metabolomics assay for cardiac metabolism and demonstration using a mouse model of dilated cardiomyopathy. Metabolomics 2016;12:59.

80. Riemersma M, Hazebroek MR, Helderman-van den Enden A, et al. Propionic acidemia as a cause of adult-onset dilated cardiomyopathy. European journal of human genetics : EJHG 2017

81. Lanfear DE, Gibbs JJ, Li J, et al. Targeted Metabolomic Profiling of Plasma and Survival in Heart Failure Patients. JACC Heart Fail 2017;5(11):823-32.

82. Shah $\mathrm{SH}$, Hunter WG. Realizing the Potential of Metabolomics in Heart Failure: Signposts on the Path to Clinical Utility. JACC Heart Fail 2017;5(11):833-36.

83. Ahmad T, Fiuzat M, Pencina MJ, et al. Charting a roadmap for heart failure biomarker studies. JACC Heart Fail 2014;2(5):477-88.

84. Jacobs $L$, Thijs $L$, Jin $Y$, et al. Heart 'omics' in AGEing (HOMAGE): design, research objectives and characteristics of the common database. J Biomed Res 2014;28(5):349-59.

85. Charron P, Arad M, Arbustini E, et al. Genetic counselling and testing in cardiomyopathies: a position statement of the European Society of Cardiology Working Group on Myocardial and Pericardial Diseases. Eur Heart J 2010;31(22):271526.

86. Pinto YM, Elliott PM, Arbustini E, et al. Proposal for a revised definition of dilated cardiomyopathy, hypokinetic non-dilated cardiomyopathy, and its implications for clinical practice: a position statement of the ESC working group on myocardial and pericardial diseases. Eur Heart $J$ 2016;37(23):1850-8.

87. Prasad SK, Tayal U. The Value of Strain in Familial Dilated Cardiomyopathy Screening. JACC Cardiovasc Imaging 2020;13(2 Pt 2):559-61.
88. Buckberg G, Hoffman JI, Mahajan A, et al. Cardiac mechanics revisited: the relationship of cardiac architecture to ventricular function. Circulation 2008;118(24):2571-87.

89. Swat SA, Cohen D, Shah SJ, et al. Baseline Longitudinal Strain Predicts Recovery of Left Ventricular Ejection Fraction in Hospitalized Patients With Nonischemic Cardiomyopathy. Journal of the American Heart Association 2018;7(20):e09841.

90. Mignot A, Donal E, Zaroui A, et al. Global longitudinal strain as a major predictor of cardiac events in patients with depressed left ventricular function: a multicenter study. J Am Soc Echocardiogr 2010;23(10):1019-24.

91. Merken J, Brunner-La Rocca HP, Weerts J, et al. Heart Failure With Recovered Ejection Fraction. J Am Coll Cardiol 2018;72(13):1557-58.

92. Ersboll M, Valeur $N$, Mogensen UM, et al. Prediction of all-cause mortality and heart failure admissions from global left ventricular longitudinal strain in patients with acute myocardial infarction and preserved left ventricular ejection fraction. J Am Coll Cardiol 2013;61(23):2365-73.

93. Smiseth OA, Torp $H$, Opdahl A, et al. Myocardial strain imaging: how useful is it in clinical decision making? Eur Heart J 2016;37(15):1196-207.

94. Knackstedt C, Bekkers SC, Schummers G, et al. Fully Automated Versus Standard Tracking of Left Ventricular Ejection Fraction and Longitudinal Strain: The FAST-EFs Multicenter Study. J Am Coll Cardiol 2015;66(13):1456-66.

95. Voigt JU, Pedrizzetti G, Lysyansky $P$, et al. Definitions for a common standard for 2D speckle tracking echocardiography: consensus document of the EACVI/ASE/Industry Task Force to standardize deformation imaging. European heart journal cardiovascular Imaging 2015;16(1):1-11. 


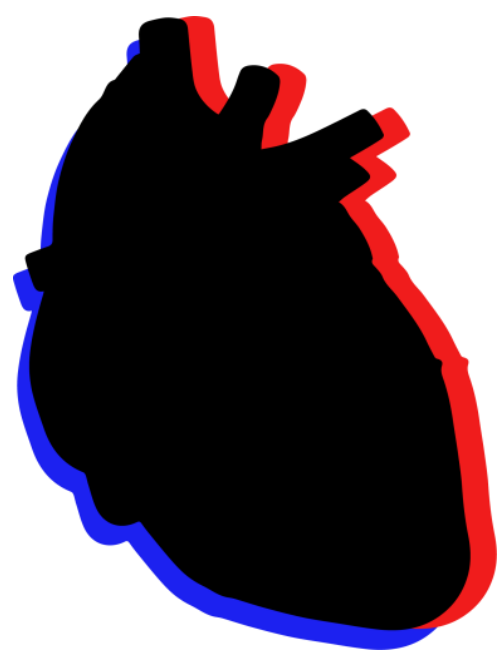

- 340 - 


\title{
CHAPTER 14
}

\author{
Summary \\ Nederlandse samenvatting \\ Valorization \\ Dankwoord \\ About the author \\ List of publications
}


- 342 - 


\section{SUMMARY}

Dilated cardiomyopathy (DCM) is a complex disease, caused by combined genetic and acquired triggers. Current definition of DCM is based solely on abnormalities in cardiac structure and function. Recommended treatment aims to reverse these cardiac abnormalities through standard heart failure therapy, treating DCM as a monomorphic disease. This does not capture the heterogeneous nature of DCM. A significant subgroup of DCM patients has a well-established genetic etiology, in which we now start to unravel the underlying pathophysiology and its clinical consequences. Currently, only the finding of a pathogenic LMNA variant may influence the clinical decision-making by the cardiologist. Yet, the genetic results do have great impact on families, as relatives are advised to undergo frequent cardiac screening to detect early cardiac abnormalities. Incomplete knowledge of disease penetrance and low sensitivity of cardiac screening tools make it difficult to specify the frequency of such screening.

Part I of this thesis discusses genetic testing in DCM, with a focus on genotypephenotype correlations. The overall genetic yield was $19 \%$ in an unselected, consecutive DCM cohort using a gene panel consisting of 48 genes (chapter 2 ). Limiting the number of genes to 17 genes did not affect the genetic yield, suggesting the necessity for re-evaluating the DCM gene panels as used in current clinical practice. Pathogenic variants are the most prevalent in titin (TTN), and are characterized by prevalent arrhythmias, increased interstitial fibrosis, but a relatively mild disease course with favorable treatment response (chapter 3 ). Truncating variants in filamin C (FLNC) have only recently been recognized as DCM-causing. DCM patients carrying pathogenic FLNC variants have a high risk for ventricular arrhythmias and cardiac sudden death (chapter 4). The arrhythmic risk of TTN and FLNC before left ventricular dysfunction develops, warrants collective efforts to evaluate multi-parametric risk stratification models. These may help to translate these clinical phenotypes into therapeutic consequences. FLNC may conclude the era of finding prevalent novel monogenic dominant forms of DCM. Less penetrant variants could alter disease expression in monogenic DCM in an oligogenic fashion. Such variants, as in PDLIM5, a gene encoding for the PDZ and LIM domain protein 5 located at the Z-disk, are candidates to alter disease expression in carriers of a variant that predisposes to disease in other genes, such as truncating variants in TTN or FLNC (chapter 5). Overall, the presence of a pathogenic genetic variant was negatively associated with treatment response, except for TTN variants (chapter 6).

Part II of this thesis links the novel genotype-phenotype knowledge from part I to the classical definition of DCM with the goal to integrate etiology, cardiac function, disease stage and comorbidities to redefine disease classification. Information from transcriptomics and metabolomics is added to provide an outlook for personalized treatment strategies. We detected a specific transcriptomic profile of $L M N A$ and TTN cardiomyopathies, and associated it with the clinical phenotype to define a more malignant sub-phenotype of TTN (chapter 7). The novel genotype-phenotype associations were put into a new perspective in chapter 8. Unsupervised clustering identified four phenogroups within DCM: two of them represent opposite poles of severity, and two of these represent distinct clinical subgroups with a high event rate irrespective of cardiac function. A primary tissue diagnosis using endomyocardial biopsies improved the prediction of therapy response in DCM patients undergoing cardiac resynchronization therapy (chapter 9). The finding of immunohistochemical cardiac inflammation in the heart was reflected in the cardiac transcriptome profile and associated 
negatively with treatment response. Metabolomics is emerging as an important tool that can aid clinicians in diagnosing disease, but also to validate genetic and transcriptomic findings. Disease severity in DCM is strongly reflected in the metabolomic profile (chapter 10). Future efforts should aim to integrate the metabolomic results with the clinical and genetic data to refine the DCM phenotypes, enhance risk stratification and identify novel therapeutic targets. Chapter 11 summarizes trials aimed at improving outcome in DCM by etiology-based treatment strategies. Few have led to implementation in the treatment guideline for DCM. Translation may still be hampered by under-recognition of the complexity of DCM in trials.

Part III of this thesis describes the implications of genetic testing for relatives of a DCM patient. Early detection of disease in (first-degree) relatives is the main rationale for performing genetic testing in DCM patients. Global longitudinal strain (GLS) analysis of echocardiographic images has a high sensitivity to detect early cardiac abnormalities in relatives with an otherwise normal left ventricular ejection fraction (LVEF) (chapter 12).

This thesis describes the clinical consequences of genetic testing in DCM (part I), integrates the genetic results with other etiologies and phenotypic manifestations to refine diagnosis and direct treatment of the disease (part II), and describes the impact of genetics on (first-degree) relatives of a patient (part III). The discussion of this thesis (chapter 13) aims to put the results in perspective of the current clinical situation, and to provide an outlook for the future of genetics in DCM. 


\section{NEDERLANDSE SAMENVATTING}

Een gedilateerde cardiomyopathie (DCM) is een complexe hartaandoening, veroorzaakt door een combinatie van genetische en verworven factoren. De huidige definitie van DCM is enkel gebaseerd op afwijkingen in cardiale structuur en functie. De aanbevolen behandeling richt zich op deze cardiale afwijkingen middels de standaard medicatie voor hartfalen. Zodoende wordt DCM als een monomorfe ziekte behandeld. Echter omvat dit niet de heterogene basis van DCM. Een significante subgroep van DCM patiënten heeft een genetische oorzaak, waarvan we nu de onderliggende pathofysiologie en klinische consequenties beginnen te ontdekken. Momenteel beïnvloed enkel de bevinding van een pathogene LMNA variant de klinische beslissingen die een cardioloog maakt. Echter hebben de genetische resultaten een grote impact op de familie, aangezien familieleden worden geadviseerd om regelmatig cardiale screening te ondergaan om zodoende cardiale afwijkingen in een vroeg stadium te ontdekken. Incomplete kennis van de ziekte-penetrantie en de lage sensitiviteit van cardiale screeningsinstrumenten maken het moeilijk om de frequentie van een dergelijke screening te specificeren.

Deel 1 van dit proefschrift bediscussieerd genetisch onderzoek in DCM, met name gericht op genotype-fenotype correlaties. De algehele opbrengst van genetisch onderzoek, wanneer we gebruik maken van een panel bestaande uit 48 genen, is $19 \%$ in een niet geselecteerd DCM cohort (hoofdstuk 2). Deze opbrengst wordt nauwelijks beïnvloedt wanneer we het aantal genen op het screeningspanel terugbrengen van 48 naar 17 genen. Dit suggereert dat we de bestaande klinische panels kritisch moeten re-evalueren. Pathogene varianten zijn het meest prevalent in titine (TTN), en worden gekarakteriseerd door frequente aritmieën, toegenomen cardiale fibrose, maar een relatief mild ziektebeloop met een goede response op behandeling (hoofdstuk 3). Truncerende varianten in filamine $C$ (FLNC) zijn recent erkend als ziekte-veroorzakend voor DCM. DCM patiënten met een pathogene FLNC variant hebben een hoog risico op ventriculaire aritmieën en plotse hartdood (hoofdstuk 4). Het aritmogene risico dat geassocieerd gaat met TTN en FLNC is aanwezig voordat de cardiale functie evident verslechterd. Collectieve samenwerking is noodzakelijk om een multiparametrisch risico model te ontwikkelen dat de patiënten die at-risk zijn beter kan identificeren. FLNC beëindigd waarschijnlijk het tijdperk van nieuwe, prevalente, monogene dominante vormen van DCM. Echter kunnen varianten met een lagere penetrantie de ziekteexpressie beïnvloeden van monogene DCM op een oligogene manier. Dergelijke varianten, zoals in PDLIM5 (een gen dat codeert voor PDZ and LIM domain protein 5 welke gelokaliseerd is bij de Z-disk), zijn kandidaten die de ziekte-expressie kunnen beïnvloeden in dragers van een predisponerende DCM variant in een ander gen, zoals TTN en FLNC (hoofdstuk 5). In het algemeen wordt de aanwezigheid van een pathogene genetische variant geassocieerd met een lagere respons op medische behandeling, behalve bij TTN varianten (hoofdstuk 6).

Deel 2 van dit proefschrift combineert de nieuwe genotype-fenotype kennis vergaard in deel 1 met de klassieke definitie van DCM met als doel het integreren van etiologie, cardiale functie, stadium van de ziekte en comorbiditeiten om zo de DCM classificatie te re-definiëren. Informatie van transcriptomics en metabolomics is toegevoegd om te werken naar een gepersonaliseerd behandelplan voor de patiënt. We ontdekten een specifiek transcript profiel in patiënten met een LMNA en TTN geassocieerde cardiomyopathie, welke geassocieerd werd met het klinisch fenotype om onderscheid te maken tussen verschillende TTN fenotypes (hoofdstuk 7). Deze nieuwe genotype-fenotype associaties zijn in een nieuw perspectief 
gebracht in hoofdstuk 8. Met behulp van clustering technieken werden vier fenogroepen geïdentificeerd in de DCM populatie: twee waren tegenovergestelde groepen in termen van ernst van hartfalen, en twee waren specifieke klinische subgroepen met een slechte prognose onafhankelijk van cardiale functie. Een primaire weefseldiagnose met endomyocardiale biopten verbeterde de succespredictie van DCM patiënten die cardiale re-synchronisatie therapie ondergingen (hoofdstuk 9). De immunohistochemische diagnose van cardiale inflammatie werd gereflecteerd in het cardiale transcript profiel en was geassocieerd met een lagere kans op succesvolle therapie. Metabolomics wordt steeds meer een belangrijk hulpmiddel dat clinici kan helpen in het diagnosticeren van ziekten, maar kan ook gebruikt worden om genetische bevindingen te valideren. De ernst van de ziekte in DCM wordt sterk gereflecteerd in het metabole profiel van een patiënt (hoofdstuk 10). Toekomstig onderzoek zal ernaar streven om de metabole resultaten te integreren met de klinische en genetische data om zo DCM fenotypes te verfijnen, risico stratificatie te verbeteren en nieuwe therapeutische aangrijpingspunten te identificeren. Hoofdstuk $\mathbf{1 1}$ is een samenvatting van alle trials die de prognose van DCM proberen te verbeteren middels behandelingen die gericht zijn op de oorzaak. Slechts enkelen van deze behandelingen zijn geïmplementeerd in de richtlijnen voor DCM. De translatie naar behandelconsequenties wordt waarschijnlijk nog steeds belemmerd doordat de complexiteit van DCM niet erkend wordt in trials.

Deel 3 van dit proefschrift beschrijft de implicaties van genetisch testen voor de familieleden van een DCM patiënt. Vroeg-detectie van ziekte in (eerstegraads) familieleden is het primaire doel van genetisch testen in DCM patiënten. Globale longitudinale strain (GLS) analyse van echocardiografische beelden heeft een hoge sensitiviteit om subtiele cardiale abnormaliteiten in familieleden te detecteren, ondanks dat ze een normale linker ventrikel ejectie fractie hebben (hoofdstuk 12).

Dit proefschrift beschrijft de klinische consequenties van genetisch testen in DCM (deel 1), integreert vervolgens de genetische resultaten met andere oorzaken en fenotypische manifestaties om zo de diagnose en behandeling van de aandoening te verfijnen (deel 2), en beschrijft de impact van genetica voor (eerstegraads) familieleden van een patiënt (deel 3). De discussie van dit proefschrift (hoofdstuk 13) plaatst de resultaten in het perspectief van de huidige klinische situatie, en geeft een vooruitzicht voor de toekomst van genetica in DCM. 


\section{VALORIZATION}

This chapter discusses the future valorization of the findings presented in this thesis. The valorization of knowledge is the relevance for social and/or economical purposes and to translate it into products, processes and innovations. The valorization of this thesis can be divided in the proposed three parts: [1] clinical consequences of genetics, [2] redefining disease diagnostics and therapeutics, and [3] screening of asymptomatic family members.

\section{Relevance}

The prevalence and incidence of chronic heart failure (HF) is likely to increase in the following decades due to ageing of the population. At present, over 240.000 people suffer from HF in the Netherlands of which over one-third has non-ischemic dilated cardiomyopathy (DCM). There are over 30.000 hospitalizations and 7.500 deaths every year due to HF in the Netherlands. Despite the improvements in therapy these past decades, the 5-year survival rate is still close to $50 \%$, which is similar to many forms of cancer. Together, the costs of health care associated with HF was 817 million euros in the Netherlands in 2017.

Current treatment strategies are primarily aimed theating the signs and symptoms of a patient, which are in general similar among all HF patients. However, not all DCM patients show the desired improvement to the general HF therapy, and therefore this group of HF patients may benefit from alternative, additional treatment options.

Genetic testing has become a routine diagnostic tool in the clinical care of DCM patients. Although the possibilities and quality of genetic testing has improved, the subsequent clinical consequences of specific gene variants often remain uncertain, thereby limiting the clinical utility of genetics. Re-evaluating the genetic testing platform in DCM, and associating the results to clinical phenotype and outcome is crucial information to go towards an (cost-)efficient genetic-first approach.

\section{Target groups}

The results presented in this thesis are relevant for patients, their relatives and for physicians, in particular cardiologists and geneticists. Increasing knowledge of genotype-phenotype associations, will make the prediction of the disease course of a specific patient more accurate, and provide possibilities for earlier intervention.

The genetic features of DCM are characterized by variable disease expression and penetrance, even within families. This leaves uncertainty for relatives who carry the pathogenic familial DCM variant, but also for the counselor which carries the responsibility for providing the best possible advise.

Unraveling the underlying pathophysiological mechanisms in various (genetic) subgroups of DCM patients will be relevant for companies interested in targeted therapies and non-invasive profiling. Although there are no targeted therapies for genetic cardiac diseases on the market yet, there is one ongoing phase 3 clinical trial investigating a therapy which is specific for LMNA carriers (NCT03439514) and myosin inhibitors have been investigated in several phase 1 and phase 2 trials for the treatment of genetic hypertrophic cardiomyopathy (HCM) patients. The increasing number of trials focusing on targeted treatment in genetic cardiomyopathies highlights the need for novel treatments for this patient group, and the willingness and commitment of research groups and companies to invest in the development. Other specific gene-related cardiomyopathies (e.g. TTN, FLNC) are likely to be future target groups for gene-directed specific therapy. 


\section{Products, processes and innovation}

The results presented in this thesis provide the opportunity to be translated in utilities. The critical re-evaluation of genes included on diagnostic screening panels and subsequent variant classification is direct knowledge which can be translated in the diagnostic process of genetic centers. Decreasing the number of genes tested, and enhancing the clarity of variant classification will limit time spent on analyzing genetic data and provide more clear test results. This will have a direct impact on the clinical work of cardiologists and geneticists, and decrease the number of false-positive genetic test results in patients.

The described research strategies and published bio-informatic analytic tools can be applied interdisciplinary. The generated transcriptomic dataset gathered from sequencing RNA isolated from cardiac samples of DCM patients, is currently one of the largest datasets in well-phenotyped DCM patients. This data was made available in the Gene Expression Omnibus (GEO) repository and is an important contribution to the research field of DCM.

This thesis presented pathophysiological differences among DCM patient subgroups. The distinct pathophysiological pathways associated with clinical phenotypes provide novel treatment targets or repurposing of existing drugs. We explored the potential of recognizing these DCM subgroups within an outpatient DCM population. In a proof-of-principle study, we generated a clinical classifier which could place every patient in one of the four unique subgroups with moderate accuracy. Such classifier based on easy accessible clinical variables carries great potential as the utility and subsequent clinical implementation has a low threshold, which will be further discussed below.

The application of speckle tracking to analyze cardiac images in more depth was an innovative approach to detect subclinical cardiac abnormalities. This additional information could help redefine the serial screening frequency in the process of cardiac screening for relatives of DCM patients, finetuning the current position statement of the European and American cardiology associations.

\section{Planning, realization and implementation}

The proposed re-evaluation of gene panels should be organized (inter)nationally to establish new position statements on a diagnostic DCM-specific gene panel. International efforts such as the Clinical Genome Resource (ClinGen) consortium and the DCM Precision Medicine Study are much needed to curate gene-disease validity, variant pathogenicity, and clinical actionability. The Dutch Society of Clinical Genetic Lab Diagnostics (VKGL) is organizing these efforts on a national scale. Re-establishing the gene-disease validity will allow us to analyze genotype-phenotype associations within large cohorts, eventually leading to gene-specific treatment plans in the guidelines for the cardiologist.

As showed in this thesis, many etiology-directed trials have been performed in the past years, although only a few had positive results with subsequent guideline implementation. These trials were conducted parallel to the ongoing unraveling of the complexity of DCM. New insights in pathophysiology and subsequent post-hoc studies of the trials show that the included patients were often too heterogeneous and might therefore not respond in the same manner to the investigated intervention. In this thesis, we provided a novel outlook towards patient selection which includes the DCM complexity. The next step is to train and validate our clinical classifier in order to place patients in specific subgroups with high accuracy. Afterwards, there are two consecutive steps to improve patient treatment: [1] investigate and enhance response to current treatment regimens and [2] uncover new treatment targets and subsequent develop novel targeted therapies. 
To specify screening recommendations for relatives of DCM patients we need to [1] investigate the disease penetrance and expression per specific gene, and [2] to determine the accuracy of speckle tracking in detecting early disease. These points should be addressed in separate studies, which will envisage large cohorts of asymptomatic carriers and long-term follow-up. Afterwards, the 'window' of early treatment should be explored, to see whether this approach can prevent (overt) DCM development.

All data is generated from observatory studies; randomized clinical trials (RCT) are necessary before treatment regimens will be adapted. Research presented in this thesis forms the basis towards basic clinical research and RCT establishing causality and optimal timing for intervention. 
- 350 - 


\section{DANKWOORD}

Het schrijven van dit laatste hoofdstuk is het meest uitdagend, wetende dat dit het meest gelezen hoofdstuk zal worden. De afgelopen jaren zijn voorbij gevlogen, maar er is geen moment geweest dat ik niet met plezier gewerkt heb aan de inhoud van dit proefschrift. Dit heb ik aan vele personen te danken. Het is dan ook niet voor niets dat de lengte van mijn dankwoord niet onder zal doen voor de lengte van mijn proefschrift.

\section{Allereerst wil ik graag mijn promotieteam bedanken:}

Beste professor Heymans, beste Stephane, het was mijn interesse in de cardiologie waardoor ik in een vroeg stadium van mijn medische carrière al bij je terecht kwam. Vanaf het eerste moment wist je mij direct te enthousiasmeren voor klinisch translationeel onderzoek. Ik ben dan ook ontzettend dankbaar voor alle kansen en vrijheid die je mij gegeven hebt om mij te ontwikkelen binnen dit vakgebied. Ook wist je me altijd een (internationaal) podium te bieden waar ik mijn werk kon presenteren en hebben we samen enkele mooie reizen mogen maken! Je onuitputtelijke enthousiasme en gedrevenheid hebben mij altijd weten te motiveren om ambitieus te denken en de opportuniteiten op te zoeken in plaats van enkel het negatieve te zien. Aan de andere kant heb je me geleerd om familie en geliefden altijd op de eerste plek te zetten! Stephane, bedankt voor deze mooie jaren, het geweldige eindresultaat, en ik ben dankbaar dat we dit de komende jaren nog kunnen doorzetten.

Beste professor Brunner, beste Han, naarmate het einde van mijn promotietraject naderde zijn onze overlegmomenten steeds frequenter geworden. Samen hebben we nieuw territorium mogen ontdekken op het gebied van genetica en DCM (van genetische frameworks tot nieuwe variant classificaties). Dit is niet altijd vlekkeloos verlopen, maar zodra ik de hoop verloren was of verdwaald was in de complexe literatuur, kon ik een telefoontje tussendoor verwachten waarna ik gemotiveerd de rode draad weer kon oppakken. Een dergelijk motivatiemoment werd niet onbenut gelaten om nog een anekdote te delen. Ik hoop in de komende jaren nog veel te mogen leren, en nog vele inspirerende anekdotes te mogen horen!

Beste dr. Krapels, beste Ingrid, gedurende de laatste maanden van mijn studie geneeskunde was jij al mijn dagelijkse begeleider. Deze rol heb je glansrijk doorgezet tijdens mijn gehele promotietraject. Maar ook daar houdt het niet op, als plaatsvervangend opleider bij de Klinische Genetica zal ik mij nog verder kunnen ontwikkelen onder jouw supervisie. Er was altijd een lage drempel om bij jou binnen te lopen of nog even te appen, waardoor ik snel nog even kon sparren over ideeën en lopend onderzoek. Jouw kritische en klinische blik kon mijn (vaak complexe en brede) analyses altijd in perspectief plaatsen en terugbrengen naar de klinische kern. De komende jaren hoop ik nog veel te leren van jouw klinische kennis, en hoe we deze kennis kunnen integreren met het onderzoek binnen de genetica!

Beste dr. Hazebroek, beste Mark, zonder jou zou dit proefschrift nooit tot stand zijn gekomen, ik ben jou ontzettend veel dank verschuldigd. Via een omweg heeft mijn academische carrière een start kunnen maken onder jouw supervisie. Hier hebben we samen de MAastricht Registry of Cardiomyopathies (de MARC, zoals je deze graag zelf noemt) tot het volgende niveau kunnen tillen! Dit hebben we voort kunnen zetten tijdens de start van mijn promotietraject. Ook buiten het academische perspectief, heb ik vele wijze levenslessen 
van jou mogen leren! Met veel plezier kijk ik terug op onze werkbesprekingen in de sportschool waar we de fundering hebben gelegd voor meerdere manuscripten die nu deel zijn van dit proefschrift.

Michiel, mijn paranimf, wat ben ik blij dat jij mij wilt bijstaan op de 'grote dag'. Ik kan mij de dag dat jij voor het eerst bij ons binnen kwam lopen als beginnende PhD'er nog goed herinneren: wat een eigenwijs mannetje was jij toch. Maar gelukkig hebben we ons al snel beter leren kennen! We hebben samen toch menig Europese stad (Barcelona, Wenen, Athene, Stockholm) mogen bezoeken voor academische (en recreatieve) doeleinden. Buiten de academische ontwikkeling die we hier hebben doorgemaakt, hebben we ook herinneringen voor het leven gecreëerd. Het is bewonderingswaardig hoe jij het voor elkaar hebt gekregen om de enige air-bnb in een buurt van Athene hebt weten te boeken die zelfs vermeden werd door elke taxichauffeur in de stad. Al betekent dit boekje het einde van ons PhD-traject samen, ik hoop dat wij nog vele jaren zullen samenwerken!

Niels, wie had gedacht dat we beide ons doctoraat zouden behalen en daarmee samen (onofficieel) in de slimste band van Nederland spelen? Vanaf het eerste moment in mijn promotie heb jij mij bijgestaan (en ook in de jaren daarvoor), en hebben we samen ideeën kunnen uitwisselen al liggen onze vakgebieden binnen andere medische kaders. Samen met Maud hebben we vele uren doorgebracht met het spelen van bordspellen, participeren in obstacle runs, en (slechte) filmavonden. Dit waren de perfecte momenten om onze PhDervaringen met elkaar te delen. Niels, ik hoop dat je altijd de persoon zult blijven die je bent: ik ken weinig mensen die zo onzelfzuchtig, behulpzaam en hartelijk zijn. Veel succes in je verdere academische carrière!

Voorzitter en de leden van de beoordelingscommissie, Prof. Dr. Paul Volders, Prof. Dr. Peter van Tintelen, Prof. Dr. Norbert Hübner, Dr. Dominik Linz en Dr. Miranda Nabben, hartelijk dank voor de kritische beoordeling van mijn proefschrift.

\section{Samenwerkingen:}

Dit proefschrift staat en valt met de vele samenwerkingen die de afgelopen jaren tot stand zijn gekomen en waar ik met veel plezier de vruchten van heb mogen plukken.

Ping, I will have to thank you first, because without you this thesis would have been only half its size! We first worked together in my second year of my PhD and since then it is a very consistent collaboration (of which many of my colleagues are jealous). It's amazing how you always keep learning and developing innovative methods to analyze data, taking me with you during the process. I think we created a solid foundation for many more years of research!

Jeannique, in 2016 zijn we samen begonnen om met twee personen een grote klinische trial uit te voeren. Dit had ik nooit, maar dan ook nooit zonder jou kunnen uitvoeren! Samen hebben we alles moeten uitzoeken, opzetten en plannen (wat soms een beetje chaotisch verliep). En wat zijn we creatief geweest met het oplossen van problemen. We mogen trots zijn op het resultaat dat wij hebben neergezet. Deze trial (en alles wat ik geleerd heb gedurende) zal ik nooit vergeten, bedankt voor alle steun en hulp! 
Marije, de eerste jaren hebben we een bureau mogen delen (soort van), en was het ontzettend fijn om 'iemand van de genetica' naast me te hebben zitten. Als nummer-1 fan van mijn muzikale carrière heb je de afgelopen jaren deze muzikale voortgang ook nauwlettend in de gaten gehouden! Het was voor mij ideaal dat ik bij Jeannique en jou altijd even kon binnenlopen om te ventileren.

Michiel (Adriaens), jij hebt me altijd weten te motiveren om met een andere blik naar de genetische data te kijken. We hebben een aantal mooie projecten lopen, en ik hoop dat we in de toekomst nog meer kunnen samenwerken. Het is niet voor niets dat ik jou altijd refereer als 'slimme Michiel' naar mijn collega's!

Blanche, Marc, Rick, Wouter, Steffie, llona, Robin, Annika en Monika: het laatste traject van mijn promotie was ik gestationeerd binnen het lab van de cardiologie aan de universiteit (al zaten we regelmatig samen tijdens de labmeetings in de jaren daarvoor). Buiten de (sterke) koffie die altijd vers klaar stond in de keuken, wil ik jullie allemaal bedanken voor de fijne samenwerking in de afgelopen jaren.

Emma, as the post-doc in the lab within our DCM group, you were always the person to go to when we wanted to discuss on the possibilities of functional studies in the lab. It was a wonderful collaboration and I'm glad we could work together on several publications. I wish you all the best in your future career in the States!

Lilian en Barbara, jullie zijn echte rotsen in de branding! Als er iets geregeld moest worden, dan was het vaak al geregeld voordat ik zelf doorhad dat het gedaan moest worden. Daarnaast stond jullie deur altijd open voor een goed gesprek, dat ga ik zeker missen. Barbara, heel veel succes binnen je nieuwe functie!

Miranda, Jan, Jo, Agnieszka, and Li-Yen, thank you for all the help with all projects involving lab work within the genetics department. I really enjoyed the collaboration and the way we could discuss about metabolism, iPScells and cardiomyocytes. I think we build a great foundation from which we can do some great research the coming years.

Arthur, Bianca, Debby, Jörgen, Kasper, Lieve, Margo, en Radek, bedankt voor het geduld, de veelvuldige overleggen en de tijd die jullie altijd voor mij hebben vrijgemaakt om samen tot de onderzoeksresultaten van deze thesis te komen. Varianten (her-)classificeren, nieuwe pipelines maken, kleuren veranderen in PCA-plots, samples naar het buitenland opsturen, zonder jullie hulp was het mij nooit gelukt. Gelukkig kunnen we deze trend de komende jaren nog verder doorzetten!

Veel dank aan de cardiologen van het MUMC, zonder jullie zou er geen data zijn om het onderzoek mee uit te voeren. Het was een voorrecht om deel te mogen nemen aan de klinische besprekingen en brainstormsessies, en in de afgelopen jaren zijn de banden tussen de cardiologie en de genetica alleen nog maar sterker geworden. In het bijzonder dank aan Christian, Vanessa, Hans-Peter, en de gehele hartfalengroep.

Sinds kort heb ik het voorrecht gekregen om deel uit te maken van een geweldig team bij de Klinisch Genetica. Gedurende mijn promotietraject ben ik regelmatig ondersteund geworden 
door de cardiogenetici, Els, Paula en Ingrid, bedankt voor alle hulp bij het benaderen van patiënten en verzamelen van informed consents en extra samples!

Naast de vele personen met wie ik binnen de muren van de universiteit heb mogen samenwerken, zijn er vele externe personen betrokken geweest bij het tot-stand-komen van dit proefschrift.

Samen met Els had ik het genoegen om een nationaal onderzoek uit te voeren met betrekking tot filamine $\mathrm{C}$. Het was duidelijke dat de lijntjes tussen de verschillende genetische centra in Nederland kort zijn, en door ieders medewerking heeft dit mogen leiden tot een mooie paper. Ik hoop dat we deze samenwerking in de komende jaren nog verder mogen uitbreiden!

Working in the field of DCM is challenging, and it is great to meet several other DCM groups in Europe. We published together on several projects, and many projects are still ongoing. We were invited and warmly received at your centers, and I hope to collaborate with all of you in the upcoming years! Thank you all: dr. Ware, dr. Tayal and prof. dr. Prasad (from London), dr. Merlo and prof dr. Sinagra (from Trieste), dr. Dominguez-Rodriguez, dr. LaraPezzi and prof. dr. Garcia-Pavia (from Madrid), and prof. dr. Pölzl (from Innsbruck).

Being part of the HOMAGE consortium (led by prof. dr. Zannad, and prof. dr. Cleland) has been a great experience for me, it was an excellent environment for a young scientist to develop as an international researcher, and a great opportunity to meet a lot of great people. Therefore I would like to thank everyone within the consortium and especially Joao for the close collaboration in multiple projects!

\section{Mijn kamergenoten:}

Ik heb ervaren dat een promotietraject onlosmakelijk verbonden is met het frequent verhuizen en wisselen van kamergenoten, resulterend in 4 verschillende werklocaties met $>20$ verschillende kamergenoten. De dynamische groep PhD-studenten bij de cardiologie kenmerkt zich door collegialiteit, hartelijkheid en ondernemendheid. Samen hebben we tientallen PhD-uitjes gehad, variërend van een wijnproeverij tot het meest fanatieke potje bubbelbal ooit! Daarom alle collegae PhD'ers hartstikke bedankt voor de mooie jaren.

Anne, jij hebt een vliegende start gemaakt binnen het DCM-team: je weet vele projecten tegelijk uit te voeren en af te ronden! Samen hebben we nog een uitdagend biomarkerproject succesvol mogen afronden in de laatste maanden, een samenwerking die zeer soepel verliep! Je hebt je goed weten te weren en te positioneren binnen het DCM-team, en het kan eigenlijk niet anders dan dat je een overvol boekje gaat krijgen!

Arantxa, wat waren wij blij dat je als miss-HFpEF op de kamer zat samen met het DCM-team! Je hebt ons goed op sleeptouw genomen door Athene, en ik keek uit naar ons bezoek aan Barcelona. Helaas gooide corona roet in het eten. Hopelijk kunnen we alsnog samen een hartfalencongres bezoeken in de toekomst! Succes met je verdediging en de laatste loodjes van je opleiding tot cardioloog!

Sophie, ontzettend leuk dat je ons team bent komen versterken! Ondanks dat je gestart bent in deze vreemde tijd, heb je al snel je draai weten te vinden binnen het team (en kun je goed 
omgaan met alle onzin die Michiel en ik er op een dag uitgooien). Heel fijn dat we de komende jaren nog kunnen samenwerken om ons onderzoek nog beter op de kaart te zetten!

Jerremy, begonnen in het koffiekamertje van de PhD-kamer waar je avonden doorbracht om strain te meten in onze DCM populatie. Hierdoor ben je co-auteur geworden op mooie papers! Nu ben je goed op weg om je eigen weg te vinden binnen de HFpEF groep. Hopelijk kunnen we komend jaar weer een mooi trio vormen om aan de Iron Man relay deel te nemen, ik hoop dat je al aan het trainen bent!

Nikki, de hardst werkende PhD-student van de groep. Een publicatie in NEJM als deel van je $\mathrm{PhD}$, iets waar wij allemaal ontzettend jaloers op zijn. Het is je van hart gegund en ook zeker verdient. Ik heb er dan ook geen twijfel bij dat dit enkel een eerste opstap is naar een mooie carrière! Vergeet ook niet de genetica mee te nemen in je toekomstige onderzoeken!

Manouk, als nieuwkomer kreeg je direct een uitdagende plek in de kamer, en mocht je beginnen aan een uitdagende studie! $\mathrm{Na}$ het vertrek van Frederique, heb jij de rol overgenomen om de planning van PhD-uitjes gaande te houden, veel dank daarvoor! Uiteindelijk heb je helemaal je weg gevonden binnen de huisartsopleiding.

Bouke, het was ons bijna gelukt om jou te integreren in het DCM-team! Bedankt dat je mij de ins- en outs hebt geleerd over het intekenen van rechterkamers op de MRI (en af en toe de hoeken van de squashbaan hebt laten zien). Heel veel succes met de volgende stappen in je opleiding en wetenschappelijk onderzoek.

Casper, in 2010 hebben onze wegen zich voor het eerst gekruist: ik werd door Stephane aan jou gekoppeld om mijn Master afstudeerstage uit te voeren. Uiteindelijk hebben we nog mogen samenwerken tijdens onze PhD-trajecten. Ondanks dat je veel zaken moest combineren als jonge specialist, was je de gangmaker van elk PhD-uitje. Ik wens je ontzetten veel succes tijdens je verdere carrière in Roermond!

Frederique, de groot-organisator van alle PhD-uitjes! Op het zelfde moment mochten wij allebei onze eerste paper in European Heart Journal reviseren, en beide zijn uiteindelijk geaccepteerd. Dit was een mijlpaal in jouw succesvolle promotie. Veel succes met je verdere carrière bij de cardiologie!

Jort, als één persoon mijn eerste periode van mijn promotietraject heeft getekend, dan ben jij dat wel. Jij hebt me aan de hand genomen en alle ins en outs binnen de cardiologie geleerd, van echo's maken tot een muis voor mijn computer vinden. Daarnaast hoefde ik je maar een berichtje te sturen en je kwam me direct uithelpen in de kliniek wanneer dat nodig mocht zijn. Ook buiten de ziekenhuismuren heb je me alleen maar goede adviezen weten te geven over schoenen, gin, sushi, en alles wat verder belangrijk is in het leven. Samen hebben we onvergetelijke congresreizen mogen maken, waar je me altijd van goede 'tips' hebt voorzien. Jort, het ga je goed in alles wat je verder zal ondernemen!

Nick, één van de weinig originele Limburgers bij ons op de kamer. Jij hebt me ontelbare keren uitgeholpen met de echocardiografie. Het is bewonderenswaardig hoe jij altijd tijd vrij hebt 
gemaakt om collega's (in de kliniek of research) uit de brand te helpen, al ging dat wel meer dan eens ten koste van je eigen werk.

Ömer, ten tijde van Temptation Island hebben we echte diepe gesprekken kunnen voeren samen met Jort. Top dat we samen nog enkele feestjes hebben kunnen bezoeken, waar je me nog wegwijs hebt weten te maken in het uitgaansleven van Maastricht. Na deze mooie tijden gaan we nu dan eindelijk beide ons doctoraat behalen! Succes met je verdere opleiding tot cardioloog!

Yvonne, ik kan me eigenlijk geen enkele borrel, uitje of etentje herinneren waar jij niet bij bent geweest. Zelfs bij mijn afscheidsetentje wist je toch de tijd te vinden om aan te sluiten! Die sociale insteek heeft onze groep een ontzettend positieve impuls gegeven, en ook mij uit mijn schulp doen kruipen! Succes met het afronden van je thesis, ik hoop dat je de positieve impuls hier in kan doorzetten.

Twan, mooi dat we nog samen een paper hebben kunnen schrijven! In het verlengde van het DCM-team hebben we goed kunnen brainstormen en de kansen weten te benutten uit de overlap tussen hartfalen en elektrofysiologie. Ik ben er helemaal voor om dit nog verder te gaan doen en de nadruk op het genetisch vlak te leggen!

Sandra, tegen de tijd dat ik begon als PhD was jij al weer een tijdje klaar en werkzaam in de kliniek, maar jouw passie voor onderzoek was ontzettend aanstekelijk. Samen hebben we nog aan een mooie paper kunnen werken, en eigenlijk overlappen onze research-technieken zich op meerdere gebieden. Mogelijk dat we dit aantal gezamenlijke publicaties nog verder kunnen uitbreiden!

Ik heb het getroffen dat ik mijn passie voor muziek maken heb kunnen aanhouden naast het onderzoek. Muziek maken doe je niet alleen, als lid van een drietal bands heb ik vele mooie momenten mogen beleven.

Tie, Tom en Fer: jullie eeuwige geduld en begrip heeft gezorgd voor een relaxte sfeer waarbij muziek maken veelal secundair is aan het samenzijn. Inmiddels hebben we samen toch al drie volledige cd's op spotify staan, en hebben we ons jaarlijkse quota aan optredens. Laten we dit nog vele jaren zo doorzetten!

Toon, Loek, Zoë, Bart en Johan: sinds 2006 hebben wij samen vele podiums mogen betreden van kleine kroegen tot grote tentfeesten. Helaas is dit nu ten einde gekomen, en zijn we allemaal onze eigen weg ingeslagen. Gelukkig hebben we nog al het visuele en audiomateriaal van deze geweldige herinneringen. Laten we samen nog $1 x$ knallen tijdens ons afscheidsoptreden in 2021!

Rim, Ruud en Niels: samen spelen we nu al meer dan 15 jaar in een band. We hebben stad en land afgereisd, in de meest indrukwekkende zalen gespeeld, $4 \mathrm{~cd}$ 's mogen opnemen en releasen, spannende momenten meegemaakt (zowel positief als negatief), maar uiteindelijk is de vriendschap die wij hebben de reden waarom wij nog steeds muziek maken. Ik hoop dat we dat nog vele jaren samen mogen doen. Zeker nu Tom ons is komen versterken!

Jelle, wat een topwerk heb je afgeleverd! Natuurlijk had ik je vorig werk al gezien, maar vanaf de eerste schets wist je direct mijn onderzoek goed te visualiseren. 
Trots ben ik op mijn 'roots' in Brachterbeek. Al heb ik mijn geboortedorp ruim 10 jaar geleden verruild voor Maastricht, ik ben altijd verbonden gebleven via de sterke muzikale connectie. Helaas hebben deze muzikale wegen zich nu afgesplitst, maar koester ik nog steeds vele mooie herinneringen gemaakt als lid van Fanfare Eensgezindheid en Joekskapel Zonger Drank Geine Klank.

Thijs, Stefan, Rico, Stefan en Bjorn, mijn vrienden van het (bijna letterlijke) eerste uur; het is uniek dat wij na decennia nog altijd zo goed bevriend zijn. Het maakt niet uit wat wij samen doen (de Zwarte Cross, weekend Ardennen, een avond met de dobbelstenen of een plafond in de keuken aanleggen..), het zijn altijd onvergetelijke momenten. En ik weet zeker dat we samen nog vele herinneringen zullen gaan maken in de komende jaren! Alles wat we nodig hebben zijn proseccoglaasjes, een set dobbelstenen en een campingstoel.

Om te voorkomen dat mijn thesis nog langer zal worden, wil ik alle ooms, tantes, neven en nichten bedanken voor de interesse die jullie altijd getoond hebben in mijn werk. Al was het niet altijd te volgen voor jullie, dit weerhield jullie nooit om te vragen wat ik ook alweer precies deed!

Lieve Joris, mijn grote broer. Ik ben ontzettend trots op alles wat je doet en de mijlpalen die je hebt bereikt. Je hebt je taak als grote broer altijd serieus genomen, en vele paden voor mij vrijgemaakt zodat ik als tweede in het gezin deze moeiteloos kon bewandelen. Ik ben blij dat we beter met elkaar overweg kunnen dan vroeger! Ik wens jou en Linda een onvergetelijk 2021!

Lieve papa en mama, jullie zijn de twee belangrijkste personen die de basis vormen voor alles wat ik bereikt heb. Ik voel mij ontzettend bevoorrecht dat ik nooit iets ben tekort gekomen in mijn jeugd (en de tijd erna), waardoor ik mij volledig op mijn toekomst kon richten. Bij al mijn diploma-uitreikingen zaten jullie op de eerste rij, en ook nu grijpen jullie elke kans aan om te laten weten hoe trots jullie zijn. Bedankt voor alle leuke momenten, goede herinneringen en alles dat jullie gedaan hebben zodat ik de persoon kon worden die ik nu ben. Bedankt voor jullie onuitputtelijke steun en liefde. Ik hou van jullie!

Sweet Olivia, in this thesis I describe my pursuit to unravel the mysteries of the heart, but I found its most important matter at Papendal in 2018 (who would ever thought that the course would be so insightful!). You have put everything in a different perspective for me, and I'm very glad that I can share all my passions and hobbies with you (although running a $12 \mathrm{~K}$ obstacle run was not your favorite). You know all the challenges related to a PhD-trajectory, and it is great that we can help each other along the way. Of course, we can't forget your contribution to this thesis: as a native English speaker, you were always the first I asked if I had any doubts about my grammar! Thank you for your everlasting support, believe and love. You're my best friend, buddy, girlfriend, and I love you so much for that! 
- 358 - 


\section{ABOUT THE AUTHOR}

Job Verdonschot was born in Maasbracht-Beek, The Netherlands, in 1988. After completing pre-university education at the Connect College in Echt, he studied Biomedical Sciences at Maastricht University. After finishing his bachelor, he continued with a masters degree in Biomedical Sciences combined with his medical training at the Faculty of Health Medicine and Life Sciences at Maastricht University, where he graduated with honors and received the MSc. and MD. degree with distinction cum laude in 2016. During his study, he completed an internship in Melbourne, Australia, and three internships at the Cardiology and Clinical Genetics departments, in the Maastricht University Medical Center+. After receiving the

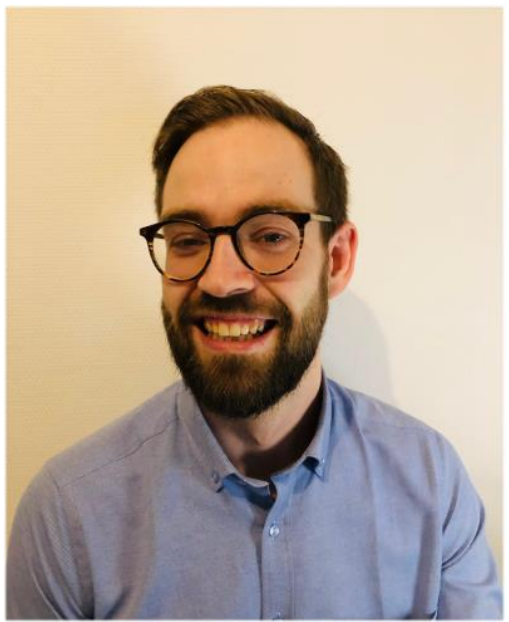
Kootstra Fellowship for talented PhD-students, he could continue as a PhD candidate in the Dilated Cardiomyopathy research group of prof. Heymans, carrying out research on the clinical implications of genetics in dilated cardiomyopathy. Job has authored over 20 publications in international peer-reviewed scientific journals, of which 15 with an impact factor above 5 (the top-ranked journals in the field of cardiology amongst them: European Heart Journal, Circulation and the Journal of the American College of Cardiology). His scientific work was presented at various national and international congresses, for which he was rewarded with multiple abstract and poster awards. In March 2020, he was awarded with the Harry Struijker-Boudier Award For Talented Academics (HS-BAFTA) which granted him the possibility to visit the group of prof. Garcia-Pavia in Madrid, Spain. In October 2020 Job started his clinical training in Clinical Genetics at the Maastricht University Medical Center (Maastricht, the Netherlands) under supervision of dr. Y.H.J.M. Arens. His current research interests build further upon his dissertation, including titin cardiomyopathies and the integration of machine learning approaches to improve our understanding of genotypephenotype correlations in DCM. 
- 360 - 


\section{LIST OF PUBLICATIONS}

1. Verdonschot JAJ*, Ferreira JP*, Wang P, Pizard A, Collier TJ, Ahmed FZ, Brunner-La Rocca HP, Clark AL, Cosmi F, Cuthbert J, Diez J, Edelmann F, Girerd N, González A, Grojean S, Hazebroek MR, Khan J, Latini R, Mamas MA, Mariottoni B, Mujaj B, Pellicori P, Peutschnigg J, Pieske B, Rossignol P, Rouet P, Staessen JA, Cleland JGF, Heymans SRB, Zannad F. Proteomic and mechanistic analysis of spironolactone in patients at risk for heart failure.

JACC: Heart Fail 2020; accepted.

2. Verdonschot JAJ, Merlo $M$, Dominguez $F$, Wang $P$, Henkens MTHM, Adriaens ME, Hazebroek MR, Mase M, Escobar LE, Cobas-Paz R, Derks KWJ, van den Wijngaard A, Krapels IPC, Brunner HG, Sinagra G, Garcia-Pavia P, Heymans SRB. Machine Learning Methods Stratify Dilated Cardiomyopathy Patients in Four Phenogroups. Risk Prediction in Three Independent European Cohorts.

Eur Heart J 2020;ehaa841.

3. Verdonschot JAJ, Merken JJ, van Stipdonk AMW, Pliger $P$, Derks KWJ, Wang $P$, Henkens MTHM, van Paassen P, Abdul Hamid MA, van Empel VPM, Knackstedt C, Luermans JGLM, Crijns HJGM, Brunner-La Rocca HP, Brunner HG, Poelzl G, Vernooy K, Heymans SRB, Hazebroek MR. Cardiac Inflammation Impedes Response to Cardiac Resynchonization Therapy in Patients with Idiopathic Dilated Cardiomyopathy.

Circ Arrhythm Electrophysiol 2020;DOI:10.1161/CIRCEP.120.008727.

4. Verdonschot JAJ, Hazebroek MR, Krapels IPC, Henkens MTHM, Raafs A, Wang P, Merken JJ, Claes GRF, Vanhoutte EK, van den Wijngaard A, Heymans SRB, Brunner HG. Implications of Genetic Testing in Dilated Cardiomyopathy.

Circ Genet Genom Precis Med 2020;13(5):476-487.

5. Verdonschot JAJ, Derks KWJ, Hazebroek MR, Wang P, Robinson EL, Adriaens ME, Krapels IPC, van den Wijngaard A, Brunner HG, Heymans SRB. Distinct Cardiac Transcriptomic Clustering in Titin and Lamin A/C-associated Dilated Cardiomyopathy Patients.

Circulation 2020;142(12);1230-1232.

6. Verdonschot JAJ*; Vanhoutte EK*; Claes GRF; Helderman-van den Enden ATJM; Hoeijmakers JGJ; Hellebrekers DMEl; de Haan A; Christiaans I; Lekanne Deprez RH; Boen HM; van Craenenbroeck EM; Loeys BL; Hoedemaekers YM; Marcelis C; Kempers M; Brusse E; van Waning JI; Baas AF; Dooijes D; Asselbergs FW; Barge-Schaapveld DQCM; Koopman P; van den Wijngaard A; Heymans SRB; Krapels IPC; Brunner HG. A Mutation Update for the FLNC gene in Myopathies and Cardiomyopathies.

Hum Mutat 2020;41(6):1091-1111. (Selected as Editor's choice article)

7. Verdonschot JAJ, Robinson EL, James KN, Mohamed MW, Claes GRF, Casas K, Vanhoutte EK, Hazebroek MR, Kringlen G, Pasierb MM, van den Wijngaard A, Glatz JFC, Heymans SRB, Krapels IPC, Nahas S, Brunner HG, Szklarczyk R. Mutations in 
PDLIM5 are rare in dilated cardiomyopathy but are emerging as potential disease modifiers.

Mol Genet Genomic Med 2020:e1049.

8. Verdonschot JAJ*, Wang P*, Van Bilsen M, Hazebroek MR, Merken JJ, Vanhoutte EK, Henkens M, Van Den Wijngaard A, Glatz JFC, Krapels IPC, Brunner HG, Heymans SRB, Bierau J. Metabolic Profiling Associates with Disease Severity in Nonischemic Dilated Cardiomyopathy.

J Card Fail 2020;26(3):212-222.

9. Verdonschot JAJ*, Merken JJ*, Brunner-La Rocca HP, Hazebroek MR, Eurlings C, Thijssen E, Wang P, Weerts J, van Empel V, Schummers G, Schreckenberg M, van den Wijngaard A, Lumens J, Brunner HG, Heymans SRB, Krapels IPC, Knackstedt C. Value of Speckle Tracking-Based Deformation Analysis in Screening Relatives of Patients With Asymptomatic Dilated Cardiomyopathy.

JACC Cardiovasc Imaging 2020;13(2 part 2):549-558.

10. Verdonschot JAJ*, Ferreira JP*,Collier T, Wang P, Pizard A, Bar C, Bjorkman J, Boccanelli A, Butler J, Clark A, Cleland JG, Delles C, Diez J, Girerd N, Gonzalez A, Hazebroek MR, Huby AC, Jukema W, Latini R, Leenders J, Levy D, Mebazaa A, Mischak H, Pinet F, Rossignol P, Sattar N, Sever P, Staessen JA, Thum T, Vodovar N, Zhang ZY, Heymans S, Zannad F. Proteomic Bioprofiles and Mechanistic Pathways of Progression to Heart Failure.

Circ Heart Fail 2019;12(5):e005897.

11. Verdonschot JAJ, Hazebroek MR, Ware JS, Prasad SK, Heymans SRB. Role of Targeted Therapy in Dilated Cardiomyopathy: The Challenging Road Toward a Personalized Approach.

J Am Heart Assoc 2019;8(11):e012514.

12. Verdonschot JAJ, Cooper LT, Heymans SRB. Parvovirus B19 in Dilated Cardiomyopathy: There Is More Than Meets the Eye.

J Card Fail 2019;25:64-66.

13. Verdonschot JAJ, Hazebroek MR, Wang P, Sanders-van Wijk S, Merken JJ, Adriaansen YA, van den Wijngaard A, Krapels IPC, Brunner-La Rocca HP, Brunner HG, Heymans SRB. Clinical Phenotype and Genotype Associations With Improvement in Left Ventricular Function in Dilated Cardiomyopathy.

Circ Heart Fail 2018;11(11):e005220.

14. Verdonschot JAJ, Hazebroek MR, Derks KWJ, Barandiaran Aizpurua A, Merken JJ, Wang P, Bierau J, van den Wijngaard A, Schalla SM, Abdul Hamid MA, van Bilsen M, van Empel VPM, Knackstedt C, Brunner-La Rocca HP, Brunner HG, Krapels IPC, Heymans SRB. Titin cardiomyopathy leads to altered mitochondrial energetics, increased fibrosis and long-term life-threatening arrhythmias.

Eur Heart J 2018;39(10):864-873. 
15. Verdonschot JAJ*, Hazebroek $M^{*}$, Merken J, Debing Y, Dennert R, Brunner-La Rocca HP, Heymans S. Relevance of cardiac parvovirus B19 in myocarditis and dilated cardiomyopathy: review of the literature.

Eur J Heart Fail 2016;18(12):1430-1441.

16. Cleland JGF, Ferreira JP, Mariottoni B, Pellicori P, Cuthbert J, Verdonschot JAJ, Peutschnigg J, Ahmed FZ, Cosmi F, Brunner-La Rocca HP, Mamas MA, Clark AL, Edelmann F, Pieske B, Khan J, McDonald K, Rouet P, Staessen JA, Mujaj B, González A, Diez J, Hazebroek MR, Heymans SRB, Latini R, Grojean S, Pizard A, Girerd N, Rossignol $P$, Collier TJ, Zannad F. The effect of spironolactone on cardiovascular function and markers of fibrosis in people at increased risk of developing heart failure: the Heart "OMics" in AGEing (HOMAGE) randomised clinical trial.

Eur Heart J 2020;ehaa758.

17. Henkens MTHM, Remmelzwaal S, Robinson EL, Ballegooijen van AJ, Barandiaran Aizpurua MA, Verdonschot JAJ, Raafs AG, Weerts J, Hazebroek MR, Sanders-Wijk van $S$, Handoko ML, den Ruijter HM, Lam CSP, de Boer RA, Paulus WJ, Empel van VPM, Vos R, Brunner-La Rocca HP, Beulens JWJ, Heymans SRB. Risk of bias in studies investigating novel diagnostic biomarkers for heart failure with preserved ejection fraction.

Eur J Heart Fail 2020. Doi: 10.1002/ejhf.1944

18. Pellicori P, Ferreira JP, Mariottoni B, Brunner-La Rocca HP, Ahmed FZ, Verdonschot JAJ, Collier T, Cuthbert JJ, Petutschnigg J, Mujaj B, Girerd N, Gonzalez A, Clark AL, Cosmi F, Staessen JA, Heymans S, Latini R, Rossignol P, Zannad F, Cleland JGF. Effects of spironolactone on serum markers of fibrosis in people at high risk of developing heart failure: rationale, design and baseline characteristics of a proof-of-concept, randomised, precision-medicine, prevention trial. The Heart OMics in AGing (HOMAGE) trial.

Eur J Heart Fail 2020. Doi: 10.1002/ejhf.1716

19. Hazebroek MR, Krapels I*, Verdonschot JAJ*, van den Wijngaard A, Vanhoutte E, Hoos $M$, Snijders L, van Montfort L, Witjens M, Dennert R, Crijns H, Brunner-La Rocca HP, Brunner HG, Heymans S. Prevalence of Pathogenic Gene Mutations and Prognosis Do Not Differ in Isolated Left Ventricular Dysfunction Compared With Dilated Cardiomyopathy.

Circ Heart Fail 2018;11(3):e004682.

20. Merken J, Hazebroek M, Van Paassen P, Verdonschot JAJ, Van Empel V, Knackstedt C, Abdul Hamid M, Seiler M, Kolb J, Hoermann P, Ensinger C, Brunner-La Rocca HP, Poelzl G, Heymans S. Immunosuppressive Therapy Improves Both Short- and Long-Term Prognosis in Patients With Virus-Negative Nonfulminant Inflammatory Cardiomyopathy.

Circ Heart Fail 2018;11(2):e004228. 
21. Merken J, Brunner-La Rocca HP, Weerts J, Verdonschot JAJ, Hazebroek M, Schummers G, Schreckenberg M, Lumens J, Heymans S, Knackstedt C. Heart Failure With Recovered Ejection Fraction.

J Am Coll Cardiol 2018;72(13):1557-1558.

22. Hazebroek MR, Moors S, Dennert R, van den Wijngaard A, Krapels I, Hoos M, Verdonschot JAJ, Merken JJ, de Vries B, Wolffs PF, Crijns HJ, Brunner-La Rocca HP, Heymans S. Prognostic Relevance of Gene-Environment Interactions in Patients With Dilated Cardiomyopathy: Applying the MOGE(S) Classification.

J Am Coll Cardiol 2015;66(12):1313-23. 